Portland State University

PDXScholar

\title{
Developing a Mixed-Methods Method to Model Elderly Health Technology Adoption with Fuzzy Cognitive Map, and its Application in Adoption of Remote Health Monitoring Technologies by Elderly Women
}

Noshad Rahimi

Portland State University

Follow this and additional works at: https://pdxscholar.library.pdx.edu/open_access_etds

Part of the Gerontology Commons, and the Technology and Innovation Commons Let us know how access to this document benefits you.

\section{Recommended Citation}

Rahimi, Noshad, "Developing a Mixed-Methods Method to Model Elderly Health Technology Adoption with Fuzzy Cognitive Map, and its Application in Adoption of Remote Health Monitoring Technologies by Elderly Women" (2018). Dissertations and Theses. Paper 4511.

https://doi.org/10.15760/etd.6395

This Dissertation is brought to you for free and open access. It has been accepted for inclusion in Dissertations and Theses by an authorized administrator of PDXScholar. Please contact us if we can make this document more accessible: pdxscholar@pdx.edu. 
Developing a Mixed-Methods Method to Model Elderly Health Technology Adoption with Fuzzy Cognitive Map, and its Application in

Adoption of Remote Health Monitoring Technologies by Elderly Women

by

Noshad Rahimi

A dissertation submitted in partial fulfillment of the requirements for the degree of

\author{
Doctor of Philosophy \\ in \\ Technology Management
}

Dissertation Committee:

Charles M. Weber, Chair

Antonie J. Jetter, Co-Chair

Katherine Wild

Wayne Wakeland

Portland State University

2018 
(C) 2018 Noshad Rahimi 


\begin{abstract}
Providing healthcare to the ever-rising elderly population has become a severe challenge and a top priority. Emerging innovations in healthcare, such as remote health monitoring technologies, promise to provide a better quality of care and reduce the cost of healthcare. However, many elderly people reject healthcare innovations. This lack of adoption constitutes a big practical problem because it keeps the elderly from benefiting from technology advances. The phenomenon is even more pronounced among elderly women, who represent the majority of the elderly population.
\end{abstract}

A plethora of studies in the field of technology adoption resulted in sound, but highly generalized theories that are too parsimonious to provide practical insight into the phenomenon of elderly healthcare technology adoption (EHTA). There is a call to arms for novel approaches that facilitate the creation of models that expand technology adoption theories to the specifics of EHTA. This dissertation is a response to this call to arms, and it contributes to modeling practice in the EHTA field. It uses fuzzy cognitive mapping to design a novel mixed-methods modeling approach. Since elderly women constitute the majority of the elderly population, this dissertation treats elderly women's health technology adoption (EWHTA) as the case-in-point. 


\section{Dedication}

To my family

To my mother who taught me unconditional love and dedication...

To my husband who taught me true friendship and kindness...

To my sister who taught me patience and grace...

To Niki who taught me how to be wise and true to myself ...

To Tara who taught me how to never lose my sparkle in life...

I could never do this without your love and support...

I Love you 


\section{Acknowledgments}

This research would not have been possible without the help and support of many whom I deeply appreciate. No one has been more important to me in the pursuit of this crazy journey than my loving family. Their love has been my inspiration and my super power.

I would like to express my deepest gratitude to my dissertation committee chair, Dr. Charles Weber, who guided me with his knowledge and intelligence. Without his persistent support and wisdom, this dissertation would not have been possible. I am greatly thankful to my dissertation committee co-chair, Dr. Antonie Jetter, who continuously supported my research with her expertise and guidance. I am grateful for the help and support of Dr. Katherine Wild and her willingness to carve out from her invaluable time for my research. I am appreciative to Dr. Wayne Wakeland to provide me with encouragement and excellent feedback on my dissertation. I am also deeply thankful to my mentor and previous academic advisor, Dr. Kocaoglu, who never stopped his loving support and kindness to me.

I had the honor and pleasure of working with many wonderful people who helped my research. I appreciate the support of Dr. Jeff Kaye and his amazing staff at ORCATECH, who generously granted me access to their wealth of expertise and resources that made my research possible. I also thank all the delightful elderly women in the Life Lab who accepted to share their stories and wisdom with me. 
I am thankful to my classmates and the wonderful faculty and staff in the Engineering Technology Management program at Portland State University and to all my amazing friends who helped me crossing the finish line.

Most importantly, thank God, who has blessed me more than I deserve. 


\section{Table of Contents}

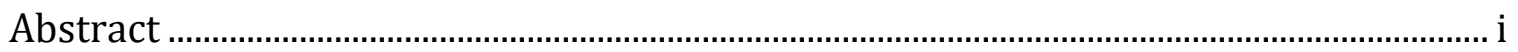

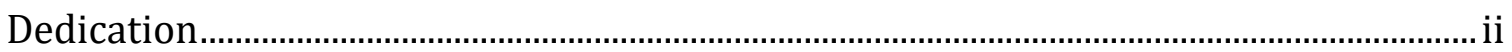

Acknowledgments ........................................................................................................ii

List of Tables......................................................................................................................

List of Figures ......................................................................................................................

List of Abbreviations ........................................................................................................

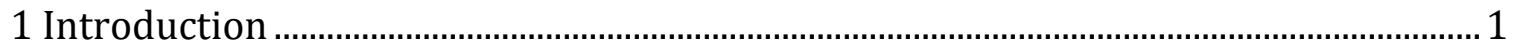

1.1. Problem Background......................................................................................... 1

1.2. Purpose of Dissertation .......................................................................................... 12

1.3. Scope of Dissertation......................................................................................... 14

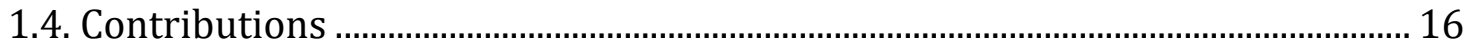

1.5. Limitations of Dissertation ..................................................................................... 17

1.6. Highlights of the Proposed Method ........................................................................ 18

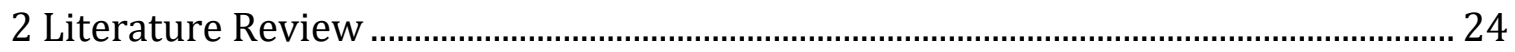

2.1. Framework of Elderly Women Health Technology Adoption ............................. 24

2.2. Literature Review in Gerontology …………………………………………...... 26

2.3. Literature Review in Human Factor Engineering (HFE) and Human Computer

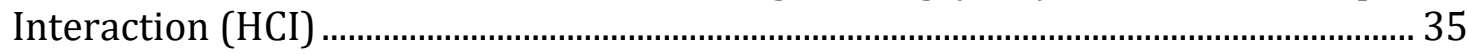

2.4. Review of Known Data Elicitation Approach in Consumer Market Research. 38

2.5. Literature Review in Health Technology Adoption................................................ 47

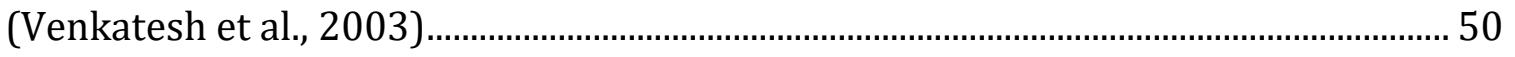

2.6. Literature Review in Modeling Methods in EHTA ............................................... 61

2.6.1. Theory_Enquiry_Quantitavie-Method (TEQn) ............................................... 63

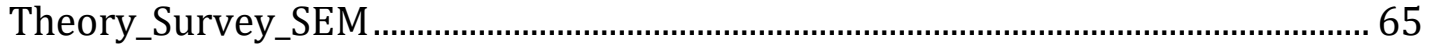

2.6.3. Integrative_Enquiry_Mixed-Method (IEM) .................................................... 66

2.6.2. Theory_Enquiry_Qualitative-Method (TEQI)_................................................. 70

2.7. Summary of the State of the Art............................................................................... 72

2.7.1. Grounded, Holistic, Flexible, Latent Needs...................................................... 76

2.7.2. Visual Display................................................................................................... 78

2.7.3. Scenario Planning...................................................................................... 80

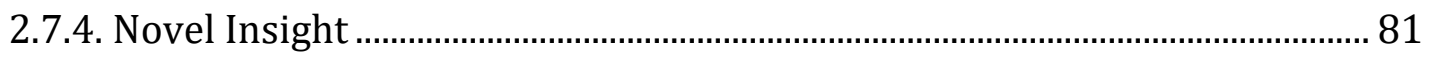




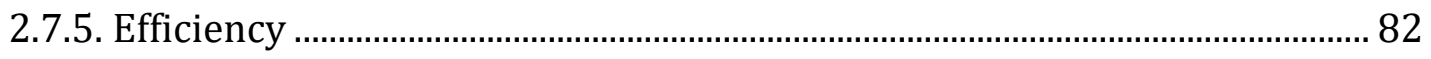

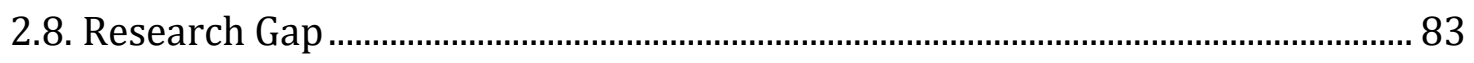

3 Proposed Research Method ................................................................................... 84

3.1. Methodological Choices...................................................................................... 86

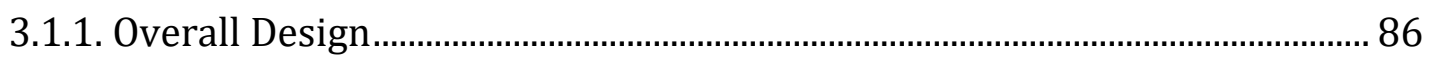

3.1.2. Choices Pertaining to Enquiry and Analysis ........................................................ 87

3.1.3. Repertory Grid Technique (RGT) as Data Elicitation Method ..................... 91

3.1.4. Fuzzy Cognitive Mapping ................................................................................... 93

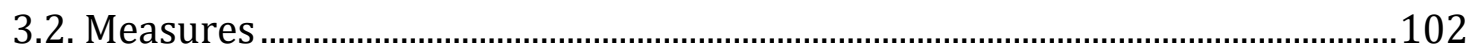

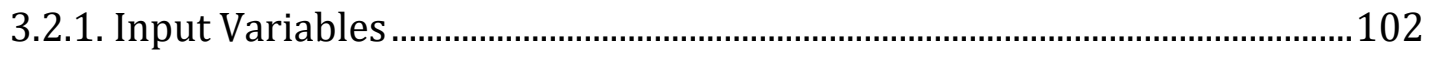

3.2.2. Intermediary Variables ............................................................................. 102

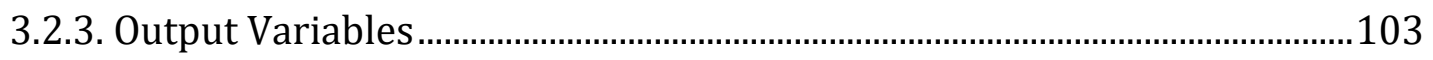

3.3. Applicable Research Settings ..............................................................................103

3.4. Proposed Research Design - Steps to Conduct the Proposed Research ..........104

3.4.1. Analysis of Objectives and Information Needs (Stage 1 of FCM Building

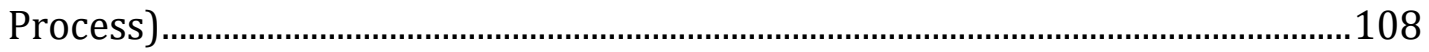

3.4.2. Analysis of Means to Fulfil Information Needs (Stage 2 of FCM Building

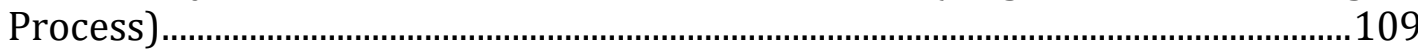

3.4.3. Knowledge Capture (Stage 3 of FCM Building Process)..............................118

3.4.4. Conceptual and Detailed Design of FCM Models (Stage 4 and 5 of FCM Building Process) ………………………………………………………………....122

3.4.5. Test and Evaluation (Stage 6 of FCM Building Process) ...............................129

4 Overview of Pilot Study …………..........................................................................132

4.1. Design of Pilot Study.........................................................................................132

4.2. Setting of Pilot Study .......................................................................................134

4.3. Findings of Pilot Study.........................................................................................136

5 The Summary of the Case Study ...............................................................................139

5.1. Measures and Variables .................................................................................142

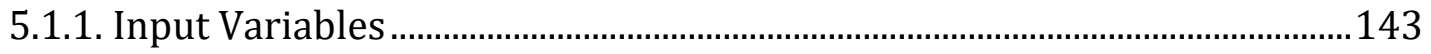

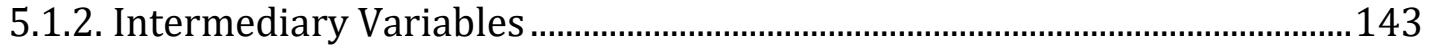

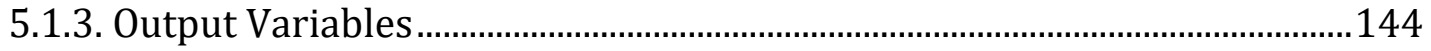

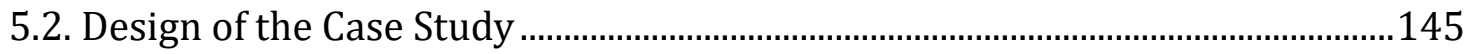

5.3. Settings for Studies ........................................................................................... 147 
5.4. Research Method Evaluation \& Subject Matter Expert Qualification 148

5.5. Method Evaluation Criteria....................................................................................149

5.6. Stages of Execution ........................................................................................... 151

6 Case Study Research ..............................................................................................155

6.1 Qualitative Data Collection (QUAL Data Collection) ...........................................158

6.1.1. Theory Validation (Research Step 1)............................................................160

6.1.2. Elderly Knowledge Capture (Research Step 2) .............................................160

6.1.3. Baseline Tech Perspective Collection (Research Step 3) .............................163

6.1.4. Gatekeeper Knowledge Capture (Research Step 4).....................................164

6.1.5. Repeat Elderly Women \& Gatekeeper Pair Knowledge Capture (Research

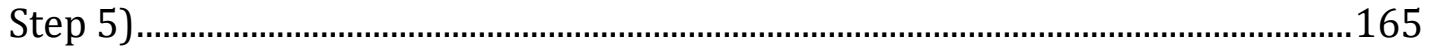

6.2 Qualitative Analysis - Model Content Building .....................................................166

6.2.1. Repertory Grid Analysis (Research Step 6) …………………………….....173

6.2.2. Content Building \& Inter-map Analysis (Research Step 7).........................181

6.2.3. Standardization across Maps (Research Step 8)..........................................194

6.2.4. Identify and Close Gaps (Research Step 9)..................................................195

6.2.5. Integration of All Standardized Maps (Research Step 10) ..........................197

6.3 Quantitative (quan) Data Collection - Converting Cognitive Maps to Fuzzy Cognitive Maps...........................................................................................................20

6.3.1. Model Structure Building (Research Step 11) ...........................................2200

6.3.2. Network Analysis of Paired FCMs (Research Step 12) ...............................204

6.4 Quantitative (quan) Analysis - Model Evaluation (Research Step 13).............209

6.4.1. Extreme Condition Tests .............................................................................. 211

6.4.2. Behavior Reproduction Tests .........................................................................216

6.4.3. Sensitivity Analysis .....................................................................................221

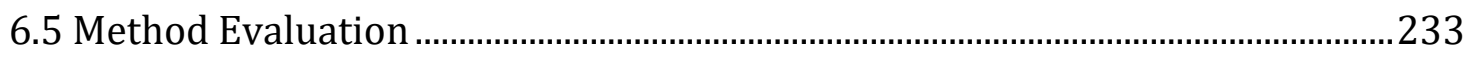

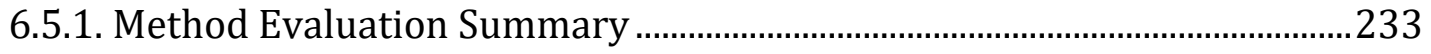

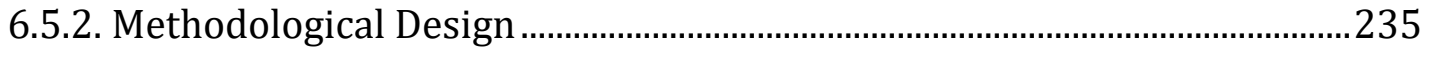

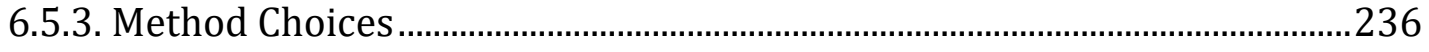

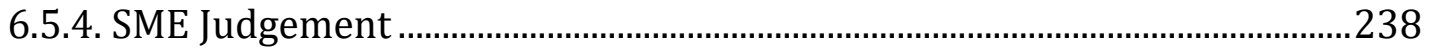

7 Application of the Simulation Model .........................................................................24

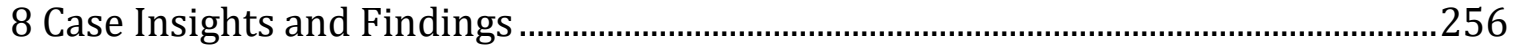


8.1. Summary of Key Drivers and Obstacles of Adoption and Their Relations (Goal

1)

8.1.1. Environmental Factors................................................................................258

8.1.2. Environmental Influences............................................................................261

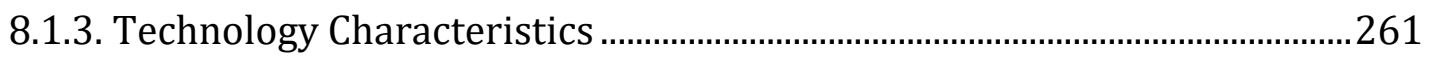

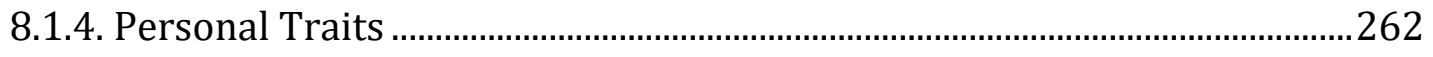

8.1.5. Elderly Perceptions ……………………………………………………....2263

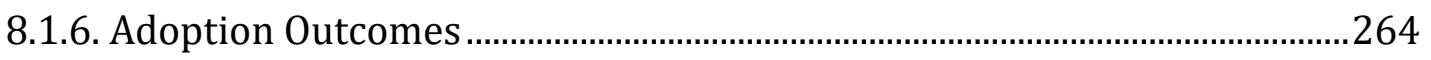

8.2. Gatekeepers' Influence on EWHTA (Goal 2) ....................................................... 265

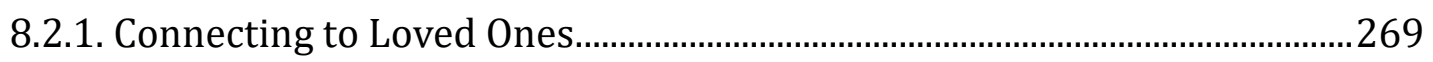

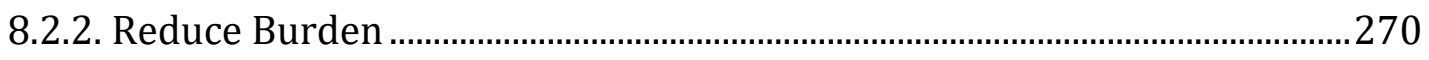

8.2.3. Giving Gatekeeper Peace of Mind...............................................................2271

8.2.4. Gifting Tech.................................................................................................. 271

8.2.5. Tech Suggestion, Introduction, and Encouragement ....................................272

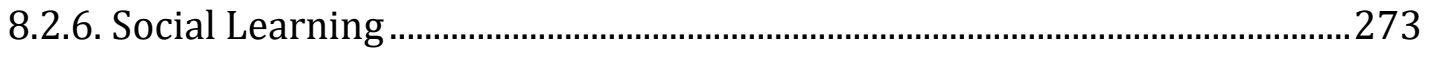

8.2.7. Continued 1-on-1 Walkthrough Support ......................................................224

8.2.8. Effectivity, Continuity \& Sufficiency of Support \& Training ........................275

8.3. Causal Quantification and Simulation (Goal 3 \& 4) ...........................................276

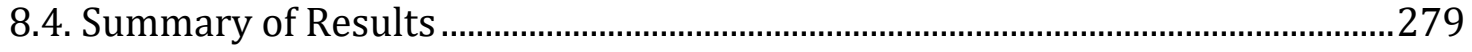

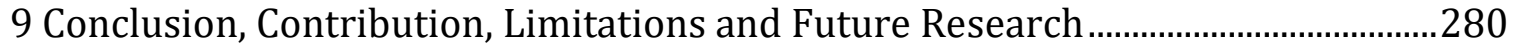

9.1. Conclusion .......................................................................................................280

9.1.1. Method Conclusion ......................................................................................280

9.1.2. Theoretical Conclusion in the Case of Elderly Women Health Technology

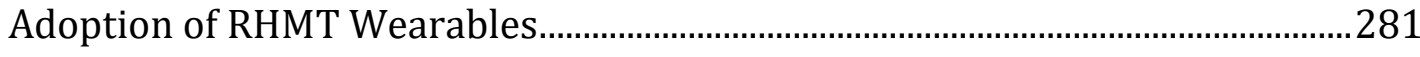

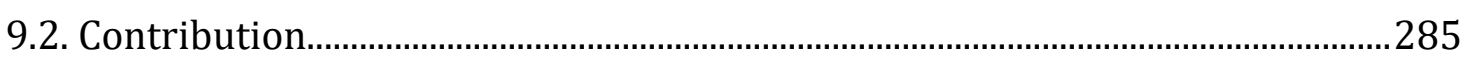

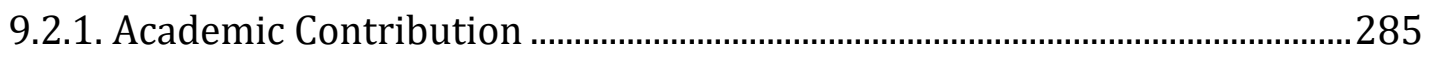

9.2.2. Contributions to Management Practice ........................................................289

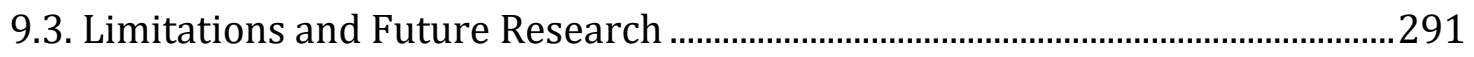

9.3.1. Research Method Limitation.........................................................................2291

9.3.2. Research Model Limitation.............................................................................293

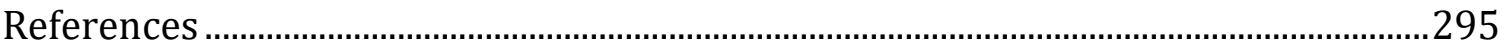

Appendix A - Data Mining in Early HTA Literatures .........................................................338

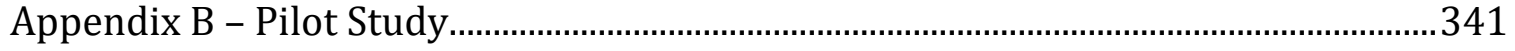




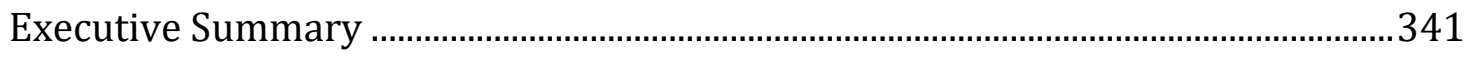

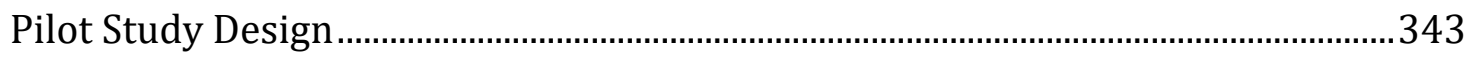

I. Analysis of Objectives and Information Needs ......................................................344

II. Analysis of Means to Fulfil Information Needs ………...........................................345

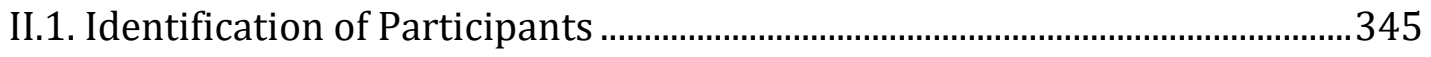

II.2. Methods Selection for knowledge Capture.......................................................346

III. Knowledge Capture .......................................................................................... 346

IV. Conceptual and Detailed Design of FCM Models ....................................................352

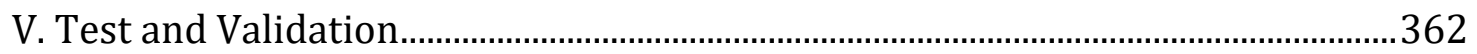

Questionnaires Used in Pilot Study.........................................................................367

Appendix C - Qualitative (QUAL) Research Data Collection Supporting Material...369

C.I. Participants Consent Form...............................................................................369

C.II. Open-ended Interview Questions .......................................................................373

Appendix D - Qualitative (QUAL) Research Analysis Supporting Material .................376

Appendix E - Quantitative (quan) Data Collection Supporting Material .....................377

E.I. Quantitative (quan) Data Collection Structured Survey Instrument..................377

E.II. Adjacency Matrices of the Elderly Women Participants.......................................404

E.III. Clustering FCM Algorithm (Aminpour, 2018) ................................................... 430

E.IV. Total Population Average Adjacency Matrices.......................................................434

E.V. Adjacency Matrix of FCM Connected.......................................................................... 436

E.VI. Adjacency Matrix of FCM Isolated …………………............................................ 438

Appendix F - Quantitative (quan) Analysis Supporting Material..................................440

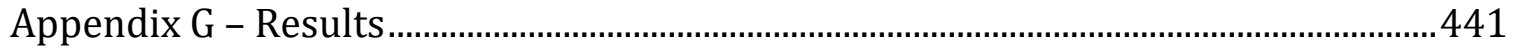

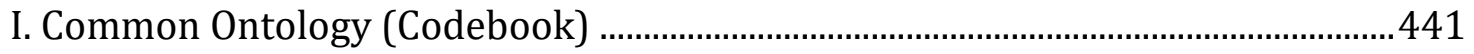

II. Resulted FCM Connected Model.....................................................................................446

III. Resulted FCMIsolated Model....................................................................................44

Appendix H - Case Study Participants' Profiles..............................................................448

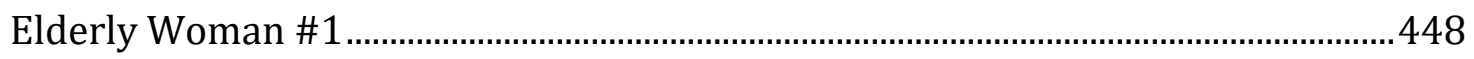

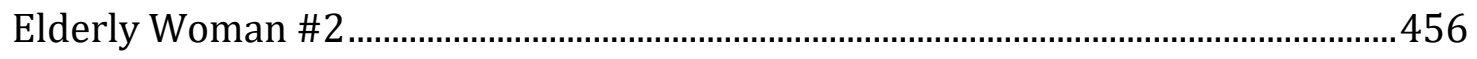

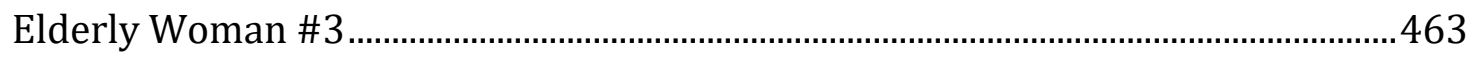

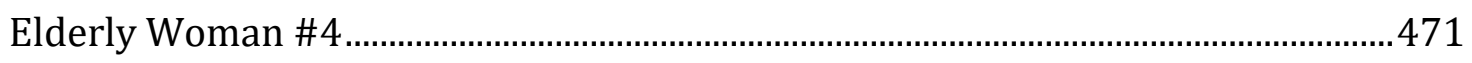

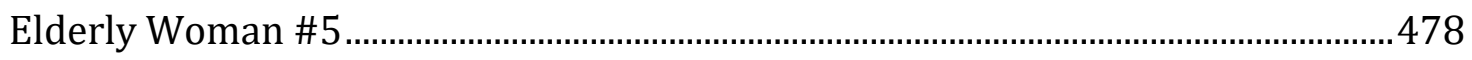




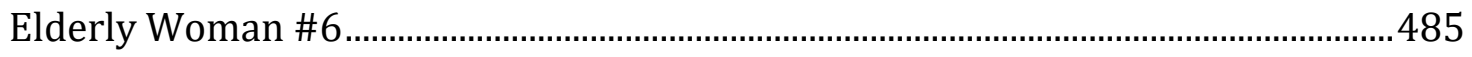

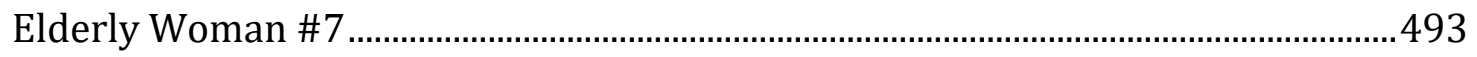

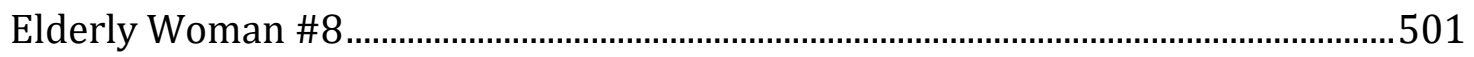

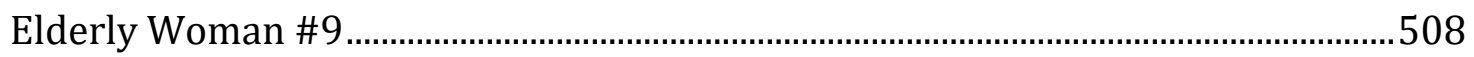

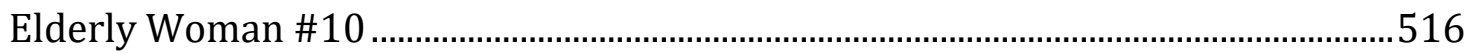

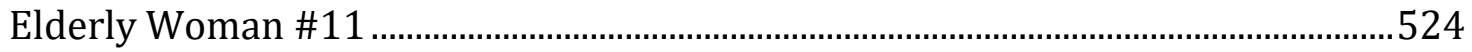

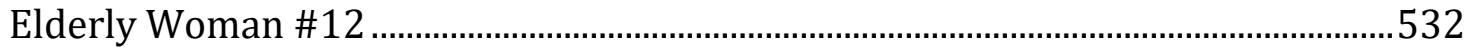

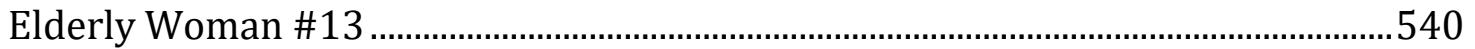

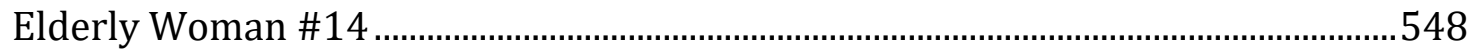

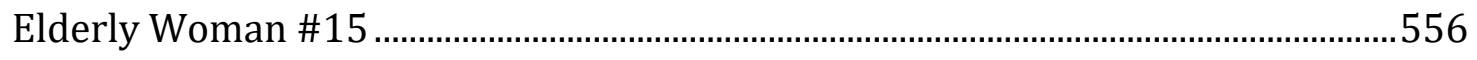




\section{List of Tables}

Table 2 - 1 The Most Popular Technology Adoption Theories in HTA .......................... 50

Table 2 - 2 Challenges in HTA research .............................................................................. 58

Table 2 - 3 Modeling methods performance based on the set criteria ............................ 76

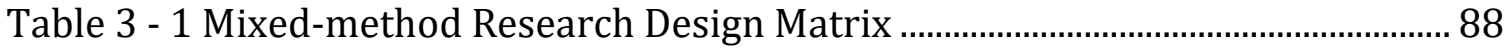

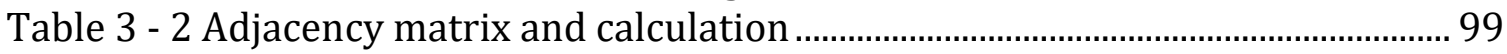

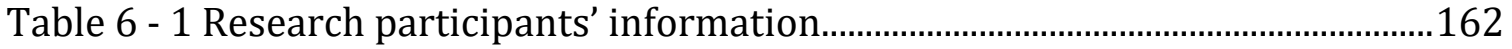

Table 6 - 2 Participants' interview duration (in minutes) ..................................................162

Table 6 - 3 Participant pairs' demographics information ................................................163

Table 6 - 4 Answer choices to quantify the edge weights and their corresponding 5-

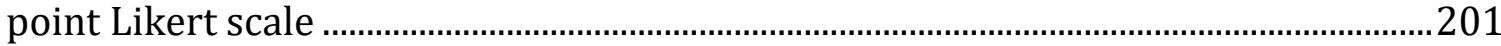

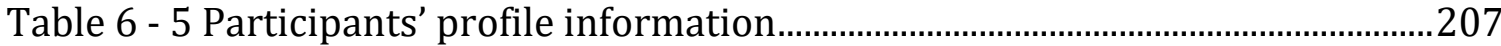

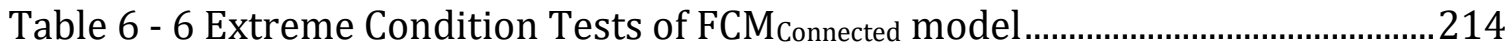

Table 6 - 7 Extreme Condition Tests of FCM Isolated model ..............................................215

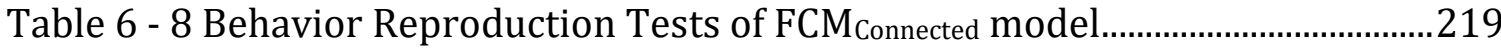

Table 6 - 9 Behavior Reproduction Tests of FCMIsolated model ........................................220

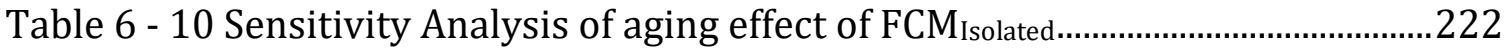

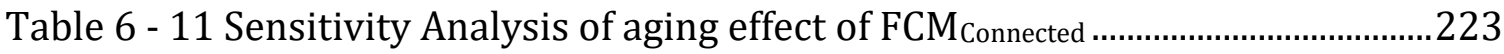

Table 6 - 12 Social Influence Sensitivity Analysis of FCM Isolated .......................................2225

Table 6 - 13 Social Influence Sensitivity Analysis of FCMConnected....................................226

Table 6 - 14 Experience Sensitivity Analysis of FCM Isolated .............................................228

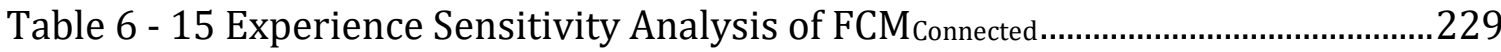

Table 6 - 16 Product Characteristics Sensitivity Analysis of FCMIsolated ......................231

Table 6 - 17 Product Characteristics Sensitivity Analysis of FCMConnected....................232

Table 6 - 18 Proposed model meets evaluation criteria...................................................235

Table 7 - 1 Simulation Results of scenario on FCMConnected .......................................250

Table 7- 2 Simulation Results of scenarios on FCM Isolated ......................................254

Table 7- 3 The interplay/prioritization of product quality \& social support...........255

Table 8 - 1 Environmental Factors of the EWHTA.................................................259

Table 8 - 2 Environmental Influences Factors of the EWHTA..................................261

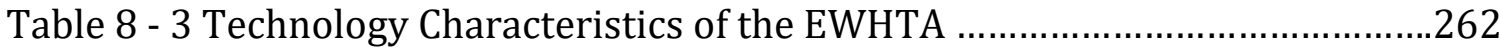

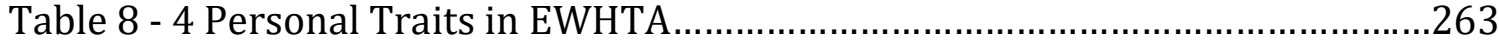

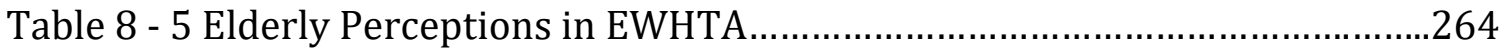

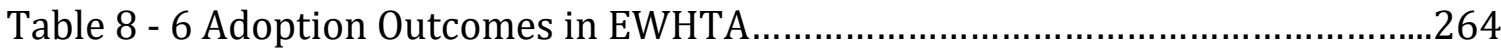




\section{List of Figures}

Figure 1 - 1 Unified Theory of Acceptance and Use of Technology (UTAUT) ................ 6

Figure 1 - 2 UTAUT in EWHTA context (Rahimi et al., 2016) ............................................ 15

Figure 2 - 1 Elderly Health Technology Adoption is at the intersection of Gerontology, Health Technology Adoption and Human Factors Engineering ............. 26 Figure 2 - 2 The reviewed popular theories applied in Health Technology Adoption

Figure 2 - 3 UTAUT with Social Influence influencing all other input factors (Rahimi et al., 2016) 55

Figure 3 - 1 A simple FCM map. 98

Figure 3 - 2 Proposed researh method: Aligning the research method steps with the mixed-method design and the fuzzy cognitive mapping ...............................................106

Figure 3 - 3 UTAUT as base cognitive model ...............................................................120 Figure 5 - 1 Case Study following Chapter 3 Research Design with an additional step 13 to evaluate the method 141

Figure 5 - 2 Activity trackers tried during this research ...............................................146 Figure 6 - 1 UTAUT as the base cognitive map to expand in each interview .............160 Figure 6 - 2 Open coding of the interview records in Atlas.ti .........................................176

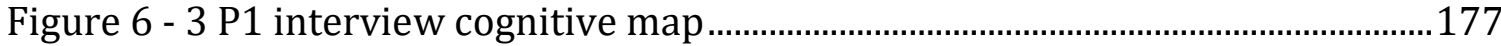

Figure 6 - 4 P1's gatekeeper interview cognitive map.........................................179

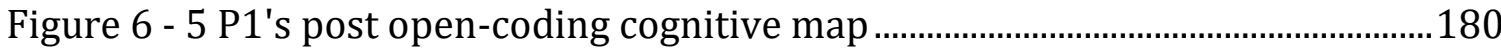

Figure 6 - 6 Consolidated open-codes in Code Manager .................................................184

Figure 6 - 7 Facilitating Condition Code Family..............................................................185

Figure 6 - 8 Parking Lot map showing the emerged concepts and their connections

Figure 6 - 9 Ease of use related concepts from P2 initial cognitive map ....................191

Figure 6 - 10 Ease of use related concepts from integrated map...................................192

Figure 6 - 11 Being frugal was formulated from opposing cases..................................194

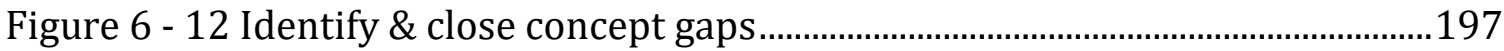

Figure 6 - 13 Integrated cognitive map.........................................................................199

Figure 6 - 14 Question 32 in quan data collection survey (complete survey in

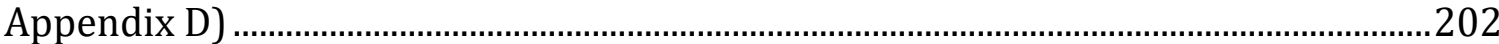

Figure 6 - 15 Question 30 in quan data collection survey (complete survey in

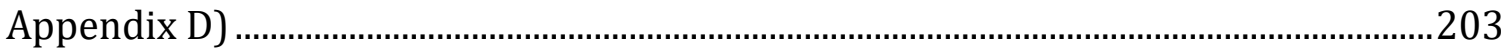

Figure 6 - 16 Output of clustering FCM based on Eigenvector similarity index........206

Figure 6 - 17 Sensitivity Analysis of aging effect of FCMIsolated ..................................222

Figure 6 - 18 Sensitivity Analysis of aging effect of FCMConnected ..............................223

Figure 6 - 19 Support Influence Sensitivity Analysis of FCMIsolated ...........................2225

Figure 6 - 20 Social Influence Sensitivity Analysis of FCMConnected...........................226

Figure 6 - 21 Experience Sensitivity Analysis of FCMIsolated .......................................228

Figure 6 - 22 Experience Sensitivity Analysis of FCMConnected...................................229

Figure 6 - 23 Product Characteristics Sensitivity Analysis of FCMIsolated .................231 
Figure 6 - 24 Product Characteristics Sensitivity Analysis of FCMConnected ...........232

Figure 7 - 1 FCMConnected model....................................................................244

Figure 7 - 2 FCMIsolated model...............................................245

Figure 8 - 1 Salient Drivers of and Obstacle Concept Categories for EWHTA..........257

Figure 8 - 2 'Environmental Factors' and 'Environmental Influences' in FCMIsolated

Figure 8 - 3 Family gatekeeping concepts of EWHTA in a segment of the two FCM models.

Figure 8 - 4 Gatekeeping (other than family's) concepts of EWHTA in a segment of

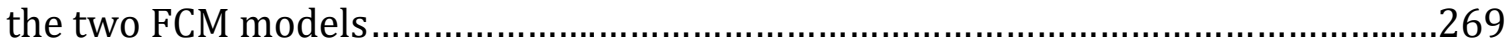

Figure 8 - 5 Concepts increasing Connecting to loved ones.....................................2270

Figure 8 - 6 Concepts increased by Connecting to loved ones.................................22

Figure 8 - 7 Concepts increasing Reduce burden ..................................................270

Figure 8 - 8 Concepts increased by Reduce burden ...............................................271

Figure 8 - 9 Concepts influencing Giving GK peace of mind.....................................271

Figure 8 - 10 Concepts influenced by Giving GK peace of mind ...............................271

Figure 8 - 11 Concepts influencing Gifting tech....................................................272

Figure 8 - 12 Concepts influenced by Gifting tech...............................................272

Figure 8 - 13 Concepts influencing Tech suggestion..............................................273

Figure 8 - 14 Concepts influenced by Tech suggestion...........................................2.273

Figure 8 - 15 Concepts influencing Social learning..............................................2.273

Figure 8 - 16 Concepts influenced by Social learning............................................274

Figure 8 - 17 Concepts influencing Continues 1-on-1 walkthrough support............274

Figure 8 - 18 Concepts influenced by Continues 1-on-1 walkthrough support.......275

Figure 8 - 19 Concepts influencing Efficiency Continuity \& Sufficiency of Support \&

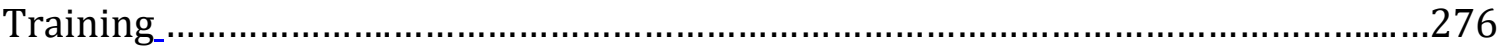

Figure 8 - 20 Adoption is directly influenced by Efficiency Continuity \& Sufficiency of

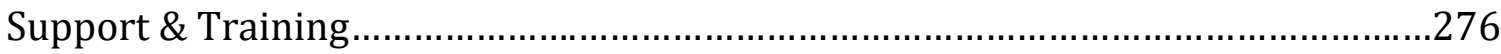

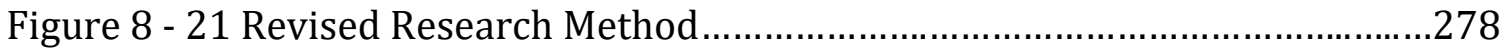




\section{List of Abbreviations}

E Elderly

EHTA Elderly Health Technology Adoption

EWHTA Elderly Woman Health Technology Adoption

EW Elderly Woman/Elderly Women

GK Elderly's Technology Gatekeeper

HCI Human Computer Interaction

HFE Human Factor Engineering/Human Factor \& Ergonomics HTA Health Technology Adoption

ICT Information and Communications Technology

QUAL/ qual Qualitative QUAN/ quan Quantitative RAA Reasoned Action Approach

RGT Repertory Grid Technique

RHMT Remote Health Monitoring Technology

SCT Social Cognitive Theory

SME Subject Matter Expert

TA Technology Adoption

TAM Technology Acceptance Model

Tech Technology TPB Theory of Planned Behavior

TRA Theory of Reasoned Action

UTAUT .Unified Theory of Acceptance and Use of Technology $\mathrm{UCD}$ User Centered Design 


\section{Introduction}

This introductory chapter describes the practical problem of poor health technology adoption by the elderly and the role technology plays in improving the quality of care for the rising elderly population. The chapter furthermore reviews how during the past several decades there have been theories conceptualized to better understand and subsequently improve technology adoption. However, these theories are too general and fail to provide practical actionable insights. The problem is particularly pertinent in the complex settings of healthcare, in which gatekeepers and agency play an important role and make and female technology adoption differ. This has resulted in the call to create new modeling methods that can contextualize the extant technology adoption theories to the specific healthcare context under study. In response to this call to arms, this dissertation develops a mixed-methods research method to model health technology adoption among elderly. The chapter describes the dissertation's scope, contribution, and limitation as well as highlights of the proposed method.

\subsection{Problem Background}

Health technology adoption in eldercare constitutes a big practical problem that needs to be solved (Crosby \& Noar, 2010). The global elderly population is growing at a rapid rate (U. S. Census Bureau, 2012; World Health Organization, 2012), especially in developed countries (United Nations, 2013). Solutions for ensuring their health and well-being are thus becoming an urgent need (United Nations, 2013; "Women and Caregiving," 2003). This need has led to a crisis that is not only 
draining the elderly quality of life, but it also bankrupting the healthcare system (Callahan, 2008).

Studies have shown that the elderly yearn for sustaining their independence (e.g., Aging, 2011; Technology and Aging, 2009; Cahillet al., 2009). Independence provides many benefits for the elderly, including a restored sense of pride, control and dignity ("Why Independence is an Important Part of Elderly Care | My Care My Home," 2013; Zimmer \& Chappell, 1994). In addition, independence of the elderly alleviates the financial burden on the children and the elderly themselves (Friedet al., 2001), as well as the entire healthcare system ("Measuring the Costs and Savings of Aging in Place," 2013).

Modern approaches to independence of the elderly tend to involve technology (Technology and Aging, 2009; BBC News, 2012). For example, Remote Health monitoring Technologies (RHMT), such as smart-home systems ("eNeighbor ${ }^{\circledR}$ Remote Monitoring System" 2009), wearables (“GoSafe Medical Alert System $®$," 2015) and specialized smartphone software ("Smartphone Apps for Senior Help" 2014), provide the infrastructure for independent living and reduce the burden of caregiving. RHMT can provide solutions through three mechanisms (Litan, 2008):

1) Better management of chronic conditions, resulting in lower needs for care;

2) Improved assessment of care needs in emergency (e.g. falls) and in everyday situations (e.g., subtle cognitive decline), leading to more targeted provision of care; and 
3) Reduced impact on caregiver schedules by enabling remote check-ins, visits, data exchange with healthcare providers, and reducing the occurrence of crises.

For these reasons, development and deployment of RHMT have been prioritized by numerous national and international initiatives. ${ }^{1}$ However, as is the case with other technologies (Morris \& Venkatesh, 2000; M. Morriset al., 2005), the elderly are slow to adopt RHMT ("Advanced Remote Patient Monitoring Systems : Market Research Report," 2013; Bowers, 2013; Cimpermanet al., 2013; Fife \& Pereira, 2011; Stojmenova et al., 2012). The prohibitive forces among elderly are strong, and often successful adoption is contingent upon strong agency (Finkelstein et al., 2006). The resulting low adoption rate limits the positive impact of RHMT.

The reasons for slow technology adoption among the elderly are multifaceted and not fully understood. Elderly Health Technology Adoption (EHTA) is adversely affected not only by the natural consequences of aging, but also by the complexity of the healthcare context (Morgan, 1998) and the adoption process itself (Crosby \& Noar, 2010). All three of these factors have to be addressed for the elderly to adopt the healthcare technologies that they need to lead a dignified life in their declining years.

\footnotetext{
${ }^{1}$ Examples of national activities are the initiatives by the Department of Health and Human Services, e.g. ("HealthCare Manager: Remote Medication and Health Plan Support System," 2011). Examples of international initiatives include Celler et al.'s (2014) and Reinkensmeyer et al.'s (2012).
} 
Each of these factors has been researched extensively within their own fields. For example, an immense amount of knowledge about "the effects of aging on human behavior" in general (Minton \& Schneider, 1985; Sterns \& Doverspike, 1989a) and technology use among elderly in particular (Morris \& Venkatesh, 2000; M. Morris et al., 2005; Pan \& Jordan-Marsh, 2010) has been gained. In addition, over four decades of research in technology adoption has resulted in an abundance of sound theories on technology adoption in organizational settings (Crosby \& Noar, 2010; Lee et al., 2003; Williams et al., 2011). It also documents the complexity of the healthcare environment and its negative impact on technology adoption (Head \& Noar, 2014). Furthermore, Human Factors Engineering (aka Human-Computer Interaction) has enabled innovation in health products that aim to be elderlyfriendly and hence adoptable (Arning \& Ziefle, 2007; Minton \& Schneider, 1985; Ong \& Lai, 2006).

Unfortunately, these individual fields of study have not been able to provide models that can explain why the elderly do not adopt technology that they need. The field of gerontology ${ }^{2}$ has yet to clearly identify what really influences the process of decision-making in technology adoption by the elderly (Chen \& Chan, 2013). Theories on technology adoption have not been able to provide actionable insights in the context of the elderly (Wild et al., 2012). Human factors engineering has

\footnotetext{
${ }^{2}$ Gerontology is the study of physical, mental and social effects of aging has discovered a great deal of information about elderly; and is one of the key fields of studies in understating EHTA. The Institute of Gerontology defines the term as: "Gerontology is the study of aging and older adults." The science of gerontology has evolved as longevity has improved. Researchers in this field are diverse and are trained in areas such as physiology, social science, psychology, public health, and policy."
} 
primarily focused on technology design-factors that reach beyond early user experience and initial adoption are rarely considered (Bannon, 1991). This disparity explains why these extensive bodies of knowledge have not been able to provide many actionable insights in the context of health technology adoption among the elderly. Evidently, a solution that meets the needs of the elderly requires an interdisciplinary approach that integrates the body of knowledge in these disparate fields.

Researchers have argued that extant theories on technology adoption such as the Reasoned Action Approach (RAA) family of theories (Fishbein \& Ajzen, 1975, 2011) and the Technology Adoption Model (TAM) (F. D. Davis, 1985) provide a sufficient foundation for studying the phenomenon of technology adoption in the healthcare environment (Rahimi et al., 2016). What is lacking, are applications of existing theories of technology adoption that address the challenges of the elderly in their specific context. As a consequence, the practical problem of health care technology adoption among the elderly remains unresolved (Bagozzi, 2007; Dwivedi et al., 2011; Heart \& Kalderon, 2013; Williams et al., 2011).

The RAA family of theories and the TAM are predecessors of the Unified Theory of Acceptance and Use of Technology (UTAUT) (Venkatesh, Morris, Davis \& Davis, 2003), which represents the consensus of leading scholars in the field of technology adoption. The UTAUT was created to be more comprehensive and inclusive, and empirical research confirmed that this is so (Balogh et al., 2013; McClellan, 2011; Robson \& Sutherland, 2012). Figure 1 -1 shows that the UTAUT 
posits that the three primal factors-performance expectancy, effort expectancy, and social influence-shape the behavioral intention. Behavioral intention and facilitating conditions directly influence the use behavior. There are also four factors--gender, age, experience and voluntariness of use--that moderate the relationships between the input and output constructs.

\section{Input Constructs}

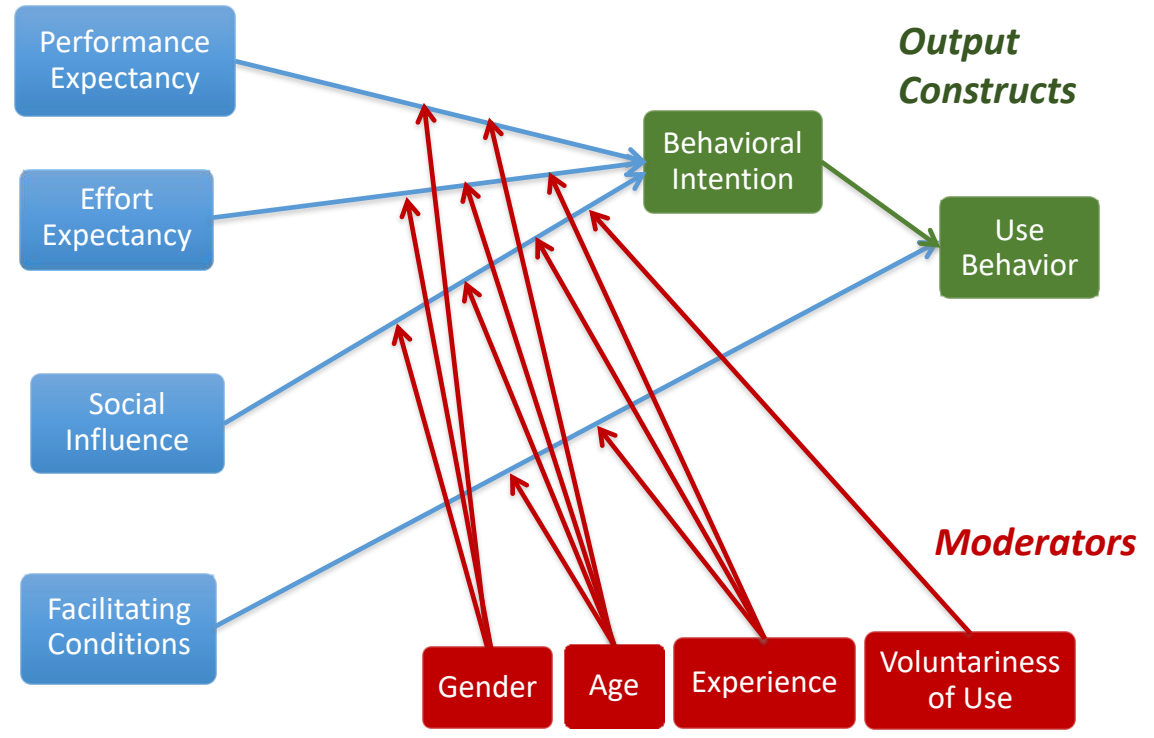

Figure 1 - 1 Unified Theory of Acceptance and Use of Technology (UTAUT)

The UTAUT, despite its growing popularity, has not performed much better in the domain of empirical research (Bagozzi, 2007; Williams et al., 2011) including healthcare (Dwivedi et al., 2011; Head \& Noar, 2014). Early tests overestimated UTAUT's predictability by suggesting that the UTAUT can explain an impressive $70 \%$ of the variance in behavior intention and about $50 \%$ in use behavior (Holden \& Karsh, 2010). However, meta-analysis showed that, like its predecessors 
(Sheeran et al., 1999; Webb \& Sheeran, 2006), the application of UTAUT in healthcare failed to capture the holistic view that is needed in practice (Bagozzi, 2007; Dwivedi et al., 2011; Rahimi \& Jetter, 2015; Williams et al., 2011).

A large body of applied research tried to remedy these shortcomings by combining theories (Karimi-Shahanjarini et al., 2013; Samoutis et al., 2008) or by adding factors to these theories (AbuShanab et al., 2010; Aggelidis \& Chatzoglou, 2009; Bagozzi, 2007; Luo et al., 2010; Robson \& Sutherland, 2012; Williams et al., 2011). However, the models that were derived from these supplemented theories delivered inconclusive results (Mohammadi \& Kaldi, 2008).

The lack of insight provided by these models is rooted in the well-known dilemma between generalizability and utility (Head \& Noar, 2014). Bagozzi (2007) argues that the main strength of TAM family of theories (including UTAUT) is their parsimony. However, due to their parsimony, these theories fail to provide any insight as to what factors form perceptions of usefulness and ease of use, and what these high-level factors mean in practice (Bagozzi, 2007). Without these insights, the research cannot provide much practical value. Evidently, applying the broadly generalizable UTAUT and all its predecessors to highly specific contexts of technology adoption including health care for the elderly (Renaud \& Van Biljon, 2008) turns out to be difficult (Bagozzi, 2007; Dwivedi et al., 2011; Rahimi \& Jetter, 2015; Williams et al., 2011).

Head and Noar posit: "When there is tension between generalisability and utility, utility should be given preference given the applied nature of the health behaviour 
field." (Head \& Noar, 2014, p. 16). Therefore, broadly generalizable theories need to be contextualized to the health behavior field, in order to be useful (Chalmers, 1982; Head \& Noar, 2014). In fact, there is a call to arms to apply sound theories of technology adoption to specific health contexts, in order to facilitate the adoption of health technologies in practice (Bagozzi, 2007; Crosby \& Noar, 2010).

Research also shows that constructs within the same theory can be highly context specific in the health behavior field (Head \& Noar, 2014; Holden \& Karsh, 2010). This mandates the development of empirically grounded models that customize the broadly based theories to specific health behavior situations. This approach would be especially compelling in EHTA, where the effects of aging, the multiplicity of stakeholders, and the complexity of health behavior generates a unique context (Christensen \& Remler, 2009).

An emerging stream of literature highlights the need to apply the latest technology adoption theories including the UTAUT to the specific health context studied (Godin \& Kok, 1996; Head \& Noar, 2014; Holden \& Karsh, 2010). Specifically, researchers have expressed the need to enhance our understanding of the independent variables that affect adoption behavior (i.e., "there is opportunity to develop the "left side of the model'" (Holden \& Karsh, 2010, p.11). A lot of efforts to do so have been made to date in the field of EHTA (Conci et al., 2009; Renaud \& Van Biljon, 2008), yet not much improvement in modeling adoption behavior among the elderly has been achieved (Morrell et al., 2004; Smith, 2004). 
Therefore, context-specific models that apply the UTAUT to the area of elderly health technology adoption must be developed.

Agency is a key issue in technology adoption by the elderly (Kwon \& Chidambaram, 2000; Mallenius, Rossi, \& Tuunainen, 2007; Renaud \& Van Biljon, 2008; Silverstone \& Haddon, 1996). For example, a great body of research shows that family members (Kwon \& Chidambaram, 2000; Mallenius et al., 2007; Renaud \& Van Biljon, 2008; Silverstone \& Haddon, 1996) and friends (Mallenius, Rossi, \& Tuunainen, 2007; Renaud \& Van Biljon, 2008) of the elderly serve as gatekeepers (Allen, 1977; Katz \& Tushman, 1981; Tushman \& Katz, 1980) for technology adoption by the elderly (Kwon \& Chidambaram, 2000; Mallenius et al., 2007; Renaud \& Van Biljon, 2008; Silverstone \& Haddon, 1996), and they influence the adoption decision critically (Guynn, 2002; Y. S. Lee, 2007; Mallenius et al., 2007; Morrell et al., 2004). They not only recommend the usage of the technology, but they also patiently teach the elderly how to use it (Rahimi et al., 2016). Often, they also purchase new technology for or gift their old technology to the elderly for which they care (Kwon \& Chidambaram, 2000; Mallenius et al., 2007; Renaud \& Van Biljon, 2008; Silverstone \& Haddon, 1996). Research findings recommend the inclusion of these unofficial care providers as a unit of analysis (Compagna and Kohlbacher, 2014).

Evidently, these gatekeepers influence all the input variables of the extant technology adoption theories. They are the most important part of the social influence construct within the UTAUT. They also affect the formation of all the other 
input constructs (as depicted in Figure $1-2$ in section 1.3). Thus, the input variables of the UTAUT are not really independent, and the contextualized model needs to provide a 'map' of their interdependency and relationships. (Rahimi et al., 2016)

Research has shown that technology adoption decision-making is different between men and women (Schaar \& Ziefle, 2011; Venkatesh \& Davis, 2000; Venkatesh, Morris, \& Ackerman, 2000; Wilkowska, Gaul, \& Ziefle, 2010). For example, while men are primarily driven by the usefulness of the technology, women value the ease of use more and are influenced by social norms (Venkatesh et al., 2000). Elderly women healthcare technology adoption (EWHTA) may thus be different from healthcare technology adoption by elderly men. To capture these intricate differences among men and women, their respective adoption practices need to be studied separately.

Gatekeeper behavior could also be gender specific. Thus, to capture the influence of gatekeepers on the elderly, four permutations of gatekeepers and the elderly need to be studied: elderly man and male gatekeeper, elderly man and gatekeeper, elderly woman and male gatekeeper, and elderly woman and gatekeeper. Since the majority of the elderly and their gatekeepers tend to be women (United Nations, 2013; "Women and Caregiving," 2003), researching the fourth of the above permutations should be given priority. This specificity not only allows for the emergence of unique perspectives of elderly women; it also enables the discovery of patterns of technology adoption among the elderly. 
Many of the challenges with the current methods are highlighted in different streams of the academic literature. These challenges include:

1. Grounding: Integrate first-hand user data (Phillips \& Zhao, 1993).

2. A need to approach the problem holistically: Cover a wide range of factors that emerge from the health context under study (Bagozzi, 2007; Head \& Noar, 2014; Ogden, 2003; Rothman, 2004).

3. Flexibility: Build flexible models that can be adjusted in response to new input (Jetter \& Kok, 2014; Kosko, 1988).

4. Latent Needs: Model latent needs (Phillips \& Zhao, 1993).

5. Need for visual formalisms: Provide cognitively simple visual representation of findings that people from different disciplines can comprehend (Creswell, 2013, ch.10).

6. Scenario Planning: Model and simulate decision scenarios (Simpson, 1992) to identify and prioritize alternative decision points based on their potential to yield a higher adoption rate (Amer et al., 2013; Glenn \& Gordon, 2009; Schoemaker, 1991; Simpson, 1992).

7. Novel Insight: Develop the capacity to provide novel insights in relation to the predictions of extant theory, and beyond (Glanz et al., 2008; Head \& Noar, 2014; Ogden, 2003; Rothman, 2004).

8. Efficiency: The method needs to be practical in regards to consideration of monetary and temporal resources and constraints (Creswell, 2013, ch.10). 
This dissertation aims to address these challenges by generating a modeling method that applies the most relevant theory for Health Technology Adoption (HTA) to visually unpack the high-level adoption constructs in complex contexts, where technology adoption is strongly influenced by agency. This decomposition is possible through qualitative data collection that provides flexibility and allows the participants to take the research through their journey of HTA. This approach leads to fresh insights by opening the black box of technology adoption, and revealing the intricacies of the paradigm in the context being studied.

\subsection{Purpose of Dissertation}

At its core, this dissertation aims to provide a research method that models technology adoption among demographic groups that rely heavily on gatekeeping for technology adoption in EHTA. This method yields a contextualized model of technology adoption, which serves as a decision tool and provides a better understanding of the drivers and obstacles in elderly health technology adoption. The model....

- Identifies the key adoption drivers and obstacles and their relations

- Illustrates how the gatekeeper influences the elderly person's adoption decision

- Represents adoption drivers and gatekeeper influences on adoption quantitatively 
- Facilitates exploration of adoption in response to various factors through simulation

The method developed in this dissertation can be applied to any elderly health technology adoption (EHTA) research. However, in this dissertation, the method proposed is applied to elderly women health technology adoption (EWHTA) since women comprise the majority of the elderly population. It is applied to the context of remote health monitoring technology (RHMT) as described in detail in section 1.1. The resulting model represents visually and quantitatively how the gatekeepers influence the elderly women's adoption decisions by capturing how these gatekeepers (which are at the center of elderly's social influence) influence the formation of perceptions of the UTAUT's main constructs-performance expectancy, effort expectancy, social influence, and facilitating condition. Thus the model should be able to answer the following questions:

$\checkmark$ How do gatekeepers influence the adoption behavior of elderly women in the adoption of an RHMT both from the point of view of intention and usage?

$\checkmark$ How does the relationship between elderly women and gatekeepers affect the elderly's perception of performance expectancy, effort expectancy and facilitating condition in the context of the adoption of an RHMT?

$\checkmark$ How does the adoption of RHMT change in response to different combinations of influencing factors? 


\subsection{Scope of Dissertation}

In this dissertation, the UTAUT is applied to the field of EHTA due to its superior performance over other extant theories (Venkatesh et al., 2003). The work does not research how other theories explain the adoption phenomenon. Should a theory with better explicatory power be found, it could, in principle, be applied and contextualized using the proposed method.

The proposed method is applicable to modeling the adoption of various technologies by a wide variety of elderly people. However, the method to be developed in this dissertation is based upon a study of the elderly, a demographic for which agency is a critical aspect of adoption. The model that results from the method to be developed may thus not apply to other demographics, for which gatekeeping is less important. Furthermore, this dissertation focuses on consumer health products that are subject to optional adoption or rejection. This would, for example, include a wearable fall detection device, but exclude a pacemaker.

As described earlier, the method proposed in this dissertation is applied in the EWHTA context to model elderly women's technology adoption decision making and how their gatekeepers influence the decision. In this complex context, there are many other important stakeholders, such as the elderly's doctor(s) and other health professionals, which are outside the scope of this research. Future studies can further investigate such stakeholder groups in the elderly's social circle, which influence their adoption of technology. 
The proposed method is not necessarily applicable to organizational settings in which gatekeepers from one firm interact with their peers in other firms to bring a technology into their firm (Allen, 1977). In the context studied by the proposed research, the gatekeeping relationship is based on a strong bond that has developed over years. This is not generally the case in most organizational settings.

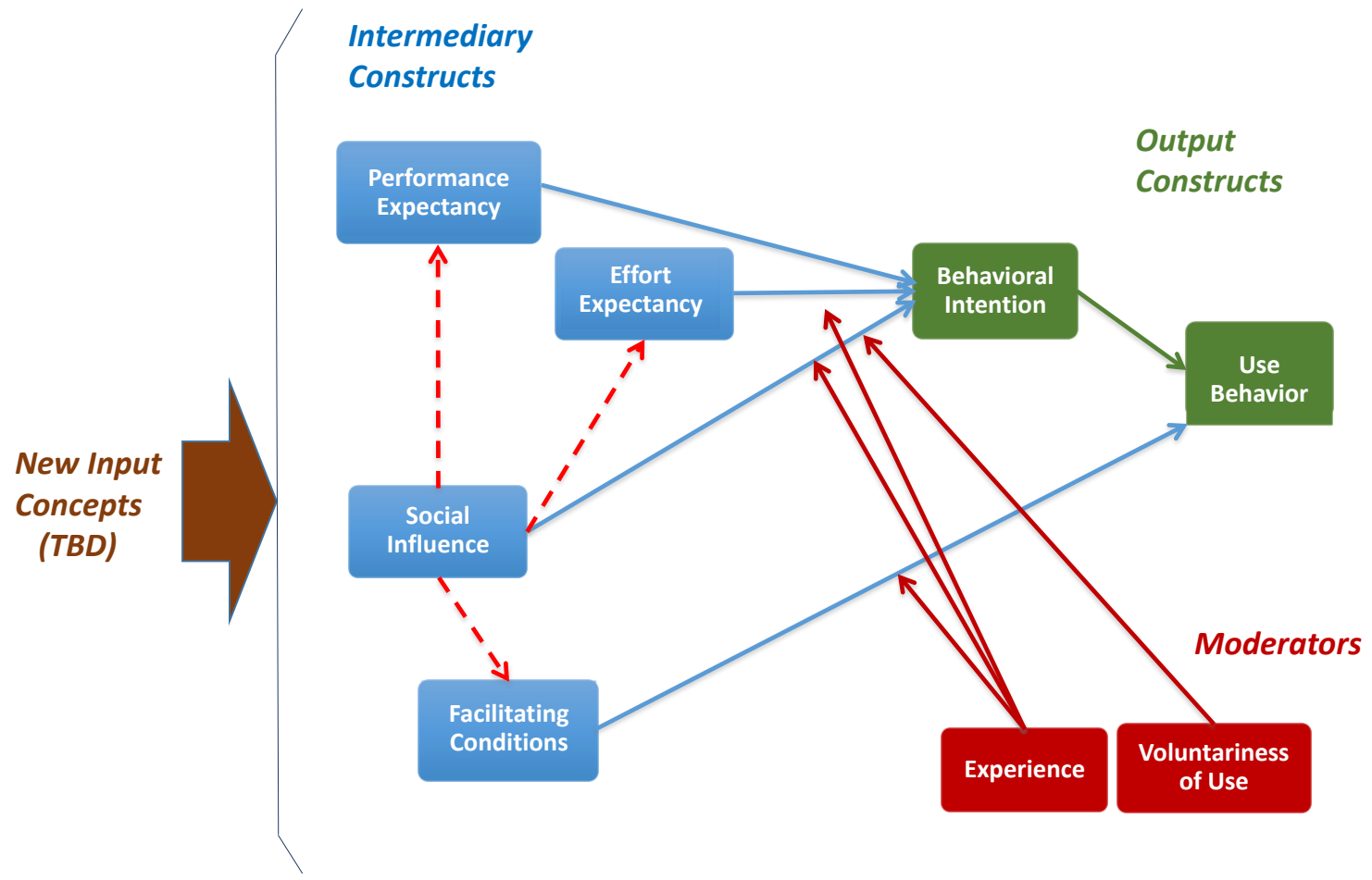

Figure 1 - 2 UTAUT in EWHTA context (Rahimi et al., 2016)

Figure 1 - 2 further delineates the scope of the proposed research. As explained earlier, previous research (Rahimi et al., 2016) has concluded that UTAUT provides the best theoretical foundation for EWHTA research. Since age and gender have already been set in this context, these moderating factors of the UTAUT have been removed. Additionally, Rahimi et al., (2016) found that, in the 
context of EWHTA, social influence affects other input constructs (doted red lines in Figure 1 - 2). Therefore, the variant of the UTAUT that is displayed in Figure 1 2 provides the theoretical foundation for future EWHTA research, including the research proposed for this dissertation.

This dissertation provides a research method that guides the creation of a model, in which the left side of this variant is developed. The original UTAUT input constructs (performance expectancy, effort expectancy, social influence, facilitating condition) will be unpacked, and additional variables (displayed in brown) will be discovered in the EWHTA context. Therefore, the original UTAUT input constructs will become intermediary constructs.

\subsection{Contributions}

Methods Contribution. The methods contribution of this dissertation is the proposed modeling method. This method can be used by academics, as well as by technology marketing and management groups in the industry, that need to research the elderly consumer market.

Contributions to management practice. The insights gained from the model described in section 1.2 will help marketers visualize the dynamics of the customer market where elderly women adopt RHMT technology. This potentially obviates the need to conduct market studies of this market segment. Managers can use this model as a tool that interprets cognition by the elderly and sheds lights into possible scenarios that lead to better adoption. For example, rollout managers 
can plan scenarios, where each scenario constitutes an alternative combination of components and/or strategies of a health technology rollout plan. FCM simulation shows the alternative plan that leads to a better adoption rate of the health product.

\subsection{Limitations of Dissertation}

Research Method Limitation. The research method has been and will continue to focus on modeling elderly health technology adoption (EHTA). In principle, the research method can be used for any group of elderly (including men). However this assertion has not been researched and remains as a limitation of this dissertation. The limitation can be corrected by repeating the research in a population of male elderly.

Model Limitation. The proposed research has been pilot tested and will be applied to cohorts of ORCATECH Life Lab program (Life Lab is explained in section 3.3. Research Setting). The Life Lab population consists of volunteers that use different RHMT technologies. Hence, it is potentially more technology savvy than the elderly population in general. The models derived in this dissertation could thus provide a more optimistic model of technology adoption; and therefore be biased and specific to this subgroup of elderly women. This level of specificity is an inherent characteristic of model building. However, the fact that the model can't be generalized to all elderly women could be considered a limitation. The research

method would have to be applied to the specific group of elderly that is under study to find a model that best reflects their mental model. 
Application Limitation. The proposed research method yields an simulation model that visually and quantitatively represents the elderly women decisionmaking during the adoption (or rejection) of a health technology. Hence, health technologies whose adoption are not optional and don't involve decision-making are out of scope. Additionally, this dissertation focuses on wearable technologies of RHMT in the context of EWHTA research. In principle, the research method can be applied to research any health technology whose adoption is optional. However, this assertion needs further testing.

Tool Limitation. The research method is based on Fuzzy Cognitive Mapping. Thus all the limitations of fuzzy cognitive mapping inherently apply to this research method.

\subsection{Highlights of the Proposed Method}

The research method proposed for this dissertation follows the guidelines for fuzzy cognitive mapping laid out by Jetter (2006). It takes advantage of FCM's ability to integrate the perspective of multiple stakeholders (here both the elderly and the gatekeepers) in quantitative models.

As mentioned in the Section 1.1, the concepts that are associated with adoption theories are too general to provide actionable insights in specific contexts including EHTA. Making sense of how these concepts apply to the phenomena under study warrants highly granular, empirically grounded approaches that study specific contexts in depth. 
The research method proposed for this dissertation follows such a guideline. It handpicks effective techniques in computer-based modeling and qualitative research approaches to facilitate modeling of technology adoption for a demographic in which agency is important. It applies mixed-method design to collect in-depth field data by Fuzzy Cognitive Mapping (FCM) (Kosko, 1986) to model EHTA. The designed mixed-method defines the steps to conduct the research in two sequential phases of qualitative followed by quantitative research to comprehensively capture elderly's mental model during the adoption decision making. As this group of users of technology products is subjected to significant influence by their technology gatekeepers, their gatekeepers are also studied.

As FCM serves as the central platform throughout the entire process of the research, it guides the phases of the research as depicted in Figure $3-2$ in Chapter 3. The first two stages of the FCM modeling decides on 1) analysis of objective and information needed, and 2) analysis of means to fulfil information needs as detailed in section 3.4.1 and 3.4.2. From there, the following phases summarize the steps that will be taken to capture knowledge (section 3.4.3), conceptualize (section 3.4.4) and then validate (section 3.4.5) the FCM as the simulation model. The research will proceed in two phases, which are detailed in chapter 3 . The first phase is qualitative, the second quantitative. Each phase includes data collection and analysis activities, which are executed sequentially. 


\section{Phase 1: Qualitative}

- Qualitative Data Collection: After identifying the participants, conduct oneon-one ethnographic data elicitation using open-ended questions and simultaneously build cognitive maps with a group of $\sim 10-20$ elderly women and their gatekeepers.

- Qualitative Analysis continues the content-building part of EHTA modeling. Each interview and cognitive map is content analyzed as part of the repertory-grid approach (George Kelly, 1955) (which is detailed in chapter 3). This qualitative analysis results in potentially new concepts and relationships, which are added to the cognitive maps. Additionally, the analysis standardizes the concepts across the maps, which results in an ontology list-a collection of all the concepts across the cognitive maps. This also allows the integration of all the cognitive maps into one composite map for the elderly and their gatekeepers.

\section{Phase 2: Quantitative}

- Quantitative Data Collection covers the structure-building part of EWHTA modeling. The researcher conducts a second set of interview with the same group of elderly women and their gatekeepers to create the structure of the model. The information from this second set of one-on-one interviews provide the degree of the influence between concepts. This phase results in the creation of the integrated FCM model. 
- Quantitative Analysis includes the testing and validating the FCM model against the original group of participants in phase 1. It also include the evaluation of the FCM model by subject matter experts. This phase results in the creation of simulation model.

At the end of quantitative phase, the simulation model is ready to be used. Different scenarios representing the decision alternatives (Simpson, 1992) are planned and tested through FCM simulation to identify and prioritize the criteria/alternatives that lead to a higher rate of adoption. For example, using the interpretive model, one can identify and prioritize the characteristics of the health product that lead to better adoption among the focused consumer demographic.

Many of the challenges with the current methods are highlighted in different streams of the academic literature. These challenges include:

1. Grounding: Integrate first-hand user data (Phillips \& Zhao, 1993).

2. A need to approach the problem holistically: Cover a wide range of factors that emerge from the health context under study (Bagozzi, 2007; Head \& Noar, 2014; Ogden, 2003; Rothman, 2004).

3. Flexibility: Build flexible models that can be adjusted in response to new input (Jetter \& Kok, 2014; Kosko, 1988).

4. Latent Needs: Model latent needs (Phillips \& Zhao, 1993).

5. Need for visual formalisms: Provide cognitively simple visual representation of findings that people from different disciplines can comprehend (Creswell, 2013, ch.10). 
6. Scenario Planning: Model and simulate decision scenarios (Simpson, 1992) to identify and prioritize alternative decision points based on their potential to yield a higher adoption rate (Amer et al., 2013; Glenn \& Gordon, 2009; Schoemaker, 1991; Simpson, 1992).

7. Novel Insight: Develop the capacity to provide novel insights in relation to the predictions of extant theory, and beyond (Glanz et al., 2008; Head \& Noar, 2014; Ogden, 2003; Rothman, 2004).

8. Efficiency: The method needs to be practical in regards to consideration of monetary and temporal resources and constraints (Creswell, 2013, ch.10).

This dissertation aims to address these challenges by generating a modeling method that applies the most relevant theory for Health Technology Adoption (HTA) to visually unpack the high-level adoption constructs in complex contexts, where technology adoption is strongly influenced by agency. This decomposition is possible through qualitative data collection that provides flexibility and allows the participants to take the research through their journey of HTA. This approach leads to fresh insights by opening the black box of technology adoption, and revealing the intricacies of the paradigm in the context being studied.

To validate and demonstrate the capability of the method, this dissertation applies the method in the compelling context of EWHTA. The method deductively decomposes the abstract primal factors of the UTAUT theory for adoption of a preadoption study of a remote health monitoring technology among elderly women. 
The research also unfolds how the elderly women's gatekeepers, who are at the core of her environmental factors, influence the formation of those main constructs. While the method is inherently qualitative, it takes advantage of FCM quantitative power during the analysis phase and validation. 


\section{Literature Review}

\subsection{Framework of Elderly Women Health Technology Adoption}

Health Technology Adoption among elderly in general (Compagna \& Kohlbacher, 2014; Walsh, 2014) and among elderly women in particular (Kurniawan, 2007; Schaar \& Ziefle, 2011) is a complex phenomenon in which numerous groups of concepts interplay. The multidisciplinary nature of such context requires approaches that integrate perspectives from the many expertise involved (Eggermont et al., 2006; Ramón-Jerónimo et al., 2013). From personal and technical to social and organizational, it has been beyond the limit of a single school of thought to comprehend the full scope of factors involved. For many decades, various bodies of scholarly research in many fields, including gerontology, information systems, and human factor engineering (i.e. computer/human interaction), have broadly studied this process in part. However, until recently, little attention had been given to the differentiating roles of gender and demographics in health technology adoption.

The complexity of EWHTA is manifold. On the one hand, health related behaviors are more than often charged by a range of emotional factors, from fear and threat to mood and worries (Compagna \& Kohlbacher, 2014; Kurniawan, 2007; Walsh, 2014), and driven by personal, moral and social norms such as sense of identity, responsibility and culture (Schaar \& Ziefle, 2011). On the other hand, technological innovations require learning and changes to the status quo that mandate modification to the processes that people in general, and the elderly in particular, 
are used to and hence comfortable with (Mallenius et al., 2007). This presents a key challenge to elderly women, who have strong desire for ease of use, simplicity and familiarity (Cerella et al., 1982; Kline, 1987; Kline \& Schieber, 1982; Plude \& Hoyer, 1985; Walsh, 2014), combined with lack of self-efficacy (Karavidas et al., 2005; Wild et al., 2012). These key factors build a strong inertia against adopting such innovations. And finally, besides lack of desire, the effect of biological aging on cognitive decline (including loss of visual and auditory acuity) gradually weakens elderly's ability to recognize and use typical modern innovations, which more than often rely on subtle cues to present information (Morris \& Venkatesh, 2000). These inconveniences result in a natural resistance to technology acceptance among this demographic.

With the compelling need for adoption of health technologies among elderly, there have been mainly three streams of research that have progressively focused on aspects of technology adoption. As depicted in Figure 2 - 1, the three interrelated disciplines of Human Factor Engineering, Gerontology and Technology Adoption each have developed and/or adopted many methodologies to systematically optimize their research processes. However, such efforts have been fragmented, and hence their findings are limited (Wagner, Hassanein, \& Head, 2010). While these competencies' objectives seems to be different in many ways, in the narrow lens of research on EHTA, their overall goal and processes intersect (Rahimi et al., 2016). These literature streams are reviewed individually, in their EHTA 
application, in the following sections with the goal of reviewing the state of the art and identifying useful research techniques and gaps that require addressing.

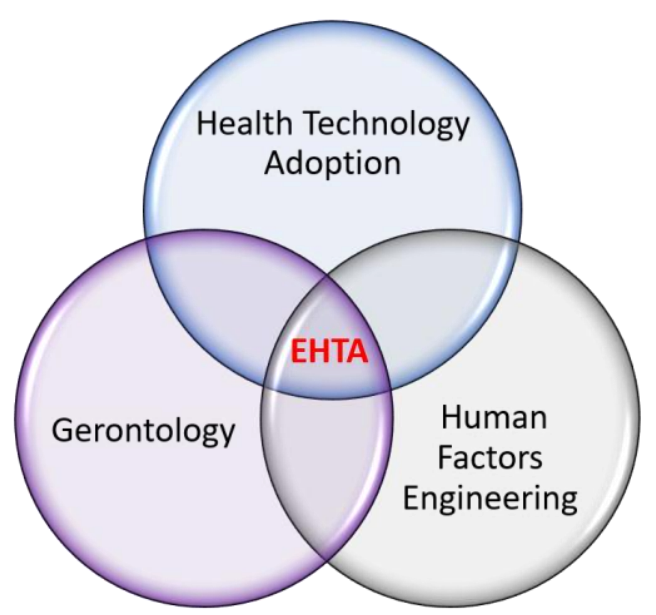

Figure 2 - 1 Elderly Health Technology Adoption is at the intersection of Gerontology, Health Technology Adoption and Human Factors Engineering

\subsection{Literature Review in Gerontology}

Since the work of Avicenna, "The Cannon of Medicine", in ancient Persia over 1500 years ago, humans have long been studying the effect of aging (Howell, 1987). Gerontology (i.e. study of old man) studies the physical, mental and social effects of aging. Through a multidisciplinary body of research, gerontology has gained an in depth understanding of different aspects of aging that can affect human behavior (Hooyman \& Kiyak, 2011). A comprehensive review of the gerontology literature that pertains to EHTA has been conducted in an earlier publication (Rahimi et al., 2016). This section discusses key aspects of that research, which specifically pertain to the proposed dissertation research. Please read the paper for a more comprehensive literature review of gerontology in relation to EHTA. 
Over the past few decades, waves of health innovations have offered the muchneeded benefits of quality, efficiency and cost savings to the health care field. However, a plethora of research papers attests to the low adoption rate of health technology among the elderly (Bowman et al., 2013; Eastman \& lyer, 2004; Maier et al., 2011; Mallenius et al., 2007; Ramón-Jerónimo et al., 2013; Renaud \& Van Biljon, 2008; Saborowski \& Kollak, 2014). The literature also suggests that technology adoption among the female elderly lags even further behind (Alburquerque-Sendin, et al., 2012; Bowman et al., 2013; Ehlers \& Huberty, 2013; Lim et al., 2011; Loe, 2010). Recent research suggests that there is still a gender gap in technology adoption (Dutta \& Omolayole, 2016; Kotze et al., 2016; Merkel et al., 2016). The effect of gender on the technology adoption rate is less experienced among the younger generations (Ling, 2002), and more pronounced among the elderly, where technology usage was found to be lower among older women (Ling, 2002). The research on gender difference in technology adoption supports the notion of a widening gap in the adoption rate between elderly women and elderly men (Merkel et al., 2016; Venkatesh and Morris (2000)). It suggests that the female elderly have a strong desire for ease of use, simplicity and familiarity. The research also showed that elderly women's strongest driver for technology adoption is ease of use, along with social norms.

A major body of literature highlights the different attitudinal dimensions that hinder technology adoption. Lack of self-efficacy with respect to technology adoption among elderly (Wild et al., 2012), which worsens with advancing age (Mallenius et 
al., 2007), is more developed among women (Zhang et al., 2014) and is particularly strong in older women (Karavidas et al., 2005; Wilkowska et al., 2010). The weak self-efficacy among the elderly (Wild et al., 2012) and women (Schaar \& Ziefle, 2011) was also a major factor that caused a range of negative attitudes toward technology adoption (Wilkowska et al., 2010). The research cited above plus the study of Karavidas et al. (2005) found anxiety to be a hindering factor in technology adoption. Other obstacles that are pronounced in EHTA are the resistance to technology change (Wilkowska et al., 2010) and satisfaction with mediocre, but old, familiar technologies (Loe, 2010, 2014). Since all these phenomena are well documented in the gerontology literature, they need to be considered in any future study of EHTA.

The gap between the body of research that has been performed independently in the field of technology adoption and in the field of gerontology leaves many questions about the process of adoption by the elderly unanswered. For instance, the elderly's need for simplicity and familiarity with their environment is well recognized in gerontology (Cerella et al., 1982; Kline, 1987; Kline \& Schieber, 1982; Plude \& Hoyer, 1985; Walsh, 2014) and is becoming an important usability factor in gerontechnology ${ }^{3}$ product design (Compagna \& Kohlbacher, 2014;

\footnotetext{
3 "Gerontechnology is an interdisciplinary field of scientific research in which technology is directed towards the aspirations and opportunities for the older persons. Gerontechnology aims at good health, full social participation and independent living up to a high age, be it research, development or design of products and services to increase the quality of life." ("What is Gerontechnology?," 2017) Fozard defines gerontechnology as the development and distribution
} 
Kurniawan, 2007). However, the study of technology adoption does not recognize these important effects of aging and their inhibitory influence on adoption behavior.

The aging process creates perceptual, cognitive and attitudinal barriers, which reduce the elderly's abilities, confidence and perception of fitness for learning new technologies (Plett \& Lester, 1991). As is known in gerontology, perception is a complex psychological process, and aging erodes both the speed and amplitude of perception (Minton \& Schneider, 1985). The elderly also tend to be slower learners (Sterns \& Doverspike, 1989b). An examination of 6,610 workers on jobrelated training, showed performance decline with age (Kubeck et al., 1996). The study found poorer training performance, less mastery of the learnt material and slower completion of final training task among older workers (Kubeck et al., 1996). These effects debilitate the elderly's own initiation of learning new technology, making their training and support needs even more important (Pan \& JordanMarsh, 2010).

Research also shows that the relative importance of adoption factors is different in elderly people than it is in younger ones. Younger persons tend to be driven by instrumentality, whereas the elderly are primarily affected by social influence and lack of self-efficacy (Morris \& Venkatesh, 2000; Morris et al., 2005). At the center of elderly's social influence with respect to technology adoption are the immense influence of the elderly's children, other family members and friends (Guynn, 2002;

of technologically based products, environments, and services that are intended to improve functioning and quality of life for aging and aged people (Fozard, 2001). 
Lee, 2007; Mallenius et al., 2007; Morrell et al., 2004). Research findings suggest that these technology gatekeepers frequently intervene in adoption, by promoting, providing, supporting and teaching the technology to the elderly (Kwon \& Chidambaram, 2000; Mallenius et al., 2007; Renaud \& Van Biljon, 2008; Silverstone \& Haddon, 1996). The role of these gatekeepers appears to be crucial; they may even become the reason for technology usage. The gatekeepers influence the elderly, by suggesting, by encouraging and by building the intention to adopt (Morrell et al., 2004). Many cases of initiating the usage of technology by the elderly were based on the combination of the gatekeeper's influence and the desire to stay connected with them (Carpenter \& Buday, 2007; Guynn, 2002; Lee, 2007; Malhotra \& Galletta, 1999). From promoting the utilitarian values of using technology, influencing elderly's choices, as well as, more than often, making the purchasing decision for the elderly and giving the technology to them, these caregivers are the primary agents that drives technology adoption (Carpenter and Buday, 2007; Conci et al., 2009; Renaud \& Biljon, 2008; Mallenius et al. 2007). In addition, these gatekeepers are often the primary source of the much needed facilitating condition because they provide continuous and convenient, but persistent technical support and training (Sterns \& Doverspike, 1989; Pan \& Jordan-Marsh, 2010).

In essence, to capture the environmental factors that affect elderly's adoption process, it is important to study the effects of the principal influencers of technology adoption, i.e. the gatekeepers who are often the elderly's children, family members 
and sometimes close friends (Mallenius et al. 2007; Carpenter and Buday 2007; Davis, 1995). The gatekeepers are the elderly's opinion leaders: they play the critical role in initiating and accelerating the adoption (Thomas, 1999), and they have the greatest impact on the elderly's adoption decision (Mallenius et al., 2007). They not only plant the seed of intention in the older people's minds; often they also start the adoption by gifting the technology (Renaud \& Van Biljon, 2008). Unless these major influences, which more than often make or break the adoption process, are investigated, building a holistic EHTA model is not possible (Compagna and Kohlbacher, 2014). ${ }^{4}$

A body of research suggests that, in addition to assistance with initiation, the gatekeepers perform an even more crucial role-maintaining the adoption by providing continuous training and support (Rahimi et al., 2016). This support strengthens elderly's self-efficacy and their ability to use the technology. Studies

\footnotetext{
${ }^{4}$ The children of the elderly are at the core of elderly care as they provide most of the elderly care (Metlife, 2011). However this caregiving comes at a big cost to them. This toll is manifold. Financially, they suffer due to decreased work hours and loss on many career-advancing opportunities, including passing up promotion, quitting jobs, retiring early or simply being away from work (National Alliance for Caregiving, 1999). And health-wise, they get physically and emotionally drained and experience higher level of health challenges as a result of caregiving (Dentinger \& Clarkberg, 2002).

Moreover, the ratio of children to elderly is decreasing dramatically due to reduced birth rates, which puts more pressure on these, often working, children (United Nations, 2013). This pressure not only stresses out the children, but also the observant elderly parent.

To a lesser degree, the friends of elderly are also a major group of unofficial caregivers (Caregiving in the U.S. 2015, 2015; Penning \& Wu, 2015).
} 
show that lack of continuous support leads to technology abandonment (due to unaddressed usability challenges), as some elderly gave the idle technology to grandchildren (Silverstone \& Haddon, 1996), and some others deserted them (Mallenius et al., 2007). The effect of the gatekeeper's training is even more salient in the case of elderly women, as Lee's research (2007) found that female users more than anything rely on the help of surrounding people to learn technology (as opposed to male users who often try it first).

A great body of the gerontology literature shows that at the center of elderly's social surroundings are family members and close friends who are the elderly's opinion leaders (Lin et al., 2013; Luijkx et al., 2015; Peek et al., 2015; Peek et al., 2014). They play the critical role both in initiating and in accelerating adoption (Luijkx et al., 2015; Thomas, 1999). Children have the greatest impact on the elderly's adoption (Mallenius et al., 2007). They are not only planting the seed of intention in the older people's minds; they are also intervening in the adoption process by gifting the technology (Renaud \& Van Biljon, 2008). Research also shows that close friends and family members (children and grandchildren in particular) strongly influence the elderly's adoption decision. This manifests itself in several dimensions: the elderly easily adopt the enthusiasm of their grandchildren for technology (Luijkx et al., 2015); they connect and communicate better with grandchildren when they use the technology (Luijkx et al., 2015; Rahimi et al., 2016); and they benefit from their grandchildren being a natural source of support (Barnard et al., 2013; Lin et al., 2013; Luijkx et al., 2015; Peek et al., 2014; 2015). 
Studying these key influencers of the elderly's technology adoption is a prerequisite for understanding the adoption. Yet, TA and gerontology research has hardly focused on these influences as a separate unit of analysis and beyond a general construct. The research of Chen and Chan (2011) highlights the need for EHTA research to include the abovementioned important biophysical and psychosocial factors to better understand technology adoption and intervene in the technology adoption process. Similarly, the systematic review of factors that influence EHTA concludes that more research is needed to capture the complexity involved in the acceptance of different types of technology for aging that is already in place (Peek et al., 2014).

Gerontology studies have yielded general cognitive insights related to adoption, but they have provided limited actionable information that pertains to the factors that specifically impact decision-making during the adoption of emerging technologies (Chen \& Chan, 2013). As a result, the field provides little guidance as to what technology designs are required to improve the expected ease of use and performance and as to which implementation strategies would lead to improved technology adoption. These practical needs are the driving force behind gerontechnology. While the gerontechnology literature primarily consists of research in new product development, it also includes a growing number of studies that present evidence of adverse manifold effects of aging on the use of technology (Hooyman \& Kiyak, 2011). 
Research in the field of gerontology has captured, in great depth, the social, cognitive, biological, and psychological impacts of aging. Elderly women, being the dominant elderly population, experience many challenges echoed by literature. These include attitudinal (Eastman \& lyer, 2004; Morris \& Venkatesh, 2000; Peine, 2009; Peine \& Herrmann, 2012; Wild et al., 2012; Wilkowska et al., 2010), informational (Peine, 2009; Peine \& Herrmann, 2012), perceptual (Cerella et al., 1982; Plude \& Hoyer, 1985; Wild et al., 2012) and cognitive barriers (Cerella et al., 1982; Kline, 1987; Kline \& Schieber, 1982; Plude \& Hoyer, 1985; Walsh, 2014) to technology adoption that often remain unaddressed and lead to rejection of the technology under consideration (Morris et al., 2005; Venkatesh et al., 2003). Beating this stumbling rate and overcoming the effect of these inhibiting forces is vital to paving the way for technology to deliver quality healthcare, and it necessitates investing major time and energy (Chappell, 1999). These underlying factors, which are driven by the social, psychological, cognitive, and biological effects of aging, are absent from the current HTA models. Without capturing these key barriers and drivers, those models are incapable of pinpointing the key factors to be addressed, in order to enable adoption.

In summary, gerontology provides limited insight into EHTA (Chen \& Chan, 2013, 2011; Peek et al., 2014). As a result, the field provides little guidance as to what technology designs are required to improve expected ease of use and performance. It also does not suggest which implementation strategies would lead to better technology adoption. Attempts to address such shortcomings have led to 
the emerging body of research in gerontechnology, which intersects the field of technology and gerontology. This cross-functional body of research has shed light on contextualizing technology for use by the elderly. However, the majority of these efforts have been on new product development, and there is much need for further research beyond innovation (Bouma et al., 2007).

The study of aging and its social, psychological, cognitive, and biological implications, in and of itself is aging; it has not been able to catch up with the rate of health technology innovations for elderly. This shortcoming has created a widening chasm between research on new product development in health-related devices and research on how the elderly may adopt such devices. This emerging gap invites multidisciplinary research that will incorporate the existing fragmented knowledge in the hitherto disparate fields of technology and gerontology, in the hope of gaining a more comprehensive understanding of EHTA. In fact, such a multidisciplinary body of research has started, but it mainly focuses on the

development of even more products (Bouma et al., 2007). This focus causes the gap between the understanding of product development for the elderly and the understanding of technology adoption by the elderly beyond the initial look and feel of the product to widen.

\subsection{Literature Review in Human Factor Engineering (HFE) and Human Computer Interaction ( $\mathrm{HCl})$}

Human Factors Engineering or Human Factors and Ergonomics (both abbreviated HFE) is the study and practice of applying the knowledge of human physic and 
psychology to design products for human use (human-factors engineering, 2016). Similarly, Human Computer Interaction $(\mathrm{HCl})^{5}$ is the study and practice of applying the knowledge from researching how people and computers. Some consider the two fields to be slightly different ("Human Factors vs Ergonomics - Are they the same?, 2005), but majority considers them the same and use the terms interchangeably. In the context of this dissertation, EHTA, both the $\mathrm{HCl}$ ("Association for Computing Machinery") and the HFE (“CHI 2016") literature refer to a similar body of knowledge and research. Therefore, $\mathrm{HFE}$ and $\mathrm{HCl}$ are going to be used synonymously and interchangeably in this text.

The prevalence of technology in human everyday life is the result of human factors engineering (Human Computer Interaction) research that has transpired over the past several decades (Myers, 1998). From the ubiquitous graphical interface used by Microsoft Windows 95 to the spectacular growth of the World-Wide Web, the recent widespread presence of technology is a direct result of the HFE body of research (Myers, 1998). As per ISO 6385, ergonomics and the study of human factors pertains to the understanding and the design of technologies that most effectively address human well-being (“ISO 6385," 2004). Hence HFE's contribution to adoption process has hinged on 1) designing and innovating

\footnotetext{
${ }^{5}$ Human-computer interaction $(\mathrm{HCl})$ is an area of research and practice that emerged in the early 1980 s, initially as a specialty area in computer science embracing cognitive science and human factors engineering. $\mathrm{HCl}$ has expanded rapidly and steadily for three decades, attracting professionals from many other disciplines and incorporating diverse concepts and approaches.("Human Computer Interaction - brief intro," 2017)
} 
technologies that would most likely be adopted, and 2) making awareness in communities that promoted the adoption of the products and services being designed (Myers, 1998).

With the ever growing global elderly population, a large portion of Human Factor Engineering (i.e. Human Computer Interaction) discipline is indeed focused on methodologies and best practices for designing and developing easy-to-use, highperformance products that are well-adapted toward the elderly's capabilities and limitations. However, as the main focus has been around technology design, considerations that hardly reach beyond early user experience and initial adoption (Bannon, 1991), thus lacking insights into how contextual settings and social influence impact adoption.

The study of technology adoption is so intertwined with technology design that studying one is often impossible without studying the other. Silverstone (Silverstone \& Haddon, 1996) has coined the widespread usage of a technology in society as Domestication. Silverstone, in the early 90's (Silverstone, 1994; Silverstone et al., 1994), posits that domestication is the critical component of completing the innovation life cycle. He further explored domestication for studying information and communication technology design and adoption (Silverstone \& Haddon, 1996). Domestication is emphasized as the critical process in which consumers define their own relationship with the innovation. The process of domestication of technology is categorized as: 1) Appropriation (obtaining), 2) Objectification (exploring), 3) Incorporation (experimenting), and 4) Conversion 
(consumption) (Silverstone \& Haddon, 1996). He states: "Domestication is anticipated in design, and design is completed in domestication" (Silverstone \& Haddon, 1996, p. 3). Hence, innovation and adoption make up the two sides of the product-life-cycle coin; the research for enhancing one side is often intertwined with addressing the other side too. Evidently, given the flux of attention to the lucrative market of aging health product innovation, there has been advancement in collecting user data, which can be used in HTA.

\subsection{Review of Known Data Elicitation Approach in Consumer Market Research}

The user-centered design methodologies aim to boost product adoption by facilitating the development of products such as health innovations that best address consumer needs. Conventional product development is conducted sequentially, from phase to phase. Functions are specialized, segmented and often performed with minimal interdisciplinary interaction. Driven by the requirement for speed and flexibility in the fierce competition of today's commerce, new holistic approaches gave life to user-centered design (UCD) philosophy. UCD more than ever pays attention to the needs, limitations and the preferences of the end users, and in relation to their living environments. One of the most pronounced changes in the evolution of new product development is the requirement gathering approach. The emerging flexible processes have become the cornerstone of the

emerging consumer product development process ("The Evolution of Market Research | RW Connect," 2014). 
The user-centered design methods are even more compelling for the aging consumers and particularly elderly women that are experiencing cognitive, biological and psychological challenges (Eastman \& lyer, 2004; Morris \& Venkatesh, 2000; Peine, 2009; Peine \& Herrmann, 2012; Wild et al., 2012; Wilkowska et al., 2010). The process of centering the product design around the elderly ensures its 'ease of use', which is the most important HTA factor among elderly women (Arning \& Ziefle, 2007; Minton \& Schneider, 1985; Morris \& Venkatesh, 2000; Ong \& Lai, 2006; Venkatesh \& Morris, 2000). The traditional product design philosophy, which generally relies on consumers changing their behavior to adopt, has evidently led to the wave of rejected products among elderly women. The premise of UCD, which centers product design and development on the elderly's cognitive, biological and psychological challenges, can break this vicious cycle. The greater the understanding of the elderly needs and desires, the greater the possibility of adopting the resulting products. This understanding can be utilized during the entire development/production process, including requirement gathering, concept generation, pre/mid and/or post-production marketing and roll out planning.

A meta-analysis of the recent health product design initiatives underlines seven categories of techniques used for the development of adoptable product (Rahimi \& Ibarra, 2014). These methods all focus on the end user's needs and desires, but some are more involved than others. These methods are discussed to identify the current challenges and opportunities in conceptualizing appropriate methods to 
research HTA among different demographics. The meta-analysis suggests that unframed inquiry methods like ethnography are the best UCD techniques for elderly population, as it allows new insight into the elderly's changing needs and unveils the details of the elderly social influences. For more detailed information, please read this earlier publication by Rahimi \& Ibarra (2014).

Consumer market research often relies on reviewing the literature to identify the needs and preferences of the elderly in their healthcare decision making from the result of prior studies. Sampietro-Colom et al. (2004) reviewed Medline and Medical Decision Making Journal publications to elicit women's preferences in their health care. Satariano et al. (2014) conducted a narrative review of research, practice, and policies from multiple fields, including information science, gerontology, engineering, housing and social services, health care and public health to obtain a holistic view for improving access and wellness in older populations. Najman and Levine's (1981) review of the literature highlighted the criticism of health care by new technologies that often prioritize life longevity over quality of life considerations. Their findings stressed the need for integration of the complex interplay of various subjective factors from different streams of studies on quality of life.

Literature. The more comprehensive streams of research consider literature as the initial and/or supplementary step and an effective way to explore prior findings. While starting research by conducting literature review is the common practice, there are some research that literature is their only source of information. The 
former type of literature review as the starting point has increasingly become the state of the art in health innovation studies for elderly, as it facilitates the integration of fragmented knowledge from various streams of research. O'Maraa et al. (2012) used literature review as a knowledge base for their qualitative study, which had two goals: raising awareness of technological intervention for managing diabetes among elderly Vietnamese women in Australia and learning lessons for future similar practices. They developed a framework in which workshops and interactive information sessions were held with the participants. This served as an effective venue to educate the community; it also brought forward fresh insights for enhancing and improving a technological intervention that resulted from the thematic qualitative analysis of those recorded sessions. In fact, many of the recent studies integrate a literature review into their studies for drawing on past systematic methods and learnings to analyze factors that are key to health technologies (Cugelman et al., 2011; Maniam et al., 2015). Evidently, a literature review provides an efficient venue to collect, integrate and build upon previous bodies of information. However, it is not sufficient for studying the troubled EHTA, a field of study that begs new insights.

Survey. The consumer market body of research contains an abundance of research conducted by survey techniques. Survey is the most popular method in gathering public information in marketing, psychology, and sociology as well as health research. 
Survey methodology provides an applied statistical method, which gathers, measures and draws inference from subjective opinion about specific constructs from a sample population. Survey instruments that are delivered via mail, phone or email/Internet have been the most popular for comparative exploratory studies in HTA. A great number of elderly health technology studies use a survey questionnaire as a quick way to capture data pertaining to behavior and environment (Liddy et al., 2008) or prioritize the value of key attributes for design requirements (Hildebrandt et al., 2015). Surveys have also been used to explore the elderly's perception of eHealth technologies (Best et al., 2015; Or et al., 2011) or find trends for elderly healthcare interventions (Xue et al., 2012). The survey instrument has been used in many exploratory studies, including the study of ageing women's preferences (Daley et al., 2011), the perception of eHealth mobile applications (Xue et al., 2012) and web-based eHealth programs (Uchida et al., 2001). Throughout these studies, it is evident that the primary issue with exploratory and descriptive survey methods is their assumption of key factors to study. Survey is a relatively a low cost technique to capture and analyze information about known factors from a large population. Nonetheless, the quality of the survey relies heavily, among other factors, on the quality of its design, on the competency of the surveyor, and on the appropriateness and engagement of its participants. This dependency makes a survey an unreliable and unfit for the study of HTA among the elderly. As elaborated earlier, the understanding of EHTA demands the discovery of novel concepts that emerge from the intersection of 
personal, societal and technological aspects of the EHTA. A novel approach called 'exploratory survey', which attempts to address the issue of presumptions in survey technique, has become increasingly popular among the users of survey methods (Pinsonneault \& Kraemer, 1993).

Systematic Observation obtains contextual information about the usage of the products in the natural environment. This qualitative technique has become a more popular alternative to the more quantitative techniques such as structural questionnaires and surveys (Ritchie, et al., 2013). There are different variations of observational research: overt, covert and active participation. In covert observation, the researchers do not identify themselves. In overt observation, the researchers identify themselves, as well as the purpose of the observation. In active participation, the researcher take an active part in the usage of the product to obtain more refine and first hand observational information.

Some observational techniques (for example, Portable Ergonomics Observation (PEO) method (Fransson-Hall et al., 1995)) are particularly beneficial for HFE applications where the assessment of high-risk issues need immediate attention. For example, during the postural observation of a new product prototype, any highrisk posture issue can be immediately identified and corrective action taken to fix the issue (Stanton et al., 2004).

An Interview is a more flexible inquiry method than the more restricted quantitative techniques such as surveys. It's one of the original and most popular methods of capturing information across different fields and its main purpose is to seek the 
person's opinion or perspective about the subject being studied (Bull \& Memon, 2000). An interview can take many forms. Depending on the research goal, an interview can be conducted in a completely unstructured format of random questioning, a semi structured way of allowing the interview to deviate from the path of the enquiry, or a fully structured and planned questioning and answer session, in which the answers are recorded and in which asking additional questions is allowed. Interviews are generally organized in three steps. They begin with open questions, which are followed by probing questions, and they end with closing questions (Stanton et al., 2004).

Verbal Protocol Analysis studies thinking in various branches of scientific research including behavior analysis (Austin \& Delaney, 1998), cognitive psychology (Crutcher, 1994) and cognitive science (Simon \& Kaplan, 1989). It gathers (the transcripts of) verbal reports and analyzes their contents to capture a person's cognition. Unlike interview techniques in which a participant is asked to answer questions, Verbal Protocol Analysis is usually conducted during the usage behavior, where the user is asked to think out loud while doing the task (Bainbridge \& Sanderson, 1995). Similar to grounded theory coding, the transcripts can be coded and analyzed in various specificity of analysis, from words or phrases to less detailed forms of coding like sentences or themes. To find the schemes, Stanton et al. $(2004,277)$ say: "Encoding the verbalizations requires the development of a mutually exclusive and exhaustive categorization scheme, which should first be tested for reliability before it is used to analyze the transcripts." 
The focus group is one of the most popular HFE and marketing data collection methods. It is a form of group interview. The goal of this method is to provide an effective way to quickly obtain information from a number of people in a welcoming and interactive way. This method has been shown to provide a safe venue for people to freely talk in response to the open ended questions raised (Stanton et al., 2004), and it is a good method for eliciting subjective data (Nielsen, 1994). However, it potentially limits data gathering. For example, participants with opinions that oppose those of the majority of the group may withhold their opinions. So may participants that consider the subject under study as too sensitive (Corlett et al., 1995, Chapter 7). When it comes to product design, an additional disadvantage of the focus group method is the fact that people are not good at imagining using a product concept that does not exist yet. This has led to the birth of "scenario-based focus group", where, in a scenario, users are asked to imagine the product being designed by connecting to an existing product (Cooper et al., 2003). This would allow the participants to collaboratively develop and incrementally enhance each other's ideas toward the product design (Corlett et al., 1995).

The repertory-grid is a technique designed to collect people's constructs, i.e. what people think about a particular topic. It is a grounded theory approach, which means that, in a bottom-up approach, the data emerges entirely from the participant. The repertory-grid is based on George Kelly's Personal Construct Theory (George Kelly, 1955), which posits that people's opinion of things around 
them are based on a collection of similar and different dimensions (i.e. personal constructs). The research is broken down to three stages - 1) observation; 2) emergence of the patterns (of similar, different and opposing artifacts); and 3) hypothesis and formation of constructs. In HFE, the resulting constructs are used as the criteria to evaluate products. The advantage of this technique is the removal of the researcher's interference from the collected perceptions. This technique has been deployed in many fields, including information systems, to obtain a fresh set of user attributes that act as evaluation criteria to ensure that the users' expectations are met (Whyte Bytheway, 1996). The repertory-grid can serve as a powerful knowledge capture technique because of its emphasis on allowing the person to interpret and explain\& about her or his experience. The unframed nature of the method allows the flow of novel information. A repertory-grid contains four parts: 1) a topic, 2) a set of elements, 3) a set of constructs, and 4) a set of ratings of elements on constructs.

In summary, ergonomists will work somewhere between the poles of scientists and practitioners with approaches based on the problem at hand (Stanton et al., 2004). Due to the reusability of their scientist-practitioner models, HFE methods are useful. There are a variety of methodological choices used in different stages of HFE product design before the development follows. These stages, which are similar across the board, are: 1) to collect, 2) to analyze and interpret, 3) to prioritize, and 4) to conceptualize the customer needs into the deliverables (Ulrich \& Eppinger, 2004). While the earlier methodologies have mainly made inferences 
from the cognitive science, the more modern ones rely on direct involvement that is centered on the users and inquiry and incorporation of their feedback into the process. The evolution of HFE has reversed innovation efforts from enhancing user experience by better technology design to enhancing technologies by designs based on the users' needs.

Despite this gradual evolution toward superior approaches, most of these methods still lack the capacity to holistically understand the elderly's paradigm and look beyond the initial phases of adoption (Hornbaek, 2006). The HFE approaches also miss the whole picture, as they only focus on the perceived needs and/or the delivery of features that the technology mandates. The key issue with such methods is that they cannot facilitate the discovery of new factors that need to be addressed. Newer methods such as ethnography do facilitate the emergence of new information and the capture of the whole picture. However, full ethnographic methods are very expensive; they demand a high degree of effort. Additionally, since they require prolonged longitudinal studies, they face the risk of attrition of participants (Dumas \& Salzman, 2006).

\subsection{Literature Review in Health Technology Adoption}

A cursory data mining of existing publications in HTA (see Appendix A) suggests that the first few decades of studies in this field have mainly focused on organizational factors, for the innovations were preeminently centered on automation and enhancement of processes in clinical settings. The data mining tables in the Appendix A highlight the prevalence of studies at the organizational 
level. They cover themes such as organizational readiness, rural hospitals and regional health delivery.

Another glimpse at the published articles in HTA (Figure A - 2 Citespace Cluster Detail of earliest 500 articles in HTA, and Figure A - 3 Subject Category of the earliest 500 articles in HTA) also suggest that elderly health technology adoption has hardly been researched in the earlier body of research. This limited search has only revealed 25 articles (from the 894 found) in gerontology and geriatrics gerontology categories of the Web of Scicence. While this is a very limited search, it is a reflection of the scarcity of early EHTA research in the context of the HTA body of research.

Rahimi and Jetter conducted an extensive review of the technology adoption literature (Rahimi \& Jetter, 2015), which looked at extant theories and their applications in health technology adoption. They found that the eleven most popular technology adoption-related theories (depicted in Figure 2 - 2) fell into two categories: human behavior theories and technology adoption theories. Human behavior theories under study included Social Cognitive Theory (SCT), the Theory of Reasoned Action (TRA), the Theory of Planned Behavior (TPB), the Reasoned Action Approach (RAA), and the Hierarchical Model of Intrinsic and Extrinsic Motivation (HMIEM). Technology adoption theories under study included the Technology Acceptance Model (TAM), the Model of PC Utilization (MPCU), Innovation Diffusion Theory (IDT), the Combined TAM \& TPB (C-TAM-TPB) theory, TAM2 and the 'Unified Theory of Acceptance and Use of Technology' 
(UTAUT). This section provides a summary of this literature review (for the complete report of the study please read (Rahimi \& Jetter, 2015)). This study covered hundreds of health technology applications of these theories. The study demonstrates that these theories are able to provide a sound foundation for HTA research. However, the study also demonstrates that these theories are unfit to capture the complexity of the healthcare context holistically.

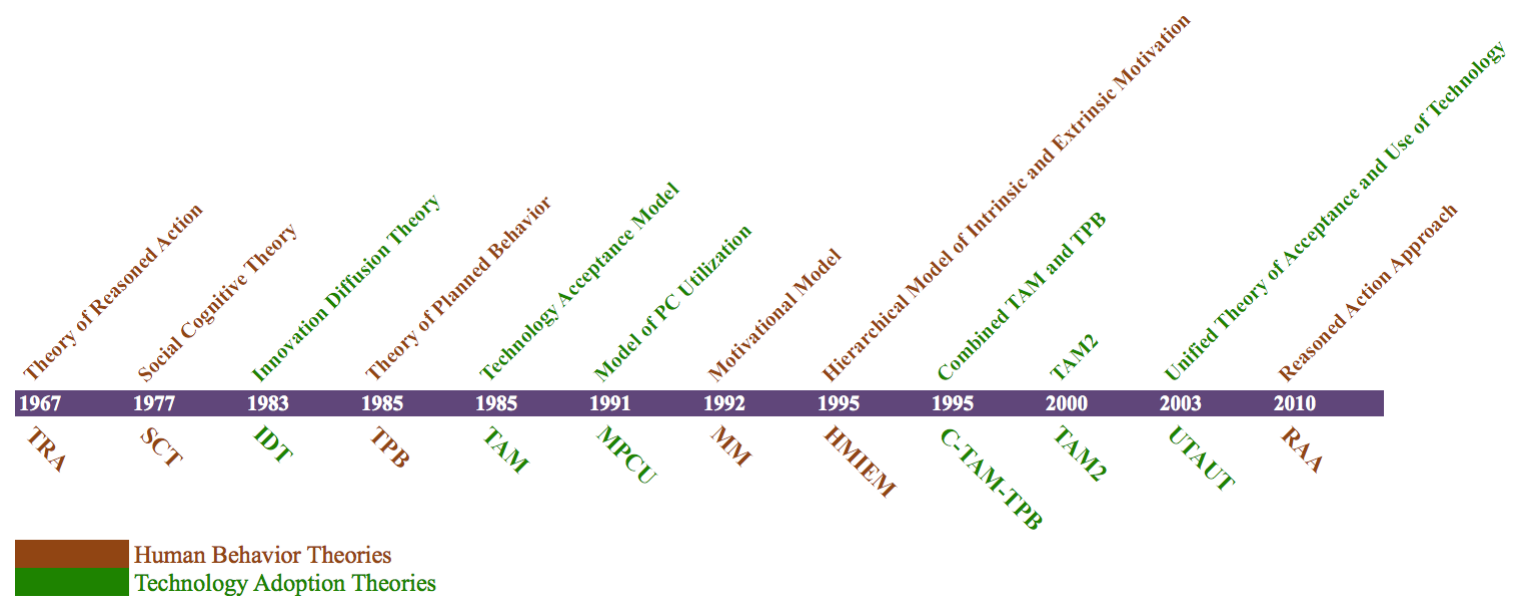

Figure 2 - 2 The reviewed popular theories applied in Health Technology Adoption

\begin{tabular}{|c|c|c|}
\hline Models & Model Description & Factors \\
\hline \multirow{2}{*}{$\begin{array}{l}\text { Theory of Reasoned } \\
\text { Action (TRA) } \\
\text { (Fishbein \& Ajzen, 1975) }\end{array}$} & \multirow{2}{*}{$\begin{array}{l}\text { Behavior intention is determined by person's } \\
\text { attitude and subjective norm(s). }\end{array}$} & $\begin{array}{l}\text { Attitude toward } \\
\text { Behavior }\end{array}$ \\
\hline & & Subjective Norm \\
\hline \multirow{3}{*}{$\begin{array}{l}\text { Theory of Planned } \\
\text { Behavior (TPB) } \\
\text { (Ajzen, 1985) }\end{array}$} & \multirow{3}{*}{$\begin{array}{l}\text { Behavior intention is determined by a } \\
\text { combination of person's attitude, subjective } \\
\text { norm(s) as well as perceived behavioral control }\end{array}$} & $\begin{array}{l}\text { Attitude toward } \\
\text { Behavior }\end{array}$ \\
\hline & & Subjective Norm \\
\hline & & $\begin{array}{l}\text { Perceived Behavioral } \\
\text { Control }\end{array}$ \\
\hline \multirow{2}{*}{$\begin{array}{l}\text { Technology Acceptance } \\
\text { Model (TAM)(Davis, 1985) }\end{array}$} & \multirow{2}{*}{$\begin{array}{l}\text { Technology acceptance behavior is driven by } \\
\text { perceived usefulness and perceived ease of use }\end{array}$} & Perceived usefulness \\
\hline & & Perceived ease of use \\
\hline \multirow{2}{*}{$\begin{array}{l}\text { Combined TAM and TPB } \\
\text { (C-TAM-TPB) } \\
\text { (Taylor \& Todd, 1995) }\end{array}$} & \multirow[t]{2}{*}{ TAM + TPB } & $\begin{array}{l}\text { Attitude toward } \\
\text { Behavior }\end{array}$ \\
\hline & & Subjective Norm \\
\hline
\end{tabular}




\begin{tabular}{|c|c|c|}
\hline & & $\begin{array}{l}\text { Perceived Behavioral } \\
\text { Control }\end{array}$ \\
\hline & & Perceived usefulness \\
\hline \multirow{6}{*}{$\begin{array}{l}\text { Model of PC Utilization } \\
\text { (MPCU) (Thompson et al., } \\
\text { 1991) }\end{array}$} & \multirow{6}{*}{$\begin{array}{l}\text { Uses of information technology are driven by } \\
\text { complexity, job-fit, long-term consequences, } \\
\text { affect toward use, social factors and the } \\
\text { facilitation conditions catering to the usage }\end{array}$} & Complexity \\
\hline & & Job-fit \\
\hline & & $\begin{array}{l}\text { Long-term } \\
\text { consequences }\end{array}$ \\
\hline & & Affect toward use \\
\hline & & Social factors \\
\hline & & $\begin{array}{l}\text { Facilitating } \\
\text { conditions }\end{array}$ \\
\hline \multirow[t]{2}{*}{$\begin{array}{l}\text { Motivational Model } \\
\text { (MM)(Davis et al., 1992) }\end{array}$} & \multirow[t]{2}{*}{$\begin{array}{l}\text { Rooted in psychological research and adapted to } \\
\text { the use of information technology, the extrinsic } \\
\text { and intrinsic motivations are key determinants in } \\
\text { IT adoption }\end{array}$} & Extrinsic motivation \\
\hline & & Intrinsic motivation \\
\hline \multirow{7}{*}{$\begin{array}{l}\text { Innovation Diffusion } \\
\text { Theory (IDT) } \\
\text { (Rogers, 2010) }\end{array}$} & \multirow{7}{*}{$\begin{array}{l}\text { Rooted in sociology, it has been applied to many } \\
\text { fields including IT. The theory highlights the } \\
\text { significance of relative advantage, ease of use, } \\
\text { image, visibility, compatibility, result } \\
\text { demonstrability and voluntariness of use as the } \\
\text { major determinants of individual technology } \\
\text { acceptance }\end{array}$} & Relative advantage \\
\hline & & Ease of use \\
\hline & & Image \\
\hline & & Visibility \\
\hline & & Compatibility \\
\hline & & $\begin{array}{l}\text { Results } \\
\text { demonstrability }\end{array}$ \\
\hline & & Voluntariness of use \\
\hline \multirow{5}{*}{$\begin{array}{l}\text { Social Cognitive Theory } \\
\text { (SCT) } \\
\text { (Bandura, 1986) }\end{array}$} & \multirow{5}{*}{$\begin{array}{l}\text { One of the most researched theories based on } \\
\text { social learning that has also been adapted to IT } \\
\text { usage. It identifies self-efficacy, performance } \\
\text { expectation, affect and anxiety to use as the } \\
\text { prominent factors driving usage. }\end{array}$} & Performance \\
\hline & & $\begin{array}{l}\text { Personal outcome } \\
\text { expectation }\end{array}$ \\
\hline & & Self-Efficacy \\
\hline & & Affect \\
\hline & & Anxiety \\
\hline \multirow{4}{*}{$\begin{array}{l}\text { Unified Theory of } \\
\text { Acceptance and Use of } \\
\text { Technology (UTAUT) } \\
\text { (Venkatesh et al., 2003) }\end{array}$} & \multirow{4}{*}{$\begin{array}{l}\text { Designed to best predict the usage of technology } \\
\text { and combines the findings of the earlier models. } \\
\text { It not only identifies performance expectancy, } \\
\text { effort expectancy, social influence and facilitation } \\
\text { conditions, but also posits that age, gender, } \\
\text { voluntariness to use and experience are } \\
\text { moderators explaining the usage of technology }\end{array}$} & $\begin{array}{l}\text { Performance } \\
\text { expectancy }\end{array}$ \\
\hline & & Effort expectancy \\
\hline & & Social influence \\
\hline & & $\begin{array}{l}\text { Facilitating } \\
\text { conditions }\end{array}$ \\
\hline
\end{tabular}

Table 2 - 1 The Most Popular Technology Adoption Theories in HTA

The theories in Table 2 - 1 are too parsimonious and simple to shed much light on

the issue at hand. They are also unable to provide actionable interventions in the 
multifaceted environment of healthcare settings (Bagozzi, 2007). For instance, while TAM (Davis, 1985) has delivered spectacular performance in general organizational settings (Bagozzi, 2007; Williams et al., 2011), it has been too simple and less than comprehensive in healthcare applications (Glanz, Rimer, \& Viswanath, 2008; Y. Lee et al., 2003).

Most of these theories assume that the intention to adopt (adoption intention) is the sole driver of the actual adoption behavior, but practice proves them wrong (Bagozzi, 2007; Glanz et al., 2008; Lim et al., 2011). A meta-analysis of 47 tests that investigated the effect of change of intention on change of behavior showed that a medium to large-sized change in intention only created a small to medium change in behavior. Therefore, the future behavioral changes should consider the non-intentional paths to action like automotive and prototype perception (Webb \& Sheeran, 2006). Additionally, it has been shown that, in health decisions, past behavior directly affects the intention to use, which current theories (Fishbein \& Ajzen, 2011; Gaither et al., 1996) do not capture.

Many of the significant challenges in the study of technology adoption/acceptance in healthcare, such as social influences (Pasick et al., 2009; Wentzel \& Wigfield, 1998), emotional factors (Baumgartner et al., 2008; Clore \& Huntsinger, 2007) and demographic characteristics (Schaar \& Ziefle, 2011; Venkatesh et al., 2000; Wilkowska et al., 2010), are not covered by the theories in this space (Albarracín et al., 2005; Wild et al., 2012). For example, age and gender are particularly important factors in technology adoption. Perceived usefulness is the most 
significant determinant of adoption among males in general, whereas perceived ease of use and social norms are the key drivers in that determine intention to use among the female elderly (Morris et al., 2005; Venkatesh \& Morris, 2000).

Research into 47 applications of social cognition theories (published between 1997 and 2001) suggested shortcomings in their conceptual contributions (Ogden, 2003). (These included the Theory of Reasoned Action -TRA (Fishbein \& Ajzen, 1975) and the Theory of Planned Behavior - TPB (Ajzen, 1985).) Specifically, Ogden's analysis showed that the conclusions obtained from such studies were more than often true by definition as opposed to by the observations derived from those studies (Ogden, 2003).

Traditionally, adoption theories have been conceptualized for organizational settings, and they have not really undergone any significant changes over the past few decades (Bagozzi, 2007; Ogden, 2003). However, over time EHTA has expanded beyond organizational issues. It involves social influences (Pasick et al., 2009; Wentzel \& Wigfield, 1998), emotional factors (Baumgartner et al., 2008; Clore \& Huntsinger, 2007) and more complex personal factors (Lee, 2007; Renaud \& Van Biljon, 2008). Existing theories provide little insight into these factors; they need to be expanded to cover many important aspects of EHTA. This difference between the current theoretical body of knowledge and the capability to understand EHTA is a gap that requires attention.

Many researchers have tried to address the gap. Their efforts have resulted in several integrated theories such as C-TAM-TPB (Taylor \& Todd, 1995) TAM2 
(Venkatesh \& Davis, 2000), RAA (Fishbein \& Ajzen, 2011), UTAUT (Venkatesh, 2003). The latest product of this theoretical evolution is the UTAUT. However, the UTAUT has proved to be less than comprehensive in explaining adoption in the healthcare context (AbuShanab et al., 2010; Aggelidis \& Chatzoglou, 2009; Bagozzi, 2007; Luo et al., 2010; Robson \& Sutherland, 2012; Williams et al., 2011).

The review of the health technology adoption literature demonstrates that, despite the abundance of studies in modeling health behavior in relation to technology (see Rahimi \& Jetter 2015), there are no major contributions in methodologies that model HTA (Crosby \& Noar, 2010; Noar \& Zimmerman, 2005). The academic literature overwhelmingly supports the use of proven theories as the most appropriate first steps to inform health research. This practice provides a strong theoretical foundation that is based upon knowledge of key factors that have been accumulated over four decades of studies of human behavior (Glanz et al., 2008). However, the intricacies of complex applications, like those of healthcare, necessitate taking additional steps. These steps should expand theories to create realistic models that can reflect many concepts and conditions specific to the issue at hand. The very parsimonious nature of the organizationally based theories doesn't allow for HTA-specific details, which compromise their utility in favor of generalizability (Bagozzi, 2007). Although their simplicity make these theories generalizable, when it comes to empirical studies, meta-analyses demonstrate that utility trumps generalizability (Chalmers, 1982). This inherent simplicity of the current theories has created inconsistent results, often leaving much of the 
variances in the human behavior unexplained (Karsh, Escoto, Beasley \& Holden, 2006; Ogden, 2003; Williams et al., 2011).

To address this shortcoming, researchers have tried to create more comprehensive models using two primary approaches: 1) Integrating several theories that are perceived to contain the most important factors relevant to the HTA application at hand (Chau \& Hu, 2002; Holden \& Karsh, 2010; Taylor \& Todd, 1995; Venkatesh \& Davis, 2000), and/or 2) using a theory as the base for the model and supplementing it with the additional key factors (AbuShanab et al., 2010; Aggelidis \& Chatzoglou, 2009; Duyck et al., 2010; Luo et al., 2010; Robson \& Sutherland, 2012; Samoutis et al., 2008; Williams et al., 2011). These approaches are effective in that they use the existing theories as existing knowledge. However, the outcome of these approaches is inconclusive, and the validity of the added factors depends on the level of knowledge of the research team.

Najman and Levine (1981) showed that there is an interrelationship between the various factors that determine technology adoption behavior. This would indicate that most technology adoption theories including the UTAUT are incomplete. A study by Rahimi et al. (2016) has documented that social influence impacts all the other input factors of the UTAUT (Figure 2 - 3). The UTAUT has to incorporate these interactions, in order for it to explain HTA. These interactions need to be captured in a model so that the expanded version of the UTAUT provides a better understanding of decision making in HTA. 


\section{Input Constructs}

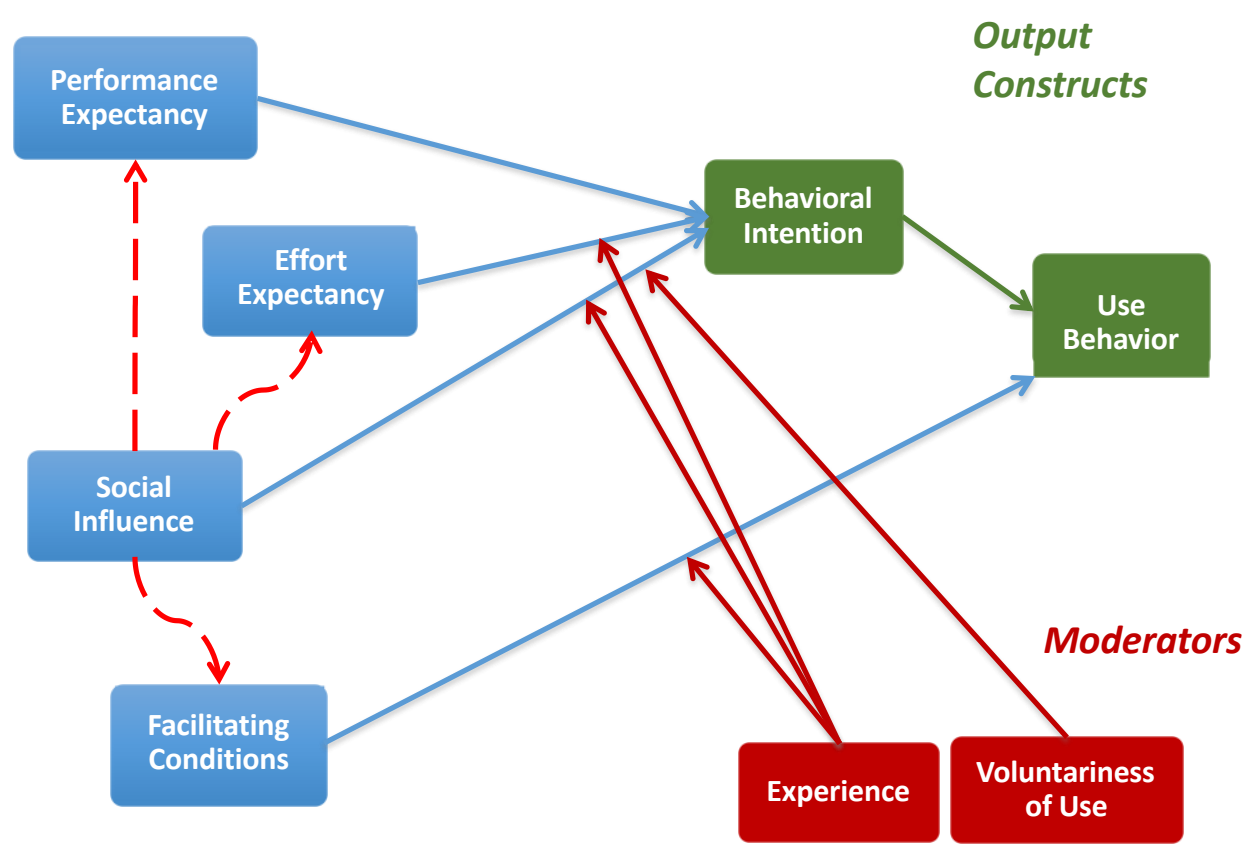

Figure 2 - 3 UTAUT with Social Influence influencing all other input factors (Rahimi et al., 2016)

A meta-analysis of health behavior research found that the performance of adoption theories varies across different health applications, as the correlation between the input and output factors depended on to the type of the health behavior being studied (Godin \& Kok, 1996; T. Webb, Joseph, Yardley, \& Michie, 2010). This finding highlights the need for modeling methodologies that can capture the specifics of HTA application. It should also cover the dynamic interplay between factors that are specific to the context being studied.

Reviewing decades of technology adoption studies provides little evidence of methodological pluralism (Crosby \& Noar, 2010; Ogden, 2003; Painter, Borba, Hynes, Mays, \& Glanz, 2008). This shortcoming exists in various complex multistakeholder fields, but it is more pressing in the study of HTA (Head \& Noar, 2014). 
This highlights the previously discussed need for applied research methods that lead to actionable insights in HTA studies (Bagozzi, 2007; Rothman, 2004). The recommendation is for the body of knowledge to be continuously informed and evolved by new observations to effectively explain, predict and intervene health behavior (Head \& Noar, 2014). The recommendation is to develop data-driven theories that are "more effective at explaining and predicting health behaviours as well as improving the ability of our interventions to change health behaviour" (Head \& Noar, 2014, p. 49).

Furthermore, when it comes to observation and interpretation, the occasional bias of the research team has also created adverse effects. There have been numerous reports of justification, rather than validation of the shortcomings of theories (Mook, 1983; Ogden, 2003). This has further reduced the practical value and the contribution of such studies (Head \& Noar, 2014; Mook, 1983). Additionally, aside from the influence of the researcher on the result (gatekeepers effect), using poor enquiry methods can also change the observation and undervalue its contribution (Bagozzi, 2007; Ogden, 2003). For instance, the design of a questionnaire with new information for participants can change or create, as opposed to access, peoples' opinion (Bagozzi, 2007; Ogden, 2003). To avoid the interference and presumption that obstruct the view to the mental model involved in the adoption decision making, the use of user-centered inquiry methods like ethnography becomes important, as their nature of observation avoids framing of the enquiry (Rahimi \& Ibarra, 2014). 
It has been common practice to conduct studies that stress the importance of basing research on proven theories. (Bagozzi, 2007; Head \& Noar, 2014; Karsh et al., 2006). As Glanz et al. (the authors of a highly cited book: "Health behavior and health education: theory, research, and practice") state: "the best theory is informed by practice; the best practice should be grounded in theory" (Glanz et al., 2008). A review of the HTA literature (Rahimi \& Jetter, 2015b), which evaluated the eleven most popular technology adoption theories, reaffirmed the importance of theoretical knowledge as the foundation for conducting technology adoption research. However this literature review also highlighted an array of shortcomings and challenges in researching health technology adoption. These issues, which are summarized in the Table $2-2$, range from theoretical unfitness and empirical challenges to quality issues. 


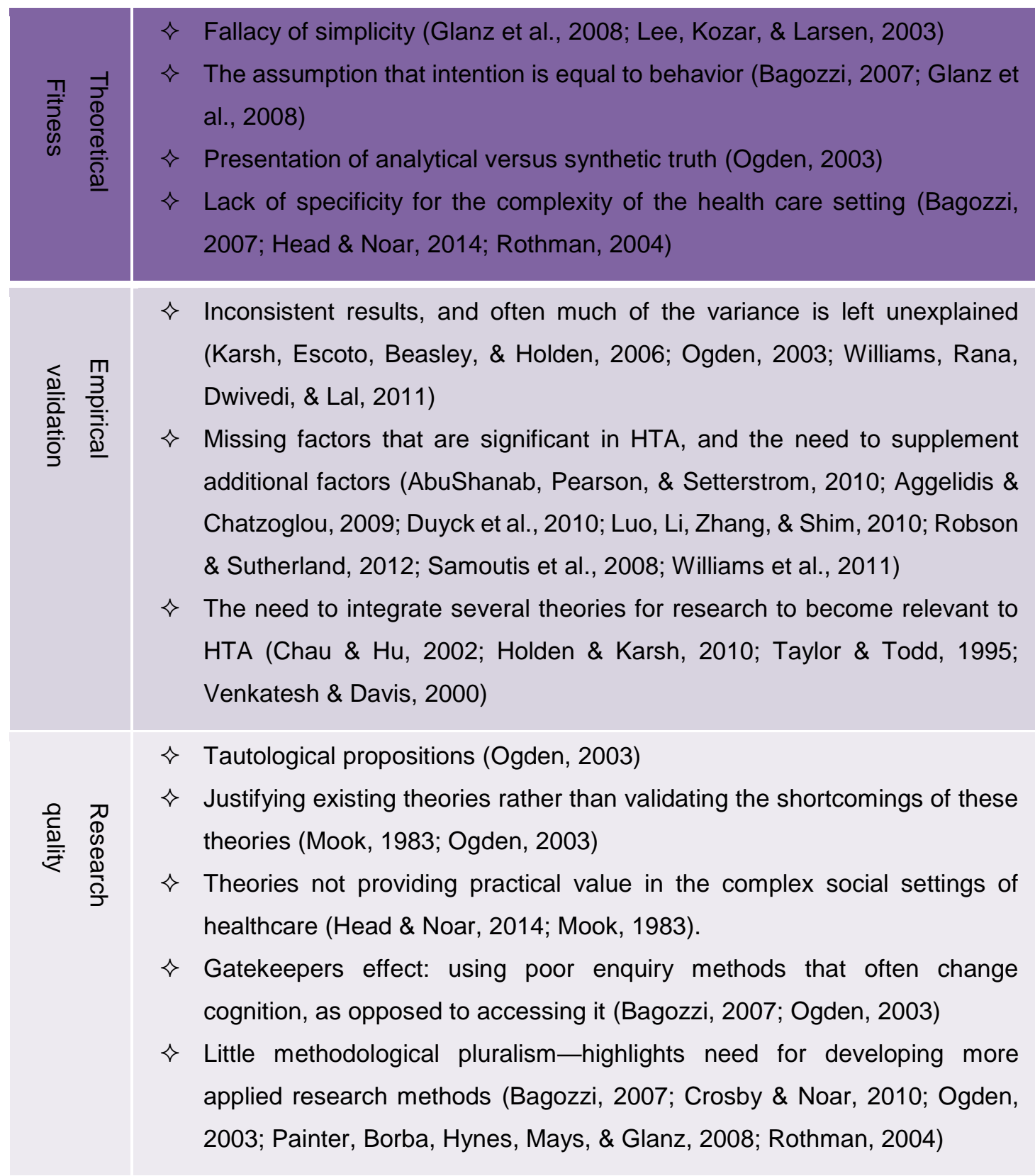

Table 2 - 2 Challenges in HTA research

In summary, a plethora of research complains about the lack of novel methodologies for studying HTA, and it reports that the era of having one unified model that can generalize the technology acceptance across applications might 
be over (Bagozzi, 2007; Godin \& Kok, 1996; Head \& Noar, 2014; Ogden, 2003). Technology adoption studies in organizational settings are becoming a thing of the past. For example, prior to $2003,27 \%$ of TA research has been around TA in organizations (Lee et al., 2003). Since the advent of the UTAUT in 2003, only a very small percentage $(2.3 \%)$ of research has been around organizational technology adoption (Williams et al., 2011). Instead, there has been a pressing need to understand the interplay of factors that are involved in the adoption of emerging health innovations that are revolutionizing healthcare delivery. (For complete review in technology adoption literature, please read Rahimi \& Jetter's publication from (2015).)

Extensive reviews of the EHTA literature show that technology adoption field provides limited guidance for elderly healthcare (Karsh et al., 2006; Williams et al., 2011). This is due to three major reasons:

I. Complex multi-stakeholder settings of healthcare are not sufficiently understood (Crosby \& Noar, 2010; Johnson, 2011; Ogden, 2003; Painter et al., 2008; Rothman, 2004);

II. The factors highlighted in TA literature are too generalized to provide empirical value in the context of healthcare (Bagozzi, 2007; Head \& Noar, 2014; Rothman, 2004); and

III. Key factors of the elderly decision making process are missing (Samoutis et al., 2008; Taylor \& Todd, 1995; Venkatesh \& Davis, 2000). 
In order to fill this knowledge gap in EHTA, promising interdisciplinary research has already begun (e.g. Council \& others, 2009). However these efforts are sporadic and of limited scope (Crosby \& Noar, 2010; Ogden, 2003). In addition, the methods for conducting holistic research are often too costly, and they require major resources, which limits their popularity ("Four Methods For Holistic Market Research," 2016; R. B. Johnson \& Onwuegbuzie, 2004). Furthermore, current methods mostly frame the enquiry, and those that rely on questionnaires may change cognition (Ogden, 2003). Often, these methods yield static information that provides little value in constantly changing environments (Guston \& Sarewitz, 2002) such as healthcare. There is much criticism about the lack of pluralism in the methods used, and there is a call to arms to actively identify the challenges and opportunities for developing better methods (Bagozzi, 2007; Crosby \& Noar, 2010). These methods could borrow advantageous features from and address shortcomings of the earlier ones.

In the pursuit of bridging the gap between the theoretical insights of scientific theories and the actionable information needed in practice, many more efforts that build scientist-practitioner models are required. These models would serve as useful tools to address the compelling need for cognitive simplicity. They could integrate and visually represent the knowledge of all stakeholders. Thus, they would be used by all stakeholders more readily. In addition, there is a need for methods that can streamline the model building process, provides structure and has the capacity for repeatability. (Stanton et al., 2004) 


\subsection{Literature Review in Modeling Methods in EHTA}

The above review of the body of research that underlies EHTA (gerontology, HFE and HTA) had identified popular approaches to modeling EHTA. It is important to note that the compelling need (identified in the earlier sections) is to conceptualize new modeling methods that contextualize EHTA. Therefore, the modeling methods that are 'theory first' or deductive (Theory $\rightarrow$ Hypothesis $\rightarrow$ Observation $\rightarrow$ Confirmation) are reviewed here. 'Theory later' or inductive approaches (Observation $\rightarrow$ pattern $\rightarrow$ tentative hypothesis $\rightarrow$ theory), which tend to build new theories, are outside the scope of this dissertation.

EHTA research in gerontology, HFE and HTA uses various combinations of data collection, analysis and validation techniques. I classify these methods into one of the three following categories: 1) the Theory_Enquiry_Quantitative-Method (TEQn); 2) the Theory_Enquiry_Qualitative-Method (TEQI); and 3) the Integrative_Enquiry_Mixed Method (IEM). These names follow the naming convention of the general stages of the deductive approach: "theory $\rightarrow$ observation_method $\rightarrow$ analysis method".

Earlier sections have established that a successful design and implementation of EHTA modeling methods should be based on a holistic understanding of the decision process of adoption by the elderly, as well as the complex interactions between the elderly and their environment. The successful method becomes a useful tool to model important factors that influence the elderly's technology use 
behavior. The model also allows the analysis of alternative characteristics of the technology under study that best fits the elderly's needs and preferences.

The review of the HTA, gerontology and HFE literature in relation to EHTA (sections 2.1 through 2.4) has highlighted the need for novel modeling methods, which extend sound TA theories to accomplish the following:

1) Contextualize the abstract factors of TA theories,

2) Incorporate key factors of the elderly decision making process, and

3) Integrate the important influencers of the elderly in the complex multistakeholder setting of EHTA.

Theory first (deductive) methods base their research on extant theory. This is unlike the inductive methods that are primarily based on observation to identify patterns that lead to, propositions, hypotheses and ultimately theory. HTA deductive modeling methods generally select a highly popular technology adoption theory (such as TAM or UTAUT) and extend it. The extension is primarily done in two ways: 1 ) merging the theory with other relevant theories (Karimi-Shahanjarini et al., 2013; Samoutis et al., 2008), 2) expanding the left side (input) of the theory by contextualizing the independent input factors of the theory (Aggelidis \& Chatzoglou, 2009; Duyck et al., 2010) (i.e. adding specific independent factors that serve as the input factors of the original independent factors). Once conceptualized, the hypothesized model is tested through some type of observation. The observation is usually through empirically collected data from the 
population under study. In these methods, the factors are usually obtained from literature (or SMEs) and the collected data is used to statistically test the significance of these factors for the application under study. These statistical tests measure the factors' relevance and forecasting power as the criterion to keep the factors in the model. This analysis leads to the conceptualization of a model that explains the most variance, and thus can be applied to the phenomenon under study.

\subsubsection{Theory_Enquiry_Quantitavie-Method (TEQn)}

The Theory_Enquiry_Quantitative-Method is arguably the most popular modeling approach in technology adoption body of research. In this combination of techniques, a popular technology adoption theory (usually TAM, but also other theories, popular at the time, such as TPB, SCT, etc. and more recently UTAUT) is extended to include the relevant factors to the study. The additional factors are derived from one theory or a combination of other theories, as well as input form subject matter experts (SMEs) and a review of the literature. The conceptualized model consists of the original theory and all the added factors suggested by the knowledge source (literature, SMEs, field data). Data are subsequently collected through a survey of the participants under study (traditionally by pen and paper or over the phone and recently using online surveys). After that, statistical methods are deployed to find the most important factor predicting the behavior. These statistical methods include factor analysis (a process in which the values of observed data are expressed as functions of a number of possible causes in order 
to find which are the most important); cluster analysis (grouping similar group of factors or variables into clusters); regression analysis (a set of quantitative techniques that estimates the relationships among variables, with focus on developing a predictive formula that predicts dependent variable(s) based on one or more independent variables/predictors); structural equation modeling (SEM, an approach that analyzes and models the structural relationship of data).

Kwon and Chidambaram applied the Theory_Enquiry_Quantitative-Method to model cell phone adoption; they integrated the TAM (Davis, 1985) and the Motivational Model (MM) (Davis et al., 1992), and they extended them with additional factors. Kwon and Chidambaram's model was contextualized and refined by survey enquiry, and subsequently analyzed by conducting multiple regression and path analysis (Kwon \& Chidambaram, 2000). Tang and Hämäläinen (2013) modeled technology adoption for Living Lab Aging Care by merging the TAM (Davis, 1985) and the theory of diffusion of innovation (Rogers, 2010), and adding factors from literature. Then the authors contextualized the resulting model based on an online survey and tested the model by multiple regression (Tang \& Hämäläinen, 2013). Similarly, in the study of the role of demographics in adoption, Lee (2010) extended TAM to include age, gender, education and income factors using literature. He, then, tested the hypothesized model with an online survey and analyzed the results using ANOVA (factor analysis, multivariate analysis of variance, and regression analysis methods) (Lee, 2010). 
Theory_Survey_SEM: This is one of the most commonly used combinations of techniques in the Theory_Enquiry_Quantitavie-Method modeling group of methods. As part of the TEQn subgroup, the theory is contextualized by expansion with context-relevant factors. From there, survey data are collected through a survey of the studied participants (traditionally by pen and paper or over the phone, and recently by using online surveys). Finally, the structural relationship of data is analyzed using Structural Equation Modeling (SEM), and the model is formed. ${ }^{6}$

Hoque \& Sorwar (2016) used Theory_Survey_SEM to extend UTAUT to understand the behavioral intentions of the elderly in their adoption of mobile health services in Bangladesh. Their EHTA model found a significant relationship between the UTAUT constructs and hedonic motivation, price value and habit. The study also identified the moderating role of gender and its influence on the adoption of mobile health technology and services. And, Rue et al. (2009) used Theory_Survey_SEM to investigate the factors affecting the elderly's participation decision in video user-created content services (self-created online videos, like those on YouTube) in Korea. They extended the TAM to include aging-related factors. Using data collected from surveying 290 online users, they analyzed the resulting model using SEM methods. The research resulted in the identification of

\footnotetext{
${ }^{6}$ Structural equation modeling (SEM) refers to a combination of mathematical models of factor analysis and multiple regression analysis. It is used to measure the structural relationship between the measured variables is the collected data and the latent constructs of the model (adoption intention and actual adoption). Examples of the application of such modeling methods follow.
} 
some of the aging-related factors such as perceived physical condition (physical age), life course events (psycho-social age), perceived user resources, prior similar experience, and computer anxiety (Ryu, Kim, \& Lee, 2009). Deng et al. (2014) also used Theory_Survey_SEM approach to explore and model the middleaged and older users' adoption of mobile health services in China. To create their hypothesized model, they extended the Theory of Planned Behavior (TPB) (Ajzen, 1985) by merging it with the Value Attitude Behavior Model (Vaske \& Donnelly, 1999) and adding four constructs that are characteristic of aging. They subsequently tested the model empirically using the data collected from a survey of 424 residents older than 40 years. Finally, they used structural equation modeling to estimate the significance of each factor (Deng et al., 2014). Similarly, Abiola (2016) and Best et al. (2015) used survey techniques for modeling of their EHTA and tested their hypothesized model using a structural equation model (Abiola, 2016; Best et al., 2015).

\subsubsection{Integrative_Enquiry_Mixed-Method (IEM)}

To alleviate the aforementioned deficiencies, researchers have introduced mixed methods to EHTA, which use the effective aspects of qualitative techniques for data collection and effective aspects of quantitative techniques for analysis and verification. This is evident by the new waves of EHTA modeling research that has used qualitative methods to extend extant theories with patterns have emerged from in-depth observation (Compagna \& Kohlbacher, 2014b; Liddy et al., 2008;

O'Mara et al., 2012). This hybrid approach allows for combining induction and 
deduction by integrating two separate studies. First, a qualitative, inductive study is conducted to find new information that was not possible by a quantitative study. The qualitative study is followed by a quantitative, deductive study, which generalizes the findings of the qualitative study (Grafton et al., 2011). This approach allows the researcher to compensate for shortcomings of each method with the strengths of the other (qualitative vs. quantitative) (Jick, 1979, as cited by Bhate 2012). It also strengthens the confidence of the research, by expanding the findings beyond one method; identifying empirical contradictions that could have been missed otherwise; and triangulating research findings (Denzin, 1978, as cited by Bhate, 2012).

The advantages of mixed-methods are particularly important in the context of social and health research, as the problems addressed in these applications are complex, and either quantitative or qualitative approaches alone are insufficient to address this complexity (Creswell, 2013, chapter 10). Since the publication of the Handbook of Mixed Methods in the Social \& Behavior Sciences (Tashakkori \& Teddlie, 2003), the method has evolved and gained momentum and become the focus of several journals (Journal of Mixed Methods Research | SAGE Journals; Mixed Methods - Journal of Ethnographic \& Qualitative Research). In recent years, mixed-methods have been increasingly welcomed and utilized in the domains of health research (Holtrop et al., 2016; Pinto et al., 2014) and gerontology research (Fink \& Beck, 2015; Vrkljan, 2009). It is also being promoted by the US department of health and human services (Coyle et al., 2016; Evidence and Evaluation | PCMH 
Resource Center). However, this approach is not commonly deployed in EHTA research. Yet, mixed-method associations (such as (MMIRA, 2016)) and scholars emphasize the need for sound research methods that utilize the strength of mixedmethod (Creswell, 2013, page 204; Vrkljan, 2009). Scholars also highlight the need and include visual capability (R. B. Johnson \& Onwuegbuzie, 2004; Onwuegbuzie \& Combs, 2010) for linking mixed-method research approaches with visual display that provide a richer understanding of our environment. They advocate for new mixed-methods that enable visual display, as that provides a powerful synthesis of inquiry and display of data and form (Dickinson, 2010).

Recent literature (e.g. Crosby \& Noar, 2010; Head \& Noar, 2014; Noar \& Zimmerman, 2005; Ogden, 2003; Painter et al., 2008; Rothman, 2004) complains about the redundancy of TA streams of research that have been reusing a priori knowledge with the false hope of finding insight in the new contexts of HTA, highlighting the need for new methods to discover new insights. Qualitative methods have been shown to facilitate new discoveries, and for many decades have been used to ground theories. As such, in recent years, they have been increasingly reconsidered for the study of HTA (Chaurasia et al., 2016; Zhang et al., 2014). This broadening of the search for the most effective techniques that can bring the much needed fresh insight has added qualitative techniques to the quantitative approaches in EHTA. The resulting mixed modeling efforts usually select a relevant TA theory and extend it using insights directly obtained from observation and involvement of the elderly under study. Although such research 
still heavily relies on related literature (gerontology, gerontechnology, psychology, cognitive and social science, etc.) to inform the study, the resulting models are closely tied to the information that has emerged from the field enquiry. Quantitative techniques are often used in this modeling research for their ability to provide effective verification and simulation of possible scenarios. A few examples of such efforts follow.

To create a predictive model for assistive technology adoption among people with Dementia (mostly elderly), Zhang et al. (Zhang et al., 2014) and (Chaurasia et al., 2016) adopted the Integrative_Enquiry_Mixed-Method research method. Although these researchers stayed away from a priori knowledge based on the criticism of its lack of predictive power, their research benefited from the literature. In this research, the conception and the contextualization phases were integrated. The resulting models were founded on the longitudinal data that the researchers collected during consultation with 40 Dementia patients and their caregivers (in the Cache County Study on Memory in Aging in Utah State University). The data collected were analyzed through several data mining algorithms to extract the most important factors. Influence diagrams were created to capture how the emerged factors influence one another. The hypothesized models were then verified by testing the significance of the factors through quantitative data analysis techniques, including statistical pair-wise comparison and stepwise regression. Thirty-one features, which represented a range of age, gender, education and details of health condition, were conceptualized. In the process of modelling 
adoption, feature selection and feature reduction were carried out, followed by identifying the best classification models.

With advancements in health technology such as the emerging Internet of Things, which generate big data, data mining and machine learning are becoming increasingly popular. Machine learning (artificial intelligence programs that learn and change based on new data) and data mining (software that examines large amounts of data to find new information) have recently entered healthcare studies, where they are expected to become prevalent (HealthITAnalytics, 2016). These practices can be used to build predictive models through finding unknown patterns and trends in health data (Kincade, 1998). They can also be used as techniques for data selection and analysis of vast data to build exploratory models to uncover previously unknown patterns (Koh, Tan, \& others, 2011). A few gerontechnology studies have already used data mining (Banaee et al., 2013), but, to date, this approach has not been deployed in EHTA research.

\subsubsection{Theory_Enquiry_Qualitative-Method (TEQI)}

In the recent years, scholars increasingly complained about the redundancy of deductive research methods that are based on presumptions of important factors (Bagozzi, 2007; Glanz et al., 2008). Quantitative analysis techniques and a conventional data collection methods (e.g. survey) lacked the ability to discover fresh insights due to their inherent presumptive framework of research design ("Weaknesses of Survey Method," 2015). By contrast, the unframed qualitative data collection and analysis techniques allow the important concepts/factors to 
surface from observation. This invited qualitative techniques, which are generally associated with inductive approaches, to enter the world of deductive model building.

In response to the call to arms to develop predictive HTA modeling (Crosby \& Noar, 2010; Head \& Noar, 2014), Renaud and Van Biljon (2008) developed a senior technology acceptance and adoption model for mobile technology based on TEQI approach. To do that, they conducted a literature study on technology adoption theories and models, as well as an investigation into the context of the senior user, to develop their model. They used earlier developed theories like Domestication of Technology (Silverstone \& Haddon, 1996) and Mobile Phone Technology Acceptance Model (MOPTAM) (van Biljon \& Kotzé, 2007), which had contextualized the UTAUT for mobile phone adoption of non-organizational users. They then added factors that the literature had marked important in elderly decision making, such as socio-economic factors. They then designed and conducted a semi-structured interview that involved recording the elderly's answers to a set of questions. Their interview questions involved guiding the elderly through a set of scenarios and design activities on a paper prototype of mobile phones. They then thematically analyzed the data collected. Data was grouped by similarity and then counted, as a means to identify the factors surfacing from the elderly, which validated the hypothesized factors (Renaud \& Van Biljon, 2008).

The downside of qualitative methods is that they are usually very time consuming and resource-intensive. Additionally, since there are fewer participants studied in 
qualitative methods, they are considered to be less representative of the population studied and less generalizable. It was thus in the interest of researchers in the HTA field and elsewhere to develop mixed methods that take advantage of the strengths of the TEQn and TEQI techniques and minimize their weaknesses.

\subsection{Summary of the State of the Art}

Researchers in human factors engineering (or Computer/Human Interaction), who focus on innovation and awareness, have made significant contributions to the adoption process. They aim to design innovative products and services that address the prospective needs, limitations and preferences of users, making these products adoptable. They also raise awareness and promote the adoption of such innovations in focused communities (O'Mara et al., 2012). However, HFE methods hardly consider the adoption beyond the initial ergonomics considerations to deliver to the needs and desires of the users.

The body of research in gerontology has resulted in an in-depth knowledge of the effects of aging, which unfolds the pronouncing factors that influence most of the elderly's adoption decision points. This information can shed light on major inhibitors that slow down the intention to adopt. They also the slow process of building perceptions about the technology's ease of use and usefulness throughout the process adoption (Silverstone \& Haddon, 1996). However, gerontology lacks the capacity to draw actionable technical insights to intervene when the elderly do not adopt technology. 
The earlier research on technology adoption has mainly assumed that intention is the sole cause of adoption. Hence, it has primarily focused on analyzing the subprocesses that pertain to the individual's intention to adopt, ignoring major influencing factors from the environment (Bagozzi, 2007; Glanz et al., 2008). Some of the research in sociology has led to a more holistic understanding of adoption in the context of society and the innovation life cycle (Rogers, 2010; Silverstone \& Haddon, 1996). However, adoption among the elderly has yet to be fully explored at an empirical level by any of these streams of research, suggesting that future EHTA research will have to integrate knowledge from all of the abovementioned fields of study. This multidisciplinary EHTA modeling has already begun, but there is a compelling need for research methods that can provide a systematic approach to create successful models in health applications (Bagozzi, 2007; Crosby \& Noar, 2010; Ogden, 2003; Painter et al., 2008; Rothman, 2004).

This broadening of the search for the most effective research methods that can bring the much needed fresh insight has revitalized qualitative techniques. However, such efforts are not systematic, and methodological frameworks that guide such research are scarce in EHTA. Thus, there is a great need for new methods that can provide a systematic way to integrate the applicable theories with the specific information of the context under study (Creswell, 2013, chapter 10), especially in EHTA.

Given the challenges discussed above, future EHTA modeling methods have to meet the following criteria, for them to be successful. 
1. Grounded: Integrate first hand user data (Phillips \& Zhao, 1993)

2. Holistic: Approach the problem holistically: cover a wide range of factors that emerge from the health context under study (Bagozzi, 2007; Head \& Noar, 2014; Ogden, 2003; Rothman, 2004)

3. Flexible: Build flexible models that can be adjusted in response to new input (Jetter \& Kok, 2014; Kosko, 1988)

4. Latent Needs: Model latent needs (Phillips \& Zhao, 1993)

5. Visual: Provide cognitively simple visual representation of findings that people from different disciplines can comprehend (Creswell, 2013, ch.10)

6. Scenario Planning: Model and simulate decision scenarios (Simpson, 1992) to identify and prioritize alternative decision points based on their potential to result in a higher adoption rate (Amer et al., 2013; Glenn \& Gordon, 2009; Schoemaker, 1991; Simpson, 1992).

7. Novel Insight: Capacity to provide novel insight in relation to and go beyond the predictions of extant theory (Glanz et al., 2008; Head \& Noar, 2014; Ogden, 2003; Rothman, 2004)

8. Efficiency: Practical in regards to consideration of monetary and temporal resources and constraints (Creswell, 2013, ch.10)

Table $2-3$ evaluates each of the aforementioned modeling categories based on the criteria set above. The most popular data collection and analysis techniques of these categories are listed in the second and third columns. The details of the evaluations of approaches follow. 


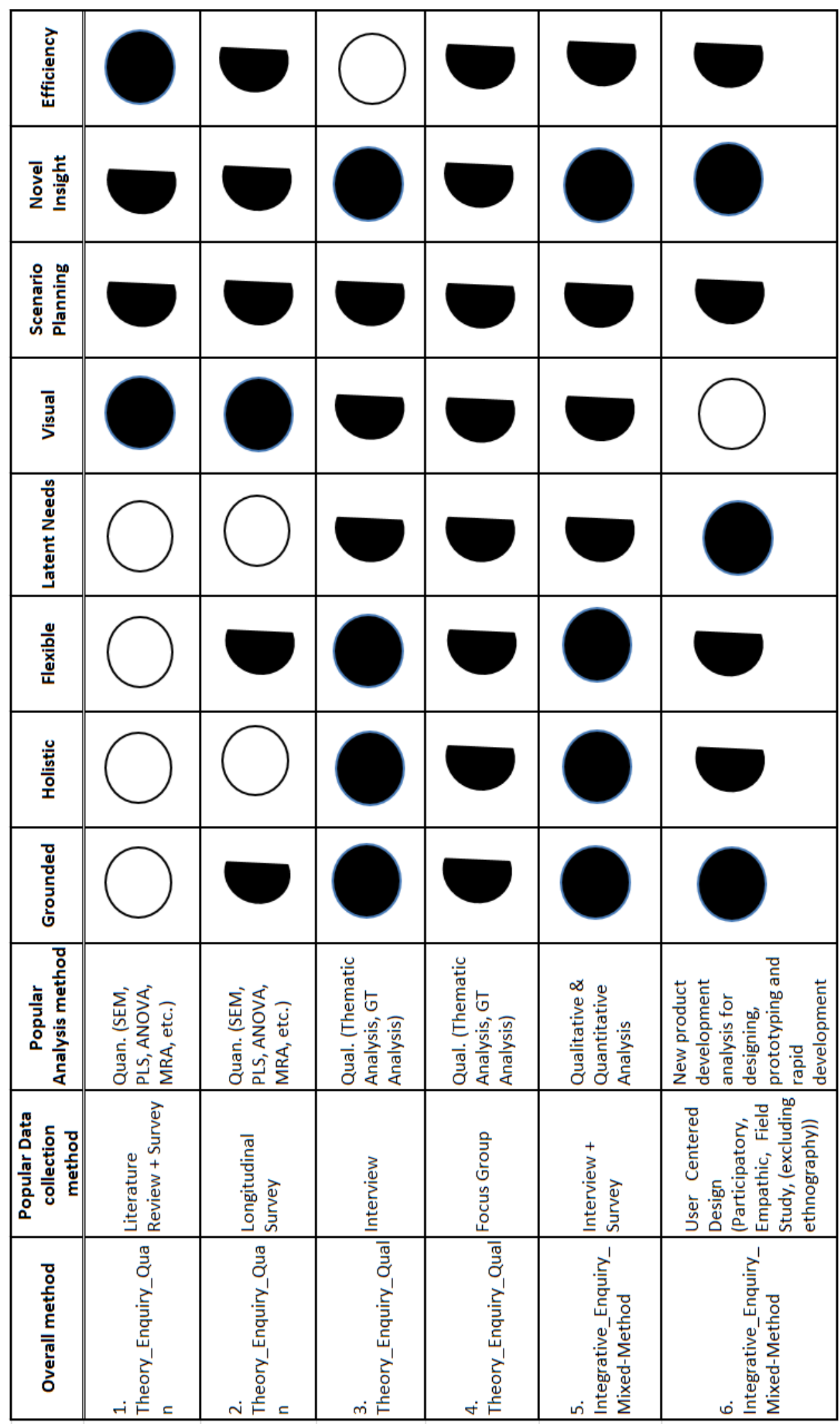




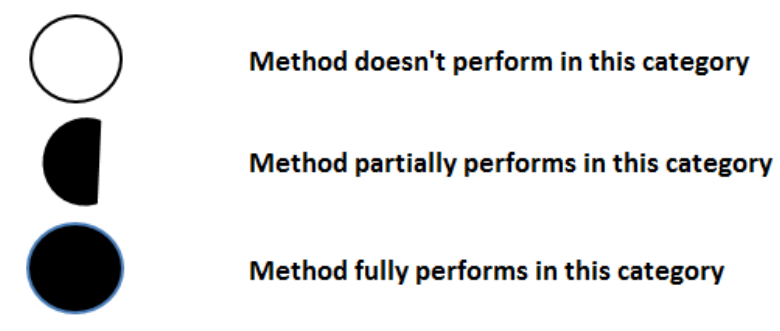

Table 2 - 3 Modeling methods performance based on the set criteria

The following paragraphs explain the evaluation of the three categories of the research approach with their most popular techniques (columns 1-3) in the above Table 2 - 3. The explanations for the first four criteria (columns 4-7) are grouped together because the performance for all these criteria is influenced by the data collection method (as listed in column 2). The details for the remaining judgement are treated separately for each of the remaining columns (8-11).

It's important to note that, the research methods generalized in row 1 through 5 are generally deployed to conceptualize a reusable model. However, the application of IEM methods in HFE (categorized in row 6) does not necessarily result in a model. Instead, research that uses these IEM methods intends to find very specific information that can be translated into product requirements.

\subsubsection{Grounded, Holistic, Flexible, Latent Needs}

The capacity of each approach to obtain and integrate first-hand data provides the opportunity to obtain the much needed information about the drivers and barriers of EHTA. Reviewing literature, which is a common practice in the TEQn methods, is a great way to obtain information from earlier research in the field. However, this information is not first hand and does not necessarily reflect the context under 
study. Additionally, survey research is bounded by the constructs being measured. Hence, the data collected relies primarily on the assumptions made, and doesn't allow for the observation of first hand data. The first popular group of Theory_Enquiry_Quan research approaches (row 1 in Table 2 - 3) refers to studies that rely on survey data collection and literature review. Therefore, they get a poor rating in Table 2 - 3 on the following criteria: grounded, holistic, flexible and latent needs. Theory_Enquiry_Quan methods that rely on a longitudinal survey (row 2 in Table 2 - 3) gather more in-depth information, as the process of gathering data at different points in time allows the researcher to capture the effect of time on the EHTA. This provides flexibility and allows more grounded observation of EHTA. However, since the participants do not have the freedom to provide the information they find important, these methods do not score highly on the "holistic" or "latent need" criteria.

A focus group creates a quick venue for obtaining much information from various people at once. However, it has been known to be less effective than approaches where data are collected from individuals. Aside from the observer dependency issue (obtained result is influenced by the researcher's understanding of the group discussion), the information obtained in the focus group is less than valuable, as people often try to agree with other participants and tend to provide pleasing opinions (Rushkoff, 2014). In fact, this has led to the abandonment of this data collection method in innovative companies like Apple ("Apple's Ive reveals design secrets," 2009). For these reasons, Theory_Enquiry_Qual methods that rely on 
focus groups as the primary data collection method (row 4 in Table 2 - 3) do not score highly on the following criteria: grounded, holistic, flexible and latent needs.

Evidently, qualitative interview techniques, such as ethnographic data collection and repertory-grid, are effective methods to obtain firsthand information directly from observations and open communications that are not framed by prior assumptions. This is why qualitative methods are now receiving recognition in healthcare research with social and cultural dimensions (Al-Busaidi, 2008; Meyer, 2000). Qualitative data collection techniques allow participants to freely articulate the meaning, experiences and views in relation to the phenomenon studied. Thus they may yield previously unknown information. This advantage earns TEQI and IEM research approaches that rely on one-on-one interviews (row 5 in Table 2 - 3) a high rating on the four criteria (in columns 4-7). In HFE, the more involved collaborative methods of user-centered design (UCD) (as detailed in section 2.3.) focus on these qualitative data collection techniques. As such, these advantages earn Integrative_Enquiry_Mixed-Methods that use such qualitative data collection techniques (row 6 in Table 2 - 3) a high rating on these criteria: grounded, holistic, flexible and latent needs.

\subsubsection{Visual Display}

One of the most important criteria in the success of a research methods is its ability to create visual models (Creswell, 2013). From the ancient anthropological discoveries of visual displays on stones and the first visual research by Dr. Snow in 1854 to the current body of literature, research has leveraged visual display 
(Dickinson, 2010). Humans recognize, perceive and evaluate visual information more effectively than numbers or words. Visual data deliver immediate and lasting impact; they provide an easy flow of information across parallel elements; and they can be used to illustrate phenomena (Tukey \& others, 1990). The importance of visual formalization in research is well recognized (Langley, 1999; A. D. Meyer, 1991; Miles \& Huberman, 1994). Miles \& Huberman extensively use diagrams and other visual tools to organize and illustrate data and their conceptual interrelations. The authors advocate for visual formulization, by stating: "Conceptual frameworks are best done graphically, rather than in text or numbers. Having to get the entire framework on a single page obliges you to specify the bins that hold the discrete phenomena, to map likely relationships, to divide the variables that are conceptually or functionally distinct, and to work with all of the information at once" (Miles \& Huberman, 1994, p. 22).

As displayed in Table 2 - 3, quantitative methods get high rating in their visual display capability, as they are mainly supplementing the visual display of the theory that they are expanding. This is not necessarily the case with the qualitative or mixed-methods, and their ability to visually display the findings is dependent on the research goal and the competency of the research team. In fact, the need for equipping mixed-method research approaches with visual display capability is highlighted by many scholars, but it has not yet been fully achieved (Dickinson, 2010). This explains why the TEQn research groups in row 1 and 2 of Table 2 - 3 has scored high whereas the TEQI (row $3 \& 4$ in Table 2 - 3 ) and the IEM methods 
that rely on interview and survey (row 5 in Table $2-3$ ) have not. In the case of HFE IEM methods that rely on UCD method for new product development (row 6 of Table 2 - 3), the goal of the research is specific to the product being designed. This specificity eliminates the need for visualization, which leads to the low rating of these research methods in the visual capability criterion.

\subsubsection{Scenario Planning}

As scenario planning is highlighted as an effective heuristic approach in complex settings (Amer et al., 2013; Schoemaker, 1991), modeling approaches that allow for scenario planning are valuable. The scenario-planning capability of models becomes important when models are used as an simulation tool. This is particularly important for practitioners in contexts with high uncertainty and an accelerating pace of change (Simpson, 1992). Such tools are advocated to be the methods of the future because they are able to capture whole ranges of detailed possibilities, and allow for simulation of possible situations to support decision making (Kahn et al., 1967; Schoemaker, 1995). Although systematic scenario planning approaches in both qualitative and quantitative research methods exist (Amer et al., 2013), they have not been used in EHTA. Since all these major categories of modeling methods have the capacity for scenario planning (even though not applied in EHTA), they get a partial rating under the scenario-planning criterion. 


\subsubsection{Novel Insight}

Health scholars are repeatedly highlighting the need for research approaches with the capacity to expand extant theory to achieve fresh insights in relation to the specific health context under study (Glanz et al., 2008; Head \& Noar, 2014; Ogden, 2003; Rothman, 2004). This is in fact essential in the complex context of EHTA. Overall, the quantitative EHTA (and TA in general) body of research has relied on framed enquiry techniques, namely survey enquiry. As detailed earlier, since this data collection technique is based on measuring the assumed constructs, it is limited and provides little freedom for the emergence of new discoveries. As such, Theory_Enquiry_Quantitative-Method approaches get a partial rating. Additionally, Theory_Enquiry_Qualitative methods that rely on focus group enquiry also get partial rating, due to the limiting characteristics of focus groups that were detailed earlier. The degree of fresh insights that Integrative_Enquiry_Mixed-Method techniques bring is often a function of the depth of enquiry and the intensity of analysis. Integrative_Enquiry_Mixed-Method techniques that focus on new product development use user-centered design enquiry to gather information that is obtained by thorough observation of the user and the environment. Although the analysis conducted rarely conceptualizes a model or goes beyond the product at hand, the insights gained are very practical and have the potential to be groundbreaking (Rahimi \& Ibarra, 2014). Additionally, the Theory_Enquiry_QualitativeMethod and Integrative_Enquiry_Mixed-Method techniques that are based on indepth enquiry of the elderly, have the potential to unfold hidden factors and other 
much needed information pertaining to EHTA decision making. For these reasons, the performance of the last three groups of techniques is rated highly for this criterion.

\subsubsection{Efficiency}

Research projects, like any other endeavors, are subject to constraints with regard to available time and money. The performance of a modeling method is rated with respect to its efficiency (Creswell, 2013, ch.10; Garmann-Johnsen, 2015). The potency of an EHTA model that is conceptualized based on a multi-year full ethnography research into the elderly's lives is evident. However, the combination of resource constraints, the speed of innovation and the compelling need to understand EHTA requires modeling methods that deliver all the previous requirements, as well as taking monetary and temporal constraints into consideration. As mentioned earlier, survey enquiry not only provides an efficient vehicle to collect information from a very large group of participants; it also makes the data analysis very easy because the data have been collected in a very organized manner and are ready for analysis. Given the availability of statistical analysis programs and tools, quantitative analysis of survey data is considered the easiest and the most readily available research approach. This justifies the high rating of the Theory_Enquiry_Quan methods (rows 1 of Table 2 - 3) in the efficiency criterion. In comparison, all other methods (rows 2 through 6 in Table 2 - 3) rate average because an extended amount of time and resources are needed for data

collection or analysis. In the case of Theory_Enquiry_Qual methods that solely rely 
on one-on-one interviews (row 3 of Table $2-3$ ), both the data collection and analysis require more time. Although such methods are adequate for conceptualizing new grounded theories, they are not very efficient for modeling research that demands timely empirical results. The rest of the methods (row 4 through 6 in Table 2 - 3) rate medium on the efficiency scale for the same reasons.

\subsection{Research Gap}

The evaluation of the state of the art in EHTA modeling techniques, which are displayed in Table 2-3, shows that there is no EHTA modeling method that currently fulfills all eight requirement of EHTA research. This presents a clear methodological gap for conducting EHTA research, which this dissertation tries to fill. 


\section{Proposed Research Method}

The analysis of enquiry methods in section 2.6 suggests that Integrative_Enquiry_Mixed-Method techniques, have the most potential to fulfill the requirements for successful modeling of EHTA. This is in line with the emerging trend to use mixed-methods approaches for healthcare modeling research. For instance, the Agency for Healthcare Research and Quality of U.S. Department of Health \& Human Services, has started to sponsor many mixed-methods healthcare studies (Al-Busaidi, 2008; Curry et al., 2013; Meyer, 2000; Palinkas et al., 2011; Zhang \& Watanabe-Galloway, 2014) and online webinars to teach how to integrate mixed-methods in health services and delivery system research ("Agency for Healthcare Research \& Quality", 2014). Additionally, the National Health Plan Collaborative (NHPC) in its "Overview of Its Origins, Accomplishments, and Lessons Learned" report states: "As the NHPC firms became more sophisticated in collecting data, the importance of a mixed-methods approach to data collection emerged..." (“Accomplishments on Targeted Activities”, 2009). I have consequently designed a mixed-methods research approach that combines ethnographic interview techniques, repertory grid technique and Fuzzy Cognitive Mapping (FCM) to contextualize the latest and most relevant technology adoption theory (UTAUT) to model EHTA.

Combining proven methods to achieve objectives is recommended (Stanton et al., 2004), and the resulting new method benefits from a pilot study that tests the validity of the method and the resulting data that it yields. The method proposed 
here has passed a preliminary test (Appendix B). I followed the proposed research method to create an EWHTA model (using RGT and FCM modeling) from a preadoption dataset, and subsequently compared the predictions of the model to actual adoption behavior using a post-adoption dataset. The pilot research confirmed the validity of the research method, by validating the EWHTA model.

This dissertation proposes to address methods gap identified in section 2.7 by designing a novel method for studying technology adoption in healthcare using Fuzzy Cognitive Mapping (FCM). It investigates the validity of this method by applying it to model EWHTA of a remote health monitoring technology. The method aims to provide a step by step deductive process for capturing knowledge from the stakeholders in the form of cognitive maps, and integrate them to a FCM model that maps out the adoption landscape among these technology users. Using FCM simulation capability, the model will be tested against the initial pre-adoption settings and validated by verifying its projected resulting outcome against the actual adoption behavior. Should the model be accurate, its empirical usefulness will be tested and verified using expert judgements.

The following six proposed research questions guide this research.

1. Can the proposed method generate a holistic yet cognitively simple visual model that represents users' mental landscapes and depicts their decision making process during health technology adoption?

2. Can the proposed method provide a mechanism to capture and model the entire adoption? 
3. Can the proposed method using FCM, successfully capture and integrate gatekeeper's influence in the complex healthcare environment?

4. Can the proposed method both breakdown generalized known TA factors in relevance to the context studied, and facilitate the emergence of new key concepts relevant to the demographics under study?

5. Can FCM simulation feature successfully simulate real-world scenarios and lead to empirically valuable insights in the context studied?

6. Can the method have the capability to provide information that is adjusted to the dynamic and changing environment and latent needs?

\subsection{Methodological Choices.}

\subsubsection{Overall Design}

Prior researchers (David, 2004; Zenobia \& Weber, 2012) present four methodological choices that a researcher has to make prior to engaging in consumer-based technology adoption research. These are: 1) theory first versus theory later; 2) process research versus variance research; 3) building theory and models in parallel or in; and 4) structural versus behavioral aspects of adoption behavior. This section uses the Zenobia-Weber (2012) framework for making methodological choices for the proposed research.

Theory First (deductive) vs. theory later (inductive): Earlier research (Rahimi \& Jetter 2015; Rahimi et al., 2016) found that the UTAUT reflects EWHTA and can 
serve as the theoretical foundation for contextualizing EHTA. This argues for the deductive approach.

Variance vs. Process Models: The focus of this dissertation is to identify detailed concepts that influence the elderly's adoption decision-making and the relationships between these concepts. The process by which the elderly make adoption decisions is beyond the scope of this dissertation. Therefore, I am pursuing variance research.

Model Building vs. Theory Building: Given that extant theory (UTAUT) is satisfactory, no new theory needs to be built. The proposed research develops an empirical model that contextualizes the UTAUT for EHTA to provide actionable insight for practitioners.

Structural vs. Behavioral Aspects of Adoption: The proposed research intends to identify the factors that influence decision making in EHTA, as well as how the relationships between the factors influence technology adoption behavior. Therefore, the model that will result from the proposed research needs to capture both the structural and the behavioral aspect of adoption.

\subsubsection{Choices Pertaining to Enquiry and Analysis}

Integrative_Enquiry_Mixed-Method Modeling: IEM methods that yield models (row 5 in Table 2 - 3) have the most potential for addressing the eight methods requirements identified in section 2.6. Mixed-methods combine the efficiency of 
both qualitative and quantitative techniques to address the requirements of today's complex contexts (like that of healthcare) (Stanton et al., 2004).

Table 3 - 1 displays different mixed-method research designs as laid out by (Johnson \& Onwuegbuzie, 2004; Morse, 1991). The conventions used in the figures are as follows: "qual" stands for qualitative; "quan" stands for quantitative; "+" stands for concurrent; " $\rightarrow$ " stands for sequential; capital letters denote high priority or weight; and lower case letters denote lower priority or weight."

\begin{tabular}{|c|c|c|}
\hline & \multicolumn{2}{|c|}{ Decision } \\
\hline & Concurrent & Sequential \\
\hline $\begin{array}{l}\text { Equal } \\
\text { Status }\end{array}$ & QUAL + QUAN & $\begin{array}{l}\text { QUAL } \rightarrow \text { QUAN } \\
\text { QUAN } \rightarrow \text { QUAL }\end{array}$ \\
\hline Dominant & QUAL + quan & $\begin{array}{l}\text { QUAL } \rightarrow \text { quan } \\
\text { qual } \rightarrow \text { QUAN }\end{array}$ \\
\hline Status & QUAN + qual & $\begin{aligned} \text { QUAN } & \rightarrow \text { qual } \\
\text { quan } & \rightarrow \text { QUAL }\end{aligned}$ \\
\hline
\end{tabular}

Table 3 - 1 Mixed-method Research Design Matrix

\subsubsection{Enquiry Approach - Qual $\rightarrow$ Quan}

The Qual $\rightarrow$ Quan approach is best suited for the proposed research because fresh insights need to be generated (Bhate 2012, see also section 2.5.3).

Qual: The enquiry starts with in-depth, qualitative data collection. I am using the ethnographic interview method combined with fuzzy cognitive mapping. The ethnographic interview (as part of RGT approach here) facilitates the generation 
of novel insights needed in EWHTA, and FCM serves as the central map that captures the mental model. This leads to the discovery of the underlying adoption factors and their relationships. Once the integrated model is created, it is judged and validated by a panel of gerontechnology and technology adoption experts. This process adds a layer of quality assurance to ensure that the simulation model is sound in all of the related perspectives.

Quan: Through second set of one-on-one interviews with the same group of participants, the quantitative data collection is conducted. This data collection allows the structure of the model to be quantitatively built. The same group of participants identifies the degree of influence of each concept on the other concepts in the model.

It's important to note that the goal of this research method is to generate a decisionmaking model that explains the elderly's adoption decision for a proposed health technology. Therefore, the research pursues a marketing research data collection approach, i.e. the elderly's mental model is captured during the pre-adoption of a health technology.

Modeling Method - FCM: The main tool for elicitation and modeling EHTA in this research method is Fuzzy Cognitive Mapping (FCM). FCM serves as the central tool throughout the entire research process, from qualitative data collection and analysis to quantitative data collection and analysis. FCM acts as the bridge between the qualitative and quantitative sub-processes of the proposed modeling method. It also meets the requirements for a modeling tool that were stated in 
section 2.6. Finally, FCM serves as both the platform and the end product of the EHTA simulation model. (FCM is described in detail in the next section.)

\subsubsection{Analysis - Qual $\rightarrow$ Quan (FCM):}

Qual: The qualitative data collection and FCM model building happens simultaneously. This approach allows for real-time clarification of the respondent's input, in which the modeler gains feedback on whether his/her interpretation of the respondent's input is correct. After the data collection, qualitative analysis is conducted (using Atlas.ti 7 ("ATLAS.ti 7 Windows | Qualitative Data Analysis with ATLAS.ti", 2017)) for each interview audio record to find the possible factors and relationships that were not captured in the FCM model during the interview. As detailed in the research steps in section 3.9, the analysis is similar to the techniques of the repertory-grid. The results of the analyses of the individual models are subsequently compared to and contrasted with each other, in order to extract potentially common themes (concepts and/or links). This endeavor yields an ontology list-a list of all the concepts that have been identified and a description of their meaning. Using the ontology list, all the FCMs are integrated into one. From there, should there be any question or concern raised by the judgement of the expert panel, the qualitative analysis is continued to find and address these concern(s).

Quan: The information is analyzed to quantify the degree of influence among the concepts. This allows for contextualize the integrated FCM maps to best reflect participants' subjective opinion. The quantitative power of the FCM simulation also 
enables further analysis and scenario planning (as interpreted by Simpson (1992)) to identify technology characteristics that lead to better adoption by the elderly.

\subsubsection{Repertory Grid Technique (RGT) as Data Elicitation Method}

To capture elderly people's mental model, the required data elicitation method need to allow the emergence of novel information. To achieve this, an adaptation of Repertory Grid Technique (RGT) is designed. As the overarching design of the proposed mixed-method modeling method, the knowledge capture follows the guiding principle of the repertory-grid elicitation approach.

Over the 60 years of development, RGT has evolved from a more rigid method of obtaining personal constructs with defined data collection technique followed by statistical analysis to study the correlations. RGT has evolved to include more diverse and inclusive approaches to extract constructs from people. Some of the RGT expansions (which are of interest here) include techniques to aggregate expert knowledge (as opposed to the personal constructs) and use "content analysis" or "frequency counts" in the analysis phase (as opposed to the original statistical analysis) (Boyle, 2005).

Gaines \& Shaw (1993) advocate the use of repertory-grid as a better tool for knowledge acquisition, and state: "What differentiates knowledge-based system development from conventional system development is the emphasis on in-depth understanding and formalization of the relations between the conceptual structures

underlying expert performance and the computational structures capable of emulating that performance." (Gaines \& Shaw, 1993, p. 1). 
Here, the RGT approach employs ethnographic interview techniques, in order to obtain in-depth knowledge from the participants, including elements of the adoption process that may not currently be represented in the literature. Ethnographic interview formats are a less rigid variant of ethnography (Agar, 1986) that allows the interviewer to participate in and steer the conversation, if deemed necessary, rather than exclusively taking an observer role (Preissle \& Grant, 2004). Additionally, the simultaneous fuzzy cognitive mapping during the interview, allows the important concepts to be mapped for further analysis.

To maximize the elicitation of important factors from the participants, the data (voice recording of the interview) is analyzed using qualitative content analysis technique (the RGT analysis) during the qualitative analysis phase of the research. The RGT is a technique designed to collect people's constructs, i.e. what people think about a particular topic. It is a grounded theory approach, which means that, in a bottom-up approach, the data emerges entirely from the participant. The repertory-grid is based on George Kelly's Personal Construct Theory (George Kelly, 1955), which posits that people's opinion of things around them are based on a collection of similar and different dimensions (i.e. personal constructs). The repertory-grid method is broken down into three stages - observation, emergence of the patterns (of similar, different and opposing artifacts); hypothesis and formation of constructs. This technique has been deployed in information systems to obtain a fresh set of user attributes that act as evaluation criteria to ensure that the users' expectations are met (Whyte \& Bytheway, 1996). It is important to note 
that repertory-grid constitutes various types of techniques that vary in their level of rigor. The designed research method in this dissertation uses a less rigorous approach of the repertory-grid, which is explained in later sections of this chapter (section 3.7.2).

The repertory-grid is based on the theory of "Personal Construct Psychology", which is based on individual and group psychological and social processes (Caputi, et al., 2011). It has been used extensively in psychological knowledge acquisition research to model the cognitive processes of human experts. As implemented in this dissertation, scholars recommend using concept mapping tools such as cognitive mapping in combination with repertory-grid techniques (Gaines \& Shaw, 1993; Vervoort, 2011).

\subsubsection{Fuzzy Cognitive Mapping}

In this dissertation, FCM will be used as the central modeling method to capture and integrate the mental models of the elderly and gatekeepers. The resulting aggregated model will support decision makers in planning the technology and the adoption process.

Technology adoption depends on how adopters perceive a situation, such as the ease of use of a technology or the likely response by members of their social network. To be able to capture perceptions, Fuzzy Cognitive Mapping (Kosko, 1986) serves as the methodological foundation of this dissertation. 


\subsubsection{FCM Background}

A fuzzy cognitive map is a type of cognitive map in which degree of causality is indicated by a real number within the range of -1 to +1 . It is a form of causal maps that depicts the respondents' subjective knowledge of or beliefs about important concepts (nodes), and their causal relationships (links) about a phenomenon under study. Concepts are typically represented as oval shapes that contain nouns, while the causal links between concepts are represented as arrows that show the direction of the causality. In decision-making contexts, the concepts of an FCM frequently represent key factors in the decision (here: technology adoption). These causal relationships are quantified to display hazy degrees of causality (Kosko, 1986). FCMs are signed diagraphs that can depict any type of causal map, permitting feedback loops, and holds no limitation on graph complexity; hence they can be a powerful methodology for modeling complex real-world phenomena that involve different groups of stakeholders (Taber, 1991; McNeill, 1994). Moreover, the cognitive maps from multiple respondents can easily be aggregated into a shared FCM. Although it presents a static picture of the aggregated mental model of the participants, an FCM's quantitative nature provides the opportunity to simulate outcomes based on multiple input scenarios (Kosko, 1992). This quantification adds computational power to the qualitative nature of the information captured in the map. It also allows for the analysis of real world scenarios and for the measurement of the model's outcomes in different potential scenarios, thus aiding decision-making (Jetter \& Kok, 2014). 


\subsubsection{FCM in technology adoption research}

There is a growing body of research that uses Fuzzy Cognitive Mapping (FCM) to gather, integrate and display the collective mental landscape of different stakeholders. FCM translates this landscape into a simulation model that can dynamically test different scenario. This allows us to understand the key factors and relationships that need to be addressed, in order to learn the best course of action (Jetter, 2006). Since its introduction (Kosko, 1986), the use of FCM has gained popularity beyond social and political science. It is used in many fields including new product development (Sperry \& Jetter, 2009), project management (Rodriguez-Repiso, Setchi, \& Salmeron, 2007), energy (A. Jetter \& Schweinfort, 2011), economics (Niskanen, 2006) and architecting medical support systems (Stylios et al., 2008).

Despite the capabilities of fuzzy cognitive maps and their growing momentum, they have not been used much to study EHTA. Until recently, it remained unclear whether FCM can successfully facilitate research in such demanding and involved context, and how it can accommodate the changing perceptions of the elderly. It was also uncertain as to how FCM could be used to gain a better understanding of the phenomena and possibly generate empirical insights. In preparation for this dissertation, my colleagues at ETM and I proposed and tested the use of FCM in EHTA research (Rahimi et al., 2016). In addition, the pilot study described in Appendix B, which demonstrates the use of FCM in EWHTA, has been conducted. 
These endeavors have proven that FCM can successfully serve as the modeling method in EHTA.

In technology adoption, this type of work has been done in educational (Hossain \& Brooks, 2008), agricultural (McRoberts et al., 2008), and healthcare organizational settings (Khoumbati, Themistocleous, \& Irani, 2006). However, none of these studies address health technology adoption in the personal realm (vs. healthcare or other organizations). Moreover, the studies do not model adoption outcomes as an FCM. Instead, they use FCM as a data acquisition tool to identify barriers and benefits of the technology that may influence adoption without simulating the actual adoption (Stach et al., 2005), or to create inputs for the Bass adoption model (Jetter, 2006). Going beyond this state-of-the-art, we model how the generally recognized factors for technology adoption (according to UTAUT) impact adoption by a specific set of stakeholders in a specific adoption setting. The resulting FCM model can be used to simulate which combination of factors leads to higher or lower adoption outcomes. These insights help technology planners modify product features and roll-out strategies in ways that increase adoption.

\subsubsection{FCM modeling}

Fuzzy Cognitive Maps (Kosko, 1986) are signed directed graphs: they consist of nodes, called "concepts" that are connected through arrows that show the direction 
of influence between concepts. A positive (negative) arrow pointing from concept A to concept B indicates that concept A causally increases (decreases) concept B. Weights can be assigned to reflect the strength of the connections. Concepts are typically verbally described and can contain hard-to-quantify concepts, such as "usefulness".

Structurally, FCMs are digraphs. As such, graph theoretical concepts apply and metrics such as density (an index of connectivity), total degree centrality (summation of all the concept links) and indegree and outdegree (the direction of the arrows) are frequently used (Özesmi \& Özesmi, 2004) to describe the overall structure of the model, as well as the position of specific concepts in the network. A high out-degree (a relatively larger number of inbound arrows than other concepts), for example, is assumed to hint at the importance of the concept as an influencer/leverage in the system.

Computationally, FCMs are regarded as a simple form of recursive neural networks (Dubois, Prade, \& Yager, 2014). Concepts are equivalent to neurons, but other than neurons, they are not either "on" (=1) or "off" (=0 or -1$)$, but can take states in-between and are therefore "fuzzy". Fuzzy concepts are non-linear functions that transform the path-weighted activations directed towards them (their "causes") into a value in $[0,1]$ or $[-1 ; 1]$. When a neuron "fires" (i.e., when a concept changes its state), it affects all concepts that are causally dependent upon it. Depending on the direction and size of this effect, and on the threshold levels of the dependent concepts, the affected concepts may subsequently change their 
state as well, thus activating further concepts within the network. Because FCMs allow feedback loops, newly activated concepts can influence concepts that have already been activated before. As a result, the activation spreads in a non-linear fashion through the FCM net until the system reaches a stable limit cycle or fixed point.

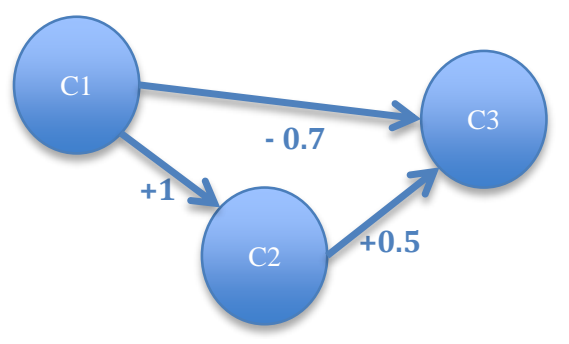

Figure 3 - 1 A simple FCM map

As an example, the simple map above displays a simple fuzzy cognitive map of three concepts and their causal relations. The causal relations in this map can be represented in the adjacency matrix (left section of the Table 3 -2). In the Figure $3-1$, the positive causality between $C 1$ and $C 2$ indicates that increasing $C 1$ results in increasing the value of $\mathrm{C} 2$; and in the same order the negative causality between $\mathrm{C} 1$ and $\mathrm{C} 3$ means that increasing $\mathrm{C} 1$ results in decreasing the value of $\mathrm{C} 3$. The higher value of causality result in a stronger effect (i.e. the increase of $\mathrm{C} 1$ has a higher positive impact on $\mathrm{C} 2$ than a negative impact on C3). An " $\mathrm{X} n$ " adjacency matrix represents the model ( $\mathrm{n}$ is the number of concepts in the model). The following adjacency matrix represents Figure 3-1 FCM. 


$$
\left[\begin{array}{ccc}
0 & 1 & -0.7 \\
0 & 0 & 0.5 \\
0 & 0 & 0
\end{array}\right] \quad\left[\begin{array}{lll}
1 & 0 & 0
\end{array}\right] \times\left[\begin{array}{ccc}
0 & 1 & -0.7 \\
0 & 0 & 0.5 \\
0 & 0 & 0
\end{array}\right]=\left[\begin{array}{lll}
0 & 1 & -0.7
\end{array}\right]
$$

Table 3 - 2 Adjacency matrix and calculation

In the event when $\mathrm{C} 1$ is activated, the values of the other concepts is calculated by the multiplication of the vector matrix and the adjacency matrix. The resulting output vector serves as the input vector for the next iteration, and thus it is multiplied with the adjacency matrix again. This process continues until the multiplication of the output vector with the adjacency matrix results in a vector that is identical to (or less than an epsilon value different than) the output vector of the earlier iteration. The output is considered stable at this point. A stop criterion is used (e.g. a maximum iteration number), in case the output can't reach the point of stability. Based on the following formula, the value of each concept (in any given time) is calculated by the summation of the values of all its influencing concepts $(\mathrm{Cj}(\mathrm{t}-1)$ multiplied by the weight of their influences $(\mathrm{Wji})$ :

$$
C i(t)=f\left(\sum_{\text {All } j \neq i} W j i \cdot C j(t-1)\right)
$$

Equation 3 - 1 Calculation of the concept values

In the above example, concept 1 is activated and after one iteration the outputs are stable, the value of concepts can represent the result of the simulation.

In Equation $3-1 S(x)$ is a squashing function that brings the concept values back to the $[-1,1]$ range. Commonly used squashing functions (aka threshold functions) (as displayed in Equation 3 - 2) such as Bivalent, Trivalent, Sigmoid, and 
Hyperbolic tangent function restrict the concept states to discrete final states-[0; $1]$ or $[-1 ; 0 ; 1]$ or to intervals $[0 ; 1]$ or $[-1 ; 1]$. Bivalent squashing functions (first equation in) can represent an increase of a concept, trivalent functions can represent an increase or a decrease of a concept and logistic functions can represent the degree of an increase or decrease of a concept (Tsadiras, 2008). This dissertation uses hyperbolic tangent function as it's a commonly used logistic function.

$$
\begin{aligned}
& \text { Bivalent function: } \\
& \text { Trivalent function: }
\end{aligned}
$$

$$
\text { Sigmoid function: } \quad f(x)=\frac{1}{1+e^{-\lambda x}}
$$

Hyperbolic tangent function: $f(x)=\tanh \lambda x=\frac{e^{\lambda x}-e^{-\lambda x}}{e^{\lambda x}+e^{-\lambda x}}$

\section{Equation 3 - 2 Some popular squashing function used in FCM simulation}

$\lambda$ is a constant that defines the slope. For $\lambda=1$, the squashing function is almost linear, which means that gradual concept changes only have a gradual impact on other concepts. Also, inputs in the interval $[-1 ; 1]$ do not map to outputs in the same range but only to approximately $80 \%$ of the maximum and minimum values, so that final concepts states are never at the extremes. For $\lambda=5$, hyperbolic tangent 
approximates a full normalization into the interval $[-1 ; 1]$ (Bueno \& Salmeron, 2009).

Concepts that are persistent over multiple iterations (e.g. Technology characteristics), rather than one-time shocks to the system (e.g. a terrorist attack), are 'clamped', which means they are reset to their initial value after each iteration.

All FCMs have "meta-rules": several input vectors - so-called input regions - lead to the same final system state (Dickerson \& Kosko, 1994a). FCMs with continuous concept states, so-called "continuous-state machines" can result in chaotic behavior (Dickerson \& Kosko, 1994b). FCMs with discrete concept states, socalled "finite state machines" result in either a fixed state vector or in a limit cycle between a number of fixed state vectors (Dickerson \& Kosko, 1994b; Stach, Kurgan, Pedrycz, \& Reformat, 2005). At this point, any new iteration delivers the same result as the prior iteration or the difference between the iterations is considered negligible because it falls under a predefined threshold for stability, epsilon $(\varepsilon)$. The number of iterations it takes until a stable fixed point or limited cycle is reached depends on squashing functions, initial state, and the structure of the FCM (Bueno \& Salmeron, 2009; Jetter, 2006; Özesmi \& Özesmi, 2004; Tsadiras, 2008). The stable end states of concepts cannot be interpreted in absolute terms, but only relative to other factors in the system or relative to other system descriptions. 


\subsection{Measures}

As the EHTA model is an FCM, the values of all the concepts, whether they are set at the input or measured as the output, are normalized to a range from -1 to 1 .

\subsubsection{Input Variables}

Earlier research (Rahimi et al., 2016) has shown that the input constructs provided by the UTAUT are interrelated. Thus they are not true input variables. The purpose of the research is to discover the input variables that explain the main UTAUT constructs - usefulness, ease of use, social influence and facilitating conditions. Therefore many of the true input variables are unknown.

Since the model is an FCM, the input variables, just like all other variables, are concepts. These concepts emerge entirely from the EHTA context. In essence, an important research goal is to discover these input variables/concepts.

\subsubsection{Intermediary Variables}

As suggested by an earlier study (Rahimi et al., 2016), gatekeepers influence all the UTAUT input constructs (depicted in Figure 2 - 3). As the research is measuring these influences and how they are formed, UTAUT input constructs become the intermediary variables of this research. 


\subsubsection{Output Variables}

The output variables are known. They are the intention to adopt and the actual adoption. Adoption intention is the propensity to adopt, whereas adoption behavior is the probability of the actual adoption of technology.

\subsection{Applicable Research Settings}

The output of this research is a model, which achieves two objectives stated in the section 1.2: 1) Identifies the key adoption drivers and obstacles and their relations, 2) Illustrates how the gatekeeper influences the elderly person's adoption decision, 3) Represents adoption drivers and gatekeeper influences on adoption quantitatively, and 4) Facilitates exploration of adoption in response to various factors through simulation. The model is geared toward explaining consumer behavior rather than organizational behavior. Thus, the model is best applied to the settings that exhibit the following characteristics.

1. Environment: Settings within the field of healthcare, which study consumer behavior rather than organizational behavior. For example the technology adoption of a health technology in an organizational setting is not targeted here, i.e. the study of the adoption of a robotic surgical device in the operating room is not modeled here.

2. Technology: Settings in which the consumer has a choice of deciding whether to adopt or reject a health care technology or service. For example, studying health technology adoption of devices that are mandated and/or 
administered by healthcare professionals do not constitute the appropriate setting here.

3. Population: Settings in which elderly consumers are faced with the choice of adoption. For example, the study of the adoption of activity trackers by young athletes is not targeted here.

\subsection{Proposed Research Design - Steps to Conduct the Proposed Research}

The proposed research will result in a methodology that addresses the needs for modeling and analyzing complex dynamic environments where the integration of various stakeholders and perspectives are demanded. This methodology is validated through the development of an empirically-grounded EWHTA model, which will be the second contribution of this dissertation. Using FCM as the methodological foundation for both extracting and modeling the adoption, the proposed method 1) integrates stakeholders' opinions, 2) triangulates data for maximum generalizability, and 3) builds quality control into the model building process for validation.

The method developed in this dissertation contextualizes the UTAUT with respect to EHTA. Capturing the influence of gatekeepers becomes a key part of this contextualization, because, in EHTA, gatekeepers exert a tremendous influence over the elderly. As a consequence, pairs that consist of an elderly person and a gatekeeper constitute the unit of analysis of the proposed research method. 
As mentioned in the Section 1.1, the concepts that are associated with TA theories are too general to be useful in the EHTA context. Making sense of how these concepts apply to the phenomena under study warrants highly granular, empirically grounded approaches that study specific contexts in depth.

The research method proposed in this dissertation follows such an approach. It handpicks effective techniques in computer-based modeling and qualitative research approaches to facilitate modeling of technology adoption for a demographic in which agency is important. It uses the ethnographic interview method in combination with a light variant of repertory-grid technique to collect indepth field data, as well as Fuzzy Cognitive Mapping (FCM) (Kosko, 1986). The combination of these techniques constructs the respective mental model of the users of technology products who are subjected to significant influence by technology gatekeepers. This is followed by quantitative techniques to integrate and create the fuzzy cognitive maps to form the technology adoption decision making tool. 


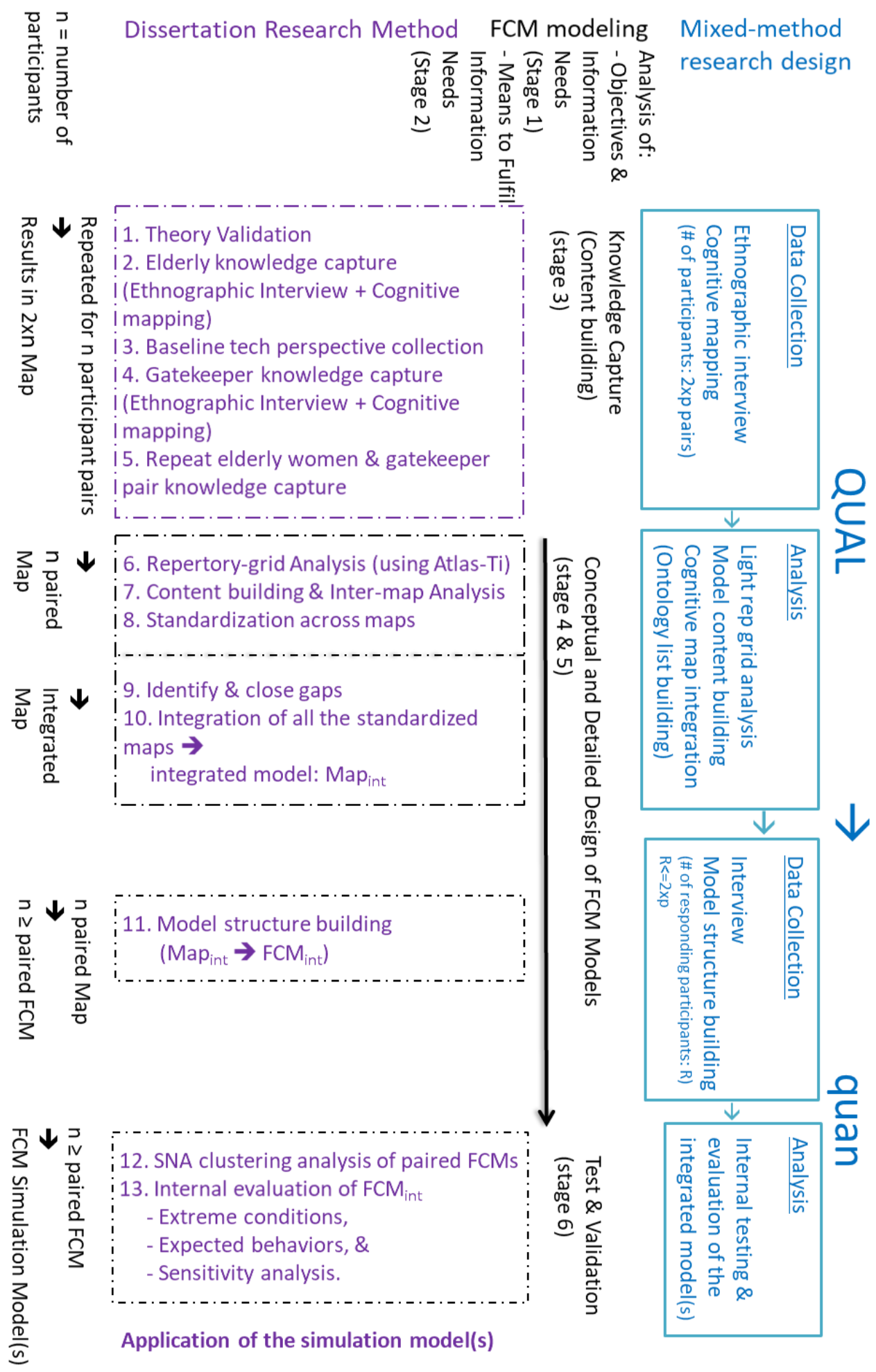

Figure 3 - 2 Proposed researh method: Aligning the research method steps with the mixedmethod design and the fuzzy cognitive mapping 
The proposed research follows a mixed-methods design, which is illustrated in Figure 3 - 2. The sequential QUAL $\rightarrow$ quan mixed-methods approach has been selected for the proposed research because of its potential ability to generate fresh insights in the complex EHTA context. The qualitative phase is abbreviated in capital letter-QUAL indicating that the qualitative part of the mixed-methods is more important than the quantitative part (abbreviated in small letters-quan). Both the QUAL and the quan phase of the proposed method contain data collection activities that are followed by analysis activities. The dominant QUAL phase consists of creating the content of the model by developing causal maps through ethnographic interview data elicitation in combination with fuzzy cognitive mapping, followed by qualitative analysis that uses a light version of repertory-grid techniques. During the subsequent quan phase, the structure of the model is created from the second set of interviews from the same group of participants (as described in section 3.9). At the end of the quan data collection, the creation of EHTA model is complete. The EHTA model is tested internally during the analysis portion of the quan phase.

The research method proposed for this dissertation basically follows the guidelines for FCM approaches laid out by Jetter (2006). It uses FCM's ability to integrate the perspective of the elderly as well as those of their gatekeepers. In addition, it has been adapted to accommodate the highly interdisciplinary nature of the proposed research. 
Jetter (2006) describes the fuzzy cognitive mapping process in terms of 6 stages: Analysis of Objectives and Information Needs (Stage 1); Analysis of Means to Fulfil Information Needs (Stage 2); Knowledge Capture (Stage 3); Conceptual and Detailed Design of FCM Models (Stages 4 and 5), and Test and Validation (Stage 6). Figure 3 - 2 illustrates that the first two stages of this process transpire prior to any data collection; the remaining stages (3 through 6 ) are executed in 12 steps. Stage 4 and Stage 5 span the analysis portion of the QUAL phase and the data collection portion of the quan phase. The final outcome of the proposed research is an EHTA simulation model that reflects the collective knowledge of all the participants from the QUAL phase and the respondents from the quan phase; it takes the form of a fuzzy cognitive map. Using this simulation model comprises the final step (13) of the proposed research. Jetter (2006) refers to what are called stages here as steps. This terminology has been changed, in order to avoid confusion with the steps that will be undertaken in the proposed research.

\subsubsection{Analysis of Objectives and Information Needs (Stage 1 of FCM Building Process)}

As stated previously (in section 1.2), the objective of this research method is to provide a robust technology adoption modeling method among demographic groups that rely heavily on gatekeeping for technology adoption. This method yields a contextualized model of technology adoption, which serves as a decision tool and provides a better understanding of the drivers and obstacles in elderly health technology adoption. The model...

- Identifies the key adoption drivers and obstacles and their relations 
- Illustrates how the gatekeeper influences the elderly person's adoption decision

- Represents adoption drivers and gatekeeper influences on adoption quantitatively

- Facilitates exploration of adoption in response to various factors through simulation

\subsubsection{Analysis of Means to Fulfil Information Needs (Stage 2 of FCM Building Process)}

\subsubsection{Identification of Participants}

In this stage, the participants are going to be selected from a variety of people that represent the consumer group under study. Since this research captures the influence of the gatekeepers on the potential users of the technology (the elderly), pairs of elderly and their gatekeepers have been identified as participants. Each pair consists of ....

$\checkmark$ One elderly who acts as the potential adopter, and

$\checkmark$ One family member or friend that has been identified by the elderly person. This person plays the role of gatekeeper for the elderly woman.

Identification of participants proceeds according to the principle of diminishing returns on learning. Pairs of elderly and gatekeepers are added until no significant insights are gained by increasing the sample of participants. According to the highly cited RGT paper by Tan and Hunter (2002), " $A$ sample size of 15 to 25 within a population will frequently generate sufficient constructs to approximate the 
"universe of meaning" regarding a given domain of discourse (Dunn et al., 1986; Ginsberg, 1989). That is, no new constructs are normally added, even if the sample size is increased." Similarly, consumer market research (Griffin \& Hauser, 1993) suggests that diminishing returns on learning should occur after interviewing from 10 to 30 participants.

\subsubsection{Methods Selection for Knowledge Capture}

Since earlier sections contain detail information about different knowledge capture techniques, this sections describes the techniques used, and explains how the knowledge is captured in the proposed research method.

During the first phase of the enquiry--the qualitative phase (Steps 1 through 10 in Fig. 8), the knowledge captured from the participants creates the content of the mental map that is the basis for creating the simulation model. During the second phase of enquiry - the quantitative phase (Step 11 through 12 in Fig. 8) -the knowledge captured from the participants creates the structure of the mental map, which reflects the degree of influence of the concepts in the model. The methods for knowledge capture are selected accordingly.

\subsection{QUAL Knowledge Capture- Repertory Grid (RGT)}

A knowledge capture method that facilitates FCM modeling is designed here based on the overarching Repertory Grid Technique (RGT) procedure. This procedure covers QUAL data collection and analysis. By executing the RGT explained here 
(i.e. the QUAL part of the mixed-method research approach), the content building part of the Fuzzy Cognitive Mapping is conducted.

It's important to highlight some of the dissimilarities between the approach designed here and the typical RGT:

1. RGT traditionally extracts constructs and their correlations; here the primary interest of knowledge capture is to find the constructs and their causal relations. So, traditionally the ratings provided by the participants create a base for correlation study, but the rating provided here is aggregated to show the degree of influence between the constructs.

2. As originated by the George Kelly's Personal Construct Theory (George Kelly, 1955), the typical RGT extracts "personal construct". Here, the RGT approach aggregates participants' constructs to construct "common grids" to study the elderly population. Hence, we cannot call the resulting constructs "personal constructs" anymore. The aggregating approach already exists in RGT ("Repertory Grid Technique", 2003).

3. The original RGT includes statistical analysis such as cluster analysis. The later methods include approaches that replace statistical analysis with a variety of techniques including frequency counting or content analysis. While these approaches are widely accepted today, it is worth noting that the approach designed in this dissertation uses content analysis (as opposed to the original popular statistical analysis). 
As the overarching procedure for the knowledge capture of the proposed mixedmethod modeling method, knowledge capture follows the guiding principle of the repertory-grid elicitation approach. This includes both the QUAL data collection and analysis as detailed here. The four phases of the RGT procedure designed here are explained and mapped to the research method as described below.

\section{Phase I- Element Selection}

The designed approach in this dissertation uses fixed elements, as suggested by extensive prior research (Rahimi \& Jetter, 2015; Rahimi et al., 2016). In the traditional RGT, participants identify both elements and constructs. However, since UTAUT has proven to provide the appropriate elements for the study of EHTA, its constructs (as depicted in Figure 3 - 3) are the elements under study here.

Phase I is conducted in step 1 of research design, when participants are introduced to the elements as proposed in UTAUT (as explained in section 3.4.3-Knowledge Capture (Stage 3)). Since concepts (i.e. the nodes) represent these elements and

all other factors derived from the research, from here on, these elements are called as concepts.

\section{Phase II - Construct Elicitation}

In this phase, using the ethnographic interview technique, participants are asked predesigned open-ended questions to provide their constructs (again: concepts). This is step 2 in the research steps (section 3.4.3.), when participants are probed 
to freely provide constructs about the fixed elements, while the researcher is simultaneously capturing them in her cognitive map.

\section{Phase III - Rating}

To measure the initial participants' perception of the technology under study, participants rate all the elements (here UTAUT constructs) in a rating grid (in the pilot study, this takes the form of Table B - 7). The rating of prospective product under evaluation will be based on a 5-point Likert scale. For example, the participants will be asked to rate the technology's ease of use, usefulness, etc. This inquiry is made during the same ethnographic interview. This is step 3 of the research steps (section 3.4.3.).

\section{Phase IV-Analysis}

This phase (steps 6 - 10 in section 3.4.4.) facilitates the augmentation and integration of the cognitive maps (the content) using the repertory-grid technique.

As Boyle (2005) states, content analysis can be used in RGT to analyze the individual grids. The content analysis approach conceptualized here, uses deep qualitative analysis (aka the grounded theory coding approach) technique to extract and aggregate constructs (i.e. map concepts) using Atlas.ti Software, as explained below.

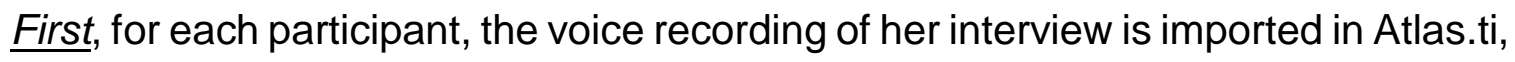
listened to and coded. The initial coding could be based on a single phrase, a sentence, or many sentences. 
Second, once the initial coding is completed, those codes are analyzed, followed by the creation of code families that collect and group the similar codes together. As such, the initial codes are assigned to the code families that best represent and generalize the initial codes' contents. This step is completed when all the initial codes have been assigned to a code family. These code families were synthesized from individual maps. So, new code families are only created when all the existing code families in that project fail to generalize the new code meaning.

$\underline{\text { Third, }}$, the concepts in the initial cognitive map of the participant are compared and contrasted with the code families, and the code families that are missing from the map are added. This action is followed by adding incoming and outgoing links for the new map concepts by reanalyzing the content (interview record).

Fourth, as the above three steps are conducted for all participants (elderly women and the gatekeepers), the code families become the standardized concepts that collect and group the participants' concepts. At the end, the code families make up the standardized concepts of the ontology list.

Fifth, all the participants' cognitive maps are standardized (i.e. the concepts are replaced by their standardized concept representatives).

This process is detailed in future sections. In a nutshell, a parking lot map is created and gradually completed during the qualitative analysis, where new concepts and relationships are incrementally added to the map. Upon the completion of the qualitative analysis, the parking lot map contains all the concepts and relationships. It is used as a model to standardize each E pair map (the map 
containing the cumulative learning of the $E$ and the E's gatekeeper). Each map is standardized by replacing each of its concepts by its corresponding concept from the parking lot map. Should there be links in the E's map that do not exist in the parking lot map, they are analyzed and added to the parking lot map. This process results in the E's pair map containing the same concepts and links that are contained in the parking lot map or a subset thereof.

Six, All the standardized maps are integrated to represent the base of the simulation model.

This is done by first expanding all the adjacency matrices of the E's pair maps, so that they contain the same concepts as the parking lot map, and they are denoted in the same order, (i.e. $\mathrm{C}_{i}$ is the same concept across all the maps). Naturally, the concepts that were newly added to these maps (from the parking lot map) have no links; in other words, the maps' adjacency matrices contain zero values in the rows and columns of these concepts. Once all the E pair maps are standardized, a simple matrix addition of their adjacency matrices represents the base of the integrated FCM model.

\subsubsection{2. quan Data Collection - Walk-through Survey}

As earlier sections have provided a detail analysis of means to fulfill information needs, this section explains how the selected method is going to be used in this part of the proposed research method. 
Once the content of the model has been built, the research method facilitates the creation of the model's structure (quantified degree of influence among concepts) using a second set of one-on-one interviews. This step corresponds to step 11 of the research method in section 3.4.4. It is conducted as follows:

First, the model is broken down to sections (e.g. 1- everything related to "Performance Expectancy" construct, etc.), and explained to the elderly in a cognitively simple narrative.

Second, the elderly is asked to identify the degree of strength of the causal relationship (influence in the output construct of the relationship) using the simple 5-point Likert scale, the elderly is then asked to assign the weight as they see appropriate.

Questions such as the following will be asked to guide the inquiry:

1. The model says there is a link between (all the input concepts to $\mathrm{C}_{\mathrm{i}}$ ) and $\left(C_{i}\right)$.

2. Can you identify how much each of these concepts contribute to $\mathrm{C}_{\mathrm{i}}$ ? (the incoming links to each concept)

3. How strong is the influence? Please pick one of the following numbers (5-point Likert scale).

1 implies "The strongest",

0.75 implies "Very strong",

0.5 implies "Somewhat strong" 


\subsection{5 implies "Not very strong"}

0 implies "Not strong at all"

"I don't know"; (alternatively, they can chose not to answer)

As explained in step 11 (in section 3.4.4.), the edge weight of each link is calculated based on the average value of the edge weights identified by all the participants for that link, unless they are contradictory. The responses are considered contradictory, if participants selected degrees of contributions that exceed 3 on the Likert scale. For example, if elderly say conceptk has no influence on conceptı (0 edge weight) and elderly say the influence of conceptk on concept $t_{k}$ is very strong/important (.75 edge weight) then the evaluation has to be studied. The study leads to potential two outcome:

- The two elderly (or more) agree on one or two (or more) close levels of contributions that do not differ more than 3 levels of Likert scale.

- The two elderly (or more) still confirm that their judgement were accurate and can't be changed, which in this case, these maps are studied to identify the clusters of similar maps (maps with similar edge weights). These clusters of similar maps are then calculated (using the same averaging formula) to separate models that each reflect the group of elderly people that agree on their judged edge weights.

Once the content and the structure of the model have been built, the integrated FCM model is ready to be tested and validated (detailed in section 3.4.5.). Upon 
successful evaluation, the integrated FCM model is ready for use as the support model for EHTA decisions. Evidently, FCM serves as a bridge between qualitative and quantitative parts of the research. FCM simulation power allows for scenario planning and interpret how they make adoption decisions. This will also support the identification of decision alternative(s) that lead to successful adoption.

The RGT approach combined with FCM model building proposed above has been pre-tested in the pilot study (described in Appendix B), as well as in an earlier publication (Rahimi et al., 2016).

\subsubsection{Knowledge Capture (Stage 3 of FCM Building Process)}

The proposed research captures the collective knowledge of elderly women and their gatekeepers. For this reason, knowledge capture requires sequential integration of the knowledge of the individual participants. Since the participants in this research are mainly elderly with limited ability to create cognitive models, the modeler interviews the participants and creates cognitive maps simultaneously. (This would be the option 4 in Table 2 of (Jetter, 2006)—alternative approaches to knowledge capture).

As the overarching QUAL design of the proposed mixed-method modeling method, knowledge capture follows the guiding principle of repertory-grid elicitation approach. Hence, the interview questions elicit information about four aspects of technology adoption: 1) health technology adoption decision-making (the topic), 2) the health technology being studied (the elements), 3) interpretation of the UTAUT

constructs and how their perceptions are formed, i.e. concepts influencing the 118 
formation of those constructs (the set of constructs), and 4) the ratings of the health technology per each UTAUT construct (a set of ratings of elements on constructs).

It is important to describe some of the different but overlapping terminologies that are used interchangeably in the qualitative data collection and analysis (in the following 13 steps on how to apply the proposed research method). The logic behind this way of explaining the research process is to create a bridge between the terminologies of the different techniques/tools that are used. The proposed research method deploys the repertory grid technique to construct fuzzy cognitive mapping models with Atlas.ti. The output of the QUAL phase is a cognitive map model. However, terminology from all three constituent techniques of the mixed method is used interchangeably:

a) FCM Terminology: Concept and link, as defined in detail in section 3.1.4.

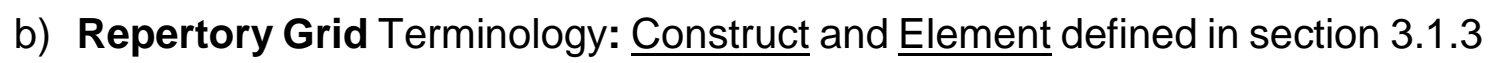
and the sections that follow. The constructs here are the UTAUT main constructs. However, since the model is an FCM map, they are also represented by FCM concepts. The element is the health technology under study. The case study studies RHMT activity tracker wearables.

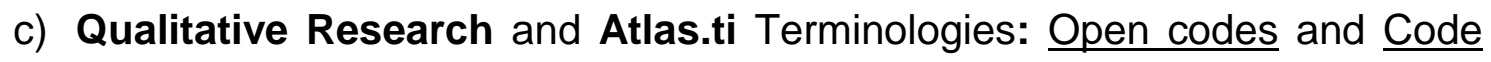
family, which are used mainly in the qualitative phases of the research steps in Atlas.ti. Open Coding is the first step in capturing the FCM concepts, in which the words/sentences/paragraphs of an interview audio recording are marked in Atlast.ti to label a piece of information. The open code could 1) 
create a new concept, 2) add to the 'grounded' factor of an existing concept, 3) create a new link between existing concepts, or 4) create both new concepts and links. A code family is a special code that is created in Atlas.ti to group existing open codes. The purpose of each code family in this research method (as created in Atlas.ti) is to create a concept that captures the underlying factor of one or many open codes that refer to one or more related FCM concepts. As a result, each code family represents one or more FCM concepts that will be added to the research model.

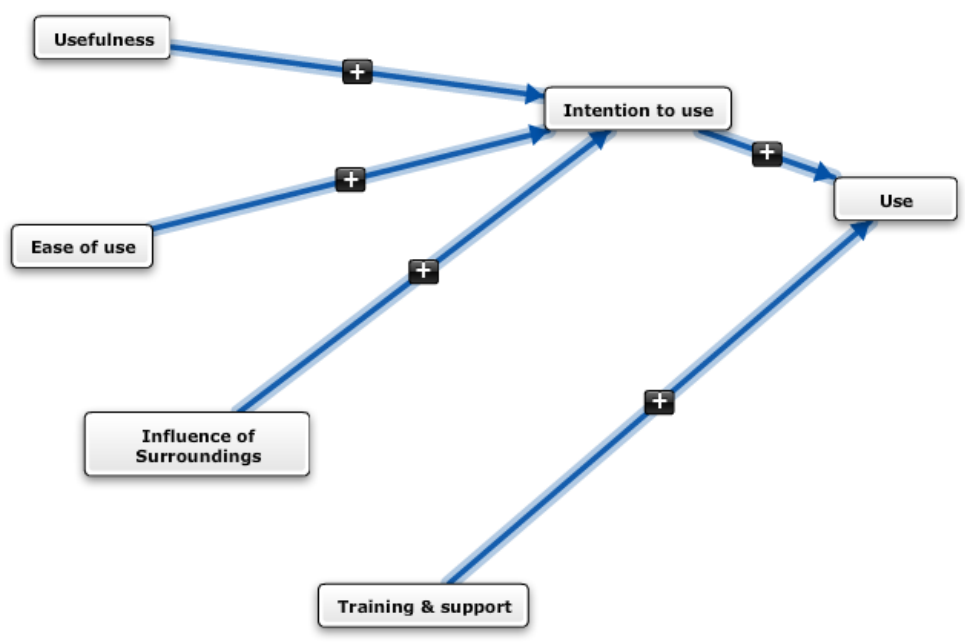

Figure 3 - 3 UTAUT as base cognitive model

Knowledge capture involves the execution of the following five steps (the ethnographic interview).

1. Theory Validation: Participants verify the validity of the UTAUT (depicted in Figure $3-3)$ in their adoption. Each participant is asked to subjectively confirm 
or object to the proposition that the main constructs of UTAUT are important and in fact influence the participant's technology adoption decision.

2. Elderly Knowledge Capture: Using predesigned, probing, open-ended questions, the participant is then asked to explain and help to develop the left side of the verified UTAUT. The researcher (i.e. the modeler) develops the basic cognitive model by expanding the UTAUT constructs (in Figure 3 - 3 ). This model is actively expanded to include the novel concepts that the participant prompts. Then, the links that depict the influence of these novel concepts on each other and on UTAUT factors are added.

3. Baseline tech perspective collection: The information about the participant's perception of the UTAUT's main constructs are gathered, as they pertain to prospective product under evaluation. This step is itemized to describe that the perceptions about the quality of the wearable device are gathered during the interview. However, this information is gathered throughout the qualitative data collection and analysis. Some of this information is captured in the initial cognitive map of the participant that is created during the interview and some of those are gathered later during the analysis. All the information gathered is captured in a separate file (elderly profile) for later evaluation of the model.

4. Gatekeeper knowledge capture: Next, the participant's gatekeeper, who is identified by the participant as the main technology influencer, is interviewed. This ethnographic interview also results in a basic cognitive map, which is created in a manner similar to the one described in step 1 and 2. However, this 
map captures the gatekeeper's perspective of how different drivers and obstacles influenc the elderly's decision during the adoption. This results in another mental model (cognitive map) of the elderly person as viewed from the perspective of the gatekeeper.

5. Repeat elderly women \& gatekeeper pair knowledge capture: The interview of the participant-gatekeeper pairs continues until incremental learning reaches diminishing returns.

\subsubsection{Conceptual and Detailed Design of FCM Models (Stage 4 and 5 of FCM Building Process)}

During the steps described in this section, all cognitive maps are augmented by qualitative analysis. The qualitative analysis follows a light version of repertory-grid analysis, as detailed below. Additionally, the analysis results in the models becoming standardized by developing an ontology list-a list of concepts that emerges from the initial models and the findings of the qualitative analysis of the repertory-grid. This process comprises a crucial stage in the integration of all hitherto generated cognitive models.

6. Repertory Grid Analysis: This step maximizes cognitive representation of the interviewee's mental model on the initial map, which resulted from each repertory-grid interview in steps 1 and 2. As such, during the analysis, the information from each elderly woman participant and her gatekeeper (the two interview recordings and maps) are studied and combined to form the augmented cognitive map for each pair. This map is qualitatively analyzed and 
updated using the recorded field data gathered in steps 1 through 3 . This analysis is conducted using Atlas.ti 7 software ("ATLAS.ti 7 Windows | Qualitative Data Analysis with ATLAS.ti", 2017). The researcher analyzes the audio recording of each interview and augments the elderly woman's cognitive model using the repertory-grid technique to collect the elderly woman's constructs. The researcher subsequently analyzes the interview with the gatekeeper, and the findings of this analysis are added to the elderly woman's cognitive map. The addition of gatekeeper's perceptions occur in a rather lengthy process. During this aggregation of the two perspectives, the following three situations can happen. They are listed in order of their likelihood of occurrence:

a. Usually, the gatekeeper's perception brings a new perspective to understanding her elderly's decision-making process, and it provides new evidence that can lead to new concepts and/or links. These are captured by open codes that later contribute to the creation of code families and links.

b. Often, the gatekeeper's perception only confirms and provides more evidence to an existing concept or link. This is sometimes captured in the same open code, meaning that during the coding of this perspective the existing code was selected as opposed to creating new one (Atlas.ti shows this by increasing the open code 'grounded' factor). When no apparent 
existing code is immediately found, a new open code is created, but later it is merged during this integration process.

c. Sometimes, the gatekeeper's perceptions contradict those expressed by the elderly. Such opposing evidence is resolved through further investigation. The original cognitive maps and, more importantly, the two interview audio records are analyzed to resolve the discrepancies ${ }^{7}$.

The resulting integrated cognitive map contains new concepts and relationships--it embodies all the constructs and relations identified by the participant pair (from both the interview records and their initial cognitive maps).

7. Content Building \& Inter-map Analysis: All maps subsequently go through iterations of inter-map analysis, a process that merges similar concepts and themes through incremental analysis. This process consists of the following steps. Related codes are grouped into more generalized, integrated codes, which are more abstract (each integrated code is represented by a code family in Atlas.ti). Next, each integrated code is analyzed to ensure that it represents all the open codes that convey the same meaning. A new, more generalized concept is created for every group of open codes that convey the accumulative

\footnotetext{
7 The later pilot study and the case study show instances in which the elderlies were too proud to state or accept the existences of some of the drivers or obstacles of the adoption. These factors were often revealed by the gatekeepers and later were confirmed during the quan data collection when the elderly had the chance to judge the degree of the influences.
} 
meaning. The original open codes are analyzed continuously to ensure that the generalized concept captures the meaning of the original codes successfully.

The concepts in each original cognitive map (generated in step 2) are compared with the new concepts (created above). All the original concepts are compared with the generalized concept and modified, if needed. In this process some new concepts are created, and some concepts are merged, in order for the granularity to become consistent. Merged concepts also have to be directly relatable to the research questions.

To generalize the models, the specific information related to the elderly participants' situations is extracted from the initial models and placed in the baseline file created in step 3 (The profiles shown in Appendix $\mathrm{H}$ are examples of such baseline files). This information includes information about the elderly states and traits such as how isolated (s)he is, how technology savvy the gatekeeper is, or what the elderly's attitude toward technology is. The participant's profile also provides information regarding some of the typical expected behaviors of the cohort being studied. Such information is used to evaluate the resulting model(s) to test whether the simulation model(s) show the same behavior. This is further described in step 12 of the research method in Behavior Reproduction Test.

8. Standardization across maps: At the end of this process, once all the cognitive maps have been analyzed and the creation of concepts and links is complete, the exhaustive list of these common concepts forms the list of 
standardized concepts. Then all the maps are standardized by replacing their concepts by their corresponding standardized representatives. The exhaustive list of these standardized concepts with their definitions make up the content of so called ontology list or codebook, two terms that are used interchangeably in this dissertation. An ontology list or codebook is defined in computer science as a formal source for naming and defining a set of entities in a particular domain. In the context of the proposed research method, the ontology list or the codebook contains all the standardized concepts and their definitions. For example, the 'ontology list'/codebook that emerged from the pilot study is depicted in Table B - 1 in Appendix B and the one that emerged from the case study is listed in Appendix G.

9. Identify \& Close gaps: Any potential gap in the way information is captured is investigated to enhance the quality of the model. These include:

a) The model needs to be studied to ensure that the concepts and links operate in a comparable temporal scale. This ensures realistic behavior (for example, an influence that takes a long time does not take affect at the same time as an influence that happens very fast). Should such situation occur, measures need to be taken to resolve such temporal disparity.

b) The concepts and links are represented in a relatively similar degree of granularity. This avoids overly detailed paths and ensures that the influences of some concepts are not exaggerated, and as a result, the behavior of the model is not skewed. Should such situation occur (e.g. 
elderly has provided much details about a particular concept), either the detailed concepts could be merged or other concepts could be dissected to match the granularity of other concepts.

c) The accumulated analysis of the cohort studied results in a comprehensive map of concepts and relationships. However, the map has to be studied to make sure common concepts and links, which have not been captured, but need to be added, are added. For example, some obvious input concepts and their links could have been missed in the research (i.e. the information received from the participants didn't provide any information about those concepts). Such concepts are added, in order to make the model more holistic.

10. Integration of all the standardized maps: The augmented maps that results from earlier steps are merged in an integrated map, Mapint. This merged map contains the collective knowledge of all elderly participants and their gatekeepers which provides a more holistic mental model of the elderly person during technology adoption decision making, which includes influence of the gatekeeper(s).

The resulting model highlights the critical drivers and barriers of the technology adoption and how these factors influence each other. The integrated model (Mapint) is a model of the elderly's adoption process, which is based on two data sources (elderly and gatekeeper). It shows how gatekeepers influence such key drivers and barriers. 
11. Model Structure building follows. In this step, the researcher gathers information pertaining to the degree of influence of each concept on other concepts. This step is the quantitative data collection phase of the research. To do this, the same groups of elderly persons who participated in the QUAL data collection are interviewed again. They are asked to subjectively validate the significance of the concepts and assign their influence on the other concepts using a 5 -point Likert scale $[0,0.25,0.5,0.75,1]$ (the respondents can refuse to provide a value for an edge weight of which they are unsure). The statements associated with the scales in the descending order are: Most important, Very important, Somewhat important, Slightly important, and Not important at all. While the choices state "important" for simplicity, the goal is to capture the strength of the edge weights and during the walkthrough the "strength" is emphasized. The valence of the links has been established in the earlier steps. The participant (after confirming the link and its valence) provides the magnitude of the influence. Since FCM edge weights inherently range from -1 to 1 , so will the Likert scale. This means that the values range from -1 to +1 in increments of 0.25 . Therefore, the possible edge weights are: $-1,-0.75,-0.5$, $-0.25,0,0.25,0.5,0.75,1$. If $R$ respondents ${ }^{8}$ provide the edge weights for every causal link of the integrated model, Mapint, then the following formula calculates

\footnotetext{
${ }^{8} \mathrm{R}$ is ideally the same as number of participants, but if some of the participants don't take part in this second interview, then $\mathrm{R}$ will be less than the number of participants.
} 
the collective value of each edge weight $\left(L_{i}\right)$. The formula simply averages all the edge weights it has received for every link.

$$
L_{i}=\frac{\sum_{r=1}^{R} l_{r . i}}{R-R_{i}^{\prime}}
$$

Equation 3 - 1 Edge weights calculation

where

$R_{i}^{\prime}$ is number of participants that didn't provide edge weight for the $i^{\text {th }}$ link

$R$ is total number of respondents

$L_{i}$ is the edge weight of the $i^{\text {th }}$ link

Once all the links of Mapint are assigned with their corresponding calculated edge weights, the Mapint becomes the fuzzy cognitive map, FCMint.

\subsubsection{Test and Evaluation (Stage 6 of FCM Building Process)}

During the process of quan data collection, the structure of the integrated model is evaluated by the participants when they are asked to identify the strength of the influence of each concept on another. For each edge weight, the degree of those influence are elicited, once the influence is confirmed. The structure of the integrated model is generated from the collective of the participants.

As Sterman states (Sterman, 2000), system models cannot be validated as they are all wrong, however their usefulness can be evaluated based on their purpose. Usefulness requires that the model adequately reflect what is known about the real-world system's structure and behavior. Accordingly, the integrated FCM 
model from the steps above ( $\left.F C M_{i n t}\right)$ needs to be tested based on the relevant criteria in system dynamics (Sterman, 2000). In addition, any potential discrepancy is investigated, and corrective actions are taken.

12. Model Evaluation of the integrated FCM (FCMint) is conducted based on the following activities.

1. Extreme Condition Tests: Models need to be robust in extreme conditions, in that under extreme conditions the models need to behave realistically (Sterman, 2000, page 869). For example, when participant perceives the technology to be not useful and not easy to use, the model can never suggest a possible adoption.

2. Behavior Reproduction Tests: Model should behave as expected. These tests can be conducted through various techniques including statistical testing. The mixed-method research design in this dissertation takes advantage of qualitative analysis of various participants within the elderly population. This qualitative analysis highlights the typical successful and unsuccessful EHTA scenarios that can be used to verify whether the model produces those expected behaviors.

3. Sensitivity Analysis: checks whether the model's conclusion change significantly when the assumptions varies in the possible range of uncertainty (Sterman, 2000, page 883). From the three types of numerical, behavior mode and policy sensitivity, the "behavior mode" is the relevant one to test EHTA models. For example, when comparing different activity 
trackers, it would be important to test how much the model behavior changes when the products features varies within the plausible range. Such simulations should not simply state the obvious. They should provide useful information that offer information about policy sensitivity or decision relevance. In other words, a model that correctly describes real-world behavior, but does not necessarily help with better decision-making because it cannot differentiate between different options, is correct but useless.

The successful validation process as described above results in FCMint becoming the EHTA simulation model, FCMIm.

13. FCM simulation model is ready to be used. The model can help practitioners to better interpret and understand the dynamics of the context under study. This better informs the best course of action that leads to better health technology adoption among the elderly women. For instance, different scenarios can be tested through simulation to identify and prioritize the characteristics of the technology group under study that lead to better adoption among the consumer demographic in focused, i.e. the elderly. Additionally, the model can provide insight to practitioners through the simulation of what-if questions. For example, simulating different marketing or technology rollout strategy scenarios can provide explanations as to what can be done to support better adoption. 


\section{Overview of Pilot Study}

A pilot study, which followed the guidelines established in chapter 3 , was conducted to test the research approach that was proposed in chapter 3 . The pilot study steps were a subset of the research method as illustrated in Figure 4 - 1. While this research is different from the main case study to research EWHTA, the information of this research has been used in the case study as will be further

described in chapter 5 and 6 . This chapter provides a summary of and Appendix B provides details of the pilot study.

\subsection{Design of Pilot Study}

Longitudinal studies complement the validity of retrospective studies (LeonardBarton, 1990). They can generate insights into the antecedents and consequences of adoption. For example, a longitudinal study can validate whether the insights of a simulation model reflect the real outcome of the scenarios that the model predicts. Thus, in contrast to what is proposed in chapter 3 , the pilot study is longitudinal. A post-adoption data collection step was added to the pilot study, in order to test whether the proposed research method yields models that correctly predict actual adoption behavior.

The variables used in the pilot study align with the measures described in section 3.2. However, arguments made in section 1.3 suggest that new input variables and intermediate variables may be discovered as a consequence of conducting the pilot study. 


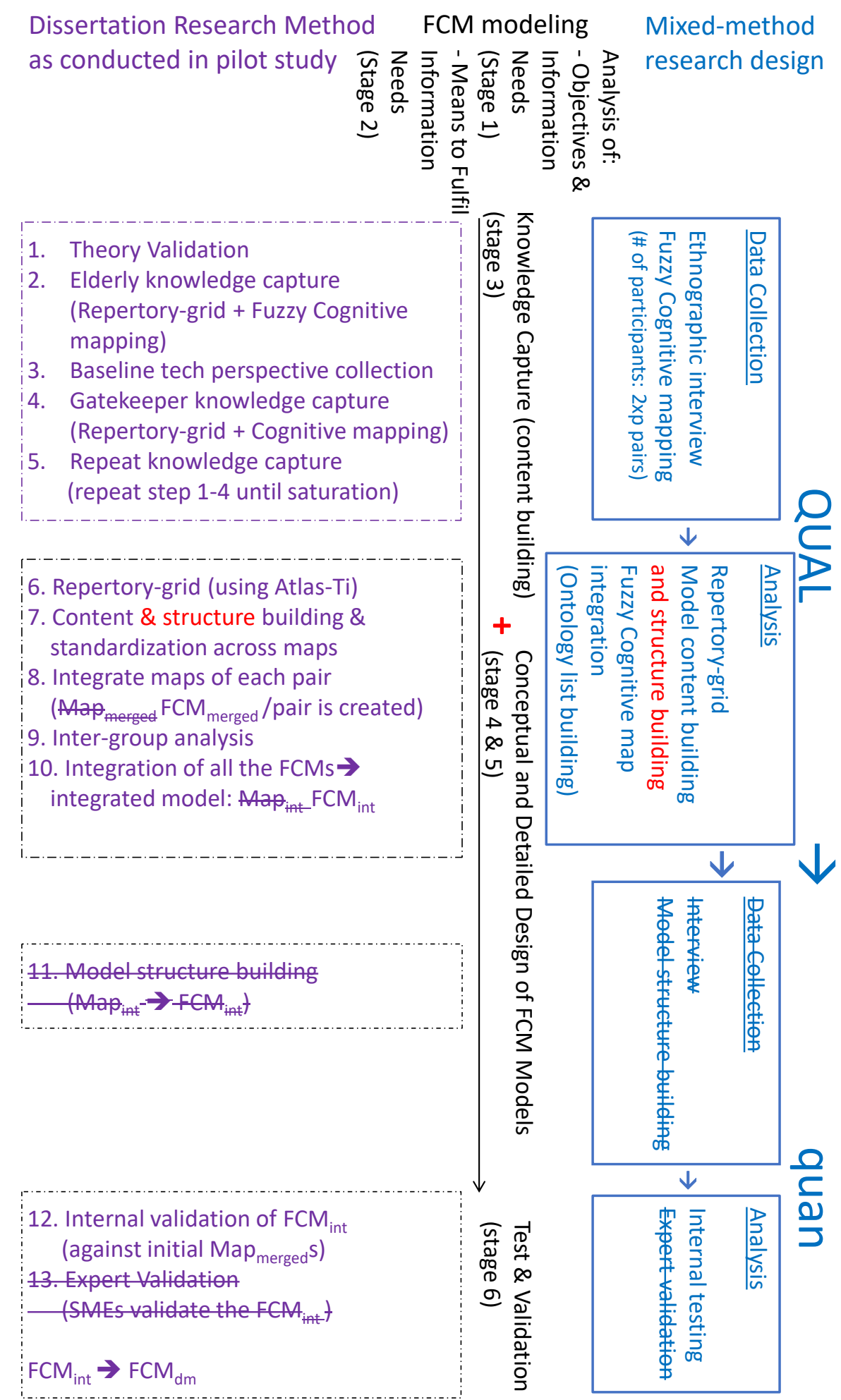

14. Decision model: $\mathrm{FCM}_{\mathrm{dm}}$ prediction was validated against the post-adoption.

Figure 4 - 1 Pilot Research Steps 


\subsection{Setting of Pilot Study}

The output of my dissertation research is a model which achieves the two objectives stated in section 1.2.:

- Identifies the key adoption drivers and obstacles and their relations

- Illustrates how the gatekeeper influences the elderly person's adoption decision

The pilot study was geared toward achieving these objectives. Thus, it should yield a model that is best applied to a setting that meets the criteria described in section 3.3. The setting of the pilot study, which is described below, meets these criteria.

1. Environment: The pilot study was set within the field of healthcare; it researched consumer behavior rather than organizational behavior. It investigated the potential adoption of a fall detector prototype called the EverWrist. (For a more detailed description of the EverWrist, please see sections 5.2 and appendix B.)

2. Technology: The consumer has a choice of deciding whether to adopt or reject the EverWrist, a health care technology/ service.

3. Population: The respondents consisted of two pairs of elderly women and their gatekeepers. Elderly women were chosen over elderly men because elderly women represent the majority of the elderly population (as suggested at the end of section 1.1). Thus, the pilot study tests the research method described in chapter 3 in the context of EWHTA. 
The pilot study was conducted in collaboration with Oregon Center for Aging and Technology (ORCATECH), an organization that researches the clinical implications of technologies for the aging population and regularly conducts field tests of RHMT products. ORCATECH is an internationally reputable, multidisciplinary organization within Oregon Health and Science University (OHSU). ORCATECH is dedicated to the development and implementation of technologies that help the elderly lead safer and more independent lives. In addition to clinical research, ORCATECH partners with a wide range of industries to consult, develop and implement elderly health technologies. ORCATECH is responsible for the Life Laboratory (Life Lab), which ORCATECH describes as follows:

"The ORCATECH Life Laboratory is a resource used to explore technologies to support independent living, to assess new behavioral markers, and to evaluate approaches for assessing neurological and other relevant health changes, all in the participant's home. The Life Lab consists of a population of community-dwelling individuals who have agreed to participate on an ongoing basis in research on technology-based health monitoring, intervention, and support of independent aging. Members of the Life Lab have the ORCATECH platform installed in their home and participate in studies ranging from new technology testing to longitudinal behavioral research." (“Life Lab”, 2017) 
The Life Lab served as an ideal setting for studying the adoption of health care technology by the elderly. ORCHATECH provides the Life Lab with the expertise in gerontology and access to the technology under study. Life Lab members serve as participants in the research. They make autonomous decisions on whether to adopt consumer health care technology or not.

ORCHATECH's field test schedule for the EverWrist determined the timing of the pilot study because recruitment of elderly/gatekeeper pairs depended on the willingness, availability and ability of elderly women from the respective ORCHATECH cohorts to participate in this research. The EverWrist field test was conducted from March to June 2016, and therefore so was the pilot study.

\subsection{Findings of Pilot Study}

The pilot study successfully validated the proposed research method by creating an EWHTA model that successfully predicted the adoption of the participants under study. The pilot research confirmed the validity of the research method, by validating the EWHTA model. The post-adoption data collection step that was added to the pilot study compared the model prediction to the actual adoption behavior. The model predicted that both the participants would not adopt the technology with the current characteristics (i.e. output variables of the modeladoption intention and adoption were both negative). The post-adoption data collection that was conducted after trial confirmed that both elderly women did not adopt the technology. Thus, the contextualized EWHTA model was successfully 
validated. Since the research method resulted in a valid model, the research method was validated in the EWHTA context.

The pilot research validated the proposed research modeling method as follows:

1) The critical importance of the gatekeeper in EWHTA became very clear. Evidently, the gatekeeper exerts significant influence over the input constructs of the UTAUT.

2) The basic model-building steps from section 3.4 were validated. In particular, the pilot study implied that the elderly woman and her gatekeeper should be analyzed together. Thus EWHTA research has to focus on the elderly-gatekeeper pair (as opposed to elderly woman by herself).

3) The pilot research resulted in an integrated FCM model that not only integrated decision making by the elderly; it also captures how her gatekeeper influences all the constructs of the adoption. This suggests that FCMs should be an important component of the proposed research.

4) The integrated FCM served as the simulation model. Its forecasting power was validated in a post-trial enquiry. The actual adoption behavior agreed with the adoption behavior predicted by the model.

Conducting the pilot study also resulted in several lessons learned that will be used in subsequent research (chapter 5). They include...

1. Organization: There are numerous files (both in electronic and in paper format) created from each step of the research. Subsequent research will 
benefit from a proper convention for systematically organizing the resulting materials.

2. Qualitative analysis provided a comprehensive framework for augmenting fuzzy cognitive mapping. The in-depth analysis of the voice recordings using repertory-grid like approach provides a robust method to ensure that the model has captured the nuances of the EWHTA context. However, this can lead to an overcrowded FCM model that is too sensitive to the input factors. As a result, an extensive effort to identify and remove the redundant links and regroup the concepts was made in the pilot study. This led to some adjustments in the steps to manage the depth of the analysis. Extensive capture of details may reach diminishing returns, or even generate many unimportant links that will have to be removed in subsequent analysis.

3. FCM Tool: The research can tremendously benefit from more mature FCM software. In this pilot study, the modeling and simulation were conducted in separate environments. This prolonged the research across the modeling steps. Future research, particularly by practitioners, demands a robust tool that can streamline model building.

A detailed description of the pilot study including the steps conducted to arrive at the decision support model can be found in Appendix B - Pilot Study. 


\section{The Summary of the Case Study}

Prior to the case study, the pilot study (as described in chapter 4), suggested a set of novel input variables and links between variables. The nature of these variables and their interdependence needed to be investigated further, in order for the research method from chapter 3 and its resulting models to be considered valid in the EHTA context. Furthermore, the findings of the pilot study were based on a data set that was gathered from two elderly women and their respective gatekeepers. This sample is too small for a method, which is supposed to yield models that are useful across the EHTA (or even EWHTA) context. Further tests of the proposed research method were consequently warranted. The case study, which is part of this dissertation and is detailed in chapter 6 , serves this purpose.

The case study research, as described in detail in chapter 6 , applies the proposed research method as outlined in chapter 3 . The goal of this research was a model that achieved the four objectives of this dissertation as stated section 1.2. The model is geared toward explaining consumer behavior rather than organizational behavior in EHTA.

The case study research investigated the adoption of activity trackers by the elderly women. This technology was chosen for the reasons described in section 3.3. Activity trackers are wearable devices that track the consumers' activities to provide feedback and promote increased physical activities. The participants in the study were elderly women that were faced with the choice of adopting or rejecting the technology in its various forms. 
The gerontechnology literature has established that gatekeepers exert a tremendous influence over elderly women. Thus, the influence of gatekeepers becomes a key part of this contextualization of the UTAUT with respect to elderly women's health technology (EWHTA). As a consequence, pairs of elderly women and their gatekeepers comprised the unit of analysis in this research, as they did in the pilot study.

Elderly women were chosen as participants over elderly men because they represent the majority of the elderly population. The cognitive capability of the elderly women constituted a key selection criterion, i.e. only elderly women who claimed to be cognitively capable of being interviewed were considered for participation. The elderly women's gatekeepers were investigated due to their (typically) critical influence in adoption decision-making by the elderly (as discussed in section 1.1.-Problem Background). Thus, in order to recruit an elderly/gatekeeper pair for the research, both the elderly woman and her designated technology gatekeeper had to be willing to participate in the research.

The case study applied the proposed research method (outlined in Chapter 3) and subsequently asked few subject matter experts to comment on the method and the usefulness of the resulting model. Figure 5 - 1 outlines the steps conducted in the case study. 


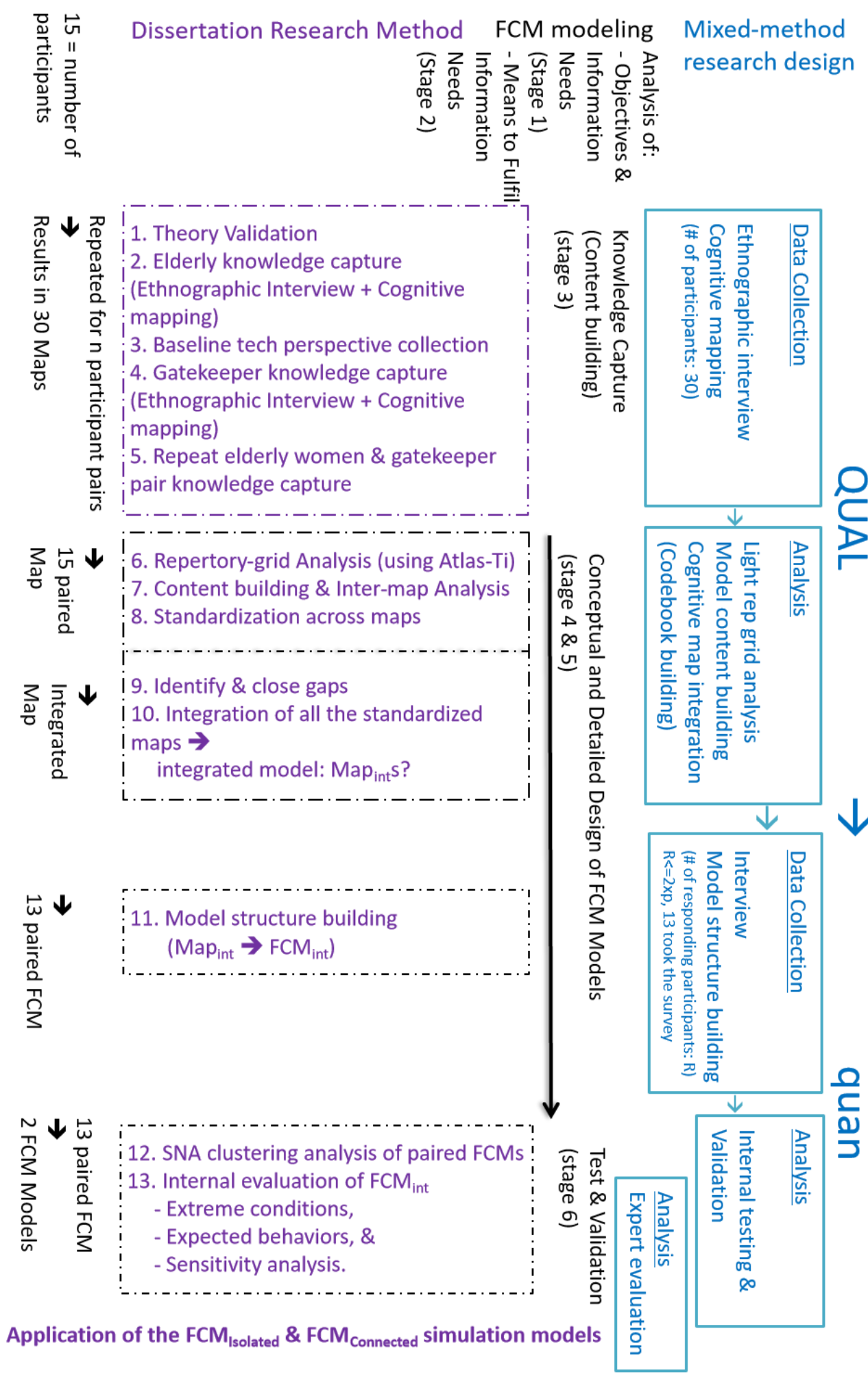

Figure 5 - 1 Case Study following Chapter 3 Research Design with an additional step 13 to evaluate the method 


\subsection{Measures and Variables}

The EWHTA model that resulted from the case study is an FCM model; hence, the values of all concepts are normalized to a range from -1 to 1 .

As detailed in chapter 6, and further to the findings of earlier publications (Rahimi

et al., 2016; Rahimi et al., In Press), a wide range of variables emerged from the research and are color coded for readability in Appendix G (I. Common Ontology(Codebook)) in chapter 6 . The following categorization combines the new concepts with the original UTAUT constructs and groups them according to their role in the model. These groups of variables are categorized in the following groups.

1. Environment Factor

2. Technology Characteristic

3. Elderly Perception

4. Environment Influence

5. Personal Characteristic

6. Tech Adoption Outcome

The six categories of concepts are described in terms of their corresponding variable types below. 


\subsubsection{Input Variables}

\subsubsection{Environment Factor}

This group of variables embodies different concepts that capture the elderly's environment. This include information about her gatekeeper, available support channels around her and her social surrounding that influence her health technology adoption. E.g. "Community tech support" or "Gatekeeper's tech savviness". One important concept that emerged here (despite not being focused on) was the "Clinician recommendation". These concepts are shaded pink in the resulted model.

\subsubsection{Technology Characteristic}

This group of variables captures the specifications and features of the technology. E.g. "Tech aesthetic appeal", "Tech cost" or "Tech usability \& accessibility". These concepts are shaded yellow in the resulted model.

\subsubsection{Intermediary Variables}

\subsubsection{Elderly Perception}

This group of concepts shows the important perceptions of the elderly woman that influence her health technology adoption. Some of these concepts are UTAUT constructs, such as "Perceived Usefulness" and "Perceived Ease of use", and some others are new emerged concepts like "Perceived needs" and "Perceived cost". These concepts are shaded green in the resulted model. 


\subsubsection{Environment Influence}

This group of concepts shows the different yet important influences that her environment has in her health technology adoption. These influences are primarily sourced by elderly's gatekeeper(s) family members and secondary by other channels of support available to and social circle around her. These concepts are shaded blue in the resulted model.

\subsubsection{Personal Characteristic}

This group of concepts captures elderly's different personal beliefs, trait, condition and efforts that are important in her adoption decision making. These range from her general attitude toward technology (such as "Positive attitude toward tech" or "Resistance to change" and the resulting "Voluntariness to use" concepts), her traits (like "Being frugal" or "self-efficacy"), her condition (like having health concern that can be remedied using technology), efforts to reach out for support ("Reaching free community support") or her intention to use the technology ("Adoption Intention"). The "Concern" concept from this category is an input variable.

\subsubsection{Output Variables}

\subsubsection{Adoption and Adoption Intention}

The only true output variable is the actual adoption behavior. Adoption and the intention to adopt are the desired output variables of the research. Adoption intention, which is also an intermediary variable, is the propensity to adopt, whereas adoption behavior is the probability of the actual adoption of technology. 
Adoption is not shaded and therefore shows as white. The other white concept is "Technical knowledge \& experience", an input variable that shows elderly's previous technical experience.

\subsection{Design of the Case Study}

While the pilot study successfully tested the overall proposed method, the EWHTA of the case study conducted here put the designed research approach to fruition. As summarized in Figure $5-1$, this research fully applied the proposed method to conceptualize EWHTA by contextualizing the most recent and comprehensive adoption theory to date, Unified Theory of Acceptance and Use of Technology (UTAUT, see Figure 1 - 1, section 1.1) (Venkatesh, 2003). This was done by integrating the perspective of the elderly women and their gatekeepers to investigate, create and validate and use the empirically emerged EWHTA model. And by doing so, it has accommodated the highly interdisciplinary nature of the proposed research.

It's important to note that the case study has added an additional step to the original method proposed in chapter 3, namely step 13--Expert Evaluation. This step allows subject matter experts to comment whether they find the method and the model useful.

The Case Study actually consists of three studies. Each study pertained to one of the activity tracker products depicted in Figure 5 - 2:

- the EverWrist fall detector ("EverWrist: A Novel Electronic Fall Monitor for Seniors | SBIR.gov," 2012) 
- the Withings activity tracker ("Nokia Steel | Activity \& Sleep Tracker

Watch," 2018)

- the Samsung Gear S2 activity tracker ("Gear S2 classic Wearables - SMR7320ZKAXAR | Samsung US," 2018)

The studies involving the EverWrist fall detectors and the Withings activity tracker were conducted in collaboration with Oregon Center for Aging and Technology (ORCATECH), an organization that researches the clinical implications of technologies for the aging population and regularly conducts field tests of RHMT products. The third study, which involved the Samsung Gear S2 activity tracker, was conducted independently of ORCATECH. Researching three products ensured that the model, which resulted from this research, was not product specific. Furthermore, aggregating the findings of three studies generated a more comprehensive understanding of the EWHTA as it pertains to wearable activity tracking devices.
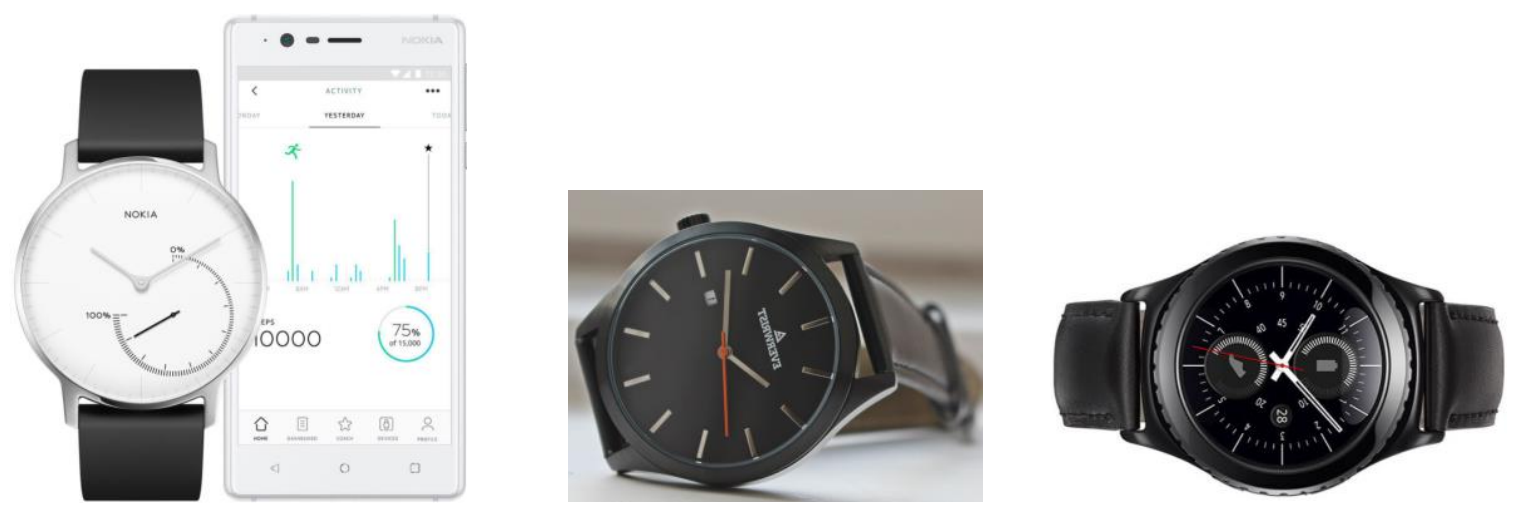

Figure 5 - 2 Activity trackers tried during this research (from left: Withings by Nokia ("Nokia Steel | Activity \& Sleep Tracker Watch," 2018), EverWrist ("EverWrist: A Novel Electronic Fall Monitor for Seniors | SBIR.gov," 2012) the picture is a newer model, and Samsung Gear S2 (“Gear S2 classic Wearables - SM-R7320ZKAXAR | Samsung US," 2018) 
ORCATECH was conducting field tests of the EverWrist and Withings activity trackers during the time that this research was performed. ORCHATECH selected a cohort of participants for each of these field tests from its 'Living Laboratory' panel of elderly volunteers. Elderly women from these two field test cohorts were chosen to participate in this dissertation research, if they met the abovementioned criteria. ORCATECH's EverWrist cohort yielded a total of two elderly/gatekeeper pairs (those discussed in the pilot study); ORCATECH's Withings cohort yielded ten pairs. The remaining three pairs came from the independent study of the Samsung Gear activity tracker.

ORCHATECH's field test schedule determined the timing of the studies in this research because recruitment of elderly/gatekeeper pairs depended on the willingness, availability and ability of elderly women from the respective ORCHATECH cohorts to participate in this research. The EverWrist study for this dissertation was conducted from March to June 2016; the study of the Withings activity tracker transpired May to August 2017. The study of the Samsung Gear tracker occurred in July 2017 , i.e. in parallel with the Withings study.

\subsection{Settings for Studies}

The majority of the participants recruited from the ORCATECH cohorts were USborn elderly women with a relatively high socio-economic status who live in upscale retirement communities. These women volunteered for ORCHTECH on a regular basis, suggesting that they are more tech savvy than the general elderly 
women population. This relatively narrow sample posed a potential issue regarding generalizability: Would a model that is derived from this group of participants explain behavior of elderly women who are less tech savvy, elderly women of lesser means, elderly women who were immigrants, and elderly women who lived at home?

In response to this question, the third study (of the Samsung Gear Tracker), was based upon a different group of participants. The participants in this study live with their family, and they are recent immigrants-they came to the USA within the last 4 years. Including this group of elderly/gatekeeper pairs introduced variability to the sample of participants that typically volunteer ORACTECH field test, both from the point of view of socio-economic status and the relationship to their gatekeepers. Hence, expanding the sample of participants broadened the context of the model that has emerged from this research. In fact, the variability of the research participants was instrumental to achieving the research goal of unpacking the gatekeepers' influence on the elderly women health technology adoption.

\subsection{Research Method Evaluation \& Subject Matter Expert Qualification}

The evaluation of the current popular modeling methods highlighted the potential benefits of mixed-methods research designs and their capacity to address the highlighted criteria (details in chapter 2). The premise of the designed mixedmethods research method in this dissertation is to offer a research approach that meets the highlighted 8 criteria (as reiterated below in section 5.5.). Additionally, the research method has primarily been validated through the pilot study. 
However, as a quick confirmation, three subject matter experts from the intersecting fields (gerontology, HFE, and TA) are asked to comment whether the

method bridges the identified gaps (as outlined in section 2.6. and reiterated below) and if the resulting model is useful. While these experts are not expected to be able to validate the entire model, they can endorse or challenge the model as it pertains to their field of expertise. They can also identify if the model violates the state of the art in their areas of expertise and whether the model generates new insights.

\subsection{Method Evaluation Criteria}

As explained in detail in chapter 2 , there is a compelling need for new research methods that facilitate the contextualization of priori TA theories in the complex elderly healthcare settings. The literature highlights the current gap and suggests that these novel methods should meet 8 criteria (as identified in section 2.6.Summary of the State of the Art). The approach described in this chapter meets these criteria.

1. Grounded: The approach integrates first hand user data from the elderly women participants and their gatekeepers.

2. Holistic: The approach treats the problem holistically: it covers a wide range of factors that emerge from the health context under study. It allows the elderly women to speak their mind freely and explain everything that influences their decision making. Additionally, collecting data from the 
elderly women's gatekeepers contributes to capturing a holistic view of the health technology adoption problem.

3. Flexible: The approach results in flexible models that can be adjusted in response to new input, as new concepts and/or links can be easily added to the model as needed.

4. Latent Needs: The approach models latent needs, because fuzzy cognitive mapping allows the researcher to integrate concepts in a manner that would not occur to the participant. This could include needs that emerge from integrating the perspective of the elderly woman with that of her gatekeeper.

5. Visual: The approach provides cognitively simple visual representation of findings that people from different disciplines can comprehend. This is possible due to the visual nature of FCM, which is enhanced by new FCM software features that allow visual filtering to capture the influences of incoming or outgoing concepts.

6. Scenario Planning: The approach takes advantage of FCM simulation capabilities to simulate what-if questions that can be turned into different scenarios. The result of simulating such scenarios can be compared and contrasted with each other. This leads to a better understanding of the effect of different exogenous factors on the adoption. Such model simulations can also help to better identify and prioritize alternative decision points based on their potential to result in a higher adoption rate. 
7. Novel Insight: The approach has the capacity to provide novel insight in relation to and go beyond the predictions of extant abstract theory.

8. Efficiency: The approach is practical in regards to consideration of monetary and temporal resources and constraints, as the model is reusable for the same category of users and technology.

\subsection{Stages of Execution}

As summarized in Figure 5 - 1, this research follows the proposed research method as detailed in chapter 3 . It is based on the two overarching steps of the designed mixed-methods method and Jetter's (2006) general FCM building practice. As chapter 6 details the research steps based on the mixed-methods stages, following texts of this section provides a summary based on the 6 stages of FCM building.

$>$ Stage 1) Analysis of Objectives and Information Needs

The first stage (2 steps) of the research has already been designed:

1. Identifies the key adoption drivers and obstacles and their relations;

2. Illustrates how the gatekeeper influences the elderly person's adoption decision. This is reflected in the model by showing how gatekeepers influence the formation of perceptions of the UTAUT's main constructs-performance expectancy, effort expectancy and facilitating condition.

$>$ Stage 2) Analysis of Means to Fulfil Information Needs

Identification of Participants 
To fulfil the needed information and as recommended by Tan and Hunter (2002), this research recruited 20 pairs of elderly woman and gatekeeper participants from which 15 sets of pairs of interviews were subsequently analyzed. Each pair consisted of:

$\checkmark$ An elderly woman who acted as the potential adopter, (she is also called any of these terms: participant, "elderly woman", "elderly", "EW","E” for short from here on).

$\checkmark$ A person (often a family member or a caregiver or friend) that was identified by the elderly woman as the primary person who influences her use of technology. This person plays the role of gatekeeper for the elderly woman. (she/he is also called any of these terms: "gatekeeper" or "GK"/"gk" for short from here on).

The principle of diminishing return on learning (which is discussed in section 3.4.2) determined how many elderly/gatekeeper pairs would be recruited for this research. Pairs were recruited and interviewed until no significant learnings were acquired, suggesting that the model generated by this research was sufficiently robust to provide insight to EWHTA. No significant learning occurred after 13 pairs were interviewed and the data that these interviews provided were analyzed. The last two pairs confirmed that insight.

As detailed in section 3.4.2, Repertory Grid technique (RGT) combined with cognitive mapping (by modeler) during the ethnographic interview were selected as the knowledge capture method. 
Stage 3) knowledge capture

The guiding detail steps as laid out in section 3.4.3 were followed to capture the knowledge of the elderly to conceptualize the EWHTA model. The selected combination of techniques proved to be effective for interviewing elderlies. As the cognitive mapping was conducted by the modeler during the interview (and not by the elderly herself) the process was not too demanding to the elderly participants. Additionally, the semi structure ethnographic interview technique provided the appropriate flexibility to the elderly and allowed them to speak freely about their own thoughts and beliefs. Consequently, in many cases elderly women chose to talk about the more familiar technologies as opposed to the technology under study (activity tracker). Although not ideal, those answers provided the information needed. However, this demanded extensive qualitative analysis to both manage the scope and contextualize to the research questions (more detail is provided in chapter 5).

$>$ Stage 4) and 5) Conceptual and Detailed Design of FCM Models

The research method proposed by this dissertation as detailed in chapter 3 section 3.9, was followed step by step to first create the content and then the structure of the integrated FCM model of EWHTA. The step 6 through 11 guided the two main phases of the research-- qualitative analysis (to create the content of the model) followed by quantitative data collection (to create the structure of the model). This is explained in detail in chapter 5 .

$>$ Stage 6) Test and Evaluation 
In this stage, the resulting model is tested, and its performance is validated using following criteria:

- Extreme conditions

- Expected behaviors

- Sensitivity analysis

Additionally, the proposed method is presented to a panel of subject matter experts to comment on the method (based on the set criteria in chapter 2 as reiterated in section 5.5.) and the usefulness of its resulting model. The findings and conclusion are described in the proceeding chapters. 


\section{Case Study Research}

Complementary to the previous chapter that provides a summary of the case study research from the perspective of the six steps (referred here as stages) of FCM modeling (Jetter, 2006), this chapter summarizes the conducted research from the perspective of the overarching research design, as well as from the perspective of the Repertory Grid Technique (RGT). These six steps of FCM modeling (Jetter, 2006) are depicted in black print in Figure 5 -1. In this chapter, the research detailed steps are marked with the corresponding steps of overarching mixedmethod research designed (in blue print), the detail research steps (in purple print) and RGT phases (in orange) depicted in Figure $5-1$.

For the qualitative phase of the research, outlined in the second stage of the research method (section 3.4.2), the combination of 'a Repertory Grid Technique (RGT) and cognitive mapping' established the means to fulfil information needs. As carefully selected and tested in the pilot study (Appendix B), the approach provided great flexibility to curb the inherent challenges involved in capturing information from the elderly demographics.

Since Repertory Grid Technique (RGT) covers a wide range of data collection and analysis approaches, it is important to identify the technique variation adopted in this research.

- The goal of the research method is to create an FCM model which inherently captures constructs and their causal relationship (detailed in 3.1.4). Therefore, unlike in the traditional RGT, the focus of this research is 
to identify the constructs and the degree of influence that they exert upon one another.

- As stated in the research design (section 3.4), the goal of the FCM model is to integrate the opinions of stakeholders. This is a departure from the original George Kelly's Personal Construct Theory (George Kelly, 1955), which focused exclusively on capturing personal constructs. This research, like a growing number of new variations of the RGT ("Repertory Grid Technique", 2003), moves beyond extracting 'personal construct'. Instead, it aggregates participants' constructs to conceptualize "common grids" to study the elderly women population.

- Due to a lack of clear understanding of the important factors in play in EHTA, this research adapts to the more recent approaches of the RGT. The approach deployed in this research opts out of statistical analysis and follows qualitative content analysis to better allow for the emergence of key factors.

For the quantitative phase of the research, which is outlined in the proposed research method (Figure 5 - 1, section 3.4.4 and section 3.4.5), this phase of the mixed-methods approach aims to conceptualize the decision support FCM model by facilitating:

1) Model structure building, in which the integrated cognitive map that resulted from the QUAL phase is converted into the FCM map of the integrated EHTA mental model. 
To achieve this, in the quan data collection phase, each edge weights (degree of causal influence of each link) of the integrated cognitive map (Mapint) is collected. This can usually be done effectively by using an online survey instrument. However, due to the cognitive limitation of the elderly and the need to maximize the accuracy of the data collected, I deployed a structured interview technique for the case study. To streamline the data collection, I designed the online survey instrument depicted in Appendix E.I). I subsequently walked every elderly participant from the QUAL phase of the research through the survey. I then entered the responses into my smartphone device.

2) Internal validation of the FCMint consists of the three tests described in section 3.4.5: 1) the extreme conditions test; 2) the behavior reproduction test; and 3) a sensitivity analysis. In the extreme conditions test, the model's integrity is tested against extreme scenarios. In the behavior reproduction test, the model's integrity is tested for typically successful and unsuccessful adoption scenarios that were observed during the QUAL phase of the research. In the sensitivity analysis, the behavior of the model is analyzed to see how varying some of the model's inputs (within a plausible range) affect the model's output. The sensitivity analysis is conducted ceteris paribus, i.e. only one variable is varied at a time.

After successful validation of the integrated FCM model, the model can be considered as the FCM decision support model and can be subsequently used by 
practitioner to conduct consumer market research and development. (FCMint $\rightarrow$ FCMdm) Detail examples of how the model is used are explained in chapter 7.

For strengthening the evaluation of the overall research. To leverage the evaluation of the overall method proposed in this dissertation, I interviewed a panel of three subject matter experts. The feedback from these experts, which primarily pertained to the usefulness of the emerged model and the overall usefulness of the research method, is discussed in section 6.5.3.

\subsection{Qualitative Data Collection (QUAL Data Collection)}

The principles of ethnographic interviewing were applied to qualitative data collection. These include (1) making the purpose of the interview explicit and known to participants; (2) repeatedly offering explanations to the participants; (3) recording the participants' explanations and asking follow-up questions; (4) acknowledging researcher's lack of knowledge about the participants' personal perspectives; and (5) using participants' everyday language. The interviews took place in the participants' homes. The elderly women were asked to subjectively define and expand on the constructs of UTAUT, based on their own perception. The gatekeepers were asked the same questions but about their perceptions of what the elderly women would perceive. Responses were audio recorded and documented as a cognitive map, using Mental Modeler software.

Upon successful recruitment, every elderly woman participant signed the consent form (listed in Appendix C, section C.I. Participants Consent Form) prior to being interviewed. Since the participants in this research are mainly elderly with limited 
ability to create cognitive models, I, acting as the modeler, simultaneously interviewed the participants and created cognitive maps. During the semistructured ethnographic interview, I asked the questions in the QUAL interview questionnaires (as listed in Appendix C, section C.II. Open-ended Interview Questions). I also guided the interview. The questions in the questionnaire focused on the specifics of the activity tracker. However, I allowed the elderly who couldn't make comments about the specifics of the AT to make general comments about technology adoption that deemed to be insightful to the study (more on this in the QUAL analysis section).

The interview followed the general format described below.

In the following 5 steps of knowledge capture, as per RGT process, the interview questions elicit information about four aspects of technology adoption that pertain to the research objectives:

1) The elderly participant's health technology adoption decision-making (the topic),

2) The health technology being studied (the elements, aka the activity tracker $(\mathrm{AT}))$,

3) Interpretation of the UTAUT constructs and how their perceptions are formed, i.e. concepts influencing the formation of those constructs (the set of constructs), and

4) The ratings of the health technology per each UTAUT construct (a set of ratings of elements on constructs). 


\subsubsection{Theory Validation (Research Step 1)}

(This step aligns with the RGT Phase I - Element Selection, and the QUAL Data Collection of the mixed-method order)

In the beginning of the interview, every elderly woman participant verified the validity of the UTAUT (as presented in Figure 6-1) in relation to their adoption. Every elderly woman who participated in the research validated the UTAUT constructs as important factors that influenced her technology adoption decision.

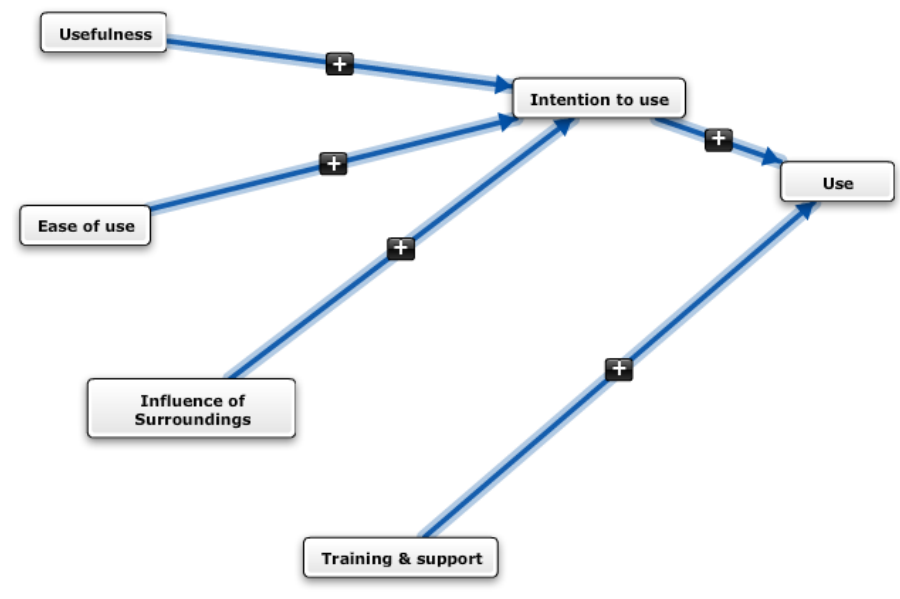

Figure 6 - 1 UTAUT as the base cognitive map to expand in each interview

\subsubsection{Elderly Knowledge Capture (Research Step 2)}

(This step aligns with the RGT Phase II - Construct Elicitation, and the QUAL Data Collection of the mixed-method order)

During QUAL Data Collection, each participant was interviewed using the semistructured one-on-one ethnographic interview technique described in section 3.4.2.2.1. Every interview was recorded to capture information for later analysis. 
During the interview, participants were asked predesigned open-ended questions (in Appendix C, section C.I.2) to provide their subjective insights. These questions were designed to probe and encourage the participants to identify the factors that influenced their adoption decision making for the activity tracker that they were evaluating (i.e. constructs in RGT, concepts in the model and contents of FCM maps). The participants were asked to freely provide constructs for the fixed elements, while the researcher was simultaneously capturing the constructs in her cognitive map. The participant also explained and helped extend the verified UTAUT's constructs. As the research focuses on the health technology adoption of elderly women, age and gender, two of the four mediating factors of the UTAUT are set here. (As the model aims to heuristically unpack UTAUT, the later QUAL analysis enables the emergence of the effects of the other two mediating factorsvoluntariness of use and experience with the model.) The participant also prompted novel concepts while the interview was being conducted. These concepts and the links that depict the influence of these novel concepts on each other and on UTAUT factors were tentatively added to the model. 


\begin{tabular}{|c|c|c|c|}
\hline $\begin{array}{l}\text { Elderly } \\
\text { Woman id }\end{array}$ & $\begin{array}{l}\text { EW Interview } \\
\text { duration (min) }\end{array}$ & Gatekeeper relationship & $\begin{array}{l}\text { Gk Interview } \\
\text { duration } \\
\text { (min) }\end{array}$ \\
\hline P1 & 56 & daughter & 46 \\
\hline P2 & 77 & daughter \& grand daughter & 53 \\
\hline P3 & 53 & $\begin{array}{l}\text { Retirement community } \\
\text { volunteer tech support }\end{array}$ & 70 \\
\hline P4 & 55 & caregiver & 47 \\
\hline P5 & 47 & daughter & 52 \\
\hline P6 & 49 & friend & 36 \\
\hline P7 & 79 & daughter & 46 \\
\hline P8 & 57 & daughter & 68 \\
\hline P9 & 48 & friend & 45 \\
\hline P10 & 43 & daughter & 77 \\
\hline P11 & 81 & husband & 66 \\
\hline P12 & 82 & daughter & 57 \\
\hline P13 & 42 & daughter & 63 \\
\hline P14 & 57 & friend & 81 \\
\hline P15 & 54 & friend & 52 \\
\hline \multicolumn{3}{|c|}{ Number of people recruited } & 40 \\
\hline \multicolumn{3}{|c|}{ Number of people interviewed } & 34 \\
\hline \multicolumn{3}{|c|}{ Number of interviews studied } & 30 \\
\hline \multicolumn{3}{|c|}{ Average age of elderly participants } & 80.7 \\
\hline \multicolumn{2}{|c|}{ Standard Deviation of age } & 10.63 Minimum-Maximum age & $65-95$ \\
\hline
\end{tabular}

Table 6 - 1 Research participants' information

\begin{tabular}{|c|c|c|c|}
\hline \multicolumn{3}{|l|}{ Average duration of interviews } & 57.97 \\
\hline $\begin{array}{l}\text { Standard Deviation of interview } \\
\text { duration }\end{array}$ & 13.30 & $\begin{array}{l}\text { Minimum-Maximum interview } \\
\text { duration }\end{array}$ & $36-82$ \\
\hline \multicolumn{3}{|c|}{ Average duration of elderly participant interviews } & 58.67 \\
\hline $\begin{array}{l}\text { Standard Deviation of elderly } \\
\text { interview duration }\end{array}$ & 13.99 & $\begin{array}{l}\text { Minimum-Maximum elderly } \\
\text { interview duration }\end{array}$ & $65-95$ \\
\hline \multicolumn{3}{|c|}{ Average duration of gatekeeper interviews } & 57.27 \\
\hline $\begin{array}{l}\text { Standard Deviation of gatekeeper } \\
\text { interview duration }\end{array}$ & 13.02 & $\begin{array}{l}\text { Minimum-Maximum } \\
\text { gatekeeper interview duration }\end{array}$ & $36-81$ \\
\hline
\end{tabular}

Table 6 - 2 Participants' interview duration (in minutes) 


\begin{tabular}{|c|c|c|c|c|c|}
\hline $\begin{array}{l}\text { Participant } \\
\#\end{array}$ & $\begin{array}{l}\text { Participant } \\
\text { Age }\end{array}$ & $\begin{array}{l}\text { Participant } \\
\text { Educations (in } \\
\text { years) }\end{array}$ & \multicolumn{2}{|c|}{ Gatekeeper relationship } & $\begin{array}{l}\text { Gatekeeper } \\
\text { Age }\end{array}$ \\
\hline P1 & 83 & 16 & \multicolumn{2}{|l|}{ daughter } & 46 \\
\hline P2 & 69 & 13 & \multicolumn{2}{|c|}{ daughter \& } & 53 \\
\hline P3 & 80 & 18 & \multicolumn{2}{|c|}{$\begin{array}{l}\text { Retirement community volunteer } \\
\text { tech support }\end{array}$} & 70 \\
\hline P4 & 94 & 18 & \multicolumn{2}{|l|}{ caregiver } & 47 \\
\hline P5 & 90 & 15 & \multicolumn{2}{|l|}{ daughter } & 52 \\
\hline P6 & 95 & 16 & \multicolumn{2}{|l|}{ friend } & 36 \\
\hline P7 & 82 & 15 & \multicolumn{2}{|l|}{ daughter } & 46 \\
\hline P8 & 90 & 20 & \multicolumn{2}{|l|}{ daughter } & 68 \\
\hline P9 & 68 & 16 & \multicolumn{2}{|l|}{ friend } & 45 \\
\hline P10 & 84 & 12 & \multicolumn{2}{|l|}{ daughter } & 77 \\
\hline P11 & 67 & 18 & \multicolumn{2}{|l|}{ husband } & 66 \\
\hline P12 & 65 & 16 & \multicolumn{2}{|l|}{ daughter } & 57 \\
\hline P13 & 71 & 12 & \multicolumn{2}{|l|}{ daughter } & 63 \\
\hline P14 & 78 & 18 & \multicolumn{2}{|l|}{ friend } & 81 \\
\hline P15 & 94 & 14 & \multicolumn{2}{|l|}{ friend } & 52 \\
\hline \multicolumn{5}{|c|}{ Average age of the elderly women participants } & 81 \\
\hline \multicolumn{3}{|c|}{$\begin{array}{l}\text { Standard Deviation of elderly participants' } \\
\text { age }\end{array}$} & 10.63 & $\begin{array}{l}\text { Minimum-Maximum } \\
\text { elderly participants' age }\end{array}$ & $65-95$ \\
\hline \multicolumn{5}{|c|}{ Average age of the gatekeepers } & 57 \\
\hline Standard De & jiation of gate & keepers' age & 13.02 & $\begin{array}{l}\text { Minimum-Maximum } \\
\text { gatekeepers' age }\end{array}$ & $36-81$ \\
\hline \multicolumn{5}{|c|}{ Average years of education of the elderly women participants } & 16 \\
\hline
\end{tabular}

Table 6 - 3 Participant pairs' demographics information

\subsubsection{Baseline Tech Perspective Collection (Research Step 3)}

(This step aligns with the RGT Phase III - Rating, and the QUAL Data Collection of the mixed-method order)

During the interview, the participant was also asked to provide her subjective measures for usefulness and ease of use (two of the UTAUT's main constructs) 
for the activity tracker being evaluated. This information was captured in the elderly profile as detailed in Appendix F- Case study Participants' Profiles. Some other information about elderlies' perceptions, traits and environmental factors, which were also captured during the QUAL data collection and the QUAL analysis, were added to the profiles.

\subsubsection{Gatekeeper Knowledge Capture (Research Step 4)}

(This step aligns with the RGT Phase II - Construct Elicitation (triangulation), and the QUAL Data Collection of the mixed-method order)

Every elderly woman participant of the QUAL data collection phase was then asked to identify the individual who most influenced her technology usage. This individual was named the technology gatekeeper, or gatekeeper (aka GK or gk) for short. The gatekeeper was then interviewed in the same fashion as the elderly woman. This is an instrumental step of the qualitative data collection, as it triangulates the knowledge capture and provides a more complete picture to how the gatekeepers influence their EWHTA decision making (research question 2 or 2).

This ethnographic interview resulted in a basic cognitive map, which is created in a manner similar to the one described in step 2. However, this map still focused on the elderly woman's decision making (from the perspective of her gatekeeper) and how the gatekeeper influences the participant's decision during the adoption. This results in another mental model (cognitive map) of the elderly person as 
viewed from the perspective of the gatekeeper. Detailed information about the QUAL data collection for each pair is found in Appendix F - Case Study Participants' Profiles.

\subsubsection{Repeat Elderly Women \& Gatekeeper Pair Knowledge Capture (Research Step 5)}

(This step aligns with the RGT Phase II - Construct Elicitation, and the QUAL Data Collection of the mixed-method order)

This step captures the repetition of the first 4 research steps for the 15 recruited pairs of elderly women and gatekeepers. The research, like any other research, faced some practical limitations, namely attrition and cognitive limitation. The challenges of capturing knowledge from a cohort of elderly with different level of cognitive decline was more pronounced. As a result, further participants recruitment became necessary and resulted in 20 pairs of the recruited participants. However, there were 15 pairs who were successfully interviewed which resulted in 30 units of qualitative interviews and 30 initial cognitive maps. The interview of the participant-gatekeeper pairs continued until incremental learning reached diminishing returns. The consumer market research literature (Griffin \& Hauser, 1993) suggests that diminishing returns should occur after interviewing 10 to 30 participants. In this research, after 13 cases (13 pairs of elderly \& gatekeepers) the last 2 cases provided little incremental learnings (more in section 5.2). 


\subsection{Qualitative Analysis - Model Content Building}

This phase is the most labor intensive and critical part of the entire research. In this phase, the content of the data collected underwent an extensive qualitative analysis. As described earlier, the qualitative content analysis, as an established RGT approach (Boyle, 2005), was the research method that was selected from the repertoire of the overarching RGT techniques. This qualitative analysis was carried out in a qualitative research software called Atlas.ti ("ATLAS.ti 7 Windows | Qualitative Data Analysis with ATLAS.ti”, 2017), which became instrumental in organizing and managing the large amount of data from different levels of coding and analysis.

Briefly, the process to obtain the constructs was conducted as follows. During open coding, concepts and their higher level categories emerged (formed as code families), predominantly using the language of the participant. During the subsequent second coding step (axial coding), these concepts and their causal relations were generalized into broader code families. During the final step, selective coding, the remaining codes were further refined to represent generalizable constructs and then standardized across all maps (Carley, 1993). During each step, the cognitive maps were updated. In total, 15 cognitive maps with standardized concept labels and meanings were generated. Each represented the learnings from a pair that consisted of an elderly woman and her gatekeeper. To preserve the insights from all participants, integration was done additively: if a concept or connection occurred in any one of the contributing maps, 
it was included, even if other contributing maps had omitted it. This integration is a complex process (Giabbanelli \& Tawfik, 2017), which has become possible through the power of qualitative aggregation (Özesmi \& Özesmi, 2004). Here, using Atlas.ti, all the open-codes that grouped and formed code families (i.e. the standardized concepts) were organized and preserved. The accumulated codes from all the interviews provided the information base from which the best abstract concepts and corresponding links were extracted.

The steps in the QUAL analysis phase, which correspond with steps 6-10 (Stage 4 - model content building as described in sections 3.4.4), facilitated the individual analysis and the subsequent cross-analysis of the 15 pairs to incrementally augment and integrate the cognitive maps (the content) of all the participants. The analysis of the content focused on extracting and aggregating constructs (i.e. map concepts) and resembled the grounded theory coding approach. This analysis was conducted in Atlas.ti software as described in the steps below.

The most important consideration during all phases of the analysis is being cognizant of the research questions/goals. This means that every piece of information needs to be actively analyzed from the lens of the research question, which makes a lot of side information irrelevant to the research. As Sterman posits:

"A model designed for a particular purpose such as understanding the business cycle or global climate change, would be much smaller, since it would be limited to those factors believed to be relevant to the question at hand. ... The resulting models could be simple 
enough so that their assumptions could be examined. The relation of these assumptions to the most important theories regarding the business cycle and climate change could then be assessed to determine how useful the models were for their intended purposes. Of course even models with well-defined purposes can be too large...In sum: Always model a problem. Never model a system."

(Sterman, 2000, page 90)

This requirement also made a large number of open-codes, which are otherwise different, synonymous, as they pertained to the phenomena under study. The purpose of the model was to show the factors that are important to the EWHTA. Hence, all other factors can be ignored or merged with respect to relevance to their effects on EWHTA. For example, different positive and negative factors emerged during the analysis of the 15 pairs. All factors influenced the elderly's voluntariness to use a technology either negatively or positively. To focus on creating a model that responded to the research questions (and not modeling the world of elderly decision making), all those factors were merged into a positive influencing factor"positive attitude toward technology", and a negative influencing factor"resistance to change".

Alizadeh and Jetter have proposed a 5-step process that guides capturing the causal-effect relationships from content (Alizadeh \& Jetter, 2017):

Step 1: Draw the raw FCM based on the original text terminology (here ethnographic interviews) 
Step 2: Consolidate the identical concepts

Step 3: Adopt consistent terminologies for conceptually similar concepts

Step 4: Tune the granularity for concepts and sub-concepts

Step 5: Identify and close the gaps

Although they (Alizadeh \& Jetter, 2017) used the literature as the means to fulfil information needed, the overarching activities to capture causal-effect relationships from content are similar. A summary of the activities conducted in this chapter are cross-referenced with these 5 steps in attempt to provide a bird's eye view of the comprehensive analysis of this chapter:

Step 1: Draw the raw FCM based on the original source:

For each pair, the voice recordings of the interviews were imported in Atlas.ti and analyzed by listening and segmenting them into codes. The initial coding (opencodes) could be based on a single phrase, a sentence, or many sentences. The concepts in the original cognitive maps, as well as the codes from both the elderly woman's and the gatekeeper's interview, provided the evidence that allowed the researcher to add the preliminary concepts and their causal connections to the elderly woman's cognitive map.

Step 2: Consolidate the identical concepts:

Once the initial coding across the 15 pairs had been completed, the resulting codes were analyzed to facilitate the creation of code families that collect and group the similar codes together. As such, the initial codes were assigned to the code families that best represented and generalized the initial codes' contents. This step 
was completed once all the initial codes had been assigned to one or many related code families. These code families were shared among the participants. So, new code families were only created when all the existing code families in that project failed to generalize the new codes' meaning.

The cognitive maps were augmented from the learnings of the code families. The concepts in the post open-coding cognitive map of the elderly women participants were compared and contrasted with the code families, and the code families that were missing from the map were added. Adding incoming and outgoing links to the new map concepts proceeds by reanalyzing the content (primarily the open codes and, if uncertain, the interview records).

Step 3: Adopt consistent terminologies for conceptually similar concepts. The earlier steps of the analysis resulted in the emergence of a set of code families that embodied the key concepts and their relations in the context of the research (and hence the model). At this point of the analysis, all the related open-codes were grouped in the created code families in Atlas.ti, and thus were easily traceable. These code families became the standardized concepts, which represent the aggregation and integration of related concepts that emerged from the participants' data. The 15 post-open-coding cognitive maps (each created from a pair case analysis) were updated and augmented using the terminology of the code families. As such, at the end of the analysis, when the code families were finalized, they made up the standardized concepts of the ontology list (aka codebook). 
All the participants' augmented cognitive maps were standardized (i.e. the concepts were replaced by a standardized concept representative that embodied the same meaning).

Step 4: Tune the granularity for concepts and sub-concepts.

The important consideration here was to model EWHTA to address the two research objectives to which this model aims to respond:

1. Identifies the key adoption drivers and obstacles and their relations;

2. Illustrates how the gatekeeper influences the elderly person's adoption decision. This is reflected in the model by showing how gatekeepers influence the formation of perceptions of the UTAUT's main constructsperformance expectancy, effort expectancy and facilitating condition.

The granularity of the emerged concepts and the decision on dissecting or grouping them together were based on the criteria set by the research goal (details provided in the next sections of this chapter).

As replacing the concepts in the 15 post-open-coding cognitive maps (in step 3 of Alizadeh and Jetter approach (2017)) it's observed that some of the maps have captured the concepts more granularly than other maps. This is rooted in the nature of ethnographic interviewing in which the participants can freely discuss details and sometimes derail from the main points. Stepping back and reanalyzing how the concepts related to the research questions set the criteria for the decision on the resolution of the concepts in the model. Once the decision on the resolution 
of the concepts were made, the concepts were merged or dissected accordingly and all the cognitive maps are updated to show the same resolution.

Step 5: Identify and close the gaps.

Upon the adjustment of the model's resolution, the comparison of the cognitive maps across the 15 cases become conceptually simpler. In this phase, the key constructs of the adoption (i.e. UTAUT constructs) were studied and compared across the maps. Conversely, each UTAUT construct was studied across the map to compare and contrast its influencing tree of concepts, in order to integrate missing concepts in each of the cognitive maps. At this point, the numerous open codes that were grouped in code families provide the basis for merging or adding concepts. Additionally, these open codes provide evidence of possible missing relations (links) that were not apparent in the participants' data, and required inferences that were not yet known. Hence, these hypotheses (mainly links) were subsequently added. Furthermore, as the cognitive maps become standardized, each concept and its influencing concepts were analyzed to ensure that no influencing concept were missing. From this analysis, missing influencing concepts became apparent and were added, in order for the cognitive maps to provide a more holistic picture of the mental model.

At this point all the cognitive maps had been standardized and could be integrated together by adding their adjacency matrices together to form the integrated model that comprised the base of the simulation model. Since the edge weights had not been quantified yet, the adjacency matrices of these cognitive maps were not fuzzy 
and only contained: a) "0" meaning there was no edge weight, i.e. no causal relation; or b) "1" meaning there was a positive causal relationship that would be quantified later, or c) "-1" meaning there was a negative causal relationship that would be quantified later. The quan data collection phase is designed to extract the value of these edge weights, as detailed in section 6.3. (step 11 of the overall research method).

The sample codes that appear in the following sections are the open codes that are brought from the qualitative analysis conducted in Atlas.ti. These open codes often start with $A, E$, or $G$ (with or without a number that shows the particular participant). They indicate the source of the open code. E stands for elderly, meaning this open-code has emerged from an elderly interview; $G$ stands for gatekeeper, meaning this open-code has emerged from a gatekeeper interview; A stands for all, meaning this open-code has emerged from at least one elderly interview and one gatekeeper. When E or $G$ is followed by 'in vivo' it means that the open code is an exact verbatim sentence from the participant.

\subsubsection{Repertory Grid Analysis (Research Step 6)}

(This step aligns with the RGT Phase IV - Analysis, and the QUAL Analysis of the mixed-method order)

\section{Step 1: Draw the raw FCM}

The research steps designed in Chapter 3,serves as the step-by-step guide for conducting the analysis, as the crucial stage in the integration of all hitherto 
generated cognitive models resulted in producing the entire content of the FCM model.

6.1. (Draw Cognitive Map 1 from elderly collected data) To maximize the cognitive representation of the interviewee's mental model, this step started the process of deep analysis of the interview voice recording. To do that, the audio recording file produced from each of the two interviews of every elderlygatekeeper pair (each case of the 15 cases), was imported into Atlas.ti. For each case study, the voice recording of the elderly woman's interview (one of the products of step 1-3) was first added to the project as a new document in Atlas.ti. The open-coding steps followed as the first step of the analysis to collect participant's constructs (RGT). Listening to the voice recording, the segments that capture concepts and/or relationships (among concepts) were selected, and the sentence describing the observation was coded. For example, the elderly woman stated that "she doesn't think the activity tracker worth its price". This segment was coded as: "E doesn't want to pay for tech". This led to the emergence of "tech cost" as a construct. Figure $6-2$ depicts the first elderly woman participant's open-coding in Atlas.ti.

6.2. (Double check Cognitive Map 1's completeness against elderly's initial cognitive map during data collection) The second product of step 1-3 was the initial cognitive map created by the modeler during the interview (like the one depicted in Figure 6-3 depicting P1 initial cognitive map). The map provided good visual cues as to the important constructs and relations that stood out 
during the interview (particularly in relevance to the main UTAUT's constructs). However, this initial cognitive map lacked the granularity to represent the relationships among those new concepts and the original constructs. This is because the maps were created during the interview and were subject to the time constraints imposed by the interview sessions and elderlies' cognitive ability. Additionally the map includes a lot of information that is very situational and only describes the participant's environment (e.g. elderly daughter is her gatekeeper). These information are removed from the model and saved in a profile that describes elderly environment and other information that will be used in the later stage to test the model (model validation). The other concepts and relationships of the cognitive map also serve as a great visual reminder of that constructs. As anticipated, the analysis of the voice recording reveals much more information than what has been captured in the cognitive map. Hence, the best practice proved to be creating a brand new cognitive map by starting from the UTAUT map (Figure 6 - 1) and building further contents (concepts and links) from the open-coding while checking to make sure none of the constructs highlighted in the original cognitive map (created during the interview) is missing. 


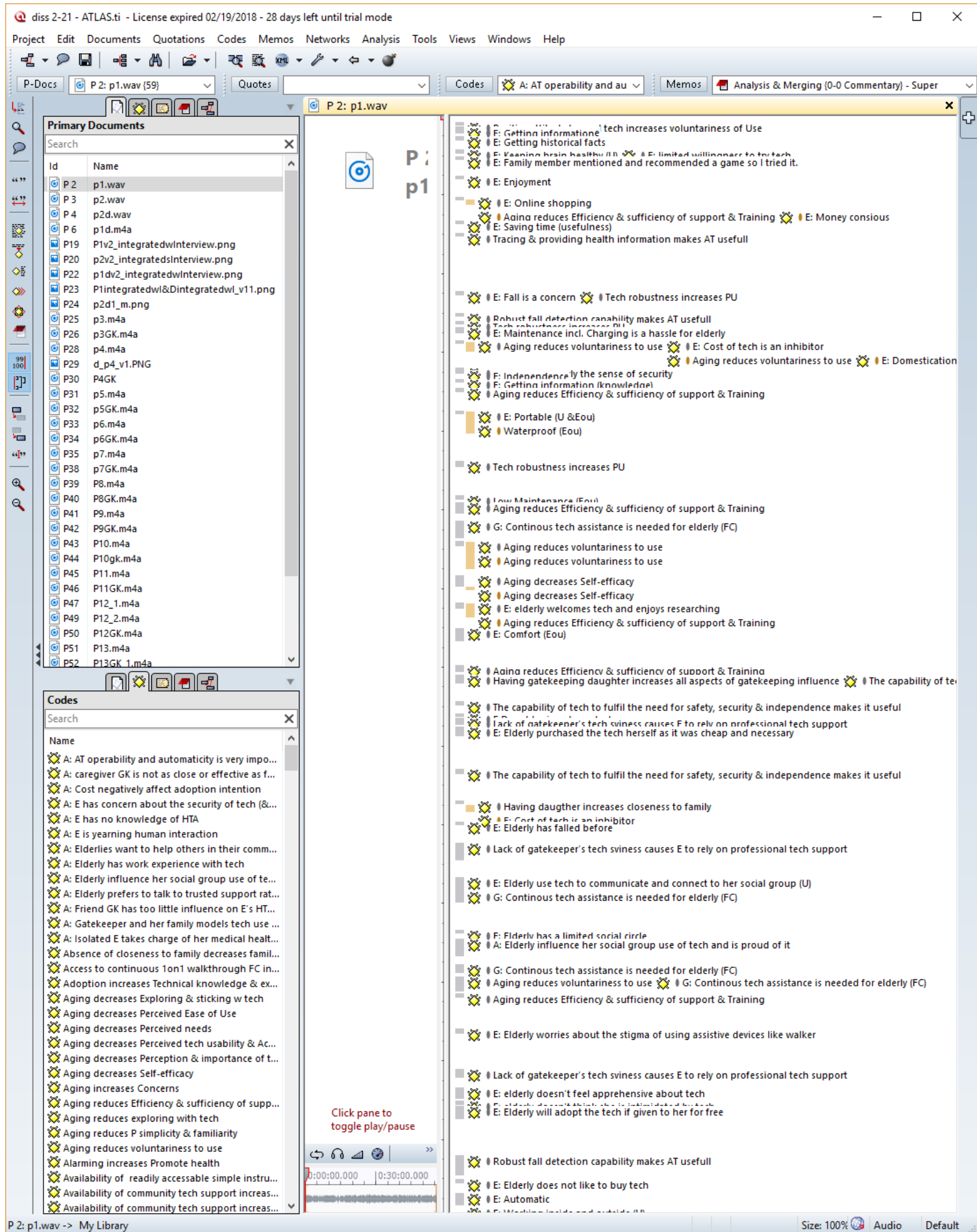

Figure 6 - 2 Open coding of the interview records in Atlas.ti 


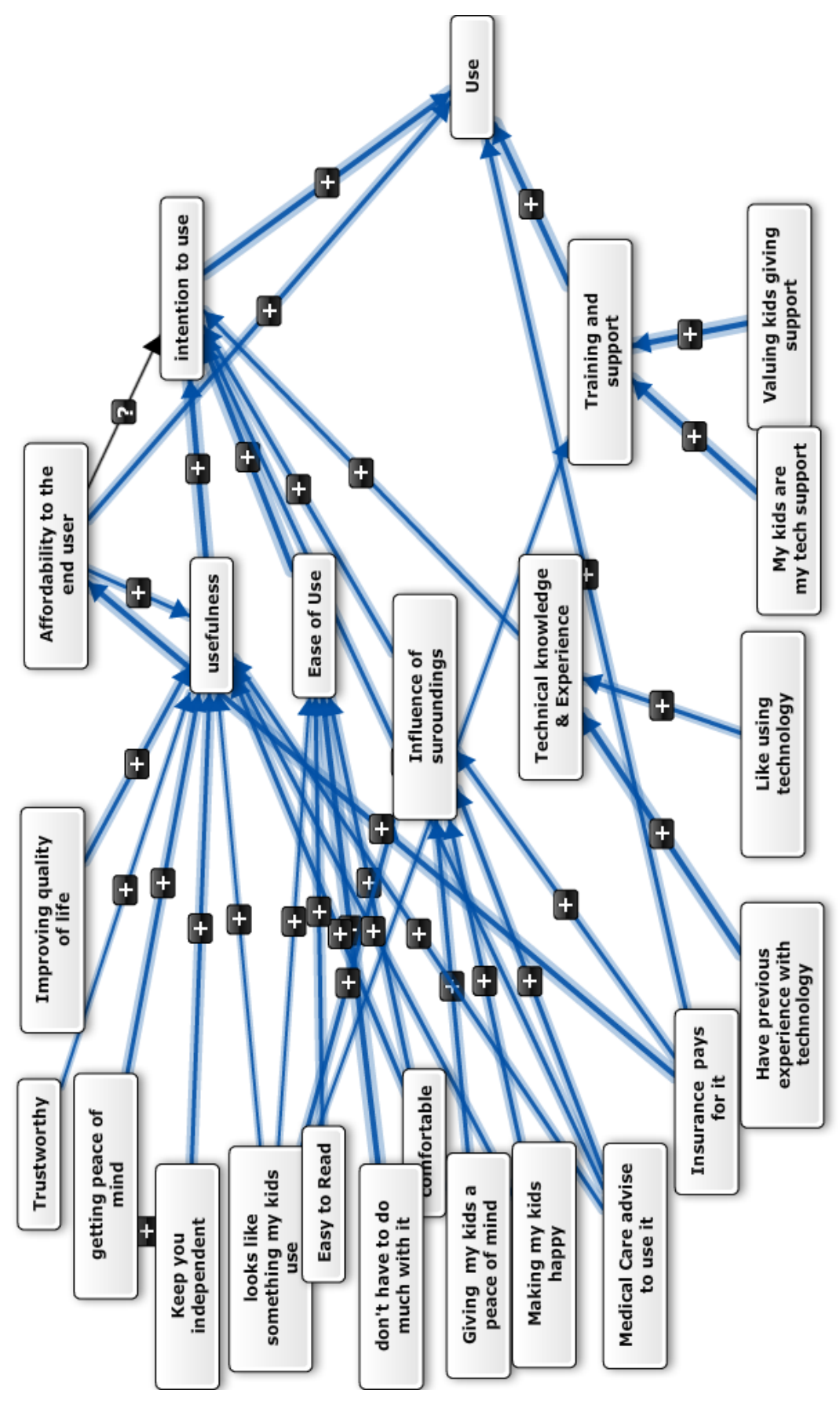

Figure 6 - 3 P1 interview cognitive map

6.3. (Augment Cognitive Map ${ }_{1}$ from secondary data source-gatekeeper) The research proceeds with the open-coding and cognitive mapping of the elderly woman's gatekeeper. This is conducted in a similar fashion to the elderly 
woman's interview (steps 6.1 and 6.2 here). The major difference of this step is that the gatekeeper's interview information still pertains to the elderly's decision making (EWHTA) from the view of the gatekeeper as the second source of information. As such, no post open-coding cognitive map is created from the gatekeeper's open-coding; and the open-coding of the gatekeeper's interview document (the interview voice recording) only contributes to the post open-coding cognitive map of the elderly woman. Figure 6 - 4) shows the resulting cognitive map from the open-coding of both the elderly woman interview document and the gatekeeper interview document with a more comprehensive set of constructs emerged from the two sources. 


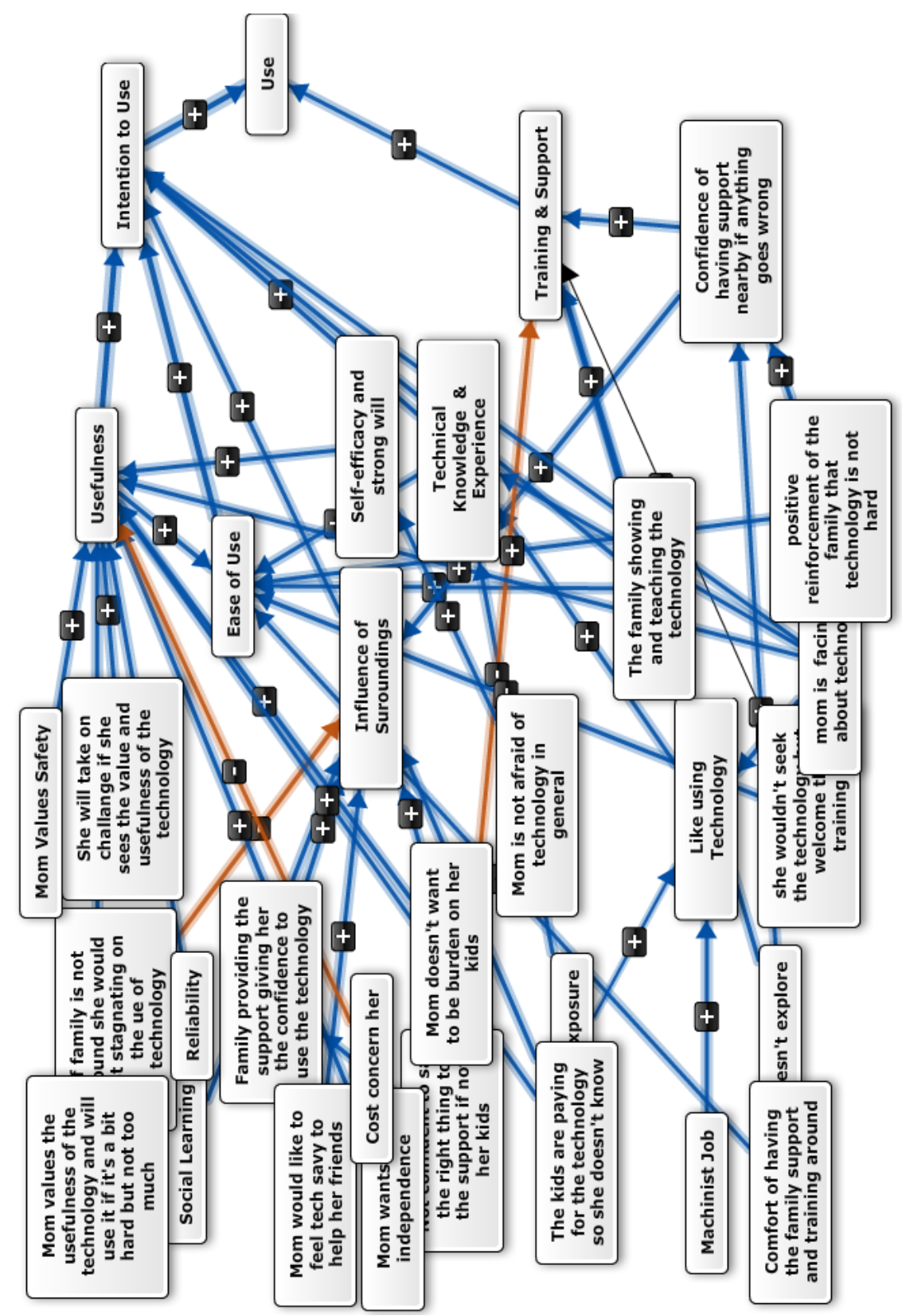

Figure 6 - 4 P1's gatekeeper interview cognitive map 


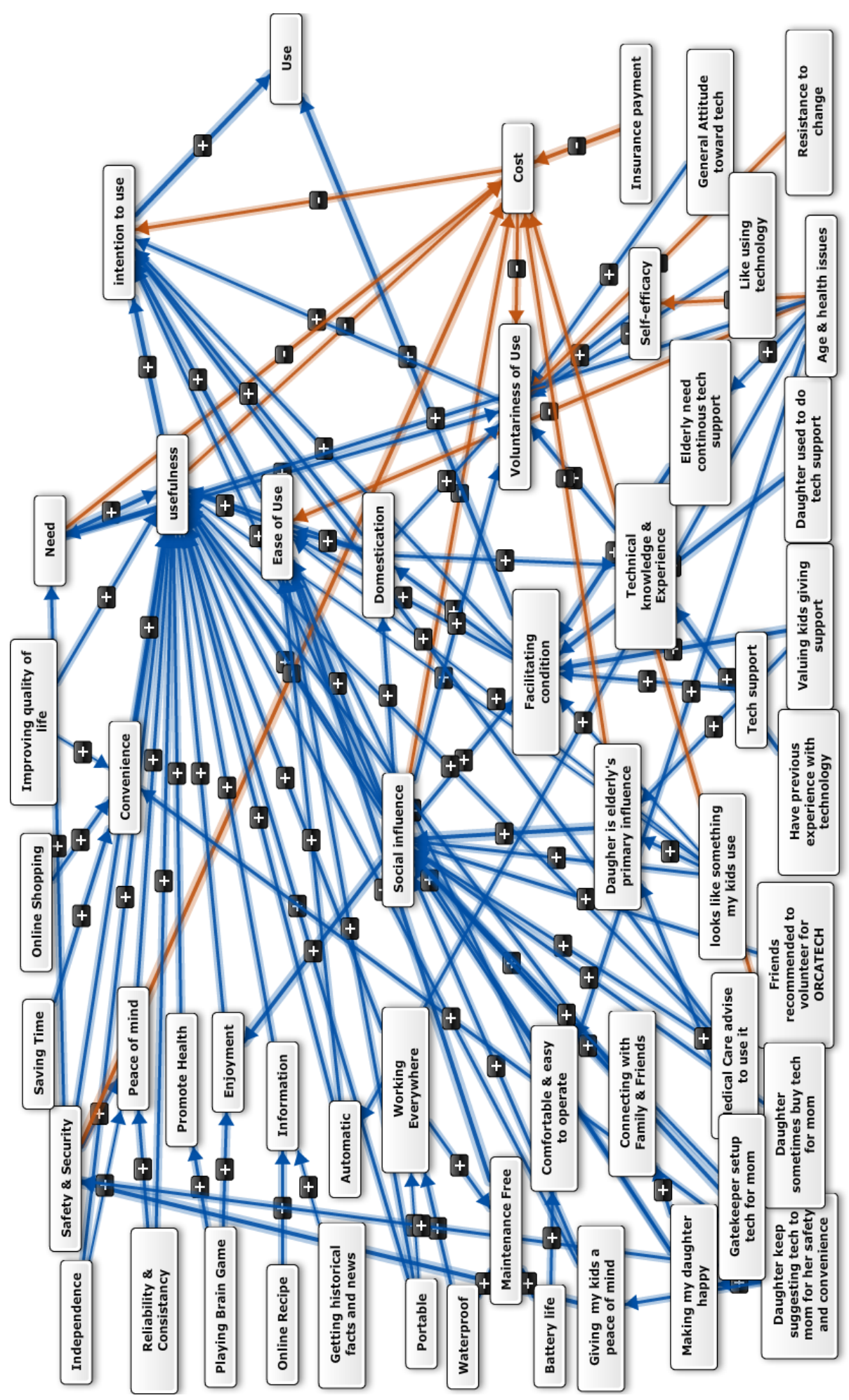

Figure 6 - 5 P1's post open-coding cognitive map 
6.4. (Draw, check and augment Cognitive Map i for all case studies ( $\mathrm{i}=2$ to 15 ) The research proceeds with the analysis of the remaining 14 pairs in the same fashion as the process described in 6.1. to 6.3.

During the iterative process of document analysis and open-coding sessions, usually one of these two scenarios occurs:

1) The extracted open-code shows an apparently new concept or relationship, which will be subsequently created. Atlas.ti adds the new open-code to its repository of all the open codes.

2) When creating an open-code, a similar open-code to the one being observed can be found in the list of the existing open-codes in Atlas.ti. It is important to reuse the existing open-code as opposed to recreating a similar one. As recommended in the qualitative analysis literature (Creswell, 2012), it is important to actively merge similar concepts to manage scope of the research. This will not only help with managing the size and scope of the list of codes, it also reduces the merging effort in the later stages of the selective coding. The software (Atlas.ti) becomes instrumental in keeping track of all the codes that can be easily searched and compared.

\subsubsection{Content Building \& Inter-map Analysis (Research Step 7)}

(This step aligns with the RGT Phase IV - Analysis, and the QUAL Analysis of the mixed-method order)

\section{Step 2: Consolidate the identical concepts}




\subsubsection{Analysis Parking Lot}

During the analysis, a new cognitive map is created by adding all the concepts that have emerged from the analysis to the base UTAUT cognitive map (Figure 6 - 1). The resulting map is called "analysis parking lot", as it parks all the incrementally learned concepts and links. This living cognitive map helps see the emerging concepts and the findings captured in Atlas.ti without the need to search in the ocean of codes. It is also used to compare and contrast the merged concepts with those concepts found during the analysis of each pair.

Comparing the parking lot map with each pair cognitive map results in one of two possible outcomes. Either the pair cognitive map lacks a concept or link that the parking lot contains, or the pair cognitive map contains a concept or link that the parking lot lacks. In the former case, the concept is added to the pair cognitive map. In the latter case, the concept is added to the parking lot.

\subsubsection{Inter-map Analysis (Research Step 7.1)}

Earlier analyses of all pairs resulted in a list of over 800 open codes, which was too large for inter-map analysis. To find the important concepts in EWHTA, the process of selective coding (RGT content analysis technique inspired by grounded theory selective coding) began by conducting an extensive comparison of all the open-codes based on the concepts they were representing. Open-codes that were 
synonymous were actively merged. This merging process helped manage the scope of the research by:

1) Reducing the number of open-codes,

2) Increasing the focus of the research on the questions it aims to answer, and

3) Removing the extra contents that are outside the scope of the research and only unnecessarily complicate the analysis.

This led to the unification of around 300 codes, which reduced the number of opencodes to around 530 (depicted in Figure 6 - 6).

The practical requirement to merge the similar codes based on the context of the research needs to be balanced against the importance of keeping their variability. This is an important consideration, since the open-codes highlight the different causal relationships among the concepts. These different, yet related codes, which are all assigned to their related code families, provide the evidence for creating links in the parking lot map and consequently in the participants' cognitive maps. For example, Figure 6 - 7 depicts the many open-codes that show how emerged 


\section{critical concept.}

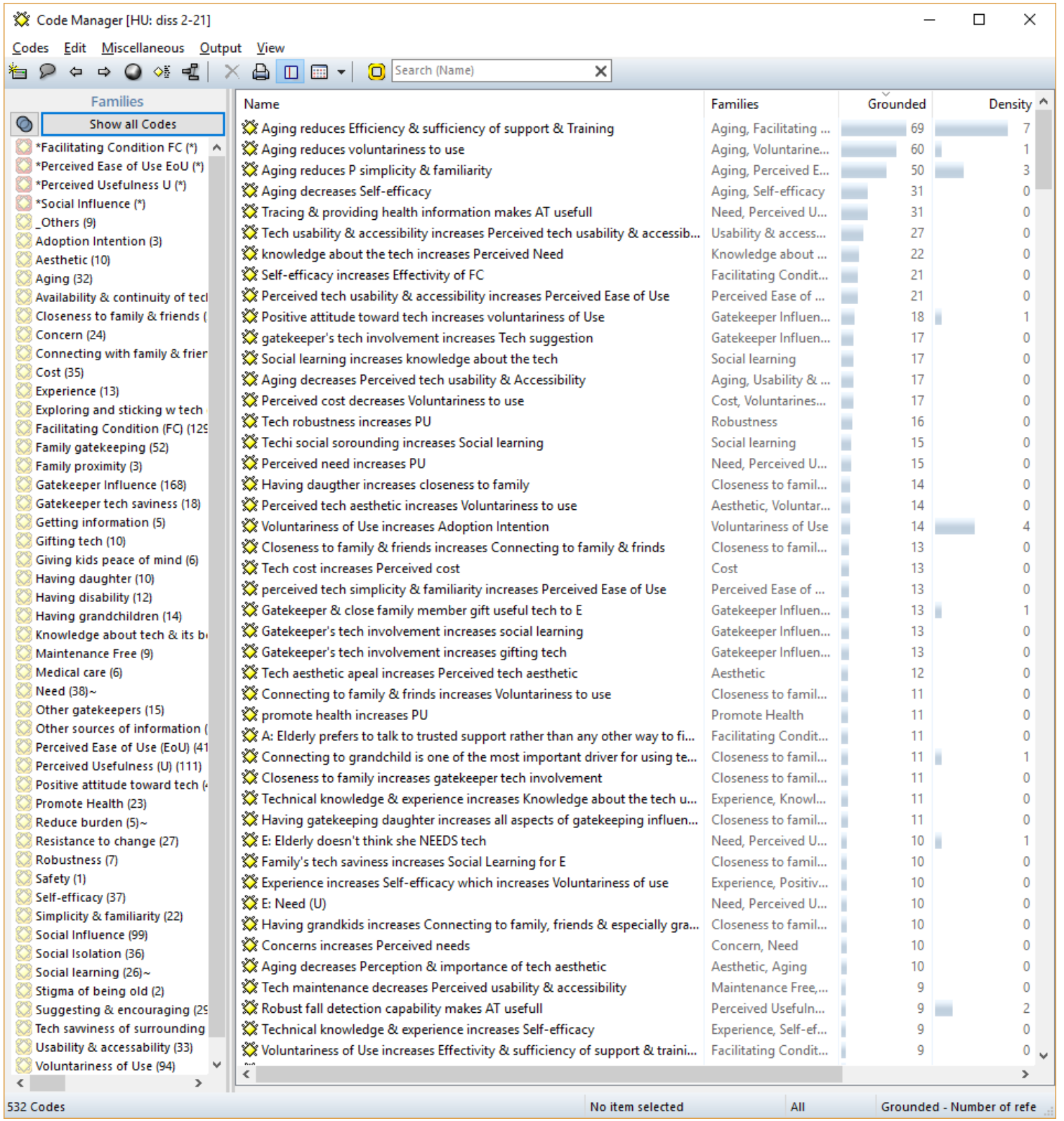

Figure 6 - 6 Consolidated open-codes in Code Manager 


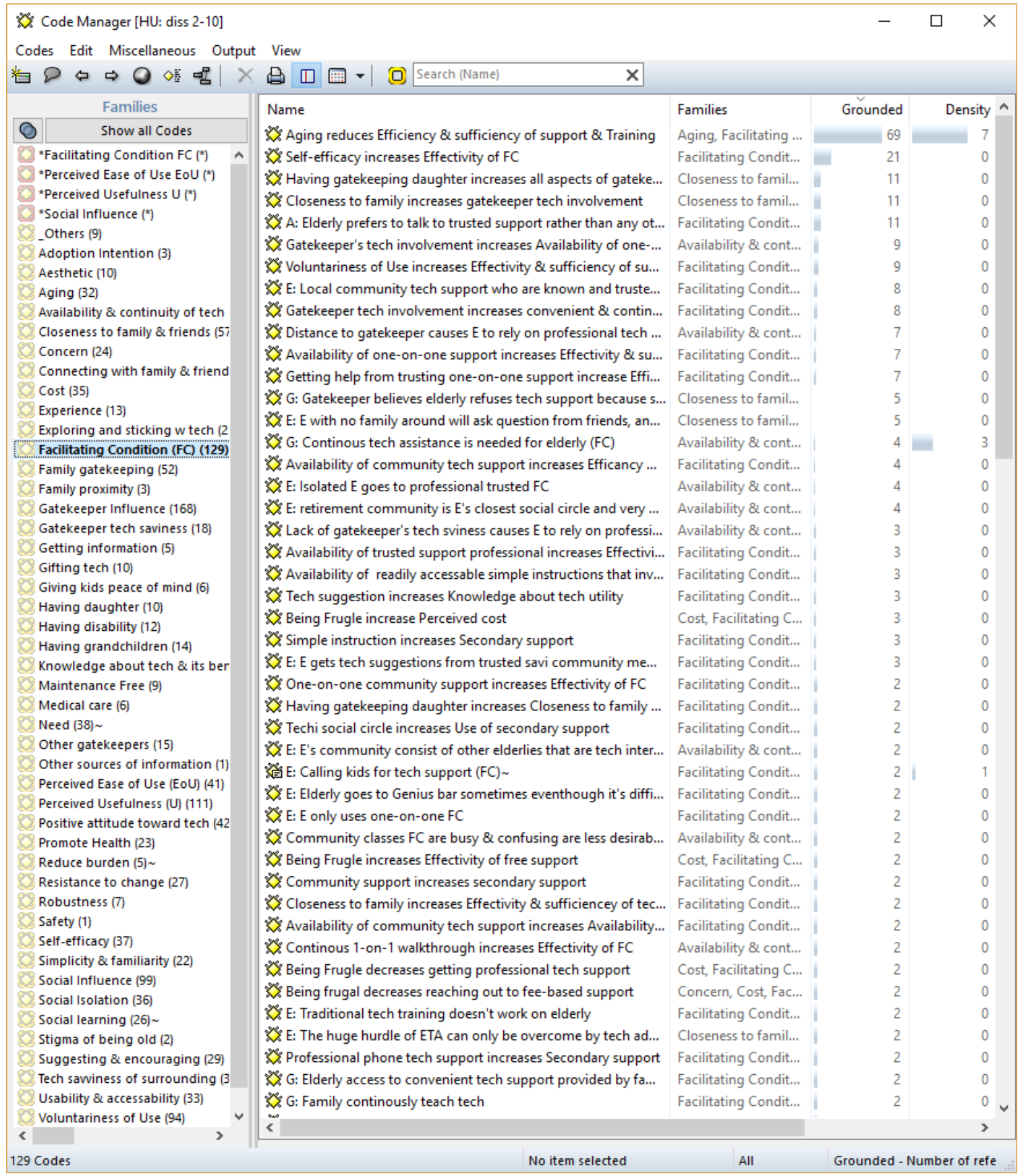

\section{Figure 6 - 7 Facilitating Condition Code Family}

\subsubsection{Merging of Synonymous Concepts (Research Step 7.2)}

\section{A wide range of keywords that are popular among the elderly and in the context of}

EWHTA such as usefulness, ease, easy, need, help, support, age, fear, mistake, 185 
self-efficacy are searched in the Atlas.ti project codes to find all the codes containing similar terms. The resulting list of open codes and others that conveyed the same meaning and rooted in the same factor were merged.

For example, the following codes were merged with the first open code:

- E: E will buy tech and use it if and only if she needs

- G: E will abandon the technology that GK and other family member has bought for her.

- E: Elderly has strong emotional attachments to her old way of life (love her books even though it's harder to read them and it's easier to read on kindle)

- E in vivo: "I'm just not interested in new technology"

- E in vivo: "I do have a smartphone but I never use it, I only carry it for emergency."

- E in vivo: "Tech doesn't fit to my life style"

- E in vivo "when one's get old, don't put new things in your elderly parents' lives, it not blessing it causes a lot of anxiety, because we don't adapt to new things"

- G: E has a high Resistance to Change

- G: E has a very strong resistance to change (pays for SP fee even though frugal but have it off all the time)

- G: E heavily relies on habit when it comes to the everyday activity 
In the world of gerontology, these open codes could be arguably rooted in various factors. However, in the context of this research all of the following related factors can be merged into one concept:

"Resistance to change decreases Voluntariness to use"

\subsubsection{Consolidation of Contradicting Concepts (Research Step 7.3)}

In some instances, a specific behavior pattern was present, and in other instances it was absent. In some instances, the behavior of elderly women could be measured by one variable. The behavior pattern was present in some women, but absent in others, or the behavior pattern was more prevalent in one woman than in another. In either situation, the behavior can be rooted in the same concept. In other words, contradicting behaviors can be rooted in the same concept. Studying the contradictions hints at the existence of or lack of the same concept. This reveals a new concept that need to be added to the cognitive map. For example, comparing the studied pairs reveals that elderly women with high self-efficacy have a much higher technology adoption rate than those with lower self-efficacy. This shows that the difference in the degree of one variable—self-efficacy—significantly influences technology adoption. Thus, all instances of open codes pertaining to self-efficacy are consolidated into one code family called "Self-efficacy". Here, in the self-efficacy code family, a group of open-codes from isolated elderlies who 
are not subject to social learning (P4, P6, and P15) highlighted how social isolation decreased elderly women's voluntariness to explore and use technology.

- G9: Isolated E isn't really exposed to tech as a result tech is foreign to her (P4)

- $E^{10}$ : Cognitive and health decline causes $E$ to become more socially isolated and just do what she enjoys and used to do (P6)

- E: depression and isolation reduces E's interest in trying or using tech (P7)

- E: Isolated E doesn't have a lot of exposure to tech (P9)

- G: Isolated E doesn't really have a GK and tech as a result is foreign to her (P6 gatekeeper)

- G: Tech rejection is more pronounced among isolated $E$ women (P6 gatekeeper)

Another group of open codes showed how the elderly woman's social interaction and social learning increase her ability and confidence to explore the new RHMT:

- E: If social circle who can figure out the RHMT E thinks she can too (P10)

- E is surrounded by close family members who are very techy which has influenced E's willingness to use tech (P12)

- Techy social circle wearing the RHMT motivates $E$ to use it too (P14)

\footnotetext{
${ }^{9}$ Reminder: G stands for gatekeeper, meaning this open-code has emerged from a gatekeeper interview.

${ }^{10}$ Reminder E stands for elderly, meaning this open-code has emerged from an elderly interview.
} 


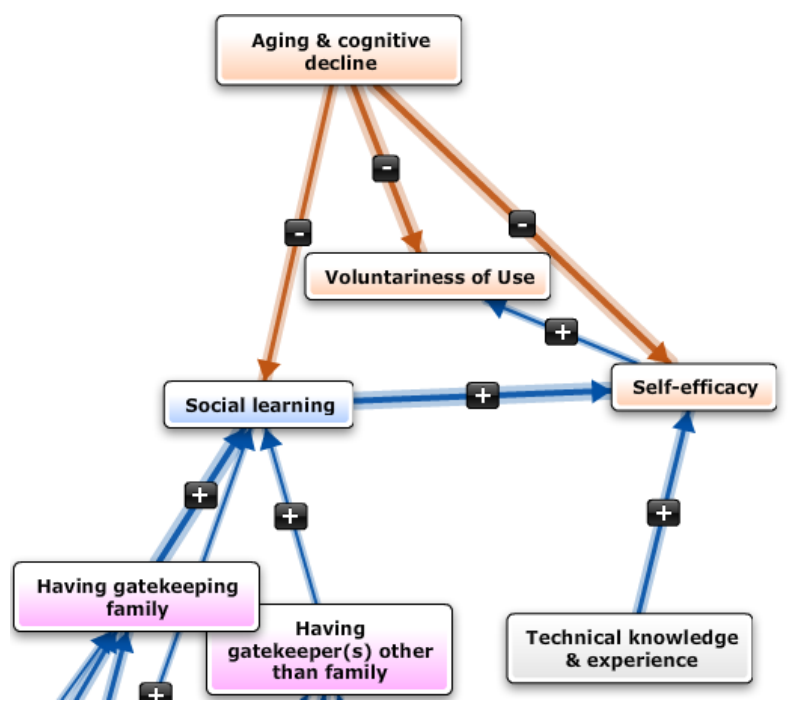

Figure 6 - 8 Parking Lot map showing the emerged concepts and their connections We know from Bandura's Social Learning theory (Bandura, 1986) and from studies of its applications in health technology adoption (e.g., Rahimi \& Jetter, 2015) that self-efficacy of the elderly is the key concept that is influenced by social learning. (Self-efficacy, in turn, influences voluntariness of use.) Moreover, in EHTA, social learning can be considered the opposite of social isolation. Hence, in EHTA, social isolation can be consolidated into one concept with social learning. This is in marked contrast with gerontology, where social isolation is an important, independent concept.

\subsubsection{Tuning the Granularity of Concepts (Research Step 7.4)}

Consistency in the resolution of concepts is an important consideration of model building (Alizadeh \& Jetter, 2017). It ensures that a highly granular set of concepts does not have a disproportionate impact on the outcome of the model. Additionally, collapsing the detailed concepts into a more general parent concept eliminates the risk of not capturing all the detailed concepts that contribute to the parent concept 
(Alizadeh \& Jetter, 2017). Moreover, it removes the potential specificity of the pairs studied.

For example, since the early stages of data collection, it became apparent that ease of use was perhaps the most important concept that presents elderly women with a challenge and about which elderly women care. Both the initial cognitive maps and the interview records highlighted granular concepts that were brought up as important. As a result, there were many concepts related to ease of use, which were captured by the initial cognitive maps (like the subsection of the model depicted in Figure 6 - 9) and the generated open-codes like those listed below. 
This was especially true for the elderly women who tested the less user-friendly activity tracker.

$\mathrm{E}:$ The battery needs to last longer

E: Longer battery life increases the ease of use

E: physical usability like bigger buttons (EoU)

E: Visual aid instead of writtings or button (EoU)

G: Easy wear of tech is important (Eou) (E.g. putting on the fall detector watch by one hand/or with familial tremor syndrom)

E: E didn't like AT because it was not easy to use.

E: Visual aid instead of writtings or button (EoU)

E: physical usability like bigger buttons (EoU)

E: Portable (U \&Eou)

G: E heavily relies on habit when it comes to the everyday activity

E: The AT needs to be waterproof

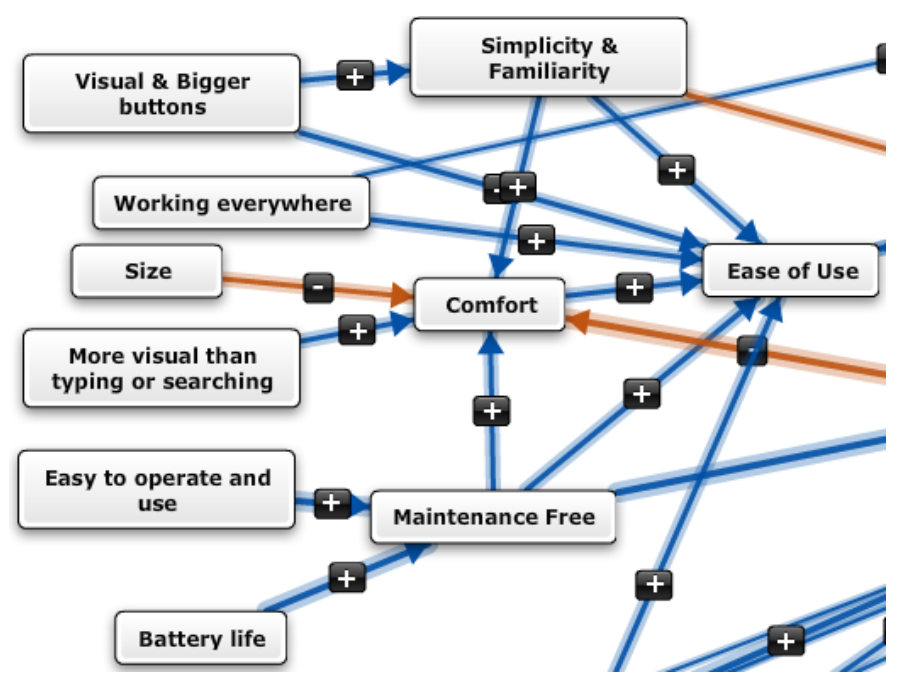

Figure 6 - 9 Ease of use related concepts from P2 initial cognitive map 
Through the iterative analysis and consideration of the granularity of other concepts (like usefulness), the resolution of concepts related to ease of use was adjusted as depicted in Figure 6 -10. All the concepts influencing the perceived ease of use collapsed into two levels of: objective technology feature categories and subjective perception of those concepts. For example, in Figure 6 - 10, the objective technology characteristics marked in yellow are Tech simplicity \& familiarity, Tech automaticity, Tech maintenance, and Tech usability \& accessibility. The subjective elderly perception categories marked in green are Perceived simplicity \& familiarity, and Perceived usability \& accessibility.

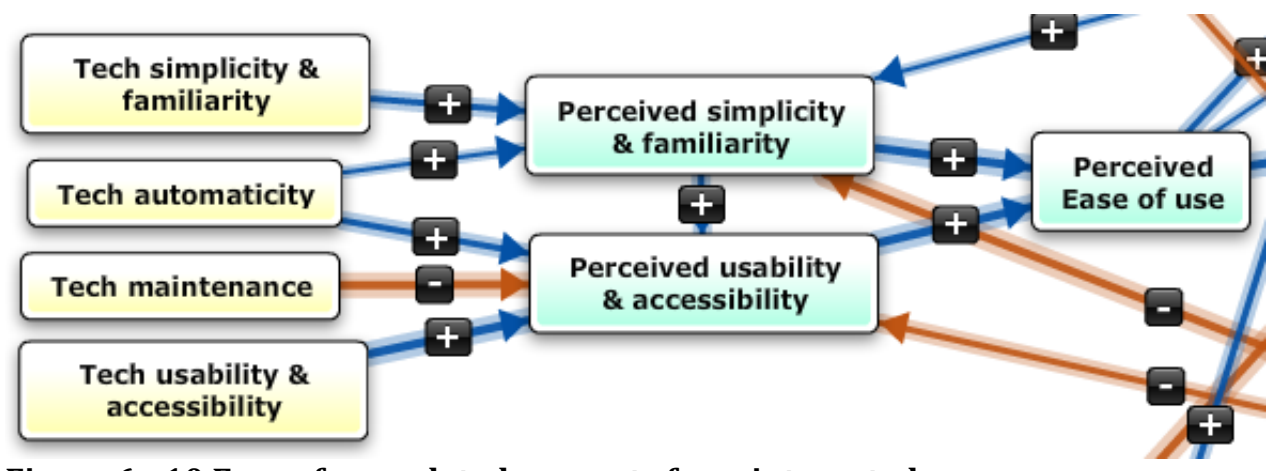

Figure 6 - 10 Ease of use related concepts from integrated map

\subsubsection{Concept Formulation from Opposing Cases (Research Step 7.5)}

As described earlier, the research recruited elderly-gatekeeper pairs that represented a variety of typical situations, and, as a result, different concepts surfaced as important in the study of the various individual pairs. During the intermodel analysis, conflicting evidence as to the importance of those concepts appeared. Some of the differences were easily justifiable. For example, free community support was an important concept in EWHTA. However, elderly women 
who were surrounded by technically savvy family members that provided continuous, one-on-one walkthrough support did not perceive the need for free community support. Thus, free community support did not appear as an important concept.

Other contrasting cases required more careful analysis. For example, for some elderly women, professional support appeared as the most important support channel, whereas for others the existence of free community support was considered most critical. Further analysis yielded the following codes:

P5 in vivo: "I would call support, if it didn't cost me an arm and a leg."

P4 in vivo: "Then you get to the cost of the support, and not everybody can do that."

These two open codes were merged into the following aggregated code that warranted the creation of a new concept-being frugal:

$\Rightarrow$ Being frugal decreases reaching out to fee-based support

This code shows that the financial constraints of elderly women and their degree of frugality have a significant impact on their propensity to use fee-based support. These two concepts are different: financial constraint is state, whereas frugality is a trait. However, they can jointly formulate the concept "being frugal", either by state or by trait, in the context of EWHTA. The introduction of this concept in the parking lot map can explain that the more frugal the elderly women, the more likely 
she will be reaching out to different free community support, as depicted in Figure $6-11$.

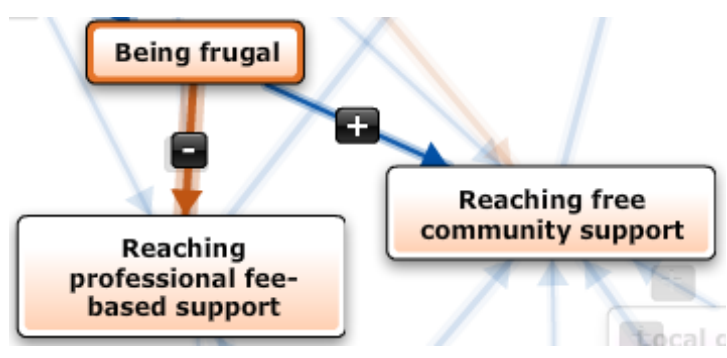

Figure 6 - 11 Being frugal was formulated from opposing cases

As observed in Figure 6 - 11, the visual filtering feature in Mental Modeler ${ }^{11}$ was instrumental in facilitating the display of all the links to and from each concept and to ensure no link is missed.

\subsubsection{Standardization across Maps (Research Step 8)}

Once the iterative analysis of the cognitive maps provided no further insight (i.e., no new concepts or links were found), the standardization process followed. At this point, all the standardized concepts had accumulated in the parking lot map, which provides an effective visual cue for all the concepts that need to be crosschecked across the cognitive maps of the various pairs. The parking lot map also has more generalizable terms for the concepts, because it has gone through iterative intermap analysis. However, at the earlier step, the focus was on the creation of the

11 Mental Modeler is a free online modeling software accessible at:

http://www.mentalmodeler.org/ that can be used to draw and simulated capture Fuzzy Cognitive Maps. 
concepts and the links and not so much the consistency across the maps. Subsequently, all the cases' cognitive maps went through accumulative standardization, where the related concepts were renamed to a concept name that represented a more standard collective meaning. At this time the meanings of the concepts were very similar, at least in the context of the research. Most of the time, the corresponding concept names in the parking lot were selected as the standard concept name, since they had gone through iterative changes during the process of content building (step 7). If there was uncertainty whether the standardized concept truly represented the corresponding concept in the study of a pair participants, their related open-codes were examined to verify that the resulting concept still reflects what the open codes suggest. This process was similar to Selective Coding in grounded theory approach.

At the end of this process, all the cognitive maps had been analyzed, and the creation of standardized concepts was complete. The exhaustive list of these common standardized concepts formed the ontology list (aka codebook), which contained all the standardized concepts and their definitions. The ontology list of the EWHTA application is depicted in Appendix G.I.

\subsubsection{Identify and Close Gaps (Research Step 9)}

The collected data may not provide the entire picture for the model and hence miss some important concepts. This necessitates an analysis of any gap that can be 
identified. Subsequently, causal hypotheses that can improve the research model are proposed.

For example, during the analysis, formation of "Knowledge about the tech utility" emerged as an important factor that contributed to technology's "Perceived Usefulness". Percieved Usefulness itself was influenced by "Exploring tech", "Tech suggestion, introduction \& encouragment", "Social learning", and "Technology knowledge \& experience". However, further study of the the concept "Knowledge about the tech utility" identified an additional gap. An elderly person may be exposed to the utility of a technology through other sources of information such as a newspaper, an infomercial or the Internet. Consequently, as depicted in Figure 
$6-12$, this identified gap was closed by adding the concept "Knowledge about the tech utility".

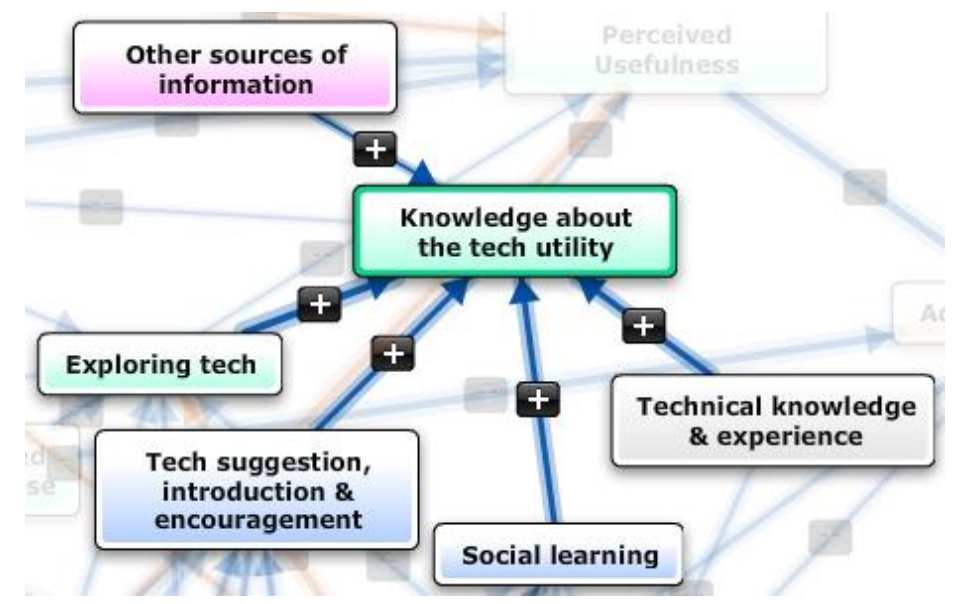

Figure 6 - 12 Identify \& close concept gaps

\subsubsection{Integration of All Standardized Maps (Research Step 10)}

Once all concepts of all cognitive maps were standardized and any potential gap was investigated and closed, the integration of the maps followed. This can be easily done by expanding every map to contain all the concepts that had emerged from the research. This was done by adding the missing concepts to the map's adjacency matrix (explained in the section 3.1.4. Fuzzy Cognitive Map) and adjusting all the concepts across the maps in the same sequence. The parking lot map described in step 7 comprises all of the concepts that emerged in the research, and, as such, their order in the parking lot map can be followed in all the 
other maps, for consistency. For example, the order of the concepts in the ontology list table (Appendix G.I) follows the same order as the parking lot map.

Integration of the standardized maps of all the cognitive models of pairs follows. All these standardized cognitive maps are integrated into one composite model that contains the collective knowledge of all elderly participants and their gatekeepers. The resulting model highlights the critical drivers and barriers to the technology adoption and how these factors influence each other. The integrated model (Mapint) also shows if and how gatekeepers influence such key drivers and barriers.

The resulting integrated model is depicted below in Figure 6 - 13 . 


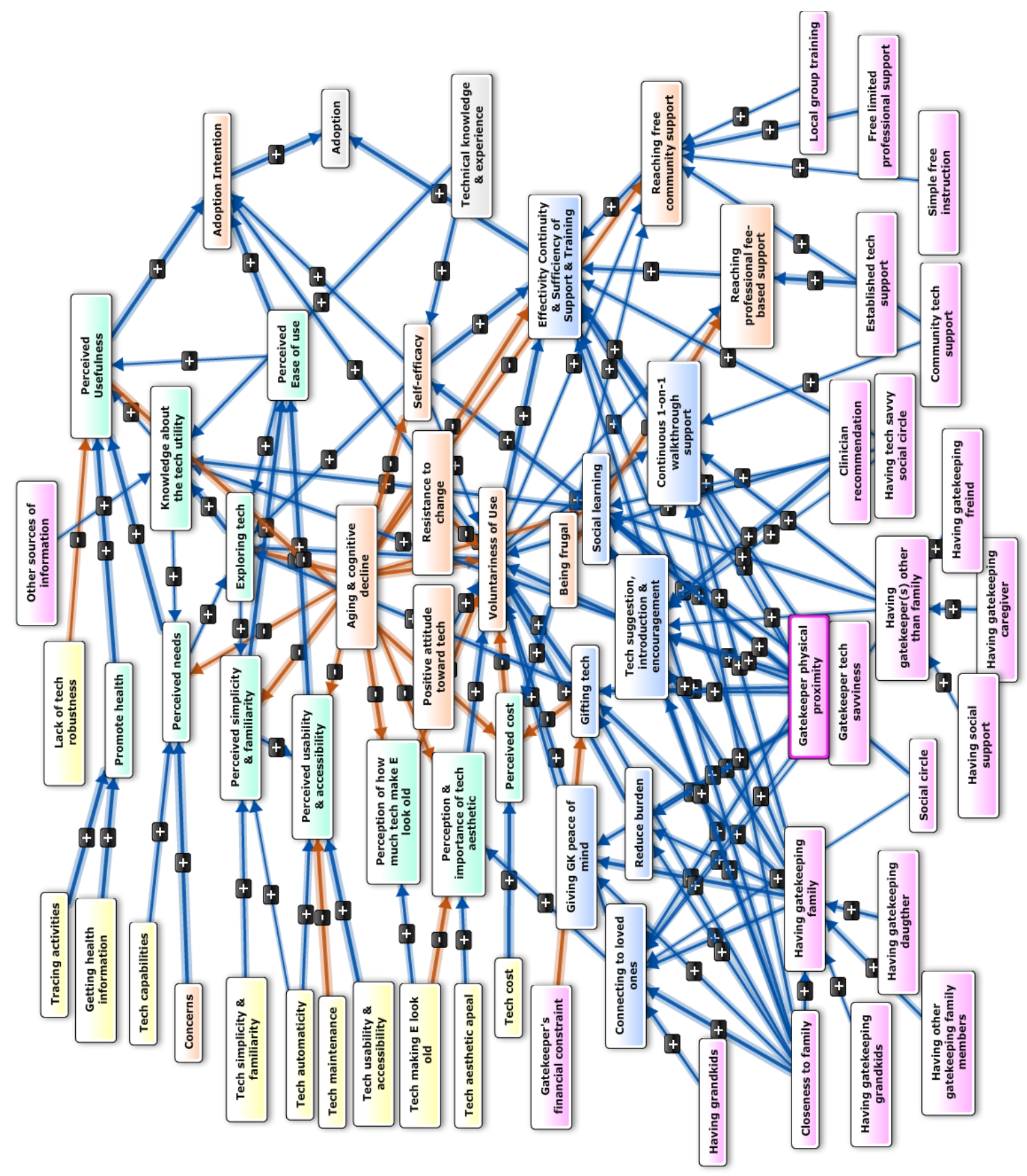

Figure 6 - 13 EWHTA integrated model 


\subsection{Quantitative (quan) Data Collection - Converting Cognitive Maps to Fuzzy Cognitive Maps}

\subsubsection{Model Structure Building (Research Step 11)}

As laid out in the step 11 of the proposed mixed-method in chapter 3 , the qualitative (QUAL) phase of the research results in the model content. Model structure building activity follows. In this step, the information pertaining to the degree of influence of each concept on other concepts is gathered. The instrument used for this data collection is a survey method administered by walking the elderly participants through the designed survey instrument (depicted in Appendix E.I). To do this, the same group of elderly women who participated in the QUAL data collection was asked to subjectively validate and quantify the causal influences of the concepts in the model. To accomplish this, they were asked to assign the importance of all the incoming influences to each concept of the model. This meant that the survey contained a question for every edge weight. As there were 127 links identified in the QUAL analysis, 127 total questions needed to be asked (i.e. 127 choices needed to be made). As depicted in the survey (Appendix E.I) model was broken down into sections in which every concept in the model and all its influencing concepts were depicted (with the focus being on the indegree links). The edge weights of the depicted indegree links were then collected by asking the elderly women to identify a degree of influence for each concept under focus. The choices corresponded to a 5-point Likert scale $[0,0.25,0.5,0.75,1]$ and a sixth choice to skip answering the question (the respondents can refuse to provide a 
value for an edge weight of which they are unsure). As detailed in the survey, the choices were as follows:

\begin{tabular}{ll}
\hline Choice & Likert Scale \\
\hline 1. Not important at all & 0 \\
2. Slightly important & 0.25 \\
3. Somewhat important & 0.5 \\
4. Very important & 0.75 \\
5. Most important & 1 \\
\hline \hline
\end{tabular}

6. I don't know

Removed from the data as unidentified

Table 6 - 4 Answer choices to quantify the edge weights and their corresponding 5-point Likert scale

The valence of the links had been established in the QUAL phase of the research. Since FCM edge weights inherently range from -1 to 1 , so do the Likert scales. This means that the values range from -1 to +1 in increments of 0.25 . Therefore, the possible edge weights are: $-1,-0.75,-0.5,-0.25,0,0.25,0.5,0.75,1$. To simplify the inquiry for the elderly participants, all the questions were turned to positive and the choices provided using positive 5-point Likert scale. This meant that the questions were designed in a way that even the negative influences were captured by the positive 5-point Likert scale. Subsequently, during the conversion of the response data to adjacency matrix of each elderly woman, the values were assigned the appropriate positive or negative edge weights. For example in survey question 32-shown below-there are two negative and two positive edge weights: 


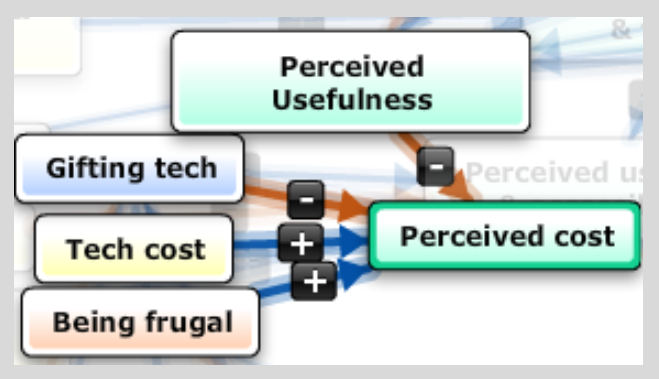

Q32 Rate the importance of these factors influencing the relative cost of the activity tracker:

$\begin{array}{cccccc}\text { Not } & \begin{array}{c}\text { Slightly } \\ \text { important } \\ \text { important }\end{array} & \begin{array}{c}\text { Somewhat } \\ \text { important }\end{array} & \begin{array}{c}\text { Very } \\ \text { important }\end{array} & \begin{array}{c}\text { Most } \\ \text { important }\end{array} & \begin{array}{c}\text { I don't } \\ \text { know }\end{array} \\ \text { at all (1) } & (2) & (3) & (4) & (5) & (6)\end{array}$

the actual cost of the activity tracker (1)

being frugal (2)

how useful it is

if it was given to you (4)

Figure 6 - 14 Question 32 in quan data collection survey (complete survey in Appendix D)

Or, in question 30 in Figure $6-15$ the effect of aging on the self-efficacy is a negative edge weight: 


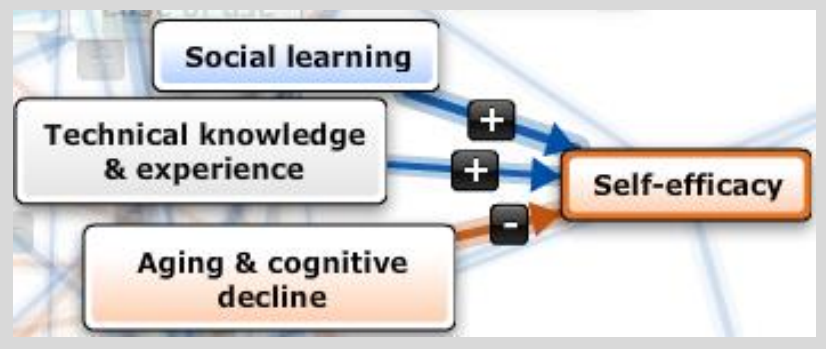

Q30 Rate the importance of these factors influencing the confidence in your ability to successfully use an activity tracker:

$\begin{array}{cccccc}\begin{array}{c}\text { Not } \\ \text { important } \\ \text { at all (1) }\end{array} & \begin{array}{c}\text { Slightly } \\ \text { important }\end{array} & \begin{array}{c}\text { Somewhat } \\ \text { important }\end{array} & \begin{array}{c}\text { Very } \\ \text { important }\end{array} & \begin{array}{c}\text { Most } \\ \text { important }\end{array} & \begin{array}{c}\text { I } \\ \text { don't } \\ \text { know }\end{array} \\ & (2) & (3) & (4) & (5) & (6)\end{array}$

seeing others use it (1)

you had working experience with technology in your past career

the older I get the less I feel I can figure technology out (3)

Figure 6 - 15 Question 30 in quan data collection survey (complete survey in Appendix D)

In these cases (and for all other negative edge weights), the choice description embodies the meaning of the negative effect, and, as such, the collected choice is translated to a negative Likert scale.

Unfortunately, two of the fifteen participants who participated in the QUAL research were not able to participate in the quan data collection. This resulted in receiving thirteen responses that were subsequently translated into thirteen adjacency 
matrices. The corresponding adjacency matrix of each elderly woman participant is listed in Appendix E.II.

\subsubsection{Network Analysis of Paired FCMs (Research Step 12)}

In the quan data collection (step 11) of the proposed method, in which Mapint is converted into the fuzzy cognitive map, FCMint, the edge weights of the final method are calculated using Equation 6 - 1. The formula simply averages all the edge weights received from the survey, removing the "I don't know" responses in the process.

$$
L_{i}=\left(\sum_{r=1}^{R} l_{r . i}\right) /\left(R-R_{i}^{\prime}\right)
$$

Equation 6 - 2 Proposed edge weights calculation formula

Where

$R_{i}^{\prime}$ is number of participants that didn't provide edge weight for the ith link

$R$ is total number of respondents

$L_{i} \quad$ is the edge weight of the $i^{\text {th }}$ link

Studying the participants' responses suggested that there was diversity in the participants' subjective judgments of the edge weights. This diversity needed to be studied to rule out factors that could reduce the integrity of the model. For example, the variability of edge weights (from the responses) could suggest that there are clusters of models and that no single one can aggregate all the elderly women's mental models. Or, it could be that, despite the elderly women being walked 
through the survey, they still didn't completely comprehend the questions. Hence, some of their responses may not have been completely accurate. To analyze this diversity of the opinions, the most relevant Network Analysis (SNA) techniques were applied to analyze the structure of every elderly woman's FCM. As depicted in the adjacency matrices in Appendix E.II. Adjacency Matrices of the Elderly Women Participants), the contents of these FCMs were almost identical and their structures varied only slightly (the only variation being the magnitude of the edge weights). These similarities between graphs make most SNA techniques, which primarily study the variation in the graphs' contents, inadequate. At the time of this dissertation, the most appropriate technique found to study network similarity for models with only slight variation in structure is Eigenvalue Method (Koutra, et al., 2011; Papadimitriou, et al., 2010). The Eigenvalue Method was implemented based on a network similarity index (eigenvector).

For my dissertation research, I deployed the algorithm listed in E.III. Clustering FCM Algorithm, which was developed by Payam Aminpour at Michigan State University (Aminpour, 2018). This algorithm implements the Eigenvalue Method in the following order. First, a reference graph is designated. For this dissertation, I designated the average adjacency matrix calculated from Equation 6 - 3; however, other matrices could have been selected. The point of this matrix is to provide a reference graph to which all other graphs are compared. Second, the similarity of the 13 pair FCMs to the reference graph (here, Average FCM) was measured. The similarity was calculated using the Eigenvector Similarity Index (ESI). A variety of 
different similarity indices exist, but the ESI is used here, because one of the important features of a graph is the vector of its eigenvalues, which contains important structural information about the graph. The ESI explains that, if two graphs possess more similar eigenvectors, then they are more similar. Third, the calculations for each pair FCM resulted in a number that represented the similarity of the pair FCM to the reference graph. This could be interpreted as each graph being a point in a line and the similarity being the distance between each FCM point and the reference point (e.g. depicted in the first section of Figure $6-16$. Finally, a clustering algorithm (K-mean) indicated that the points that were close together formed a cluster. The output of the execution of this algorithm on the average FCM and the 13 participant pairs' FCMs is depicted in Figure 6 - 16.

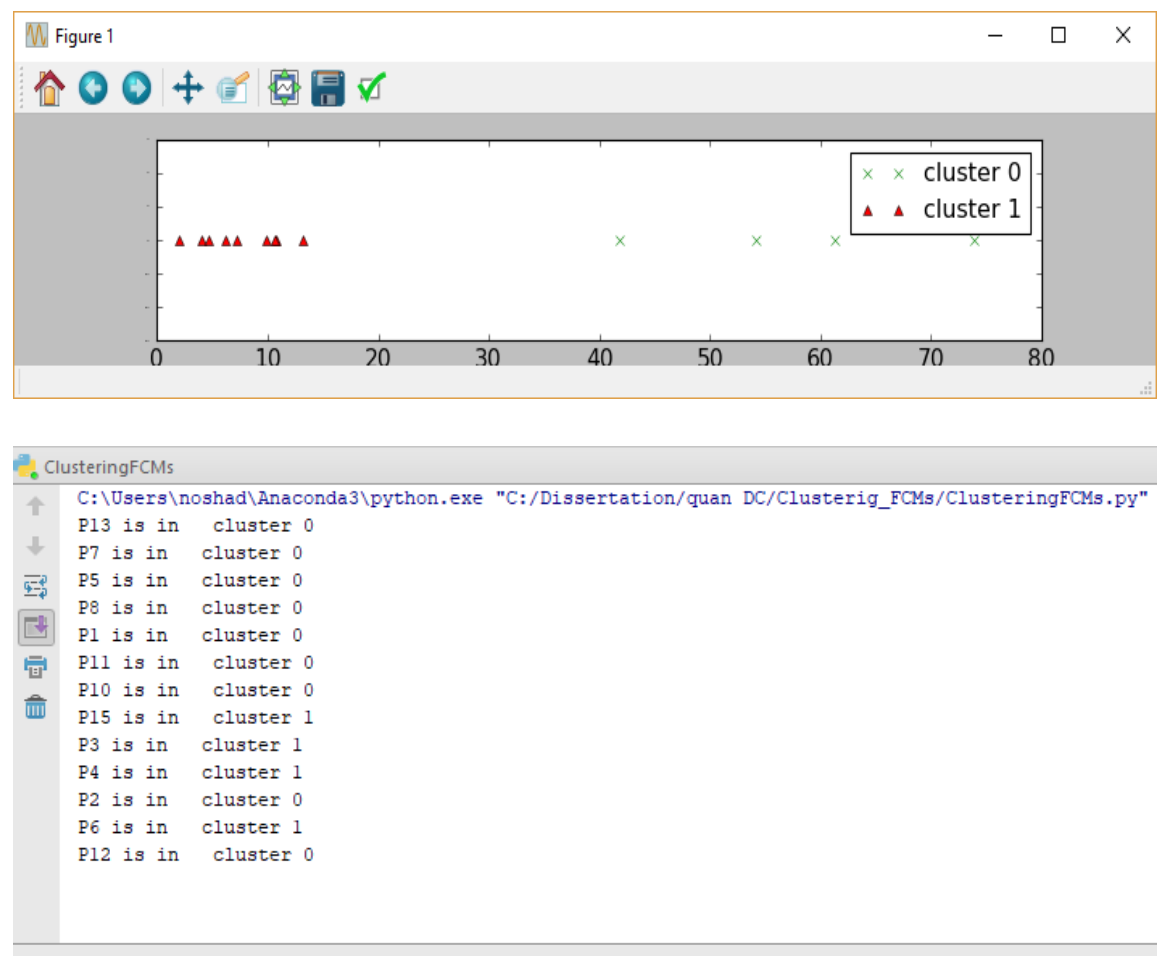

Figure 6 - 16 Output of clustering FCM based on Eigenvector similarity index 
This suggested that the case study data set consists of two clusters of FCMs:

- First Cluster: $P_{1}, P_{2}, P_{5}, P_{7}, P_{8}, P_{10}, P_{11}, P_{12}, P_{13}$

- Second Cluster: $P_{3}, P_{4}, P_{6}, P_{15}$

To understand why the structure of these two clusters of FCMs are different and if this difference is rooted in the elderlies' demographic or environmental factors, the profiles of the participants were reanalyzed. To do this, different identifiers of the population of elderly women in the study were itemized and values between [$100 \%$ to $100 \%]$ were assigned to show the presence and the strength of each identified factor [-100\% being the least and $100 \%$ being the most].

\begin{tabular}{|c|c|c|c|c|c|c|c|c|c|c|c|c|c|}
\hline 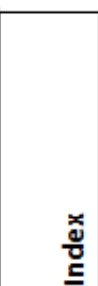 & $\stackrel{\square}{8}$ & 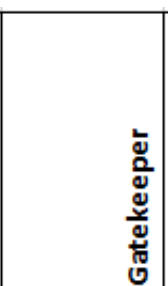 & 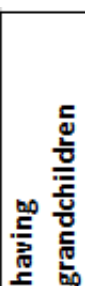 & 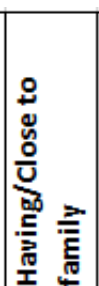 & 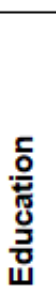 & 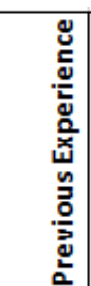 & 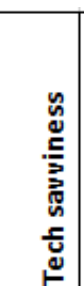 & 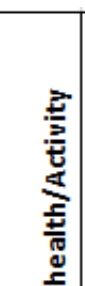 & 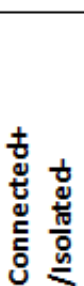 & 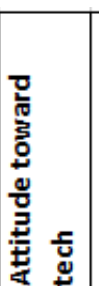 & 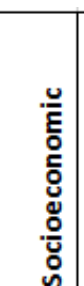 & 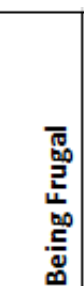 & o \\
\hline P1 & 83 & Daughter & Yes & $70 \%$ & 16 & $0 \%$ & $-50 \%$ & $50 \%$ & $70 \%$ & $-50 \%$ & $0 \%$ & $90 \%$ & R. Community \\
\hline P2 & 69 & Daughter(rs & Yes & $50 \%$ & 13 & $30 \%$ & $50 \%$ & $50 \%$ & $20 \%$ & $50 \%$ & $30 \%$ & $80 \%$ & R. Community \\
\hline P3 & 80 & - R.C.Tech & No & $0 \%$ & 18 & $50 \%$ & $40 \%$ & $50 \%$ & $-100 \%$ & $-100 \%$ & $50 \%$ & $50 \%$ & R. Community \\
\hline P4 & 94 & - caregiver & No & $-100 \%$ & 18 & $30 \%$ & $-50 \%$ & $-40 \%$ & $-100 \%$ & $100 \%$ & $0 \%$ & $70 \%$ & R. Community \\
\hline P5 & 90 & Daughter & No & $60 \%$ & 15 & $20 \%$ & $30 \%$ & $30 \%$ & $50 \%$ & $80 \%$ & $50 \%$ & $90 \%$ & R. Community \\
\hline P6 & 95 & - Friend & No & $\mid-100 \%$ & 16 & $-100 \%$ & $-70 \%$ & $0 \%$ & $-100 \%$ & $80 \%$ & $80 \%$ & $60 \%$ & R. Community \\
\hline P7 & 82 & Daughter & Yes & $40 \%$ & 15 & $70 \%$ & $60 \%$ & $50 \%$ & $40 \%$ & $80 \%$ & $90 \%$ & $0 \%$ & R. Community \\
\hline P8 & 90 & Daughter & Yes & $0 \%$ & 20 & $0 \%$ & $-70 \%$ & $50 \%$ & $20 \%$ & $-70 \%$ & $50 \%$ & $90 \%$ & R. Community \\
\hline P10 & 84 & Daughter & Yes & $100 \%$ & 12 & $20 \%$ & $-50 \%$ & $50 \%$ & $80 \%$ & $80 \%$ & $80 \%$ & $20 \%$ & R. Community \\
\hline P11 & 67 & - husband & No & $40 \%$ & 18 & $100 \%$ & $80 \%$ & $80 \%$ & $10 \%$ & $100 \%$ & $-20 \%$ & $100 \%$ & house \\
\hline P12 & 65 & Daughter & Yes & $90 \%$ & 16 & $10 \%$ & $10 \%$ & $70 \%$ & $80 \%$ & $100 \%$ & $-20 \%$ & $80 \%$ & house \\
\hline P13 & 71 & Daughter & Yes & $80 \%$ & 12 & $0 \%$ & $-70 \%$ & $60 \%$ & $90 \%$ & $100 \%$ & $0 \%$ & $70 \%$ & R. Community \\
\hline P14 & 78 & - Friend & Yes & $30 \%$ & 18 & $70 \%$ & $40 \%$ & $100 \%$ & $60 \%$ & $-70 \%$ & $80 \%$ & $0 \%$ & R. Community \\
\hline P15 & 94 & - Neighbor & No & $-90 \%$ & 14 & $70 \%$ & $50 \%$ & $20 \%$ & $-100 \%$ & $40 \%$ & $50 \%$ & $40 \%$ & R. Community \\
\hline
\end{tabular}

Table 6 - 5 Participants' profile information 
Table $6-5$ shows that the major difference between the two clusters is the elderly woman's degree of connectedness/isolation. In other words, the elderly women in the first cluster were all well connected to their family and had family gatekeepers who immensely influenced their technology adoption. On the other hand, the second cluster's participants were all socially isolated $(-100 \%$ score in the connected criterion). They hardly had gatekeepers who could motivate and facilitate their health technology adoption. This suggested that the integration of the FCMs should happen at the cluster level. There should be two models, one for each cluster, where each model respectively represents the collective mental model of each group of elderly women.

The two models were calculated based on the average formula of the participants' adjacency matrices in each cluster. The results of these calculations are displayed in Appendix E.V. Adjacency Matri and Appendix E.VI. Adjacency Matri. The Connected Cluster Adjacency Matrix in Appendix E.V has no "social circle" node, as all the edge weights associated with this concept equal 0 for this cluster. All the edge weights that differ from those of Total Population Adjacency Matrix in Appendix E.VI. are highlighted in these two cluster matrices. 


\subsection{Quantitative (quan) Analysis - Model Evaluation (Research Step 13)}

The Network Analysis of the data arrived from the quan data collection suggested that not all of elderly women's mental models can be integrated into one aggregate model. Fascinatingly, it became apparent that the participants' mental models identify two market segments, namely, socially isolated elderly women and socially connected elderly women. This warranted the division of the data set into two corresponding segments. Therefore, two FCM models, each of which aggregated one of the identified clusters, were created by following the Equation 6 - 2 Proposed

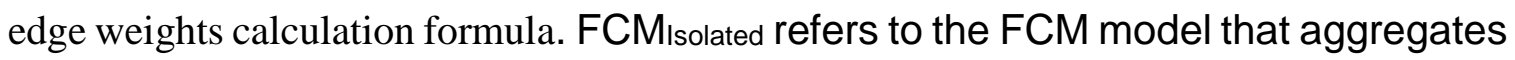
the group of isolated elderly women and FCMconnected refers to the FCM model that aggregates the group of connected elderly women.

In this phase of the research, the resulted FCM models should be validated using the evaluation scenarios detailed in the following three sections. As Sterman posits:

"all models are wrong... all models, mental or formal, are limited, simplified representations of the real world. They differ from reality in ways large and small, infinite in number."

"No model has ever been or ever will be thoroughly validated. . . "Useful," "illuminating," "convincing," or "inspiring confidence" are more apt descriptors applying to models than "valid."

(Sterman, system dynamics page 846) 
This highlights the fact that no model can be fully validated. However, since this dissertation claims to have proposed a useful empirical model building method, the claim can be validated by testing the usefulness of the resulted models. The following groups of tests (Sterman, 2000), as laid out in the chapter 3, can provide the adequate measures to test the integrity of the models.

In the three following sections, detailed information about the data set, like the elderly profiles in Appendix $\mathrm{H}$ is useful in the scenario planning.

Each one of the following sections embodies a set of scenarios that need to be simulated. Since these sets of scenarios need to be simulated per each FCM model, each section contains two tables that each summarize the simulations for an FCM model. For example, Table 6 - 6 shows the information about simulations of the FCMconnected model and Table 6 - 7 Extreme Condition Tests of FCMlsolated model for the extreme condition tests scenarios. The rows in these tables denote two types of concepts: input concepts (aka start vectors) and output concepts. The concepts in the start vectors section represent the minimum set of concepts that best represents the scenarios. They are set as the start vector, and they are set to stay unchanged throughout the simulation. All other concepts are not displayed in these tables. Their values have been set to zero initially, but they change as the simulations progress. The concepts in the output section represent the behavior of the model.

The first three columns of each table respectively denote concept type, concept number in the model adjacency matrices and concepts names. The remaining columns refer to the scenarios under consideration. Every number in the table 210 
represents the value of the particular concept for the scenario of that column. The elements of the output section denote the values of the output concepts that did not change by more than 0.001 (epsilon value) from iteration to iteration. For each simulation, the number of iterations that resulted in stable outputs is recorded in the row marked as "Stability Iteration \#".

\subsubsection{Extreme Condition Tests}

The FCM models need to be tested under extreme conditions to ensure they behave realistically (Sterman, 2000, page 869). For this, both FCM Isolated and FCMconnected were simulated for the following extreme scenarios, in order to ensure the model's behavior reflects reality.

ECT- Scenario 1: The technology is neither useful nor is it easy to use.

Expected models behavior: The model should suggest neither intention nor adoption.

ECT- Scenario 2: Elderly has no access to established support channels.

Expected models behavior: The model should suggest no adoption, regardless of intention to adopt.

ECT- Scenario 3: Elderly has a very negative attitude toward technology. Expected models behavior: The model should suggest no adoption intention.

ECT- Scenario 4: Elderly has a strong resistance to change her way of life (i.e. adopt technology).

Expected models behavior: The model should suggest no adoption intention. 
ECT- Scenario 5: Elderly is too old.

Expected models behavior: The model should suggest no adoption intention.

ECT- Scenario 6: The technology is objectively excellent both in usefulness and ease of use.

Expected models behavior: The model should suggest adoption intention.

ECT- Scenario 7: Elderly has full access and actively reaches out to the established support channels.

Expected models behavior: The model should suggest adoption.

ECT- Scenario 8: Elderly has a very positive attitude toward technology. Expected models behavior: The model should suggest adoption intention.

ECT- Scenario 9: Elderly has no resistance to adopting new technology. Expected models behavior: The model should suggest adoption intention.

These scenarios are simulated in Excel following the process detailed in section 3.1.4. Fuzzy Cognitive Mapping section. (A typical FCM simulation in depicted in the Figure $F-1$. Table 6 - 6 provides the details extreme condition tests simulations

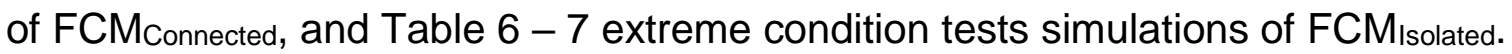
Each of the 9 columns, on the right of the tables, show the details of one of the scenarios designed above. For each scenario, the minimum set of concepts that best represent the scenario are set as the start vector and are set to stay unchanged throughout the simulation. The two output variables (Adoption and Adoption Intention) are recorded when their values became stable through the 
iterations of the simulation. The values considered stable when their changes from the last iteration of the simulation were less than 0.001 (epsilon).

The evaluations of the two models' integrity in the extreme condition tests are both successful as the simulation results of the 9 described scenarios are aligned with 
the expected behaviors. This can be observed by comparing the expected

\begin{tabular}{|c|c|c|c|c|c|c|c|c|c|c|c|}
\hline & 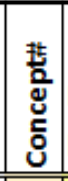 & $\begin{array}{l}\text { Extreme Condition Tests of } \\
\mathrm{FCM}_{\text {Connected }}\end{array}$ & 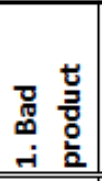 & 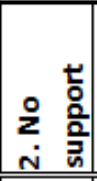 & 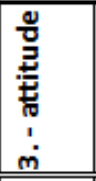 & 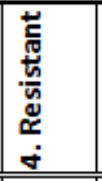 & $\begin{array}{l}\text { 등 } \\
8 \\
\stackrel{\circ}{-} \\
\dot{n}\end{array}$ & 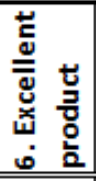 & 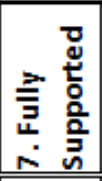 & 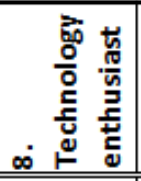 & 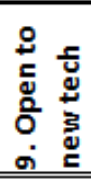 \\
\hline \multirow{32}{*}{ 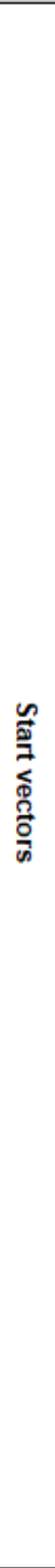 } & c5 & Tech automaticity & -1 & & & & & 1 & & & \\
\hline & c6 & Getting health information & -1 & & & & & 1 & & & \\
\hline & c12 & Aging \& cognitive decline & & & & & 1 & & & & \\
\hline & c15 & Tech usability \& accessibility & -1 & & & & & 1 & & & \\
\hline & c17 & Tech capabilities & -1 & & & & & 1 & & & \\
\hline & c19 & Tracing activities & -1 & & & & & 1 & & & \\
\hline & c21 & Tech simplicity \& familiarity & -1 & & & & & 1 & & & \\
\hline & c24 & Gatekeeper physical proximity & & -1 & & & & & 1 & & \\
\hline & c25 & Tech cost & 1 & & & & & & & & \\
\hline & c27 & Tech maintenance & 1 & & & & & & & & \\
\hline & c28 & Tech making E look old & 1 & & & & & -1 & & & \\
\hline & c30 & Community tech support & & -1 & & & & & 1 & & \\
\hline & c33 & Lack of tech robustness & 1 & & & & & -1 & & & \\
\hline & c34 & Having gatekeeping daugther & & & & & & & 1 & & \\
\hline & c36 & Gatekeeper tech savviness & & -1 & & & & & 1 & & \\
\hline & c39 & Local group training & & & & & & & 1 & & \\
\hline & c41 & Tech aesthetic apeal & -1 & & & & & 1 & & & \\
\hline & c42 & Clinician recommendation & & 0 & & & & & & & \\
\hline & c44 & Positive attitude toward tech & & & -1 & & & & & -1 & \\
\hline & 045 & Resistance to change & & & & 1 & & & & & 1 \\
\hline & c50 & $\begin{array}{l}\text { Free limited professional } \\
\text { support }\end{array}$ & & 0 & & & & & 1 & & \\
\hline & c51 & Having tech savvy social circle & & 0 & & & & & & & \\
\hline & c52 & Established tech support & & 0 & & & & & 1 & & \\
\hline & c53 & Simple free instruction & & 0 & & & & & 1 & & \\
\hline & c54 & Having gatekeeping caregiver & & 0 & & & & & & & \\
\hline & c55 & Closeness to family & & 0 & & & & & 1 & & \\
\hline & c56 & Having gatekeeping freind & & 0 & & & & & & & \\
\hline & c57 & Having gatekeeping grandkids & & 0 & & & & & 1 & & \\
\hline & c59 & Having grandkids & & 0 & & & & & & & \\
\hline & c60 & $\begin{array}{l}\text { Having other gatekeeping } \\
\text { family members }\end{array}$ & & 0 & & & & & 1 & & \\
\hline & c61 & Other sources of information & & 0 & & & & & & & \\
\hline & c62 & \begin{tabular}{|l} 
Gatekeeper's financial \\
constraint
\end{tabular} & & 0 & & & & & & & \\
\hline \multirow{3}{*}{ 异 } & \# & Stability Iternation \# & 9 & 10 & 5 & 23 & 8 & 8 & 9 & 23 & 23 \\
\hline & c1 & Adoption & -0.847 & -0.887 & -0.506 & -0.718 & -0.896 & 0.830 & 0.893 & 0.718 & 0.718 \\
\hline & c2 & Adoption Intention & -0.945 & $\mid-0.896$ & -0.325 & $\mid-0.598$ & -0.960 & 0.938 & 0.916 & 0.598 & 0.598 \\
\hline
\end{tabular}

Table 6 - 6 Extreme Condition Tests of FCMconnected model 
behaviors posited earlier with the output values as listed in the bottom two rows

\begin{tabular}{|c|c|c|c|c|c|c|c|c|c|c|c|}
\hline & $\begin{array}{l}\text { 拉 } \\
\text { 엉 } \\
\text { 엉 } \\
\end{array}$ & $\begin{array}{l}\text { Extreme Condition Tests } \\
\text { of } \mathrm{FCM}_{\text {|solated }}\end{array}$ & 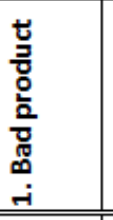 & $\begin{array}{l}\text { 늠 } \\
\frac{0}{2} \\
\frac{3}{3} \\
0 \\
2 \\
\dot{2} \\
\end{array}$ & 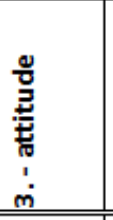 & 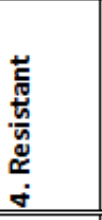 & $\begin{array}{l}\text { 흥 } \\
\therefore \\
\stackrel{\circ}{\bullet} \\
\text { ம் } \\
\end{array}$ & 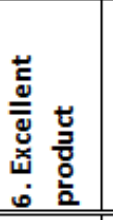 & 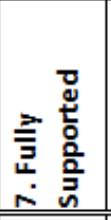 & 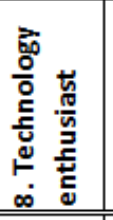 & 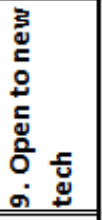 \\
\hline \multirow{33}{*}{ 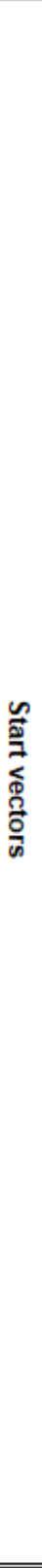 } & c5 & Tech automaticity & -1 & & & & & 1 & & & \\
\hline & c6 & Getting health information & -1 & & & & & 1 & & & \\
\hline & c12 & Aging \& cognitive decline & & & & & 1 & & & & \\
\hline & c15 & Tech usability \& accessibility & -1 & & & & & 1 & & & \\
\hline & c17 & Tech capabilities & -1 & & & & & 1 & & & \\
\hline & $\mathrm{c} 19$ & Tracing activities & -1 & & & & & 1 & & & \\
\hline & c21 & Tech simplicity \& familiarity & -1 & & & & & 1 & & & \\
\hline & $\mathrm{c} 24$ & $\begin{array}{l}\text { Gatekeeper physical } \\
\text { proximity }\end{array}$ & & -1 & & & & & 1 & & \\
\hline & c25 & Tech cost & 1 & & & & & & & & \\
\hline & c27 & Tech maintenance & 1 & & & & & & & & \\
\hline & $\mathrm{c} 28$ & Tech making E look old & 1 & & & & & -1 & & & \\
\hline & c30 & Community tech support & & -1 & & & & & 1 & & \\
\hline & c33 & Lack of tech robustness & 1 & & & & & -1 & & & \\
\hline & c34 & Having gatekeeping daugther & & & & & & & 1 & & \\
\hline & c36 & Gatekeeper tech savviness & & -1 & & & & & 1 & & \\
\hline & c39 & Local group training & & & & & & & 1 & & \\
\hline & C41 & Tech aesthetic apeal & -1 & & & & & 1 & 1 & & \\
\hline & C42 & Clinician recommendation & & 0 & & & & & 1 & & \\
\hline & c44 & Positive attitude toward tech & & & -1 & & & & & -1 & \\
\hline & $\mathrm{C45}$ & Resistance to change & & & & 1 & & & & & 1 \\
\hline & c50 & support & & 0 & & & & & 1 & & \\
\hline & c51 & circle & & 0 & & & & & & & \\
\hline & c52 & Established tech support & & 0 & & & & & 1 & & \\
\hline & $\mathrm{c53}$ & Simple free instruction & & 0 & & & & & 1 & & \\
\hline & c54 & $\begin{array}{l}\text { Having gatekeeping } \\
\text { caregiver }\end{array}$ & & 이 & & & & & & & \\
\hline & c55 & Closeness to family & & 0 & & & & & 1 & & \\
\hline & c56 & Having gatekeeping freind & & 0 & & & & & & & \\
\hline & c57 & $\begin{array}{l}\text { Having gatekeeping } \\
\text { grandkids }\end{array}$ & & 0 & & & & & 1 & & \\
\hline & c59 & Having grandkids & & 0 & & & & & & & \\
\hline & c60 & $\begin{array}{l}\text { Having other gatekeeping } \\
\text { family members }\end{array}$ & & 0 & & & & & 1 & & \\
\hline & c61 & $\begin{array}{l}\text { Other sources of } \\
\text { information }\end{array}$ & & 0 & & & & & & & \\
\hline & c62 & $\begin{array}{l}\text { Gatekeeper's financial } \\
\text { constraint }\end{array}$ & & 0 & & & & & & & \\
\hline & c63 & Social circle & & 0 & & & & & & & \\
\hline \multirow{3}{*}{$\begin{array}{l}\text { 을 } \\
\text { Ð } \\
\text { 三 }\end{array}$} & \# & Stability Iternation \# & 7 & 9 & 18 & 22 & 7 & 8 & 12 & 18 & 23 \\
\hline & c1 & Adoption & -0.877 & -0.893 & -0.828 & -0.786 & -0.899 & 0.870 & 0.878 & 0.828 & 0.786 \\
\hline & $\mathrm{c} 2$ & Adoption Intention & -0.944 & -0.920 & -0.706 & -0.629 & -0.964 & 0.939 & 0.841 & 0.706 & 0.629 \\
\hline
\end{tabular}

Table 6 - 7 Extreme Condition Tests of FCM Isolated model 
In Table $6-6$ and Table $6-7$.

\subsubsection{Behavior Reproduction Tests}

Models should behave as expected. During the qualitative analysis phase of the case study of this dissertation, some dominant themes were identified (detailed in the participants' profiles Appendix $\mathrm{H}$. These themes highlights the typical successful and unsuccessful EHTA scenarios that can be used to verify whether the model produces those same expected behaviors. Following scenarios and their expected behaviors are proposed for this step of the models' evaluation.

\section{For isolated elderlies:}

BRT- Scenario 1: The positive effects of technology experience trumps social isolation. In other word, if the elderly woman has technology experience, she will adopt a good technology even if she is socially isolated.

BRT- Scenario 2: The effects of positive attitude toward technology trumps social isolation. In other word, if the elderly woman has positive attitude toward technology, she will adopt a good technology even if she is socially isolated.

BRT- Scenario 3: Those socially isolated elderly women who have negative attitude toward technology will not adopt.

BRT- Scenario 4: Those socially isolated elderly women who have no previous technology experience has a really low chance of technology adoption.

For connected elderlies:

BRT- Scenario 1: The positive effects of technology experience trumps the weak gatekeeping influences and support. In other word, if the elderly woman has 
technology experience, she will adopt a good technology even if she is not close to her family and her gatekeeping support is weak.

BRT- Scenario 2: The effects of positive attitude trumps the weak gatekeeping influences and support. In other word, if the elderly woman has technology experience, she will adopt a good technology even if she is not close to her family and her gatekeeping support is weak.

BRT- Scenario 3: Elderly woman with strong Gatekeeping influence will adopt a good product even if she is socially isolated otherwise.

BRT- Scenario 4: Strong Gatekeeping effects trump lack of access to other support channels.

For both group:

BRT- Scenario 5: As identified in the analysis, the recommendation of a technology by the elderly women's clinician is very important in her decision to adopt and hence both models simulating should show this salience (I.e. if clinician recommends the technology both the models should indicate adoption.

BRT- Scenario 6: While strong gatekeeping influence for connected EW can trump inexperience, strong facilitating conditions for the isolated EW, in the absence of other driving forces, can't by itself lead to technology adoption.

BRT-Scenario 7: In each group younger elderly women have much higher chance of technology adoption than the older elderly women, however a connected EW has a higher chance of adoption than an isolated EW. To test this scenario, two 
similar scenarios with different values for aging concepts are simulated for each group and values are compared.

Similar to the extreme condition tests scenarios, these scenarios were simulated in Excel following the process detailed in the section 3.1.4. Fuzzy Cognitive Mapping. Table $6-8$ and Table $6-9$ show details parameters of the simulations. In these tables, the columns in white, show the details of each of the scenarios designed above. For each scenario, the minimum set of concepts that best represent the scenario are set as the start vector and are set to stay unchanged throughout the simulation. The two output variables (Adoption and Adoption Intention) are recorded when their values became stable through the iterations of the simulation. The values considered stable when their changes from the last iteration of the simulation were less than 0.001 (epsilon). For each simulation the number of iterations that resulted in stable outputs are recorded in the row marked as "Stability Iteration \#".

The results of the simulations show that both the FCM Isolated and FCMconnected models are successfully reproducing the expected behaviors. This can be observed by comparing the expected behaviors posited earlier with the output values as listed in the bottom two rows in Table $6-8$ and Table $6-9$. 


\begin{tabular}{|c|c|c|c|c|c|c|c|c|c|c|}
\hline & 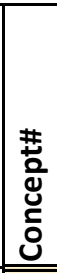 & $\begin{array}{l}\text { Behavior Reproduction } \\
\text { Tests of FCM }\end{array}$ & 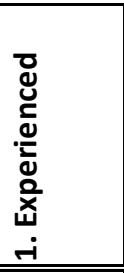 & 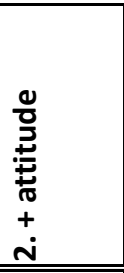 & 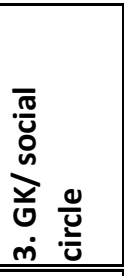 & 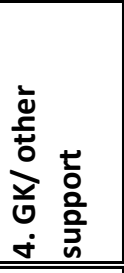 & 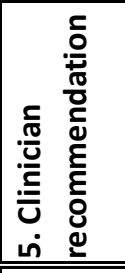 & 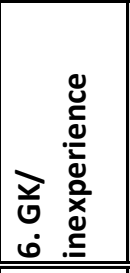 & 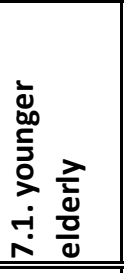 & $\begin{array}{l}\frac{\lambda}{2} \\
\frac{0}{0} \\
\frac{0}{0} \\
\frac{1}{0} \\
\frac{0}{0} \\
\text { ํ. } \\
\end{array}$ \\
\hline \multirow{22}{*}{ 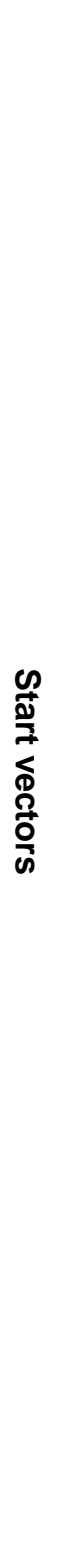 } & c12 & Aging \& cognitive decline & & & & & & & 0.5 & 1 \\
\hline & c13 & $\begin{array}{l}\text { Technical knowledge \& } \\
\text { experience }\end{array}$ & 1 & & & & & -1 & & \\
\hline & c17 & Tech capabilities & 0.5 & 0.5 & 0.5 & 0.5 & 0.5 & 0.5 & 0.5 & 0.5 \\
\hline & c24 & $\begin{array}{l}\text { Gatekeeper physical } \\
\text { proximity }\end{array}$ & 0 & 0 & 1 & 1 & & 1 & & \\
\hline & c30 & Community tech support & & & & -1 & & & & \\
\hline & c34 & $\begin{array}{l}\text { Having gatekeeping } \\
\text { daugther }\end{array}$ & 0 & 0 & 1 & 1 & & 1 & & \\
\hline & c36 & Gatekeeper tech savviness & 0 & 0 & 1 & 1 & & 1 & & \\
\hline & c39 & Local group training & & & & -1 & & & & \\
\hline & c42 & $\begin{array}{l}\text { Clinician } \\
\text { recommendation }\end{array}$ & & & & & 1 & & & \\
\hline & c44 & $\begin{array}{l}\text { Positive attitude toward } \\
\text { tech }\end{array}$ & & 1 & & & & & & \\
\hline & $c 45$ & Resistance to change & & & & & & & & \\
\hline & c50 & $\begin{array}{l}\text { Free limited professional } \\
\text { support }\end{array}$ & & & & -1 & & & & \\
\hline & c51 & $\begin{array}{l}\text { Having tech savvy social } \\
\text { circle }\end{array}$ & 0 & 0 & -1 & & & & & \\
\hline & c52 & Established tech support & & & & -1 & & & & \\
\hline & c53 & Simple free instruction & & & & -1 & & & & \\
\hline & c54 & $\begin{array}{l}\text { Having gatekeeping } \\
\text { caregiver }\end{array}$ & & & -1 & & & & & \\
\hline & c55 & Closeness to family & 0 & 0 & 1 & 1 & & 1 & & \\
\hline & c56 & Having gatekeeping freind & & & -1 & & & & & \\
\hline & c57 & $\begin{array}{l}\text { Having gatekeeping } \\
\text { grandkids }\end{array}$ & 0 & 0 & 1 & 1 & & 1 & & \\
\hline & c59 & Having grandkids & 0 & 0 & 1 & 1 & & 1 & & \\
\hline & c60 & $\begin{array}{l}\text { Having other gatekeeping } \\
\text { family members }\end{array}$ & & & 1 & 1 & & 1 & & \\
\hline & c61 & $\begin{array}{l}\text { Other sources of } \\
\text { information }\end{array}$ & & & & -1 & & & & \\
\hline \multirow{3}{*}{ 을 } & $\#$ & Stability Iteration \# & 12 & 15 & 9 & 9 & 12 & 11 & 22 & 9 \\
\hline & c1 & Adoption & 0.85219 & 0.79802 & 0.89419 & 0.89292 & 0.8378 & 0.87345 & 0.63287 & -0.8939 \\
\hline & $c 2$ & Adoption Intention & 0.904 & 0.78548 & 0.92869 & 0.92712 & 0.847 & 0.80409 & 0.65774 & -0.9483 \\
\hline
\end{tabular}

Table 6 - 8 Behavior Reproduction Tests of FCMConnected model 


\begin{tabular}{|c|c|c|c|c|c|c|c|c|c|c|}
\hline & 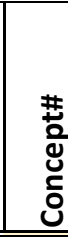 & $\begin{array}{l}\text { Behavior Reproduction } \\
\text { Tests of } \mathrm{FCM}_{\text {Isolated }}\end{array}$ & 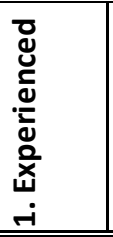 & 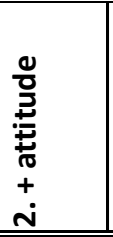 & 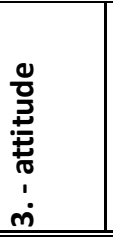 & 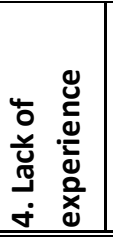 & 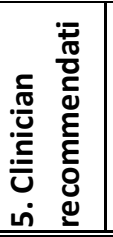 & 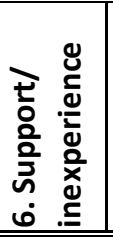 & 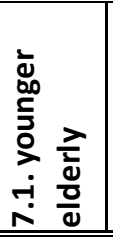 & 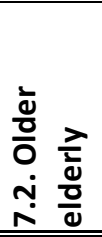 \\
\hline \multirow{22}{*}{ 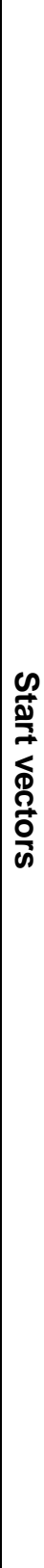 } & c12 & Aging \& cognitive decline & & & & & & & 0.5 & 1 \\
\hline & c13 & $\begin{array}{l}\text { Technical knowledge \& } \\
\text { experience }\end{array}$ & 1 & & & -1 & & -1 & & \\
\hline & c17 & Tech capabilities & 0.5 & 0.5 & 0.5 & 0.5 & 0.5 & 0.5 & 0.5 & 0.5 \\
\hline & c24 & $\begin{array}{l}\text { Gatekeeper physical } \\
\text { proximity }\end{array}$ & 0 & 0 & 0 & 0 & & & & \\
\hline & c30 & Community tech support & & & & & & 1 & & \\
\hline & c34 & $\begin{array}{l}\text { Having gatekeeping } \\
\text { daugther }\end{array}$ & 0 & 0 & 0 & 0 & & & & \\
\hline & c36 & $\begin{array}{l}\text { Gatekeeper tech } \\
\text { savviness }\end{array}$ & 0 & 0 & 0 & 0 & & & & \\
\hline & c39 & Local group training & & & & & & 1 & & \\
\hline & c42 & Clinician recommendation & & & & & 1 & & & \\
\hline & c44 & $\begin{array}{l}\text { Positive attitude toward } \\
\text { tech }\end{array}$ & & 1 & -1 & & & & & \\
\hline & c45 & Resistance to change & & & & & & & & \\
\hline & c50 & $\begin{array}{l}\text { Free limited professional } \\
\text { support }\end{array}$ & & & & & & 1 & & \\
\hline & c51 & $\begin{array}{l}\text { Having tech savvy social } \\
\text { circle }\end{array}$ & 0 & 0 & 0 & 0 & & & & \\
\hline & c52 & Established tech support & & & & & & & & \\
\hline & c53 & Simple free instruction & & & & & & & & \\
\hline & c54 & $\begin{array}{l}\text { Having gatekeeping } \\
\text { caregiver }\end{array}$ & & & & & & & & \\
\hline & c55 & Closeness to family & 0 & 0 & 0 & 0 & & & & \\
\hline & c56 & Having gatekeeping freind & & & & & & & & \\
\hline & c57 & $\begin{array}{l}\text { Having gatekeeping } \\
\text { grandkids }\end{array}$ & 0 & 0 & 0 & 0 & & & & \\
\hline & c59 & Having grandkids & 0 & 0 & 0 & 0 & & & & \\
\hline & c60 & $\begin{array}{l}\text { Having other gatekeeping } \\
\text { family members }\end{array}$ & & & & & & & & \\
\hline & c61 & $\begin{array}{l}\text { Other sources of } \\
\text { information }\end{array}$ & & & & & & 1 & & \\
\hline \multirow{3}{*}{$\frac{\text { 을 }}{\frac{7}{0}}$} & \# & Stability Iteration \# & 11 & 14 & 9 & 12 & 12 & 15 & 14 & 7 \\
\hline & c1 & Adoption & 0.8785 & 0.8557 & -0.606 & -0.827 & 0.8501 & -0.225 & 0.3748 & -0.896 \\
\hline & c2 & Adoption Intention & 0.9168 & 0.8175 & -0.133 & -0.700 & 0.753 & -0.508 & 0.3056 & -0.945 \\
\hline
\end{tabular}

Table 6 - 9 Behavior Reproduction Tests of FCM Isolated model 


\subsubsection{Sensitivity Analysis}

In this section, using sensitivity analysis technique, the stability of the conclusion of the models checked to see if their conclusions are changed significantly when the assumptions varies in the possible range of uncertainty (Sterman, 2000, page 883). As the two EWHTA models here are behavior models, the "behavior mode" is the relevant type of sensitivity analysis that should be conducted here. The following important concepts are considered for analyzing the sensitivity of the behavior of both the FCMIsolated and FCMconnected models.

SA-1 Scenarios: How stable are the models' behaviors when age varies from -1 (youngest) to 1 (oldest).

The older the elderly woman the less likely she will adopt a technology. To do this, each FCM model should be simulated for a range of values for "aging" concept, and the simulation result should show a decreasing value trend as the value of aging increases.

As per the simulation results (Adoption and Adoption Intention) in Table 6 -10 and

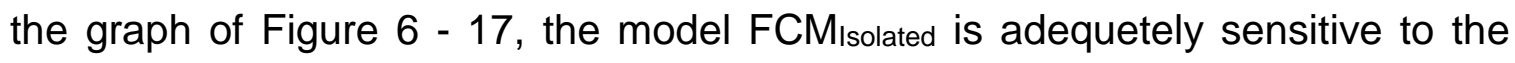
changes in the "Aging and cognitive decline" concept. Similarly, the simulation results (Adoption and Adoption Intention) in Table 6-11 and the graph of Figure $6-18$, , both shows that the model FCMconnected is adequetely sensitive to the changes in the "Aging and cognitive decline" concept. 


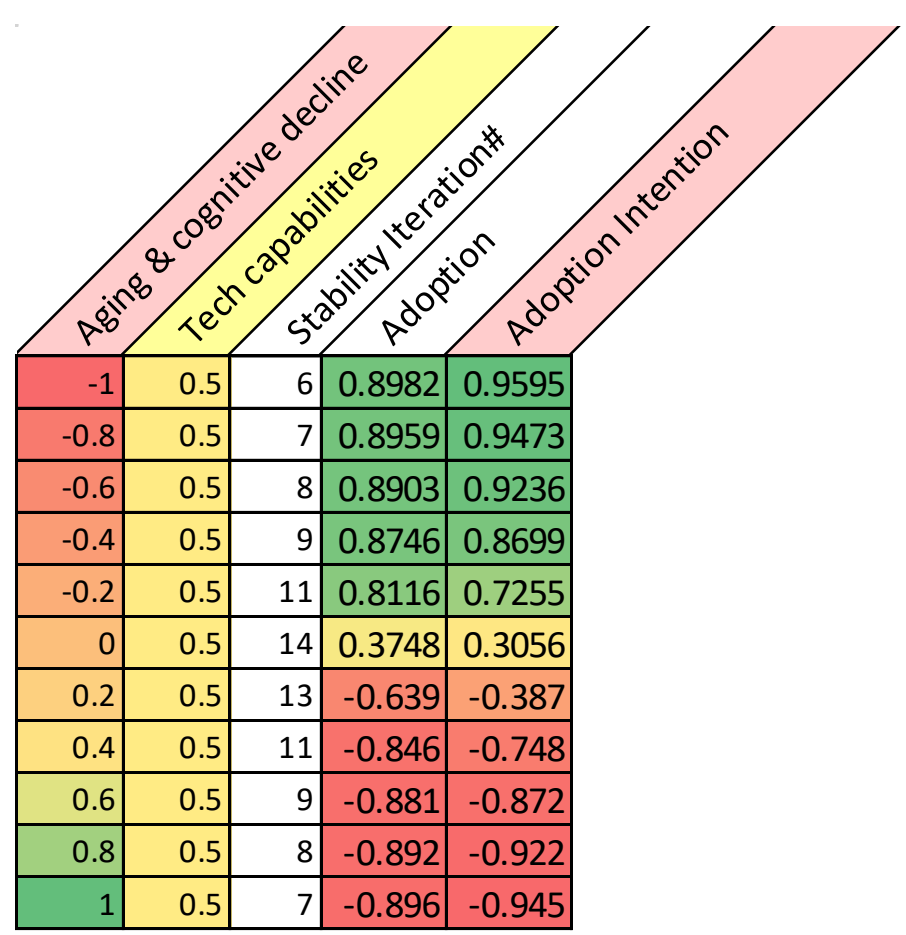

Table 6 - 10 Sensitivity Analysis of aging effect of FCM Isolated

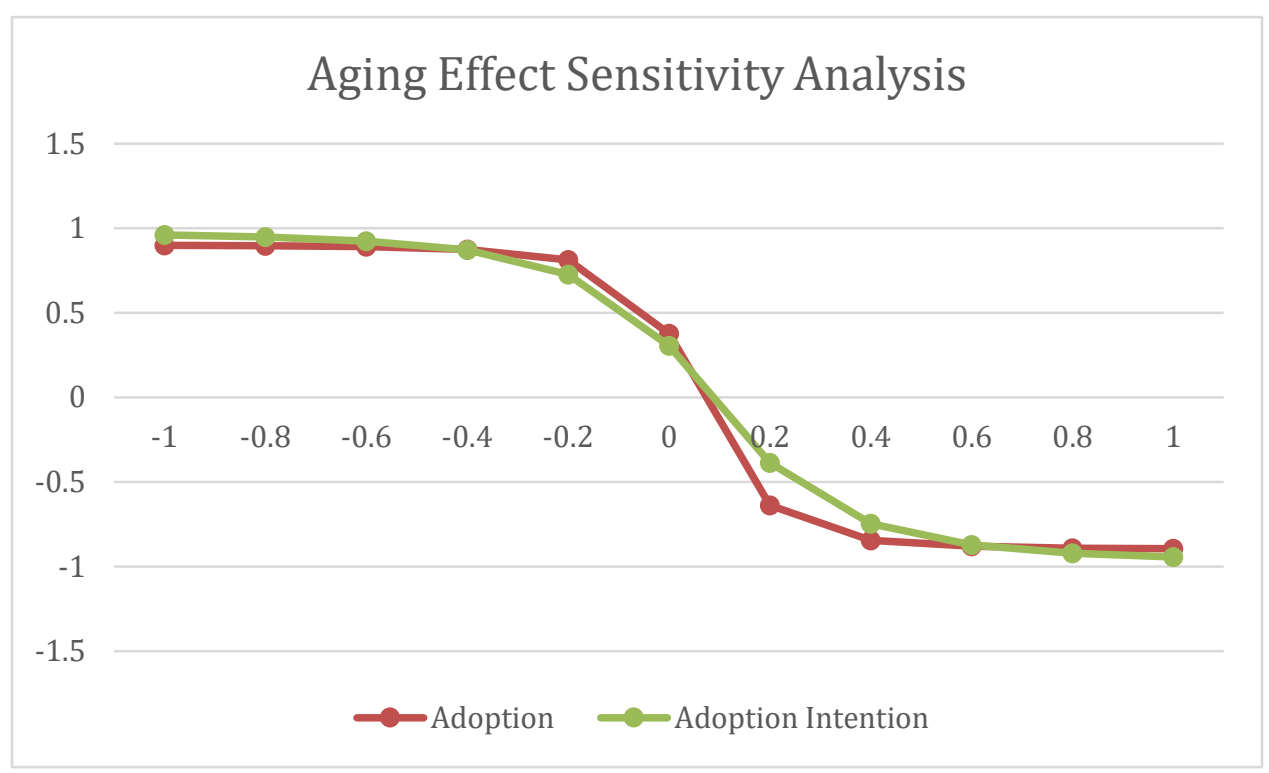

Figure 6 - 17 Sensitivity Analysis of aging effect of FCM Isolated 


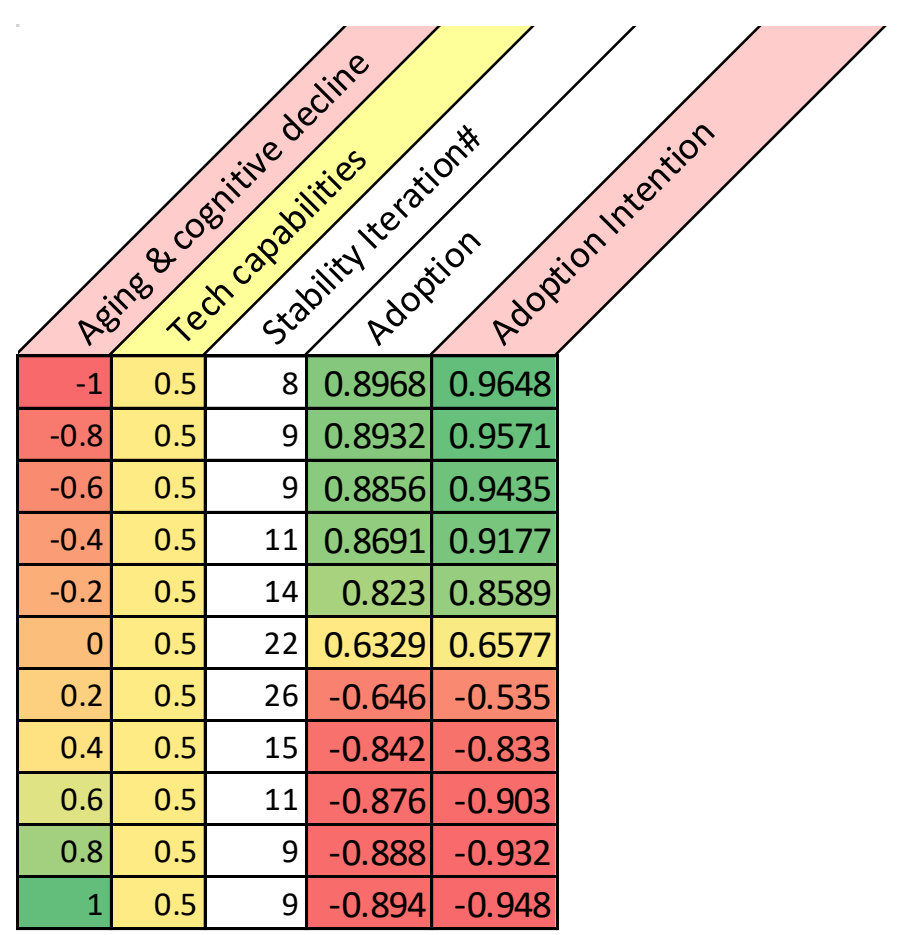

Table 6 - 11 Sensitivity Analysis of aging effect of FCM Connected

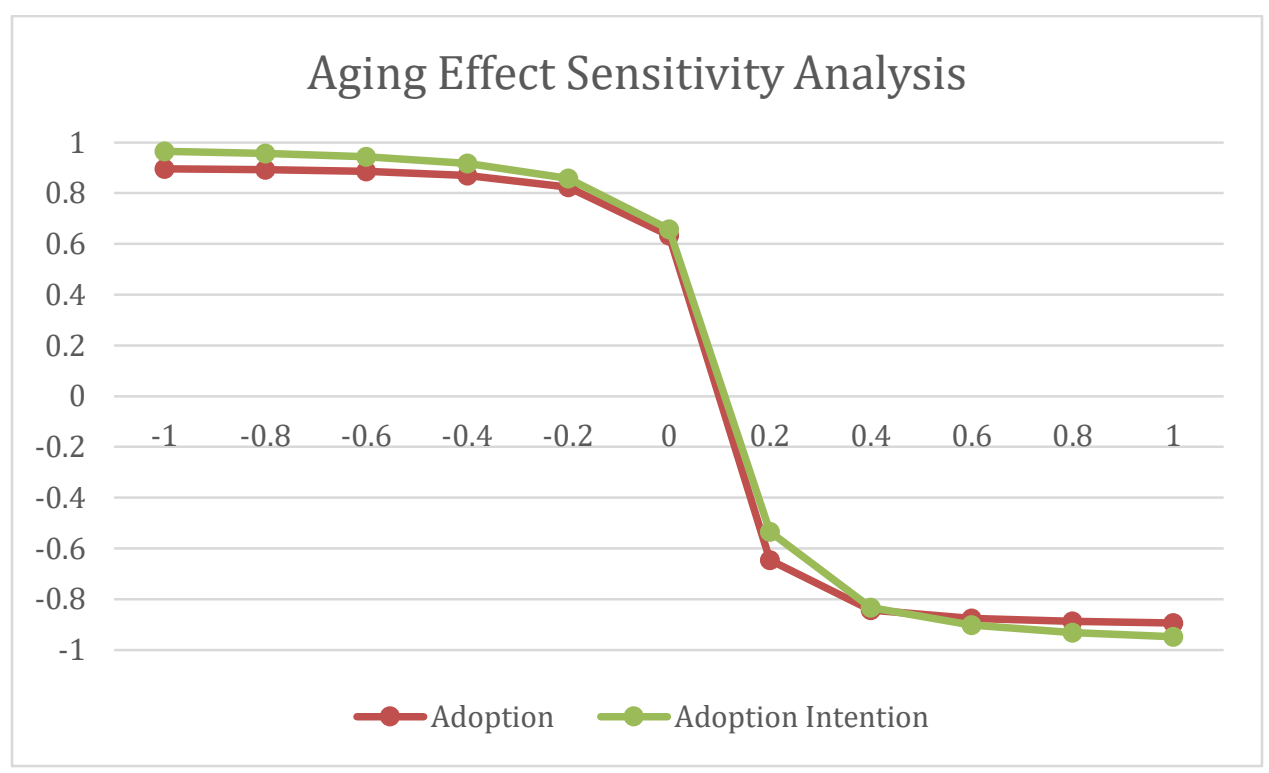

Figure 6 - 18 Sensitivity Analysis of aging effect of FCM Connected 
SA-2 Scenarios: How stable are the models' behaviors when social influence varies.

What is here referred to as social influence are the different forces that positively influence EWHTA outcome. For the two groups modeled in FCM Isolated and FCMconnected, these influences are coming from different environmental factors. In the case of isolated elderly women, these influences are coming mainly from the support channels. In the case of connected elderly women, these influences are exert by the EW's tech savvy social circle, and particularly her immediate family members. Subsequently, the simulations of each of FCMisolated and FCMconnected are conducted by capturing simulation results when these influences vary in the possible range of -1 to 1 .

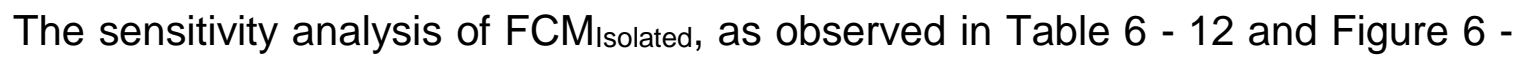
19 Support Influence Sensitivity Analysis of FCMIsolated the sensitivity analysis of the FCMconnected can depict the technology adoption challenge of isolated elderly women. The sharp contrast between the technology adoption rate of the two elderly group is evident in the graphs of this sensitivity analysis. Conversely, the simulation results of the FCMconnected social influence sensitivity analysis as shown in Table $6-13$. 


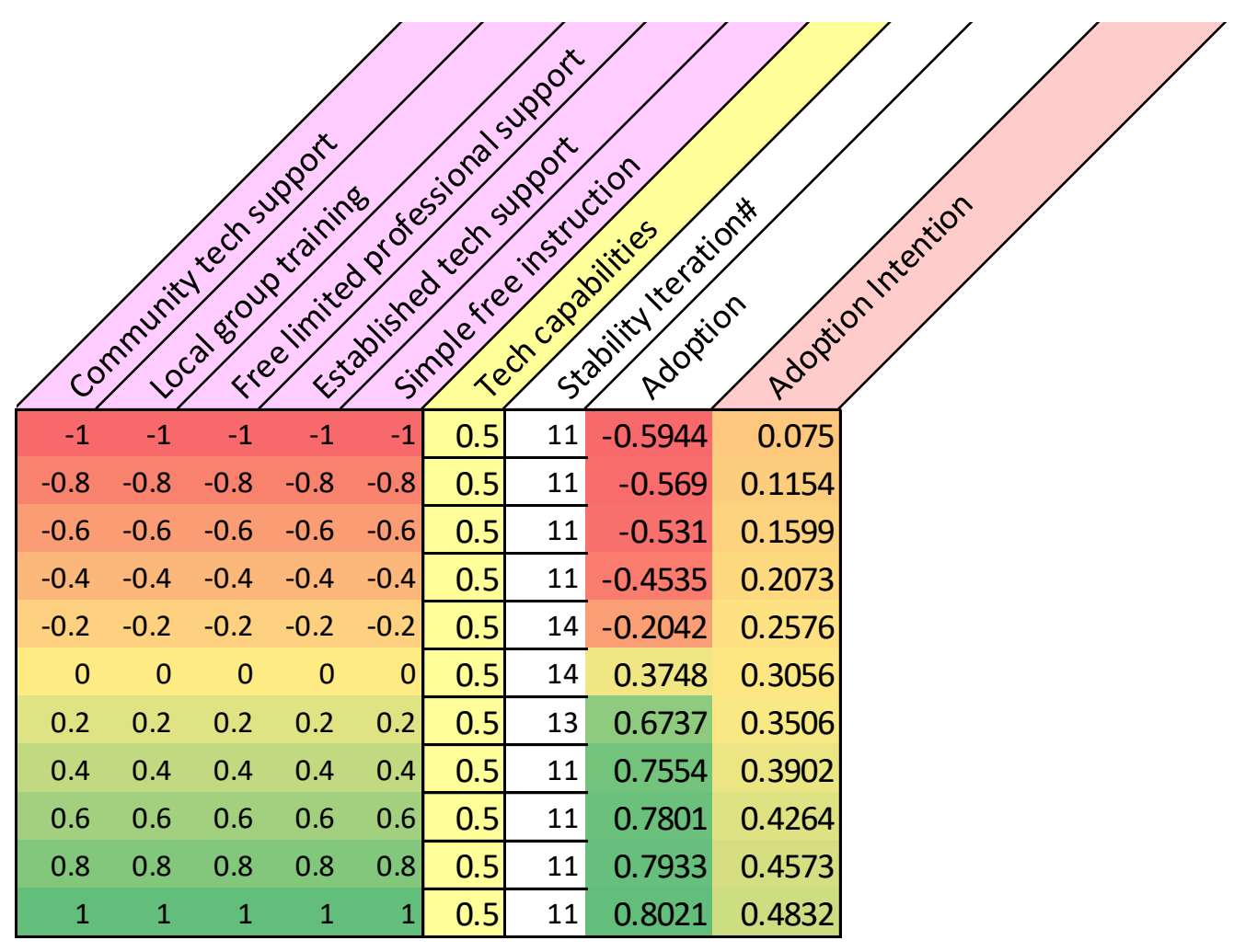

Table 6 - 12 Social Influence Sensitivity Analysis of FCMIsolated

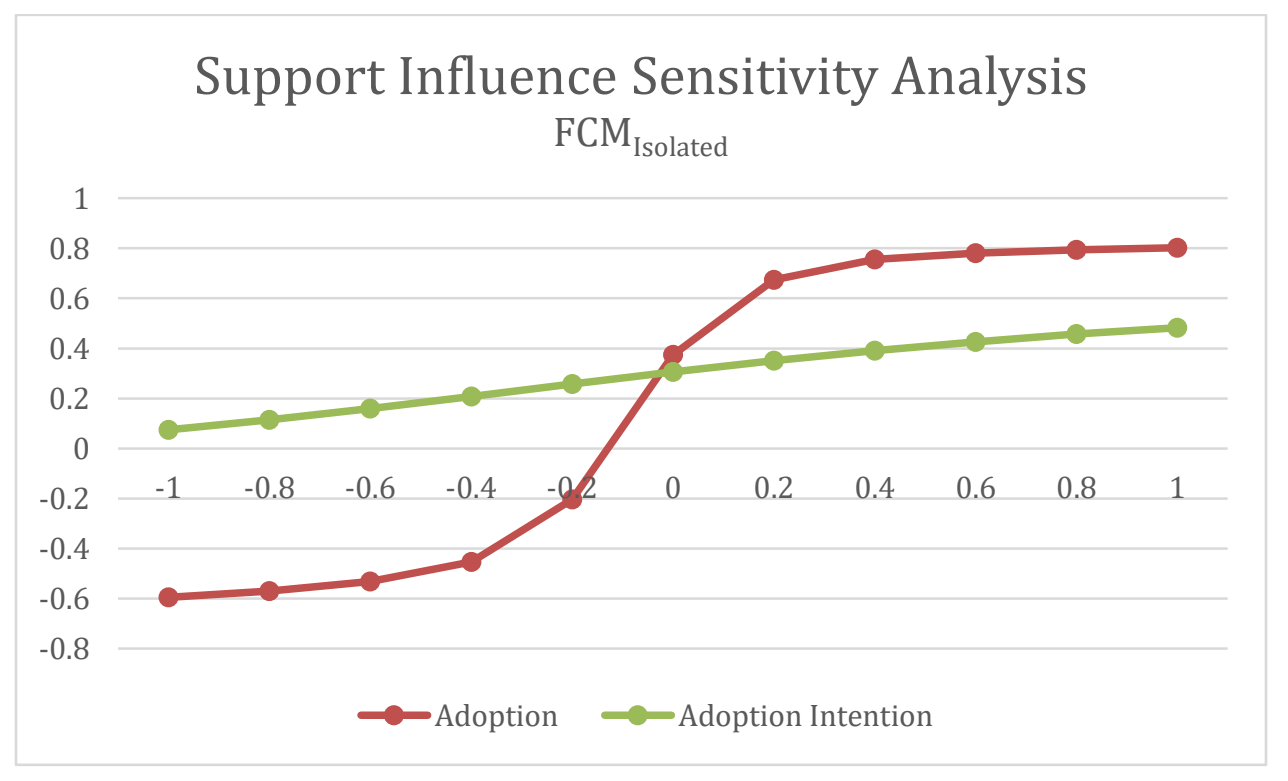

Figure 6 - 19 Support Influence Sensitivity Analysis of FCM Isolated $_{\text {Is }}$ 


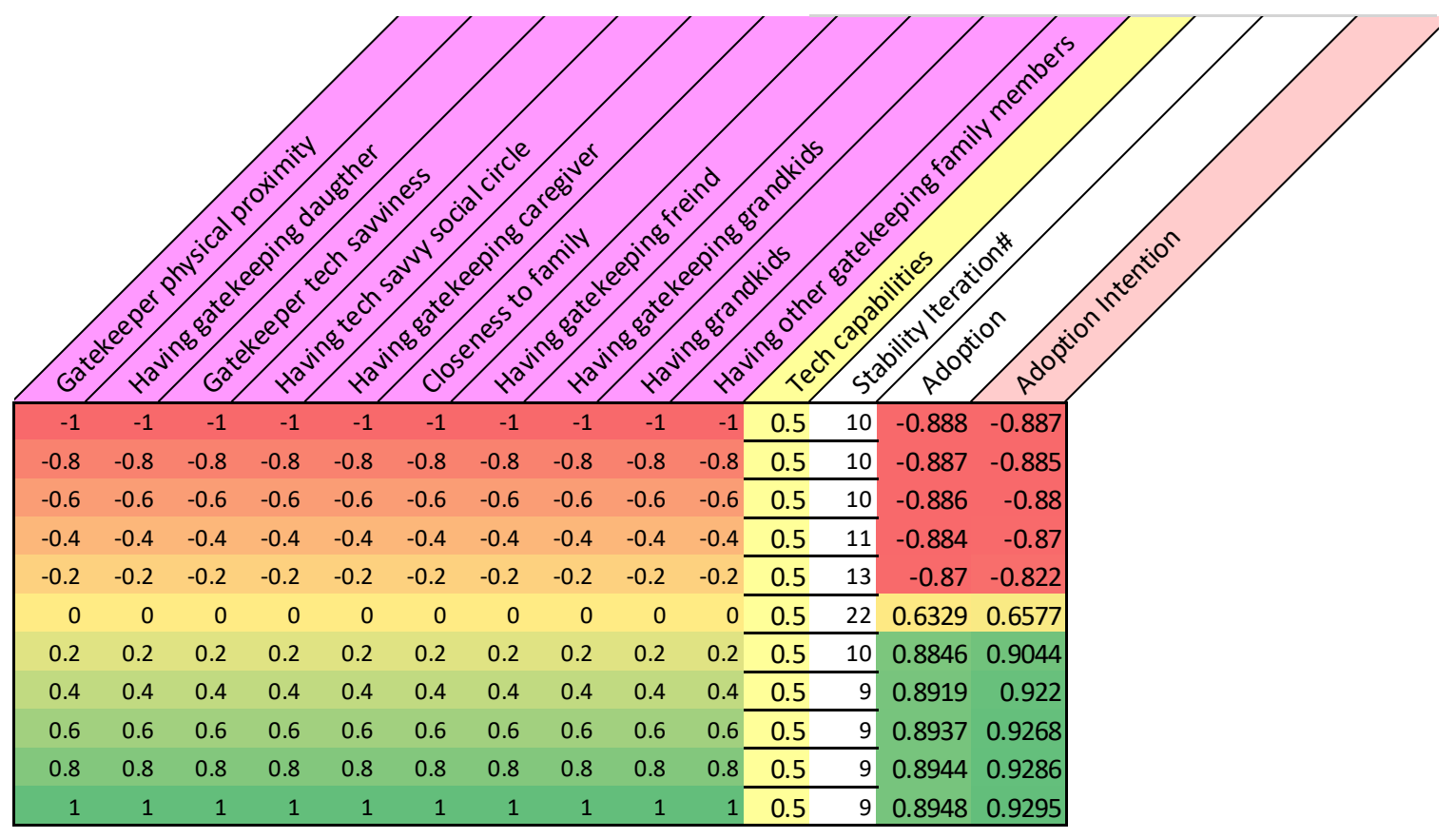

Table 6 - 13 Social Influence Sensitivity Analysis of FCMconnected

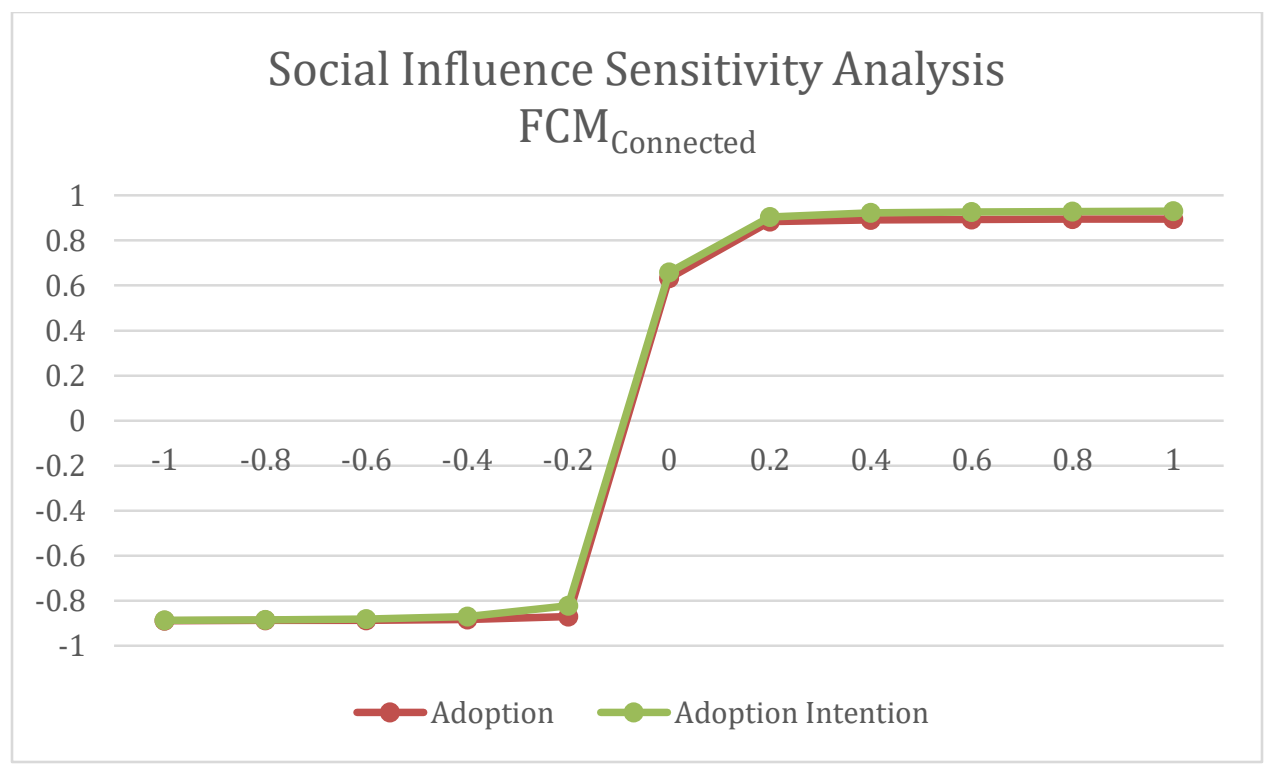

Figure 6 - 20 Social Influence Sensitivity Analysis of FCMconnected

Figure $6-21$ shows how sharply gatekeeping influences can increase the rate of the EWHTA, as both the adoption intention and adoption are clamped back to 
under 1 even for a moderate influence of 0.4 degree. The sharp sensitivity of the TA output concepts hints the colossal effect of gatekeeping influence.

SA-3 Scenarios: How stable are the models' behaviors when the level of experience changes.

Similar to the earlier set of scenarios, the variations in the model behaviors are captured for the varying level of experience in the possible range of -1 to 1 .

These sensitivity analysis scenarios simulations suggest that while even little experience can significantly increase TA among connected elderly women, isolated elderly women need a lot of technology experience to warrant TA. This is evident by comparing Figure 6 - 21 and Figure 6 - 22 simulations start to plateau near maximum from a little degree of experience. 


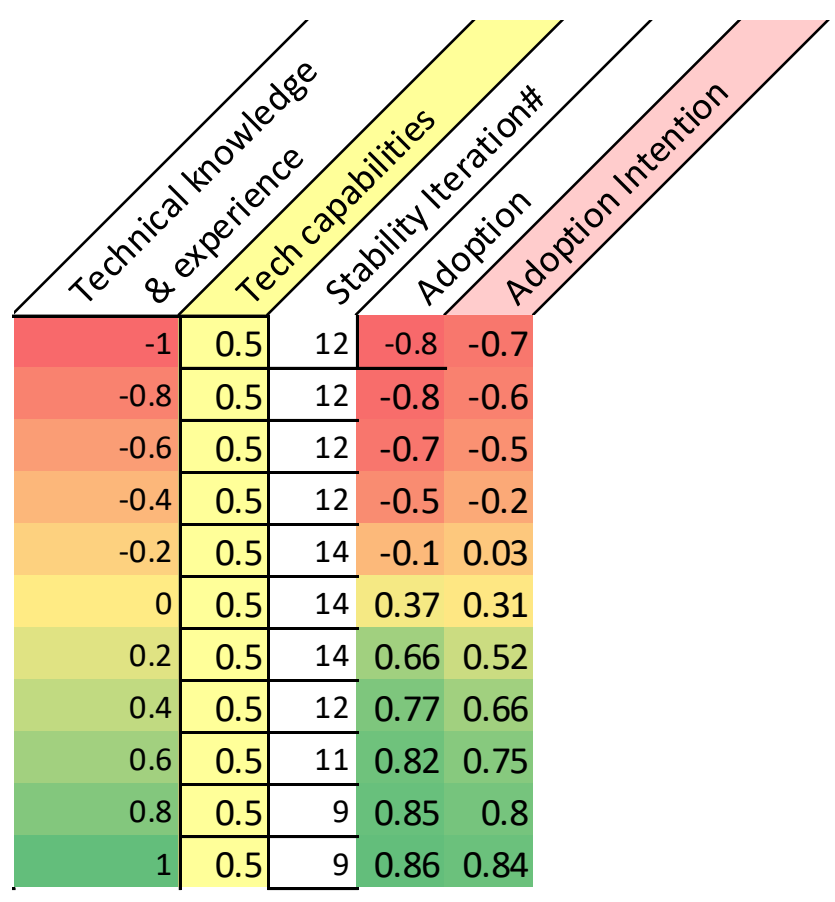

Table 6 - 14 Experience Sensitivity Analysis of FCM Isolated

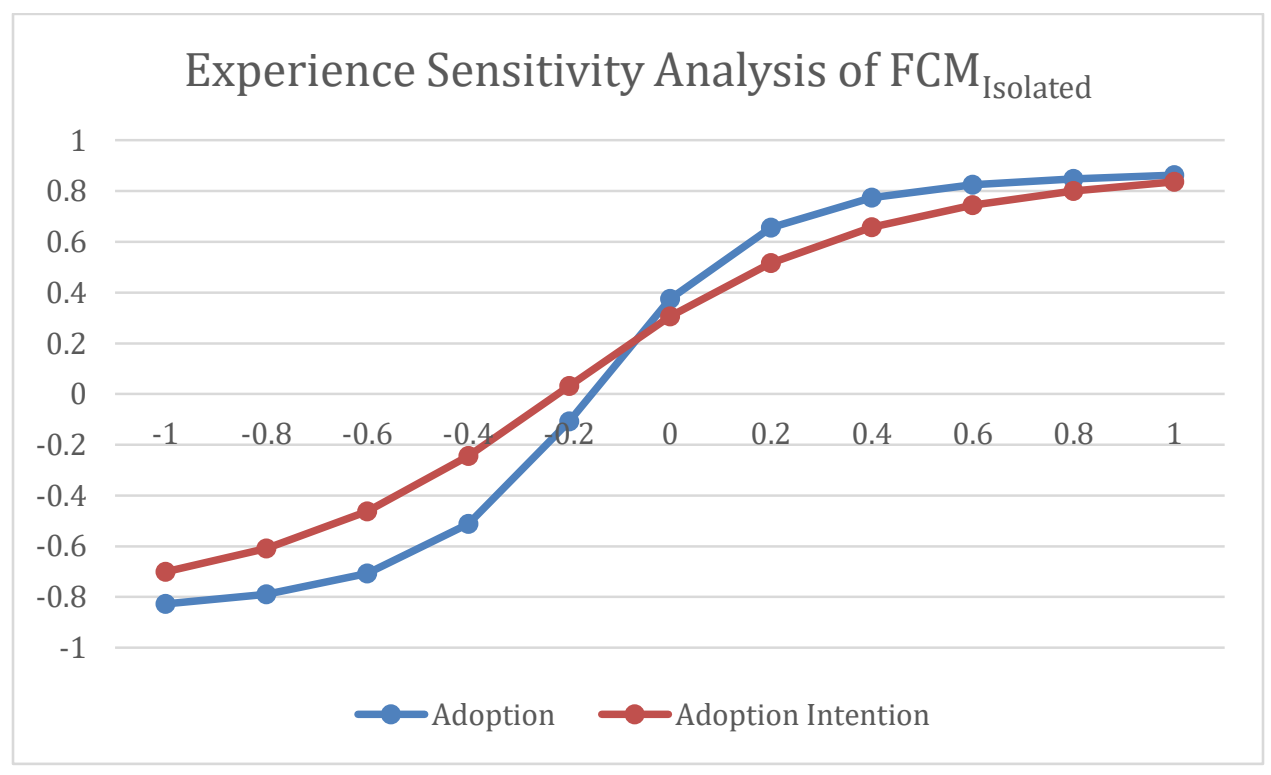

Figure 6 - 21 Experience Sensitivity Analysis of FCM Isolated 


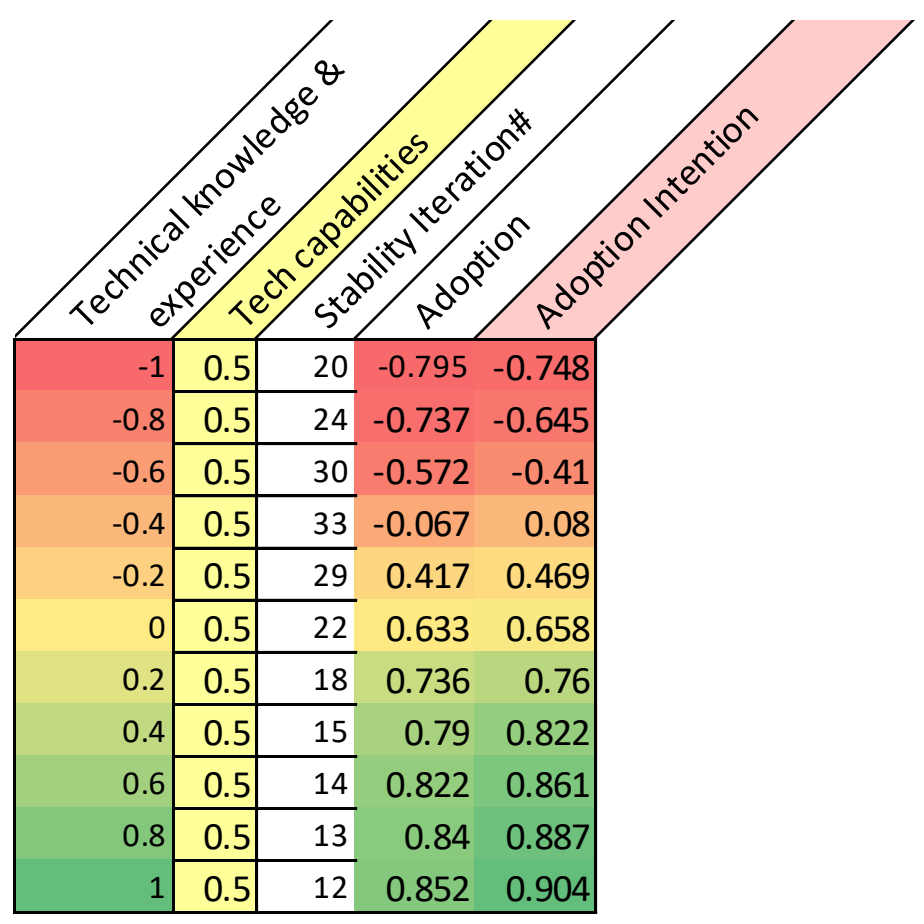

Table 6 - 15 Experience Sensitivity Analysis of FCMConnected

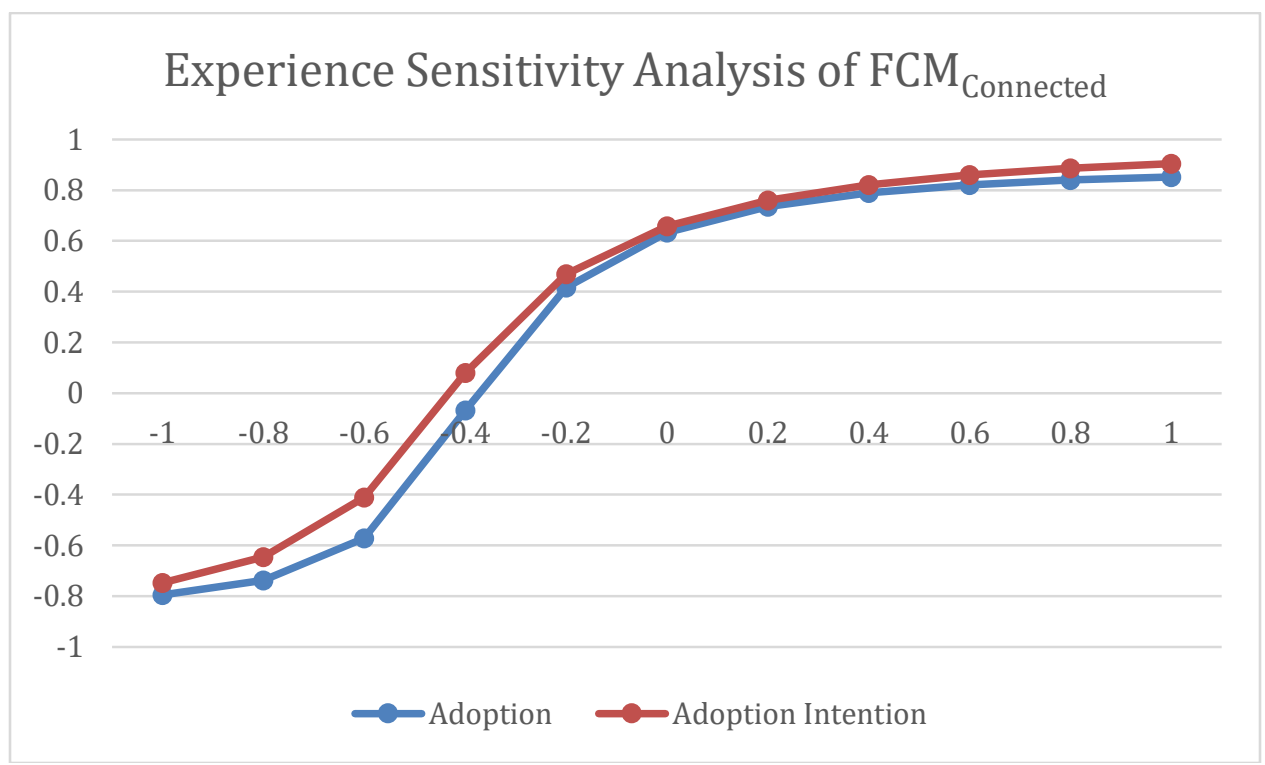

Figure 6 - 22 Experience Sensitivity Analysis of FCMConnected

SA-4 Scenarios: How stable are the models' behaviors when product features vary. 
Theoretically, the better quality and more suitable the product the more likely EW will adopt it. To do this, each FCM model should be simulated for a range of technology characteristics (concepts in yellow) which influence perceived usefulness and ease of use in the possible range of -1 to 1 to see the change in the behavior of the models. It's important to note that while some of the characteristics are the desirable and some undesirable, so when changing the valence of the numbers should be assigned accordingly. Tech automaticity, getting health information, Tech usability \& accessibility, Tech capabilities, Tracing activities, Tech simplicity \& familiarity, and Tech aesthetic appeal are all desirable concepts. Tech cost, Tech maintenance, Tech making E look old, and Lack of tech robustness are the undesirable concepts and when the desirable concepts are assigned high numbers, these concepts are assigned the same number with the opposite valence (as observed in the simulations start vectors on the left side of the stability iteration \# in Table 6 - 16 and Table 6 - 17).

The results of these set of scenarios depicted a similar trend to that of the earlier sensitivity analysis scenarios, in which start vectors simulating more desirable

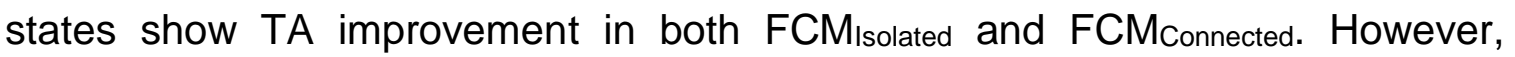

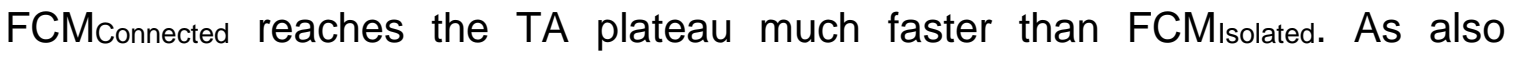
suggested in an earlier publication (Rahimi et al., In Press) and in line with the reality, little improvement in products make more significant TA improvement in the connected elderly women than in the isolated elderly women. 


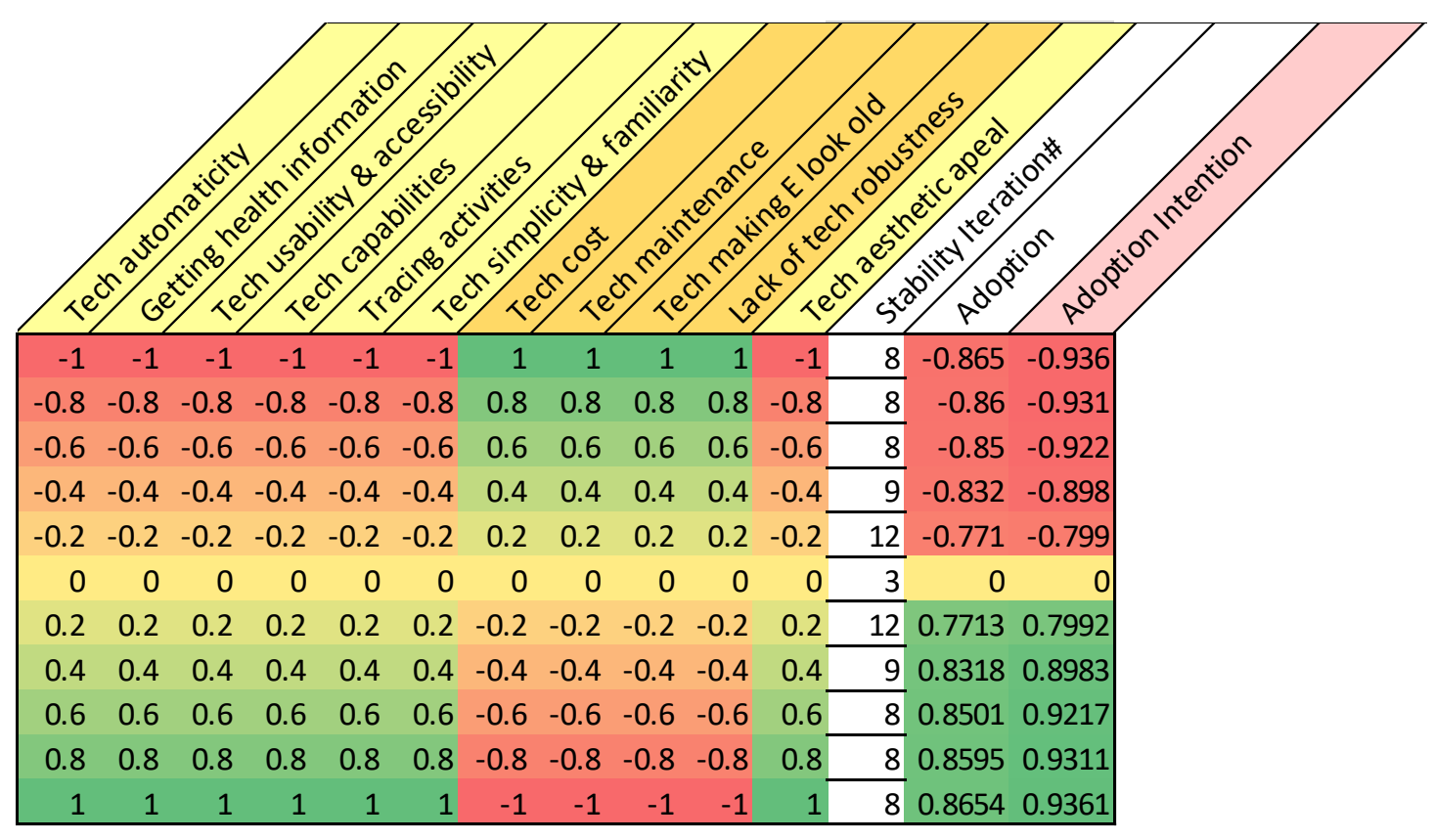

Table 6 - 16 Product Characteristics Sensitivity Analysis of FCM Isolated

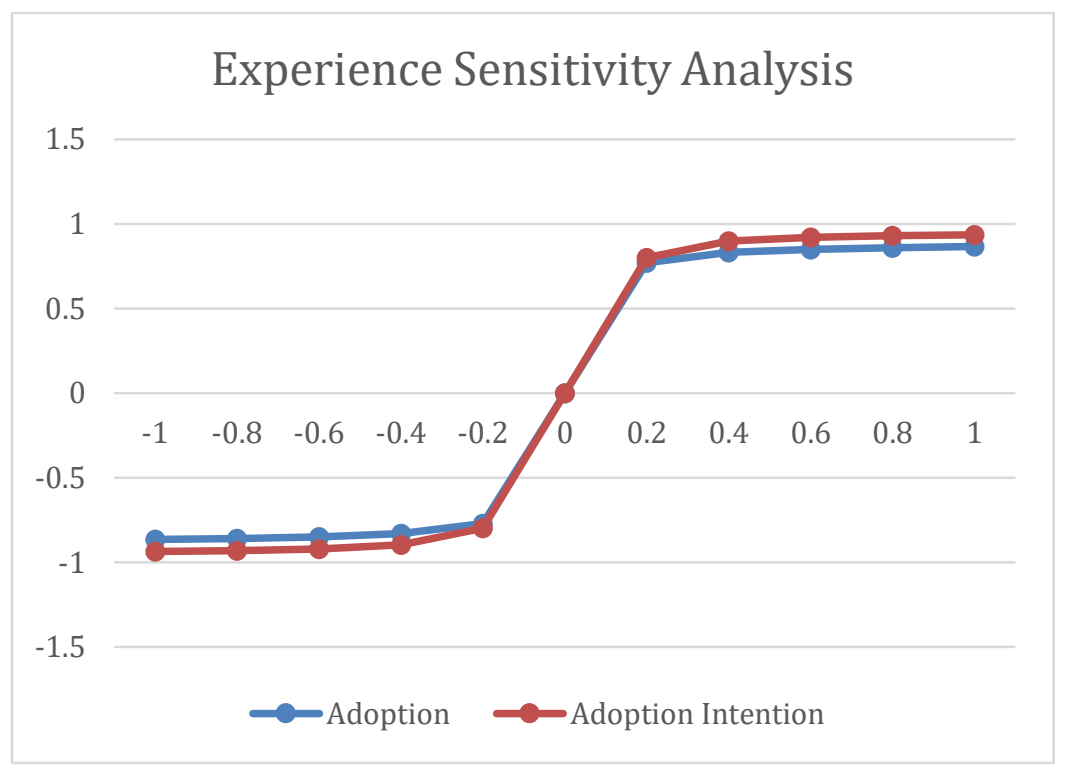

Figure 6 - 23 Product Characteristics Sensitivity Analysis of FCM Isolated 


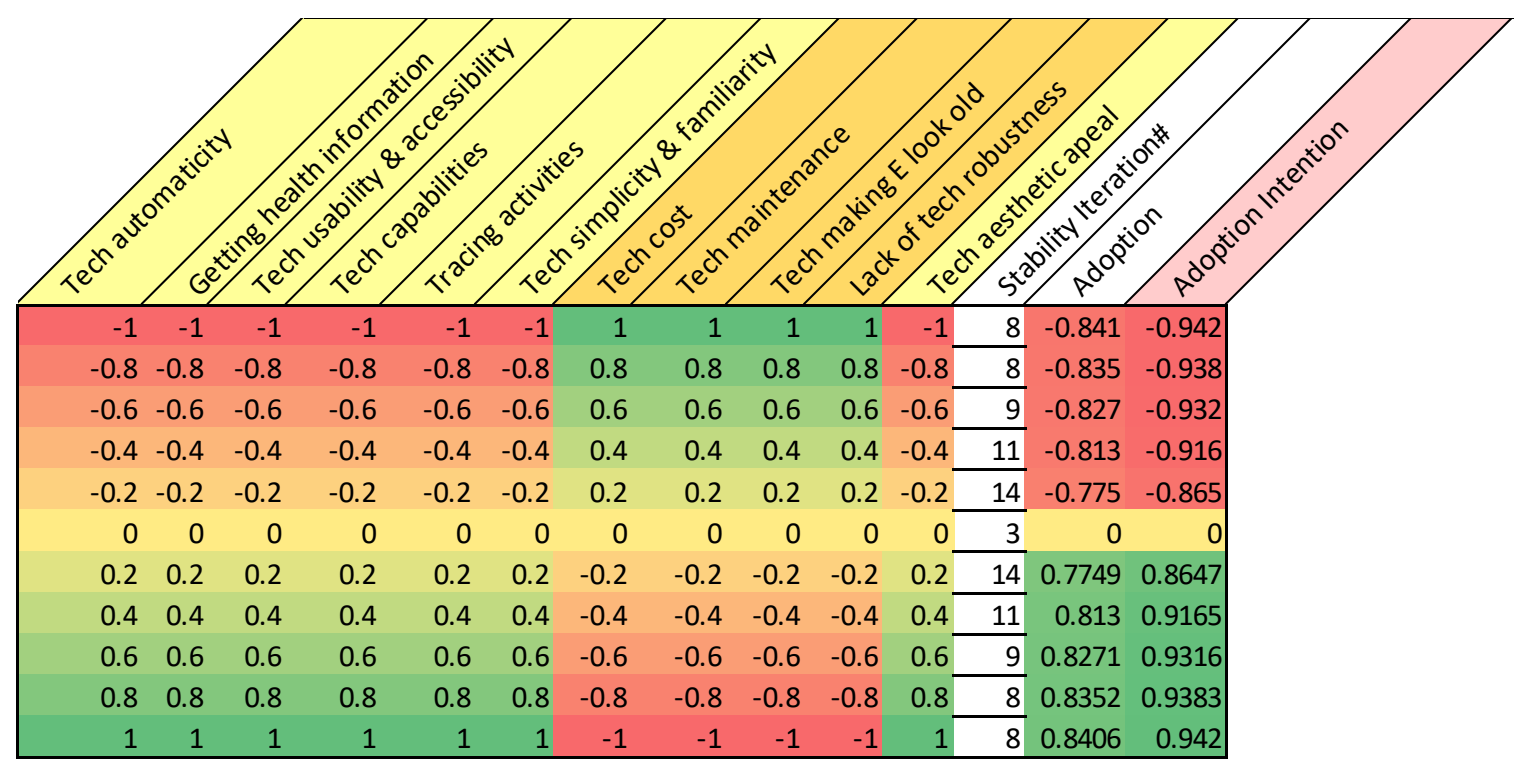

Table 6 - 17 Product Characteristics Sensitivity Analysis of FCMconnected

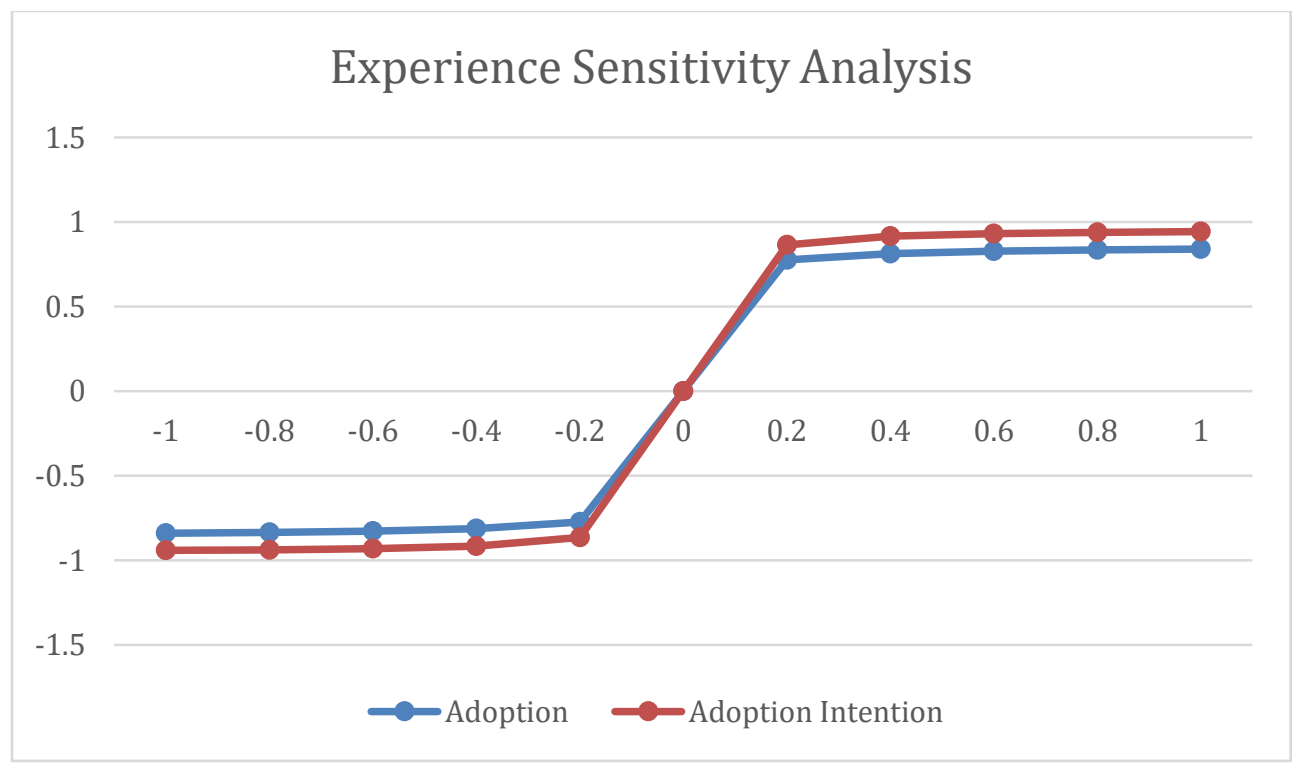

Figure 6 - 24 Product Characteristics Sensitivity Analysis of FCM Connected

The execution of the three levels of model validation criteria-extreme condition tests, behavior reproduction test, and sensitivity analysis conducted here, all depicted the alignment of their behavior with reality. Therefore it's concluded that 
the integrity of the two FCM models are validated and they can be successfully used for scenario planning to answer what-if questions and supporting decision making.

\subsection{Method Evaluation}

\subsubsection{Method Evaluation Summary}

The case study, as detailed in the first four earlier sections of this chapter (Sections $6.1,6.2,6.3,6.4)$, presented a successful implementation of the proposed mixedmethod research method. The integrity of the emerged FCM models were successfully evaluated by simulation the models for extreme conditions, expected behavior, and sensitivity analysis. As such the FCM models are ready to be used as simultion models.

This dissertation takes an additional step beyond the proposed research method. In this dissertation, subject matter experts are asked to comment on the overall research method and the usefulness of the FCM model. This enhances the validity of the method, as the model will be judged by the actual practitioners in the field of EHTA.

This dissertation aims to respond to the call to arms to overcome method scarcity in the field of EHTA by creating and applying an empirical research method. As

detailed in chapter 2, the need for such empirical research methods is rooted in inability of abstract technology adoption theories to provide actionable insight. Hence, the claim of this dissertation to have contributed to plurality of research methods that can conceptualize general technology adoption theories, in order to 
provide detailed understanding of the factors involved in EHTA, need to be assessed.

In the following sections of this chapter, the proposed method is evaluated based on the criteria that emerged from the extensive literature review summarized in chapter 2 (specifically section 2.6-Summary of the State of the Art).

The careful hand selection of the different techniques used in the proposed method was a result of investigating best tools that can fulfill the requirement for successful research methods. The following sections discuss some of these characteristics that fulfilled the highlighted gaps that serve as criteria for effective research method.

The proposed method is evaluated based on the criteria that are displayed in Table 6 - 18. The columns in this table denote the requirements for a useful, empirically grounded model, as determined by the review of the literature in section 2.7. Henceforth in this section, each requirement is underlined when mentioned. The rows are the steps taken to ensure that the proposed model facilitates the delivery of these requirements. These steps consist of overall methodological design approaches, method technique choices and the SME judgements. The checkmarks indicate that a particular attribute of this method has satisfied a specified requirement. 


\begin{tabular}{|c|c|c|c|c|c|c|c|c|}
\hline Method Evaluation & $\begin{array}{l}\text { D } \\
\frac{1}{0} \\
\frac{1}{7} \\
0 \\
\frac{0}{0}\end{array}$ & 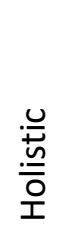 & $\frac{\frac{0}{0}}{\frac{\pi}{x}}$ & 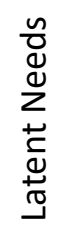 & $\begin{array}{l}\bar{\pi} \\
\overline{5} \\
\stackrel{5}{5}\end{array}$ & 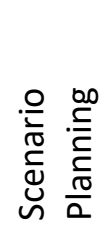 & 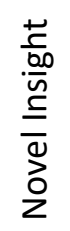 & 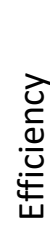 \\
\hline \multicolumn{9}{|l|}{ Method design } \\
\hline Theory first approach & & & & & & & $\sqrt{ }$ & $\sqrt{ }$ \\
\hline $\begin{array}{l}\text { Including gatekeeper's } \\
\text { perspective }\end{array}$ & $\sqrt{ }$ & $\sqrt{ }$ & & $\sqrt{ }$ & & & & \\
\hline \multicolumn{9}{|l|}{ Methodological choice } \\
\hline Fuzzy Cognitive Mapping & & & $\sqrt{ }$ & & $\sqrt{ }$ & & & \\
\hline Ethnographic Interview & $\sqrt{ }$ & & & & & & & \\
\hline Mixed-method approach & & $\sqrt{ }$ & & $\sqrt{ }$ & & & & \\
\hline FCM Modeling \& Simulation & & & & & & $\sqrt{ }$ & & \\
\hline SME judgement & & $\sqrt{ }$ & $\sqrt{ }$ & $\sqrt{ }$ & $\sqrt{ }$ & $\sqrt{ }$ & $\sqrt{ }$ & $\sqrt{ }$ \\
\hline
\end{tabular}

Table 6 - 18 Proposed model meets evaluation criteria

\subsubsection{Methodological Design}

Including gatekeeper data collection to the research: Including the insights from both the elderly women and their gatekeepers in the qualitative phase of the research facilitates emergence of learning that is empirically grounded and improves data triangulation. This practice also enhances the method's capacity to identify latent needs and find holistic data that emerges from integrating the perspective of the elderly woman with that of her gatekeeper. For example, elderly's desire to connect with loved ones through the use of wearables referred to a latent needs that would not have been discovered, if the gatekeeper had not participated in the research. 
Theory first approach: As per earlier investigations (Rahimi \& Jetter 2015; Rahimi et al., 2016), UTAUT proved to be the most appropriate theoretical foundation for contextualizing EHTA. Therefore, it was selected as the baseline that the proposed method would extend. This deductive approach of the research method enabled quick contextualization and expansion of the known main constructs proven to be critical in technology adoption. This approach leveraged the efficiency of the research method by finding the salient novel insights without the need to search for the main constructs that pertain to the adoption. This is particularly important for this proposed method, which is intended to become a practical consumer market research method that can be used by essentially any researcher interested in the EHTA industry. These researchers may not necessarily be aware of the extant theories. Thus, having theory-first-based research method at their disposal would increase the efficiency of their efforts at building consumer market research models.

\subsubsection{Method Choices}

Fuzzy Cognitive Mapping, Modeling and Simulation: These criteria are well aligned with the inherent modeling and simulation-ready characteristics of Fuzzy Cognitive Mapping. Fuzzy cognitive mapping yields directed graphs, enhance the modeler's ability to visualize key concepts in EHTA. This is readily apparent in the case study in chapter 6, which yielded to two integrated FCM models depicted in

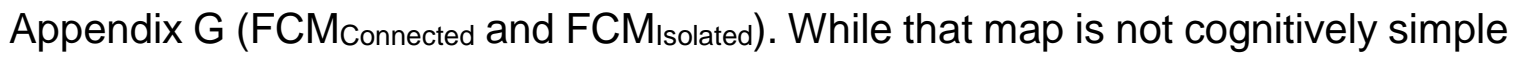
to view in its entirety, the filtering tools provided by the presentation software can 
highlight a subset of the map to show one segment at a time. This capability can be seen in some of the figures in section 6.2 (e.g. Figure 6 - 11), as it has been used in the QUAL analysis section.

Fuzzy Cognitive Mapping has also been proven to provide a flexible framework for capturing knowledge (Liu, 2003). A concept or a causal link that emerges in later stages of analysis can be added to a well-established model without complications. It can also be tested easily by using simulations. Furthermore, the simulation capability of FCM is best suited for scenario planning, which helps with addressing what-if scenarios (see chapter 7).

Mixed-method approach: The proposed research method deploys a mixedmethods approach, which takes advantage of the benefits provided by qualitative and quantitative research methods. The qualitative research phase (QUAL) was designed to best extract grounded and holistic information without preconceived assumptions of key concepts. The qualitative research method's capacity to extract grounded insights was the reason it was selected as the dominant component of the proposed research method. During the subsequent quantitative research phase (quan) quantified weights were assigned to the causal links that had emerged during the earlier qualitative research. This facilitated the creation of the quantified part of the FCM, which allows for simulation and scenario planning.

Ethnographic Data Collection Method: As mentioned in chapter 2, there were numerous complaints raised in the EHTA literature about a lack of understanding of how the elderly perceive health technology and how they decide on whether to 
reject or adopt. The ability of a modeling approach to integrate holistic, first hand user data was highlighted as an important requirement to address the lack of information in EHTA. This requirement became the compelling reason for selecting ethnographic interviews in the repertory-grid techniques that were used for the qualitative phase of the mixed-method. Additionally, some concepts that were not the subject of the enquiry, emerged from ethnographic data collection method. Some of these concepts ultimately pointed out some of the elderly women's latent needs. These subtle references would not have surfaced, were it not for the free data inquiry that characterizes this data collection technique and the deep analysis nature of qualitative research. This resulted in the emergence of information that would not have occurred to the participants. For example, the emergence of the concept "Tech making elderly look old" pointed to a latent needs that would not have emerged without conducting an ethnographic interview.

\subsubsection{SME Judgement}

To enhance the credibility of the proposed method evaluation and the empirical value of the resulting FCM decision support model, the informal judgment of three subject matter expert in the field of EHTA was sought. From the feedback received, the research obtained a sanity check and a set of suggested scenarios for the model to test and discuss the expected behavior suggested by the model.

This additional informal inquiry served as an unbiased judgment of the practical usefulness of the proposed research method and the model that resulted from the EWHTA case study. It also substantiated the value of the method as a consumer 
market research method, and in doing so enhanced the credibility of my dissertation.

The three subject matter experts (SMEs) who were interviewed had an academic background and industry experience in the intersecting fields of EHTAgerontology, health technology product development and adoption. This ensured the best judgement regarding the method and the model's practical usefulness. The first SME was the director of one of the most credible elderly health technology research institution in United States. He has also worked as a practitioner in the field of EHTA over the past $20+$ years. In that role, he routinely evaluates RHMT products and makes decisions pertaining to their rollout. The second SME, a professor of neurology in OHSU School of Medicine, is an active researcher and practitioner who has also been working closely with different RHMT products in the field studies with elderly. The third SME is a PSU professor and a practitioner in the field of elderly health product development. As the cofounder of an innovative RHMT development company, he determines the characteristics and features of that company's next generation health technology products.

In each interview, the subject matter expert was walked through the model to illustrate the important motivators and inhibitors that emerged from this research: 1) the factors that influence an elderly woman's perception of a wearable device's usefulness and ease of use; 2) how her social surroundings influence these perceptions, her intention to adopt, and her actual usage behavior; 3) how her previous experience and personal traits play into her decision making. The model really interested the experts; they judged the model to be useful in their line of 239 
business and identified that this would be a good "one stop shop" for understanding the elderly woman's world view. They mentioned that the insights gained can support better decision making when it comes to: 1) planning the next wearable product; 2) deciding on purchasing the most suitable wearable that has the best chance of adoption among a group of elderly; 3) designing the best marketing strategy to target people with elderly parents, and 4) developing the best marketing strategies for creating advertisements that emphasize the influence categories captured in the model. Additionally, the experts pointed out few "what-if" questions that were used as scenarios in subsequent simulations. These applications of the model are detailed in Chapter 7.

Furthermore, the SMEs were asked to comment on the proposed method and whether it meets the criteria demanded by the literature. Is it holistic and flexible? Does it have the ability to capture latent needs? Does it generate visual representations? Does it allow for scenario planning? Does it have the capacity to provide novel insights? Is it efficient?

Two of the three SMEs were aware of UTAUT. All three mentioned that they lack any systematic approach for conducting consumer market research for the elderly. The experts posited that they mainly rely on their experience and usually expect a technology to be adopted if it is not obtrusive, if it has low cost, and if the targeted consumers exhibit a high self-efficacy. The technology role out manager mentioned that their organization rates products with pluses and minuses according to the above categories for consideration. The organization also identified other drivers such as no interference, no cost to their consumer, no 240 
switching costs, no new way of doing it, and no learning requirement. The first SME wondered about the generalizability of the model across gender. In addition, he was interested in the model's behavior for different hyper-segmented markets (e.g. athletic elderly vs. those who care about the technologies' aesthetic appeal). The second SME also mentioned that, in her current practice, she considers the main constructs of UTAUT, but no contextualized information or systematic methodology for planning is used. She mentioned that a model like this could be helpful. While the model in its entirety was hard to comprehend, the software filtering feature facilitated a simple visual representation of elderly's mental model. She particularly liked the capability of honing in on a concept and seeing how it is influenced by and influences other concepts. During the walkthrough, she agreed with the accuracy of the relationships highlighted in the model, which aligned with her expertise as a gerontologist.

The SME, additionally, stated that the learning from conducting this method and the resulting model make it possible for decision makers without a prior academic background to gain insights into effective marketing approaches and strategies. For example, promotion of such products among middle-aged people (gatekeepers) with elderly parents could be an effective advertisement. Additionally, the concepts in the blue category (in the model depicted in Figure 6 13), which are the different gatekeeping influences, can show the important themes upon which the advertisement could focus. The second SME stated that the model not only shows to whom one should market wearables; it also shows us how to market them. 
The third SME with the expertise in HFE also expressed interest in the model and in knowing factors that help EHTA. His practice is particularly interested in learning how different features of technology should be prioritized based on the elderly's perception and preference. When I was using the filtering feature of Mental Modeler to show him each section of the model, he often stopped me and asked about the concepts and relationships. He mentioned that the insights were fascinating and very helpful when it comes to better understanding his consumers, their preferences and their needs. He liked the detailed information provided by the model. For example, when he saw the concept "Tech making E look old", he mentioned that their wearable has a problem with that. He said that they need to fix their wearable's look, and wish he had considered this concept before. He expressed concern that new health product developments, particularly in smaller innovative companies like his, are done by engineers who have no idea about these intricacies. He also mentioned that models like the one produced by the case study could be very helpful in helping them understand the elderly needs and preferences. He jokingly asked where we were last year when the company was debating the features of their wearable. He also stated that, going forward, for small companies that innovate wearables, computer-based models like this are efficient tools that are instrumental to their decision making.

In a nutshell, the SME judgement of both the proposed EHTA method and the case study EWHTA model was a high passing grade. They liked both the theoretical insight that the model provided and its capability to simulate what-if scenarios to obtain actionable insights. The model presented them a novel insight about the 242 
fact that those theoretical constructs are interdependent and how elderly's social life and the environment influence their technology adoption. In general, they judged both the method and the model to be useful for the study of elderly health technology adoption. 


\section{Application of the Simulation Model}

The two FCM models were successfully validated in chapter 6 and formed the decision support models, as depicted here in Figure $7-1$ and Figure $7-2$.

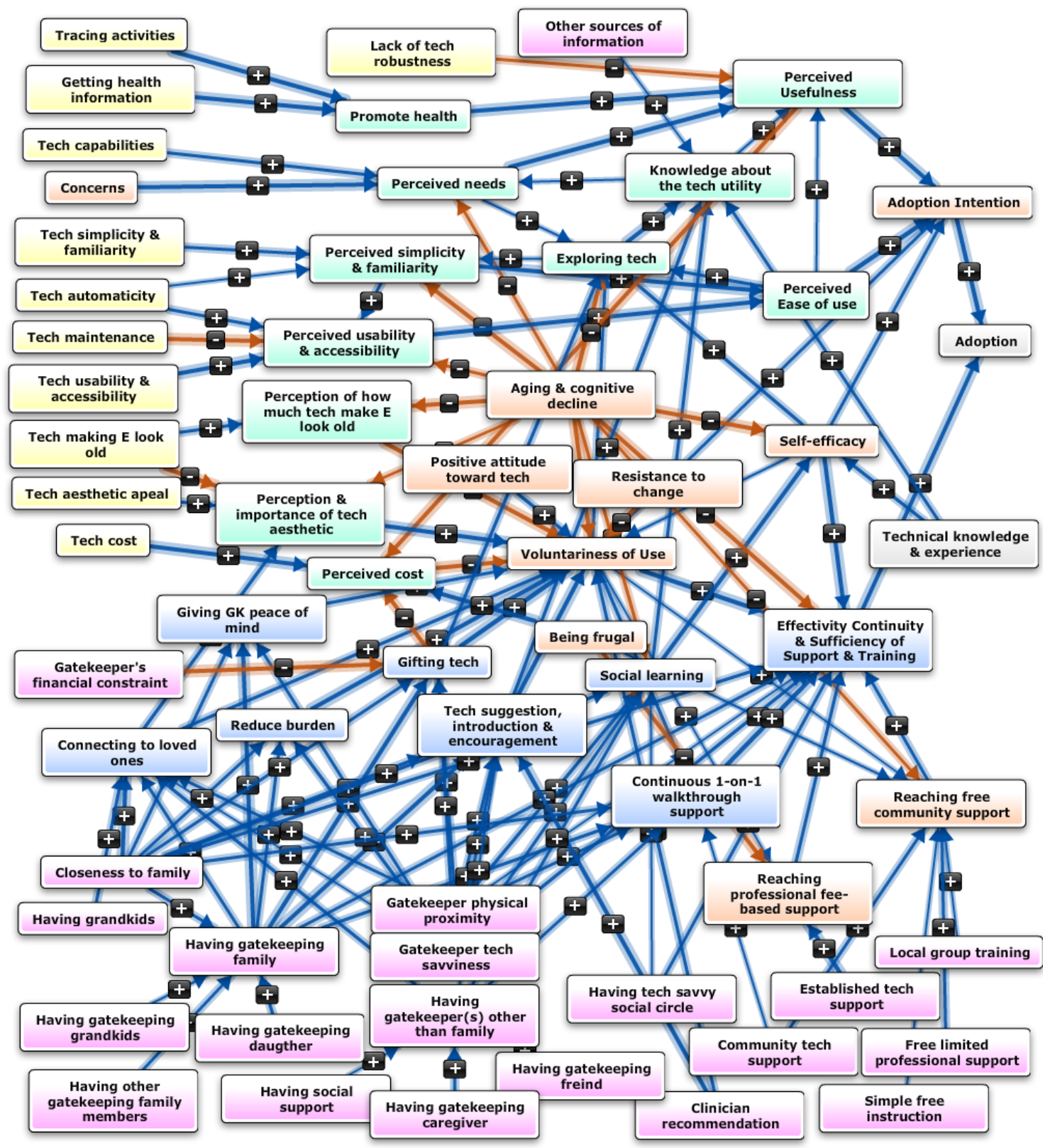

Figure 7 - 1 FCM Connected model 


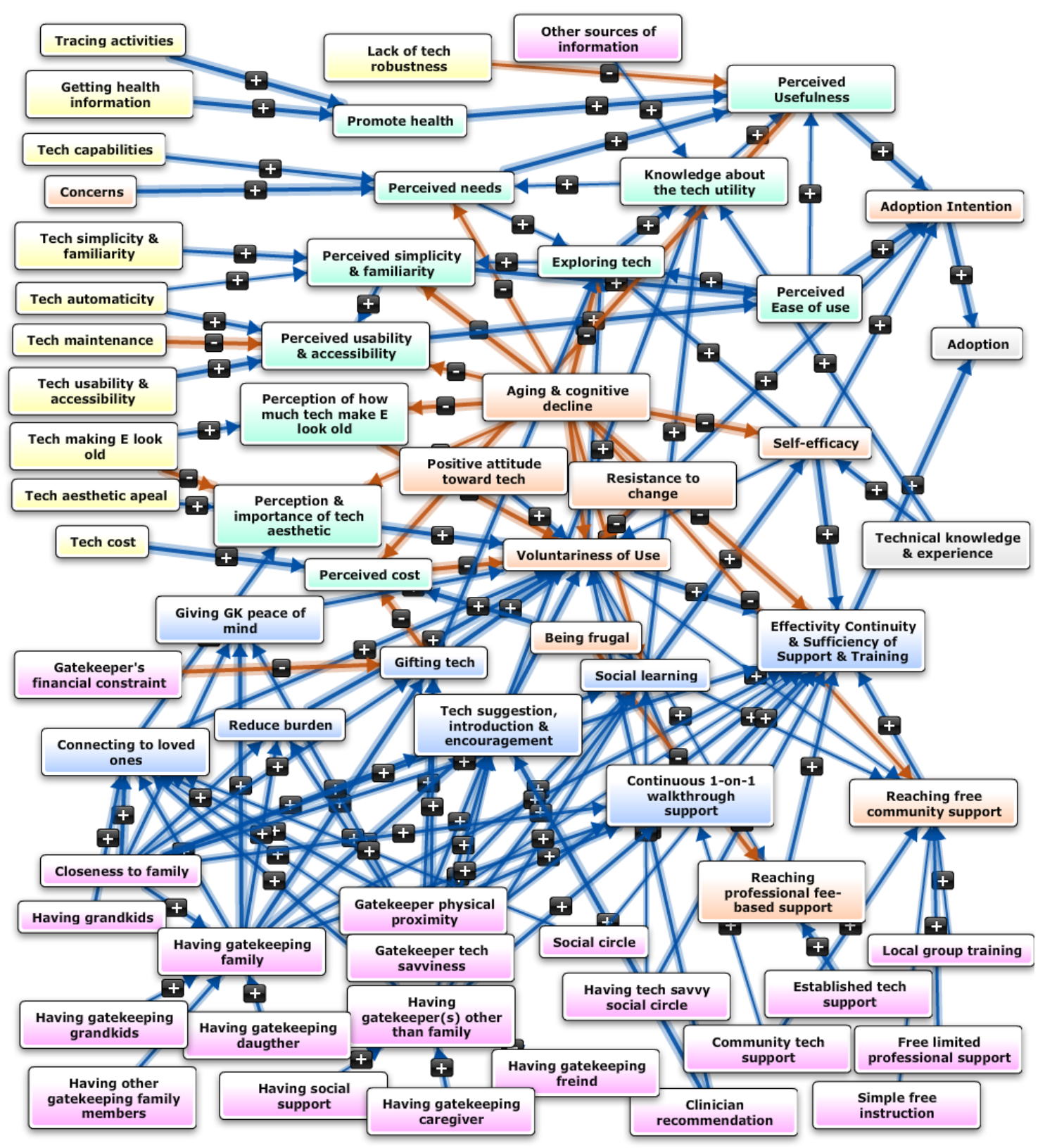

Figure 7 - 2 FCM Isolated model

These models show that technology adoption is impacted by various factors such as product characteristics (concepts in yellow), different facilitating conditions (concepts in pink), the elderly women's personal experience (in white) and the 
elderly women's personal traits (concepts in peach color). Some of these factors like product features, support and training channels can be controlled and determined by decision makers at the companies that develop the products. However, some salient factors, namely social support by family members and other gatekeepers, are largely beyond the control of product companies. For example, can a good technology product make up for unfavorable social support, i.e., can we expect technology adoption by a growing number of isolated elderly? And, can gatekeeper support make up for the quirks and drawbacks of emergent RHMT wearable, which is not yet fully mature?

The two FCM models can be used to explore these questions by simulating scenarios in consideration of different combinations of feature sets and social support. In alignment with scenario planning conducted in an earlier publication (Rahimi et al., 2018), the following ten scenarios were simulated. As detailed in sections 3.1.4, all simulations run with a hyperbolic tangent squashing function (Papageorgiou \& Salmeron, 2014) and clamping of concepts (Kosko, 1986); GK stands for gatekeeper. The results are reported in Table 7- 1 and Table 7- 2.

In the first four scenarios, it's assumed that the technology is an average ("good enough") product that was usable but had some flaws, while varying the social influences. The product was configured as follows:

- Medium operating range (i.e. works in the house but not at the far end of the yard). This is identified by setting "Tracing activity" to 0 (This concept was set to 0.5 for scenarios with better product in scenarios 5 to 10 ). 
- works relatively reliably. This is identified by setting "Lack of Robustness" to 0.5 in scenarios 1 through 4 . This concept was set to 0.25 in scenarios 5 through 10 , which represented a more robust product than the one in scenarios 1 through 4.

- has features that promote healthy behavior (e.g. step counter). This is identified by setting "Getting health information" to 0.5 scenarios 1 through 4 . This concept was set to 0.75 in scenarios 5 through 10 , which represented an activity tracker that showed health measures better than the one scenarios 1 through 4 .

Since these scenarios consider various degrees of EW's social factors, the

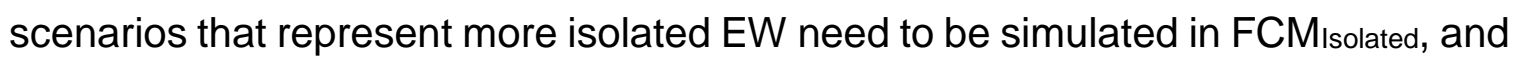
the ones reflect more connected EW should be simulated in FCMConnected. Different pink concepts in the start vectors section of Table 7-1 and Table 7- 2 shows the values that were set to best represent EW facilitating conditions. The results are listed in the two bottom rows of the tables.

Here is the summary of the 10 scenarios that tries to answer the questions. The scenarios that represent the isolated EW are naturally simulated in FCM $\mathrm{Isolated}_{\text {and }}$ the scenarios that represent the connected EW are simulated in FCMconnected. Here is the list of scenarios:

1. OK product but no support

2. OK product with good gatekeeping support

3. OK product but low gatekeeping support

4. OK product but little gatekeeping support 
5. Better product with good community support

6. product but little gatekeeping support

7. Better product with no gatekeeping support

8. 8a. Better product with better technology support (e.g. EW lives in a retirement community with technology support).

8a. Better product with better social support (e.g. EW has close family).

9. Better product with good community training ${ }^{12}$ and relatively good social support

10. Better product with perfect community training

Scenario 1-the baseline scenario—refers to EW situations with no social support; therefore, the appropriate model for simulating this scenario is the FCM simulation results in the lowest negative adoption intention and adoption (listed in Table 7-2), suggesting that adoption is very unlikely.

Scenario 2 reflects strong influence by gatekeepers, who can provide encouragement and facilitate the use of technology; the appropriate model for simulating this scenario is the FCMconnected. These gatekeepers tend to be family members who had technology knowledge of their own. Hence, the maximum value of 1 is assigned to the GK concepts in this scenario. The elderly felt close to their gatekeepers and interacted with them frequently during visits and phone calls;

\footnotetext{
12 'Community Training \& Support' represents any type of technology training and support that is received for free such as the free technology training and support that is often available in retirement communities. The terms Community Training and Community Support can be used interchangeably.
} 
they had no fear of asking naïve questions. Scenario 2 resulted in the highest adoption intention and adoption rate.

To reflect different family gatekeeping influence, family gatekeeping concepts are initialized with varying values from 0.1 -the lowest to 1 -the highest: 'Gatekeeper physical proximity', “Having gatekeeping daughter', 'Closeness to family', 'Having gatekeeping grandkids', 'Having grandkids', 'Having other gatekeeping family members'. To reflect different levels of gatekeeping expertise 'Gatekeeper tech savviness' concept is initialized with varying values from 0.1 -the lowest to 1 -the highest.

Scenarios 3 and 4 explored the effect of situations with lower gatekeeper support (tested with 0.5 in scenario 3 and 0.1 in scenario 4). These scenarios show that the lower the gatekeeping support, the less internally motivated the elderly woman tends to be. Similarly, the lower the gatekeeping support, the less likely she is to have the resources to learn and use the new unfamiliar technology. Both of these scenarios predict lower adoption outcomes (the lower the facilitating condition, the lower the chance of adoption).

I subsequently checked if strong product features offset low levels of gatekeeper support: so, in scenario 5 , scenario 4 was modified to reflect improved product features. This yielded a slightly higher adoption intention and adoption rate for scenario 5 than the one that resulted from scenario 4. However, the adoption rate for scenario 5 lagged far behind the adoption rate for scenario 2, which involved the adoption of a less attractive product, but exhibited moderate levels of 
gatekeeper support and expertise. This showed that the importance of facilitating conditions can trump the importance of the improvement in the quality of the product.

\begin{tabular}{|c|c|c|c|c|c|c|}
\hline 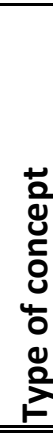 & 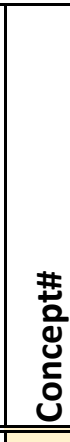 & Application of $\mathrm{FCM}_{\text {Connected }}$ & 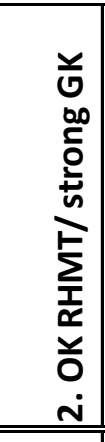 & 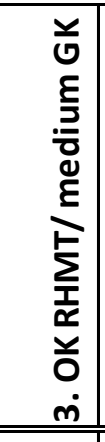 & 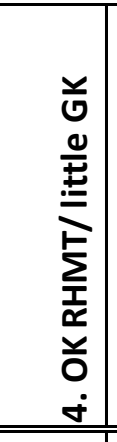 & 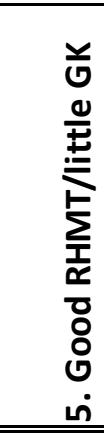 \\
\hline \multirow{11}{*}{ 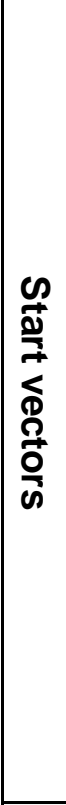 } & c12 & Getting health information & 0.5 & 0.5 & 0.5 & 0.75 \\
\hline & c13 & Aging \& cognitive decline & 0.5 & 0.5 & 0.5 & 0.5 \\
\hline & c17 & Tracing activities & 0 & 0 & 0 & 0.5 \\
\hline & c24 & $\begin{array}{l}\text { Gatekeeper physical } \\
\text { proximity }\end{array}$ & 1 & 0.5 & 0.1 & 0.1 \\
\hline & c30 & Lack of tech robustness & 0.5 & 0.5 & 0.25 & 0.25 \\
\hline & c34 & Having gatekeeping daugther & 1 & 0.5 & 0.1 & 0.1 \\
\hline & c36 & Gatekeeper tech savviness & 1 & 0.5 & 0.1 & 0.1 \\
\hline & c39 & Closeness to family & 1 & 0.5 & 0.1 & 0.1 \\
\hline & c42 & $\begin{array}{l}\text { Having gatekeeping } \\
\text { grandkids }\end{array}$ & 1 & 0.5 & 0.1 & 0.1 \\
\hline & c44 & Having grandkids & 1 & 0.5 & 0.1 & 0.1 \\
\hline & c45 & $\begin{array}{l}\text { Having other gatekeeping } \\
\text { family members }\end{array}$ & 1 & 0.5 & 0.1 & 0.1 \\
\hline \multirow{3}{*}{ 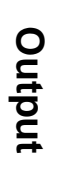 } & \# & Stability Iteration \# & 15 & 17 & 17 & 17 \\
\hline & c1 & Adoption & 0.785 & 0.743 & -0.454 & -0.351 \\
\hline & c2 & Adoption Intention & 0.423 & 0.319 & $-0.716 \mid$ & -0.636 \\
\hline
\end{tabular}

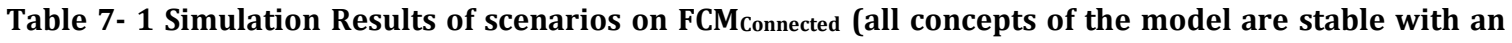
epsilon of 0.001); the initial value for every other concepts is zero)

Scenario 6 simulated the adoption of a better product without any social support.

Hence, the appropriate model for simulating this scenario is the FCM Isolated. This, 
again, resulted in a very low adoption outcome, suggesting that improvements to the product do not increase the chances of adoption as effectively as social support does. So, to some extent, gatekeeper support can compensate for product features. This was confirmed in the field, as the isolated elderly women didn't even want to adopt a better activity tracker, even after they had tried it. The elderly women reported that family support not only helps them use technology that they find difficult to understand-it is also the main driver of their adoption decision.

Since the EWs' family dynamics arguably can't be changed, in the subsequent scenarios, we try to explore the effectiveness of facilitating conditions provided by sources other than family in isolated EWHTA. These other sources (secondary facilitating conditions) are any other people that help the EW with tech training and support, including professional (for fee) or free tech support channels and the people around the EW who help free of charge. The models have captured the different tech support channels in concepts: "Community tech support", "Local group training", "Free limited professional support", "Established tech support" and "Simple free instruction". Additionally, the two models embody some concepts to capture the gatekeeping effects of EW's social surrounding (other than family). These concepts include "Having tech savvy social circle", "Having gatekeeping friend" and "Social circle". Therefore, these scenarios set the values of these concepts to what is displayed in Table 7-2.

In scenario 7, a moderate level of formal support channels are examined to see if these support channels can help overcome the adoption gap for isolated elderly 
who, arguably, would benefit most from RHMT. In scenario 7, the values of these concepts are set to 0.5 as displayed in Table $7-2$. Scenario 7 shows a rate of adoption that is higher than that in scenario 6 , yet much lower than that in the family gatekeeper scenarios (scenarios 2 and 3 ). This is a realistic outcome: even a well-designed training service likely cannot provide the multitude of functions that family or a friend's social support provides. This suggests that these support channels, just like the influences induced by the family gatekeepers, should find ways to actively engage the EW and don't assume that just their presence in the community is enough for the EW to reach out and benefit from them.

As observed in the case study, isolated elderly women often suffer from a lack of tech adoption due to many factors including lack of motivation, exposure and support. The varying degree of isolation (or social surrounding) is examined hereafter, in scenario 8 to 10 , to understand how it influences EWHTA.

Scenario 8 examines the effect of the limited social surrounding of the isolated EW with no close family. The concepts "Having tech savvy social circle" and "Having gatekeeping friend" and "Social circle" is set to 0.5 to represent an EW who may have few tech savvy friend and may still get out of her house to attend to some social activities (like going to church or grocery shopping). The simulation of FCM Isolated suggests that she would have much higher chance of HTA compared to those without this limited exposure and support (Table 7-2). This is in line with the observation in the field in the case study. This result could be due to the fact that these people from the EW's social circle influence the EW endogenously, even 
if they cannot provide as much technical information as the formal support channels do. They effectively deliver social learning that increases her self-efficacy and product awareness to the EW. They are also available to provide low barrier, jargon-free support that is not intimidating to the EW. EW in the case study mentioned that they were worried about asking naïve questions from professional tech support or in the training classes.

Scenarios 9 and 10 represent the adoption of a good RHMT product in a better social environment than scenario 7. In scenario 9, the EW is less isolated and has a relatively technology savvy limited social circle, as well as moderate access to support channels. In scenario 10, the EW has full access to these environmental factors and is hardly isolated. 


\begin{tabular}{|c|c|c|c|c|c|c|c|c|c|c|c|}
\hline 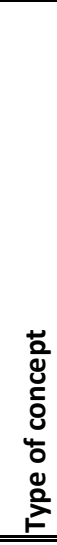 & 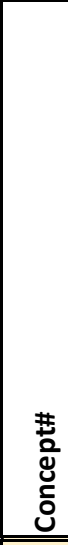 & Application of FCM Isolated $_{\text {. }}$ & 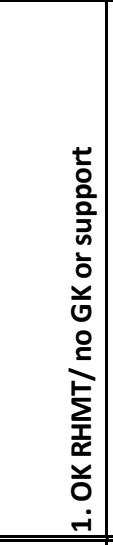 & 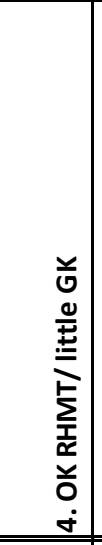 & 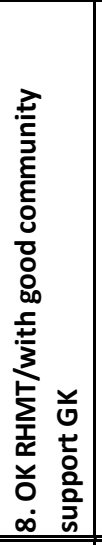 & 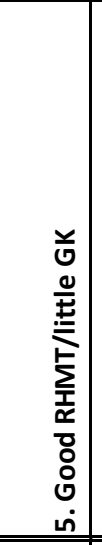 & 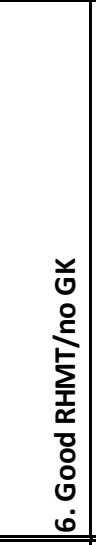 & 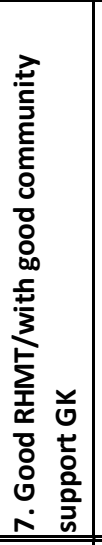 & 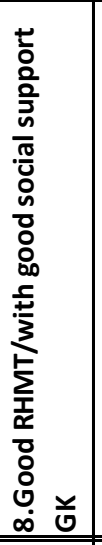 & 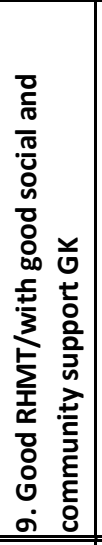 & 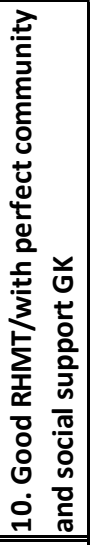 \\
\hline \multirow{12}{*}{ 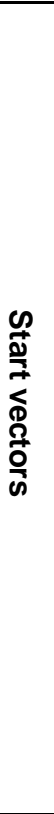 } & c6 & $\begin{array}{l}\text { Getting health } \\
\text { information }\end{array}$ & 0.5 & 0.5 & 0.5 & 0.75 & 0.75 & 0.75 & 0.75 & 0.75 & 0.75 \\
\hline & c12 & Aging \& cognitive decline & 0.5 & 0.5 & 0.5 & 0.5 & 0.5 & 0.5 & 0.5 & 0.5 & 0.5 \\
\hline & c19 & Tracing activities & 0 & 0 & 0 & 0.5 & 0.5 & 0.5 & 0.5 & 0.5 & 0.5 \\
\hline & c30 & Community tech support & 0 & 0.1 & 0.5 & 0.1 & 0 & 0.5 & & 0.5 & 1 \\
\hline & c33 & Lack of tech robustness & 0.5 & 0.5 & 0.5 & 0.25 & 0.25 & 0.25 & 0.25 & 0.25 & 0.25 \\
\hline & c39 & Local group training & 0 & 0.1 & 0.5 & 0.1 & 0 & 0.5 & & 0.5 & 1 \\
\hline & c50 & $\begin{array}{l}\text { Free limited professional } \\
\text { support }\end{array}$ & 0 & 0.1 & 0.5 & 0.1 & 0 & 0.5 & & 0.5 & 1 \\
\hline & c51 & $\begin{array}{l}\text { Having tech savvy social } \\
\text { circle }\end{array}$ & 0 & 0.1 & & 0.1 & 0 & & 0.5 & 0.5 & 1 \\
\hline & c52 & Established tech support & 0 & 0.1 & 0.5 & 0.1 & 0 & 0.5 & & 0.5 & 1 \\
\hline & c53 & Simple free instruction & 0 & 0.1 & 0.5 & 0.1 & 0 & 0.5 & & 0.5 & 1 \\
\hline & c56 & $\begin{array}{l}\text { Having gatekeeping } \\
\text { freind }\end{array}$ & 0 & 0.1 & & 0.1 & 0 & & 0.5 & 0.5 & 1 \\
\hline & c62 & Social circle & & 0.1 & & 0.1 & & 0.5 & 0.5 & 1 & 1 \\
\hline \multirow{3}{*}{$\begin{array}{l}\text { 을 } \\
\text { 융 } \\
\text { 을 }\end{array}$} & $\#$ & Stability Iteration \# & 10 & 10 & 11 & 11 & 10 & 12 & 10 & 10 & 10 \\
\hline & c1 & Adoption & -0.8827 & -0.867 & -0.802 & -0.844 & -0.867 & -0.568 & 0.684 & 0.699 & 0.787 \\
\hline & c2 & Adoption Intention & -0.8917 & -0.873 & -0.892 & -0.770 & -0.815 & -0.752 & 0.165 & 0.165 & 0.422 \\
\hline
\end{tabular}

Table 7- 2 Simulation Results of scenarios on FCM Isolated (all concepts of the model are stable with an epsilon of 0.001 ); The initial value for every other concepts is zero) Here the more formal support channel concepts are displayed in a lighter shade of pink

While the simulation of both these scenarios suggests a higher chance of adoption, naturally scenario 10 suggests the best rate of adoption. These are both in line with empirical evidence from the field, and suggest that the more we can surround the elderly with tech savvy peers, the higher her chance of adoption. One actionable insight from these scenarios could be that the decision makers should 
recruit well-connected tech savvy EW from the EW communities to act as technology advocate to model, motivate and support EWHTA in those communities.

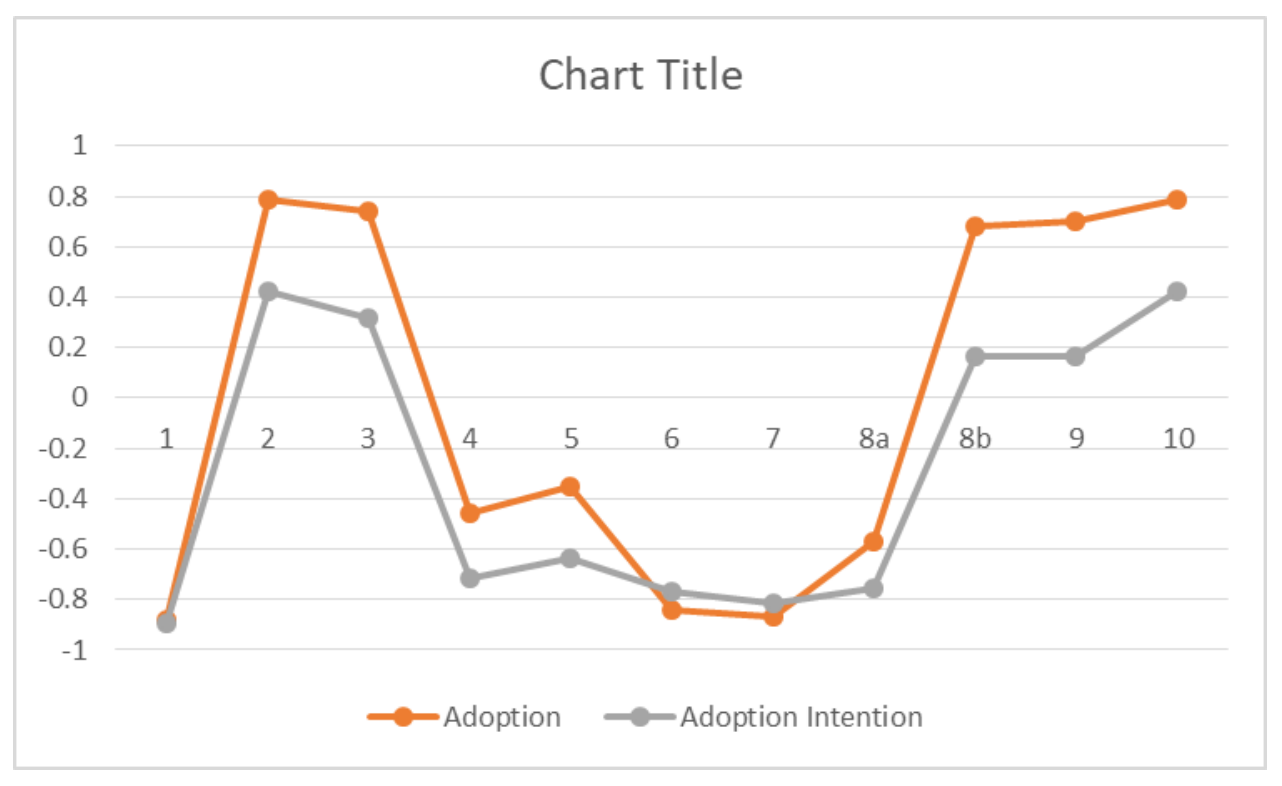

Table 7- 3 The interplay/prioritization of product quality \& social support 


\section{Case Insights and Findings}

This dissertation set out to apply the proposed method to model EWHTA to achieve the goals identified earlier (listed in section 1.2). The purpose of the case study was to...

1. Identifies the key adoption drivers and obstacles and their relations

2. Illustrates how the gatekeeper influences the elderly person's adoption decision

3. Represents adoption drivers and gatekeeper influences on adoption quantitatively

4. Facilitates exploration of adoption in response to various factors through simulation

This chapter provides detailed information showing how the case study was able to achieve all four above goals by the two FCM models that emerged from the case study (depicted in Figure $7-1$ and Figure 7 - 2). The information provided in this chapter in its entirety provides information about goal 1 . as summarized in the next section (8.1). Section 8.2 explains the findings in respect to goal 2 by explaining the six environmental influences highlighted in the two FCM models resulted from that case study. Section 8.3 describes the findings that addresses goal 3 and 4 .

\subsection{Summary of Key Drivers and Obstacles of Adoption and Their Relations (Goal 1)}

The integrated FCM models that resulted from the 'elderly women and their gatekeeper' pairs map out the contextualized model of the UTAUT for the two 
groups of EWHTA: connected (Figure $7-1$ ) and isolated (Figure $7-2$ ). They showed how new inputs and intermediary factors contextualize the original abstract constructs of the UTAUT. The mixed-method research method facilitated the extension of the original input constructs of the UTAUT to show how they form and how they affect the output constructs of UTAUT in the context of EWHTA. These FCM models show that technology adoption is impacted by various factors, including product characteristics, different facilitating conditions, and the elderly women's personal experience and traits. As detailed in Appendix G, the following six categories of concepts were identified as the salient drivers of and obstacles to the elderly women's health technology adoption decision-making:

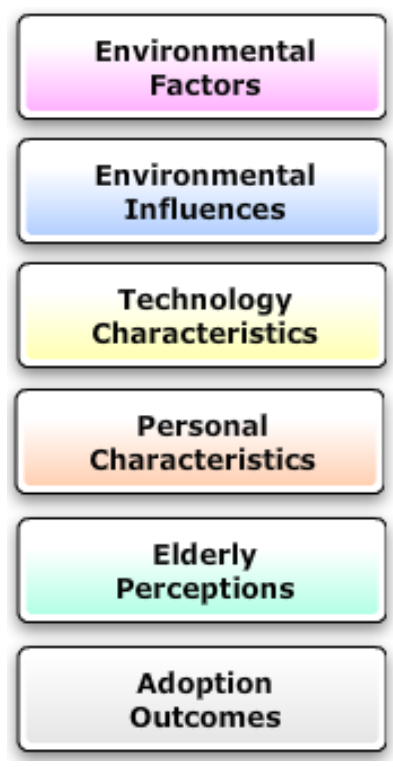

Figure 8 - 1 Salient Drivers of and Obstacle Concept Categories for EWHTA 


\subsubsection{Environmental Factors}

These concepts embody the important EWHTA environmental factors that influence EWHTA. They consist of 21 concepts listed in Table 8 - below.

The case study found 14 concepts (number $1-12$ and $19-20$ in Table 8 - ) that are directly related to EW social surroundings. In the context of EWHTA, social isolation is a significant psychosocial determinant of technology decision making to the point that isolated elderly have a different mental model as those of the connected elderly women. This is in line with other behavioral studies (Giabbanelli, et al., 2012), but this case study highlights its importance in EWHTA. The most important social circle factors of EW are their gatekeeping concepts, which are discussed in section 8.2 in response to the research question 2 . The case study also found 7 factors that make up EW's support resources outside her social circle (number 13 - 17 in Table 8 - ), should the EW have access to them. These include the official support channels form community technology support, the fee-based professionals EW hires, the training classes in the community to the limited nonofficial help of vendors to step-by-step instruction manuals. The case study also found another concept-Clinician recommendation, which was found to have an enormous influence in EWHTA. A segment of the FCM Isolated model that maps out

environmental and how they influence other concepts is displayed in Figure 8-2 below. The corresponding segment of FCMconnected model is similar but doesn't have the social circle concept as identified negligible for the socially connected group of EW. 


\begin{tabular}{|c|c|c|}
\hline \multicolumn{2}{|c|}{ Gatekeeping Factors } & $\begin{array}{l}\text { Concept \# } \\
\text { in Adjacency } \\
\text { Matrices }\end{array}$ \\
\hline 1 & Gatekeeper physical proximity & $\mathrm{C} 24$ \\
\hline 2 & Gatekeeper tech savviness & $\mathrm{C} 36$ \\
\hline 3 & Having gatekeeping daughter & $\mathrm{C} 34$ \\
\hline 4 & Having gatekeeping grandkids & $\mathrm{C} 57$ \\
\hline 5 & Closeness to family & $\mathrm{C} 55$ \\
\hline 6 & Having gatekeeping family & C35 \\
\hline 7 & Having grandkids & C59 \\
\hline 8 & Having other gatekeeping family members & $\mathrm{C} 60$ \\
\hline 9 & Having gatekeeper(s) other than family & C58 \\
\hline 10 & Having gatekeeping caregiver & C54 \\
\hline 11 & Having gatekeeping friend & C56 \\
\hline 12 & Gatekeeper's financial constraint & C62 \\
\hline \multicolumn{3}{|c|}{ Support resources } \\
\hline 13 & Community tech support & $\mathrm{C} 30$ \\
\hline 14 & Established tech support & C52 \\
\hline 15 & Local group training & C39 \\
\hline 16 & Free limited professional support & C50 \\
\hline 17 & Simple free instruction & C53 \\
\hline \multicolumn{3}{|c|}{ Social circle other than family } \\
\hline 18 & Clinician recommendation & $\mathrm{C} 42$ \\
\hline 19 & Having tech savvy social circle & $\mathrm{C} 51$ \\
\hline 20 & Social circle & C63 \\
\hline \multicolumn{3}{|c|}{$\begin{array}{l}\text { Media \& other source of learning about the } \\
\text { wearable }\end{array}$} \\
\hline 21 & Other sources of information & C61 \\
\hline
\end{tabular}

Table 8 - 1 Environmental Factors of the EWHTA 


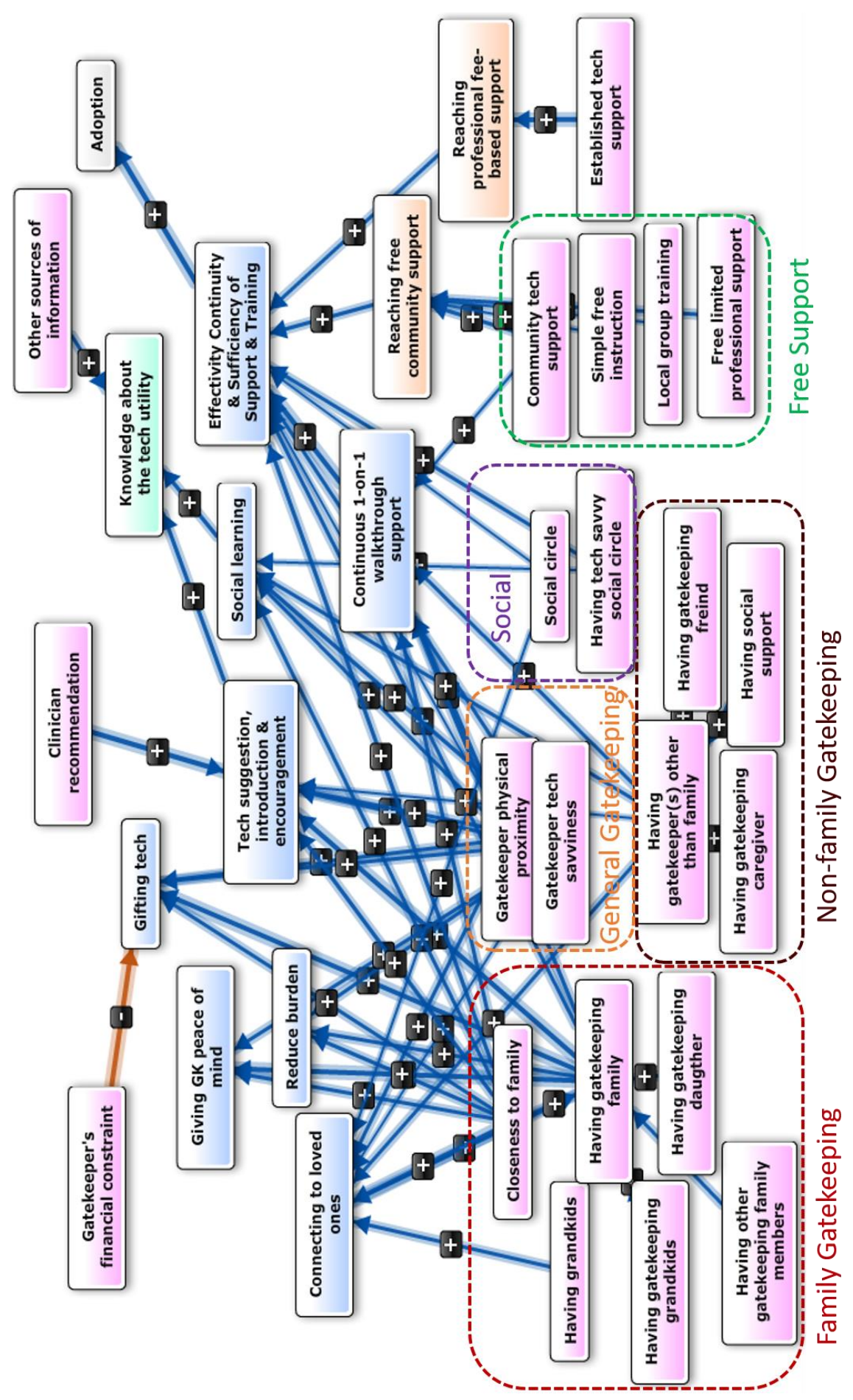

Figure 8 - 2 'Environmental Factors' and 'Environmental Influences' in FCM Isolated 


\subsubsection{Environmental Influences}

The dominant QUAL phase of the proposed method facilitated the extraction of different types of influence that the environmental factors exert on EWHTA. These concepts, which are defined in Appendix G, encapsulate different influences from the environmental factors that affect EW's attitudes, perceptions, and decision toward EWHTA. More information on this category is provided in the response to question 2 in section 8.2 .

\begin{tabular}{|llc|}
\hline Environmental Influences & $\begin{array}{l}\text { Concept \# } \\
\text { in Adjacency Matrices }\end{array}$ \\
\hline 1 & Effectivity, Continuity \& Sufficiency of Support \& Training & C4 \\
\hline 2 & Social learning & $\mathrm{C} 11$ \\
\hline 3 & Tech suggestion, introduction \& encouragement & $\mathrm{C} 18$ \\
\hline 4 & Connecting to loved ones & $\mathrm{C} 31$ \\
\hline 5 & Reduce burden & $\mathrm{C} 37$ \\
\hline 6 & Gifting tech & $\mathrm{C} 38$ \\
\hline 7 & Giving GK peace of mind & $\mathrm{C} 43$ \\
\hline 8 & Continuous 1-on-1 walkthrough support & $\mathrm{C} 49$ \\
\hline
\end{tabular}

Table 8 - 2 Environmental Influences Factors of the EWHTA

\subsubsection{Technology Characteristics}

The concepts described in this section embody the important characteristics of the wearable device, which influence EWHTA. They consist of 11 concepts listed in Table 8 - 3 below.

These concepts, which are described in the codebook in Appendix G, represent the relative degree in which the product is scored in the specified criterion that the concept represents in relation to EWHTA. 


\begin{tabular}{|c|c|c|}
\hline \multicolumn{2}{|c|}{ Technology Characteristics } & \multirow{2}{*}{$\begin{array}{r}\text { Concept \# } \\
\text { in Adjacency Matrices } \\
\text { C5 }\end{array}$} \\
\hline 1 & Tech automaticity & \\
\hline 2 & Getting health information & C6 \\
\hline 3 & Tech usability \& accessibility & C15 \\
\hline 4 & Tech capabilities & $\mathrm{C} 17$ \\
\hline 5 & Tracing activities & C19 \\
\hline 6 & Tech simplicity \& familiarity & $\mathrm{C} 21$ \\
\hline 7 & Tech cost & $\mathrm{C} 25$ \\
\hline 8 & Tech maintenance & $\mathrm{C} 27$ \\
\hline 9 & Tech making E look old & $\mathrm{C} 28$ \\
\hline 10 & Lack of tech robustness & C33 \\
\hline 11 & Tech aesthetic appeal & C41 \\
\hline
\end{tabular}

Table 8 - 3 Technology Characteristics of the EWHTA

\subsubsection{Personal Traits}

The concepts described in this section embody the traits of the EW that influence EWHTA. They consist of 11 concepts listed in Table 8 - and capture the collective attitudinal and conditional traits of the elderly as well as her tendencies and efforts. Like others, these concepts are described in the codebook in Appendix G. 


\begin{tabular}{|rl|r|}
\hline Personal Traits & $\begin{array}{r}\text { Concept \# } \\
\text { in Adjacency Matrices }\end{array}$ \\
\hline 1 & Adoption Intention & $\mathrm{C} 2$ \\
\hline 2 & Self-efficacy & $\mathrm{C} 7$ \\
\hline 3 & Aging \& cognitive decline & $\mathrm{C} 10$ \\
\hline 4 & Voluntariness of Use & $\mathrm{C} 12$ \\
\hline 5 & Exploring tech & $\mathrm{C} 20$ \\
\hline 6 & Concerns & $\mathrm{C} 32$ \\
\hline 7 & Positive attitude toward tech & $\mathrm{C} 44$ \\
\hline 8 & Resistance to change & $\mathrm{C} 45$ \\
\hline 9 & Being frugal & $\mathrm{C} 46$ \\
\hline 10 & Reaching free community support & $\mathrm{C} 47$ \\
\hline 11 & Reaching professional fee-based support & $\mathrm{C} 48$ \\
\hline
\end{tabular}

Table 8 - 4 Personal Traits in EWHTA

\subsubsection{Elderly Perceptions}

These concepts capture EW's perceptions about the different aspects of the product being studied. The two key concepts of-Perceived Ease of Use and Perceived Usefulness are UTAUT constructs and are the most important perceptions and the determinants of the EW adoption intention. This category consists of the 10 concepts listed in Table 8 -, which are described in the codebook in Appendix G. 

Elderly Perceptions
Concept \#
in Adjacency Matrices

\begin{tabular}{|rlc}
\hline 1 & Perceived Ease of use & C3 \\
\hline 2 & Perceived needs & C8 \\
3 & Promote health & C9 \\
4 & Perceived simplicity \& familiarity & C14 \\
\hline 5 & Perceived usability \& accessibility & C16 \\
\hline 6 & Knowledge about the tech utility & C22 \\
\hline 7 & Perceived Usefulness & C23 \\
\hline 8 & Perceived cost & C26 \\
\hline 9 & Perception of how much tech make E look old & C29 \\
10 & Perception \& importance of tech aesthetic & C40
\end{tabular}

Table 8 - 5 Elderly Perceptions in EWHTA

\subsubsection{Adoption Outcomes}

These concepts capture EW's past or current adoption outcomes. The past adoptions ('Technical knowledge \& experience' concept) increase the chance of a current outcome of adoption by increasing EW's self-efficacy and general product awareness. The adoption outcome is the result of strong adoption intention, as well as effective, continuous and sufficient support and training. Both concepts are described in the codebook in Appendix G (I. Common Ontology (Codebook)).

\begin{tabular}{|c|c|}
\hline Adoption Outcome & $\begin{array}{r}\text { Concept \# } \\
\text { in Adjacency Matrices }\end{array}$ \\
\hline 1 Adoption & $\mathrm{C} 1$ \\
\hline 2 Technical knowledge \& experience & $\mathrm{C} 13$ \\
\hline
\end{tabular}

Table 8 - 6 Adoption Outcomes in EWHTA 


\subsection{Gatekeepers' Influence on EWHTA (Goal 2)}

In the context of EWHTA, the gatekeepers are those people who open the door to technology for the EW. They often become the ambassador of the technology. As described in Chapter 2, the literature suggests that this influence is important for EWHTA, and without it the elderly typically do not adopt the technology. The case study highlights this importance and even suggest that the mental model of the

elderly is different based on the degree of presence or absence of these gatekeepers' influences. The answer to the research question 2 follows based on the findings of the case study as captured in the two FCM models in Figure $7-1$ and Figure $7-2$.

Gatekeepers are divided to two groups: family gatekeepers and non-family gatekeepers. The EW who benefit from having family gatekeepers experience a much higher rate of technology adoption than those with non-family gatekeepers. The two clusters that emerged from the quan data collection vividly attested to this. The group that clustered in the category of 'isolated' with the lower observed adoption rate where those without family gatekeepers. The group that clustered in the category of 'connected' with the higher observed adoption rate were those with family gatekeepers. In fact, in the case of FCMconnected, the effect of a techy social circle still is important, but it is negligible when compared to the colossal effects of family gatekeeping. The two models from the case study_FCMconnected (Figure 7 -1) and FCM Isolated (Figure 7 -2)—illustrate how these two types of gatekeeping influence EWHTA. 
"Family gatekeeping": In the context of the case study, the most important drivers of this category, are the family members, particularly grandkids but more frequently daughters and sometimes other family members like sons. They induce immense influence on mom's (grandma's) HTA. The degree of this influence depends on:

1) Whether the EW has a daughter, older grandkids or any other family members that can help her. This is captured by the concept "Having gatekeeping family", which is caused by one or more of the following concepts: "Having gatekeeping grandkids", "Having other gatekeeping family members", and "Having gatekeeping daughter".

2) How close and connected she is with her family, as captured by the concept "Closeness to family".

3) If she has one or more grandkids to motivate her to connect with them, as captured by concept "Having grandkids".

4) How technically savvy her primary technology helper is, as captured by concept "Gatekeeper tech savviness".

5) And finally, how near to EW this family technology helper lives and how often she/he visits or calls.

These concepts positively influence many concepts that increase EW's willingness to use the technology (as captured by concept "Voluntariness of Use") and her perception of Usefulness and Ease of Use of the wearable (as captured by concept "Perceived Usefulness" and "Perceived Ease of Use"). The immense influence of 
strong family gatekeeping are categorized in eight ways as depicted in Figure 8 2 (influence concepts group are colored in blue) and described in detail in the next eight subsections.

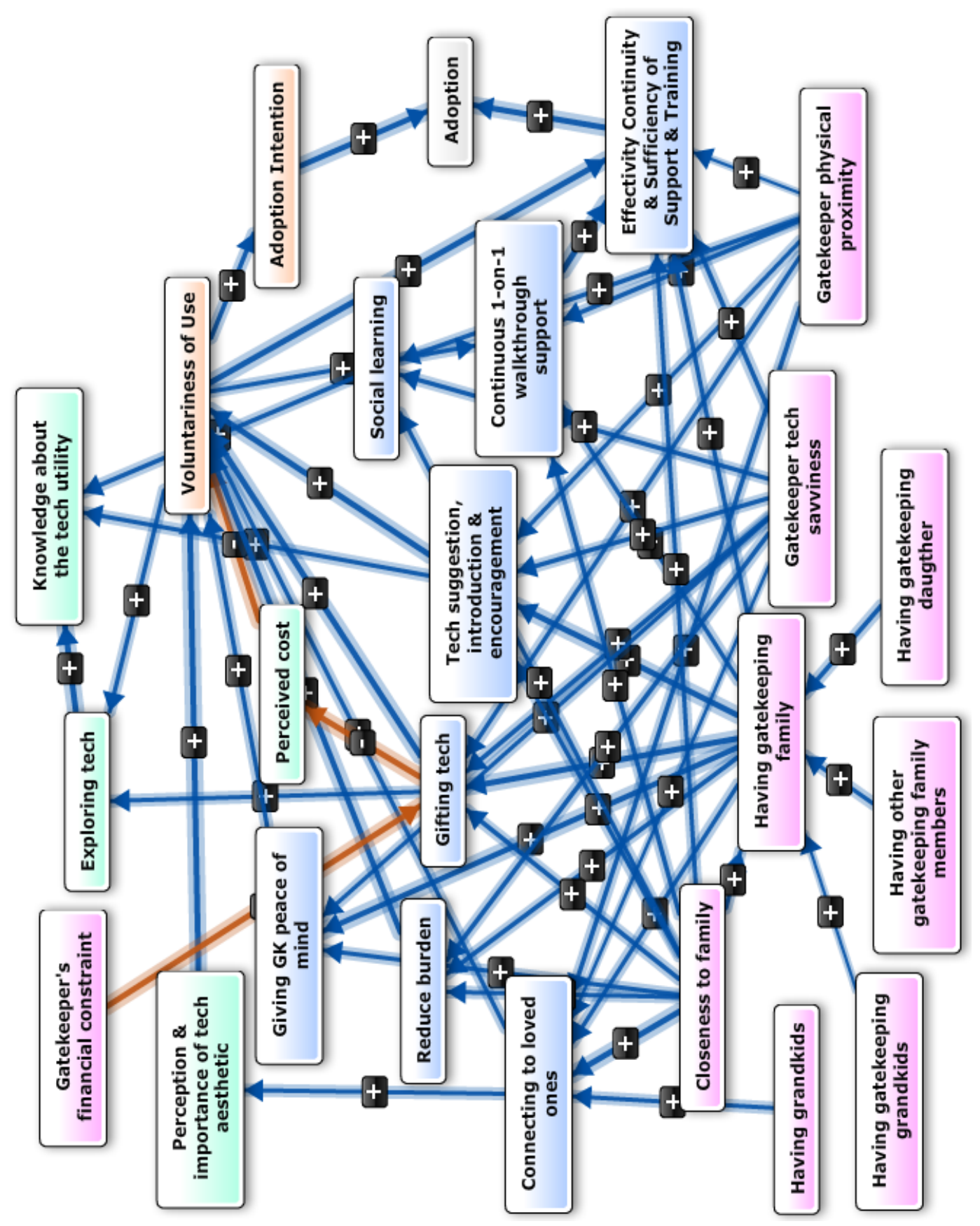

Figure 8 - 3 Family gatekeeping concepts of EWHTA in a segment of the two FCM models 267 
In the case of EW without family gatekeepers, the presence of non-family gatekeepers is still influential in her EWHTA. Figure $8-2$ depicts the role of one or multiple non-family gatekeeper(s). This is captured in the FCM models by the concept "Having gatekeeper(s) other than family", which accumulates the effects of "Having gatekeeper friend", "Having gatekeeping caregiver" and "Having social support". The presence of one or more of these input concepts increases the strength of "Having gatekeeper(s) other than family", which has an effect that is similar to the influence exerted by the family gatekeeping concepts, but much smaller in magnitude. Although they don't have as much influence on the 'environmental influence' group of factors as the family gatekeepers do, these nonfamily gatekeeping influences still contribute to "Continuous one-on-one walkthrough support", "Social learning", "Tech suggestion, introduction \& encouragement" and "Connecting to loved ones". For the non-family gatekeepers, the role of other two concepts discussed above, namely "Gatekeeper tech savviness" and "Gatekeeper physical proximity", is similar to having family gatekeepers. The more technology savvy the gatekeepers are and the closer they live, the more they can positively influence EWHTA. 


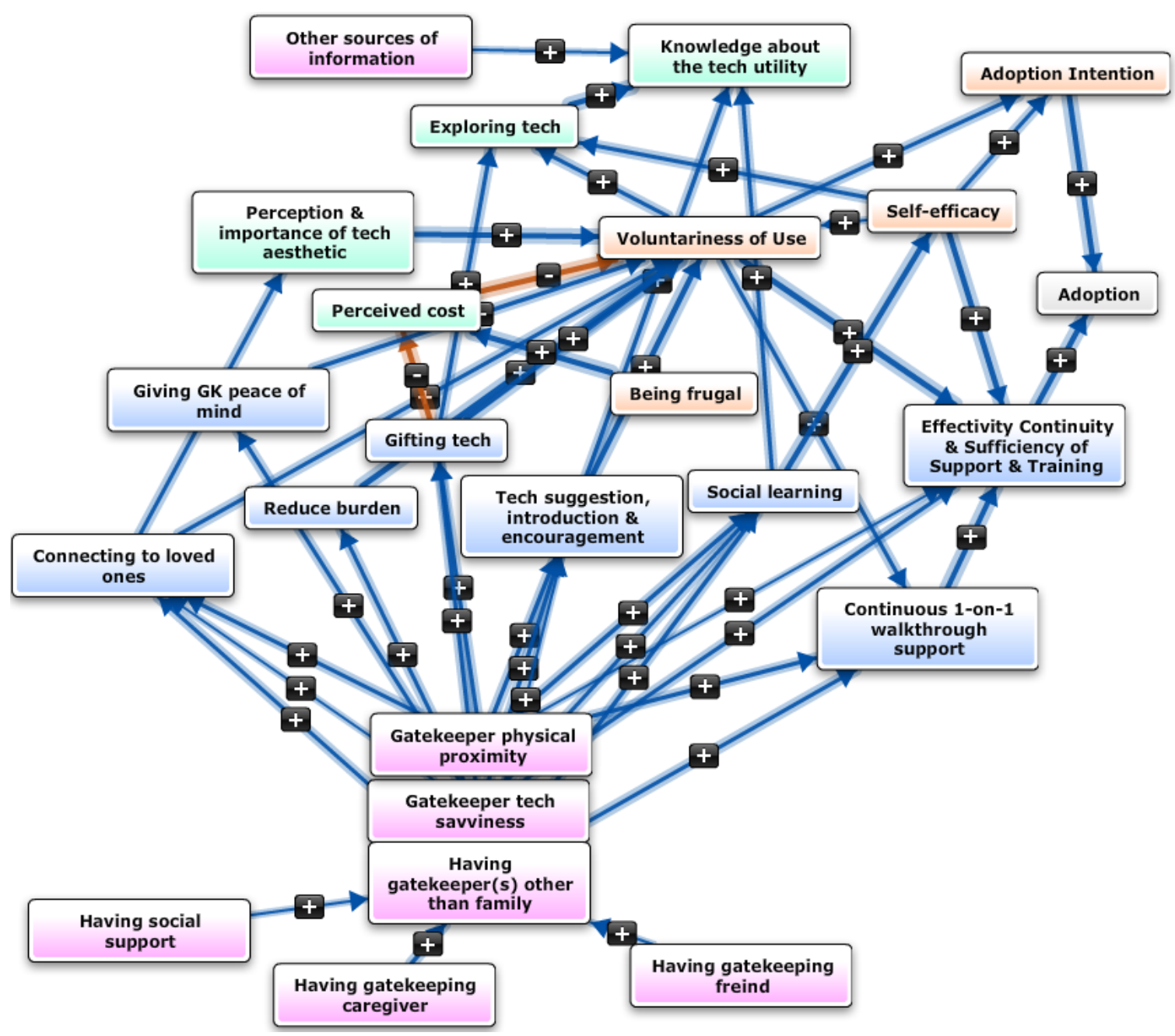

Figure 8 - 4 Gatekeeping (other than family's) concepts of EWHTA in a segment of the two FCM models

These gatekeeping influences are discussed in below subsections.

\subsubsection{Connecting to Loved Ones}

EW feels that by wearing the wearable she will better connect with her younger family members and can look more cool and up to date with technology. Consequently, this concept increases EW's "Voluntariness to use" and "Perception \& importance of tech aesthetic". 


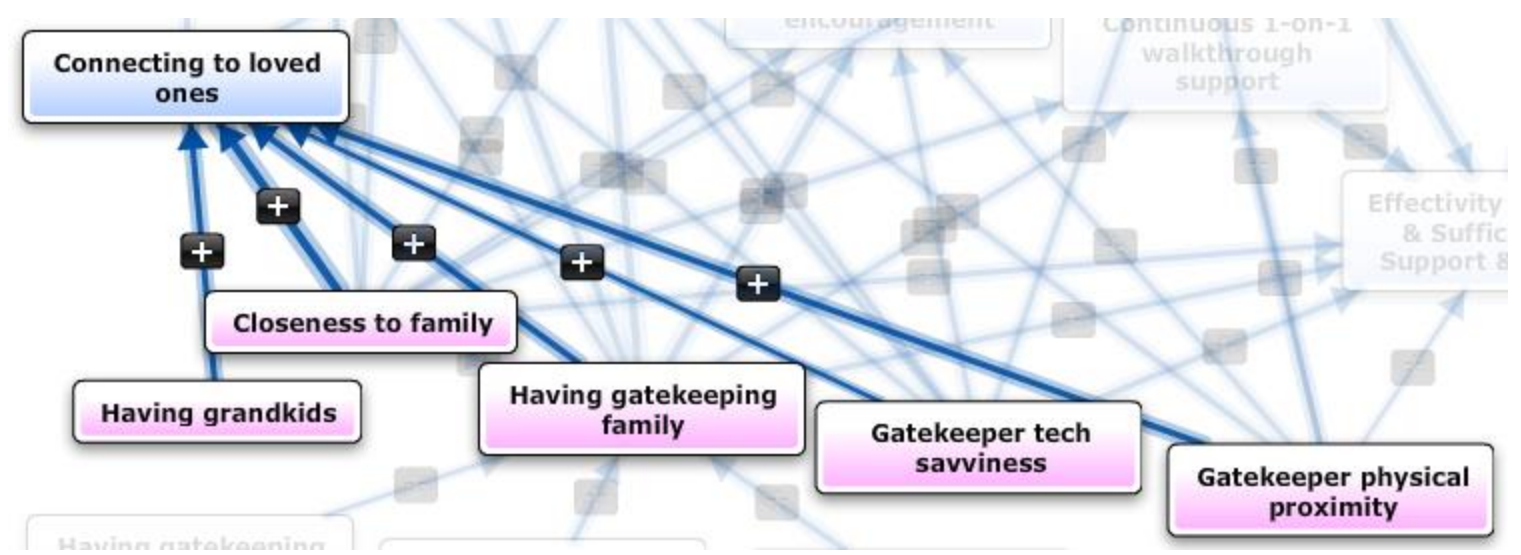

Figure 8 - 5 Concepts increasing Connecting to loved ones

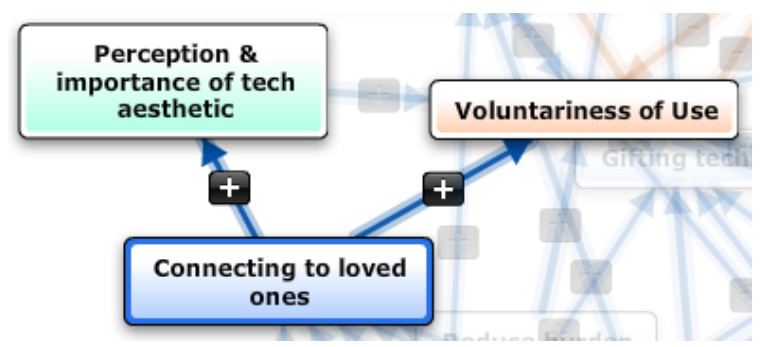

Figure 8 - 6 Concepts increased by Connecting to loved ones

\subsubsection{Reduce Burden}

The closer the EW is to her family and the more technology savvy the family is, the more she feels that using the wearable can reduce her burden on them. The desire to reduce the burden on her family gatekeeper(s) increases her voluntariness of use.

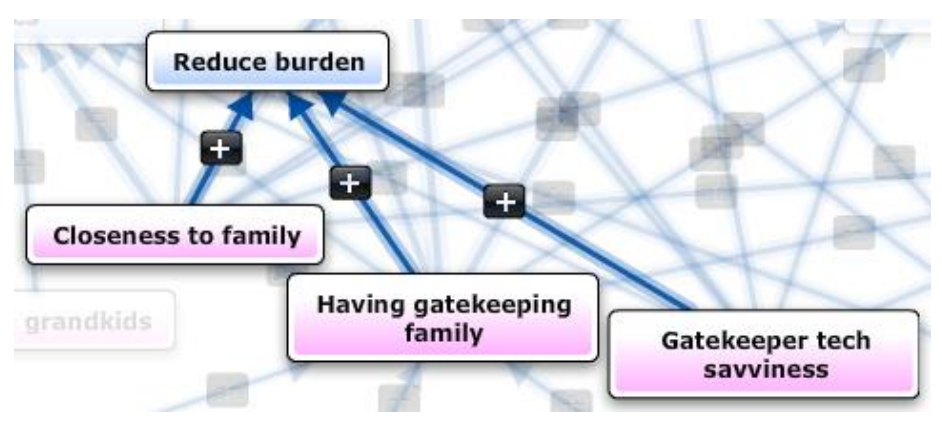

Figure 8 - 7 Concepts increasing Reduce burden 


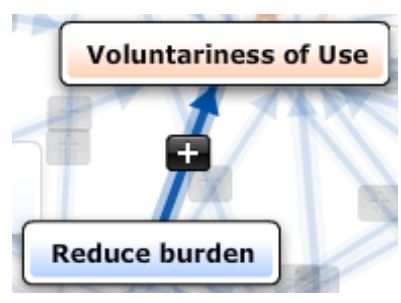

Figure 8 - 8 Concepts increased by Reduce burden

\subsubsection{Giving Gatekeeper Peace of Mind}

The closer the EW is to her family members and the more technology savvy they are, the more she feels that using the wearable can give them peace of mind. This desire of the EW increases her 'Voluntariness of Use'.

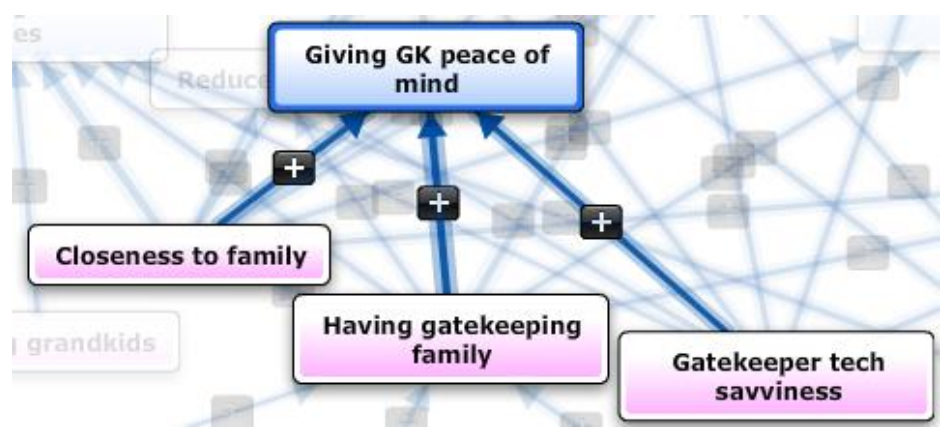

Figure 8 - 9 Concepts influencing Giving GK peace of mind

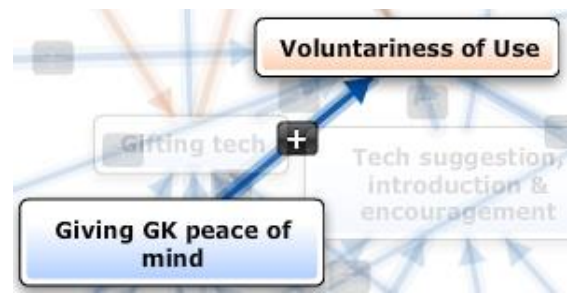

Figure 8 - 10 Concepts influenced by Giving GK peace of mind

\subsubsection{Gifting Tech}

The closer the family is to EW both emotionally and physically and the more technology savvy the family is, the greater the chances are that she gets a 
technology gift such as a wearable. While the family members' financial constraints reduce their means to give EW technology gifts, they are the primary source for supplying EW with new or hand-me-down technologies. These technology gifts not only remove the cost barrier to $\mathrm{EW}$ and increase her voluntariness of use; they become conveniently available for her to try and explore.

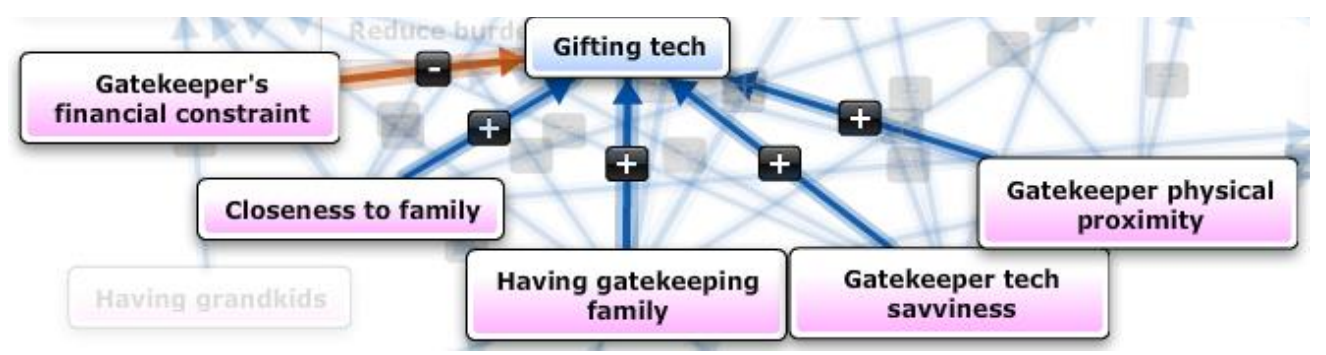

Figure 8 - 11 Concepts influencing Gifting tech

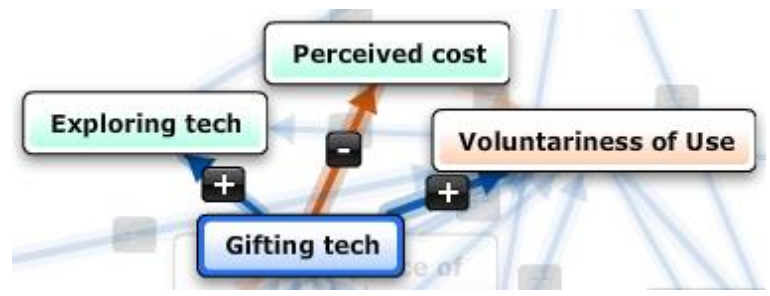

Figure 8 - 12 Concepts influenced by Gifting tech

\subsubsection{Tech Suggestion, Introduction, and Encouragement}

The closer the family is to the EW, both emotionally and physically, and the more technology savvy the family members are, the more impactful their suggestions and opinions will be. When they see a wearable that they find useful for EW, they often not only suggest it to her; they also introduce to her and encourage her to use it. This group of concepts increases EW's product awareness and her willingness to use the technology. 


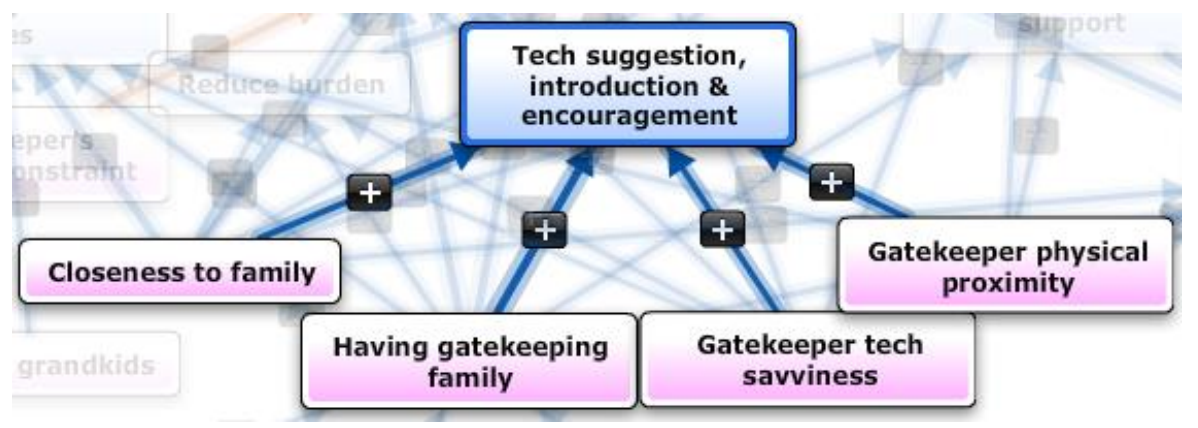

Figure 8 - 13 Concepts influencing Tech suggestion

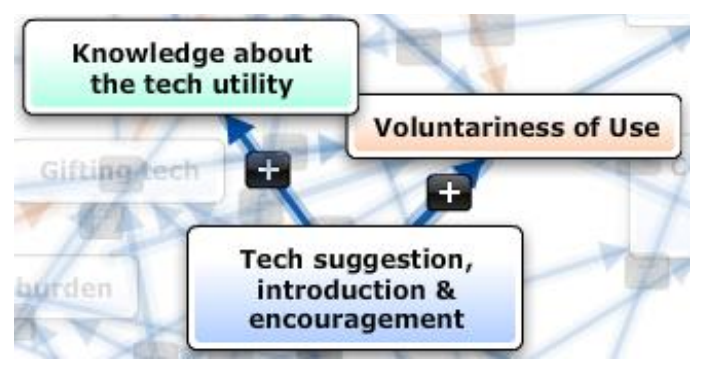

Figure 8 - 14 Concepts influenced by Tech suggestion

\subsubsection{Social Learning}

The closer the family is to the EW, both emotionally and physically, and the more technology savvy the family members are, the more the EW is exposed to the technology and learn from others how to use it. This social learning increases the EW's confidence in her ability to figure out and use the technology, and it increases her product awareness.

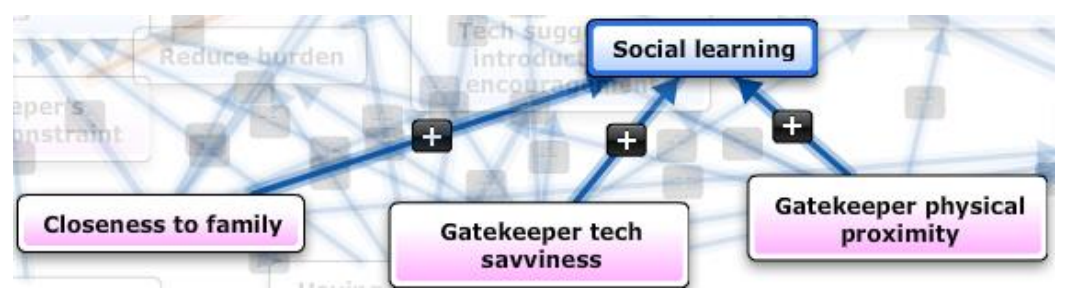

Figure 8 - 15 Concepts influencing Social learning 


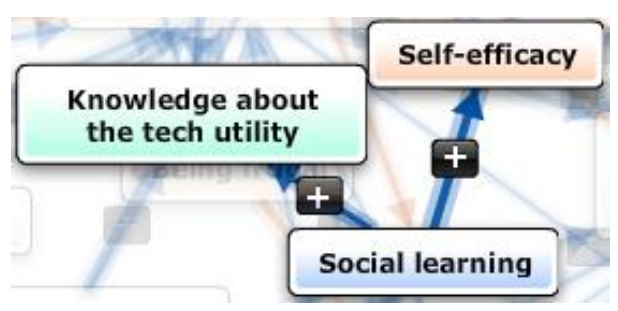

Figure 8 - 16 Concepts influenced by Social learning

\subsubsection{Continued 1-on-1 Walkthrough Support}

The family gatekeeper(s) deliver(s) the most effective technology support. Their support is continuous, convenient and free of technology jargon. The EW, who is too proud and worried about asking naïve questions from other people, often waits for the time she can talk to her family to get their support. The closer the family is to EW, both emotionally and physically, and the more technology savvy the family members are, the more continuous one-on-one walkthrough support she gets. Figure 8 - 17 shows that the FCM models suggest that this effective support is also increased by the strength of the EW's voluntariness of use and the possible presence of community tech support. Continued one-on-one walkthrough support strongly increases the effectiveness, continuity and sufficiency of support and training (aka Facilitating Condition).

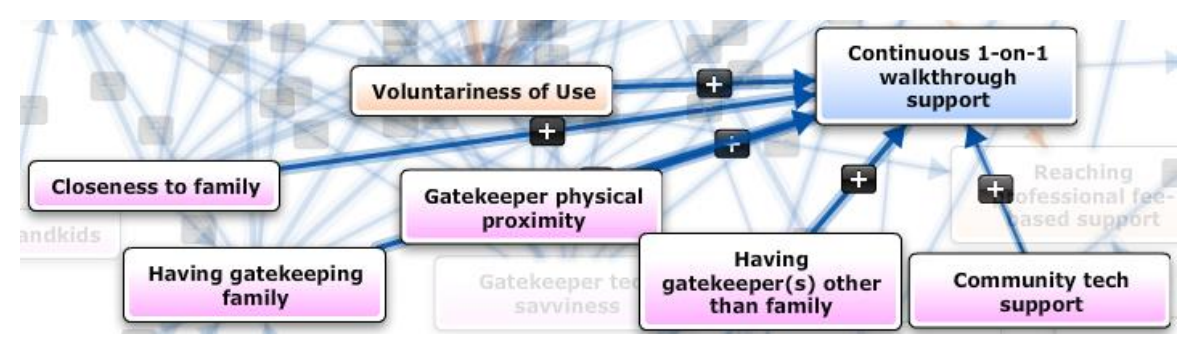

Figure 8 - 17 Concepts influencing Continues 1-on-1 walkthrough support 


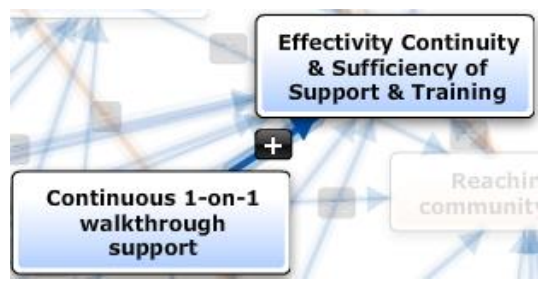

Figure 8 - 18 Concepts influenced by Continues 1-on-1 walkthrough support

\subsubsection{Effectivity, Continuity \& Sufficiency of Support \& Training}

The two FCM models suggests that EW's internal drivers and gatekeeping influences increase the accumulating facilitating condition (a main construct of UTAUT). Figure 8 - 19 shows that the older the EW gets and/or the more her cognitive ability declines, the less effective and sufficient the support and training around her becomes. However, there are internal and external factors that can compensate for the negative influence of aging. These include the stronger internal drivers of high self-efficacy and voluntariness of use. Additionally, the available support resources—should she reach out to them—can increase the accumulative facilitation condition. These resources include the fee-based and free support channels, as well as the EW's technology savvy social circle. While the aforementioned drivers (particularly EW's internal factors) are key, the support and facilitation received from family gatekeeper(s) remain essential. These influences are captured in the FCM models by the causal links from the following concepts: having gatekeeping family, closeness to family, gatekeeper tech savviness, gatekeeper physical proximity, and Continuous 1-on-1 walkthrough support. Efficiency Continuity \& Sufficiency of Support \& Training directly increase adoption behavior, as is posited by the UTAUT. 


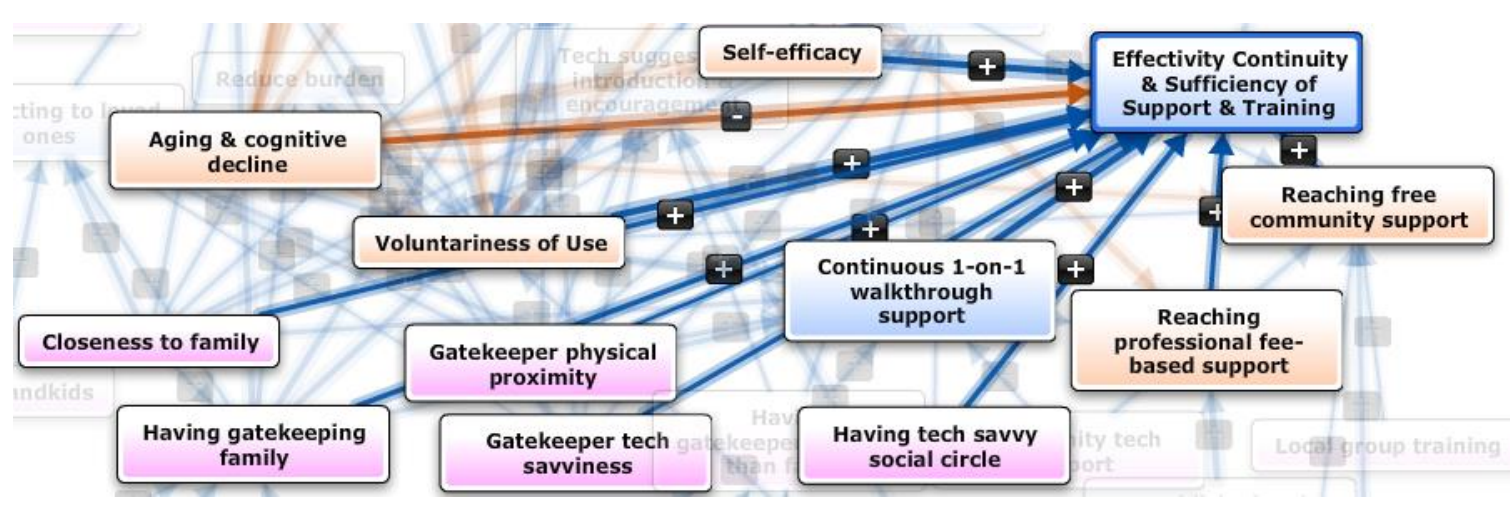

Figure 8 - 19 Concepts influencing Efficiency Continuity \& Sufficiency of Support \& Training

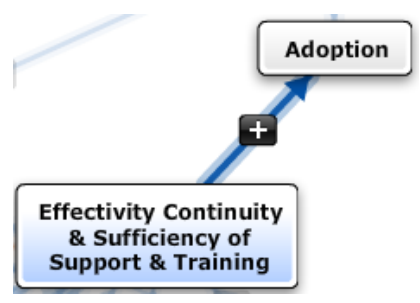

Figure 8 - 20 Adoption is directly influenced by Efficiency Continuity \& Sufficiency of Support \& Training

\subsection{Causal Quantification and Simulation (Goal 3 \& 4)}

The designed mixed-method of this dissertation has not only expanded the UTAUT to contextualize elderly health technology adoption, it has quantified the strength of the various drivers and influences in the EWHTA context studied. It additionally, found that the degree of causal relationships of the identified drivers and barriers are different among clusters of elderly. The application of the method in the case study, identified two clusters-socially isolated and connected-in which the degree of the influences of drivers and influences are similar. This led to the creation of the two FCMisolated and FCMconnected models. The possibility of FCM clustering was not anticipated and therefore was not originally considered as a 
research step in the proposed method. However, the quantitative nature of FCM structure (value of edge weights) hinted the variability of the models that demanded clustering. Given the graph nature of FCM models, Network Analysis techniques were conducted. The Eigenvector Similarity Index (ESI) found two clusters of similar FCM models; and further analysis of the two clusters found the common theme among the two clusters to be the degree in which each group is socially connected/isolated. As a result an additional step is added to the research method in which Network Analysis (SNA) is carried to study the emerged FCM maps to identify potential clustering of the models. The new enhanced mixedmethods research method is illustrated in Figure 8 - 21 Figure 8 - 21 Revised Research Method), which summarizes the details steps, conducted in the case study as detailed in chapter 6 .

Despite the initial assumption, the case study found that the causal influences of the emerged drivers and barriers of the adoption are different among elderly. Conducting Network Analysis (as detailed in section 6.4.) identified two cluster of models in which the degrees of influences are different which subsequently they integrated in the two models. These quantitative variations are captured by the different edge weights in the FCM models' adjacency matrices in Appendix E.V and E.VI. These matrices represents adoption drivers and gatekeeper influences on adoption quantitatively and can be easily simulated using any tool that can conduct matrix multiplication, including MS Excel or software packages. As depicted in the earlier chapters (Chapter 6, section 6.4 , and chapter 7 ) these quantitative models allowed 


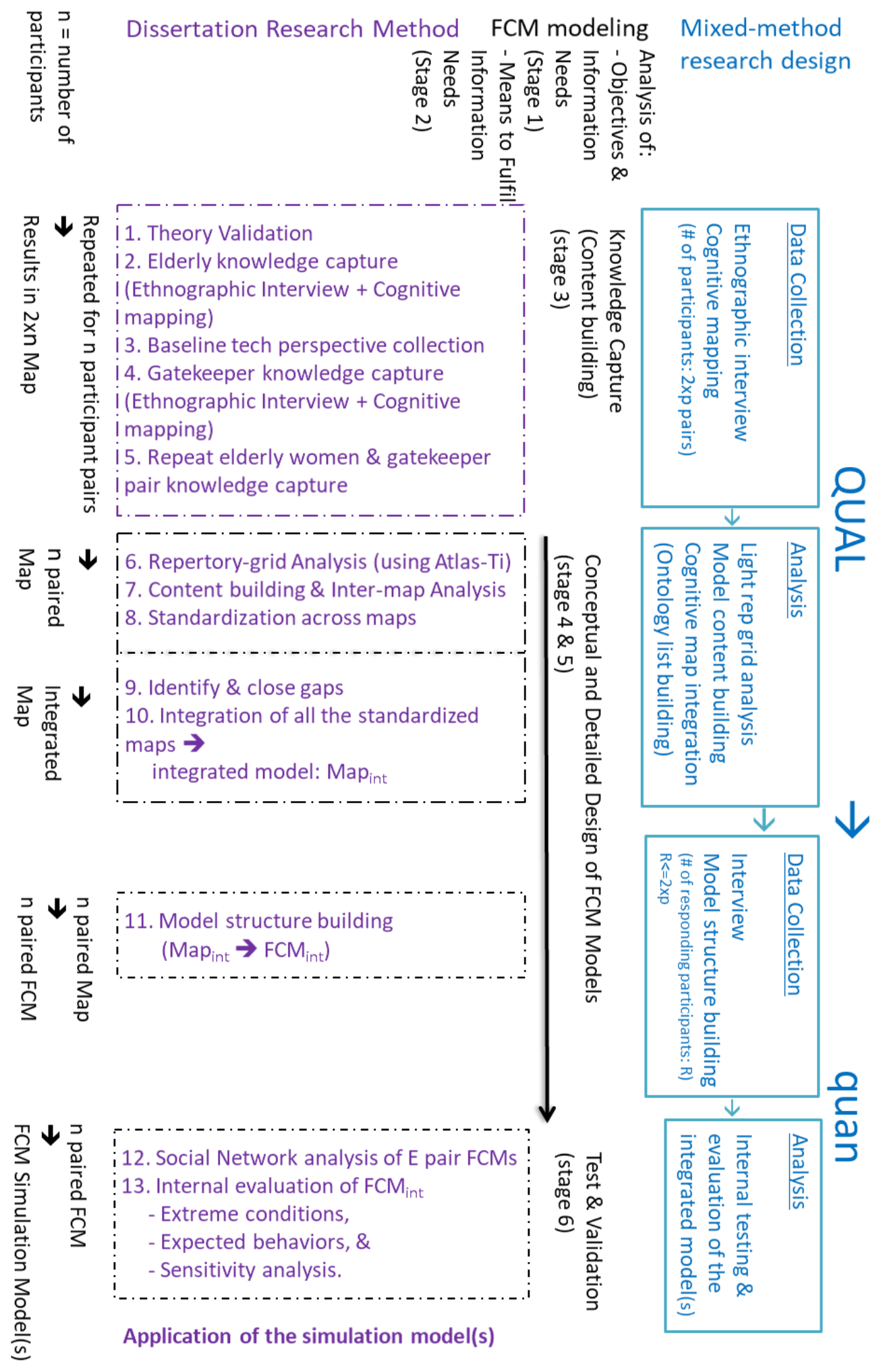

Figure 8 - 21 Revised Research Method 
many scenarios to be simulated. The resulted values provided a means to explore how different degrees of various factors influenced the elderly women intention and usage of an activity tracker wearable. As became apparent, better gatekeeping support and advocacy affect the elderly women usage of the wearable more than improvement in the product characteristics.

\subsection{Summary of Results}

This chapter provided detailed information that successfully addressed the four research goals laid out in chapter 2. The proposed research method was applied to the case of EWHTA which resulted in two FCM models. These two FCM models provide detail insights into the elderly women decision making by 1) mapping out the salient drivers and obstacles of their health technology adoptions, and 2) by facilitating scenario planning and simulation which helps answer 'What-if?' questions. By doing so, the conducted case study proved that my research delivers a robust health technology adoption modeling method that can readily be applied to the study of the elderly. The method yields a simulation model that can be applied to the study of essentially any group of elderly people. 


\section{Conclusion, Contribution, Limitations and Future Research}

\subsection{Conclusion}

\subsubsection{Method Conclusion}

First, this dissertation delivered a soft analytics method, which contextualizes theoretical insights from health technology adoption by combining ethnographic interviews and FCM (Chapter 3). It integrates the stakeholders' views, overcomes data scarcity, and provides specificity to health technology adoption theory. The empirical grounding for this method is provided by a case study, in which elderly women decide whether to adopt a wearable remote health monitoring technology (Chapter 5 and 6).

Second, from conducting the successful case study with its resulting fruitful models, it is concluded that the designed method is a robust research approach for studying elderly health technology adoption. The research method was further enhanced by adding a research step to conduct Network Analysis to find possible clustering of the models.

Third, based on the highlighted need, this dissertation, designed a method that delivered the means to discover hyper-segments of a consumer market. The method is based on the consumers' mental model, and it shows how consumers adopt RHMT. The clustering technique used in the designed methods is not biased to any conventional market segmentation on how to micro-segment consumers; it 
allows the consumer judgment to become the criterion for clustering. This became possible through the quantification of the influences in Fuzzy Cognitive Mapping.

Fourth, the careful selection of the effective techniques in the designed method combines the advantages of qualitative research and quantitative research. This synergistic combination provides the means for the resulting models to capture deep insights of the context studied and facilitate exploring the adoption using scenario planning and simulation.

Fifth, this dissertation proved the usefulness of fuzzy cognitive map as a modeling method to contextualize technology adoption. By doing so, this dissertation pioneered the use of fuzzy cognitive mapping in modeling health technology adoption outside organizational contexts. FCM computational power allowed exploration of adoption behavior in response to various factors.

Sixth, the designed method substantiated the usefulness of fuzzy cognitive mapping as a modeling method to study health technology adoption in the consumer market. The inherent network nature of the FCM model enabled the utilization of the emerging Network Analysis techniques to facilitate consumer market segmentation.

\subsubsection{Theoretical Conclusion in the Case of Elderly Women Health Technology Adoption of RHMT Wearables}

First, this dissertation found social isolation as a significant psychosocial determinant of technology adoption decision making to the point that isolated 
elderly women have a different decision-making model as those of the connected elderly women. This is in line with other health behavioral studies (e.g., Giabbanelli et al., 2012). Now it also known in EHTA.

Second, the application of the designed method found an array of drivers and inhibitors that are salient to EWHTA. As detailed in Chapter 8, 63 concepts among the socially isolated EW, and 62 concepts among the socially connected EW were found. These concepts grouped into six concept categories in the context of EWHTA-environmental factors, environmental influences, technology characteristics, personal traits, elderly perceptions, adoption outcomes.

Third, Environmental factors are very important in EWHTA. Gatekeeping influences exerted by the elderly women's family are essential in the elderly women health technology adoption. Additionally, Elderly women repeatedly state that if their clinician recommend using the wearable, they will adopt it. This highlights the strength of the clinician's influence in the elderly's health related decision-making. Therefore, clinician endorsement is highlighted as one of the effective strategies to promote and encourage the use of the technology.

Fourth, Cost remains to be an important factor in EWHTA decision which is missing from UTAUT (because it was conceptualized for organizational TA). Removing the cost factor (e.g. given by the insurance) is a big step toward their adoption.

Fifth, Experience, Self-efficacy, need, attitude toward tech, gatekeeper network that provide the effective facilitating conditions are some of the most salient drivers of adoption as aging and cognitive decline is the most salient obstacle of EWHTA. 
This explains why elderly women who have close nearby tech savvy family enjoy the best adoption rate.

Sixth, the case study illustrates that environmental factors immensely affect the elderly women's adoption behavior. Gatekeeping effects in particular have a colossal impact on the elderly women's perception, willingness to use and actual usage of the RHMT wearable. The group of concepts pertaining to family gatekeeping reaches the pinnacle of environmental influences. All these environmental factors increase elderly women's voluntariness of use, product awareness, social learning, exposure to the product, and ability to use the technology. The environmental influence group of concepts demonstrates the many possible ways that these gatekeepers exert their influences as described below.

Gatekeepers often suggest and/or introduce the product and encourage their use. This influence is stronger from the group of family gatekeeping concepts, when compared to the influence exerted from the non-family gatekeeping concepts. In other words, family gatekeeping influences are stronger causal factors in adoption, and those elderly with family have a higher health technology adoption rate.

Gatekeepers often provide social learning to the elderly by the very usage of the product themselves.

Usually only the family gatekeepers initiate the process of technology adoption by gifting new or hands-me-down technologies. 
Often the gatekeepers are EW's family members, and, in most cases, these are the middle-aged daughters who are very busy with many responsibilities. They often become the reason for the technology adoption, particularly where using the wearable can give the family peace of mind and reduce the burden on them. As these two categories of influences are observed among elderly with family gatekeepers, these influences contribute to the higher adoption rate among the elderly women with family gatekeepers.

EW, particularly those who are close to their family members and are regularly visited by them, like to keep up with the family and create a sense of connection to these loved ones' world, which is filled with technologies. Hence, they become motivated to use the technology to better connect to their loved ones. While this is more pronounced with EW with grandchildren, the effect still exists with other family members and, to a lesser degree, with her social circle at large.

The most important influence of the gatekeepers is the support and training they provide to the elderly. The gatekeepers and, more than anyone, the gatekeepers within the family provide the most effective form of support to the elderly women. Training and support are one-on-one and tailored to the needs and level of knowledge of the EW, and they are continuously and conveniently provided to her. Additionally, she is comfortable asking for help from these gatekeepers, as their instructions are jargon free and the EW doesn't worry about asking naïve questions. 
The more official means of support and training—either fee-based or free-are the other forms of support and training for EW. These channels are particularly important for those with weaker other gatekeeping support. The existence of these channels becomes ineffective for those elderly who are extremely isolated and often too depressed or separated from the society to reach out to any technology support. However, in the case of isolated EW who are less separated or depressed or have higher self-efficacy, these support channels have direct influence in their adoption decision.

\subsection{Contribution}

\subsubsection{Academic Contribution}

The main contribution of this dissertation is the proposed modeling method. As detailed in chapter 2, the literature highlights a gap in research methods for contextualizing highly generalized technology adoption theories. This dissertation responds to the call to arms for innovative research methods that can model technology adoption based on the specifics of the context being studied. This is particularly compelling in the field of EWHTA, where the pertinent extant literature cannot explain why elderly women choose not to adopt technology that would improve their personal condition.

This dissertation has proposed a novel research method that facilitates the creation of contextualized models that can explain EWHTA. This method can be used by academics, but its primary intent is to provide a consumer marketing 
research tool for technology management groups that need to study the elderly consumer market. While this method has been applied to the context of EWHTA, it is designed to be applied to other cases of EHTA and even HTA in general. Additionally, the method is not fundamentally restricted to any particular context.

Furthermore, the method designed in the dissertation provides the ability to quantify the degrees in which each of the drivers and barriers influence each other and ultimately influence adoption outcome. This provides a tool that can offer fresh insights into the context being studied.

First, the research method conceptualized in this dissertation is an effective empirical modeling method that adds to the method pluralism demanded in the HTA literature (as described in Chapter 2). This was proven by both the pilot study (detailed in Chapter 4 and Appendix B) and the case study research (detailed in Chapter 5 through 7) that was conducted in this dissertation.

Second, this dissertation successfully responded to the call to arms for conceptualizing empirical research methods that contribute to the plurality of empirical EHTA research methods. The research method contributes to the body of health technology adoption research by translating abstract theoretical insights into models that represent any case in the EHTA domain. The resulting models can help close the gap between theory and practice and provide detailed actionable insights to practitioners. In doing this, the conceptualized research method delivered a successful means of utilizing FCM by building quantitative simulation models based on in-depth qualitative techniques. In doing so, it 
extended the current health technology adoption research, and created a new way to discover much-needed practical insights to EHTA body of research.

Third, the design research method used in-depth qualitative techniques to build holistic FCM simulation models. By doing this, the method brings FCM research back to its origins of cognitive mapping. It also applies FCM research to health technology adoption in the elderly consumer market, a domain new to the FCM body of research. In doing this, the method extends the current FCM body of research.

Fourth, the resulting FCM models reached the four research goals by opening the black box of elderly women health technology adoption. The models...

1. Identify the key adoption drivers and obstacles and their relations, as it did in the case study by the emergence of the 63 concepts in six overall categories.

2. Illustrate how the gatekeeper influences the elderly person's adoption decision, as it did in the case study by highlighting the eight ways described.

3. Represent adoption drivers and gatekeeper influences on adoption quantitatively. The models in the case study showed these drivers for the two groups of the elderly women. The model also showed how strong each of these drivers and influences were.

4. Facilitate exploration of adoption in response to various factors through simulation, as the case study FCM models have demonstrated. Both the FCM Isolated and FCMconnected simulated many extreme conditions and expected behaviors to evaluate whether their behaviors are indeed in line with those of 
reality. The models further simulated ranges of assumptions to ensure their conclusions are not changed significantly (Chapter 6). Additionally, the models simulated adoption outcomes for different product characteristics, as well as for different levels and types of social support (Chapter 7).

Managers in charge of developing new RHMT products and their rollout strategies have evaluated the models and considered them 'insightful decision support models'. These experts stated that the models can help identify suitable feature sets based on offering a deep understanding of the decision-making dynamics by the elderly (Section 6.5).

Fifth, the method that was developed and tested in this dissertation has generated models that have identified two market segments for EWHTA—socially isolated elderly women and socially well-connected elderly women. However, in principle, the method could be applied to studying the adoption behavior of any group of individuals within their particular immediate social context. The method would first generate maps of the mental models of individuals, which integrate perspectives of the individual and his/her primary influencers. These models could be subsequently analyzed and integrated into one or more composite model, which reflects the aggregated perspective of all the constituent individual models. This composite model then characterizes the adoption behavior of the whole group.

Sixth, as the case study in this dissertation has shown, the method may generate multiple clusters of mental models, where each cluster reflects a different adoption 
pattern. Generating a composite model for each cluster may identify a market segment that is based on the adoption behavior that the composite model reflects.

In principle, this approach can be extended to analyze hyper-segmented markets. The method could be applied to a large group of consumers, and a multitude of cluster models can result from this effort. Each of these models would represent a hyper-segment of the market.

\subsubsection{Contributions to Management Practice}

First, the application of the designed method in the context of EWHTA of wearable activity trackers found an array of insights that were acknowledged by experts and practitioners in EHTA. This application particularly shows the importance technology gatekeepers and highlights the importance of including gatekeepers as a unit of study in the study of EHTA, which current health research addresses insufficiently (Chapter 2). This research suggests that practitioner and those conducting elderly consumer market of RHMT wearables to be aware of various gatekeeping influences and consider them in their planning and strategies. For example, as one of the experts who evaluated the FCM models mentioned, good commercials of such technologies should advertise wearables to the family members of elderly women.

Second, the research method developed in this dissertation intends to provide a better alternative to the conventional market studies by guiding consumer research. The research method yields visual model(s) that not only illustrate(s) but 
also simulate(s) practical insights for technology decision makers. The insights gained from the models help marketers explore the dynamics of the consumer market with various environmental factors in which elderly adopt RHMT technology. As the research results in simulation models, they can be reused repeatedly and support future planning and applications.

Third, the models from applying the designed method provides the practitioner ability to quantify the degrees in which each of the drivers and barriers influence each other and the overall adoption. This provides a tool to help decision makers explore the adoption in different settings and for different market segments of the elderly to gain actionable insights. The model helps the technology decision makers in several ways. New product developers can use this model as a simulation tool that explores possible scenarios for adoption, allowing them to determine specifications that improve the adoption rate of the product. Health technology delivery managers can use the model to plan a technology rollout that achieves a more successful adoption. For example, the model could serve as a guide for planning a successful training program for the users of the product, as it did in the case study.

Fourth, it should be noted that the arguments about generalizability from the previous section are not restricted to theory. The method developed in this dissertation is largely independent of context. Thus, practitioners can apply the method to identify market segments and product features, as well as developing product rollout strategies. For example, one of the SMEs that were interviewed for 
the evaluation of the usefulness of the research method mentioned that it would have been great if we could identify hyper-segments of the EW consumer market. This hyper-segmentation could help him cater toward their needs and preferences in a way that the elderly would not reject the wearable. Such market segmentation based on the cluster of adoption patterns occurred organically in the case study, which showed that the EWHTA is different based on psychosocial factors. The hyper-segmentation could happen should the participants represent those detailed segments.

\subsection{Limitations and Future Research}

Like any other research, this dissertation is subject to limitations, which can be overcome by further research. A list of limitations is provided in this section.

\subsubsection{Research Method Limitation}

First, the comprehensive nature of the mixed-method approach in the proposed research method, and particularly the dominant qualitative phase, is labor intensive. Although the resulting method provides an efficient reusable model for the context of the study, creating the models is not a trivial task. Interviews are time-consuming, as are multiple rounds of coding and re-coding, which are needed to capture the breadth of insights while also standardizing the meaning of concepts. Several researchers are currently working on online-based data acquisition tools for FCM (Giabbanelli \& Tawfik, 2017; Pfaff et al., 2015). While they will likely not replicate the exploratory depth of ethnographic interviewing and qualitative analysis of the proposed research method, these approaches may 291 
prove to be less labor intensive. This will make it possible to scale FCM projects to more participants without extensive effort.

Second, the model and simulation scenarios were designed to enhance our understanding of the role of the informal gatekeeper and on his/her impact on adoption, given different product feature sets. Future research may also want to include formal gatekeepers, such as healthcare professionals. These gatekeepers, according to comments during ethnographic interviews (as captured in the models), exert immense influence on EWHTA. To achieve a better understanding of technology adoption among a group of elderly and gatekeepers, this interplay of influence can be modeled by combining FCM and agent-based modeling, as proposed by (Giabbanelli, Gray, \& Aminpour, 2017). The elderly, their informal gatekeepers and medical professionals can each be represented by group-specific FCM that encode the behavior of the agents. The agent-based model defines the rules of interactions between the agents, thus shaping an artificial market (Zenobia et al., 2009).

Third, the research method focused on the elderly and the technology gatekeepers to facilitate the modeling of the elderly woman's decision making and hence her perceptions. This naturally excluded the dynamics between different and sometimes contradictory product characteristics (such as the relation between battery life of and active display of information on the wearable). Future work can explore tradeoffs between different features, the impact of different training interventions, and outcomes that are contingent upon social support provided at 
different stages of the adoption process. By uncovering these fundamental dynamics of technology adoption, this follow-on research could enhance the ability of analysts of big data (e.g. tracked RMHT usage data) to ascribe meaning to their analyses.

Fourth, a potential drawback of the resulting FCM model(s) could be a lack of flexibility, which may make it impossible to simulate the outcomes of innovative product designs or rollout strategies that were unknown at the model building stage (i.e. they don't involve the concepts captured in the model). Some of these limitations can be overcome by follow-on studies that add the missing concepts or links. However, this proposition requires further testing.

\subsubsection{Research Model Limitation}

The case study demonstrated and validated the research method by applying it to the case of EWHTA. The resulting models are subject to limitations that can be addressed by future research. Some of these limitations are listed below.

First, the case study research has been conducted with female participants. This was in response to the TA literature suggesting that the adoption patterns of the two genders are different and need to be studied separately to better understand intricacies. Thus, the models that resulted from this dissertation cannot be considered validated for elderly men. This deficiency can be corrected by repeating the research in a population of male elderly.

Second, the proposed research has been conducted mainly with participants and respondents of the ORCATECH Life Lab program. These respondents often 293 
represent a population of middle-class residents of Portland, Oregon, USA. However, the proposed research method should, in principle, be applicable to any EHTA context (e.g., different socio-economic groups, regions and countries). The research method would have to be validated in other contexts for which an application is under consideration.

Third, the case study in this dissertation focused on wearable technologies in the context of EWHTA research. In principle, the research method can be applied to any health technology whose adoption is optional. Thus, the proposed research method should not be used in applications where adoption is not voluntary.

Fourth, the research method is based on Fuzzy Cognitive Mapping. Thus, all the limitations of fuzzy cognitive mapping inherently apply to this research method. For example, FCM cannot capture temporal lags. One particular concept may thus influence downstream concepts at different times (one link could be activated within a minute; another within a year). Approaches to working around this limitation have been suggested (e.g. Jetter, 2006). However, these approaches have not been validated in the proposed research method. 


\section{References}

Abiola, O. Y. (2016). The Moderator Effect Of Income And Social Status, Social Connectedness And Physical Activity On Healthy Ageing Amongst Older Adults In Ibadan Metropolis, Nigeria. African Journal For The Psychological Studies Of Social Issues, 19(2), 135-149.

AbuShanab, E., Pearson, J. M., \& Setterstrom, A. J. (2010). Internet banking and customers' acceptance in Jordan: the unified model's perspective. Communications of the Association for Information Systems, 26(1), 23.

Advanced Remote Patient Monitoring Systems : Market Research Report. (2013). Retrieved from http://www.kaloramainformation.com/Advanced-Remote-Patient$7450566 /$

Agar, M. (1986). Speaking of ethnography (Vol. 2). London: Sage.

Agency for Healthcare Research \& Quality. (2017). Retrieved March 23, 2017, from https://www.ahrq.gov/

Aggelidis, V. P., \& Chatzoglou, P. D. (2009). Using a modified technology acceptance model in hospitals. International Journal of Medical Informatics, 78(2), 115-126.

Aging, N. I. on. (2011, July 12). There's No Place Like Home - For Growing Old. Retrieved October 9, 2016, from https://www.nia.nih.gov/health/publication/theres-no-place-home-growing-old Ajzen, I. (1985). From Intentions to Actions: A Theory of Planned Behavior. In P. D. J. Kuhl \& D. J. Beckmann (Eds.), Action Control (pp. 11-39). Springer Berlin 
Heidelberg. Retrieved from

http://link.springer.com.proxy.lib.pdx.edu/chapter/10.1007/978-3-642-69746-3_2

Albarracín, D., Gillette, J. C., Earl, A. N., Glasman, L. R., Durantini, M. R., \& Ho, M.-H. (2005). A Test of Major Assumptions About Behavior Change: A Comprehensive Look at the Effects of Passive and Active HIV-Prevention Interventions Since the Beginning of the Epidemic. Psychological Bulletin, 131(6), 856-897. https://doi.org/10.1037/0033-2909.131.6.856

Alburquerque-Sendin, F., Barberio-Mariano, E., Brandao-Santana, N., Rebelatto, D. A. N., \& Rebelatto, J. R. (2012). Effects of an adapted physical activity program on the physical condition of elderly women: an analysis of efficiency. Revista Brasileira De Fisioterapia, 16(4), 328-336.

Al-Busaidi, Z. Q. (2008). Qualitative research and its uses in health care. Sultan Qaboos University Medical Journal, 8(1), 11-19.

Alizadeh, Y., \& Jetter, A. (2017). Content Analysis using Fuzzy Cognitive Map (FCM), A Guide to Capturing Causal Relationships from Secondary Sources of Data. Presented at the PICMET, Portland, OR, USA.

Allen, T. J. (1977). Managing the flow of technology: technology transfer and the dissemination of technological information within the $\mathrm{R}$ and $\mathrm{D}$ organization. Retrieved from http://www.osti.gov/scitech/biblio/7100210

Amer, M., Daim, T. U., \& Jetter, A. (2013). A review of scenario planning. Futures, 46, 23-40.

Aminpour, P. PyFCM: python for fuzzy cognitive mapping. (2018). 
Apple's Ive reveals design secrets. (2009). Retrieved November 28, 2016, from http://www.macworld.com/article/1141509/jonathan_ive_london.html

Arning, K., \& Ziefle, M. (2007). Understanding age differences in PDA acceptance and performance. Computers in Human Behavior, 23(6), 2904-2927.

Association for Computing Machinery. (2017). Retrieved January 4, 2017, from http://www.acm.org/

ATLAS.ti 7 Windows | Qualitative Data Analysis with ATLAS.ti. (2017). Retrieved March 1, 2017, from http://atlasti.com/product/v7-windows/

Bagozzi, R. P. (2007a). The Legacy of the Technology Acceptance Model and a Proposal for a Paradigm Shift. Journal of the Association for Information Systems, 8(4), 3.

Bagozzi, R. P. (2007b). The Legacy of the Technology Acceptance Model and a Proposal for a Paradigm Shift. Journal of the Association for Information Systems, 8(4), 3.

Bainbridge, L., \& Sanderson, P. (1995). Verbal protocol analysis (Vol. 7). ch. Retrieved from https://books.google.com/books?hl=en\&lr=\&id=_Uq-hONFDUC\&oi=fnd\&pg=PA169\&dq=Bainbridge+and+Sanderson+verbal+protoc ol+analysis\&ots=JGaqBOvGzv\&sig=CIhLXfbIHdzDvROQLEB7ivWsrw4

Balogh, A. P., Siakas, K. V., Koinig, S., Ekert, D., Coakley, D., Colomo-Palacios, R., \& Kostoglou, V. (2013). Social Media Networker: A New Profile for a New Market. In T. Matsuo \& R. Colomo-Palacios (Eds.), Electronic Business and Marketing (pp. 137-146). Springer Berlin Heidelberg. Retrieved from http://link.springer.com.proxy.lib.pdx.edu/chapter/10.1007/978-3-642-37932$1 \_10$ 
Banaee, H., Ahmed, M. U., \& Loutfi, A. (2013). Data mining for wearable sensors in health monitoring systems: a review of recent trends and challenges. Sensors, 13(12), 17472-17500.

Bandura, A. (1986). Social foundations of thought and action. Englewood Cliffs, NJ Prentice Hall.

Bannon, L. (1991). From human factors to human actors: The role of psychology and human-computer interaction studies in system design. Design at Work: Cooperative Design of Computer Systems, 25-44.

Baumgartner, H., Pieters, R., \& Bagozzi, R. P. (2008). Future-oriented emotions: conceptualization and behavioral effects. European Journal of Social Psychology, 38(4), 685-696.

BBC News. (2012). How tech can help the elderly stay independent. Retrieved October 9, 2016, from http://news.bbc.co.uk/2/hi/programmes/click_online/9745189.stm

Best, R., Souders, D. J., Charness, N., Mitzner, T. L., \& Rogers, W. A. (2015). The Role of Health Status in Older Adults' Perceptions of the Usefulness of eHealth Technology. In International Conference on Human Aspects of IT for the Aged Population (pp. 3-14). Springer. Retrieved from http://link.springer.com/chapter/10.1007/978-3-319-20913-5_1

Bhate, K. (2012). An investigation of the influence of customer perceived-value, company brand equity and loyalty/rewards programs of OEM (original equipment manufacturer) inkject supplies on consumer buying behaviour to promote purchasing of OEM supplies. Dublin Business School. Retrieved from http://esource.dbs.ie/handle/10788/356 
Bouma, H., Fozard, J. L., Bouwhuis, D. G., \& Taipale, V. T. (2007). Gerontechnology in perspective. Gerontechnology, 6(4), 190-216.

Bowers, L. A. (2013). Remote patient-monitoring technology still faces reimbursement roadblock. Retrieved October 9, 2016, from http://www.ltlmagazine.com/article/remote-patient-monitoring-technology-stillfaces-reimbursement-roadblock

Bowman, S., Hooker, K., Steggell, C. D., \& Brandt, J. (2013). Perceptions of Communication and Monitoring Technologies Among Older Rural Women: Problem or Panacea? Journal of Housing For the Elderly, 27(1-2), 48-60. https://doi.org/10.1080/02763893.2012.754814

Boyle, T. A. (2005). Improving team performance using repertory grids. Team Performance Management: An International Journal, 11(5/6), 179-187.

Bueno, S., \& Salmeron, J. L. (2009). Benchmarking main activation functions in fuzzy cognitive maps. Expert Systems with Applications, 36(3, Part 1), 5221-5229. https://doi.org/10.1016/j.eswa.2008.06.072

Bull, R., \& Memon, A. (2000). Handbook of the psychology of interviewing. Wiley. Cahill, E., Lewis, L. M., Barg, F. K., \& Bogner, H. R. (2009). “You Don’t Want to Burden Them" Older Adults' Views on Family Involvement in Care. Journal of Family Nursing, 15(3), 295-317. https://doi.org/10.1177/1074840709337247

Callahan, D. (2008). health care costs and medical technology. The Hastings Center. Retrieved from http://wvvw.thehastingscenter.org/uploadedFiles/Publications/Briefing_Book/heal th\%20care $\% 20$ costs $\% 20$ chapter.pdf 
Caputi, P., Viney, L. L., Walker, B. M., \& Crittenden, N. (2011). Personal construct methodology. John Wiley \& Sons. Retrieved from https://books.google.com/books?hl=en\&lr=\&id=7Y62SkKRriIC\&oi=fnd\&pg=PT $8 \& \mathrm{dq}=$ Gaines, + Brian+R.\%3B+Shaw,+Mildred+L.G.+(2011).+\%22Computeraided+constructivism\%22.+In+Caputi,+Peter\%3B+Viney,+Linda+L.\%3B+Walke r,+Beverly+M.\%3B+Crittenden,+Nadia.+Personal+construct+methodology.+Mal den,+MA:+John+Wiley+\%26+Sons\&ots=YY2cVebt4-

\&sig=qrHb_JfBbnewOifintU-tD6zNVI

Caregiving in the U.S. 2015. (2015). United States. Retrieved from http://www.caregiving.org/wpcontent/uploads/2015/05/2015_CaregivingintheUS_Final-Report-June4_WEB.pdf

Carley, K. (1993). Coding Choices for Textual Analysis: A Comparison of Content Analysis and Map Analysis. Sociological Methodology, 23, 75-126. https://doi.org/10.2307/271007

Carpenter, B. D., \& Buday, S. (2007). Computer use among older adults in a naturally occurring retirement community. Computers in Human Behavior, 23(6), 30123024.

Celler, B. G., Sparks, R., Nepal, S., Alem, L., Varnfield, M., Li, J., ... Jayasena, R. (2014). Design of a multi-site multi-state clinical trial of home monitoring of chronic disease in the community in Australia. BMC Public Health, 14. https://doi.org/10.1186/1471-2458-14-1270 
Cerella, J., Poon, L. W., \& Fozard, J. L. (1982). Age and iconic read-out. Journal of Gerontology, 37(2), 197-202.

Chalmers, A. F. (1982). What is this thing called science? : an assessment of the nature and status of science and its methods (2nd ed..). StLucia, Qld: University of Queensland Press.

Chappell, N. L. (1999). Receptivity to new technology among older adults. Disability \& Rehabilitation, 21(5-6), 222-230.

Chau, P. Y. K., \& Hu, P. J.-H. (2002). Investigating healthcare professionals' decisions to accept telemedicine technology: an empirical test of competing theories. Information \& Management, 39(4), 297-311. https://doi.org/10.1016/S03787206(01)00098-2

Chaurasia, P., McClean, S. I., Nugent, C. D., Cleland, I., Zhang, S., Donnelly, M. P., ... Tschanz, J. (2016). Modelling assistive technology adoption for people with dementia. Journal of Biomedical Informatics, 63, 235-248.

https://doi.org/10.1016/j.jbi.2016.08.021

Chen, K., \& Chan, A. H. (2013). Use or Non-Use of Gerontechnology—A Qualitative Study. International Journal of Environmental Research and Public Health, 10(10), 4645-4666.

Chen, K., \& Chan, A. H. S. (2011). A review of technology acceptance by older adults. Gerontechnology, 10(1), 1-12.

CHI 2016. (2017). Retrieved January 4, 2017, from https://chi2016.acm.org/wp/ 
Christensen, M. C., \& Remler, D. (2009). Information and communications technology in US health care: why is adoption so slow and is slower better? Journal of Health Politics, Policy and Law, 34(6), 1011-1034.

Cimperman, M., Brenčič, M. M., Trkman, P., \& de Leonni Stanonik, M. (2013). Older Adults' Perceptions of Home Telehealth Services. Telemedicine Journal and EHealth, 19(10), 786-790. https://doi.org/10.1089/tmj.2012.0272

Clore, G. L., \& Huntsinger, J. R. (2007). How emotions inform judgment and regulate thought. Trends in Cognitive Sciences, 11(9), 393-399.

Compagna, D., \& Kohlbacher, F. (2014). The limits of participatory technology development: The case of service robots in care facilities for older people. Technological Forecasting and Social Change. Retrieved from http://www.sciencedirect.com/science/article/pii/S0040162514002376

Conci, M., Pianesi, F., \& Zancanaro, M. (2009). Useful, social and enjoyable: Mobile phone adoption by older people. In Human-Computer Interaction-INTERACT 2009 (pp. 63-76). Springer. Retrieved from http://link.springer.com/chapter/10.1007/978-3-642-03655-2_7

Cooper, L., Baber, C., Langford, J., \& McDonagh, D. (2003). Focused groups: scenariobased discussions. Focus Groups: Supporting Effective Product Development, 129.

Corlett, E. N., Wilson, J. R., \& CORLETT, N. (1995). Evaluation of Human Work, 2nd Edition (Chapter 7). CRC Press.

Council, N. R., \& others. (2009). Computational technology for effective health care: immediate steps and strategic directions. National Academies Press. Retrieved 302 
from

https://books.google.com/books?hl=en\&lr=\&id=HUAXQzIPxV8C\&oi=fnd\&pg= PT1\&dq=elderly+health+technology+adoption+interdisciplinary+research\&ots=S i9Vytz9Ke\&sig=U4zXMphOxDOm0kwqAr2dAeLJaWY

Coyle, C. E., Schulman-Green, D., Feder, S., Toraman, S., Prust, M. L., Plano Clark, V. L., \& Curry, L. (2016). Federal Funding for Mixed Methods Research in the Health Sciences in the United States: Recent Trends. Journal of Mixed Methods Research, 1558689816662578. https://doi.org/10.1177/1558689816662578

Creswell, J. W. (2012). Qualitative inquiry and research design: Choosing among five approaches. Sage. Retrieved from https://books.google.com/books?hl=en\&lr=\&id=Ykruxor10cYC\&oi=fnd\&pg=PR 1\&dq=Creswell,+J.+W.+(1998).+Qualitative+Inquiry+and+Research+Design:+C hoosing+Among+Five+Traditions. + Thousand+Oaks, + CA.:+Sage\&ots=4bk1kEnAn\&sig=srtYdLJOAM-Kaohk7BHF7_NxhFY

Creswell, J. W. (2013). Research design: Qualitative, quantitative, and mixed methods approaches. Sage publications. Retrieved from https://books.google.com/books?hl=en\&lr=\&id=EbogAQAAQBAJ\&oi=fnd\&pg= PR1\&dq=RESEARCH+DESIGN+Qualitative,+Quantitative,+and+Mixed+Metho ds+Approaches+JOHN+W .+CRESWELL+UNIVERSITY+OF+NEBRASKALINCOLN\&ots=caiOoWMuDc\&sig=esOyfMFJMG_PoBzQMdQRCAaEKLs

Crosby, R., \& Noar, S. M. (2010a). Theory development in health promotion: are we there yet? Journal of Behavioral Medicine, 33(4), 259-263. 
Crosby, R., \& Noar, S. M. (2010b). Theory development in health promotion: are we there yet? Journal of Behavioral Medicine, 33(4), 259-263.

Cugelman, B., Thelwall, M., \& Dawes, P. (2011). Online interventions for social marketing health behavior change campaigns: a meta-analysis of psychological architectures and adherence factors. Journal of Medical Internet Research, 13(1), e17.

Curry, L. A., Krumholz, H. M., O'cathain, A., Clark, V. L. P., Cherlin, E., \& Bradley, E. H. (2013). Mixed methods in biomedical and health services research. Circulation: Cardiovascular Quality and Outcomes, 6(1), 119-123.

Daley, A., Stokes-Lampard, H., Wilson, S., Rees, M., Roalfe, A., \& MacArthur, C. (2011). What women want? Exercise preferences of menopausal women. Maturitas, 68(2), 174-178.

Davis, D. M. (1995). Cellular Telephones and Pagers in Hawaii: Cultural Influences on Personal Use Patterns. Hawaii, USA: University of Hawaii. Retrieved from Department of Communication.

Davis, F. D. (1985). A technology acceptance model for empirically testing new end-user information systems: Theory and results. Massachusetts Institute of Technology. Retrieved from http://dspace.mit.edu.proxy.lib.pdx.edu/handle/1721.1/15192

Davis, F. D., Bagozzi, R. P., \& Warshaw, P. R. (1992). Extrinsic and intrinsic motivation to use computers in the workplace1. Journal of Applied Social Psychology, 22(14), 1111-1132. 
Deng, Z., Mo, X., \& Liu, S. (2014). Comparison of the middle-aged and older users' adoption of mobile health services in China. International Journal of Medical Informatics, 83(3), 210-224.

Dentinger, E., \& Clarkberg, M. (2002). Informal Caregiving and Retirement Timing among Men and Women Gender and Caregiving Relationships in Late Midlife. Journal of Family Issues, 23(7), 857-879. https://doi.org/10.1177/019251302236598

Denzin, N. K. (1978). The research act: A theoretical orientation to sociological methods. McGraw-Hill, New York._2012, 'Triangulation, 2, 80-88.

Dickerson, J., \& Kosko, B. (1994). Virtual words as Fuzzy Dynamical Systems. In Technology for Multimedia, B. Sheu (Ed.) (pp. 1-35). IEEE.

Dickinson, W. B. (2010). Visual displays for mixed methods findings. SAGE Handbook of Mixed Methods in Social \& Behavioral Research. 2nd Ed. Thousand Oaks, CA: Sage.

Dubois, D. J., Prade, H., \& Yager, R. R. (2014). B. Kosko, Adaptive Inference in Fuzzy Knowledge Networks. In Readings in fuzzy sets for intelligent systems. Morgan Kaufmann.

Dumas, J. S., \& Salzman, M. C. (2006). Usability assessment methods. Reviews of Human Factors and Ergonomics, 2(1), 109-140.

Dunn, W. N., Cahill, A. G., Dukes, M. J., \& Ginsberg, A. (1986). The policy grid: A cognitive methodology for assessing change dynamics. Policy Analysis: Perspectives, Concepts, and Methods. 
Dutta, S., \& Omolayole, O. (2016). Are There Differences Between Men and Women in Information Technology Innovation Adoption Behaviors: A Theoretical Study. The Journal of Business Diversity, 16(1), 106.

Duyck, P., Pynoo, B., Devolder, P., Voet, T., Adang, L., Ovaere, D., \& Vercruysse, J. (2010). Monitoring the PACS implementation process in a large university hospital—discrepancies between radiologists and physicians. Journal of Digital Imaging, 23(1), 73-80.

Dwivedi, Y. K., Rana, N. P., Chen, H., \& Williams, M. D. (2011). A Meta-analysis of the Unified Theory of Acceptance and Use of Technology (UTAUT). In Governance and Sustainability in Information Systems. Managing the Transfer and Diffusion of IT (pp. 155-170). Springer. Retrieved from http://link.springer.com/chapter/10.1007/978-3-642-24148-2_10

Eastman, J. K., \& Iyer, R. (2004). The elderly's uses and attitudes towards the Internet. Journal of Consumer Marketing, 21(3), 208-220.

Eggermont, S., Vandebosch, H., \& Steyaert, S. (2006). Towards the desired future of the elderly and ICT: Policy recommendations based on a dialogue with senior citizens. Poiesis \& Praxis, 4(3), 199-217.

Ehlers, D. K., \& Huberty, J. (2013). Middle-Aged Women's Preferred Theory-Based Features in Mobile Physical Activity Applications. Journal of Physical Activity \& Health. Retrieved from http://europepmc.org/abstract/med/24368818

eNeighbor® Remote Monitoring System| Healthsense Senior Care. (2014). Retrieved June 25, 2014, from http://www.healthsense.com/index.php/products/remotemonitoring/eneighbor 
EverWrist: A Novel Electronic Fall Monitor for Seniors | SBIR.gov. (2017). Retrieved March 3, 2017, from https://www.sbir.gov/sbirsearch/detail/388925

Evidence and Evaluation | PCMH Resource Center. (2017). Retrieved January 11, 2017, from https://pcmh.ahrq.gov/page/evidence-and-evaluation

Fife, E., \& Pereira, F. (2011). Digital home health and mHealth: Prospects and challenges for adoption in the U.S. In FITCE Congress (FITCE), 2011 50th (pp. 1-11). https://doi.org/10.1109/FITCE.2011.6133431

Fink, A., \& Beck, J. C. (2015). Developing and Evaluating a Website to Guide Older Adults in Their Health Information Searches A Mixed-Methods Approach. Journal of Applied Gerontology, 34(5), 633-651.

Finkelstein, S. M., Speedie, S. M., \& Potthoff, S. (2006). Home telehealth improves clinical outcomes at lower cost for home healthcare. Telemedicine Journal \& EHealth, 12(2), 128-136.

Fishbein, M., \& Ajzen, I. (1975). Belief, attitude, intention and behavior: An introduction to theory and research. Retrieved from http://trid.trb.org.proxy.lib.pdx.edu/view.aspx?id=1150648

Fishbein, M., \& Ajzen, I. (2011). Predicting and changing behavior: The reasoned action approach. Taylor \& Francis. Retrieved from http://books.google.com/books?hl=en\&lr=\&id=2rKXqb2ktPAC\&oi=fnd \&pg=PR $2 \& d q=$ Ajzen + fishbein + Theory + of + Reasoned + Action $+($ TRA $)+\& o t s=z 9 c q W H o p$ mm\&sig=OZf837VW3HdF99Vxh_9If4Axxf4 
Four Methods For Holistic Market Research. (2016). Retrieved February 24, 2017, from http://www.themarketingbureau.co.nz/_blog/Stories/post/Four_Methods_For_Hol istic_Market_Research/

Fozard, J. L. (2001). Gerontechnology and perceptual motor-function: New opportunities for prevention, compensation, and enhancement. Gerontechnology, 1(1), 5-24.

Fransson-Hall, C., Gloria, R., Kilbom, \AAsa, Winkel, J., Karlqvist, L., \& Wiktorin, C. (1995). A portable ergonomic observation method (PEO) for computerized online recording of postures and manual handling. Applied Ergonomics, 26(2), 93100.

Fried, T. R., Bradley, E. H., Williams, C. S., \& Tinetti, M. E. (2001). Functional disability and health care expenditures for older persons. Archives of Internal Medicine, 161(21), 2602-2607.

Gaines, B. R., \& Shaw, M. L. (1993). Knowledge acquisition tools based on personal construct psychology. The Knowledge Engineering Review, 8(01), 49-85.

Gaither, C. A., Bagozzi, R. P., Ascione, F. J., \& Kirking, D. M. (1996). A reasoned action approach to physicians' utilization of drug information sources. Pharmaceutical Research, 13(9), 1291-1298.

Garmann-Johnsen, N. (2015). CBPM in E-health. Universitetet i Agder, Kristiansand. Retrieved from https://www.researchgate.net/publication/287106434_CBPM_in_Ehealth_Exploring_and_Modelling_the_Role_of_InterOrganizational_Coordination_and_Collaborative_Business_Process_Management _in_e-Health_Innovation 
Gear S2 classic Wearables - SM-R7320ZKAXAR | Samsung US. (2018). Retrieved February 18, 2018, from http://www.samsung.com/us/mobile/wearables/smartwatches/samsung-gear-s2classic-black-sm-r7320zkaxar/

George Kelly. (1955). Retrieved December 21, 2016, from http://ksi.cpsc.ucalgary.ca/PCP/Kelly.html

Giabbanelli, P. J., Gray, S. A., \& Aminpour, P. (2017). Combining fuzzy cognitive maps with agent-based modeling: Frameworks and pitfalls of a powerful hybrid modeling approach to understand human-environment interactions. Environmental Modelling \& Software, 95, 320-325.

Giabbanelli, P. J., \& Tawfik, A. A. (2017). In Overcoming the PBL Assessment Challenge: Design and Development of the Incremental Thesaurus for Assessing Causal Maps (ITACM)| SpringerLink. Springer. Retrieved from https://link.springer.com/article/10.1007/s10758-017-9338-8

Giabbanelli, P. J., Torsney-Weir, T., \& Mago, V. K. (2012). A fuzzy cognitive map of the psychosocial determinants of obesity. Applied Soft Computing, 12(12), 37113724.

Ginsberg, A. (1989). Construing the business portfolio: A cognitive model of diversification. Journal of Management Studies, 26(4), 417-438.

Glanz, K., Rimer, B. K., \& Viswanath, K. (2008). Health behavior and health education: theory, research, and practice. John Wiley \& Sons. Retrieved from http://books.google.com.proxy.lib.pdx.edu/books?hl=en\&lr=\&id=WsHxyj710Ug C\&oi=fnd\&pg=PR5\&dq=Glanz, + K., + Rimer, + B. + K., $+\% 26+$ Viswanath,$+K .+(200$ 309 
8).+Theory,+research,+and+practice+in+health+behavior+and+health+education \&ots=EWNWQ8LtVM\&sig=f1t1beEeI9FDu24EXRJwPub8mwo

Glenn, J. C., \& Gordon, T. J. (2009). Futures Research Methodology-Version 3-0. Editorial desconocida. Retrieved from http://library.teachthefuture.org/wpcontent/uploads/2016/04/Futures-Research-Methodology-Version-3.pdf

Godin, G., \& Kok, G. (1996). The theory of planned behavior: a review of its applications to health-related behaviors. American Journal of Health Promotion, 11(2), 87-98.

GoSafe Medical Alert System | Philips Lifeline ${ }^{\circledR}$. (2015). Retrieved July 23, 2015, from http://www.lifelinesys.com/content/lifeline-products/get-life-gosafe

Grafton, J., Lillis, A. M., Grafton, J., Lillis, A. M., \& Mahama, H. (2011). Mixed methods research in accounting. Qualitative Research in Accounting \& Management, 8(1), 5-21.

Griffin, A., \& Hauser, J. R. (1993). The voice of the customer. Marketing Science, 12(1), $1-27$.

Guston, D. H., \& Sarewitz, D. (2002). Real-time technology assessment. Technology in Society, 24(1), 93-109.

Guynn, J. (2002). Internet connects seniors to rest of the world. Knight Ridder Tribune Business News, 19, 1.

Head, K. J., \& Noar, S. M. (2014). Facilitating progress in health behaviour theory development and modification: the reasoned action approach as a case study. Health Psychology Review, 8(1), 34-52. https://doi.org/10.1080/17437199.2013.778165 
HealthCare Manager: Remote Medication and Health Plan Support System. (2011).

Retrieved October 9, 2016, from https://www.sbir.gov/sbirsearch/detail/370285

HealthITAnalytics. (2016). Big Data, Artificial Intelligence, IoT May Change Healthcare in 2017. Retrieved January 16, 2017, from http://healthitanalytics.com/news/bigdata-artificial-intelligence-iot-may-change-healthcare-in-2017

Heart, T., \& Kalderon, E. (2013). Older adults: are they ready to adopt health-related ICT? International Journal of Medical Informatics, 82(11), e209-231. https://doi.org/10.1016/j.ijmedinf.2011.03.002

Hildebrandt, J., Brauner, P., \& Ziefle, M. (2015). Smart textiles as intuitive and ubiquitous user interfaces for smart homes. In International Conference on Human Aspects of IT for the Aged Population (pp. 423-434). Springer. Retrieved from http://link.springer.com/chapter/10.1007/978-3-319-20913-5_39

Holden, R. J., \& Karsh, B.-T. (2010). The Technology Acceptance Model: Its past and its future in health care. Journal of Biomedical Informatics, 43(1), 159-172. https://doi.org/10.1016/j.jbi.2009.07.002

Holtrop, J. S., Potworowski, G., Green, L. A., \& Fetters, M. (2016). Analysis of Novel Care Management Programs in Primary Care: An Example of Mixed Methods in Health Services Research. Journal of Mixed Methods Research, 1558689816668689. https://doi.org/10.1177/1558689816668689

Hooyman, \& Kiyak. (2011). Social Gerontology: A Multidisciplinary Perspective. Retrieved December 1, 2016, from https://www.pearsonhighered.com/program/Hooyman-Social-Gerontology-AMultidisciplinary-Perspective-9th-Edition/PGM110443.html 
Hoque, M., \& Sorwar, G. (2016). Factors Influencing mHealth Acceptance among Elderly People in Bangladesh. arXiv Preprint arXiv:1606.00874. Retrieved from http://arxiv.org/abs/1606.00874

Hornbaek, K. (2006). Current practice in measuring usability: Challenges to usability studies and research. International Journal of Human-Computer Studies, 64(2), 79-102.

Hossain, S., \& Brooks, L. (2008). Fuzzy cognitive map modelling educational software adoption. Computers \& Education, 51(4), 1569-1588.

Howell, T. H. (1987). Avicenna and His Regimen of Old Age. Age and Ageing, 16(1), 58-59. https://doi.org/10.1093/ageing/16.1.58

Human Computer Interaction. (2017). Retrieved January 4, 2017, from https://www.interaction-design.org/literature/book/the-encyclopedia-of-humancomputer-interaction-2nd-ed/human-computer-interaction-brief-intro

Human Factors - -Human Computer-Interaction : Psychology. (2017). Retrieved January 4, 2017, from https://psychology.rice.edu/Content.aspx?id=94

Human Factors vs Ergonomics - Are they the same? (2005). Retrieved January 4, 2017, from https://ergoweb.com/forums/topic/human-factors-vs-ergonomics-are-theythe-same/

human-factors engineering. (2016). Retrieved January 4, 2017, from https://www.britannica.com/topic/human-factors-engineering ISO 6385. (2004). Retrieved December 15, 2016, from https://www.iso.org/obp/ui/\#iso:std:iso:6385:ed-2:v1:en 
IV. Accomplishments on Targeted Activities. (2009). Retrieved March 23, 2017, from /research/findings/final-reports/nhpceval/4.html

Jetter, A., \& Schweinfort, W. (2011). Building scenarios with Fuzzy Cognitive Maps: An exploratory study of solar energy. Futures, 43(1), 52-66.

Jetter, A.J. (2006). Fuzzy Cognitive Maps for Engineering and Technology Management: What Works in Practice? In Technology Management for the Global Future, PICMET 2006 (Vol. 2, pp. 498-512).

Jetter, A.J., \& Kok, K. (2014). Fuzzy Cognitive Maps for futures studies-A methodological assessment of concepts and methods. Futures, 61, 45-57.

Jetter, Antonie J., \& Sperry, R. C. (2013). Fuzzy cognitive maps for product planning: using stakeholder knowledge to achieve corporate responsibility. In System Sciences (HICSS), 2013 46th Hawaii International Conference on (pp. 925-934). IEEE. Retrieved from http://ieeexplore.ieee.org.proxy.lib.pdx.edu/abstract/document/6479945/

Jick, T. D. (1979). Mixing qualitative and quantitative methods: Triangulation in action. Administrative Science Quarterly, 24(4), 602-611.

Johnson, R. (2011). Factors affecting intent to use consumer generic tests: a revised technology acceptance model. Retrieved from http://137.215.9.22/handle/2263/24001

Johnson, R. B., \& Onwuegbuzie, A. J. (2004). Mixed methods research: A research paradigm whose time has come. Educational Researcher, 33(7), 14-26.

Journal of Mixed Methods Research | SAGE Journals. (2017). Retrieved January 11, 2017, from http://journals.sagepub.com/home/mmr 
Kahn, H., Wiener, A. J., \& others. (1967). year 2000; a framework for speculation on the next thirty-three years. Retrieved from http://agris.fao.org/agrissearch/search.do?recordID=US201300314687

Karavidas, M., Lim, N. K., \& Katsikas, S. L. (2005). The effects of computers on older adult users. Computers in Human Behavior, 21(5), 697-711.

Karimi-Shahanjarini, A., Rashidian, A., Omidvar, N., \& Majdzadeh, R. (2013). Assessing and Comparing the Short-Term Effects of TPB Only and TPB Plus Implementation Intentions Interventions on Snacking Behavior in Iranian Adolescent Girls: A Cluster Randomized Trial. American Journal of Health Promotion, 27(3), 152-161. https://doi.org/10.4278/ajhp.110311-QUAN-113

Karsh, B.-T., Escoto, K. H., Beasley, J. W., \& Holden, R. J. (2006). Toward a theoretical approach to medical error reporting system research and design. Applied Ergonomics, 37(3), 283-295.

Katz, R., \& Tushman, M. (1981). An investigation into the managerial roles and career paths of gatekeepers and project supervisors in a major $\mathrm{R} \& \mathrm{D}$ facility. $R \& D$ Management, 11(3), 103-110. https://doi.org/10.1111/j.14679310.1981.tb00458.x

Khoumbati, K., Themistocleous, M., \& Irani, Z. (2006). Evaluating the adoption of enterprise application integration in health-care organizations. Journal of Management Information Systems, 22(4), 69-108.

Kincade, K. (1998). Data mining: digging for healthcare gold. Insurance \& Technology, 23(2), 2-7. 
Kline, D. W., \& Schieber, F. J. (1982). Visual persistence and temporal resolution. Aging and Human Visual Function, 231-244.

Kline, Donald W. (1987). Ageing and the spatiotemporal discrimination performance of the visual system. Eye, 1(2), 323-329.

Koh, H. C., Tan, G., \& others. (2011). Data mining applications in healthcare. Journal of Healthcare Information Management, 19(2), 65.

Kosko, B. (1986). Fuzzy cognitive maps. International Journal of Man-Machine Studies, $24(1), 65-75$.

Kosko, B. (1988). Hidden patterns in combined and adaptive knowledge networks. International Journal of Approximate Reasoning, 2(4), 377-393.

Kosko, B. (1992). Neural networks and fuzzy systems: a dynamical systems approach to machine intelligence. Diskette 2. Diskette. Prentice-Hall.

Koutra, D., Parikh, A., Ramdas, A., \& Xiang, J. (2011). Algorithms for graph similarity and subgraph matching. In Proc. Ecol. Inference Conf.

Kubeck, J. E., Delp, N. D., Haslett, T. K., \& McDaniel, M. A. (1996). Does job-related training performance decline with age? Psychology and Aging, 11(1), 92.

Kurniawan, S. (2007). Mobile phone design for older persons. Interactions, 14(4), 24-25.

Kwon, H. S., \& Chidambaram, L. (2000). A test of the technology acceptance model: the case of cellular telephone adoption. In System Sciences, 2000. Proceedings of the 33rd Annual Hawaii International Conference on (p. 7-pp). IEEE. Retrieved from http://ieeexplore.ieee.org/xpls/abs_all.jsp?arnumber=926607

Langley, A. (1999). Strategies for theorizing from process data. Academy of Management Review, 24(4), 691-710. 
Lee, J.-W. (2010). The roles of demographics on the perceptions of electronic commerce adoption. Academy of Marketing Studies Journal, 14(1), 71.

Lee, Y., Kozar, K. A., \& Larsen, K. R. (2003). The technology acceptance model: past, present, and future. Communications of the Association for Information Systems, 12(1), 50.

Lee, Y. S. (2007). Older Adults' User Experiences with Mobile Phones: Identification of User Clusters and User Requirements. Virginia Polytechnic Institute and State University. Retrieved from http://scholar.lib.vt.edu/theses/available/etd09172007-135013/

Leonard-Barton, D. (1990). A dual methodology for case studies: Synergistic use of a longitudinal single site with replicated multiple sites. Organization Science, 1(3), $248-266$.

Liddy, C., Dusseault, J. J., Dahrouge, S., Hogg, W., Lemelin, J., \& Humber, J. (2008). Telehomecare for patients with multiple chronic illnesses Pilot study. Canadian Family Physician, 54(1), 58-65.

Life Lab. (2014). Retrieved November 11, 2014, from http://www.ohsu.edu/xd/research/centers-institutes/orcatech/tech/life-lab.cfm

Lim, S., Xue, L., Yen, C. C., Chang, L., Chan, H. C., Tai, B. C., ... Choolani, M. (2011). A study on Singaporean women's acceptance of using mobile phones to seek health information. International Journal of Medical Informatics, 80(12), e189e202. 
Lin, Y.-C., Liang, J.-C., Yang, C.-J., \& Tsai, C.-C. (2013). Exploring middle-aged and older adults' sources of Internet self-efficacy: A case study. Computers in Human Behavior, 29(6), 2733-2743.

Ling, R. (2002). Adolescent girls and young adult men: Two sub-cultures of the mobile telephone. Revista de Estudios de Juventud, 52, 33-46.

Litan, R. E. (2008). Vital signs via broadband: Remote health monitoring transmits savings, enhances lives. Better Health Care Together. Retrieved from http://www.broadbandillinois.org/uploads/cms/documents/litan.pdf

Liu, Z.-Q. (2003). Fuzzy cognitive maps in GIS data analysis. Soft Computing, 7(6), 394401. https://doi.org/10.1007/s00500-002-0228-0

Loe, M. (2010). Doing it my way: old women, technology and wellbeing. Sociology of Health \& Illness, 32(2), 319-334.

Loe, M. (2014). Comfort and medical ambivalence in old age. Technological Forecasting and Social Change. Retrieved from http://www.sciencedirect.com/science/article/pii/S0040162514001346

Luijkx, K., Peek, S., \& Wouters, E. (2015). “Grandma, You Should Do It—It's Cool” Older Adults and the Role of Family Members in Their Acceptance of Technology. International Journal of Environmental Research and Public Health, 12(12), 15470-15485. https://doi.org/10.3390/ijerph121214999

Luo, X., Li, H., Zhang, J., \& Shim, J. P. (2010). Examining multi-dimensional trust and multi-faceted risk in initial acceptance of emerging technologies: An empirical study of mobile banking services. Decision Support Systems, 49(2), 222-234. 
Maier, C., Laumer, S., \& Eckhardt, A. (2011). Technology adoption by elderly people-an empirical analysis of adopters and non-adopters of social networking sites. In Theory-Guided Modeling and Empiricism in Information Systems Research (pp. 85-110). Springer. Retrieved from http://link.springer.com/chapter/10.1007/9783-7908-2781-1_5

Malhotra, Y., \& Galletta, D. F. (1999). Extending the technology acceptance model to account for social influence: Theoretical bases and empirical validation. In Systems Sciences, 1999. HICSS-32. Proceedings of the 32nd Annual Hawaii International Conference on (p. 14-pp). IEEE. Retrieved from http://ieeexplore.ieee.org/xpls/abs_all.jsp?arnumber=772658

Mallenius, S., Rossi, M., \& Tuunainen, V. K. (2007). Factors affecting the adoption and use of mobile devices and services by elderly people-results from a pilot study. 6th Annual Global Mobility Roundtable, 31. Retrieved from http://citeseerx.ist.psu.edu/viewdoc/download?doi=10.1.1.130.2463\&rep=rep1\&t ype $=$ pdf

Maniam, A., Dhillon, J. S., \& Baghaei, N. (2015). Determinants of patients' intention to adopt diabetes self-management applications. In Proceedings of the 15th New Zealand Conference on Human-Computer Interaction (pp. 43-50). ACM. Retrieved from http://dl.acm.org/citation.cfm?id=2808059 McClellan, M. A. (2011). Clinician Acceptance of Electronic Medical Records (EMRs): Relating Personality Factors to Continuance Intention. UNIVERSITY OF MINNESOTA. Retrieved from http://conservancy.umn.edu/handle/11299/162236 
McNeill, D. (1994). Fuzzy logic: The revolutionary computer technology that is changing our world. Simon and Schuster. Retrieved from https://books.google.com/books?hl=en\&lr=\&id=mKq8tzavONkC\&oi=fnd\&pg=P A9\&dq=Mcneill,+D.+(1994).+Fuzzy+logic:+The+revolutionary+computer+techn ology+that+is+changing+our+world.+New+York:+Simon+\%26+Schuster\&ots $=1$ TyH0t4Zbl\&sig=RYTnlq-cOwxT4IUBWEu2a6_hs3U

McRoberts, N., Franke, A. C., \& others. (2008). A diffusion model for the adoption of agricultural innovations in structured adopting populations. Retrieved from https://ideas.repec.org/p/ags/saclwp/61117.html

Measuring the Costs and Savings of Aging in Place. (2013). [Evidence Matters]. Retrieved October 9, 2016, from https://www.huduser.gov/portal/periodicals/em/fall13/highlight2.html

Merkel, S., Enste, P., Hilbert, J., Chen, K., Chan, A., \& Kwon, S. (2016). Technology Acceptance and Aging. Kwon, S.(Hg.): Gerontechnology, 2. Retrieved from https://books.google.com/books?hl=en\&lr=\&id=7yYODAAAQBAJ\&oi=fnd\&pg $=\mathrm{PA} 335 \& \mathrm{dq}=$ elderly $+\% 2 \mathrm{~B}+\% 22$ technology + adoption $\% 22+\% 2 \mathrm{~B}+\% 22 \mathrm{gender}+\mathrm{ga}$ p\%22\&ots=EMViibJdDr\&sig=oNUIIR5pAVm752e1YXArgUd1zwY

Metlife. (2011). The MetLife Study of Caregiving Costs to Working Caregivers. Retrieved October 9, 2016, from http://www.caregiving.org/wpcontent/uploads/2011/06/mmi-caregiving-costs-working-caregivers.pdf Meyer, A. D. (1991). Visual data in organizational research. Organization Science, 2(2), 218-236. 
Meyer, J. (2000). Using qualitative methods in health related action research. British Medical Journal, 320(7228), 178.

Miles, M. B., \& Huberman, A. M. (1994). Qualitative data analysis: An expanded sourcebook. Sage. Retrieved from http://books.google.com/books?hl=en\&lr=\&id=U4lU_wJ5QEC\&oi=fnd\&pg=PR12\&dq=Miles+and+huberman\&ots=kDTF5KPQO\&sig=o7kpRU7bJtHQXs5KSpXFaFt4Mfg

Minton, H. L., \& Schneider, F. W. (1985). Differential psychology. Waveland PressInc. Mixed Methods - Journal of Ethnographic \& Qualitative Research. (2017). Retrieved January 12, 2017, from http://www.jeqr.org/mixed-methods

MMIRA - Home. (2017). Retrieved January 11, 2017, from http://mmira.wildapricot.org/ Mohammadi, S., \& Kaldi, A. (2008). Adoption of iris-based authentication. In Industrial Engineering and Engineering Management, 2008. IEEM 2008. IEEE International Conference on (pp. 1582-1586). IEEE. Retrieved from http://ieeexplore.ieee.org/xpls/abs_all.jsp?arnumber=4738138

Mook, D. G. (1983). In defense of external invalidity. American Psychologist, 38(4), 379. Morgan, D. L. (1998). Practical strategies for combining qualitative and quantitative methods: Applications to health research. Qualitative Health Research, 8(3), 362376.

Morrell, R. W., Mayhorn, C. B., \& Echt, K. V. (2004). Why older adults use or do not use the internet. Gerotechnology: Research and Practice in Technology and Aging. New York: Springer, 71-85. 
Morris, M. G., \& Venkatesh, V. (2000). Age differences in technology adoption decisions: Implications for a changing work force. Personnel Psychology, 53(2), $375-403$.

Morris, M. G., Venkatesh, V., \& Ackerman, P. L. (2005). Gender and age differences in employee decisions about new technology: An extension to the theory of planned behavior. Engineering Management, IEEE Transactions on, 52(1), 69-84.

Morris, M., Intille, S. S., \& Beaudin, J. S. (2005). Embedded assessment: Overcoming barriers to early detection with pervasive computing. In Pervasive Computing (pp. 333-346). Springer. Retrieved from http://link.springer.com.proxy.lib.pdx.edu/chapter/10.1007/11428572_20

Morse, J. M. (1991). Approaches to qualitative-quantitative methodological triangulation. Nursing Research, 40(2), 120-123.

Myers, B. A. (1998). A brief history of human-computer interaction technology. Interactions, 5(2), 44-54.

Najman, J. M., \& Levine, S. (1981). Evaluating the impact of medical care and technologies on the quality of life: a review and critique. Social Science \& Medicine. Part F: Medical and Social Ethics, 15(2-3), 107-115.

National Alliance for Caregiving. (1999). The MetLife Juggling Act Study Balancing Caregiving with Work and the Costs Involved. Retrieved October 9, 2016, from http://www.caregiving.org/data/jugglingstudy.pdf

Nielsen, J. (1994). Usability inspection methods. In Conference companion on Human factors in computing systems (pp. 413-414). ACM. Retrieved from http://dl.acm.org/citation.cfm?id=260531 
Niskanen, V. (2006). Application of fuzzy linguistic cognitive maps to prisoner's dilemma. Int'l Journal of Innovative Computing, Information and Control, 2(1), $139-152$.

Noar, S. M., \& Zimmerman, R. S. (2005). Health Behavior Theory and cumulative knowledge regarding health behaviors: are we moving in the right direction? Health Education Research, 20(3), 275-290.

Nokia Steel | Activity \& Sleep Tracker Watch. (2018). Retrieved February 18, 2018, from https://health.nokia.com/us/en/steel?gclid=Cj0KCQiA5aTUBRC2ARIsAPoPJk9 OEpfudSr5IMZ_NhYihndIFQS2rYeMjEHxCbQXVdzi6EAziuXS64aAjNCEALw_wcB\#shopsection/shop?screen=desc

Nuno David, M. B. M. (2004, June 30). The Structure and Logic of Interdisciplinary Research in Agent-Based Social Simulation. Retrieved March 1, 2017, from http://jasss.soc.surrey.ac.uk/7/3/4.html

Ogden, J. (2003). Some problems with social cognition models: a pragmatic and conceptual analysis. Health Psychology, 22(4), 424.

O’Mara, B., Gill, G. K., Babacan, H., \& Donahoo, D. (2012). Digital technology, diabetes and culturally and linguistically diverse communities: A case study with elderly women from the Vietnamese community. Health Education Journal, 71(4), 491-504.

Ong, C.-S., \& Lai, J.-Y. (2006). Gender differences in perceptions and relationships among dominants of e-learning acceptance. Computers in Human Behavior, 22(5), 816-829. 
Onwuegbuzie, A. J., \& Combs, J. P. (2010). Emergent data analysis techniques in mixed methods research: A synthesis. Handbook of Mixed Methods in Social and Behavioral Research, 2.

Or, C. K., Karsh, B.-T., Severtson, D. J., Burke, L. J., Brown, R. L., \& Brennan, P. F. (2011). Factors affecting home care patients' acceptance of a web-based interactive self-management technology. Journal of the American Medical Informatics Association, 18(1), 51-59.

Özesmi, U., \& Özesmi, S. L. (2004). Ecological models based on people’s knowledge: a multi-step fuzzy cognitive mapping approach. Ecological Modelling, 176(1-2), 43-64. https://doi.org/10.1016/j.ecolmodel.2003.10.027

Painter, J. E., Borba, C. P., Hynes, M., Mays, D., \& Glanz, K. (2008). The use of theory in health behavior research from 2000 to 2005: a systematic review. Annals of Behavioral Medicine, 35(3), 358-362.

Palinkas, L. A., Horwitz, S. M., Chamberlain, P., Hurlburt, M. S., \& Landsverk, J. (2011). Mixed-methods designs in mental health services research: A review. Psychiatric Services, 62(3), 255-263.

Pan, S., \& Jordan-Marsh, M. (2010). Internet use intention and adoption among Chinese older adults: From the expanded technology acceptance model perspective. Computers in Human Behavior, 26(5), 1111-1119.

Papadimitriou, P., Dasdan, A., \& Garcia-Molina, H. (2010). Web graph similarity for anomaly detection. Journal of Internet Services and Applications, 1(1), 19-30.

Papageorgiou, E. I., \& Kontogianni, A. (2012). Using fuzzy cognitive mapping in environmental decision making and management: a methodological primer and an 323 
application. International Perspectives on Global Environmental Change. InTech,

Germany. Retrieved from http://www.intechopen.com/source/pdfs/27194/InTechUsing_fuzzy_cognitive_mapping_in_environmental_decision_making_and_mana gement_a_methodological_primer_and_an_application.pdf

Papageorgiou, Elpiniki I., \& Salmeron, J. L. (2014). Methods and Algorithms for Fuzzy Cognitive Map-based Modeling. In Fuzzy Cognitive Maps for Applied Sciences and Engineering (pp. 1-28). Springer, Berlin, Heidelberg. https://doi.org/10.1007/978-3-642-39739-4_1

Pasick, R. J., Burke, N. J., Barker, J. C., Joseph, G., Bird, J. A., Otero-Sabogal, R., ... others. (2009). Behavioral theory in a diverse society: Like a compass on Mars. Health Education \& Behavior, 36(5 suppl), 11S-35S.

Peek, S. T., Luijkx, K. G., Rijnaard, M. D., Nieboer, M. E., van der Voort, C. S., Aarts, S., ... Wouters, E. J. (2015). Older adults' reasons for using technology while aging in place. Gerontology, 62(2), 226-237.

Peek, S. T. M., Wouters, E. J. M., van Hoof, J., Luijkx, K. G., Boeije, H. R., \& Vrijhoef, H. J. M. (2014). Factors influencing acceptance of technology for aging in place: a systematic review. International Journal of Medical Informatics, 83(4), 235248. https://doi.org/10.1016/j.ijmedinf.2014.01.004

Peine, A. (2009). Understanding the dynamics of technological configurations: A conceptual framework and the case of Smart Homes. Technological Forecasting and Social Change, 76(3), 396-409.

Peine, A., \& Herrmann, A. M. (2012). The sources of use knowledge: Towards integrating the dynamics of technology use and design in the articulation of 324 
societal challenges. Technological Forecasting and Social Change, 79(8), 14951512.

Penning, M. J., \& Wu, Z. (2015). Caregiver Stress and Mental Health: Impact of Caregiving Relationship and Gender. The Gerontologist, gnv038. https://doi.org/10.1093/geront/gnv038

Pfaff, M. S., Drury, J. L., \& Klein, G. L. (2015). Crowdsourcing Mental Models using DESIM (Descriptive to Executable Simulation Modeling). Presented at the International Conference on Natualistic Decision Making, McLean, VA: The MITRE Corporation.

Phillips, B., \& Zhao, H. (1993). Predictors of assistive technology abandonment. Assistive Technology, 5(1), 36-45.

Pinsonneault, A., \& Kraemer, K. (1993). Survey research methodology in management information systems: an assessment. Journal of Management Information Systems, 10(2), 75-105.

Pinto, R. M., Wall, M. M., \& Spector, A. Y. (2014). Modeling the Structure of Partnership Between Researchers and Front-Line Service Providers: Strengthening Collaborative Public Health Research. Journal of Mixed Methods Research, 8(1), 83-106. https://doi.org/10.1177/1558689813490835

Plett, P. C., \& Lester, B. T. (1991). Training for older people: a handbook. International Labour Organization. Retrieved from https://books.google.com/books?hl=en\&lr=\&id=6-rEAo1kg8C\&oi=fnd\&pg=PR5\&dq=Training+for+older+people:+a+handbook\&o ts=qtjzB8tzKO\&sig=AMW_taByNClQLW9hp5gNzaZREQA 
Plude, D. J., \& Hoyer, W. J. (1985). Attention and performance: Identifying and localizing age deficits. Aging and Human Performance, 47-99.

Preissle, J., \& Grant, L. (2004). Fieldwork traditions: Ethnography and participant observation. Foundations for Research: Methods of Inquiry in Education and the Social Sciences, 161-180.

Rahimi, N., \& Ibarra, M. (2014). A Review of Multiple User Center Design Methods for New Product Development in Smart and Connected Health Applications. In 2014 Proceedings of PICMET'14 (pp. 3498-3510). IEEE.

Rahimi, N., \& Jetter, A. (2015). Explaining health technology adoption: past, present, future. In Management of Engineering and Technology (PICMET), 2015 Portland International Conference on (pp. 2465-2495). IEEE. Retrieved from http://ieeexplore.ieee.org/abstract/document/7273030/

Rahimi, N., Jetter, A. J., Weber, C. M., \& Wild, K. (2018). Soft Data Analytics with Fuzzy Cognitive Maps: Modeling Health Technology Adoption by Elderly Women. In Advanced Data Analytics in Health (pp. 59-74). Springer, Cham.

Rahimi, N., Jetter, A., \& Weber, C. M. (2016). An Inductive Ethnographic Study in Elderly Woman Technology Adoption and the role of her children. Presented at the PICMET, Hawaii, USA: IEEE.

Ramón-Jerónimo, M. A., Peral-Peral, B., \& Arenas-Gaitán, J. (2013). Elderly persons and Internet use. Social Science Computer Review, 31(4), 389-403.

Renaud, K., \& Van Biljon, J. (2008). Predicting technology acceptance and adoption by the elderly: a qualitative study. In Proceedings of the 2008 annual research conference of the South African Institute of Computer Scientists and Information 326 
Technologists on IT research in developing countries: riding the wave of technology (pp. 210-219). ACM. Retrieved from

http://dl.acm.org/citation.cfm?id=1456684

Repertory Grid Technique. (2017). Retrieved March 29, 2017, from http://www.epistemics.co.uk/Notes/184-0-0.htm

Ringland, G., \& SCHWARTZ, P. P. (1998). Scenario planning: Managing for the future. John Wiley \& Sons. Retrieved from http://documents.irevues.inist.fr/handle/2042/30191

Ritchie, J., Lewis, J., Nicholls, C. M., \& Ormston, R. (2013). Qualitative research practice: A guide for social science students and researchers. Sage.

Robson, P., \& Sutherland, K. E. (2012). Public relations practitioners and social media: themes in a global context. In WORLD PUBLIC RELATIONS FORUM 2012 (p. 103). Retrieved from http://www.globalalliancepr.usi.ch/website/sites/default/files/nolie/WPRF/WPRF \%202012/WPRF12_RESEARCH-COLLOQUIUMPROCEEDINGS.pdf\#page $=103$

Rodriguez-Repiso, L., Setchi, R., \& Salmeron, J. L. (2007). Modelling IT projects success with fuzzy cognitive maps. Expert Systems with Applications, 32(2), 543559.

Rogers, E. M. (2010). Diffusion of innovations. Simon and Schuster. Retrieved from http://books.google.com/books?hl=en\&lr=\&id=v1ii4QsB7jIC\&oi=fnd\&pg=PR15 $\& d q=$ diffusion + of + innovations $+\& o t s=D J-$ rrQQu6W\&sig=LuIG_jfA5UoqfdV3U77NNaVK8fE 
Rothman, A. J. (2004). "Is there nothing more practical than a good theory?”: Why innovations and advances in health behavior change will arise if interventions are used to test and refine theory. The International Journal of Behavioral Nutrition and Physical Activity, 1(1), 11. https://doi.org/10.1186/1479-5868-1-11

Rushkoff, D. (2014). Get back in the box.

Ryu, M.-H., Kim, S., \& Lee, E. (2009). Understanding the factors affecting online elderly user's participation in video UCC services. Computers in Human Behavior, 25(3), 619-632. https://doi.org/10.1016/j.chb.2008.08.013

Saborowski, M., \& Kollak, I. (2014). “How do you care for technology?”-Care professionals' experiences with assistive technology in care of the elderly. Technological Forecasting and Social Change. Retrieved from http://www.sciencedirect.com/science/article/pii/S0040162514001632

Samoutis, G. A., Soteriades, E. S., Stoffers, H. E., Zachariadou, T., Philalithis, A., \& Lionis, C. (2008). Designing a multifaceted quality improvement intervention in primary care in a country where general practice is seeking recognition: the case of Cyprus. BMC Health Services Research, 8, 181. https://doi.org/10.1186/14726963-8-181

Sampietro-Colom, L., Phillips, V. L., \& Hutchinson, A. B. (2004). Eliciting women's preferences in health care: A review of the literature. International Journal of Technology Assessment in Health Care, 20(02), 145-155.

Satariano, W. A., Scharlach, A. E., \& Lindeman, D. (2014). Aging, place, and technology: Toward improving access and wellness in older populations. Journal of Aging and Health, 26(8), 1373-1389. 
Schaar, A. K., \& Ziefle, M. (2011). Smart clothing: Perceived benefits vs. perceived fears. In Pervasive Computing Technologies for Healthcare (PervasiveHealth), 2011 5th International Conference on (pp. 601-608). IEEE. Retrieved from http://ieeexplore.ieee.org/xpls/abs_all.jsp?arnumber=6038875

Schoemaker, P. J. (1991). When and how to use scenario planning: a heuristic approach with illustration. Journal of Forecasting, 10(6), 549-564.

Schoemaker, P. J. (1995). Scenario planning: a tool for strategic thinking. Sloan Management Review, 36(2), 25.

Sheeran, P., Abraham, C., \& Orbell, S. (1999). Psychosocial correlates of heterosexual condom use: a meta-analysis. Psychological Bulletin, 125(1), 90.

Silverstone, R. (1994). Television and everyday life. Routledge. Retrieved from https://books.google.com/books?hl=en\&lr=\&id=DwaKAgAAQBAJ\&oi=fnd\&pg $=$ PP1\&dq=Silverstone, + R.+Television+and+Everyday + Life,+ Routledge,+ Londo n\&ots=0pLLAwbHfg\&sig=DCbEo75joz3M62FT_KaHggZ3Vgw

Silverstone, R., \& Haddon, L. (1996). Design and the domestication of ICTs: technical change and everyday life. Communicating by Design: The Politics of Information and Communication Technologies, 44-74.

Silverstone, R., Hirsch, E., \& Morley, D. (1994). Information and communication technologies and the moral economy of the household. Consuming Technologies. Media and Information in Domestic Spaces. Retrieved from https://books.google.com/books?hl=en\&lr=\&id=VSKAgAAQBAJ\&oi=fnd\&pg=PA13\&dq=\%27Information + and+Communicatio n+Technologies+and+the+Moral+Economy+of+the+Household $\% 27,+$ in+Silverst 329 
one,+R.+and+Hirsch,+E.(eds.)+Consuming+Technologies,+Routledge,+London \&ots=I7Vf_Tqf-k\&sig=4FrC4yGGHdn46scfdzKTXpp7DmI

Simon, H. A., \& Kaplan, C. A. (1989). Foundations of cognitive science. Retrieved from http://psycnet.apa.org/psycinfo/1990-97026-001

Simpson, D. G. (1992). Key lessons for adopting scenario planning in diversified companies. Planning Review, 20(3), 10-48.

Smartphone Apps for Senior Help - Medical Alert Comparison. (2015). Retrieved July 23, 2015, from http://www.medicalalertcomparison.com/articles/smartphoneapps-for-senior-help/

Smith, A. (2014). Older Adults and Technology Use. Retrieved October 3, 2014, from http://www.pewinternet.org/2014/04/03/older-adults-and-technology-use/

Sperry, R., \& Jetter, A. (2009). Theoretical framework for managing the front end of innovation under uncertainty. In Management of Engineering \& Technology, 2009. PICMET 2009. Portland International Conference on (pp. 2021-2028). IEEE. Retrieved from http://ieeexplore.ieee.org/abstract/document/5261940/

Stach, W., Kurgan, L., Pedrycz, W., \& Reformat, M. (2005). Genetic learning of fuzzy cognitive maps. Fuzzy Sets and Systems, 153(3), 371-401. https://doi.org/10.1016/j.fss.2005.01.009

Stanton, N. A., Hedge, A., Brookhuis, K., Salas, E., \& Hendrick, H. W. (2004). Handbook of human factors and ergonomics methods. CRC Press. Retrieved from https://books.google.com/books?hl=en\&lr=\&id=RApSggShPc8C\&oi=fnd\&pg=P P1\&dq=Handbook + of + Human + Factors+and+Ergonomics + Methods\&ots $=x c 3 j$ th KWaB\&sig=k0am2vGGU370DG_stYPBqJ0EmEE 330 
Sterman, J. D. (2000). Business dynamics: systems thinking and modeling for a complex world.

Sterns, H. L., \& Doverspike, D. (1989). Aging and the training and learning process. Retrieved from http://psycnet.apa.org/psycinfo/1989-98681-008

Stojmenova, E., Debevc, M., Zebec, L., \& Imperl, B. (2012). Assisted living solutions for the elderly through interactive TV. Multimedia Tools and Applications, 66(1), 115-129. https://doi.org/10.1007/s11042-011-0972-1

Stylios, C. D., Georgopoulos, V. C., Malandraki, G. A., \& Chouliara, S. (2008). Fuzzy cognitive map architectures for medical decision support systems. Applied Soft Computing, 8(3), 1243-1251.

Taber, R. (1991). Knowledge processing with fuzzy cognitive maps. Expert Systems with Applications, 2(1), 83-87.

Tan, F. B., \& Hunter, M. G. (2002). The repertory grid technique: A method for the study of cognition in information systems. Mis Quarterly, 39-57.

Tang, T., \& Hämäläinen, M. (2013). An empirical investigation of living lab ageing care innovations adoption in Chinese healthcare industry. In 2013 International Conference on Engineering, Technology and Innovation (ICE) IEEE International Technology Management Conference (pp. 1-12). https://doi.org/10.1109/ITMC.2013.7352706

Tashakkori, A., \& Teddlie, C. (2003). Handbook of Mixed Methods in Social \& Behavioral Research. SAGE. 
Taylor, S., \& Todd, P. A. (1995). Understanding Information Technology Usage: A Test of Competing Models. Information Systems Research, 6(2), 144-176. https://doi.org/10.1287/isre.6.2.144

Technology and Aging. (2009). Technologies to Help Older Adults Maintain Independence: Advancing Technology Adoption. Retrieved October 9, 2016, from http://www.techandaging.org/briefingpaper.pdf

The Evolution of Market Research | RW Connect. (2014). Retrieved February 22, 2017, from https://rwconnect.esomar.org/change-in-the-marketing-research-disciplineevolution-paradigm-shift-and-the-emergence-of-human-intelligence/

Thomas, W. (1999). Accelerating the Diffusion of Innovations Using Opinion Leaders. Retrieved from http://www.researchgate.net/profile/Thomas_Valente/publication/254077617_Acc elerating_the_Diffusion_of_Innovations_Using_Opinion_Leaders/links/547df346 0cf241bf4b5b9722.pdf

Thompson, R. L., Higgins, C. A., \& Howell, J. M. (1991). Personal computing: toward a conceptual model of utilization. MIS Quarterly, 125-143.

Tsadiras, A. K. (2008). Comparing the inference capabilities of binary, trivalent and sigmoid fuzzy cognitive maps. Information Sciences, 178(20), 3880-3894.

Tukey, J. W., \& others. (1990). Data-based graphics: visual display in the decades to come. Statistical Science, 5(3), 327-339.

Tushman, M. L., \& Katz, R. (1980). External communication and project performance: An investigation into the role of gatekeepers. Management Science, 26(11), 10711085. 
U. S. Census Bureau, D. I. S. (2012). The Older Population in the United States: 2012.

Retrieved June 21, 2014, from

http://www.census.gov/population/age/data/2012.html

Uchida, H., Hata, Y., Matsuura, S., Tsuchikawa, T., Morotomi, Y., \& Aoyam, H. (2001). Rough set based knowledge discovery of interface for the Internet usage among Japanese elderly women. In International Conference on Computational Intelligence (pp. 749-754). Springer. Retrieved from http://link.springer.com/chapter/10.1007/3-540-45493-4_73

Ulrich, K. T., \& Eppinger, S. D. (2004). Product design and development. 2004. Boston, MA: McGraw-Hill/Irwin.

United Nations. (2013). World Population Ageing 2013. New York, NY, US: United Nations. Retrieved from http://www.un.org/en/development/desa/population/publications/pdf/ageing/Worl dPopulationAgeing2013.pdf

van Biljon, J., \& Kotzé, P. (2007). Modelling the Factors That Influence Mobile Phone Adoption. In Proceedings of the 2007 Annual Research Conference of the South African Institute of Computer Scientists and Information Technologists on IT Research in Developing Countries (pp. 152-161). New York, NY, USA: ACM. https://doi.org/10.1145/1292491.1292509

Vaske, J. J., \& Donnelly, M. P. (1999). A value-attitude-behavior model predicting wildland preservation voting intentions. Society \& Natural Resources, 12(6), 523537. 
Venkatesh, V., \& Davis, F. D. (2000). A theoretical extension of the technology acceptance model: four longitudinal field studies. Management Science, 46(2), 186-204.

Venkatesh, V. M. G. D. G. B. D. F. D. (2003). User Acceptance of Information Technology: Toward a Unified View. MIS Quarterly, 27(3), 425-478.

Venkatesh, V., \& Morris, M. G. (2000). Why don't men ever stop to ask for directions? Gender, social influence, and their role in technology acceptance and usage behavior. MIS Quarterly, 115-139.

Venkatesh, V., Morris, M. G., \& Ackerman, P. L. (2000). A longitudinal field investigation of gender differences in individual technology adoption decisionmaking processes. Organizational Behavior and Human Decision Processes, 83(1), 33-60.

Venkatesh, V., Morris, M. G., Davis, G. B., \& Davis, F. D. (2003). User acceptance of information technology: Toward a unified view. MIS Quarterly, 425-478.

Vervoort, J. M. (2011). Framing futures: Visualizing perspectives on social-ecological. Wageningen University. Retrieved from http://library.wur.nl/WebQuery/wurpubs/fulltext/280504

Vrkljan, B. H. (2009). Constructing a Mixed Methods Design to Explore the Older Driver-Copilot Relationship. Journal of Mixed Methods Research, 3(4), 371385. https://doi.org/10.1177/1558689809336843

Wagner, N., Hassanein, K., \& Head, M. (2010). Computer use by older adults: A multidisciplinary review. Computers in Human Behavior, 26(5), 870-882. 
Walsh, D. A. (2014). Iconic memory and attentional processes in the aged. In New Directions in Memory and Aging (PLE: Memory): Proceedings of the George A. Talland Memorial Conference (p. 153). Psychology Press. Retrieved from https://books.google.com/books?hl=en\&lr=\&id=DP-

KAwAAQBAJ\&oi=fnd\&pg=PA153\&dq=Iconic+memory+and+attentional+proce sses+in+the+aged+DA+Walsh+\%26+prasse\&ots=zu0nGT4kQF\&sig=ZyDRgKR-dZFUIVWQ0EkzV4K-Rg

Weaknesses of Survey Method. (2015). Retrieved February 24, 2017, from http://www.opentextbooks.org.hk/ditatopic/29587

Webb, T., Joseph, J., Yardley, L., \& Michie, S. (2010). Using the internet to promote health behavior change: a systematic review and meta-analysis of the impact of theoretical basis, use of behavior change techniques, and mode of delivery on efficacy. Journal of Medical Internet Research, 12(1), e4.

Webb, T. L., \& Sheeran, P. (2006). Does changing behavioral intentions engender behavior change? A meta-analysis of the experimental evidence. Psychological Bulletin, 132(2), 249.

Wentzel, K. R., \& Wigfield, A. (1998). Academic and Social Motivational Influences on Students’ Academic Performance. Educational Psychology Review, 10(2), 155175. https://doi.org/10.1023/A:1022137619834

What is Gerontechnology? | Geron Technology. (2017). Retrieved March 12, 2017, from http://gerontechnologie.nl/what-is-gerontechnology/

Why Independence is an Important Part of Elderly Care | My Care My Home. (2013). Retrieved October 31, 2016, from 
http://www.mycaremyhome.co.uk/2013/06/27/why-independence-is-animportant-part-of-elderly-care/

Whyte, G., \& Bytheway, A. (1996). Factors affecting information systems' success. International Journal of Service Industry Management, 7(1), 74-93.

Wild, K. V., Mattek, N. C., Maxwell, S. A., Dodge, H. H., Jimison, H. B., \& Kaye, J. A. (2012). Computer-related self-efficacy and anxiety in older adults with and without mild cognitive impairment. Alzheimer's \& Dementia, 8(6), 544-552. https://doi.org/10.1016/j.jalz.2011.12.008

Wilkowska, W., Gaul, S., \& Ziefle, M. (2010). A Small but Significant Difference-The Role of Gender on Acceptance of Medical Assistive Technologies. Springer. Retrieved from http://link.springer.com/chapter/10.1007/978-3-642-16607-5_6

Williams, M., Rana, N., Dwivedi, Y., \& Lal, B. (2011). Is UTAUT really used or just cited for the sake of it? A systematic review of citations of UTAUT's originating article. Retrieved from http://aisel.aisnet.org/ecis2011/231/

Women and Caregiving: Facts and Figures. (2003). Retrieved June 25, 2014, from https://caregiver.org/women-and-caregiving-facts-and-figures

World Health Organization. (2012). Public - Private Partnership Announces Immediate 40 Percent Cost Reduction for Rapid TB Test. Retrieved from http://www.who.int/tb/features_archive/GeneXpert_press_release_final.pdf

Xue, L., Yen, C. C., Chang, L., Chan, H. C., Tai, B. C., Tan, S. B., ... Choolani, M. (2012). An exploratory study of ageing women's perception on access to health informatics via a mobile phone-based intervention. International Journal of Medical Informatics, 81(9), 637-648. 
Zadeh, L. A. (1965). Fuzzy sets. Information and Control, 8(3), 338-353.

Zenobia, B. A., \& Weber, C. M. (2012). Bridging the gap between artificial market simulations and qualitative research in diffusion of innovation. International Journal of Innovation and Technology Management, 9(04). Retrieved from http://www.worldscientific.com/doi/abs/10.1142/S0219877012500265

Zenobia, B., Weber, C., \& Daim, T. (2009). Artificial markets: A review and assessment of a new venue for innovation research. Technovation, 29(5), 338-350.

Zhang, S., McClean, S. I., Nugent, C. D., Donnelly, M. P., Galway, L., Scotney, B. W., \& Cleland, I. (2014). A Predictive Model for Assistive Technology Adoption for People With Dementia. IEEE Journal of Biomedical and Health Informatics, 18(1), 375-383. https://doi.org/10.1109/JBHI.2013.2267549

Zhang, W., \& Watanabe-Galloway, S. (2014). Using mixed methods effectively in prevention science: Designs, procedures, and examples. Prevention Science, $15(5), 654-662$.

Zhang, X., Guo, X., Lai, K.-H., Guo, F., \& Li, C. (2014). Understanding gender differences in m-health adoption: a modified theory of reasoned action model. Telemedicine Journal and E-Health: The Official Journal of the American Telemedicine Association, 20(1), 39-46. https://doi.org/10.1089/tmj.2013.0092

Zimmer, Z., \& Chappell, N. L. (1994). Mobility restriction and the use of devices among seniors. Journal of Aging and Health, 6(2), 185-208. 


\section{Appendix A - Data Mining in Early HTA Literatures}

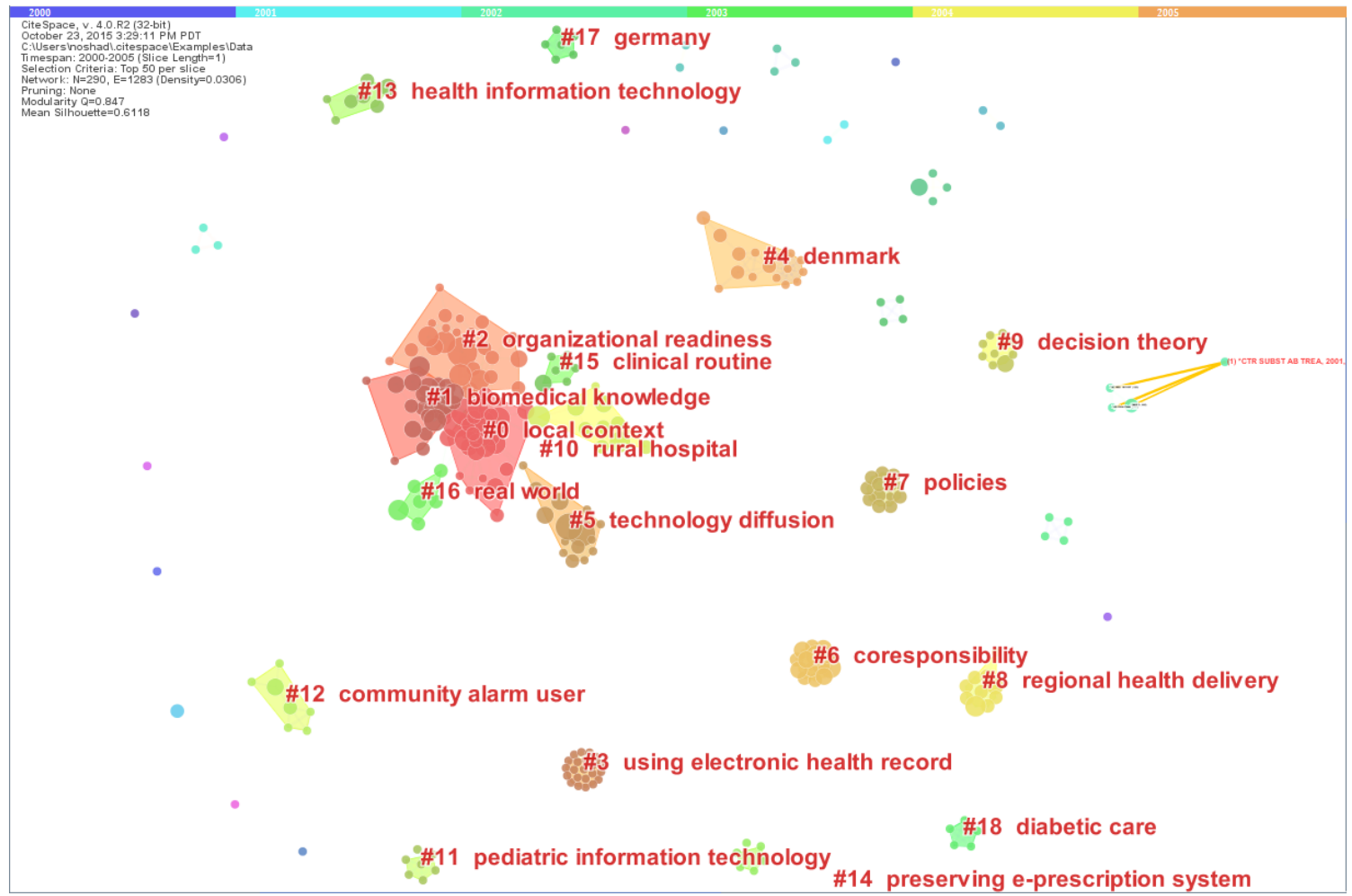

Figure A - 1 Citespace Cluster Analysis of earliest 500 articles in HTA

Selected the oldest $512 \mathrm{HTA}$ articles from Web of Science based on the search criteria of:

TS $=\left(\right.$ Health and technolog ${ }^{*}$ and $\left(\right.$ adopt $^{*}$ or accept ${ }^{\star}$ or diffus $\left.{ }^{*}\right)$ not injur ${ }^{\star}$ not cardi ${ }^{*}$ not HIV* not cancer ${ }^{*}$ not pregnan* not nutrit* not MRI not musc*) and $\mathrm{TI}=\left(\right.$ smart $^{*}$ or technolog* or intelligent or tele* or e?health or EMR or EHR or "electronic health")

Refined by: RESEARCH DOMAINS: ( SCIENCE TECHNOLOGY) AND RESEARCH DOMAINS: ( SCIENCE TECHNOLOGY) AND DOCUMENT TYPES: ( ARTICLE ) AND LANGUAGES:( ENGLISH ) AND RESEARCH AREAS: ( HEALTH CARE SCIENCES SERVICES OR MEDICAL INFORMATICS OR COMPUTER SCIENCE OR COMMUNICATION OR TELECOMMUNICATIONS OR GERIATRICS GERONTOLOGY OR BEHAVIORAL SCIENCES OR PUBLIC ENVIRONMENTAL OCCUPATIONAL HEALTH OR MEDICAL INFORMATICS OR ENGINEERING OR ROBOTICS OR TELECOMMUNICATIONS OR OPERATIONS RESEARCH MANAGEMENT SCIENCE OR GERIATRICS GERONTOLOGY OR REHABILITATION ) AND RESEARCH DOMAINS: ( SCIENCE TECHNOLOGY) 


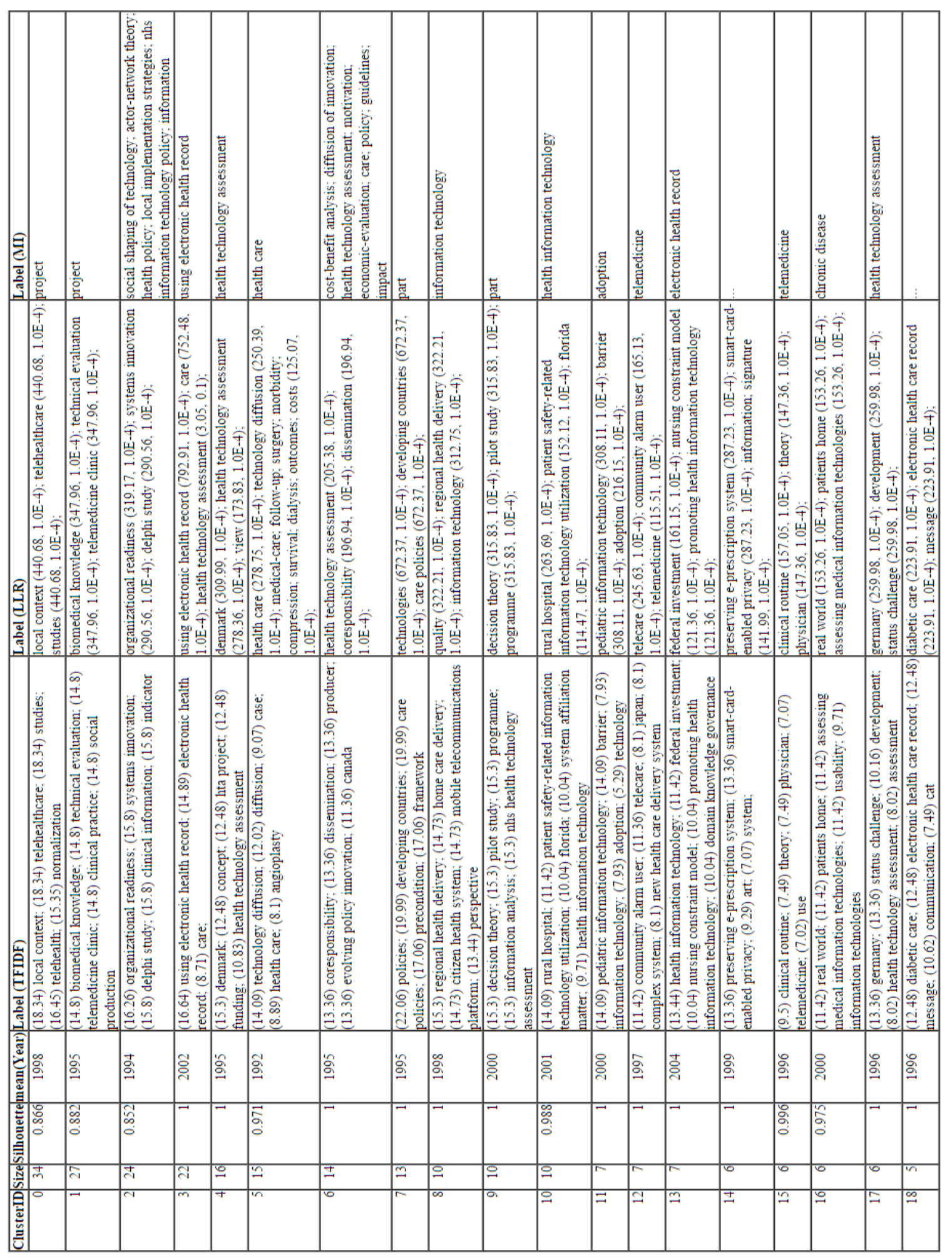

Figure A - 2 Citespace Cluster Detail of earliest 500 articles in HTA 


\begin{tabular}{|c|c|c|c|c|}
\hline $\begin{array}{l}\rightarrow \text { View Records } \\
\times \text { Exclude Records }\end{array}$ & Field: Web of Science Categories & Record Count & $\%$ of 894 & Bar Chart \\
\hline$\square$ & HEALTH CARE SCIENCES SERVICES & 427 & $47.763 \%$ & 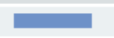 \\
\hline$\square$ & MEDICAL INFORMATICS & 268 & $29.978 \%$ & 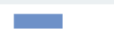 \\
\hline$\square$ & COMPUTER SCIENCE INFORMATION SYSTEMS & 178 & $19.911 \%$ & - \\
\hline$\square$ & HEALTH POLICY SERVICES & 176 & $19.687 \%$ & 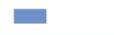 \\
\hline$\square$ & PUBLIC ENVIRONMENTAL OCCUPATIONAL HEALTH & 162 & $18.121 \%$ & ש \\
\hline$\square$ & INFORMATION SCIENCE LIBRARY SCIENCE & 82 & $9.172 \%$ & 更 \\
\hline$\square$ & COMPUTER SCIENCE INTERDISCIPLINARY APPLICATIONS & 75 & $8.389 \%$ & - \\
\hline$\square$ & MANAGEMENT & 40 & $4.474 \%$ & I \\
\hline$\square$ & BUSINESS & 32 & $3.579 \%$ & I \\
\hline$\square$ & ECONOMICS & 29 & $3.244 \%$ & I \\
\hline$\square$ & ERGONOMICS & 27 & $3.020 \%$ & I \\
\hline$\square$ & PSYCHOLOGY MULTIDISCIPLINARY & 26 & $2.908 \%$ & $\mathbf{I}$ \\
\hline$\square$ & SOCIAL SCIENCES BIOMEDICAL & 24 & $2.685 \%$ & I \\
\hline$\square$ & ENVIRONMENTAL SCIENCES & 22 & $2.461 \%$ & I \\
\hline$\square$ & COMPUTER SCIENCE CYBERNETICS & 21 & $2.349 \%$ & I \\
\hline$\square$ & ENGINEERING INDUSTRIAL & 21 & $2.349 \%$ & I \\
\hline$\square$ & MEDICINE GENERAL INTERNAL & 19 & $2.125 \%$ & I \\
\hline$\square$ & ENGINEERING BIOMEDICAL & 18 & $2.013 \%$ & I \\
\hline$\square$ & ENGINEERING ENVIRONMENTAL & 18 & $2.013 \%$ & I \\
\hline$\square$ & COMPUTER SCIENCE THEORY METHODS & 17 & $1.902 \%$ & I \\
\hline$\square$ & OPERATIONS RESEARCH MANAGEMENT SCIENCE & 16 & $1.790 \%$ & 1 \\
\hline$\square$ & GERONTOLOGY & 15 & $1.678 \%$ & I \\
\hline$\square$ & PSYCHOLOGY EXPERIMENTAL & 14 & $1.566 \%$ & 1 \\
\hline$\square$ & SOCIAL SCIENCES INTERDISCIPLINARY & 14 & $1.566 \%$ & 1 \\
\hline$\square$ & TELECOMMUNICATIONS & 14 & $1.566 \%$ & 1 \\
\hline$\square$ & COMPUTER SCIENCE ARTIFICIAL INTELLIGENCE & 12 & $1.342 \%$ & I \\
\hline$\square$ & ENGINEERING ELECTRICAL ELECTRONIC & 10 & $1.119 \%$ & 1 \\
\hline$\square$ & GERIATRICS GERONTOLOGY & 10 & $1.119 \%$ & I \\
\hline$\square$ & ENGINEERING MANUFACTURING & 9 & $1.007 \%$ & I \\
\hline$\square$ & PHARMACOLOGY PHARMACY & 9 & $1.007 \%$ & I \\
\hline$\square$ & COMPUTER SCIENCE SOFTWARE ENGINEERING & 7 & $0.783 \%$ & I \\
\hline$\square$ & EDUCATION EDUCATIONAL RESEARCH & 7 & $0.783 \%$ & 1 \\
\hline$\square$ & PSYCHOLOGY APPLIED & 7 & $0.783 \%$ & 1 \\
\hline$\square$ & COMPUTER SCIENCE HARDWARE ARCHITECTURE & 6 & $0.671 \%$ & । \\
\hline$\square$ & ENGINEERING MECHANICAL & 6 & $0.671 \%$ & I \\
\hline$\square$ & PLANNING DEVELOPMENT & 6 & $0.671 \%$ & । \\
\hline$\square$ & MATHEMATICAL COMPUTATIONAL BIOLOGY & 5 & $0.559 \%$ & 1 \\
\hline$\square$ & REHABILITATION & 5 & $0.559 \%$ & । \\
\hline$\square$ & COMMUNICATION & 4 & $0.447 \%$ & I \\
\hline$\square$ & PSYCHOLOGY & 3 & $0.336 \%$ & 1 \\
\hline$\square$ & DEMOGRAPHY & 2 & $0.224 \%$ & 1 \\
\hline$\square$ & ENGINEERING MULTIDISCIPLINARY & 2 & $0.224 \%$ & 1 \\
\hline$\square$ & SOCIAL ISSUES & 2 & $0.224 \%$ & 1 \\
\hline
\end{tabular}

Figure A - 3 Subject Category of the earliest 500 articles in HTA 


\section{Appendix B - Pilot Study}

\section{Executive Summary}

To investigate the feasibility and utility of the proposed method, this research applied the method (proposed in this dissertation) to study elderly women testers of EverWrist, a prototype of a remote health monitoring technology. EverWrist is a wristwatch developed by Barron \& Associates and founded by America's Seed Fund under the NIH contract R44AG032160-03 ("EverWrist: A Novel Electronic Fall Monitor for Seniors | SBIR.gov," 2012). To conduct this research, Barron \& Associates partnered with the Oregon Center for Aging and Technology (ORCATECH). ORCATECH conducted a field study in which elderly participants tested and reported on EverWrist. The purpose of the study was twofold: 1) to determine the false alarm and missed detection rate of falls using EverWrist; and 2) to quantify the circumstances that led to false alarms and missed detection of falls. The elderly women volunteers of a cohort of Life Lab (described in section 3.3) field tested this novel electronic fall detection monitor for seniors.

Through collaboration with ORCATECH, I gained access to the cohort of elderly who tested EverWrist. The pilot research consisted of a longitudinal study of two elderly women from the EverWrist project and the gatekeepers that they identified. (The research method is described in 3.5 through 3.7.) The longitudinal study was conducted in two phases: preadoption (prior to the trial of EverWrist) and post-adoption (immediately after the trial of EverWrist). Elderly women were chosen as participants, because they represent the majority of the elderly population. Moreover, the elderly women's gatekeepers were investigated due to their (typically) critical influence in adoption decision-making by the elderly. 
The pilot research was conducted based on steps 1 to step 12 of chapter 3 (pre-adoption data collections), as described in section 3.8, 3.9 and 3.10. Due to the scope of the pilot study, step 13, expert validation, and step 14, crowd validation of forecasting capability, were not conducted. Instead of conducting a second set of interview to build the structure of the model (step 11), I, acting as the modeler, assigned the weights of the model links (edge weights) based on the information gathered from the initial data collection (step 1 to 6). Immediately after the trial period of EverWrist (one month), a post adoption interview was conducted with each of the two elderly women, in order to identify the post-trial behavior. In particular, the actual intention and decision to adopt the technology were elicited. To test the accuracy of the simulation model forecasting capability, the resulting simulation models were simulated for each elderly scenario. Consequently, the simulation results, the values of the simulation model's output variables-adoption and adoption intention—were compared to the actual "adoption intention" and "adoption" decision of the two elderly women. It is important to note that the simulations of the resulting simulation model were in agreement with the findings of the post-adoption interview. Therefore, the pilot study successfully verified the appropriateness of the research method proposed for modeling EWHTA.

Although the study's questions mainly focused on EverWrist, most of the answers went beyond EverWrist. The respondents described factors that pertained to their approach to making technology adoption decisions in general. Therefore, it's important to note that while EverWrist served as the stimulus to initiate the conversation, the resulting simulation model explains a broader pattern of technology adoption by the elderly women. 


\section{Pilot Study Design}

This pilot study tested the proposed method by applying the method to the trial of EverWrist, an RHMT wearable, among elderly women. In this study, the proposed methodology is validated through the development of an empirically-grounded EWHTA model. The pilot study applies the proposed method (in chapter 3), except for the qualitative data collection step and the expert validation that verify the viability of the method and the validity of resulting model.

\section{Unit of Analysis}

The unit of analysis is the detailed relationship between the underlying factors of the adoption (as outlined in UTAUT), how gatekeeper influences these factors, and the propensity of elderly to adopt health technology.

As detailed in chapter 3 , the research method proposed in this dissertation follows the guidelines for Fuzzy Cognitive Mapping laid out by Jetter (2006). It takes advantage of FCM's ability to integrate the perspective of multiple stakeholders (here elderly and the gatekeepers). In addition, it has been adapted to accommodate the highly interdisciplinary nature of the proposed research.

As explained in the Section 1.1, the concepts that are associated with theories derived from individual disciplines are too general to be useful in the multidisciplinary context of EHTA. Making sense of how the general concepts of adoption theories apply to EWHTA warrants a highly granular, empirically grounded approach that studies these specific contexts in depth. The research method proposed for this dissertation follows such an approach. The designed mixed-method approach has handpicked effective techniques in computer-based modeling and qualitative research approaches to facilitate modeling of technology adoption for a demographic in which agency is important. It uses repertory- 
grid to collect in depth, pre-adoption field data and Fuzzy Cognitive Mapping (FCM) (Kosko, 1986) to model the mental model of users of technology products, who are subjected to significant influence by technology gatekeepers. This is followed by qualitative and then quantitative techniques to analyze and validate the fuzzy cognitive maps to form the technology adoption decision making model.

The following sections lay out the steps that were taken to conduct the research in the six stages of fuzzy cognitive mapping (Jetter, 2006): I) Analysis of Objectives and Information Needs; II) Analysis of Means to Fulfil Information Needs; III) Knowledge Capture; IV \& V) Conceptual and Detailed Design of FCM Models; and VI) Test and Validation.

\section{Analysis of Objectives and Information Needs}

The objective of this pilot research is to test the proposed method capability to provide a robust technology adoption modeling method among demographic groups that rely heavily on gatekeeping for technology adoption. This pilot study focused on two pairs of elderly women and their gatekeepers to contextualize a EWHTA model, which serves as a decision tool and provides a better understanding of the drivers and obstacles specific to the elderly women. Similar to the objectives described in section 3.6 (however focusing on the elderly women), the model ...

5. Identifies the key adoption drivers and obstacles among elderly women;

6. Unpacks how these drivers and obstacles are interrelated and how they influence the adoption; and

7. Illustrates how gatekeepers influence elderly women's adoption decision. This is reflected in the model by showing how gatekeepers influence the formation of 
perceptions of the UTAUT's main constructs-performance expectancy, effort expectancy and facilitating condition.

Thus the model should be able to answer the following questions in the context of EWHTA:

$\checkmark$ How do gatekeepers influence the adoption behavior of elderly women, both from the point of view of intention and usage?

$\checkmark$ How does the relationship between elderly women and gatekeepers affect the elderly's perception of performance expectancy, effort expectancy and facilitating condition?

\section{Analysis of Means to Fulfil Information Needs}

\section{II.1. Identification of Participants}

In the pilot study, the goal was to test the methods capability to integrate various mental models and stay accurate to each one. Therefore, the pilot recruited two pairs of participants:

$\checkmark$ Two elderly women who act as the potential adopters

$\checkmark$ The identified gatekeeper for each elderly woman. They both identified one of their daughters as their gatekeepers.

This combination generated four FCM models-two elderly women mental models and two elderly mental women mental models from the perspective of their gatekeepers. Having more than one pair of participants allowed the integration steps to be tested and validated. 


\section{II.2. Methods Selection for knowledge Capture}

The information about the knowledge capture method is identical to that of the research method detailed in section 3.7.2.

\section{Knowledge Capture}

Knowledge capture follows the steps laid out in section 3.8. However, due to the limited scope of the pilot study, the content and structure of the FCM models were created at the same time. (This will be different in the proposed method.) As detailed in section 3.8, the proposed research method will develop an FCM simulation model in two separate steps of: 1) content building by developing causal maps during the first set of interviews and qualitative analysis (QUAL phase); 2) structure building by the second set of interviews (quan data collection as described in the step 5. (Model Structure Building)). In the pilot research, model building was entirely based on qualitative data collection, where both the content and the structure of the FCM simulation model were built during the repertory-grid interview and the qualitative analysis. The process was conducted as follows.

1. Participants confirmed the validity of the UTAUT (as depicted in Figure 6 - ) constructs, and verified that these constructs in fact influence the participant's technology adoption decision.

2. The participant was then asked to explain and help develop the left side of the verified UTAUT by answering the predesigned, probing, open-ended questions in Table B - 6 Repertory-grid open-ended questions of the pilot study (presented at the end of this appendix). The researcher developed the basic cognitive model by expanding the UTAUT constructs. This model was actively 
expanded to include the novel concepts that participant prompted. Then, the links that depicted the influence of these concepts on each other and on UTAUT factors were added.

3. To measure the initial participants' perception of the technology, they were asked to quantitatively rate (based on a 5-point Likert scale) their perception of the UTAUT's main constructs, as they pertain to prospective product under evaluation. For example, she was asked to rate the technology's ease of use, usefulness, etc. by filling out the participants perception questionnaire in Table $B-7)$.

The initial fuzzy cognitive maps of the two elderly women's mental model are depicted below.

4. Next, the participant's gatekeeper, who was identified by the participant as the main technology influencer, was interviewed. Similar to step 1 and 2 , the repertory-grid data elicitation combined with fuzzy cognitive mapping also resulted in a basic fuzzy cognitive map of the elderly woman's mental model and a voice recording of the interview. This map captured how the gatekeeper influenced the participant's decision during the adoption. This resulted in another mental model (fuzzy cognitive map) of the elderly person as viewed from the perspective of her gatekeeper.

5. The interview of the participant-gatekeeper pairs was conducted for two pairs.

The initial (pre-adoption) fuzzy cognitive maps of the mental models of the two elderly women, which represent the point of view of the gatekeepers, are depicted below. 


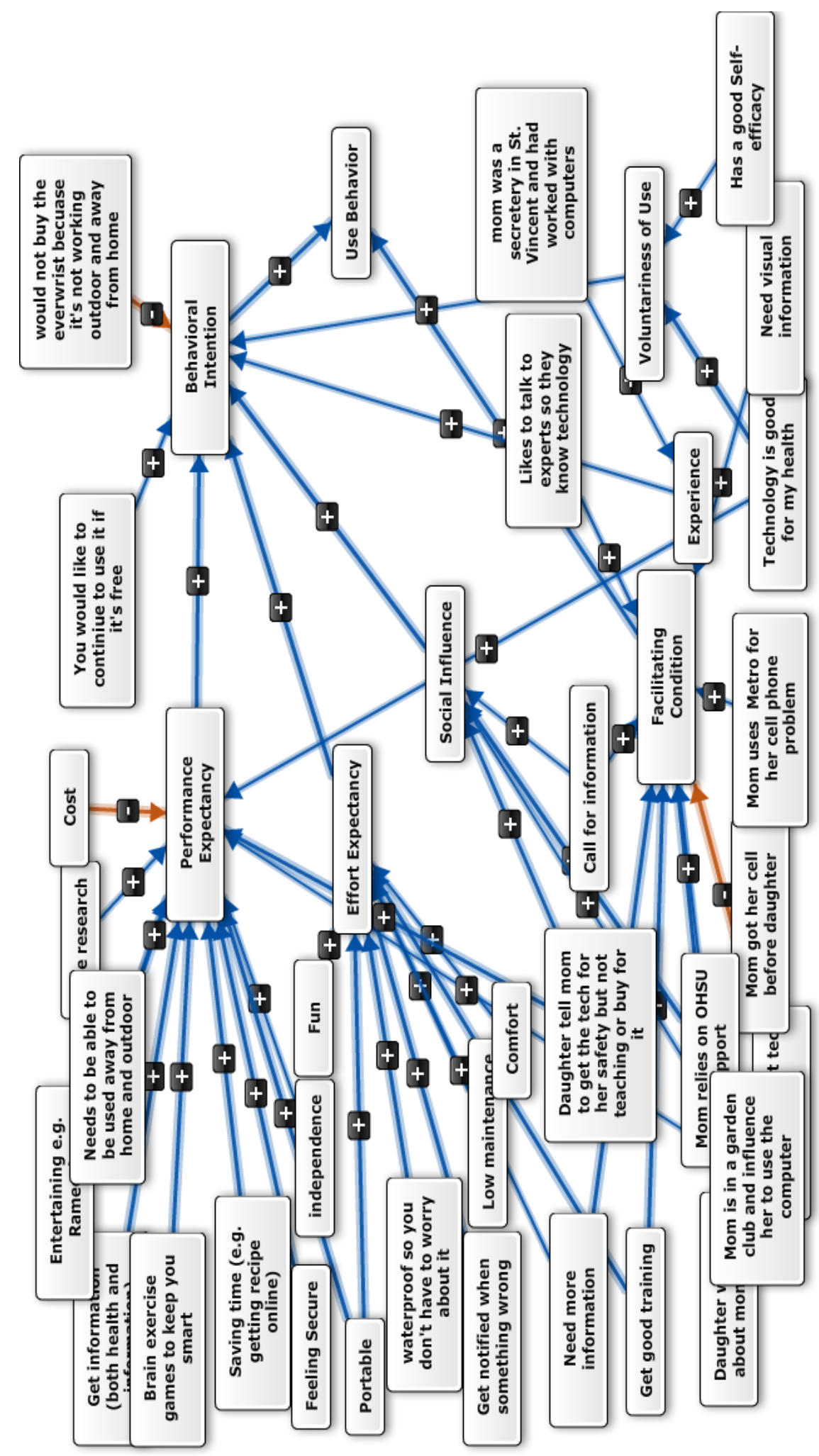

Figure B - 1 The Initial FCM of Elderly Woman \#1 


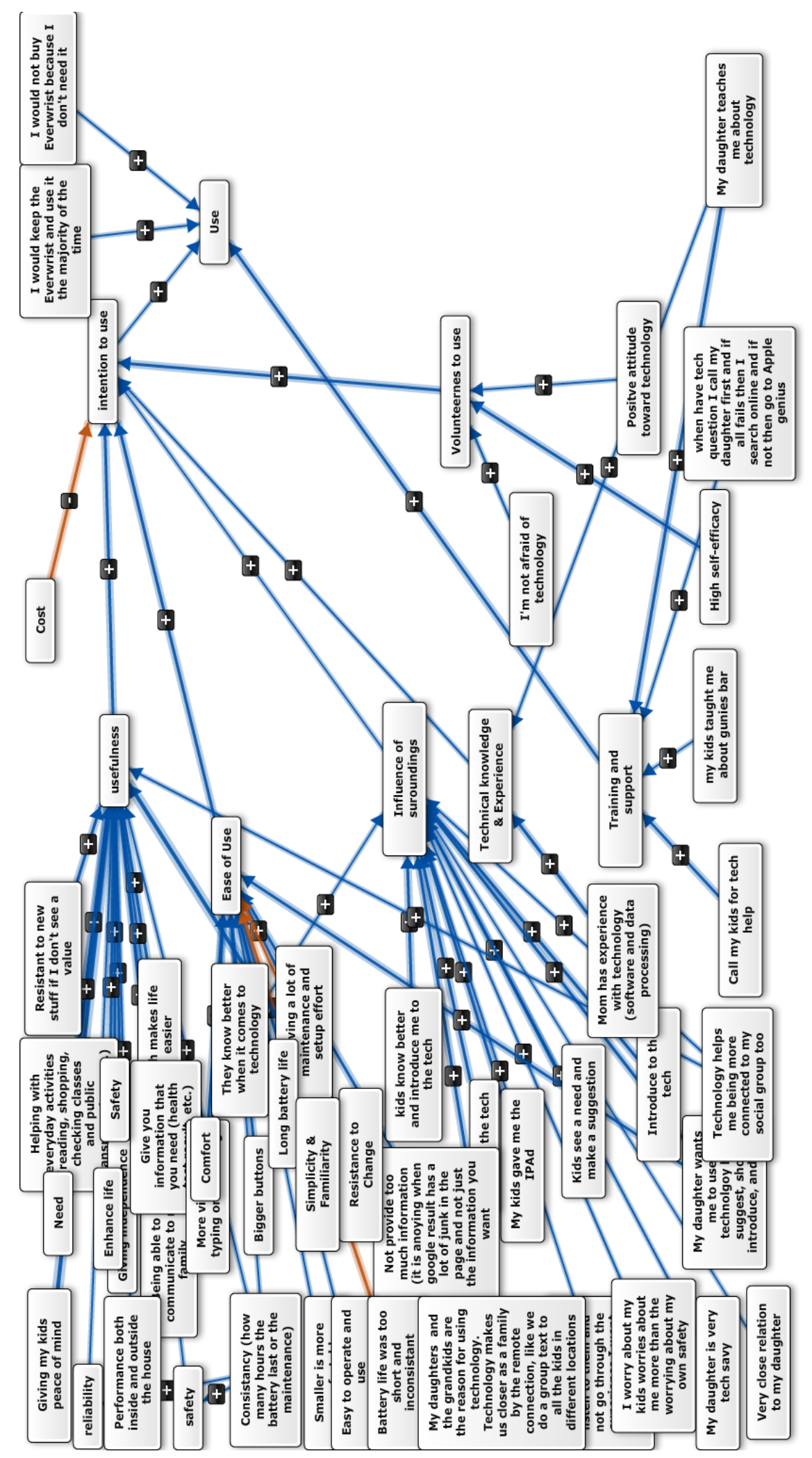

Figure B - 2 The Initial FCM of Elderly Woman \#2 


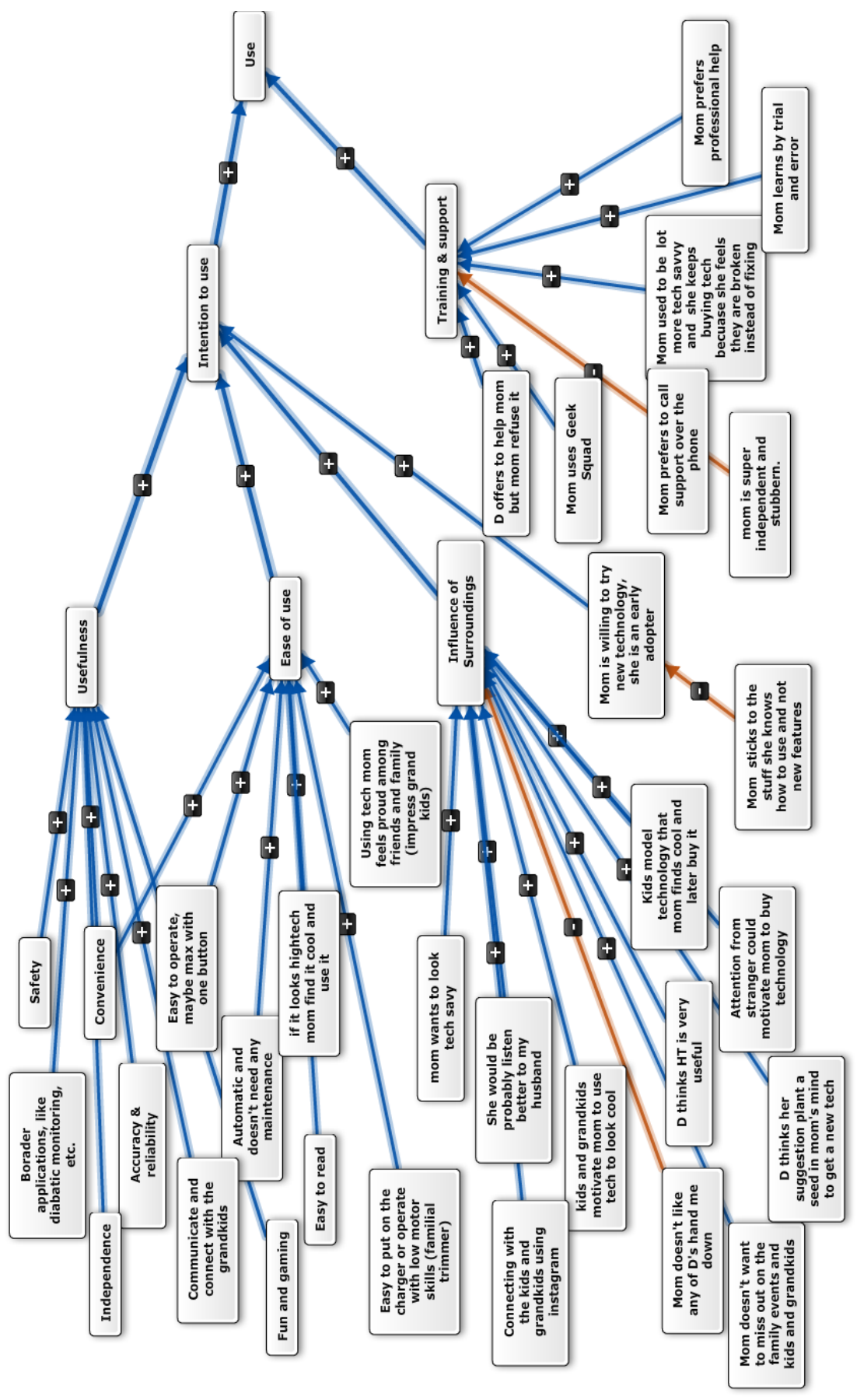

Figure B - 3 The Initial FCM of Elderly Woman \#1 by Gatekeeper 350 


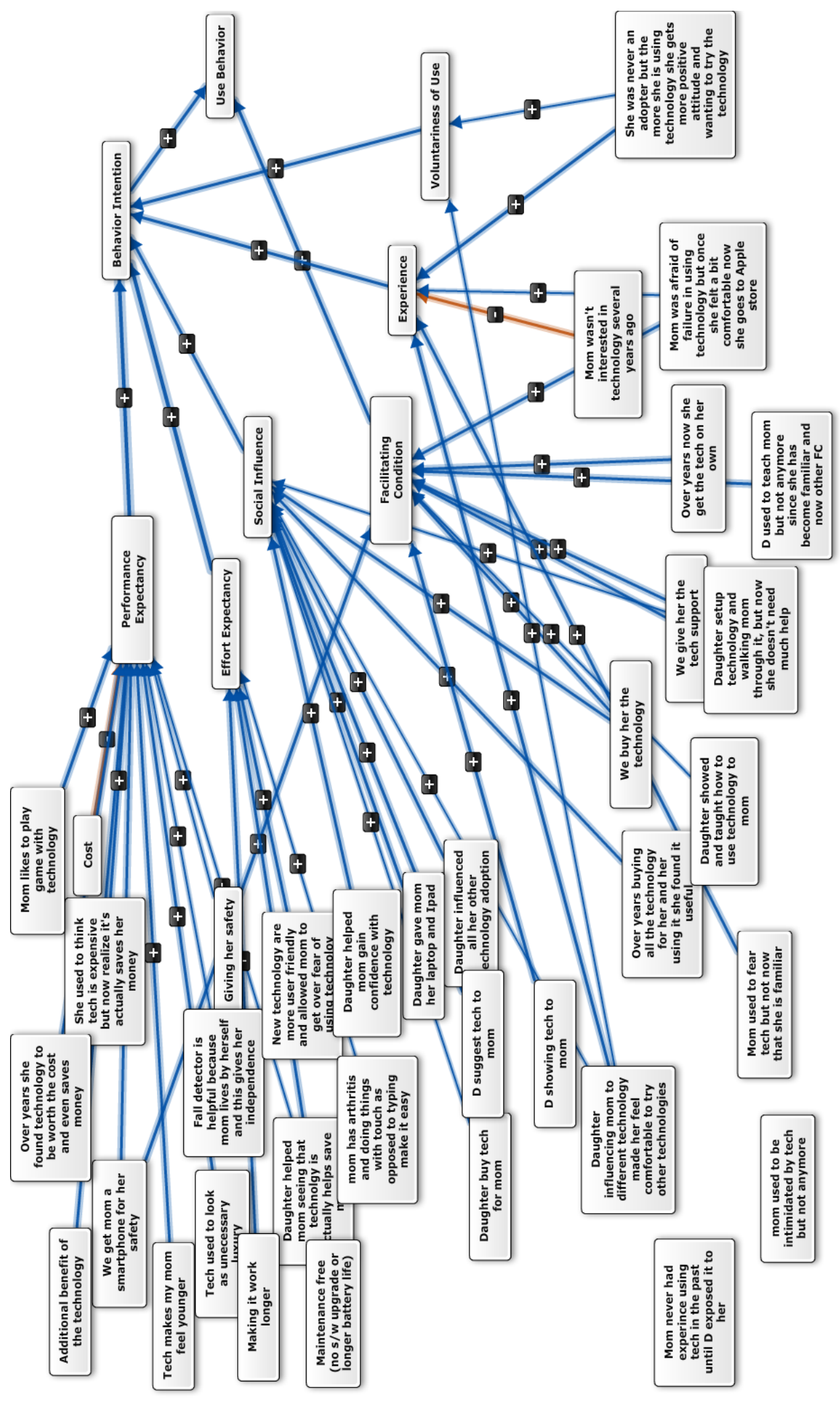

Figure B - 4 The Initial FCM of Elderly Woman \#2 by Gatekeeper

351 


\section{Conceptual and Detailed Design of FCM Models}

During the steps of this section, all four models were augmented by the qualitative analysis part of the repertory grid that contributed to the content of the cognitive map. Additionally, the models were standardized through developing an ontology list - a list of the concepts that emerged from the FCMs and the qualitative codes from the interviews. This process served as a key step in the integration of all the FCM models and formation of the FCM simulation model.

6. This step maximized the cognitive representation of each of the four interviewee's mental models on the initial fuzzy cognitive maps. To manage the scope of the pilot study, I estimated the edge weights based on the information received during the interview. This was done instead of conducting the second set of interview (to create the structure of the model) in a later step. Some of the edge weights were assigned during the interview part of the repertory-grid (in step 1 and 2); and some other during the qualitative analysis, when additional concepts were added to the participant's map (using Atlas-Ti 7 software (Figure )). This was conducted by analyzing the voice recording of each interview and augmenting the participant's FCM model using repertory-grid techniques. This approach generated new constructs from qualitative analysis of the interview, which were subsequently added to the emerging mental model. (As described in section 2.3., the repertory-grid is a grounded-theory elicitation technique that collects people's constructs through three stages-observation, emergence of patterns and formation of constructs.) The repertory-grid technique was used due to its capability to obtain fresh set of user information (Whyte \& Bytheway, 1996). As shown in Figure, this process generated and grouped 250 codes that 
yielded new concepts and relationships that were added to the initial FCM. The integrity of the augmented map was then tested against the participant's initial perception measures of EverWrist (collected in step 3). 


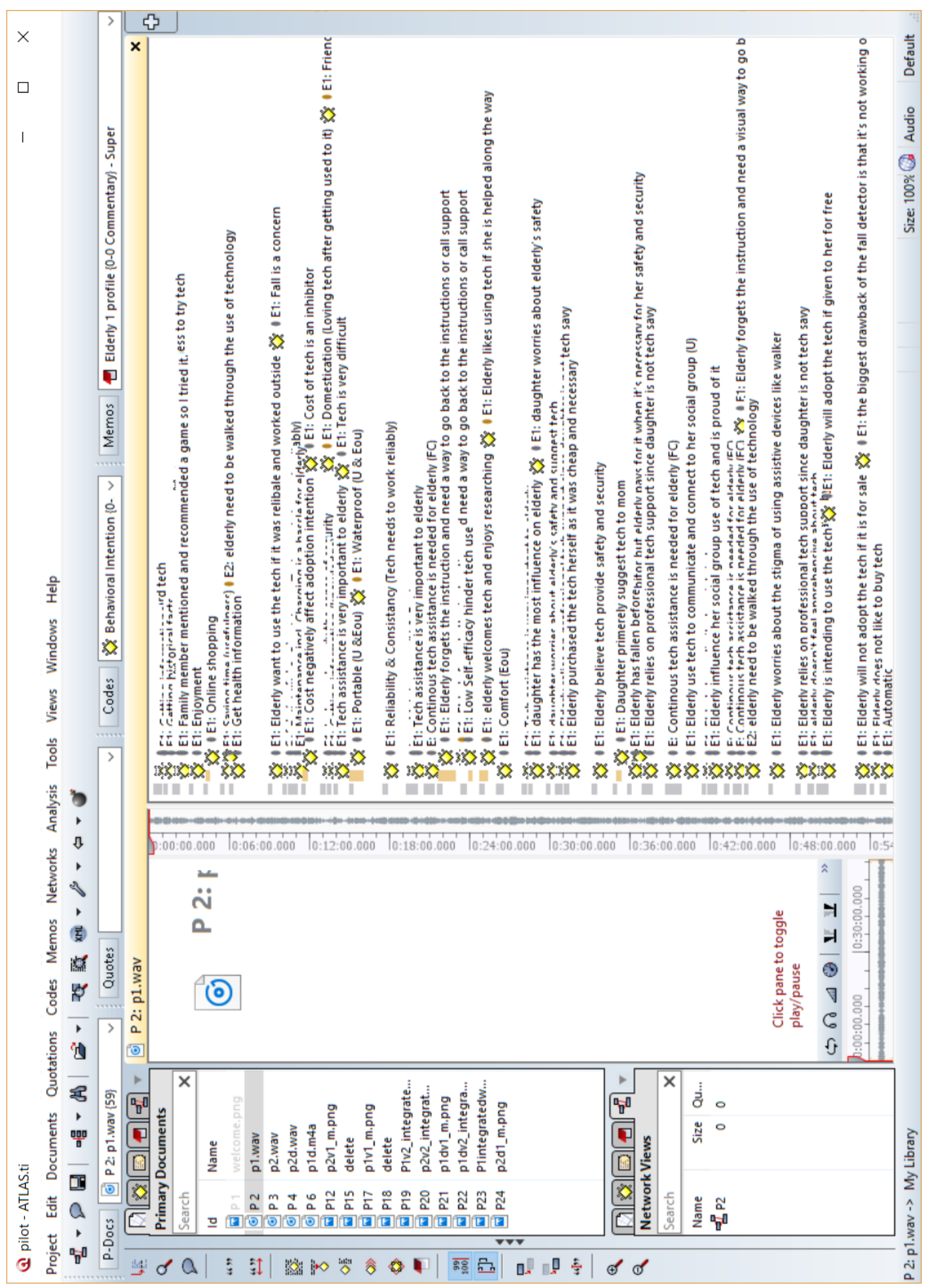

Figure B - 5 Step 6 of research method - Qualitative Analysis of each interview 354 


\begin{tabular}{|c|c|c|c|c|}
\hline \multicolumn{2}{|l|}{ 20de Manager [HU: pilot] } & - & \multirow[t]{2}{*}{$\square$} & \multirow[t]{2}{*}{$\times$} \\
\hline \multicolumn{3}{|c|}{ 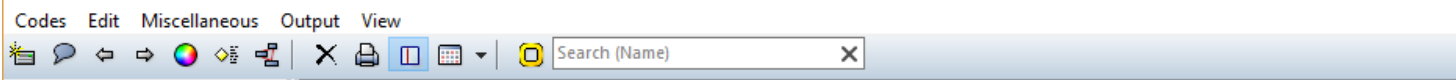 } & & \\
\hline Families & Name & \multicolumn{2}{|c|}{ Grounded } & \multirow[t]{2}{*}{$\wedge$} \\
\hline Show all Codes & Behavioral Intention & & 0 & \\
\hline \multirow{43}{*}{$\begin{array}{l}\text { Cost (11) } \\
\text { E1 (62) } \\
\text { E2 (71) } \\
\text { Ease of Use (23) } \\
\text { Elderly (133) } \\
\text { Facilitating Condition (31) } \\
\text { G1 (49) } \\
\text { G2_(39) } \\
\text { Gatekeeper (88) } \\
\text { Gatekeeper Influence (64) } \\
\text { Social Influence (63) } \\
\text { Usefulness (68) } \\
\text { Voluntariness to Use (45) }\end{array}$} & \& Convenience \& Comfort (U) & 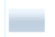 & 2 & \\
\hline & D1: Elderly is tech savy & in & 1 & \\
\hline & 2.: D1: Elderly is willing to learn and use the tech to connect to grandchild & in & 1 & \\
\hline & \& D1: G Accuracy \& Raliability (U) & a & 1 & \\
\hline & D1: G Attention can motivate elderly to purchase tech (SI) & & 0 & \\
\hline & D1: G Attention from stranger could motivate elderly to purchase tech (SI) & a & 1 & \\
\hline & D1: G Automatic (Eou) & i & 1 & \\
\hline & 2 D1: G Easy wear of tech (Eou) (E.g. putting on the fall detector watch by one hand or with familial tremor sy... & 道 & 1 & \\
\hline & D1: G Elderly does not care about look of tech (SI) & i & 1 & \\
\hline & 2*: D1: G Elderly doesn't explore all the functionalities of the tech (Vou) & + & 2 & \\
\hline & 2. D1: G Elderly doesn't like to get hands me down tech from family & a & 1 & \\
\hline & 2 D1: G Elderly enjoys interaction with stranger (SI) & i & 1 & \\
\hline & D1: G Elderly get support form tech support (FC) & a & 1 & \\
\hline & 2 D1: G Elderly get support from Geek squad (FC) & 四 & 1 & \\
\hline & D1: G Elderly gets help from where she buys tech (FC) & 道 & 1 & \\
\hline & 2 D1: G Elderly has a positive attitude toward tech and is willing to use tech & i & 1 & \\
\hline & 2 D1: G Elderly has experience w tech & 国 & 1 & \\
\hline & 2 D1: G Elderly is afraid of missing out on the family and especially grandkids' life (SI) & 国 & 1 & \\
\hline & 2 D1: G Elderly is really trying to connect with the grandkids and is willing to learn \& use new tech needed (SI) & i & 1 & \\
\hline & D1: G Elderly is stubborn and doesn't listen to gatekeeper (Resistance) & a & 1 & \\
\hline & 2.: D1: G Elderly is worried about looking weak infront of the family if ask for help (SI) & 四 & 1 & \\
\hline & D1: G Elderly occasionally accept tech support from Gatekeeper (FC) & 道 & 1 & \\
\hline & 2 D1: G Elderly relies on professional tech support (FC) & i & 1 & \\
\hline & 2. D1: G Elderly think anyting tech looks cool (Aesthetic \& SI) & 清 & 1 & \\
\hline & 2. D1: G Elderly wants to deny her low self efficacy and instead of trying to troubleshoot tech she just buys an... & 国 & 1 & \\
\hline & 20. D1: G Elderly wants to look tech savy in her social circle and friends (SI) & 泟 & 1 & \\
\hline & 20. D1: G Elderly wants to look tech savy in her social circle and specially infront of her grandkids (SI) & i & 1 & \\
\hline & 2 D1: G Entertainment (U) & 四 & 1 & \\
\hline & 2 D1: G Low Maintenace (Eou) & 运 & 1 & \\
\hline & 2 D1: G Operate easy (doesn't require a lot of fine motor) (Eou) & i & 1 & \\
\hline & 201: G Simple to operate (Eou) & 清 & 1 & \\
\hline & 2. D1: Gatekeeper and her family models tech use to elderly (SI) & I & 1 & \\
\hline & 2: D1: Gatekeeper believes elderly needs fall detector & 迺 & 1 & \\
\hline & 20. D1: Gatekeeper believes elderly refuses tech support because she is very independent, stubborn and proud & rit & 2 & \\
\hline & 201: Datekeeper believes in the usefulness of tech & & 0 & \\
\hline & D1: Gatekeeper believes tech offers convenience (U) & 四 & 1 & \\
\hline & 2. D1: Gatekeeper believes that elderly is not tech savy and struggles with tech & i & 1 & \\
\hline & 2: D1: Gatekeeper encourages TA to keep elderly independent & i & 1 & \\
\hline & 2 D1: Gatekeeper encourages TA to keep elderly safe & i & 1 & \\
\hline & D1: Gatekeeper has a very positive view of tech & i & 1 & \\
\hline & D1: Gatekeeper is interested in HT that keep elderly safe (fall detection, blood monitoring) (U) & a & 1 & \\
\hline & D1: Gatekeeper is not very involve in elderly's fall detector use & 四 & 1 & \\
\hline & D1: Gatekeeper is tech savy & 国 & 1 & $\checkmark$ \\
\hline$<$ & $<$ & & & $>$ \\
\hline 250 Codes & [1] D1: Gatekeeper believes elderly All & ame & & \\
\hline
\end{tabular}

Figure B - 6250 Codes that is integrated into the FCM model

7. The four FCM maps went through iterations of accumulative standardization across maps, where similar concepts and themes were merged and their links were consolidated. This led to the creation of an ontology list (all the standardized concepts and their definition as listed in Table B - 1). As part of the effort to generalize the models, the information related to the elderly's profile was 
extracted from the models and placed in separate files (as presented in Figure ).

These profiles later helped validate the integrity of the resulting simulation model.

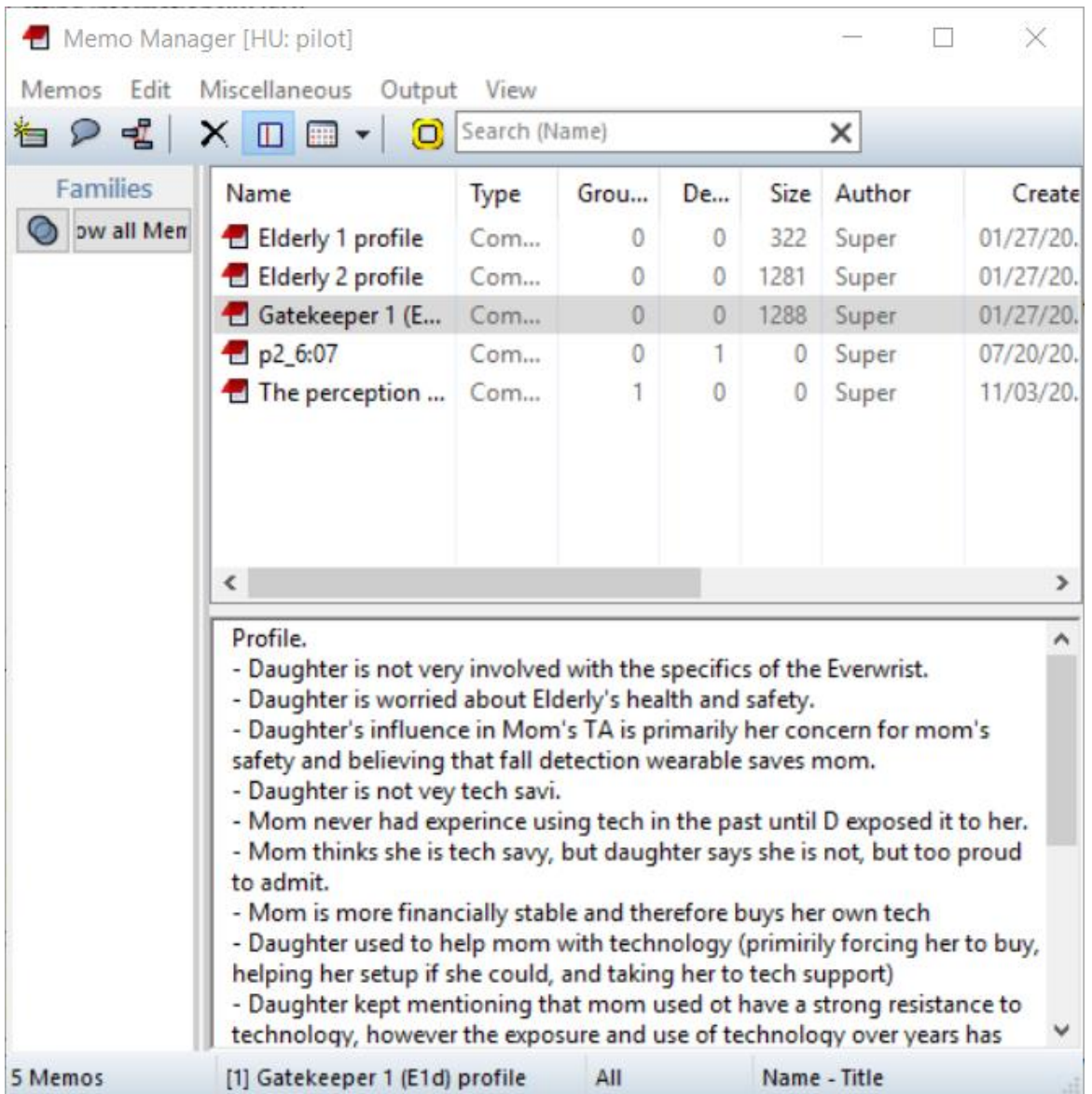

Figure B - 7 Profile of each elderly is created from the qualitative analysis of the interviews

$\begin{array}{llc}\text { Concept } & \text { Description } & \text { Category } \\ \text { Usefulness } & \text { Elderly's perception of how useful the technology is } & \text { Personal } \\ \text { Tech Characteristics } & \text { Personal } \\ \text { Ease of Use } & \text { Elderly's perception of how easy it is to use the technology } & \text { Tech Characteristics } \\ \text { Social Influence } & \text { The influence of the people around the elderly in the technology use } & \text { Social Influence } \\ \text { Technical knowledge } & \text { The technical knowledge and experiences of the elderly with ICT } & \text { Personal } \\ \text { \& Experience } & & \text { Any type of training and tech support provided to elderly }\end{array}$




\begin{tabular}{|c|c|c|}
\hline Adoption Intention & Elderly's intention to adopt the technology & Output Variable \\
\hline Adoption & Elderly's acceptance and usage of the technology & Output Variable \\
\hline Comfort & Elderly's desire for comfort & $\begin{array}{l}\text { Personal } \\
\text { Tech Characteristics }\end{array}$ \\
\hline Maintenance free & $\begin{array}{l}\text { The degree to which the technology does not need care and } \\
\text { maintenance (Charging, resetting, etc.) }\end{array}$ & Tech Characteristics \\
\hline $\begin{array}{l}\text { Giving family peace of } \\
\text { mind }\end{array}$ & $\begin{array}{l}\text { The degree to which the technology provides services that gives elderly's } \\
\text { family peace of mind }\end{array}$ & Tech Characteristics \\
\hline Independence & Technology's capability to fulfill elderly's need \& desire for independence & $\begin{array}{l}\text { Personal } \\
\text { Tech Characteristics }\end{array}$ \\
\hline Perceived cost & The result of the cost/benefit analysis of the technology & Personal \\
\hline Medical Care & $\begin{array}{l}\text { The advice or recommendation of the medical professional about using } \\
\text { the technology }\end{array}$ & Social Influence \\
\hline Usability & $\begin{array}{l}\text { The degree in which the technology is designed ergonomically (\& meets } \\
\text { elderly's cognitive condition) }\end{array}$ & Tech Characteristics \\
\hline Working everywhere & $\begin{array}{l}\text { Technology's geographical range of operation (Inside and outside of } \\
\text { place of residence) }\end{array}$ & Tech Characteristics \\
\hline Waterproof & Technology's capacity to operate when in contact with water & Tech Characteristics \\
\hline Safety & Technology's capability to fulfill elderly's need for safety & $\begin{array}{l}\text { Personal } \\
\text { Tech Characteristics }\end{array}$ \\
\hline Saving Time & The relative amount of time saved as a result of using the technology & Tech Characteristics \\
\hline $\begin{array}{l}\text { Reliability \& } \\
\text { Consistency }\end{array}$ & The degree in which the technology operates consistently and reliably & Tech Characteristics \\
\hline Voluntariness of Use & Elderly's inherent willingness to use the technology & Personal \\
\hline Positive attitude & The degree in which the elderly think positively about the use of the & Personal \\
\hline toward tech & technology & \\
\hline Portability & $\begin{array}{l}\text { The degree of the portability of the technology (how easy it is to } \\
\text { carry/wear the tech. E.g. elderly thinks the wearable around the neck is } \\
\text { easier to wear than around the wrist) }\end{array}$ & Tech Characteristics \\
\hline $\begin{array}{l}\text { Convenience \& } \\
\text { enhance life }\end{array}$ & The degree in which the technology enhances elderly's quality of life & Tech Characteristics \\
\hline
\end{tabular}




\begin{tabular}{|c|c|c|}
\hline Self-efficacy & Elderly's ability and confidence in successfully using the technology & Personal \\
\hline Promote Health & The degree in which the technology provides health benefits & Tech Characteristics \\
\hline General Information & The degree in which the technology provides useful information & Tech Characteristics \\
\hline Enjoyment & $\begin{array}{l}\text { The degree in which the technology provides joy (E.g. gaming, reading, } \\
\text { socializing, etc.) }\end{array}$ & Personal \\
\hline Connecting to family & The degree in which the technology provides joy (E.g. gaming, socializing, & Social Influence \\
\hline (\& Friends) & etc.) & Tech Characteristics \\
\hline Social learning & $\begin{array}{l}\text { Learning about the technology use by seeing the technology use (from } \\
\text { modeling) }\end{array}$ & Social Influence \\
\hline Convenient, persistent & The existence of a tech support that is convenient, available and & Social influence \\
\hline$\&$ available tech & dedicated to the elderly's learning (often provided by the elderly's & Facilitating Condition \\
\hline support & gatekeeper, friends and family members) & \\
\hline Resistance to change & $\begin{array}{l}\text { Elderly's inherent resistance to any change in her living environment } \\
\text { (often intensifies with aging) }\end{array}$ & Personal \\
\hline Friends \& family & $\begin{array}{l}\text { The influence of the people who are close to the elderly in her } \\
\text { technology use }\end{array}$ & Social Influence \\
\hline Domestication & The period when elderly tries the technology & Facilitating Condition \\
\hline $\begin{array}{l}\text { Suggesting \& } \\
\text { encouraging }\end{array}$ & The act of suggesting and encouraging the use of the technology & Social Influence \\
\hline $\begin{array}{l}\text { Convenient Tech } \\
\text { support }\end{array}$ & $\begin{array}{l}\text { The existence of a tech support that is reachable and not intimidating by } \\
\text { elderly }\end{array}$ & Facilitating Condition \\
\hline Automatic & $\begin{array}{l}\text { The degree in which the use of technology doesn't require elderly's } \\
\text { action and involvement }\end{array}$ & Tech Characteristics \\
\hline $\begin{array}{l}\text { Gatekeeper tech } \\
\text { savviness }\end{array}$ & The degree in which elderly's gatekeeper is tech savvy & Social Influence \\
\hline Gifting tech & $\begin{array}{l}\text { The possibility and frequency in which the elderly receive technology for } \\
\text { free }\end{array}$ & Social Influence \\
\hline Accessibility (Visual \& & The degree in which the technology is designed so it can be easily used & Tech Characteristics \\
\hline Bigger buttons) & by elderly with cognitive decline and special needs & \\
\hline More visual than & The degree in which the technology is designed so it can operate using & Tech Characteristics \\
\hline yping or sea & des & \\
\hline
\end{tabular}




\begin{tabular}{|c|c|c|}
\hline Existential needs & Elderly's perception of her needs & Personal \\
\hline Simplicity \& familiarity & $\begin{array}{l}\text { The degree in which the elderly desire for her life to be simple and her } \\
\text { surroundings to be familiar }\end{array}$ & Personal \\
\hline Size & The bulkiness of the technology & Tech Characteristics \\
\hline Battery life & $\begin{array}{l}\text { The period of time the technology operates before it needs to be } \\
\text { recharged }\end{array}$ & Tech Characteristics \\
\hline Preference of first & Elderly's preference for asking for tech support from her gatekeepers & Social influence \\
\hline $\begin{array}{l}\text { asking gatekeeper for } \\
\text { tech support }\end{array}$ & first and only ask other sources if gatekeeper is not available & Facilitating Condition \\
\hline Medical information & $\begin{array}{l}\text { The degree in which the technology provides useful health information } \\
\text { (One of the most important type of information elderly seeks) }\end{array}$ & $\begin{array}{l}\text { Personal } \\
\text { Tech Characteristics }\end{array}$ \\
\hline $\begin{array}{l}\text { Fear of asking naive } \\
\text { questions }\end{array}$ & $\begin{array}{l}\text { Elderly's inherent reluctance and fear about asking tech questions due to } \\
\text { low self-efficacy about tech knowledge }\end{array}$ & Personal \\
\hline $\begin{array}{l}\text { Trusting gatekeeper } \\
\text { knowledge \& }\end{array}$ & $\begin{array}{l}\text { Elderly's degree of trust to her gatekeeper that leads to the immense } \\
\text { influence of gatekeeper on elderly's TA decision }\end{array}$ & Personal \\
\hline judgement & & \\
\hline Tech cool factor & Elderly's pride to look tech savvy and cool in her social surroundings & $\begin{array}{l}\text { Personal } \\
\text { Social Influence }\end{array}$ \\
\hline Aging & The effect of human aging & Personal \\
\hline Reduce Burden & Elderly's desire to reduce burden on her gatekeeper and family members & $\begin{array}{l}\text { Personal } \\
\text { Social Influence }\end{array}$ \\
\hline $\begin{array}{l}\text { Continuity \& } \\
\text { availability of support }\end{array}$ & $\begin{array}{l}\text { The degree in which tech support is continuous and available to the } \\
\text { elderly }\end{array}$ & Facilitating Condition \\
\hline
\end{tabular}

Table B - 1 Pilot Study Ontology List

8. For every participant-gatekeeper pair, the augmented FCM maps (resulted from step 6 \& 7) were merged into a single, merged FCM ( $\left.\mathrm{FCM}_{\text {merged }}\right)$ that provided a more holistic mental model for each elderly woman during technology adoption decision making. 
The steps 6, $7 \& 8$ were repeated for the two elderly-gatekeeper pairs, which resulted in an $\mathrm{FCM}_{\text {merged }}$ model for every pair (a total of two $\mathrm{FCM}_{\text {merged). }}$

9. Inter-group analysis then became the focus of the study. Patterns of adoption were studied as part of the analysis of the models. These patterns provided insights into the intricacies of the elderly women's adoption decision making and how the adoption decision was influenced by the gatekeepers.

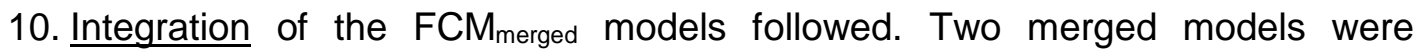
integrated into one model that contained the collective knowledge of the elderlygatekeeper pairs. The resulting model highlighted the critical drivers and barriers of the technology adoption and how these factors influenced each other. The integrated model, FCM $_{\text {int }}$ (as depicted in Figure B - 8) also shows how gatekeepers influence such key drivers and barriers.

11. Model Structure building: this process was conducted in parallel with the model content building during the qualitative data collection and analysis as detailed above. As such, this step in pilot study did not follow the step 11 of the proposed method. This work-around was conducted to manage the scope of the pilot study. Instead, during the qualitative analysis, the degree of the influence of the concepts (structure) were extracted from the information from the first set of interview.

The resulting integrated model $\left(F C M_{i n t}\right)$ formed the simulation model $\left(F C M M_{d m}\right)$ depicted below. 


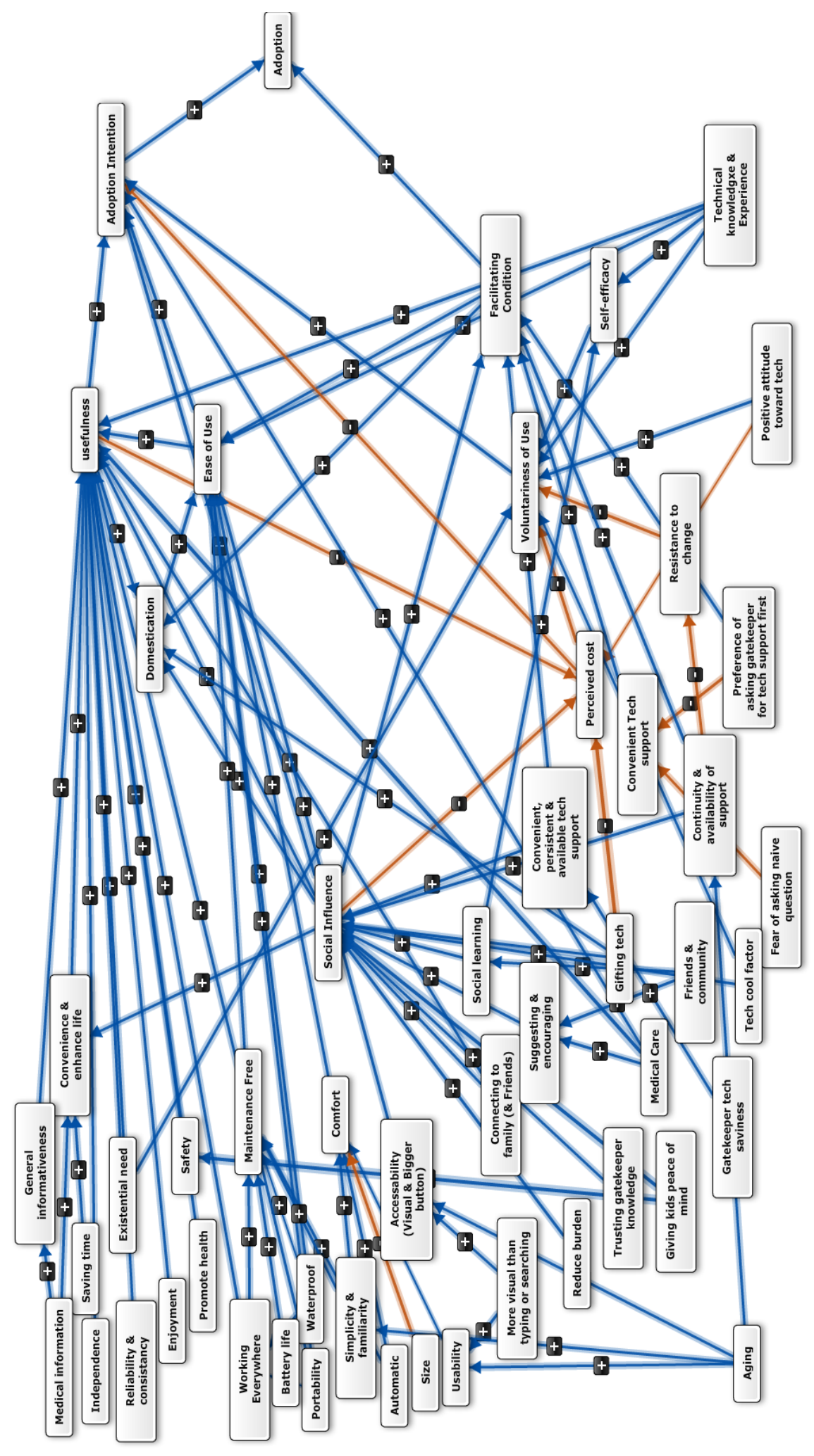

Figure B - 8 The Integrated FCM $_{\text {int }}$ (Simulation model) 


\section{Test and Validation}

The following validations were conducted to test the simulation model on each participant's profile to ensure it successfully represents the initial FCM models.

12. Internal Validation of the integrated FCM was then conducted. This was done by testing the model's outcome for each elderly woman-gatekeeper pair to validate the integrity of the model. To do this, the model behavior was tested against each elderly's initial perception of the technology (the filed profile depicted in Table B - 7). For each pair, the behavior of the integrated FCM complied with that of the FCMmerged (The adoption intention and adoption resulting values successfully reflected the information captured in the elderly interview). This step resulted in the FCMint and consequently the simulation model: $\mathrm{FCM}_{\mathrm{dm}}$.

The pilot study did not perform step 13: Expert Validation of the integrated FCM due to the limited nature of the study. The $\mathrm{FCM}_{\text {int }}$ consequently served as the simulation model: $\mathrm{FCM}_{\mathrm{dm}}$

\section{Post-adoption interview}

To validate the accuracy of the simulation model, i.e. the FCM $\mathrm{dm}^{\text {'s }}$ predictability, the pilot study conducted a post-adoption interview of the elderly women to capture the actual adoption behavior and test that the simulation model could successfully predict that behavior. To do this, I went back to the elderly women after the trial period of the EverWrist and talked to them about their experience. I then created the post-adoption profile for each elderly woman (elderly woman \#1's depicted in Table B - 2, and elderly woman \#2's in Table ). The quantified input vectors were assigned a number (from -1 to +1) that best represented elderly's situation. From there the simulation model behavior 
was tested against each elderly woman's initial profile in Table and Table. This was done using FCM simulation. For each elderly-gatekeeper pair, the profile was translated into a set of input concepts that best presented each profile (input vectors as displayed in the value column in Table and Table ). As displayed in Table B - 4 Elderly Woman \#1 EverWrist Trial Simulationand Table B - 5 Elderly Woman \#2 EverWrist Trial Simulation, the FCM simulations for both sets of input vectors resulted in negative adoption intention and adoption values. These results were in line with the post-adoption profile, and therefore the simulation model generated by the proposed research method accurately predicted the adoption behavior of its participants.

\begin{tabular}{|c|c|c|c|}
\hline post adoption interview & & Value & Profile \\
\hline Comfort & 8 & -1 & \multirow{13}{*}{$\begin{array}{l}\text { P1 likes to use a fall detector since she has fallen in the past, and her } \\
\text { daughter is encourage her to use one for her safety; however she } \\
\text { doesn't like Everwrist because it doesn't work outside. She is also } \\
\text { unhappy about Everwrist inconsistent performance. It sometimes work } \\
\text { and sometimes doesn't. The battery keep getting out of charge and } \\
\text { she has no idea when she needs to recharge it. She is also } \\
\text { complaining that the watch is too bulky and big and not having the } \\
\text { safety strap, make the band get stuck to everything. She also has to } \\
\text { often push it underneath her cloths since she is worried that the watch } \\
\text { get wet and breaks. Her number one complain is Everwrists inability } \\
\text { to work outside as she thinks she might fall outside and worrried. } \\
\text { Elderly stated that she is not adoption the technology and wouldn't } \\
\text { continue using it even if it was given to her for free. She was thinking } \\
\text { of getting a fall detector contracted by Walmart that some of her } \\
\text { friends have got (it works everywhere and connected to a monitoring } \\
\text { center (monthly payment contract for the monitoring center operation) }\end{array}$} \\
\hline Maintenance free & 9 & -0.8 & \\
\hline Giving family peace of mi & 10 & & \\
\hline Usability & 14 & -1 & \\
\hline Working everywhere & 15 & -1 & \\
\hline Waterproof & 16 & -1 & \\
\hline Reliability \& consistency & 19 & -1 & \\
\hline Convenient tech support & 35 & & \\
\hline Gatekeeper tech sa & 37 & & \\
\hline Existential needs & 41 & 0.5 & \\
\hline Battery life & 44 & -0.7 & \\
\hline Aoption intentio & & $\mathrm{n}$ & \\
\hline Adoption & & & \\
\hline
\end{tabular}

Table B - 2 Elderly Woman \#1 Post-adoption profile 


\begin{tabular}{|c|c|c|c|}
\hline Post adoption interview & C\# & Value & Profile \\
\hline Comfort & 8 & -1 & P2 doesn't think she immediately needs a fall \\
\hline Maintenance free & 9 & -0.8 & detector, however she wouldn't object it if it \\
\hline Giving family peace of mind & 10 & 0.7 & was given to her. However, she would not \\
\hline Usability & 14 & -1 & adopt Everwrist since she didn't like it's \\
\hline Working everywhere & 15 & -1 & performance. She complains about it not \\
\hline Waterproof & 16 & -1 & working consistently. The bank of the watch \\
\hline Reliability \& consistency & 19 & -1 & bothers her and she doens't think it's easy to \\
\hline Convenient tech support & 35 & & operate. \\
\hline Gatekeeper tech saviness & 37 & & All her adoptions have been resulted from \\
\hline Size & 43 & 0.6 & daughter's SI (from suggesting, gifting, training, \\
\hline Battery life & 44 & -0.7 & modeling, ) \\
\hline Fear of asking naïve question & 47 & 0.8 & Additionally, daughter is not involved with the \\
\hline Adoption Intention & no & & Everwrist trial as it's been administrated by \\
\hline Adoption & no & & ORCATECH. \\
\hline
\end{tabular}

\section{Table B - 3 Elderly Woman \#2 Post-adoption profile}

In summary, the pilot study has successfully validated the proposed research method. Evidently, it resulted in a simulation model that successfully reflected its participants' mental models. The simulation model can simulate different planned scenarios and consequently provide insight as to what scenarios lead to successful adoption. For example, it can simulate different product characteristics to identify the characteristics needed for the health product that will most likely be adopted. 


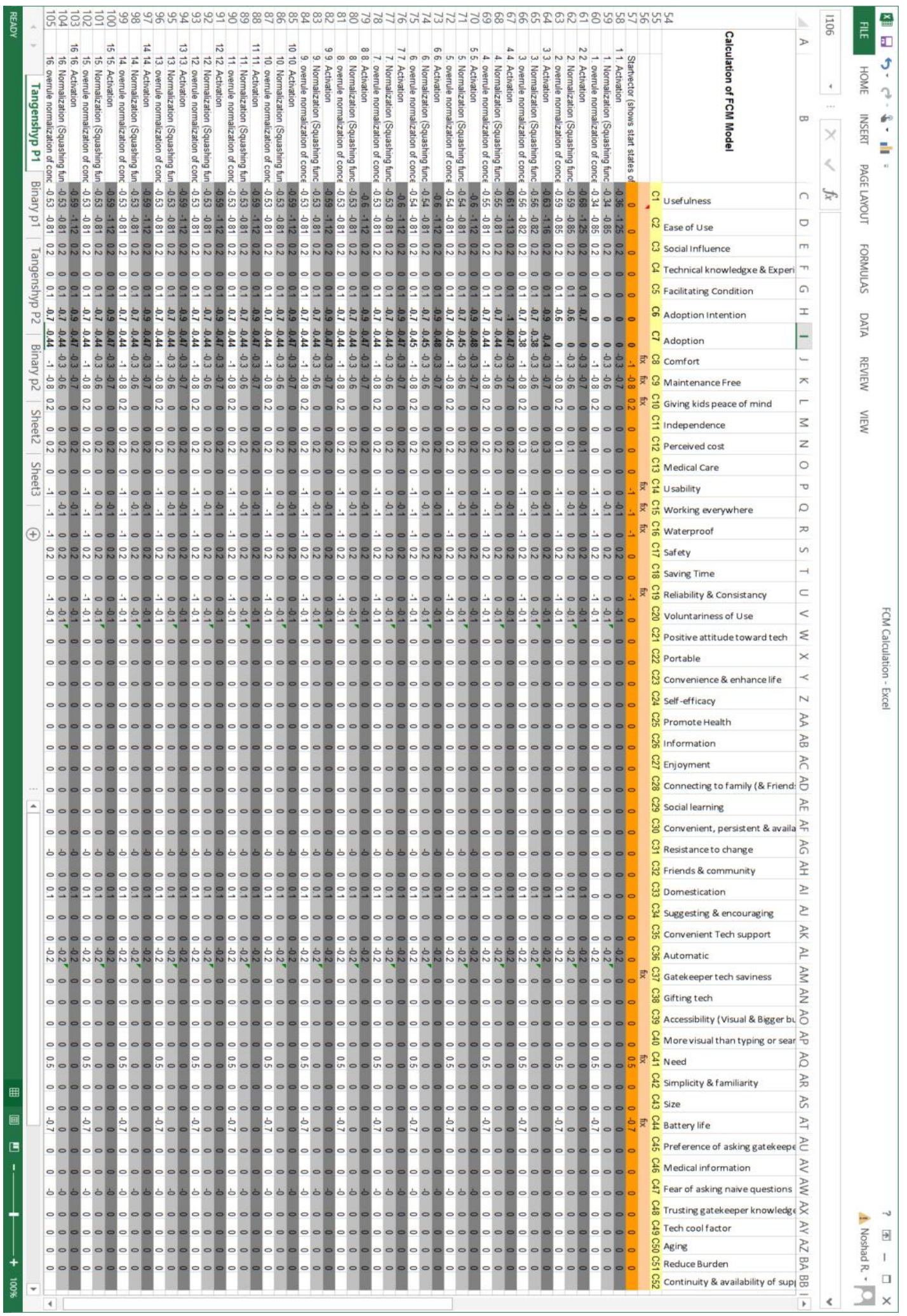

Table B - 4 Elderly Woman \#1 EverWrist Trial Simulation 


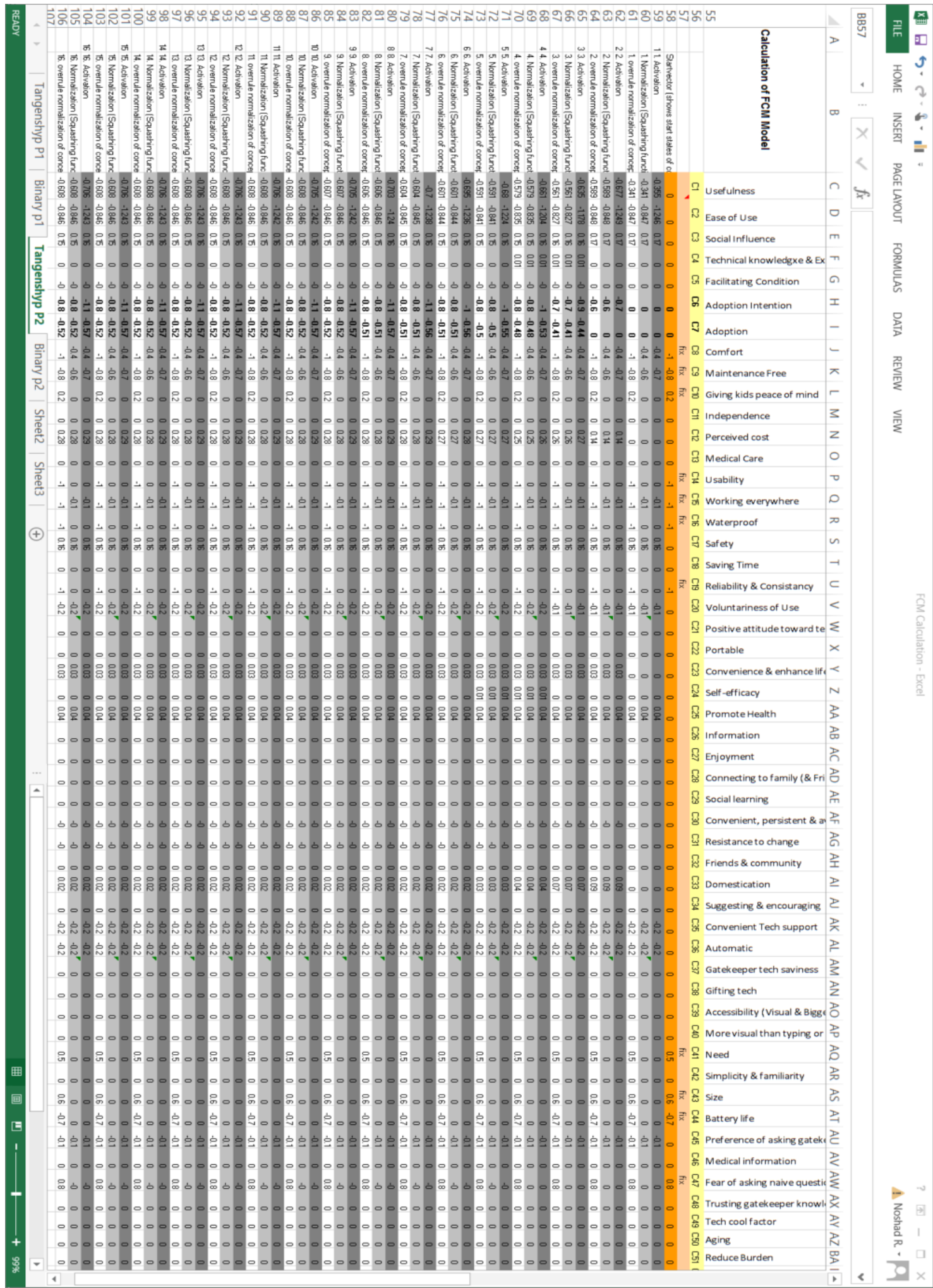

Table B - 5 Elderly Woman \#2 EverWrist Trial Simulation 


\section{Questionnaires Used in Pilot Study}

\begin{tabular}{|c|c|}
\hline \multicolumn{2}{|c|}{ Q\# Category } \\
\hline 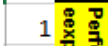 & How useful do you think technology is in keeping you healthy? How useful is Everwrist to you? \\
\hline 20 & Why is it useful? What makes it useful? \\
\hline $3 \frac{y^{3}}{2}$ & What are the performances you are interested in? \\
\hline 4 & What is a useful fall detector like, to you? \\
\hline $5 \frac{8}{2} \mathrm{~m}$ & How easy to use Everwrist is, for you? \\
\hline $6 \stackrel{2}{\circ}$ & What are the challenges? Anything you find hard? \\
\hline $7 \frac{3}{3}$ & If you could change anything about Everwrist, what would you do to make it easier? \\
\hline 8 & How easy is it to learn to operate technology? Everwrist? \\
\hline $9 \stackrel{్ ట ్}{\circ}$ & Is using technology is a bad/good idea in general? \\
\hline 10 8 $\bar{z}$ & How did you decide to try Everwrist? Did you volunteer by yourself or someone made you? \\
\hline 11 飠岁 & Is using the device fun? \\
\hline 12 & Do you like working with Everwrist? How about with technology in general? \\
\hline $13 \check{\varrho}$ & Who has the most influence in your decisions? Who has the most influence in your decision to use technology? Everwrist? \\
\hline 14 䰹 & How close are you to your kids? Daughter? \\
\hline $15 \bar{\Xi}$ & Do your kids think you should use technology? How about using Everwrist? \\
\hline $16 \stackrel{\vec{c}}{\bar{c}}$ & If they do, How do they generally influence your technology use? \\
\hline 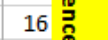 & By suggestion? \\
\hline $16^{\infty}$ & By showing and making you familiar with technology? \\
\hline 16 & By teaching you how to use it or being available to answer any questions you might have? \\
\hline 16 & By buying or giving you the technology? \\
\hline 17 & Are your kids tech savvy? How much do they use technology? \\
\hline 18 & Do your kids have a lot of experience with technology? How do they like using technology? \\
\hline 19 & Do you think they have any influence in making you feel that technology is useful? \\
\hline 20 & Do you think they have any influence in making you feel that technology can be easy? \\
\hline 21 & How about your other social surrounding, do they have any influence or support your technology usage? \\
\hline 22 \& & Do you have the knowledge necessary to use the device? \\
\hline 23 言高 & How do you learn how to use technology? Do you call help desk/call your kids/read manual/trial and error? \\
\hline $24 \stackrel{\overrightarrow{0}}{g}$ & Do you have the resources you need to use technology? \\
\hline 25 & Are your kids available to help you use the system? \\
\hline $26 \underset{\nexists 灬}{\stackrel{\Phi}{\#}}$ & Can you usually complete a task with technology without help? \\
\hline 27 通 & Can you usually complete a task with technology if you have someone to call if needed? \\
\hline $28^{2}$ & Can you usually complete a task with technology if you have enough time? \\
\hline 29 & What type of assistance do you prefer to have? Professionals (like: Apple Genius bar) or friends or your kids? \\
\hline $30 \geqq$ & Do you feel apprehensive about using technology? \\
\hline $31 \frac{\mathrm{x}}{\mathrm{g}} \mathrm{g}$ & Do you ever fear to make a mistake with technology? (e.g. hitting a wrong key and loosing information) \\
\hline 32 & Do you hesitate to use technology for that fear? \\
\hline 33 & Is the device/technology in anyway intimidating to you? \\
\hline 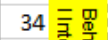 & Are you intending to use Everwrist consistently during the next month? \\
\hline 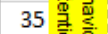 & If they give you the device, do you predict to continue using it? And for how many more months? \\
\hline $36 \overline{\mathrm{g}}$ & If they sell you the device, would you buy and use it? Why/why not? \\
\hline 37 㸚 & Do you have a lot of experience with technology in general? Can you explain? \\
\hline $38 \stackrel{0.0}{\sigma}$. & In the past (e.g. job), did you use technology a lot? \\
\hline $39 \stackrel{0}{\mathrm{D}}$ & What other technologies do you use? \\
\hline
\end{tabular}

Table B - 6 Repertory-grid open-ended questions of the pilot study 


\section{Participant perception questionnaire}

Please provide your perspective of the EverWrist fall detection (scale of 0 to 100). Feel free

to talk about it.

1 How useful the product seem to you?

2 How easy is it to use it?

3 How much influence your family (your daughter or study partner) have on you in using EverWrist?

4 How much influence other people around you might have on you in using EverWrist

(E.g. your friends using it)?

5 How important is to have training and help in using EverWrist?

6 How much experience do you have with technology?

7 Would you consider yourself tech savvy?

8 How much do you enjoy or like to work with technology? Would you volunteer to use EverWrist (or other technologies) for its benefits, if it was entirely up to you?

9 If at the end of the trial you are given the EverWrist, how would you rate your intention to use it?

10 If at the end of the trial you are given the EverWrist, what is the probability that you would actually keep using it?

Table B - 7 Participants perception questionnaire of the pilot study 


\section{Appendix C - Qualitative (QUAL) Research Data Collection Supporting Material}

\section{C.I. Participants Consent Form}

\section{The Portland State University \\ Consent to Participate in Research}

\section{Technology Adoption among Senior Women}

You are being asked to participate in a research study by Antonie Jetter, who is the Principal Investigator from the Department of Engineering and Technology Management, at Portland State University. This research is studying how women accept healthcare technologies. You are being asked to participate in this study because you are either:

- A woman of $65+$ years of age and intending to tryout a remote health monitoring watch, or

- The study partner of a women who is intending to tryout a remote health monitoring watch

This form will explain the research study, and will also explain the possible risks as well as the possible benefits to you. If you have any questions, please ask one of the study investigators. 


\section{What will happen if I decide to participate?}

We will contact you to set up time and location for an interview that is convenient to you. Most meetings will be held during regular office hours in your home or a public meeting space most convenient to you. During the interview, we will ask you to freely explain your opinion about the health technologies you have used and/or will likely to use, and explain about your motivations, concerns and preferences in regards to them. During these conversations we will help you to practice and will draw your graphical mental model (“cognitive maps") where we draw important concepts you mention and draw links between them to show their influence on each other. The interview will be audio recorded to make sure that your comments are recorded accurately.

\section{How long will I be in this study?}

Participation in this study will take 2 to 3 hours in total. We will ask to interview you twice (1-1.5 hours for each interview), once before and once after the trial of a remote health monitoring product.

\section{What are the risks or side effects of being in this study?}

Some people may find it difficult to talk to researchers or other participants about their decision-making process or their needs and preferences in regards to technologies. They may experience stress. You are free to stop participating in this study at any time and for any reason. Though we try to accommodate different needs 
and schedules, you may be inconvenienced by the study participation. Though unlikely, there is the risk of a possible loss of privacy and confidentiality in a research study. For more information about risks and discomforts, ask the investigator.

\section{What are the benefits to being in this study?}

You may not receive a direct benefit from participating in this research. However, some people find sharing their information and learning about cognitive maps a valuable experience. We hope that this study will contribute to learning ways to better acceptance of technologies and result in improving women's health.

\section{How will my information be kept confidential?}

We do our best to protect the security of all your personal information, but we cannot guarantee confidentiality of all study data. To keep your information as safe as possible, we will store audio files and all other research data on the password protected PSU server and use special coding to protect your information. All the audio files and any other identifiable information will be destroyed immediately after the transcription and analysis (approximately within a year from data collection). To protect confidentiality, your name will not be used in a written copy of the interview / focus group discussion.

We plan to publish the results of the study, but we will not include any information that would identify you. Your name will not be used in any published reports about this study. Information contained in your study records is used by study staff. The 
Portland State University Institutional Review Board (IRB) and/or other entities may be permitted to access your records, and there may be times when we are required by law to share your information. It is the investigator's legal obligation to report child abuse, child neglect, elder abuse, harm to self or others or any life-threatening situation to the appropriate authorities, and; therefore, your confidentiality will not be maintained.

\section{Will I be paid for taking part in this study?}

You will not be paid for taking part in this study and will have to pay for your own travel expenses. If you come by car to a university location, we will be able to reimburse your parking on university assigned parking lots.

\section{Can I stop being in the study once I begin?}

Yes, you can stop participating in this study at any time.

\section{Whom can I call with questions or complaints about this study?}

If you have any questions, concerns or complaints at any time about the research study, Antonie Jetter, or her associates will be glad to answer them at 503-725-4663.

\section{Whom can I call with questions about my rights as a research participant?}

If you have questions regarding your rights as a research participant, you may call the PSU Office for Research Integrity at (503) 725-2227 or 1(877) 480-4400. The IRB is a group of people from PSU and the community who provide information about safety and ethical issues related to research involving human participants. For more information, you may also access the IRB website at https://sites.google.com/a/pdx.edu/research/integrity.

\section{CONSENT}


By signing this consent form, you are agreeing that:

- You have read this consent form (or it has been read to you)

- You have had an opportunity to ask questions and all questions have been answered to your satisfaction

- You understand that you may leave the study at any time

- You understand that you are not waving any of your legal rights as a research participant

- Your participation in this research is completely voluntary.

- You have received a copy of the consent form for your record.

Name of Adult Subject (print)

Signature of Adult Subject

Date

\section{INVESTIGATOR SIGNATURE}

This research study has been explained to the participant and all of his/her questions have been answered. The participant understands the information described in this consent form and freely consents to participate.

Name of Investigator/ Research Team Member (type or print)

(Signature of Investigator/ Research Team Member)

Date

\section{C.II. Open-ended Interview Questions}

\begin{tabular}{|c|c|c|}
\hline Category & Q\# & Question \\
\hline \multirow{6}{*}{ 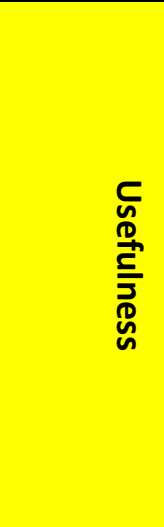 } & 1 & How useful do you think technology is in keeping you healthy? \\
\hline & & $\begin{array}{l}\text { Are you familiar with Activity Tracking? Do you belive it would provide any } \\
\text { health benefit? If yes, what? }\end{array}$ \\
\hline & 2 & $\begin{array}{l}\text { How useful is activity tracking to you? Why is it useful? What makes it } \\
\text { useful? ( }\end{array}$ \\
\hline & 3 & What are the activity measures you are interested in? \\
\hline & & $\begin{array}{l}\text { - Do you think the type of data this watch collects will benefit you, your } \\
\text { loved ones or your clinician? }\end{array}$ \\
\hline & 5 & $\begin{array}{l}\text { What challenges do you think there are in using the devise? In general or in } \\
\text { particular to an activity tracker? }\end{array}$ \\
\hline
\end{tabular}




\begin{tabular}{|c|c|c|}
\hline & 6 & If you could change anything about the watch, what would that be? \\
\hline & & - What would you do to make it easier? \\
\hline & 7 & How easy is it for you to learn to operate a technology? watch? \\
\hline \multirow{4}{*}{ 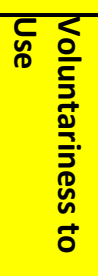 } & 8 & $\begin{array}{l}\text { Who has the most influence in your technological decisions? and medical } \\
\text { decisions? }\end{array}$ \\
\hline & 9 & How did you decide to volunteer to test this device? \\
\hline & 10 & Do you think using the device will be fun? What did you enjoy about it? \\
\hline & 11 & Do you like wearing this watch? How about with technology in general? \\
\hline \multirow{11}{*}{ 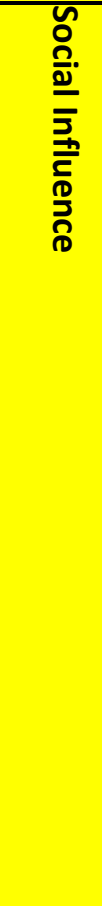 } & 12 & $\begin{array}{l}\text { How close are you to your family and friends? What is the most important } \\
\text { social community you have? }\end{array}$ \\
\hline & 13 & $\begin{array}{l}\text { Do your kids think you should use technology? How about using Activity } \\
\text { Tracker? }\end{array}$ \\
\hline & 14 & If they do, How do they generally influence your technology use? \\
\hline & 15 & By suggestion? \\
\hline & 16 & By showing and making you familiar with technology? \\
\hline & 17 & $\begin{array}{l}\text { By teaching you how to use it or being available to answer any questions } \\
\text { you might have? }\end{array}$ \\
\hline & 18 & By buying or giving you the technology? \\
\hline & 19 & Are your family or friends tech savvy? How much do they use technology? \\
\hline & 20 & $\begin{array}{l}\text { Do your family/friends have a lot of experience with technology? How do } \\
\text { they like using technology? }\end{array}$ \\
\hline & 21 & $\begin{array}{l}\text { Do you think they have any influence in making you feel that technology is } \\
\text { useful or easy to use? }\end{array}$ \\
\hline & 22 & $\begin{array}{l}\text { How about your other social surrounding, do they have any influence or } \\
\text { support your technology usage? }\end{array}$ \\
\hline \multirow{4}{*}{ 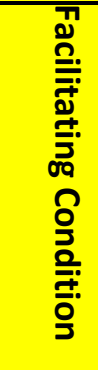 } & 23 & $\begin{array}{l}\text { How do you usually learn to use a technology? Do you read manual, ask } \\
\text { someone (who)? Trial \& Error? Or call/go to help desk? }\end{array}$ \\
\hline & 24 & $\begin{array}{l}\text { How do you learn how to use technology? Do you call help desk/call your } \\
\text { social circle/read manual/trial and error? }\end{array}$ \\
\hline & 25 & Do you have the resources you need to use technology? \\
\hline & 26 & $\begin{array}{l}\text { Are your social circle available to help you use the system? (what system, } \\
\text { tracker?) }\end{array}$ \\
\hline \multirow{6}{*}{ 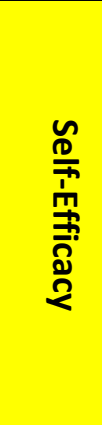 } & 27 & Can you usually use technology without help? \\
\hline & 28 & Can you usually use technology if you have someone to call if needed? \\
\hline & 29 & Can you usually complete a task with technology if you have enough time? \\
\hline & 30 & $\begin{array}{l}\text { What type of assistance do you prefer to have? Professionals (like Apple } \\
\text { Genius bar) or friends or your kids? }\end{array}$ \\
\hline & 31 & $\begin{array}{l}\text { Do you ever fear to make a mistake with technology? (e.g. hitting a wrong } \\
\text { key and losing information) }\end{array}$ \\
\hline & 32 & Do you hesitate to use technology for that reason? \\
\hline
\end{tabular}




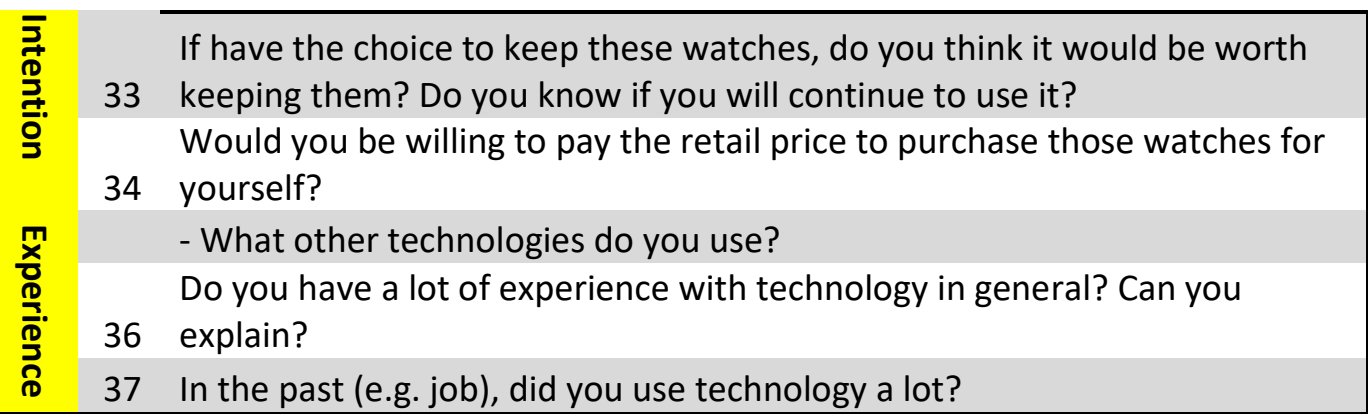




\section{Appendix D - Qualitative (QUAL) Research Analysis Supporting Material}

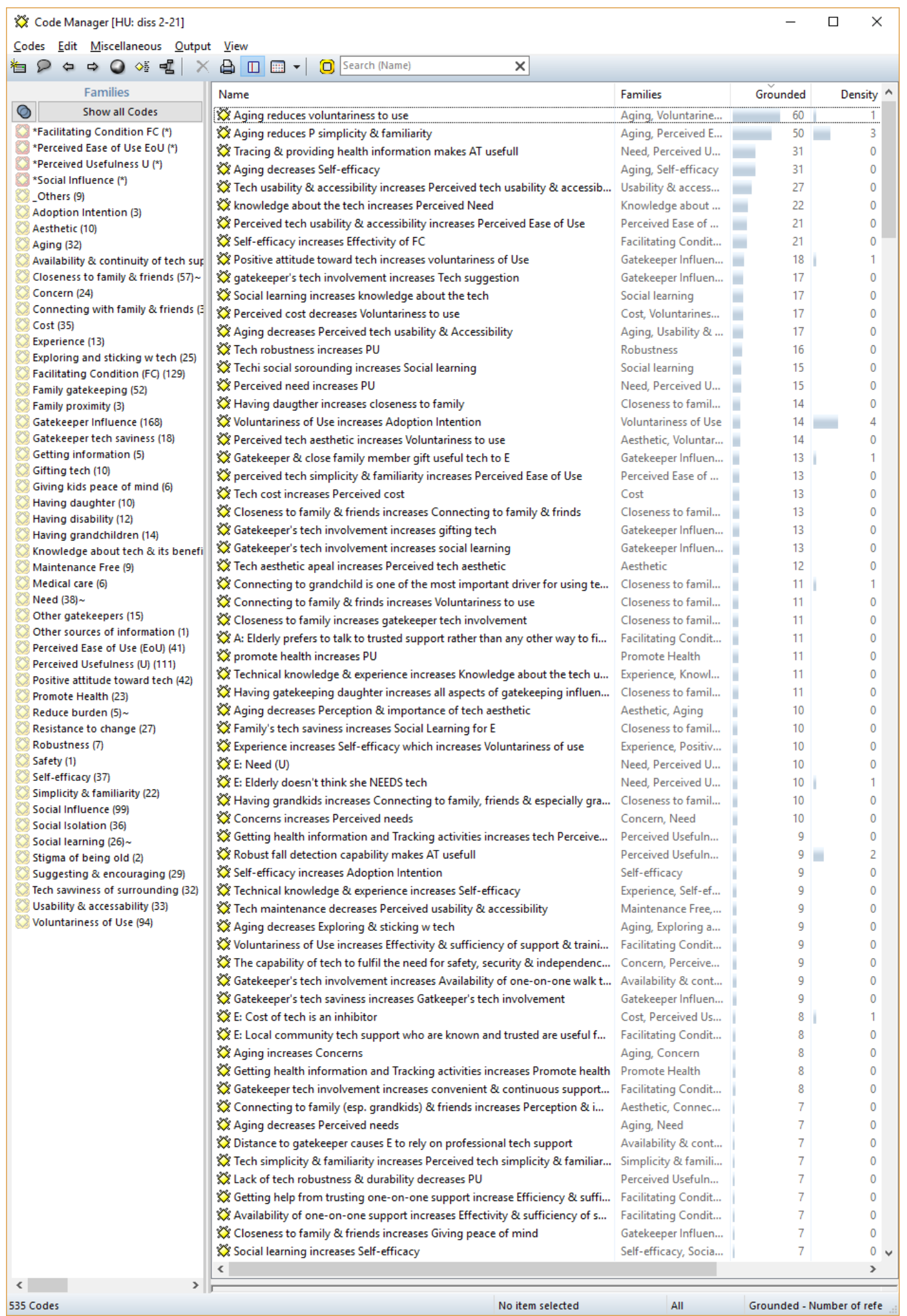

Figure D - 1 Case Study Open Codes on the right and Code families in left from Atlas.ti 


\section{Appendix E - Quantitative (quan) Data Collection Supporting Material}

\section{E.I. Quantitative (quan) Data Collection Structured Survey Instrument}

It's worth to note that the choices in the multiple choices ask the participants to identify the degree of the 'importance' of the links as opposed to the 'strength' of the links. This is mainly due to the elderly choice of the wordings as observed during the ethnographic interviews. Although the two words have different meaning, in the context of this inquiry they were used interchangeably as I walked the elderly through the survey.

\section{The survey}

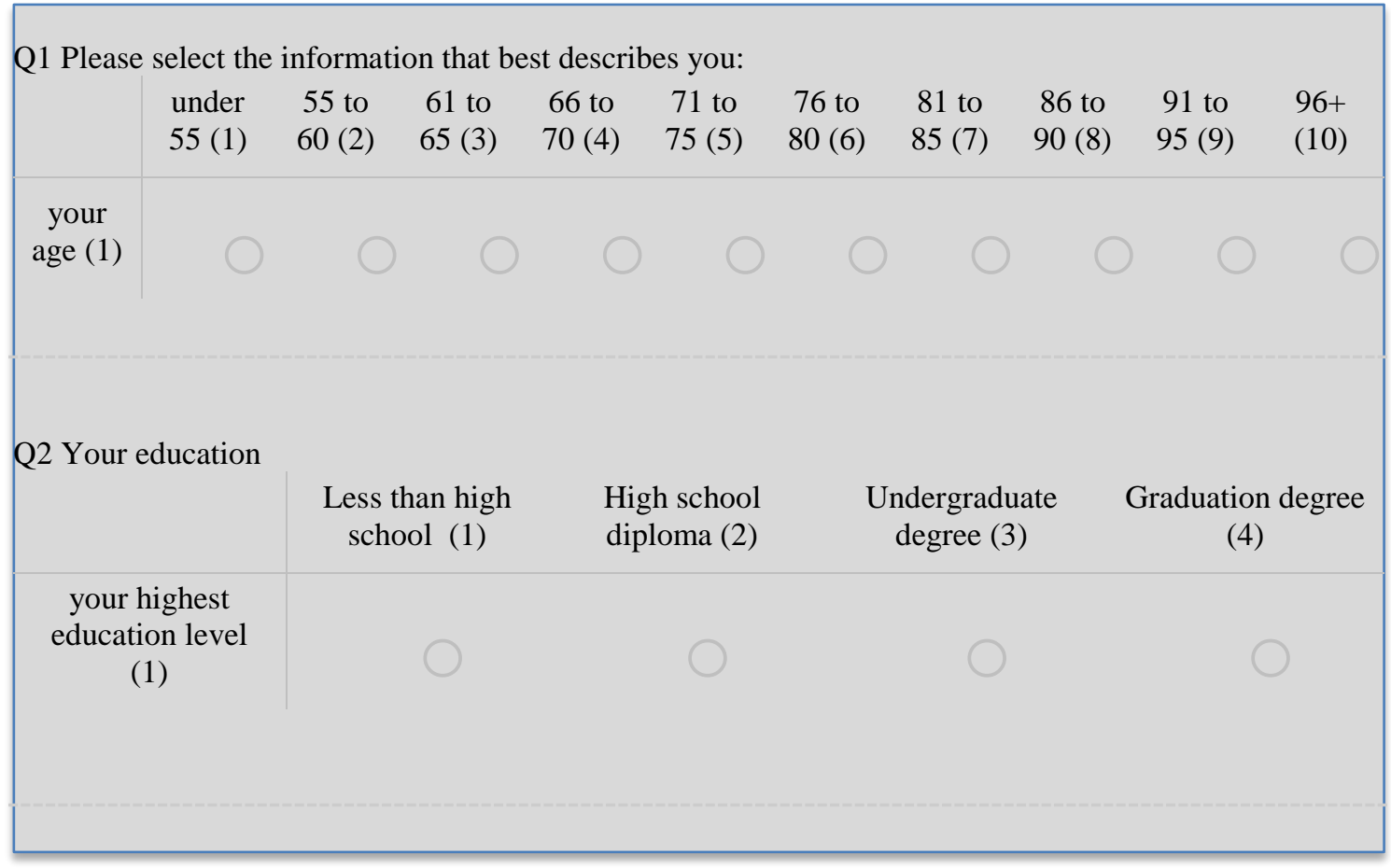




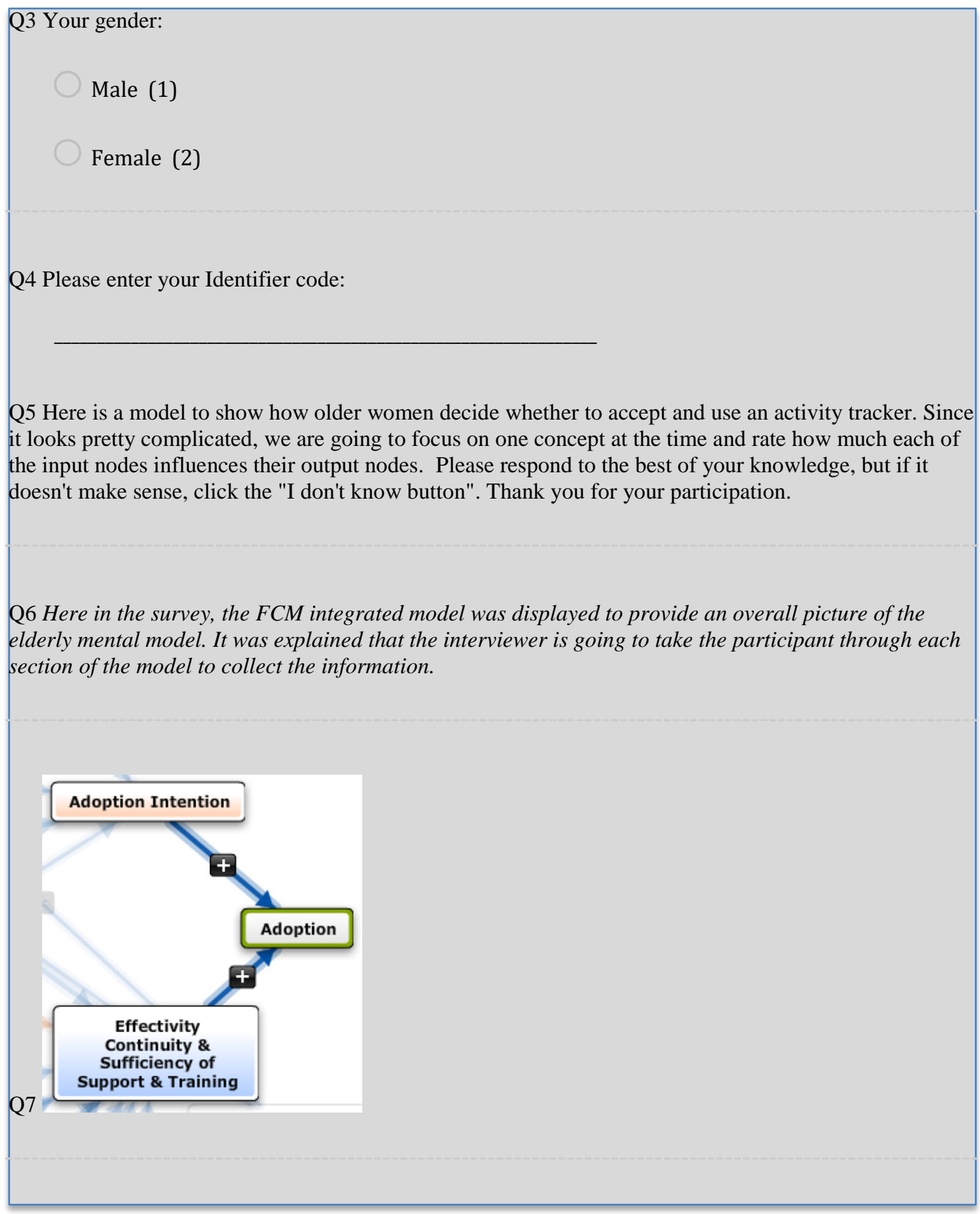




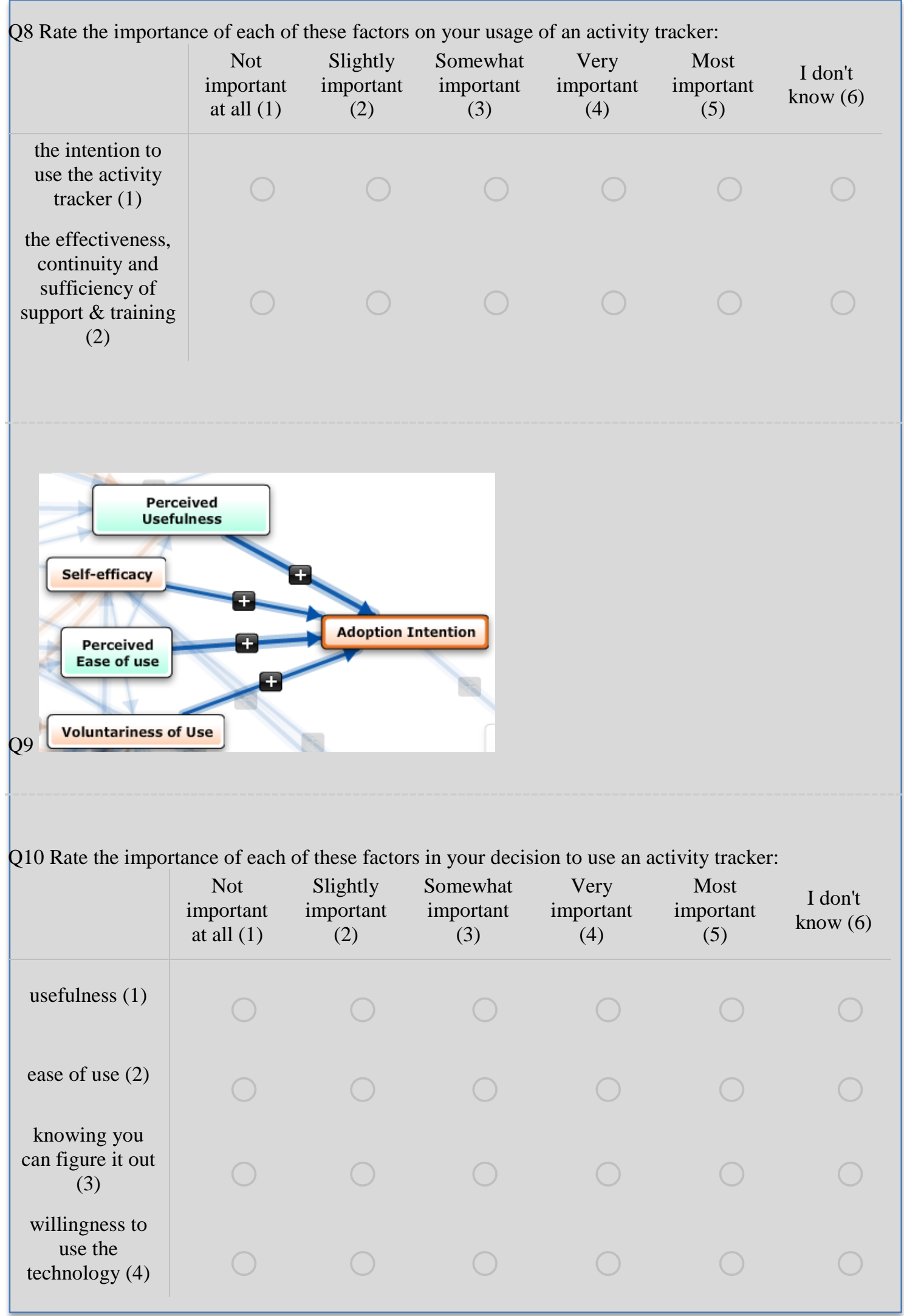




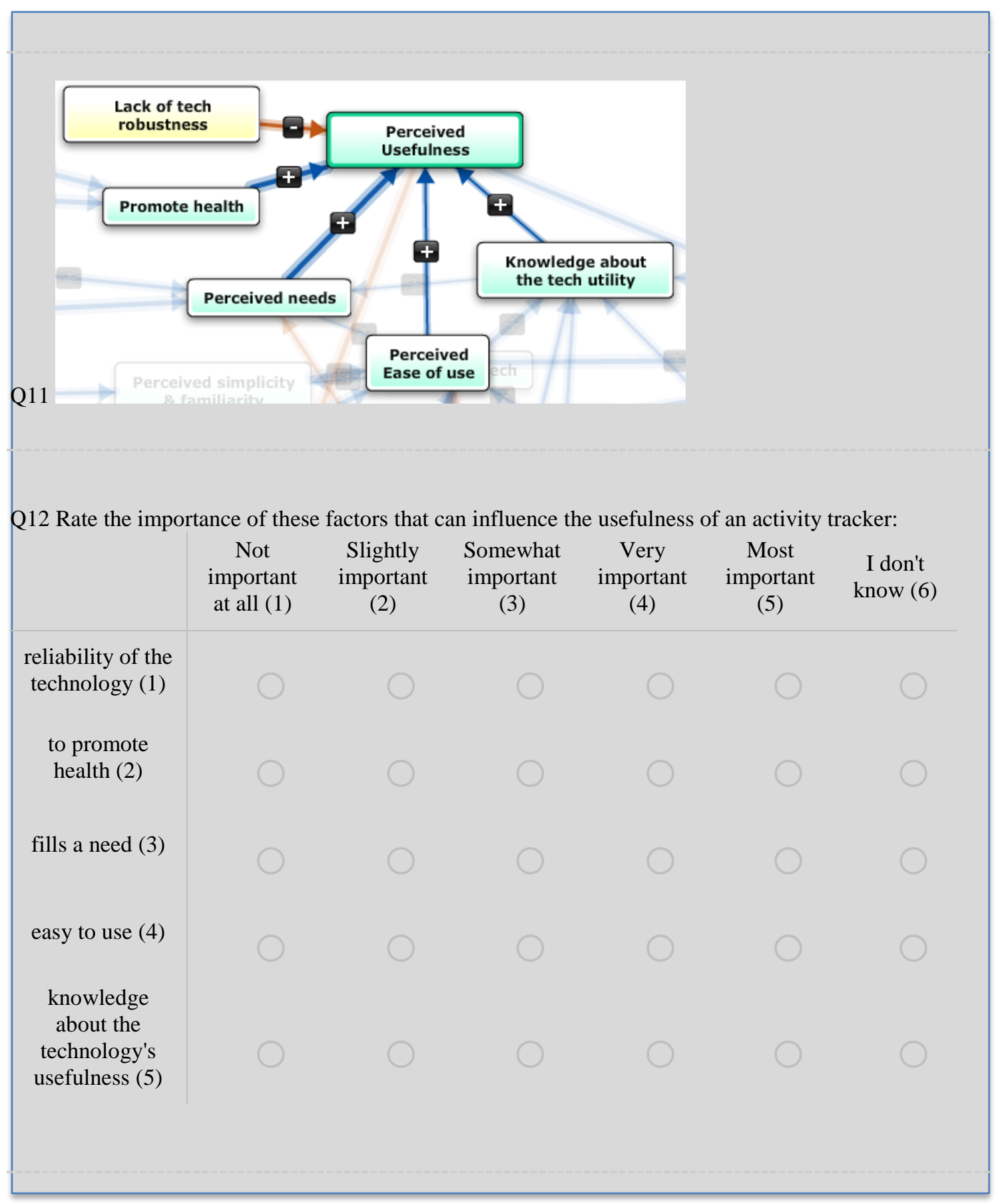




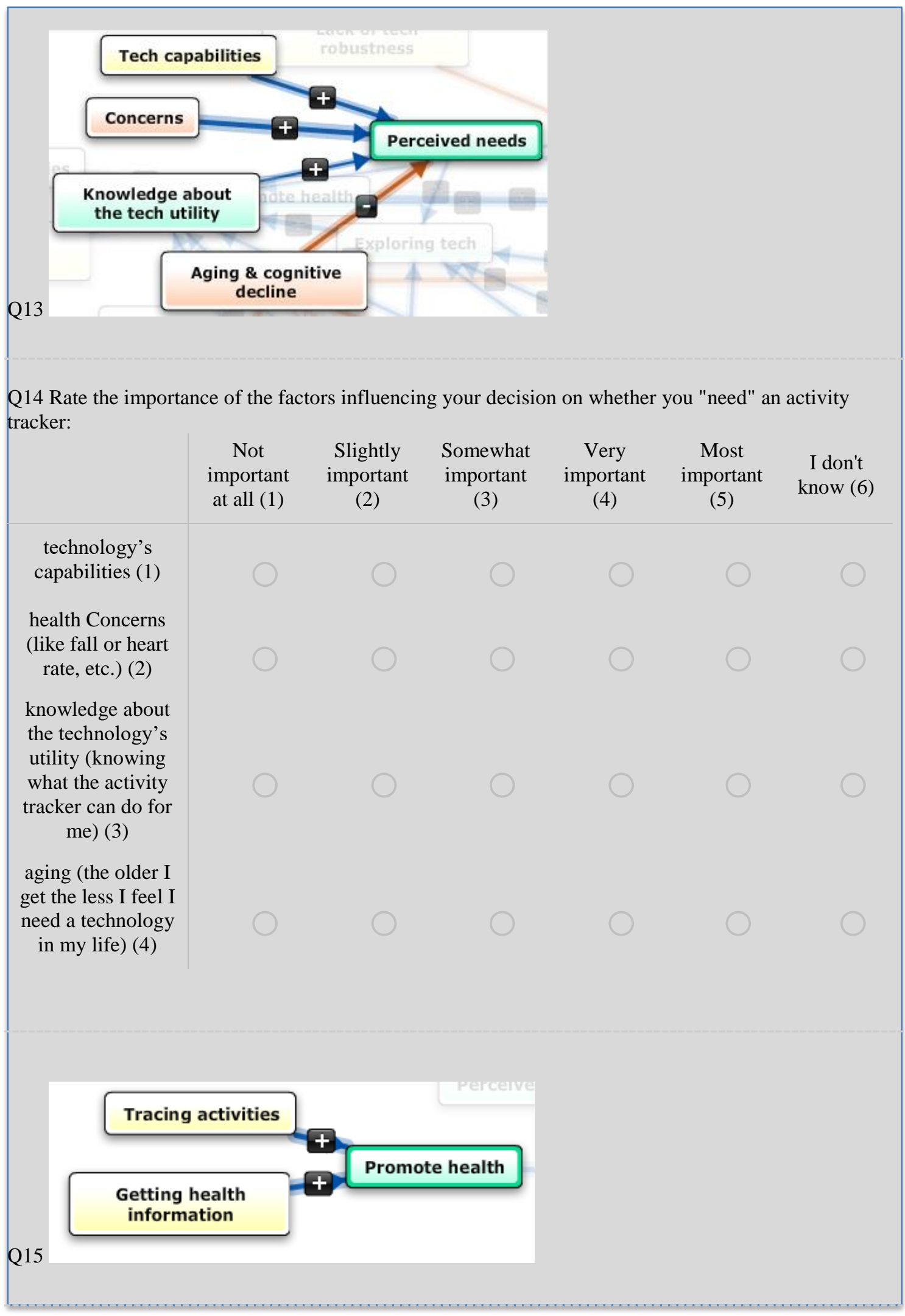


Q16 Rate the importance of the factors influencing your decision on whether an activity tracker promotes your health:

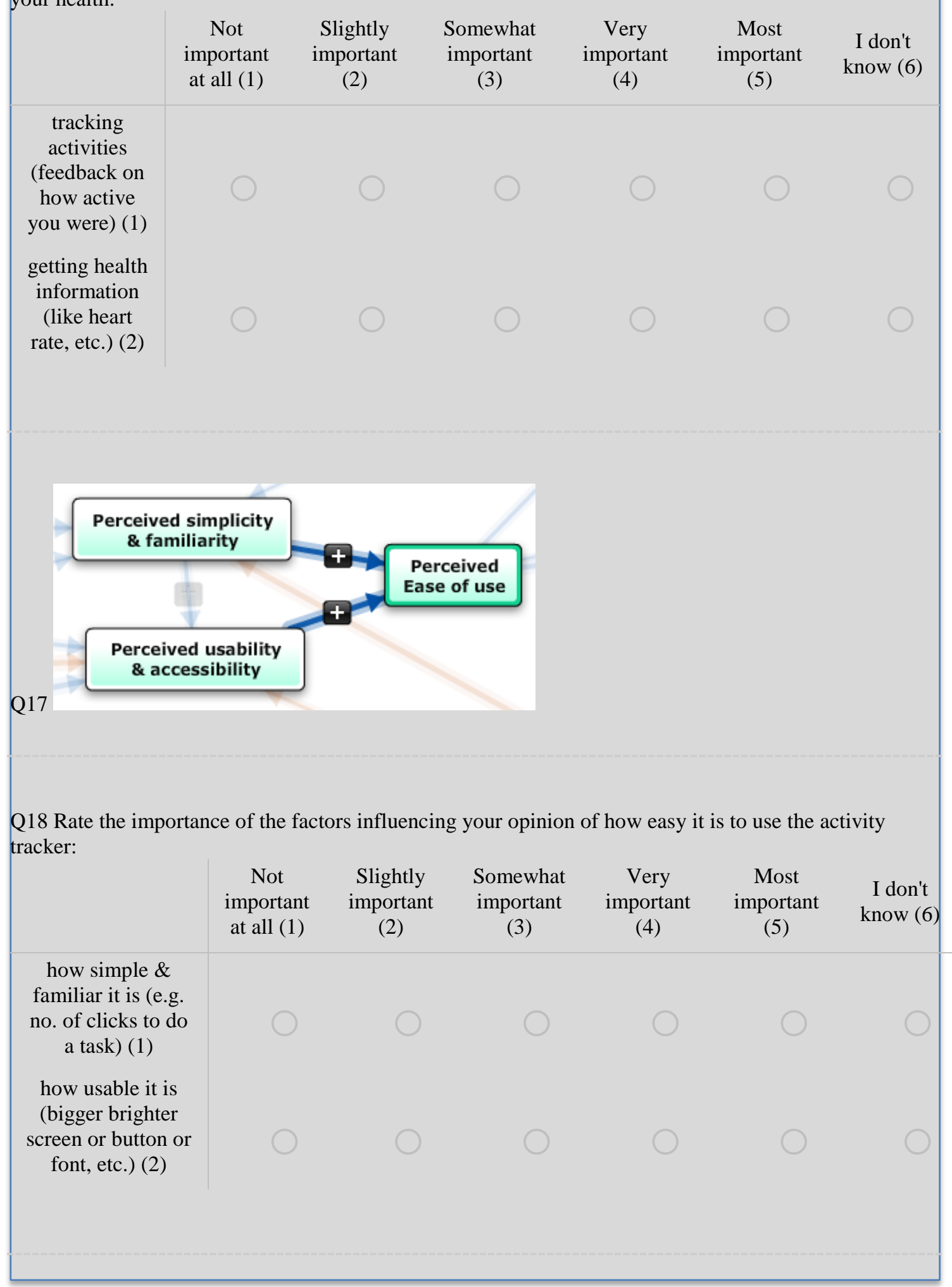




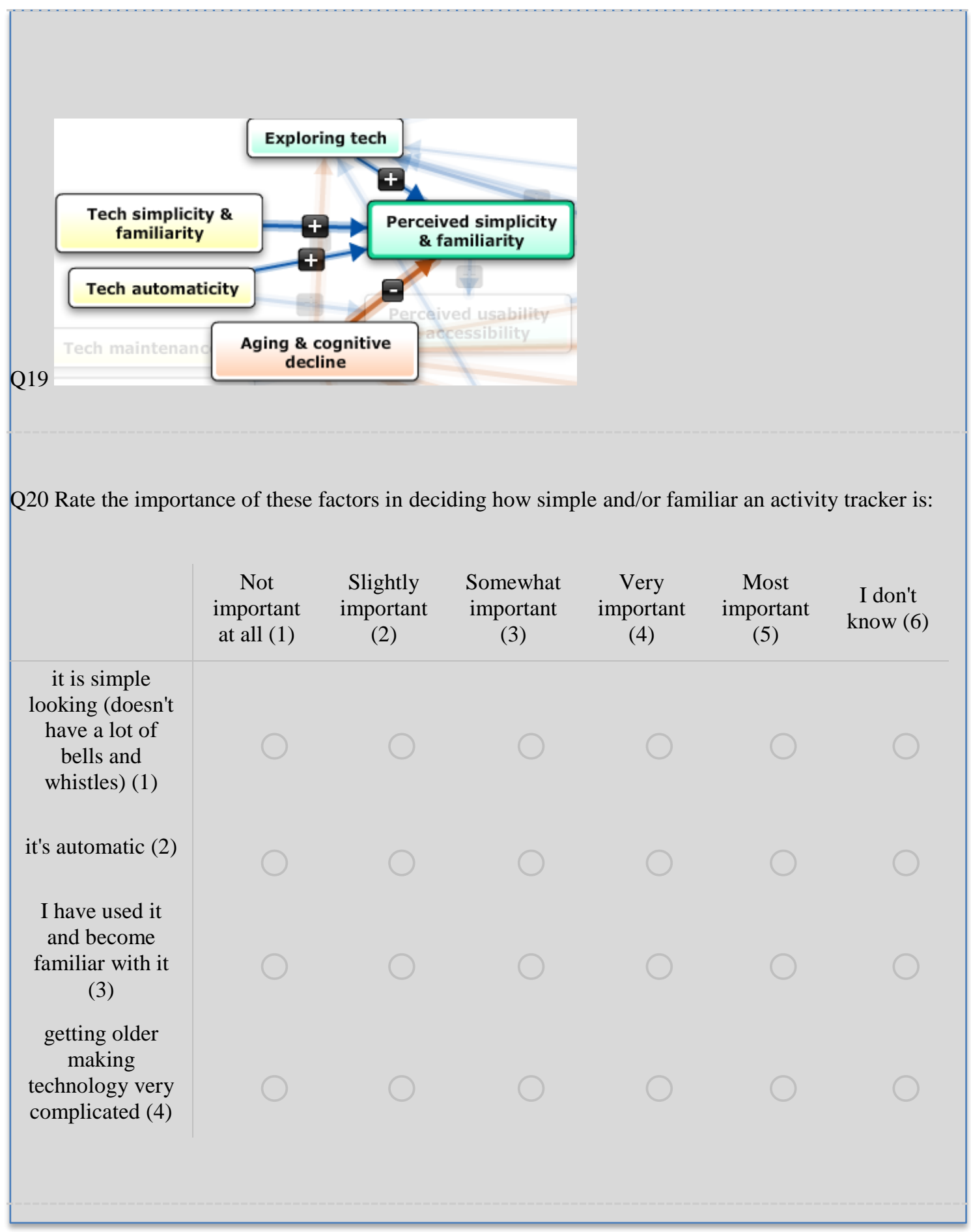




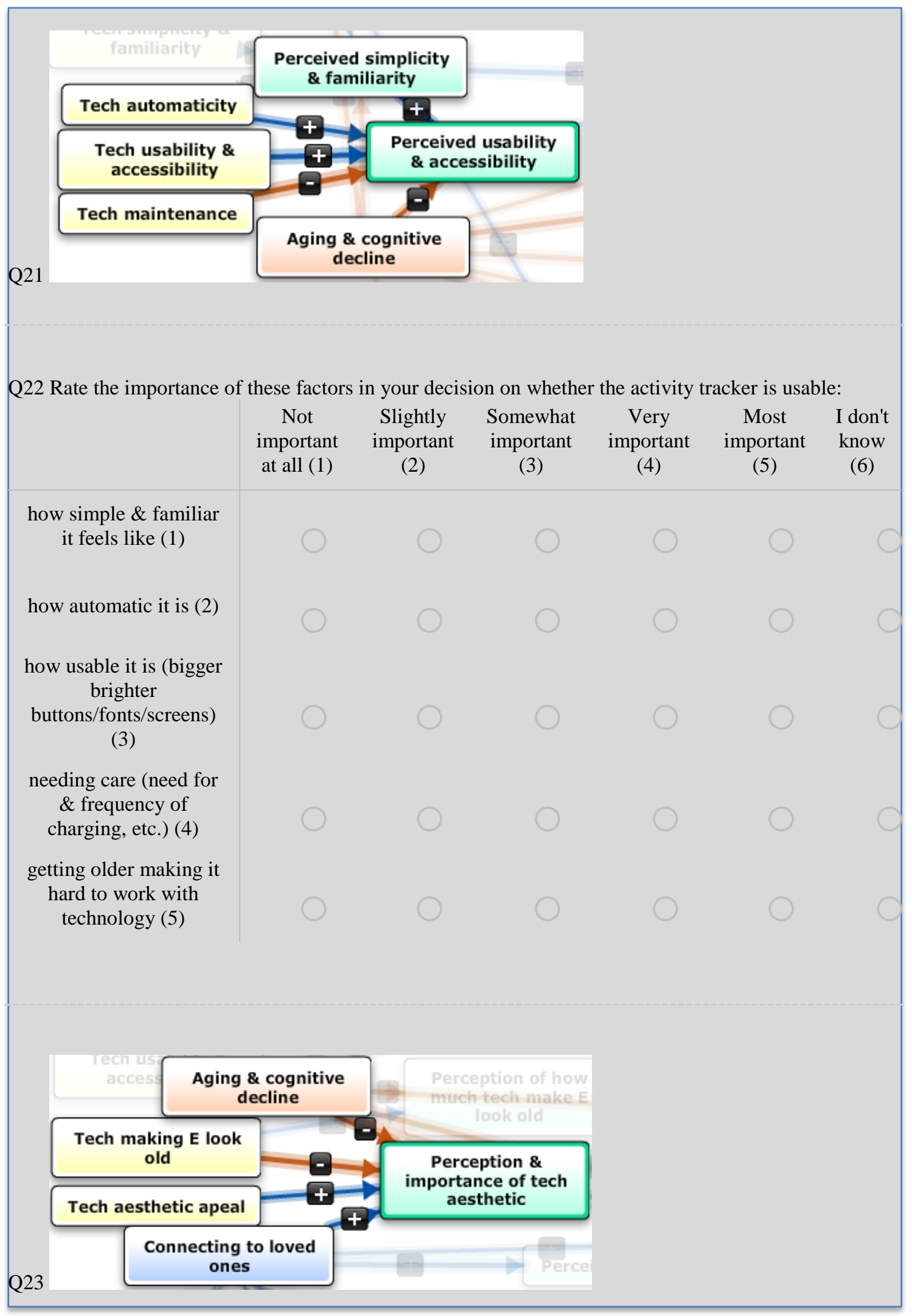


Q24 Rate the importance of these influencing factors in your decision on whether the activity tracker looks good enough that you would like to wear it:

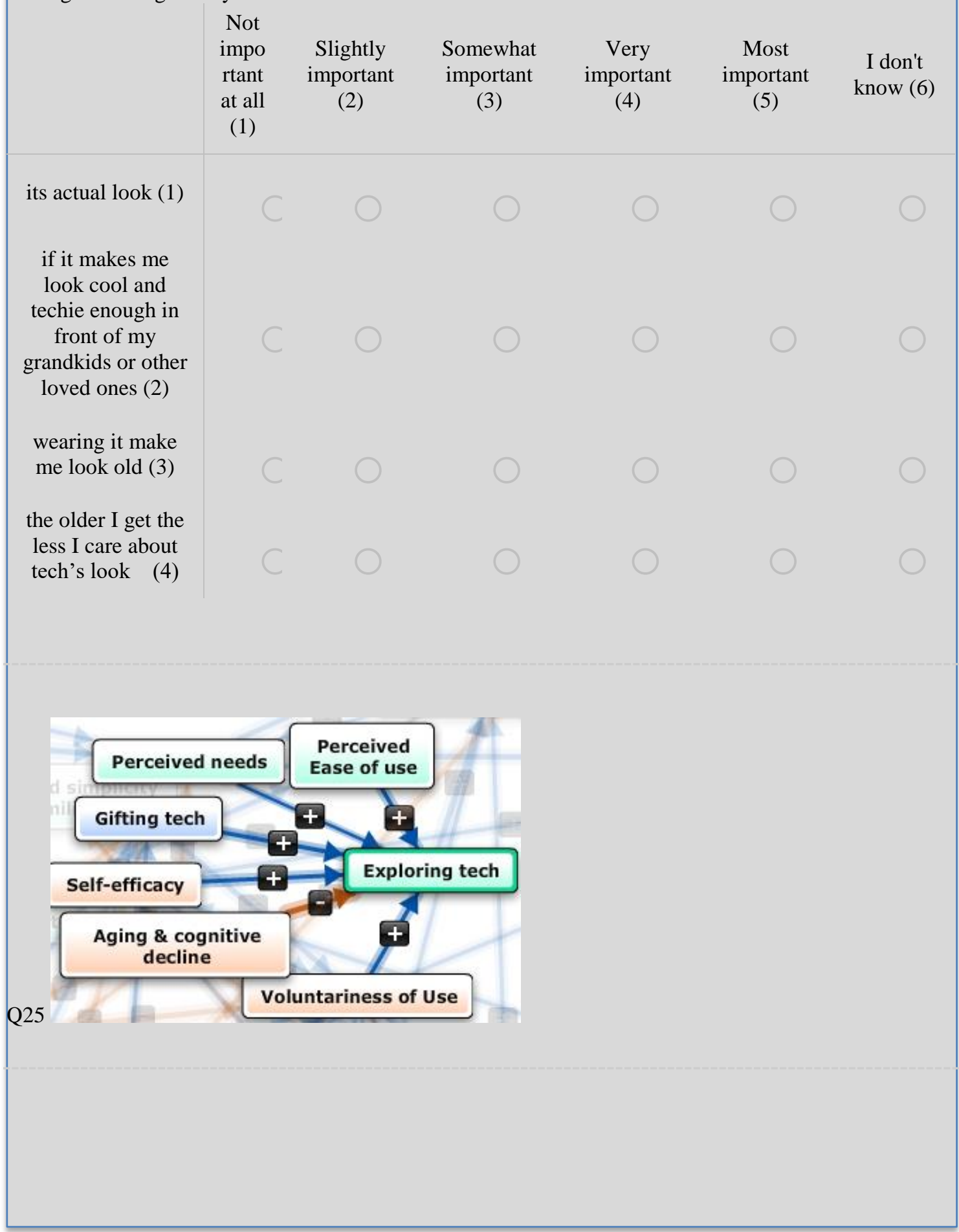


Q26 Rate the importance of these influencing factors that make you decide whether to try \& explore an activity tracker:

\begin{tabular}{|c|c|c|c|c|c|}
\hline $\begin{array}{l}\text { Not } \\
\text { import } \\
\text { ant at }\end{array}$ & $\begin{array}{l}\text { Slightly } \\
\text { important } \\
\text { (2) }\end{array}$ & $\begin{array}{c}\text { Somewhat } \\
\text { important } \\
\text { (3) }\end{array}$ & $\begin{array}{c}\text { Very } \\
\text { important } \\
\text { (4) }\end{array}$ & $\begin{array}{c}\text { Most } \\
\text { important } \\
\text { (5) }\end{array}$ & $\begin{array}{c}\text { I don't } \\
\text { know (6) }\end{array}$ \\
\hline
\end{tabular}

how easy it is to use (1)

if I need it (2)

having the technology (somebody gave it to you) (3)

if I know I can figure it out (4) willingness to using it (5)

the older I get the less I want to try a new technology

(6)

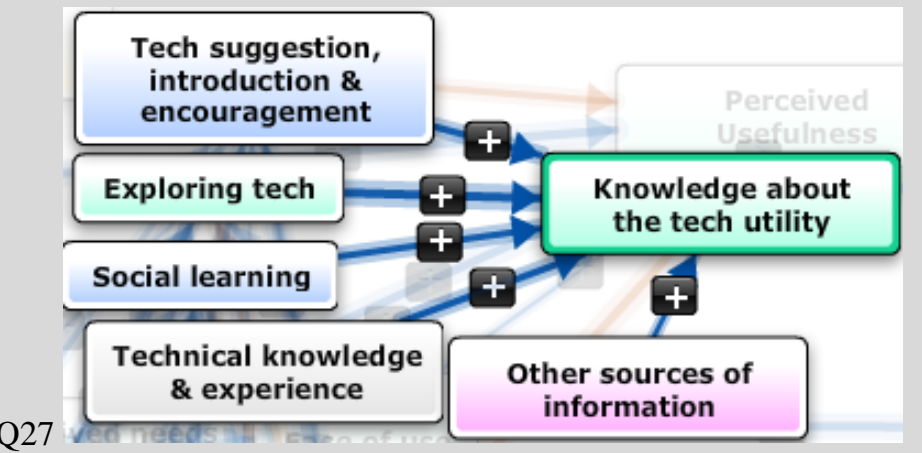




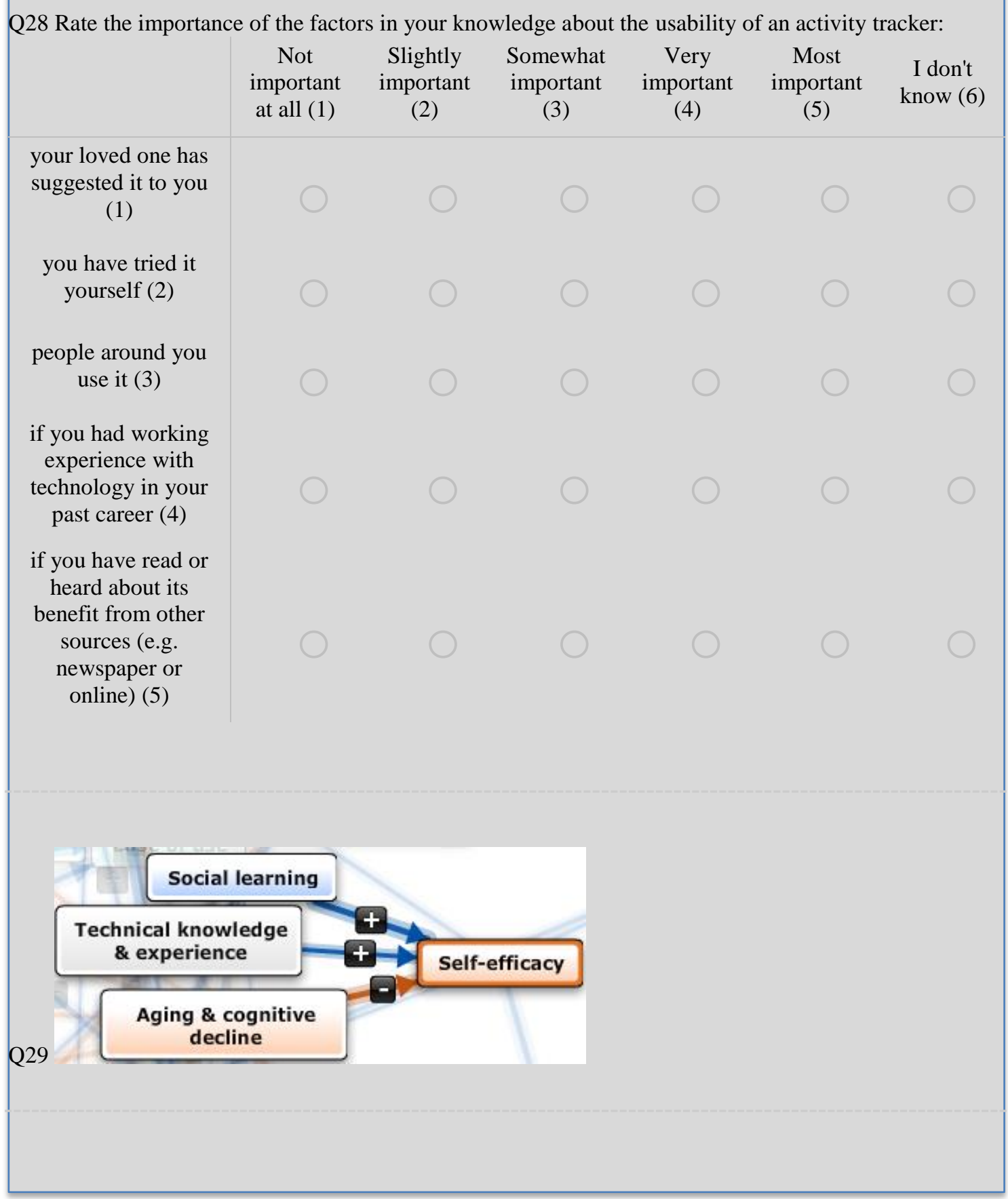


Q30 Rate the importance of these factors influencing the confidence in your ability to successfully use an activity tracker:

\begin{tabular}{cccccc}
$\begin{array}{c}\text { Not } \\
\text { important } \\
\text { at all (1) }\end{array}$ & $\begin{array}{c}\text { Slightly } \\
\text { important }\end{array}$ & $\begin{array}{c}\text { Somewhat } \\
\text { important }\end{array}$ & $\begin{array}{c}\text { Very } \\
\text { important }\end{array}$ & $\begin{array}{c}\text { Most } \\
\text { important }\end{array}$ & $\begin{array}{c}\text { I don't } \\
\text { know (6) }\end{array}$ \\
\hline & $(3)$ & $(4)$ & $(5)$ &
\end{tabular}

seeing others use it (1)

you had working experience with technology in your past career (2) the older I get the less I feel I can figure technology out

(3)

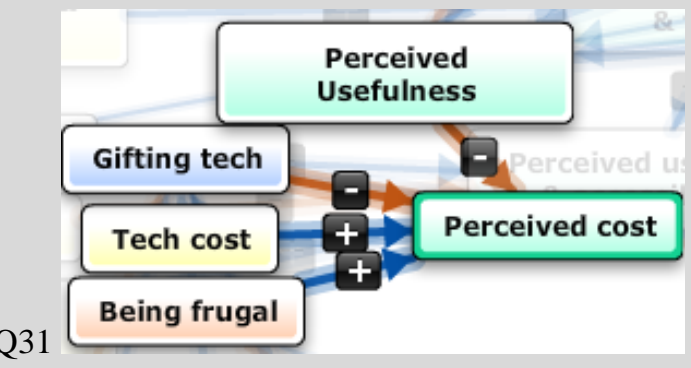


Q32 Rate the importance of these factors influencing the relative cost of the activity tracker:

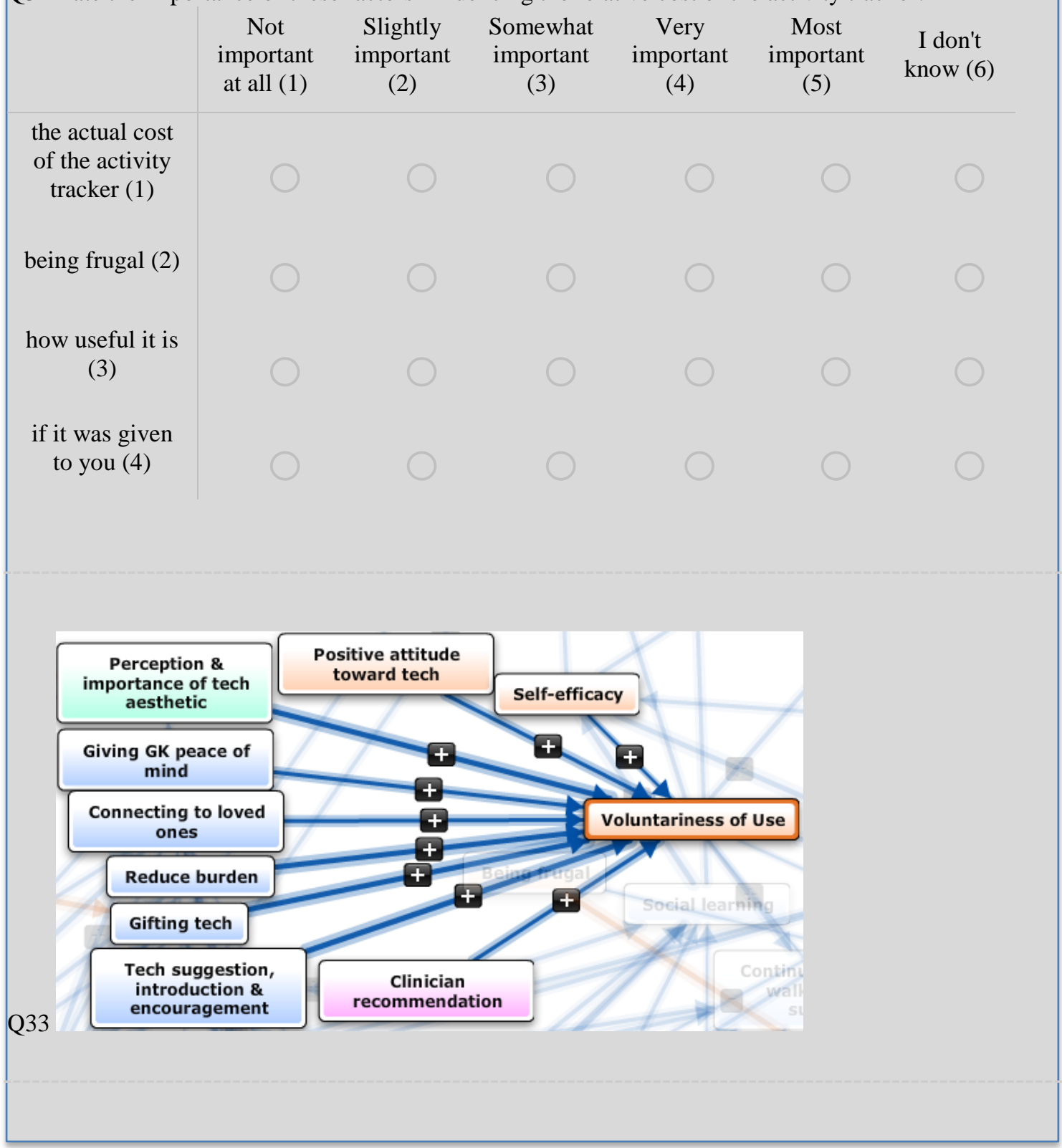


Q34 Rate the importance of these factors in influencing your willingness to use the activity tracker:

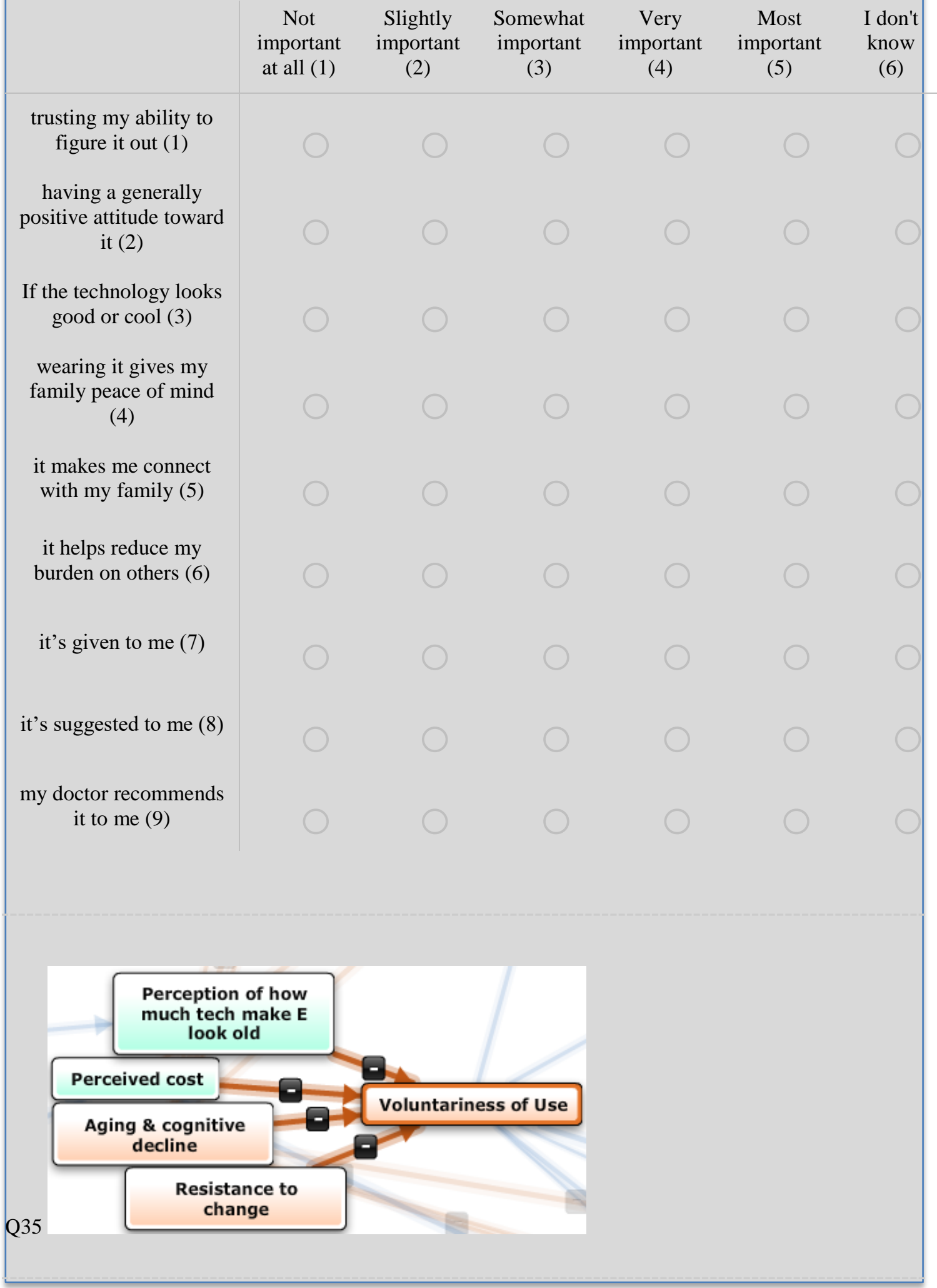


Q36

Rate the importance of these factors that decrease your willingness to use the activity tracker:

$\begin{array}{cccccc}\text { Not } & \text { Slightly } & \text { Somewhat } & \text { Very } & \text { Most } & \text { I don't } \\ \text { important } & \text { important } & \text { important } & \text { important } & \text { important } & \text { know (6) } \\ \text { at all (1) } & (2) & (3) & (4) & (5) & \end{array}$

it makes me

look too old

(1)

it's too

expensive for what it does

(2)

getting older

not wanting to change my lifestyle (4)

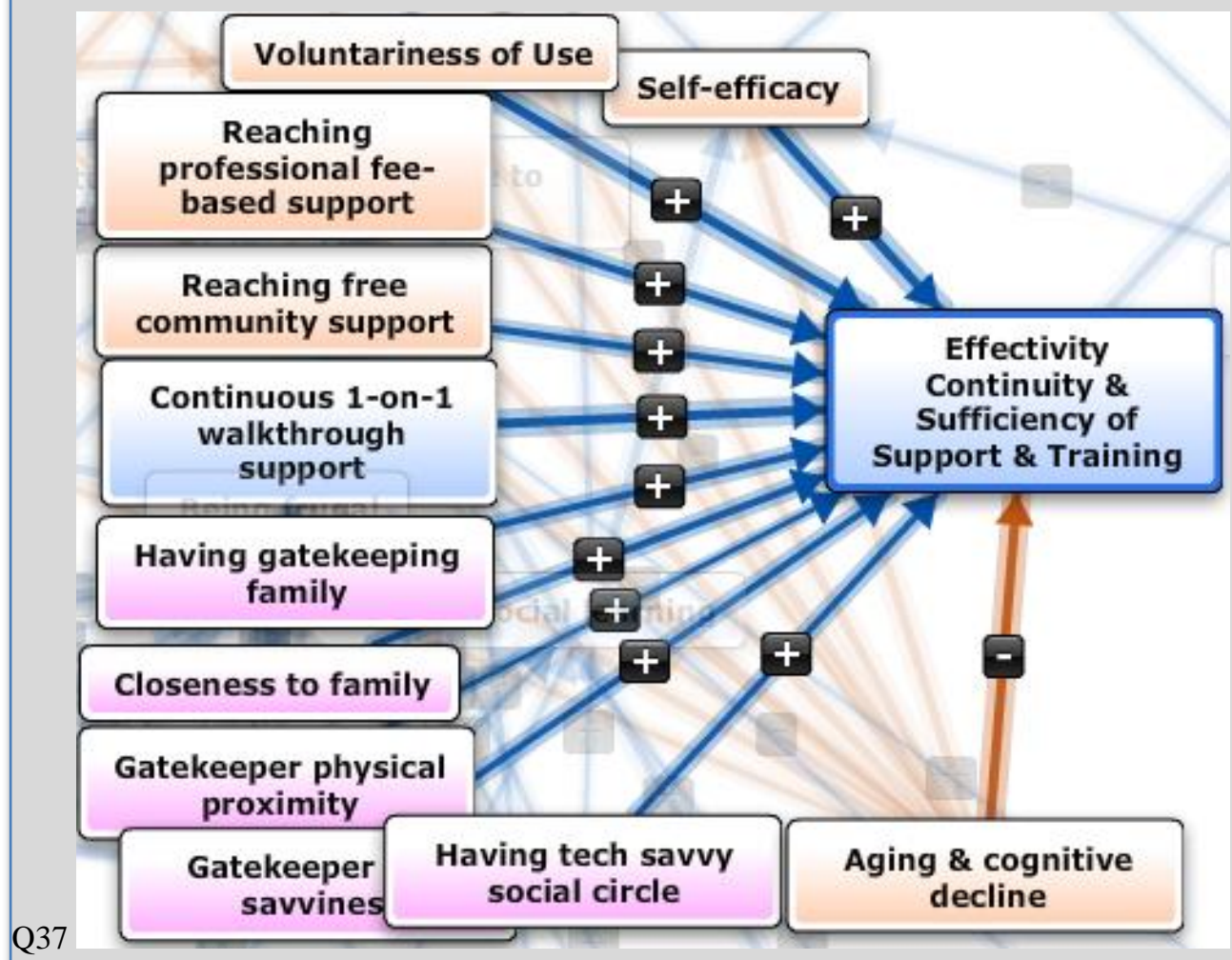


Q38 Rate the importance of these factors in influencing the effectiveness and adequacy of training and technological support:

$\begin{array}{cccccc}\begin{array}{c}\text { Not } \\ \text { important } \\ \text { at all (1) }\end{array} & \begin{array}{c}\text { Slightly } \\ \text { important }\end{array} & \begin{array}{c}\text { Somewhat } \\ \text { important }\end{array} & \begin{array}{c}\text { Very } \\ \text { important }\end{array} & \begin{array}{c}\text { Most } \\ \text { important }\end{array} & \begin{array}{c}\text { I don't } \\ \text { know (6) }\end{array} \\ & & (3) & (4) & (5) & \end{array}$

trusting your

ability to figure

technology out (1)

your willingness to use and figure our

technology (2)

reaching out to professional support (3)

reaching out to free community support

having access to a continuous one-onone support (5)

having a family that helps with technology (6)

being connected to your family (7)

having a techie loved one who lives close by (8)

how techie your loved one is (9)

having techie people around you (10)

the older you get the less effective the support gets

(11) 


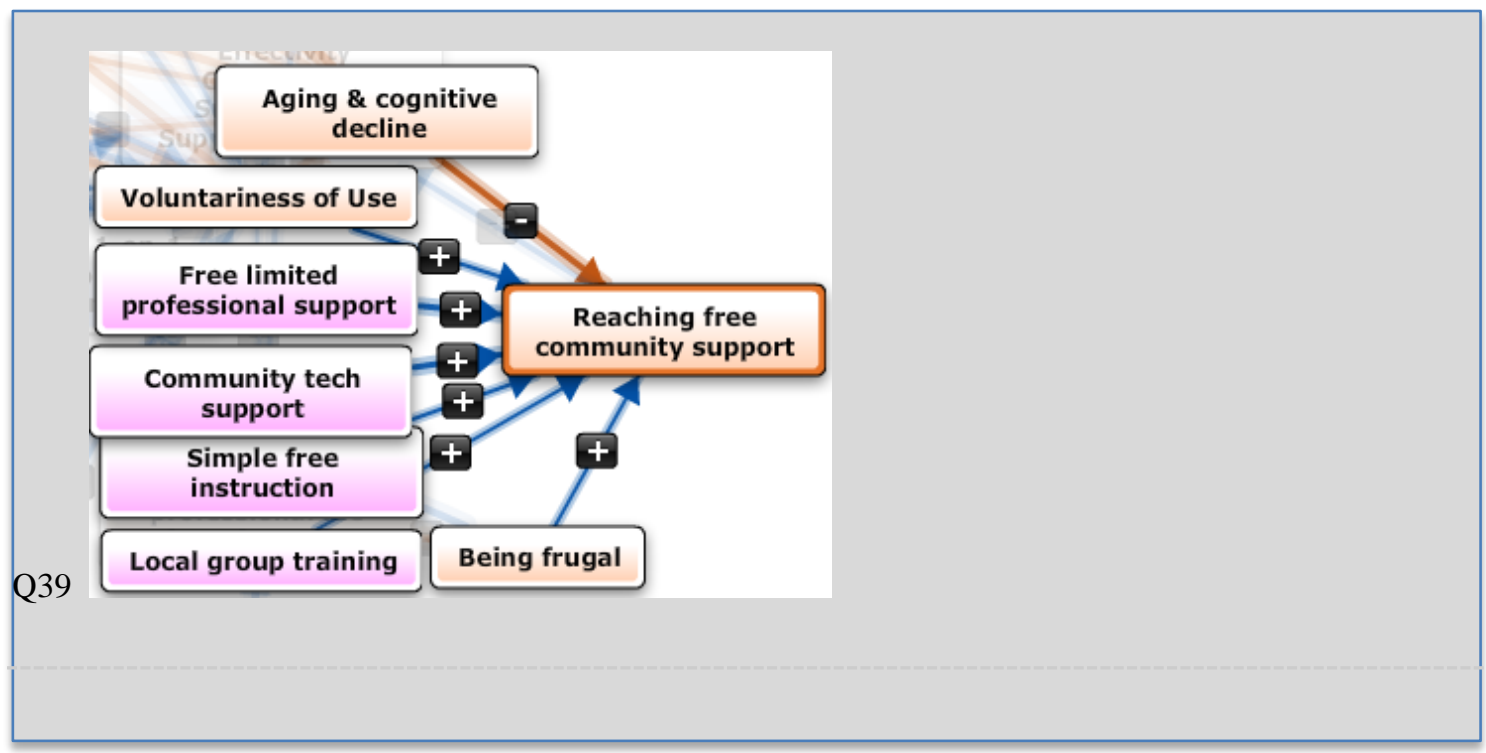


Q40 Rate the importance of these factors that influence reaching out to free support:

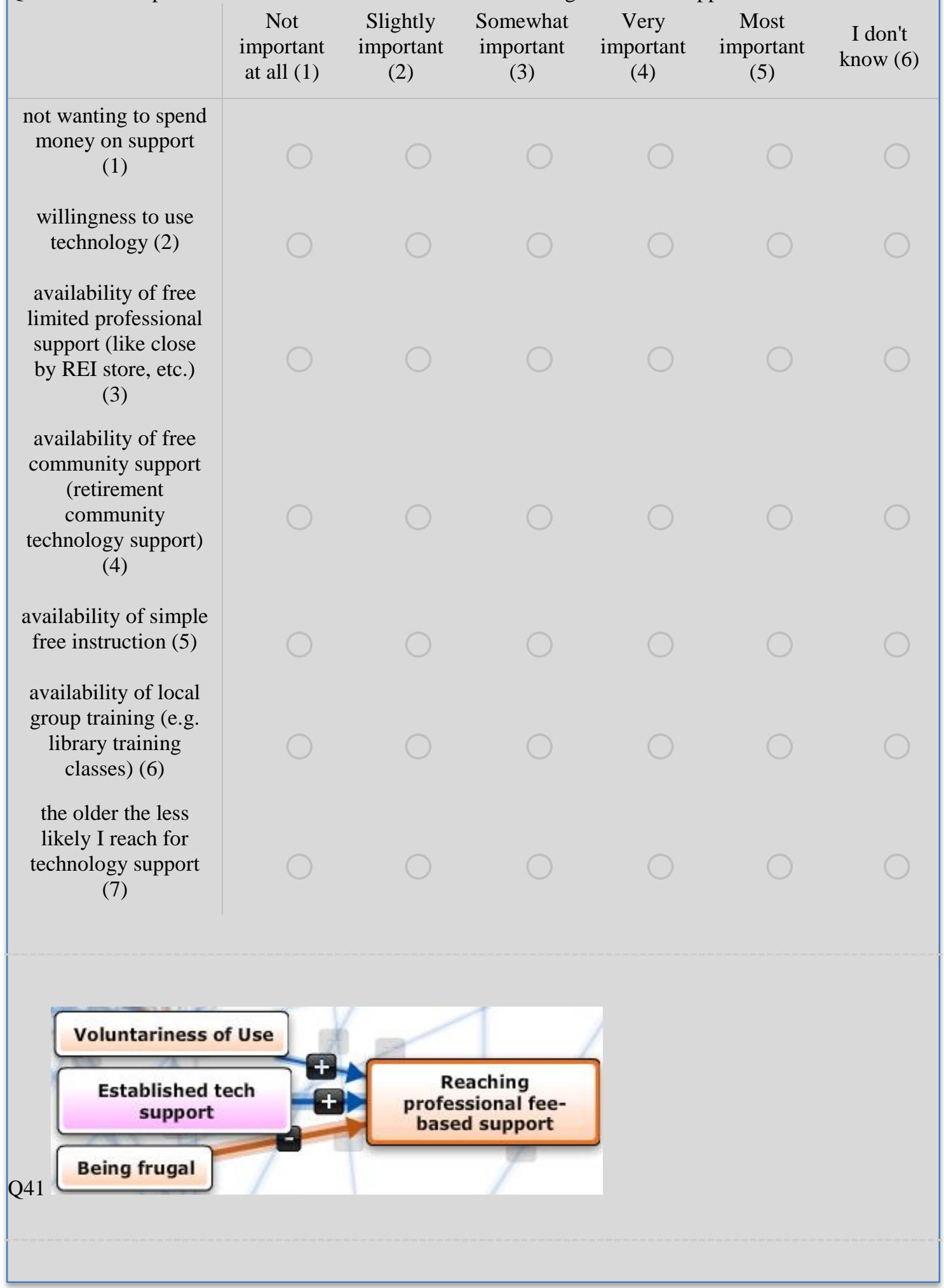




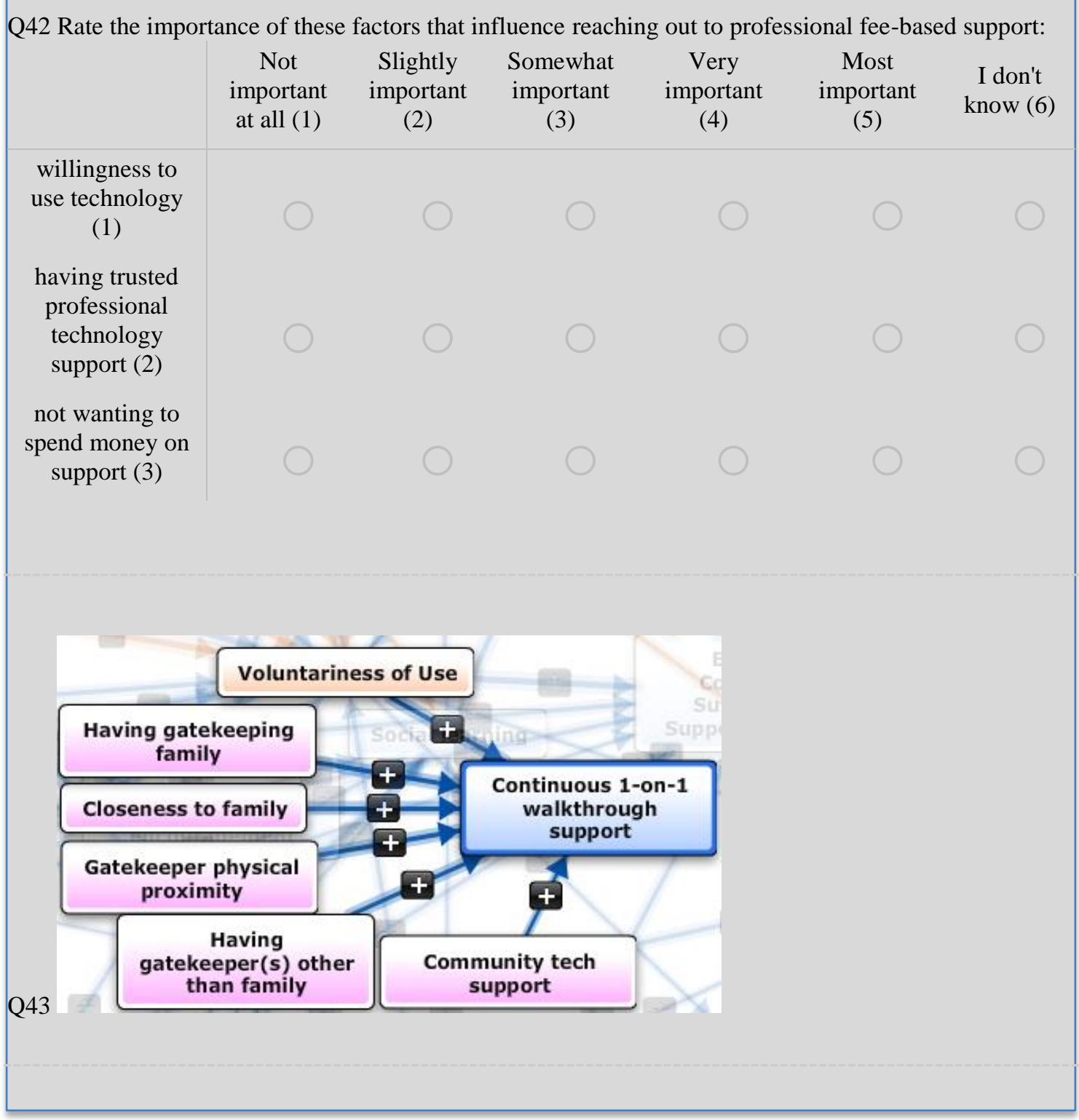


Q44 Rate the importance of these factors that influence the availability of continuous one-on-one support:

$\begin{array}{cccccc}\begin{array}{c}\text { Not } \\ \text { important at } \\ \text { all (1) }\end{array} & \begin{array}{c}\text { Slightly } \\ \text { important }\end{array} & \begin{array}{c}\text { Somewhat } \\ \text { important }\end{array} & \begin{array}{c}\text { Very } \\ \text { important }\end{array} & \begin{array}{c}\text { Most } \\ \text { important }\end{array} & \begin{array}{c}\text { I don't } \\ \text { know (6) }\end{array} \\ & & (3) & (4) & (5) & \end{array}$

willingness to use technology

(1)

having a family that helps with technology (2)

being close to family (3)

your techie helper lives close by (4)

having a techie person who is not family (5)

availability of community technology support (6)

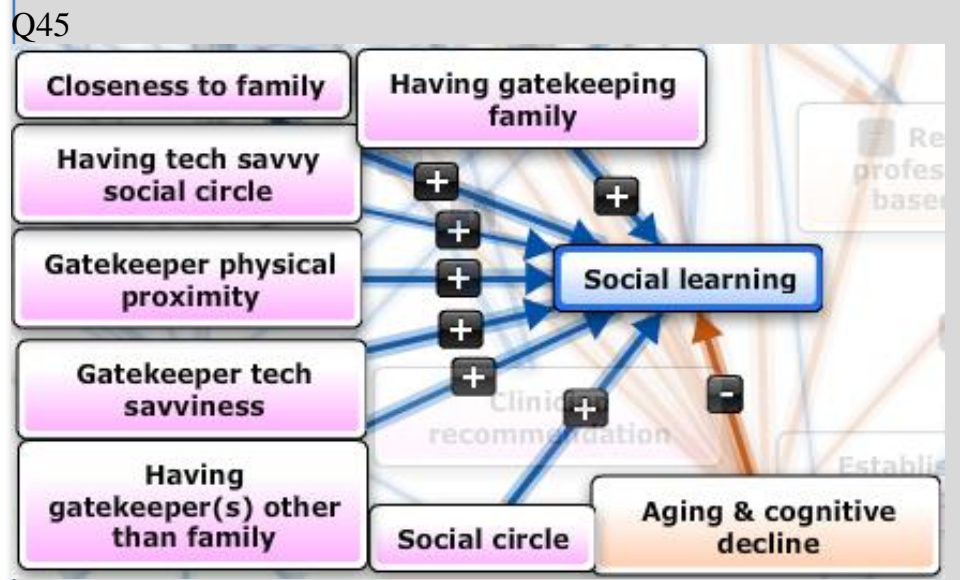


Q46 Rate the importance of these factors that influence your learning from your surroundings:

\begin{tabular}{|c|cccccc} 
& $\begin{array}{c}\text { Not } \\
\text { important } \\
\text { at all (1) }\end{array}$ & $\begin{array}{c}\text { Slightly } \\
\text { important } \\
(2)\end{array}$ & $\begin{array}{c}\text { Somewhat } \\
\text { important } \\
(3)\end{array}$ & $\begin{array}{c}\text { Very } \\
\text { important } \\
(4)\end{array}$ & $\begin{array}{c}\text { Most } \\
\text { important } \\
(5)\end{array}$ & $\begin{array}{c}\text { I don't } \\
\text { know (6) }\end{array}$ \\
$\begin{array}{c}\text { having a techie } \\
\text { person(s) in your } \\
\text { family (1) }\end{array}$ \\
$\begin{array}{c}\text { how emotionally } \\
\text { close you are to } \\
\text { your family (2) }\end{array}$ \\
$\begin{array}{c}\text { your techie help } \\
\text { lives close by (3) } \\
\text { how technical } \\
\text { your techie } \\
\text { person is (4) } \\
\text { having a techie } \\
\text { person(s) outside } \\
\text { your family (5) } \\
\text { having techie } \\
\text { social circle (6) }\end{array}$ \\
$\begin{array}{c}\text { having social } \\
\text { circle (7) }\end{array}$ \\
the older you are \\
the less you care \\
what others do (8)
\end{tabular}

\section{Q47}

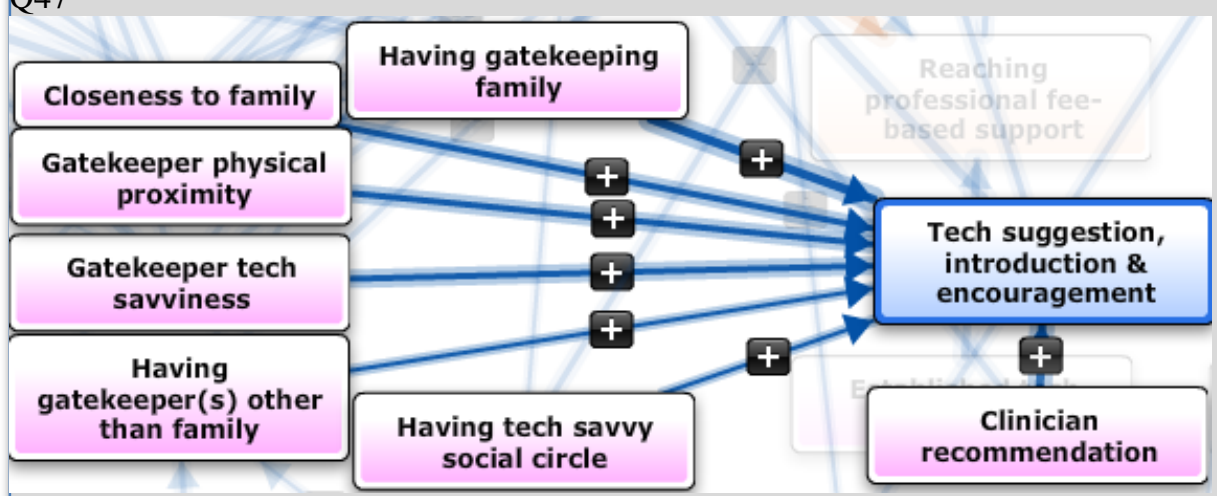


Q48 Rate the importance of these factors that influence getting effective technology advice from your surroundings:

$\begin{array}{cccccc}\begin{array}{c}\text { Not } \\ \text { important }\end{array} & \begin{array}{c}\text { Slightly } \\ \text { important }\end{array} & \begin{array}{c}\text { Somewhat } \\ \text { important }\end{array} & \begin{array}{c}\text { Very } \\ \text { important }\end{array} & \begin{array}{c}\text { Most } \\ \text { important }\end{array} & \begin{array}{c}\text { I don't } \\ \text { know (6) }\end{array} \\ \text { (2) } & (3) & (4) & (5) & \end{array}$

\begin{abstract}
having a techie person(s) in your family helping you
\end{abstract} (1)

being emotionally close to your family who is suggesting technology (2) your techie help who lives close by suggesting it (3)

how technical your techie person is (4)

having a techie person(s) outside your family (5) having techie social circle (6)

your doctor recommending the technology (7) 


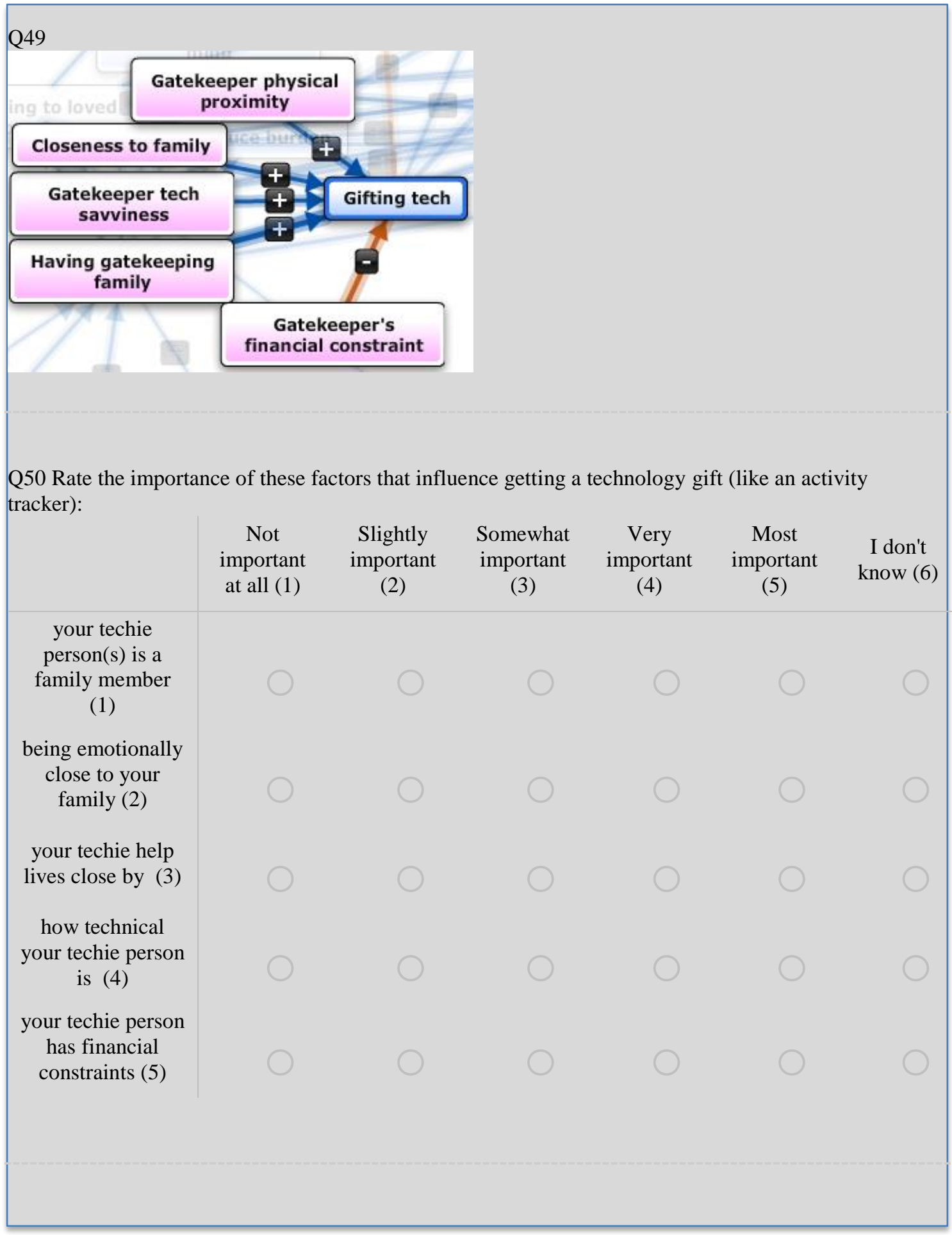




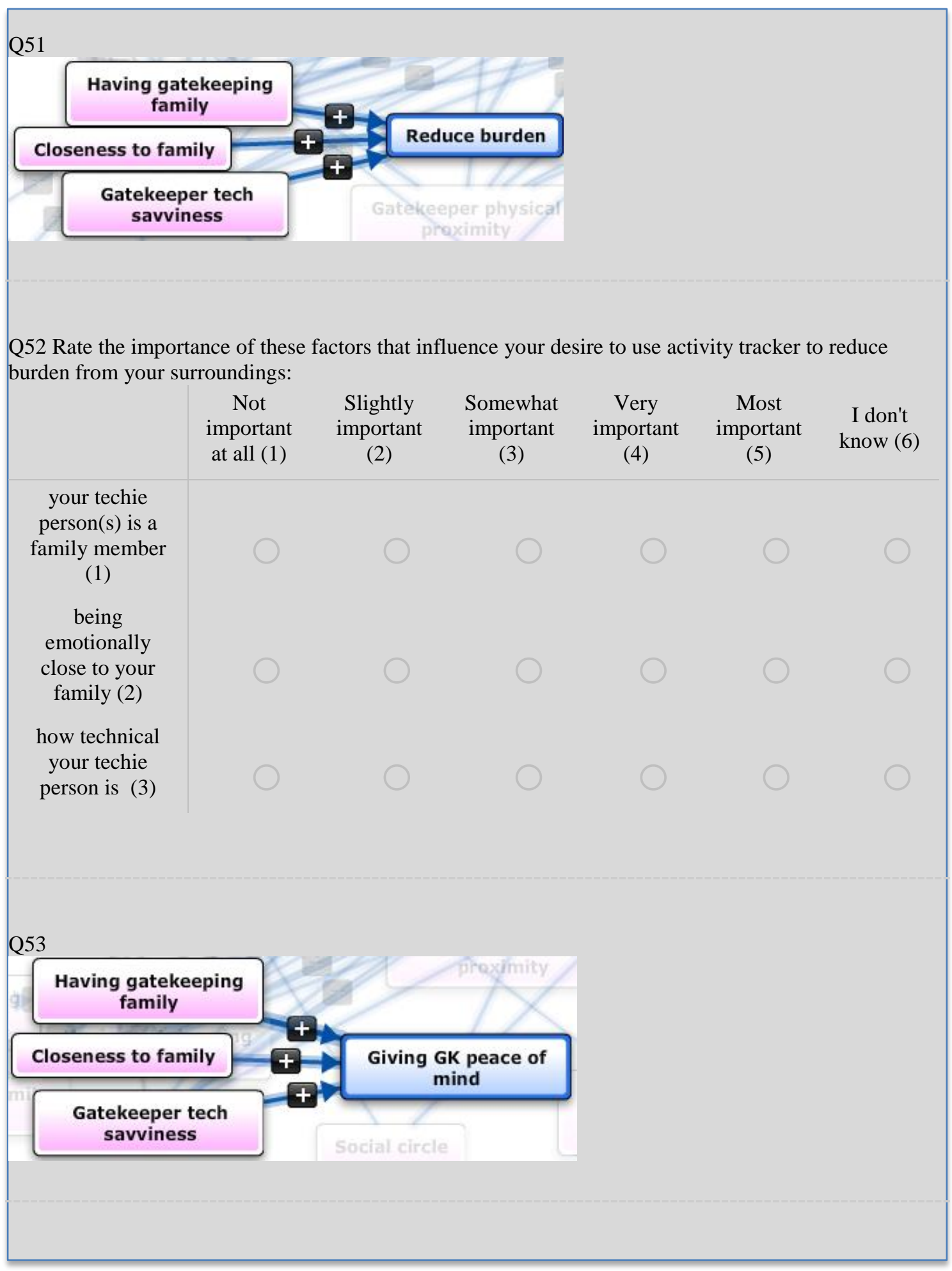


Q54 Rate the importance of these factors that influence your decision to use activity tracker to give your loved ones peace of mind:

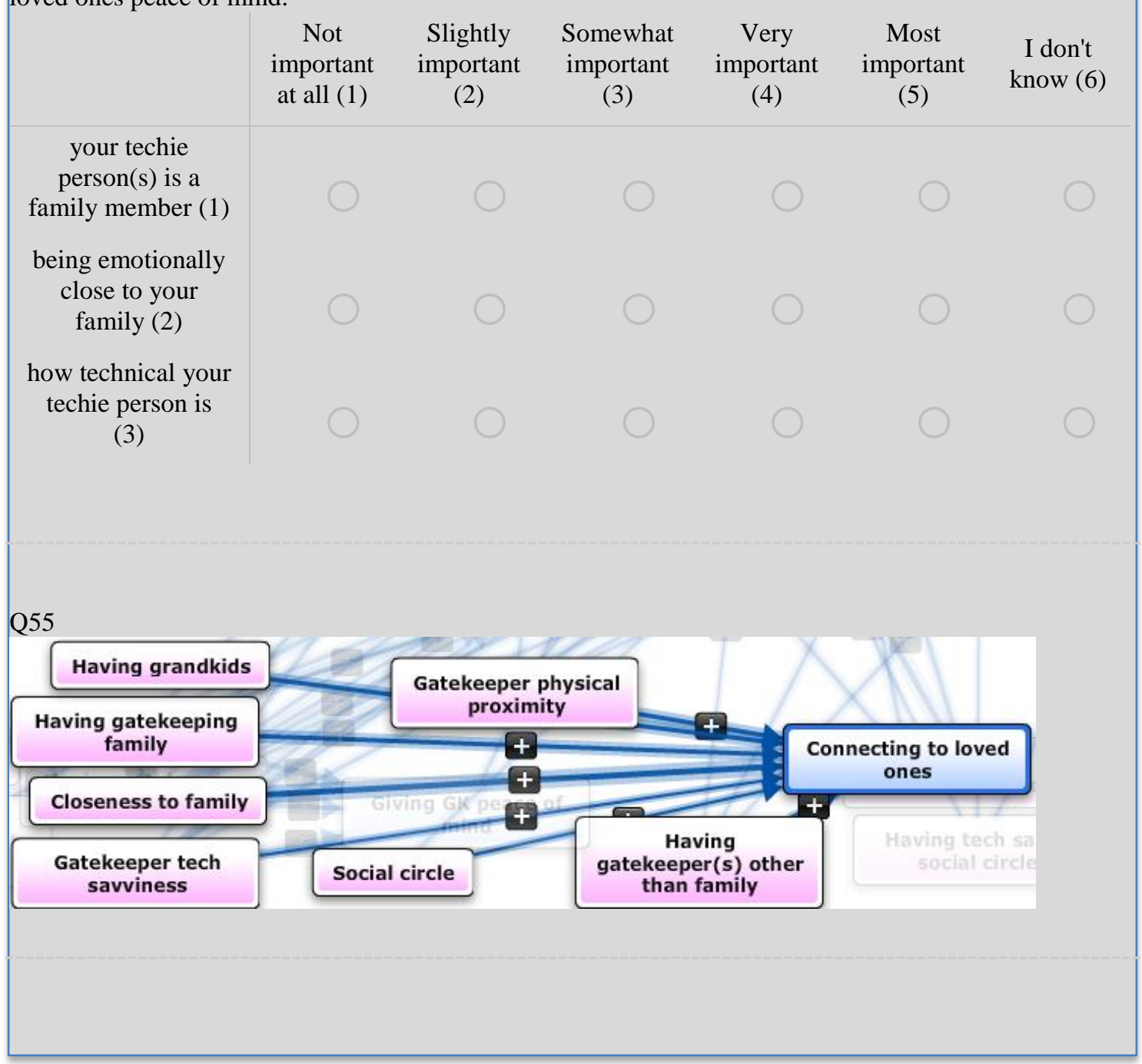


Q56 Rate the importance of these factors that influence your desire to use activity tracker to better connect with your loved ones:

$\begin{array}{cccccc}\begin{array}{c}\text { Not } \\ \text { important } \\ \text { at all (1) }\end{array} & \begin{array}{c}\text { Slightly } \\ \text { important }\end{array} & \begin{array}{c}\text { Somewhat } \\ \text { important }\end{array} & \begin{array}{c}\text { Very } \\ \text { important }\end{array} & \begin{array}{c}\text { Most } \\ \text { important }\end{array} & \begin{array}{c}\text { I don't } \\ \text { know (6) }\end{array} \\ & & (3) & (4) & (5) & \end{array}$

having grandchildren

(1)

having a social circle

(2)

your techie person(s)

is a family member

(3)

your techie person is not family (4)

being emotionally

close to your family

(5)

your technology support techie person lives close by (6)

how technical your techie person is (7)

Q57

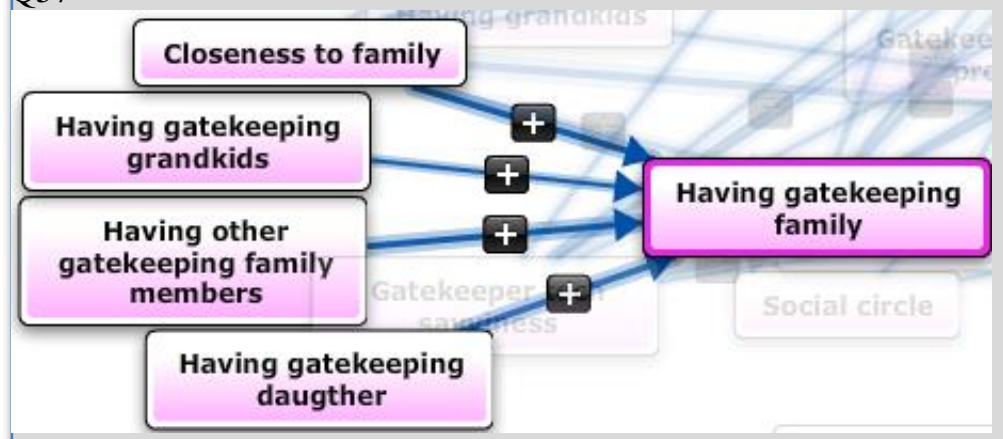


Q58 Rate the importance of these factors in having a family member(s) who can help you with technology:

$\begin{array}{cccccc}\begin{array}{c}\text { Not } \\ \text { important }\end{array} & \begin{array}{c}\text { Slightly } \\ \text { important }\end{array} & \begin{array}{c}\text { Somewhat } \\ \text { important }\end{array} & \begin{array}{c}\text { Very } \\ \text { important }\end{array} & \begin{array}{c}\text { Most } \\ \text { important }\end{array} & \begin{array}{c}\text { I don't } \\ \text { know (6) }\end{array} \\ \text { (2) } & (3) & (4) & (5) & \end{array}$

being emotionally

close to your family

(1)

having a techie grandchild(ren) who help(s) with technology (2)

having a techie daughter who helps with technology (3)

having other techie family member(s) who help with technology (4) 


\section{E.II. Adjacency Matrices of the Elderly Women Participants}

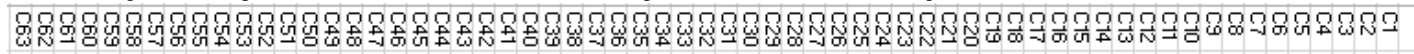

00000000000000000000000000000000000000000000000000000000001010

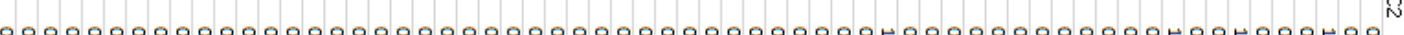
1. 000000000000000000000000000000000000000000 ñ 000 in 0 ñ 0000000000000 in 00000000 ñ๊o0 oñ

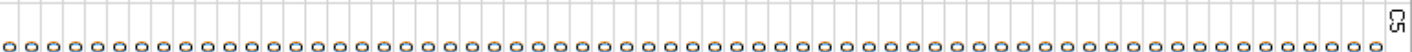
| 0000000000000000000000000000000000000000000000000000000000000000 00000000000000000000000000000000000000000000000000 vinin viñ 000000000

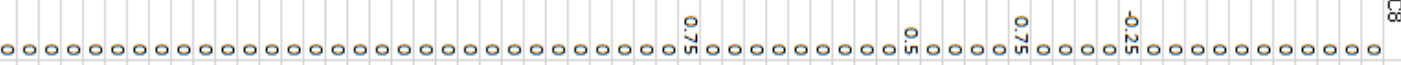
00000000000000000000000000000000000000000004 i⿱n. 000000000000000000 in

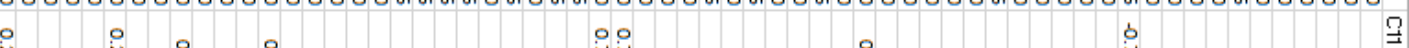

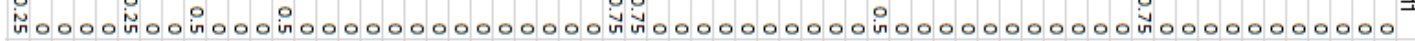
$000000000000000000000000000000000000000000000000000000000000000 \frac{7}{N}$ $000000000000000000000000000000000000000000000000000000000000000 \frac{?}{\omega}$

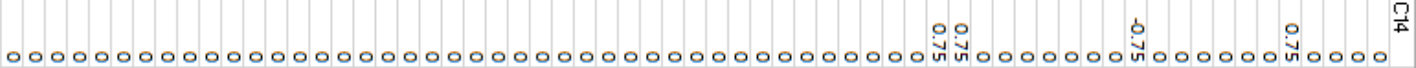
$000000000000000000000000000000000000000000000000000000000000000 \frac{?}{4}$

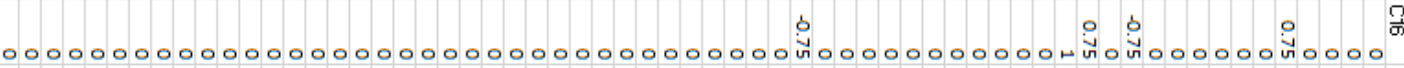
$000000000000000000000000000000000000000000000000000000000000000 \frac{7}{=1}$

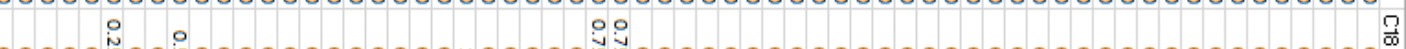

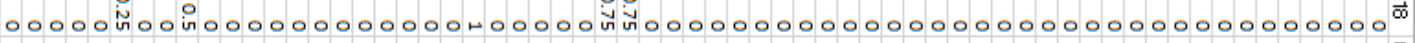
$000000000000000000000000000000000000000000000000000000000000000 \frac{?}{\omega}$ $000000000000000000000004000000000000000000000000 i 0 n$ in 0

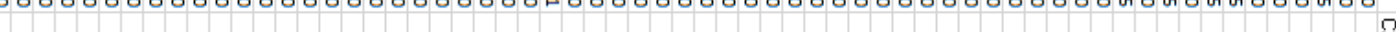
$000000000000000000000000000000000000000000000000000000000000000-\frac{1}{3}$

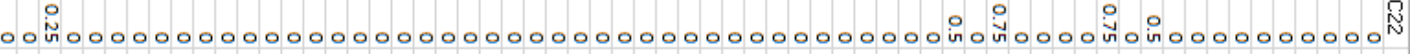

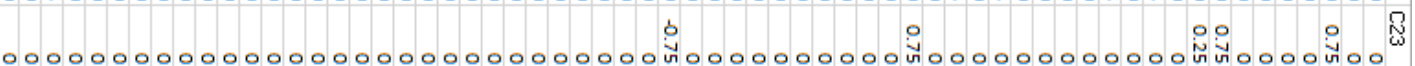
N $000000000000000000000000000000000000000000000000000000000000000 \frac{N}{1}$ 00000000000000000000000000000000000000000000000000000000000000001 00000000000000000 iño0000001000000000000 ஸ் $000000000000000000000000000000000000000000000000000000000000000-7$ 000000000000000000000000000000000000000000000000000000000000000

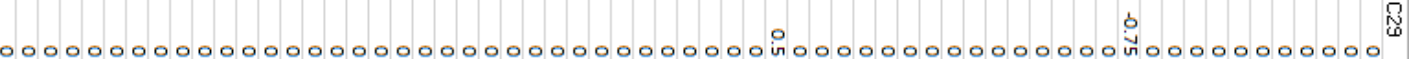
000000000000000000000000000000000000000000000000000000000000009

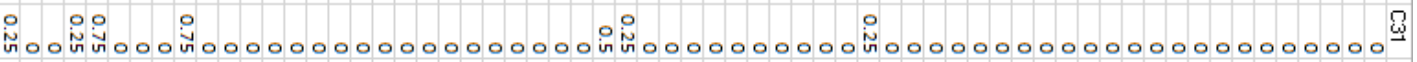
$000000000000000000000000000000000000000000000000000000000000000 \mathrm{~W}$ $000000000000000000000000000000000000000000000000000000000000000 \mathrm{~W}$ $000000000000000000000000000000000000000000000000000000000000000 \ldots$

\section{Table E - 1 Elderly Woman P1 Adjacency Matrix (first half)}




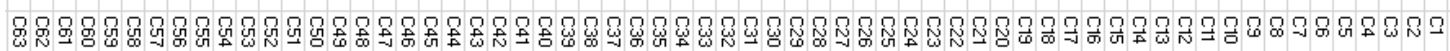

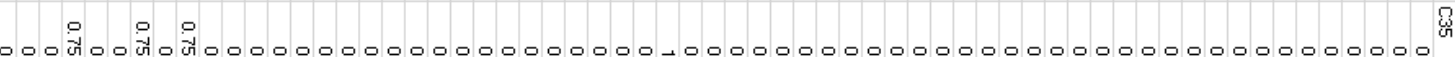
| 000000000000000000000000000000000000000000000000000000000000000

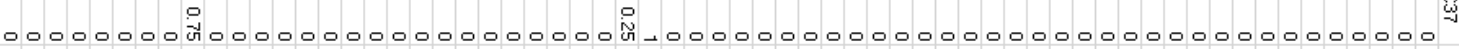

1) ○ й $00000000000000000000000000000000000000000000000000000000000000 \underline{W}$

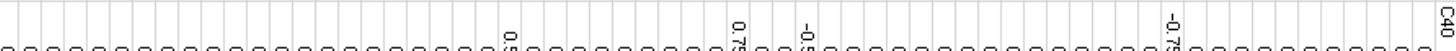

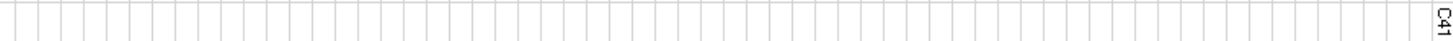
000000000000000000000000000000000000000000000000000000000000000 $00000000000000000000000000000000000000000000000000000000000000 \hat{N}$

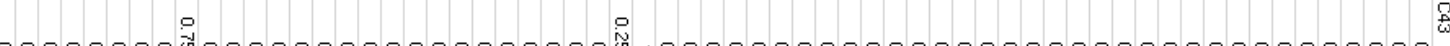

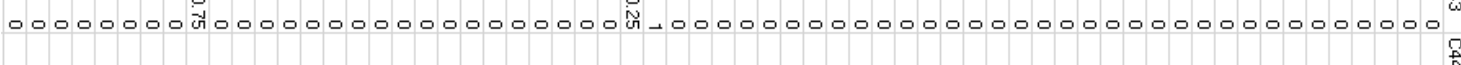
000000000000000000000000000000000000000000000000000000000000000 $000000000000000000000000000000000000000000000000000000000000000 \hat{i n}$

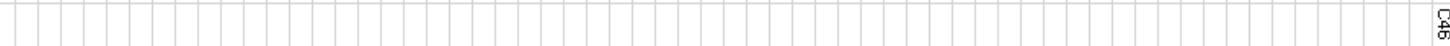
1

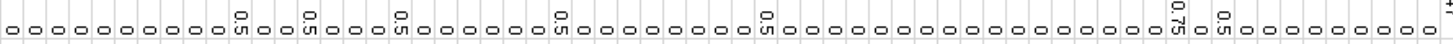

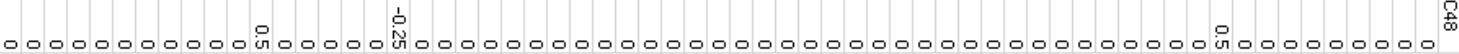

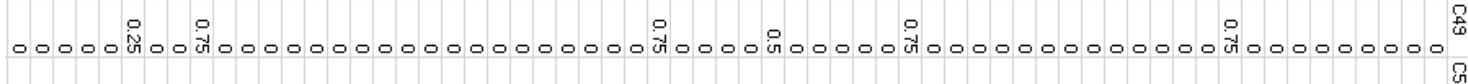
000000000000000000000000000000000000000000000000000000000000000 | 1 (1 | 000000000000000000000000000000000000000000000000000000000000000 $0000000000000000000000000000000000000000000000000000000000000 N$

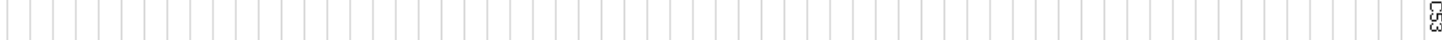
000000000000000000000000000000000000000000000000000000000000000 000000000000000000000000000000000000000000000000000000000000000 $000000000000000000000000000000000000000000000000000000000000000 \hat{~}$

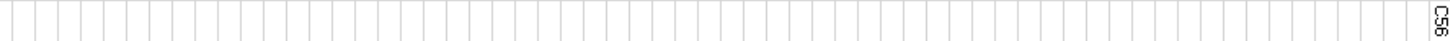
00000000000000000000000000000000000000000000000000000000000000 000000000000000000000000000000000000000000000000000000000000000

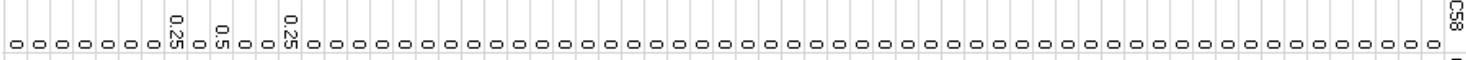
| 000000000000000000000000000000000000000000000000000000000000000 000000000000000000000000000000000000000000000000000000000000000 00000000000000000000000000000000000000000000000000000000000000 | 000000000000000000000000000000000000000000000000000000000000000 000000000000000000000000000000000000000000000000000000000000000

\section{Table E - 2 Elderly Woman P1 Adjacency Matrix (second half)}



$00000000000000000000000000000000000000000000000000000000000 \tilde{U}_{0} 00$

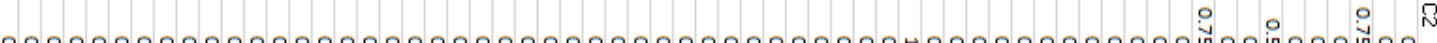

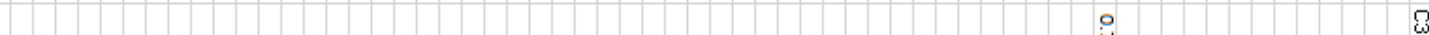
0000000000000000000000000000000000000000000000010 V̌ 0000000000000

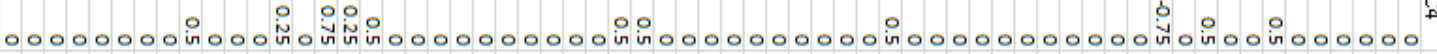

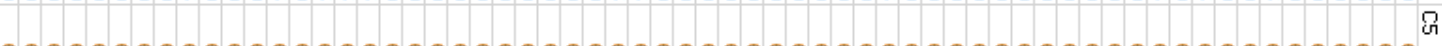
000000000000000000000000000000000000000000000000000000000000000 00000000000000000000000000000000000000000000000000000000000000 0000000000000000000000000000000000000000000000000010 iñ 000000000

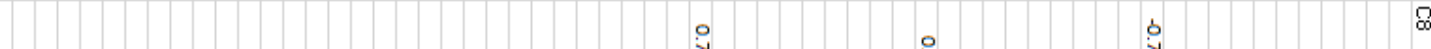
0000000000000000000000000000000 ñ.00000000ino00010000 ñ 00000000000

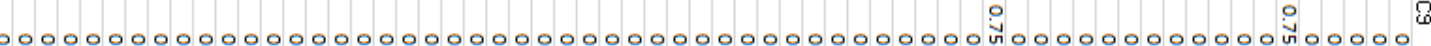
00000000000000000000000000000000000000000000 ñ 000000000000 ñ 00000

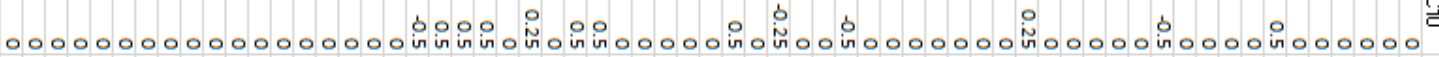

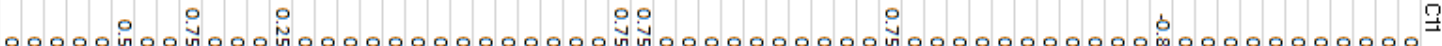
$000000000000000000000000000000000000000000000000000000000000000 \frac{?}{N}$ $000000000000000000000000000000000000000000000000000000000000000 \frac{7}{\omega}$

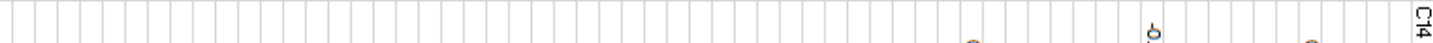
00000000000000000000000000000000000000000010 in 000000 iñ 0000000000 $000000000000000000000000000000000000000000000000000000000000000 \frac{?}{4}$

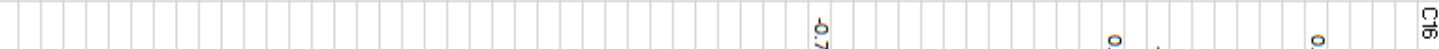

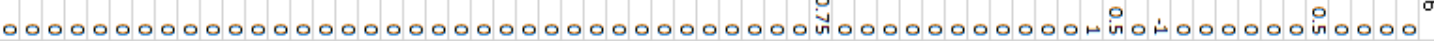
$000000000000000000000000000000000000000000000000000000000000000 \frac{9}{=1}$ 00000 iño 0 iño0 000000000 in 00000 in 00000 nn 00 ñ 000000000000 in 00000 in nn 0000000000 nn 00000000000000000000000 $000000000000000000000000000000000000000000000000000000000000000 \bar{l}$

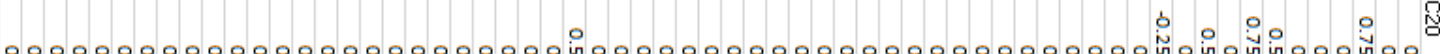

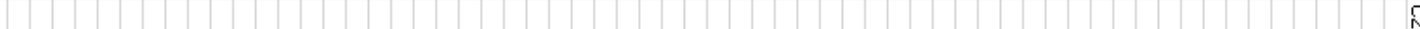
$000000000000000000000000000000000000000000000000000000000000000-1$

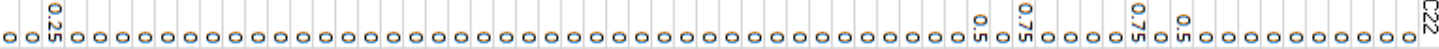

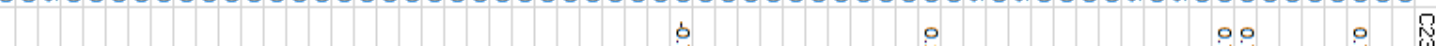
000000000000000000000000000000 ñ 000000000 ñ 000000000000 ที่ $000000000000000000000000000000000000000000000000000000000000000 \AA$ $000000000000000000000000000000000000000000000000000000000000000 \hat{N}$ 0000000000000000 in 0000001000000000000 iño 00000000000000000000000 $000000000000000000000000000000000000000000000000000000000000000-1$ 0000000000000000000000000000000000000000000000000000000000000000 | 00000000000000000000000000000000000 in 000000000000000 ที่00000000000 0000000000000000000000000000000000000000000000000000000000000009

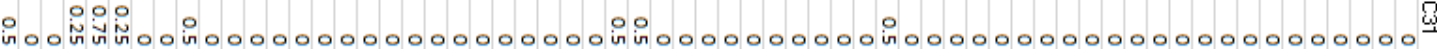
000000000000000000000000000000000000000000000000000000000000000 胥 $000000000000000000000000000000000000000000000000000000000000000 \bar{\Phi}$ $000000000000000000000000000000000000000000000000000000000000000 \frac{W}{A}$

Table E - 3 Elderly Woman (P2) Adjacency Matrix (first half) 


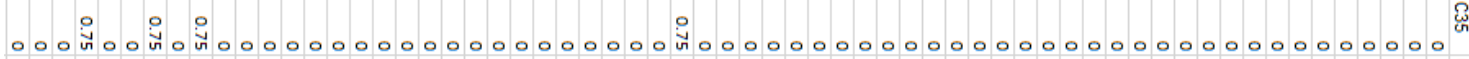
喥 (5)

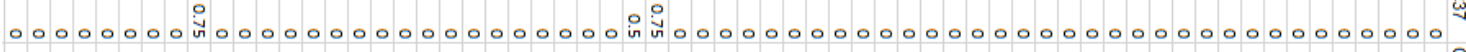
0 0000000000000000000000000000000000000000000000000000000 每 000000000000000000000 in 00000000 iñ 0 in 0000000000000001000000000000 $00000000000000000000000000000000000000000000000000000000000000 \pm$ f

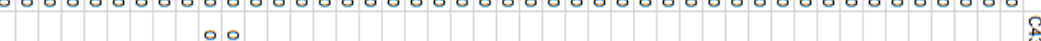
00000000 iñ 00000000000000000 iñ $00000000000000000000000000000000000000000000000000000000000000 \AA$

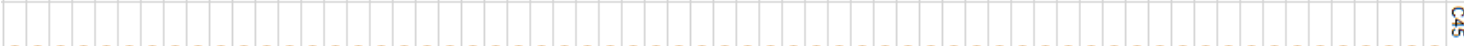
000 o 000000000000000000000000000000000000000000000000000000000000000

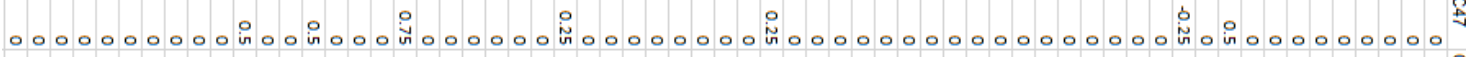

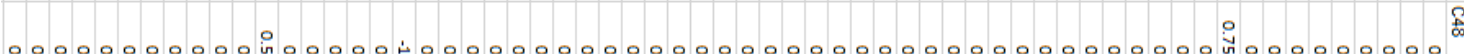

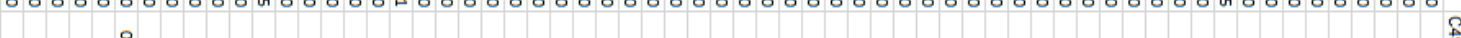
0000 惫 0 in 000000000000000000 in 0000 in 00000 in 0000000000000 in $000000000 \hat{0}$ 000000000000000000000000000000000000000000000000000000000000000 10.0 而 000 o 000000000000000000000000000000000000000000000000000000000000000 $000000000000000000000000000000000000000000000000000000000000 \tilde{m}$ 1.00

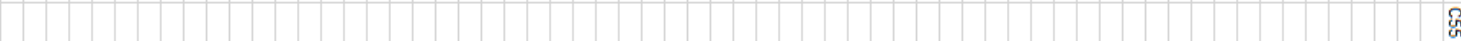
000000000000000000000000000000000000000000000000000000000000000 $00000000000000000000000000000000000000000000000000000000000000 \%$

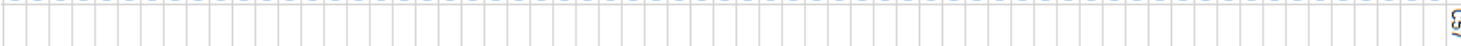
000000000000000000000000000000000000000000000000000000000000000

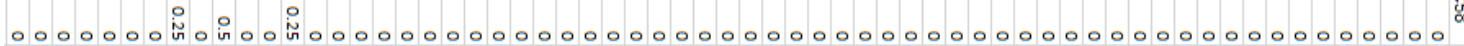
00000000000000000000000000000000000000000000000000000000000000

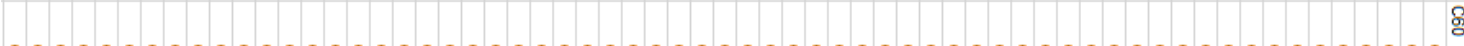

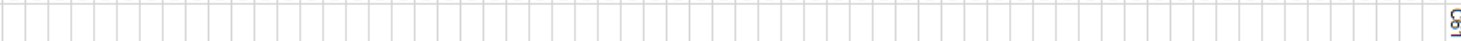
000000000000000000000000000000000000000000000000000000000000000 $00000000000000000000000000000000000000000000000000000000000000 \AA$ 0.0000000000000000000000000000000000000000000000000000000

Table E - 4 Elderly Woman (P2) Adjacency Matrix (second half) 

0000000000000000000000000000000000000000000000000000000000 in 000 年

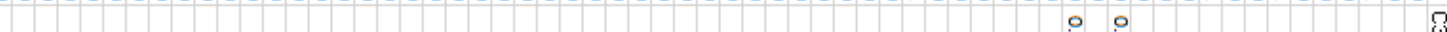
00000000000000000000000000000000000000000000000 ที่0 ñ.0000000000000 o 何 000000000000000000000000000000000000000000000000000000000000000 0000000000000000000000000000000000000000000000000000000000000

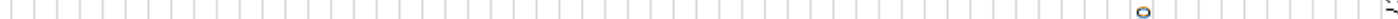
000000000000000000000000000000000000000000000000001540000000000 000000000000000000000000000000010000000001000010000 in 00000000000 1. 0000000000000000000000000000000000000000000041000000000000100000

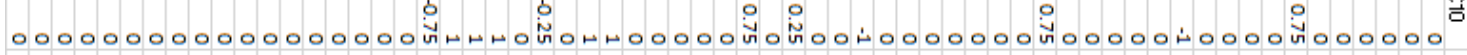

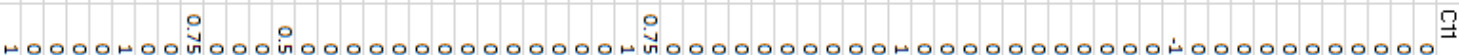

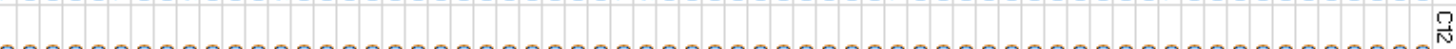
$000000000000000000000000000000000000000000000000000000000000000 \frac{9}{2}$ $000000000000000000000000000000000000000000000000000000000000000 \frac{9}{\omega}$

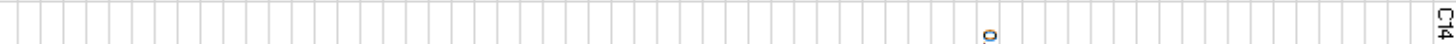
0000000000000000000000000000000000000000001 Uี่0000000100000010000 $000000000000000000000000000000000000000000000000000000000000000 \frac{9}{\sqrt{n}}$

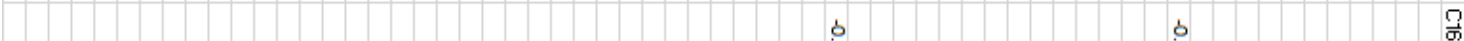

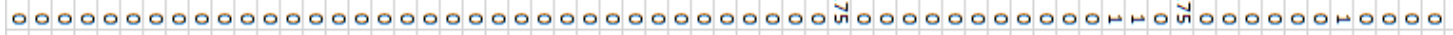
$000000000000000000000000000000000000000000000000000000000000000 \frac{1}{=1}$

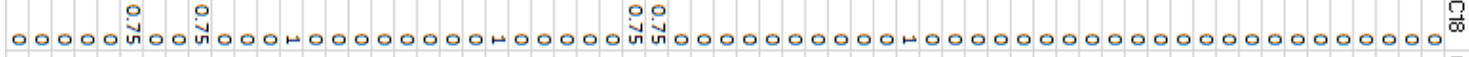
$000000000000000000000000000000000000000000000000000000000000000 \frac{T_{1}^{1}}{10}$

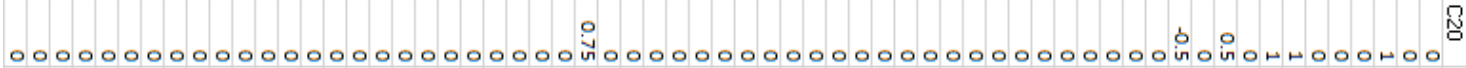
$000000000000000000000000000000000000000000000000000000000000000 \frac{N}{N}$ 0010000000000000000000000000000000000000000101000010 in 0000000000 000000000000000000000000000000100000000001000000000000150000100 $000000000000000000000000000000000000000000000000000000000000000 \AA$ 000000000000000000000000000000000000000000000000000000000000000 N 00000000000000000ที่00000001000000000000 ที่010000000000000000000000芯

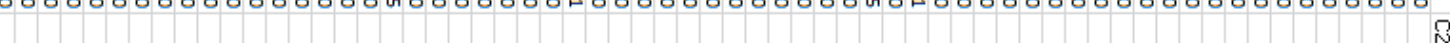
$000000000000000000000000000000000000000000000000000000000000000^{-1}$ $00000000000000000000000000000000000000000000000000000000000000 \AA$ 0000000000000000000000000000000000ino00000000000000 ñ 00000000000 $000000000000000000000000000000000000000000000000000000000000000 \overline{\mathscr{W}}$

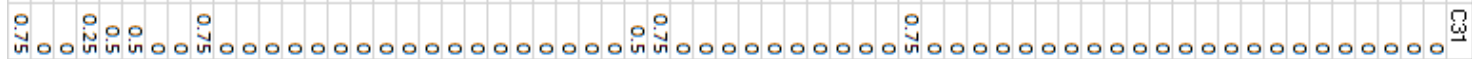
$000000000000000000000000000000000000000000000000000000000000000 N$ $000000000000000000000000000000000000000000000000000000000000000 \omega$ $000000000000000000000000000000000000000000000000000000000000000 \AA$

\section{Table E - 5 Elderly Woman (P3) Adjacency Matrix (first half)}




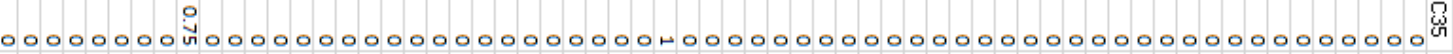

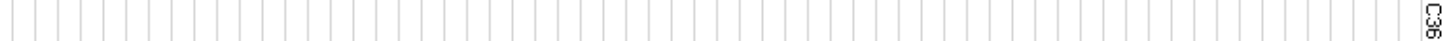
000000000000000000000000000000000000000000000000000000000000000

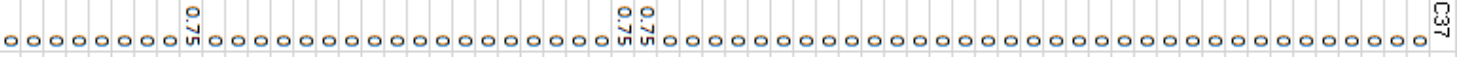
0 0000000000000000000000000000000000000000000000000000000000000004

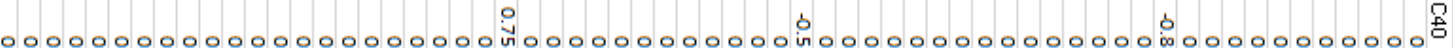

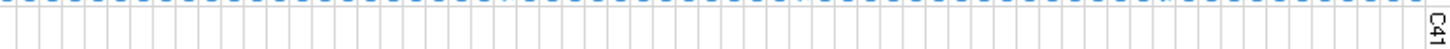
000000000000000000000000000000000000000000000000000000000000000 $000000000000000000000000000000000000000000000000000000000000000 \frac{0}{1}$ 00000000 ที่00000000000000000 ที่ ที 00000000000000000000000000000000 $000000000000000000000000000000000000000000000000000000000000000 \$$ $000000000000000000000000000000000000000000000000000000000000000 \frac{f}{1}$ 年 0000000000000000000000000000000000000000000000000000000000000000

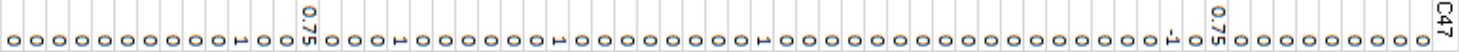
000000000001000001000000000000000000000000000000000004000000000 点

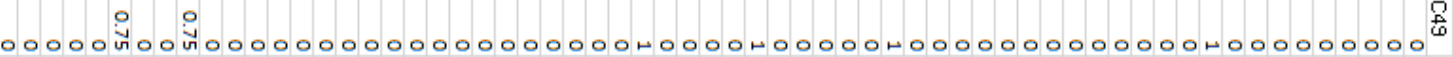

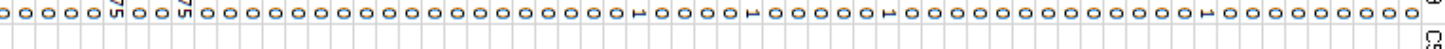
000000000000000000000000000000000000000000000000000000000000000 乌ీ $000000000000000000000000000000000000000000000000000000000000000 \mathfrak{G}^{-1}$ $000000000000000000000000000000000000000000000000000000000000000 N$ $000000000000000000000000000000000000000000000000000000000000000 \AA$ $000000000000000000000000000000000000000000000000000000000000000 \frac{1}{1}$ 000000000000000000000000000000000000000000000000000000000000000 留 贶 0000000000000000000000000000000000000000000000000000000000000000 00000000000000000000000000000000000000000000000000000000000000090 0000000 iñoino iñ 0000000000000000000000000000000000000000000000000 $000000000000000000000000000000000000000000000000000000000000000,1]$ 00000000000000000000000000000000000000000000000000000000000009 0000000000000000000000000000000000000000000000000000000000000009 000000000000000000000000000000000000000000000000000000000000000 000000000000000000000000000000000000000000000000000000000000000 R $000000000000000000000000000000000000000000000000000000000000000 \$$

\section{Table E - 6 Elderly Woman (P3) Adjacency Matrix (second half)}


0000000000000000000000000000000000000000000000000000000000100 00000000000000000000000000000000000000001000000000000 i⿱n.

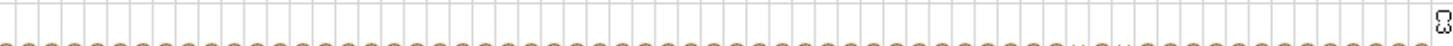
000000000000000000000000000000000000000000000001010000000000000

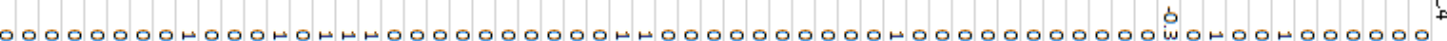

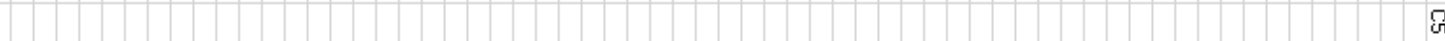
000000000000000000000000000000000000000000000000000000000000000

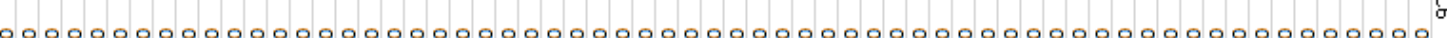

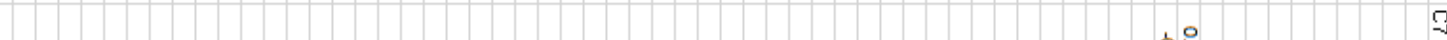
000000000000000000000000000000000000000000000000010 in 0000000000 0000000000000000000000000000000 iñ 00000000 vino 000 vino 000 iñ 00000000000 (1: 000000000000000000000000000000000000000000001000000000000100000

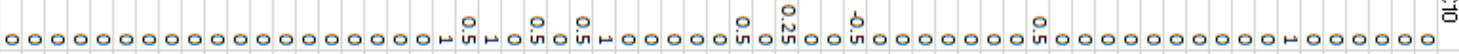
in 0 o 㲖 $000000000000000000000000000000000000000000000000000000000000000 \frac{7}{1}$ $000000000000000000000000000000000000000000000000000000000000000 \frac{?}{\omega}$

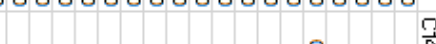
000000000000000000000000000000000000000000 in $00000000000000000000000000000000000000000000000000000000000000 \frac{?}{\mathfrak{l}}$

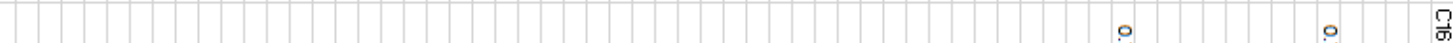
0000000000000000000000000000000000001000000000001 Uี० $000000000000000000000000000000000000000000000000000000000000000 \frac{7}{7}$

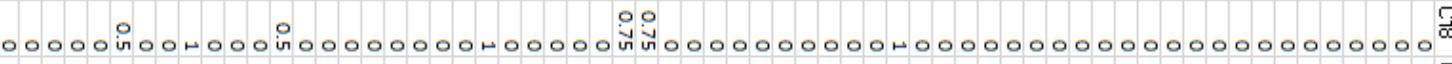
$000000000000000000000000000000000000000000000000000000000000000 \frac{9}{1}$

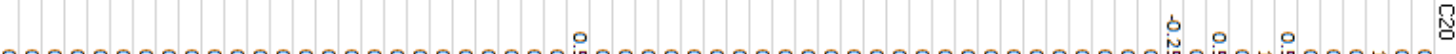
$000000000000000000000000000000000000000000000000000000000000000 \frac{3}{3}$ iv

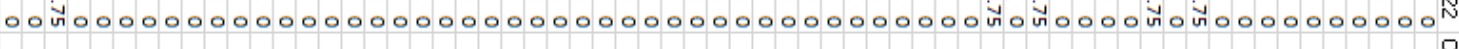
00000000000000000000000000000010000000000 ñ 000000000000110000 iñ 00 W $000000000000000000000000000000000000000000000000000000000000000 \AA$ 000000000000000000000000000000000000000000000000000000000000000 Nin $000000000000000001000000010000000000001010000000000000000000000 \AA$ $000000000000000000000000000000000000000000000000000000000000000-7$ 0000000000000000000000000000000000000000000000000000000000000000

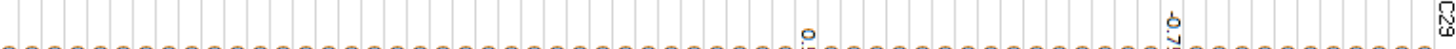
00000000000000000000000000000000000 in 0000000000000004100000000000

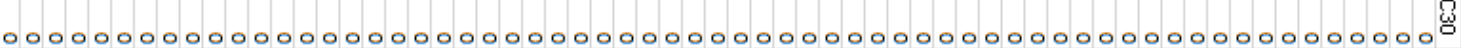

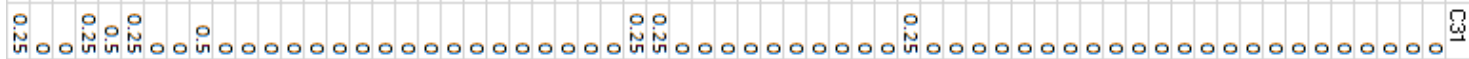
$000000000000000000000000000000000000000000000000000000000000000 \mathfrak{W}^{0}$ $000000000000000000000000000000000000000000000000000000000000000 \omega$ $000000000000000000000000000000000000000000000000000000000000000 \frac{W}{4}$

\section{Table E - 7 Elderly Woman (P4) Adjacency Matrix (first half)}




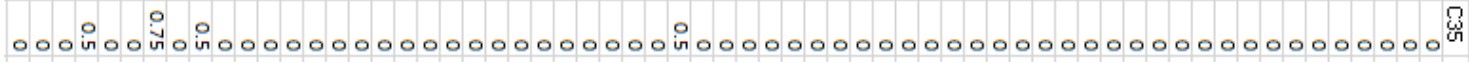
(1.000. 000000000000000000000000000000000000000000000000000000000000000 000000001000000000000000000 iñ

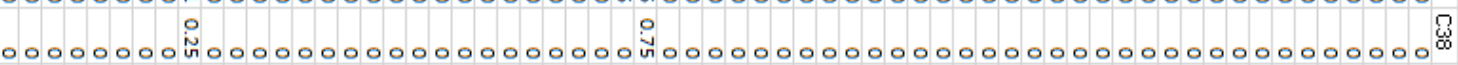
$000000000000000000000000000000000000000000000000000000000000000 \%$

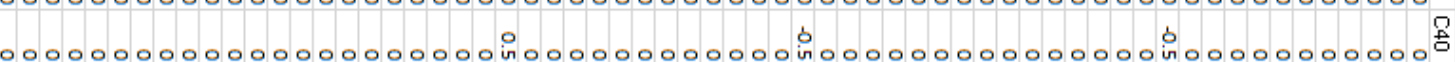
I. 000000000000000000000000000000000000000000000000000000000000000 000000000000000000000000000000000000000000000000000000000000000 N 00000000 iñ 00000000000000000 iñ 000000000000000000000000000000000000000000000000000000000000000 000000000000000000000000000000000000000000000000000000000000000 000000000000000000000000000000000000000000000000000000000000000 f́n $000000000000000000000000000000000000000000000000000000000000000 \frac{f}{f}$

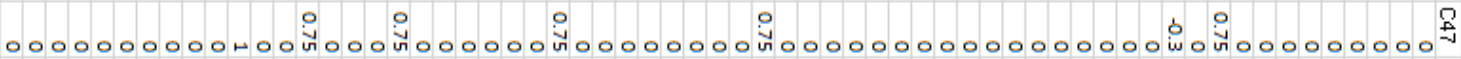
00000000000100000 in $000000000000000000000000000000000001000000000 \frac{f}{\infty}$

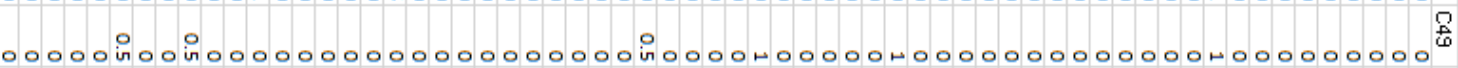
000000000000000000000000000000000000000000000000000000000000000 0000000000000000000000000000000000000000000000000000000000000009 000000000000000000000000000000000000000000000000000000000000000.35

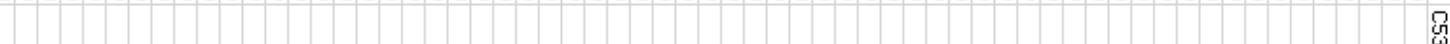
000000000000000000000000000000000000000000000000000000000000000 $000000000000000000000000000000000000000000000000000000000000000 \AA$ $000000000000000000000000000000000000000000000000000000000000000 \ln ^{1}$ 000000000000000000000000000000000000000000000000000000000000000 唒 $000000000000000000000000000000000000000000000000000000000000000^{9}$ 0000000000000000000000000000000000000000000000000000000000000000 000000000000000000000000000000000000000000000000000000000000000 ⿹ 000000000000000000000000000000000000000000000000000000000000000 解 0000000000000000000000000000000000000000000000000000000000000009 000000000000000000000000000000000000000000000000000000000000000 思 000000000000000000000000000000000000000000000000000000000000000
$00000000000000000000000000000000000000000000000000000000000000 \%$

Table E - 8 Elderly Woman (P4) Adjacency Matrix (second half) 
000000000000000000000000000000000000000000000000000000000010100 0.0 0 i 0000000000000000000000000000000000000000000000101000000000000

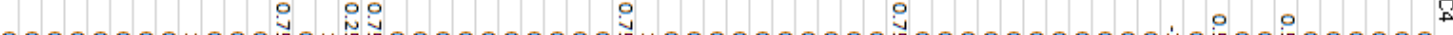
00000000म000nOH nn 000000000000000000000000000000000000000000000000000000000000000 $00000000000000000000000000000000000000000000000000000000000000 \pi$

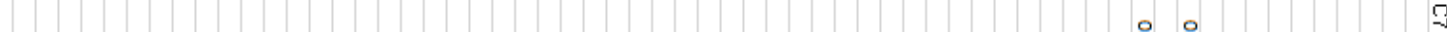
0000000000000000000000000000000000000000000000000041540000000000

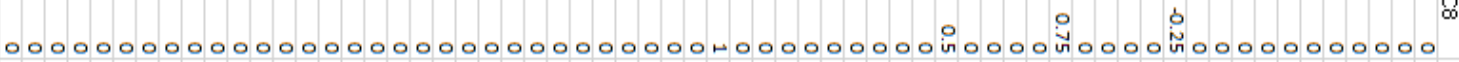
12 L 00000000000000000000000000000000000000000000 in 000000000000 vin 0000

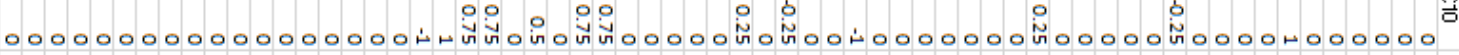

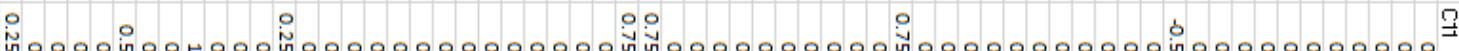

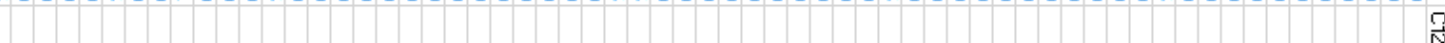
$000000000000000000000000000000000000000000000000000000000000000 \frac{2}{2}$ $000000000000000000000000000000000000000000000000000000000000000 \frac{?}{\omega}$

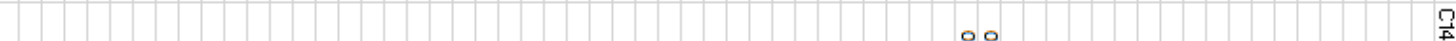
000000000000000000000000000000000000000000 Uี่ $000000000000000000000000000000000000000000000000000000000000000 \frac{?}{\sqrt{n}}$

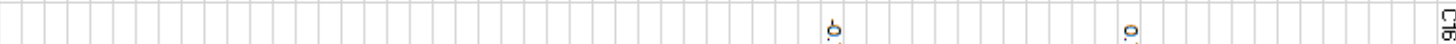
000000000000000000000000000000000000 ñ $000000000014 n 0100000010000$ $000000000000000000000000000000000000000000000000000000000000000 \frac{7}{7}$

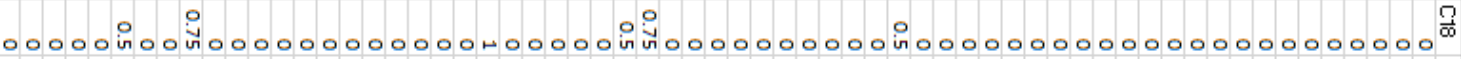
$000000000000000000000000000000000000000000000000000000000000000 \frac{?}{\Phi}$

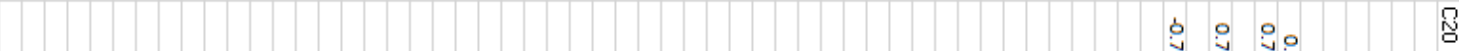
000000000000000000000000010000000000000000000000000 जี้ $000000000000000000000000000000000000000000000000000000000000000 \frac{3}{3}$

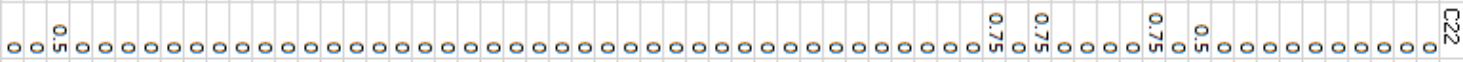

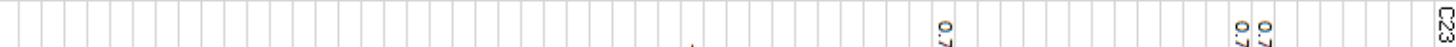
00000000000000000000000000000010000000000 ที 00000000000 ที่ $000000000000000000000000000000000000000000000000000000000000000 \frac{N}{3}$ 000000000000000000000000000000000000000000000000000000000000000 N 000000000000000001000000010000000000001010000000000000000000000 న్ $000000000000000000000000000000000000000000000000000000000000000 \sqrt{3}$ $000000000000000000000000000000000000000000000000000000000000000 \%$ 1. 0000000000000000000000000000000000 in 00000000000000 iñ 000000000 $000000000000000000000000000000000000000000000000000000000000000 \underline{\omega}$ 00000000 in 00000000000000000 in 000000000000000000000000000000000000000000000000000000000000000 W $000000000000000000000000000000000000000000000000000000000000000 \bar{W}$

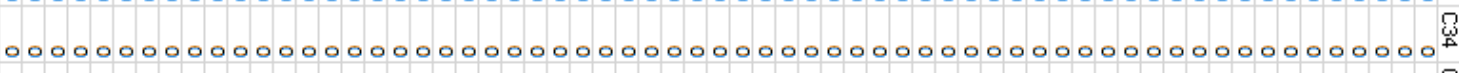

\section{Table E - 9 Elderly Woman (P5) Adjacency Matrix (first half)}



$00000000000000000000000000000000000000000000000000000000000000 \bar{W}$

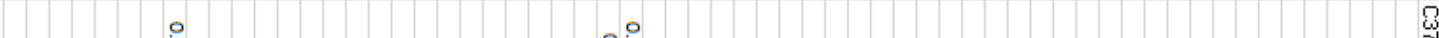
00000000 ที่000000000000000000ํํํำ00000000000000000000000000000000

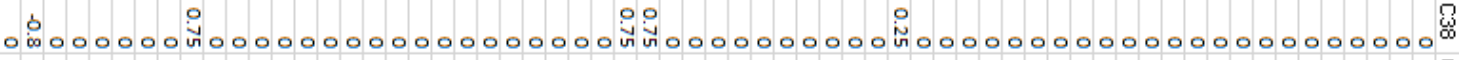
$000000000000000000000000000000000000000000000000000000000000000 \$$

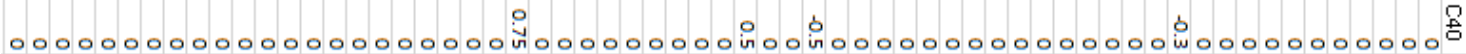

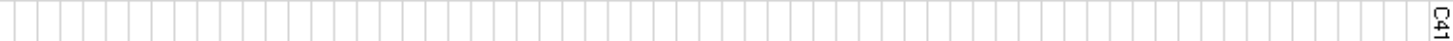
000000000000000000000000000000000000000000000000000000000000000 000000000000000000000000000000000000000000000000000000000000000 A 00000000 iño00000000000000000iñ $0000000000000000000000000000000000 \omega$ $000000000000000000000000000000000000000000000000000000000000000 \frac{1}{f}$ 0000000000000000000000000000000000000000000000000000000000000000 $000000000000000000000000000000000000000000000000000000000000000 \hat{f}$ $000000000000000000000000000000000000000000000000000000000000000 \frac{1}{f}$

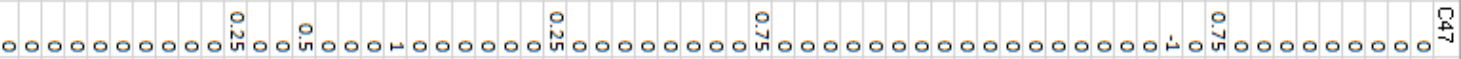
00000000000 in 0000100000000000000000000000000000000000 in $000000000 \frac{1}{10}$

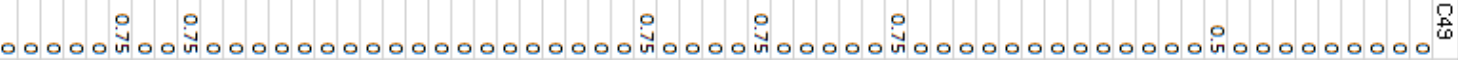
000000000000000000000000000000000000000000000000000000000000000 乌̂ 000000000000000000000000000000000000000000000000000000000000000 留 000000000000000000000000000000000000000000000000000000000000000 依 芯 $000000000000000000000000000000000000000000000000000000000000000 \frac{1}{n}$ 000000000000000000000000000000000000000000000000000000000000000 ñn 000000000000000000000000000000000000000000000000000000000000000 婴 $000000000000000000000000000000000000000000000000000000000000000-15$

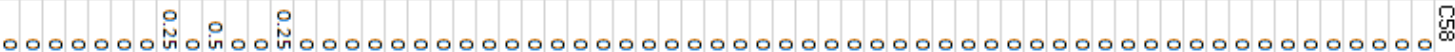
000000000000000000000000000000000000000000000000000000000000000 I 0000000000000000000000000000000000000000000000000000000000000009 000000000000000000000000000000000000000000000000000000000000009 000000000000000000000000000000000000000000000000000000000000000 思 $000000000000000000000000000000000000000000000000000000000000000 \%$

Table E - 10 Elderly Woman (P5) Adjacency Matrix (second half) 

00000000000000000000000000000000000000000000000000000000000 in 0 in

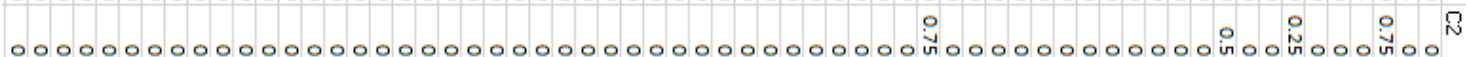

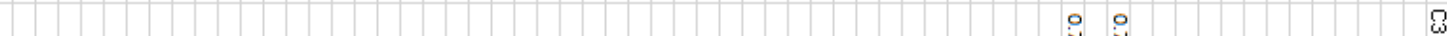
00000000000000000000000000000000000000000000000 Vี 0 Vี 0000000000000 il

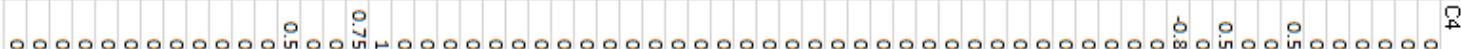

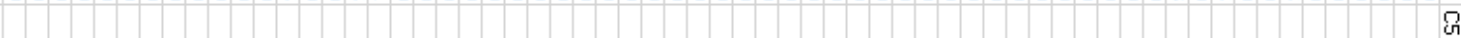
000000000000000000000000000000000000000000000000000000000000000 000000000000000000000000000000000000000000000000000000000000000 00000000000000000000000000000000000000000000000 in in in 000000000 0000000000000000000000000000000 iñ 00000000 in 0000 iñ 000 iñ 0000000000

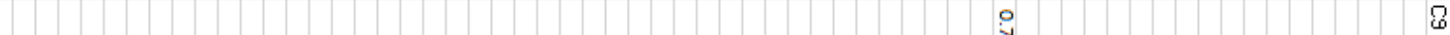
00000000000000000000000000000000000000000000 ví000000000000100000

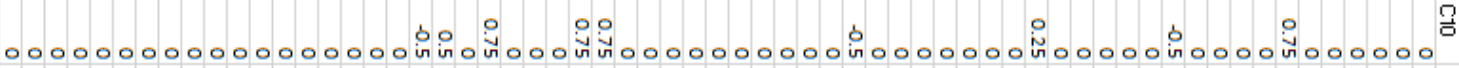
$000000000000000000000000000000000000000000000000000000000000000 ?$ $000000000000000000000000000000000000000000000000000000000000000 \frac{9}{\frac{7}{3}}$ $000000000000000000000000000000000000000000000000000000000000000 \frac{?}{\omega}$

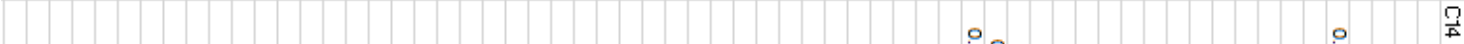
000000000000000000000000000000000000000000 iñ 00000001000000 in 0000 $00000000000000000000000000000000000000000000000000000000000000 \frac{?}{\mathrm{l}}$

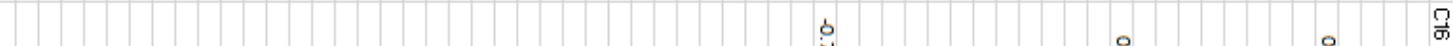
00000000000000000000000000000000000 iñ 00000000001 in 0 10 00000ino000 $000000000000000000000000000000000000000000000000000000000000000 \frac{9}{7}$ $000000000000000000000100000000000000000000000000000000000000000 \frac{?}{\frac{1}{1}}$ $000000000000000000000000000000000000000000000000000000000000000 \frac{T}{\omega}$

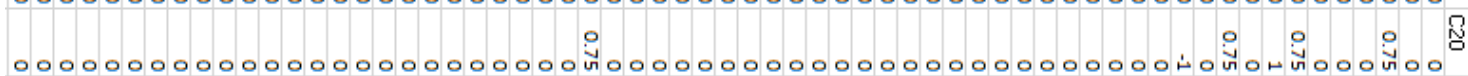
000000000000000000000000000000000000000000000000000000000000000, - 0 in 0000000000000000000000000000000000000000 iño00000inoñ 0000000000 N $00 \mathrm{n} 0000000000000000000000000000000000000000 \mathrm{n} 000000 \mathrm{~m} 0 \mathrm{n} 0000000000$ 000000000000000000000000000000 ñ 000000000 iñ 000 in 00000000 in 10000 iñ 00 in $000000000000000000000000000000000000000000000000000000000000000 \AA$ $000000000000000000000000000000000000000000000000000000000000000 \mathrm{~N}$

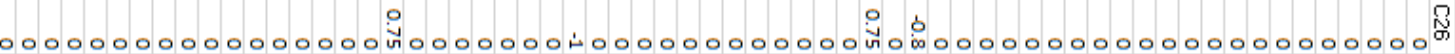
及 $000000000000000000000000000000000000000000000000000000000000000^{-1}$ 00000000000000000000000000000000000000000000000000000000000000000 0000000000000000000000000000000000 in $000000000000000 v 100000000000$ $00000000000000000000000000000000000000000000000000000000000000 \Phi$ $00000000000000000000000000000000000000000000000000000000000000 \underline{\Xi}$ $000000000000000000000000000000000000000000000000000000000000000 N$

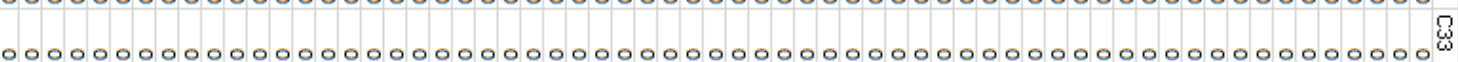
$00000000000000000000000000000000000000000000000000000000000000+\frac{W}{7}$

Table E - 11 Elderly Woman (P6) Adjacency Matrix (first half) 
$000000000000000000000000000000000000000000000000000000000000000 \%$

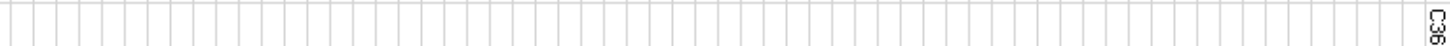
000000000000000000000000000000000000000000000000000000000000000 000000000000000000000000000000000000000000000000000000000000000 $000000000000000000000000000000000000000000000000000000000000000 \%$ $000000000000000000000000000000000000000000000000000000000000000 \frac{W}{4}$

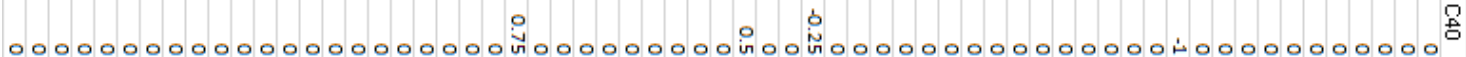

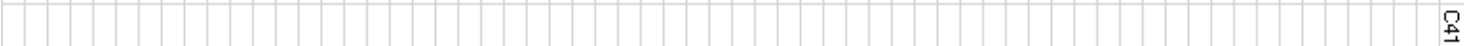
$000000000000000000000000000000000000000000000000000000000000000-$ $000000000000000000000000000000000000000000000000000000000000000 \frac{R}{1}$ $00000000000000000000000000000000000000000000000000000000000000 \frac{?}{\omega}$ 000000000000000000000000000000000000000000000000000000000000000 000000000000000000000000000000000000000000000000000000000000000 000000000000000000000000000000000000000000000000000000000000000 if $00000000000000000000000000000000000000000000000000000000000000 \frac{7}{8}$

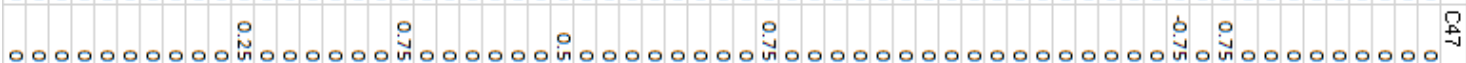

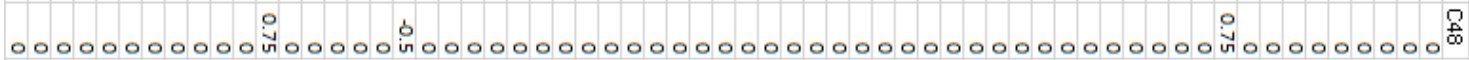

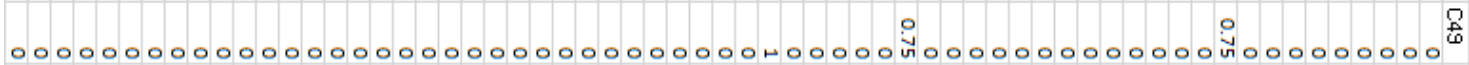
000000000000000000000000000000000000000000000000000000000000000 留

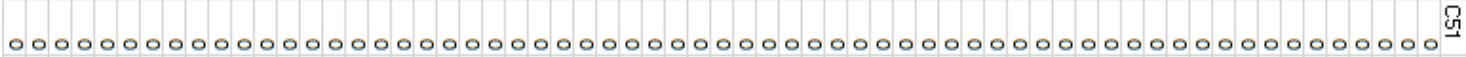
00000000000000000000000000000000000000000000000000000000000000 帒

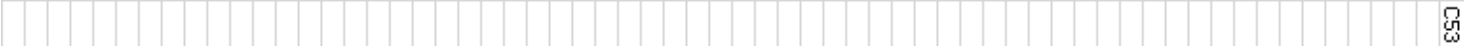
000000000000000000000000000000000000000000000000000000000000000 $00000000000000000000000000000000000000000000000000000000000000 \frac{n}{1}$ 000000000000000000000000000000000000000000000000000000000000000 in 00000000000000000000000000000000000000000000000000000000000000 婴 $000000000000000000000000000000000000000000000000000000000000000-1$

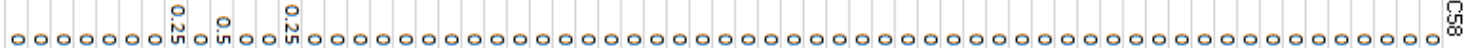
留 0000000000000000000000000000000000000000000000000000000000000009 0000000000000000000000000000000000000000000000000000000000000009 000000000000000000000000000000000000000000000000000000000000000 $000000000000000000000000000000000000000000000000000000000000000 \frac{9}{N}$ $000000000000000000000000000000000000000000000000000000000000000 \$$

Table E - 12 Elderly Woman (P6) Adjacency Matrix (second half) 

0000000000000000000000000000000000000000000000000000000000010040 年

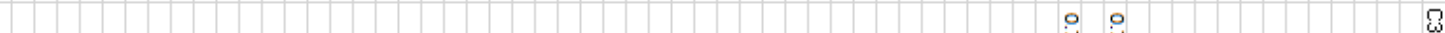
00000000000000000000000000000000000000000000000 vు 0 ñ 0000000000000

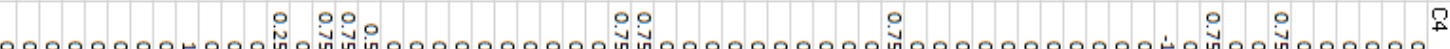
们 00000000000000000000000000000000000000000000000000000000000000 000000000000000000000000000000000000000000000000000000000000000

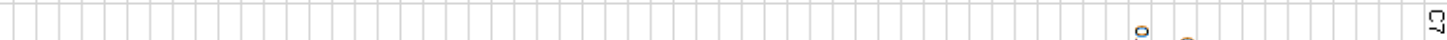
0000000000000000000000000000000000000000000000000 iñ 0000000000000000000000000000 in 00000000 iño0 0 iñ 00000000000000 10

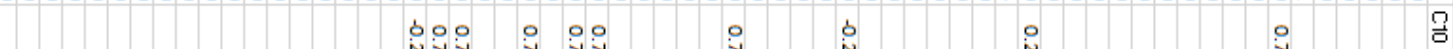

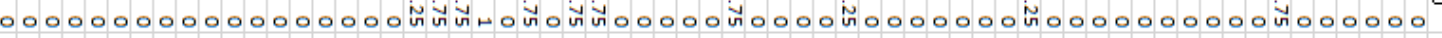

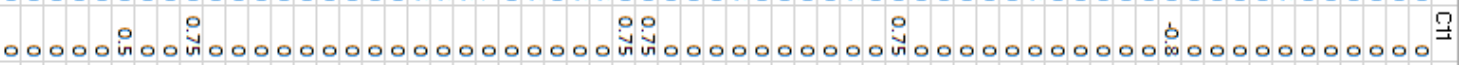
$000000000000000000000000000000000000000000000000000000000000000 \frac{9}{1}$ $000000000000000000000000000000000000000000000000000000000000000 \frac{?}{\omega}$

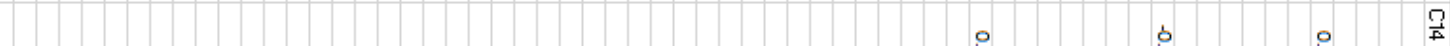
0000000000000000000000000000000000000000001 ñ.0000000 ñ 000000 ज̃ 0000 $000000000000000000000000000000000000000000000000000000000000000 \frac{7}{\sqrt{n}}$ 000000000000000000000000000000000000 in 00000000000 in $000000000000000000000000000000000000000000000000000000000000000=\frac{7}{1}$

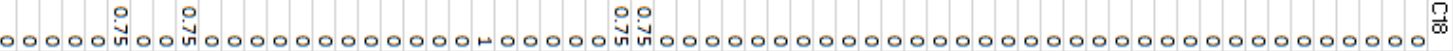
00000 Vn 0 N 000000000000100000 vin 000000000000000000000000000000000000 $00000000000000000000000000000000000000000000000000000000000000 \frac{\vec{A}}{1}$ 0000000000000000000000000 in 0000000000000000000000000 in 0 in 01000 in 000 $000000000000000000000000000000000000000000000000000000000000000 \Omega$ 00 in 0000000000000000000000000000000000000000 in

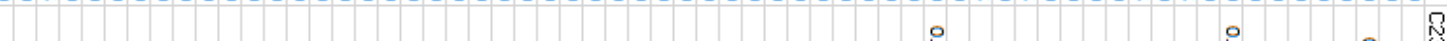
0000000000000000000000000000010000000000 vñ 00000000000 int 0000 in 000 $000000000000000000000000000000000000000000000000000000000000000+$ 000000000000000000000000000000000000000000000000000000000000000 N̂n 00000000000000000 in 0000001000000000000 iñ $000000000000000000000000000000000000000000000000000000000000000-1$ 00000000000000000000000000000000000000000000000000000000000000000

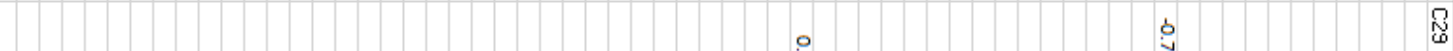
00000000000000000000000000000000000 in 000000000000000 ñ 00000000000 000000000000000000000000000000000000000000000000000000000000000,

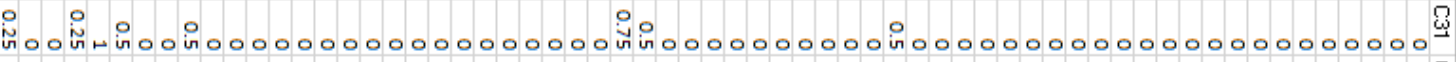
$000000000000000000000000000000000000000000000000000000000000000 N$ $000000000000000000000000000000000000000000000000000000000000000 \$$ $000000000000000000000000000000000000000000000000000000000000000 \frac{\mathbb{W}}{-}$

Table E - 13 Elderly Woman (P7) Adjacency Matrix (first half) 


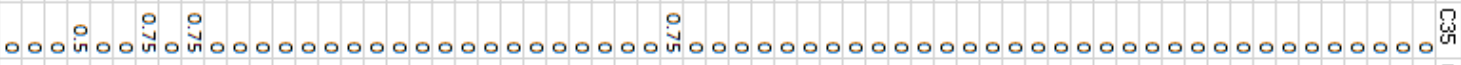

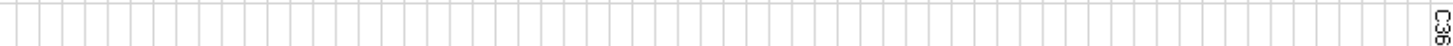
000000000000000000000000000000000000000000000000000000000000000

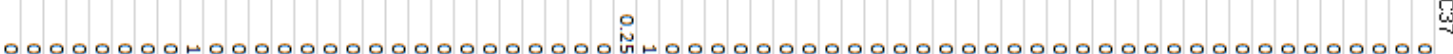
0 $000000000000000000000000000000000000000000000000000000000000000 \%$

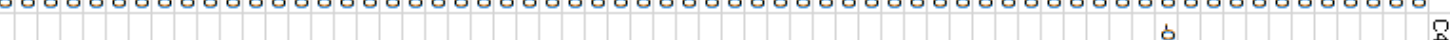
000000000000000000000000000000000000000000000000000 जั000000000000 E

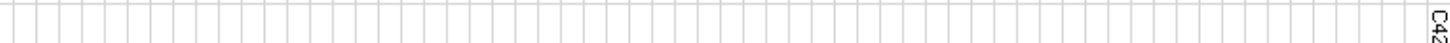
$000000000000000000000000000000000000000000000000000000000000000^{n}$ 000000001000000000000000000 iñ $10000000000000000000000000000000000 \frac{?}{\omega}$ $000000000000000000000000000000000000000000000000000000000000000 \$$ $000000000000000000000000000000000000000000000000000000000000000 \hat{f}$ $000000000000000000000000000000000000000000000000000000000000000 \frac{f}{f}$

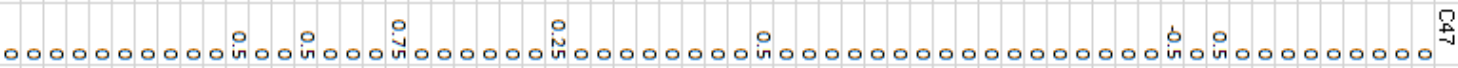
00000000000100000000000000000000000000000000000000000 ज̃̃000000000

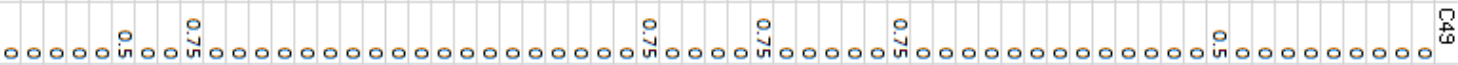
000000000000000000000000000000000000000000000000000000000000000 乌 0000000000000000000000000000000000000000000000000000000000000009 000000000000000000000000000000000000000000000000000000000000000 S

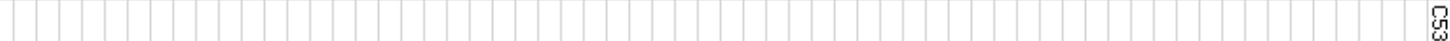
000000000000000000000000000000000000000000000000000000000000000 000000000000000000000000000000000000000000000000000000000000000 T 000000000000000000000000000000000000000000000000000000000000000 年 00000000000000000000000000000000000000000000000000000000000000 每 $000000000000000000000000000000000000000000000000000000000000000-\frac{n}{9}$

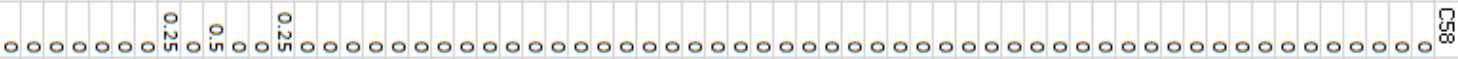
000000000000000000000000000000000000000000000000000000000000000 N 0000000000000000000000000000000000000000000000000000000000000009 $00000000000000000000000000000000000000000000000000000000000000 \frac{9}{9}$ 000000000000000000000000000000000000000000000000000000000000000 思 $000000000000000000000000000000000000000000000000000000000000000 \frac{1}{9}$

Table E - 14 Elderly Woman (P7) Adjacency Matrix (second half) 

00000000000000000000000000000000000000000000000000000000000 iñ

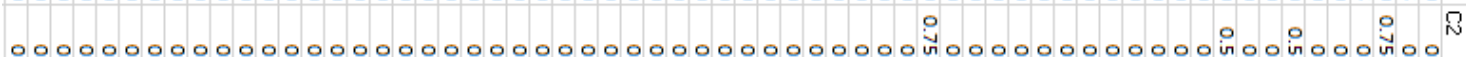

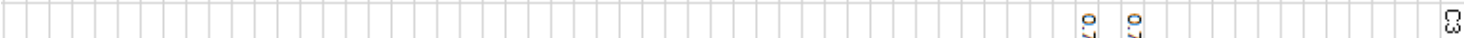
00000000000000000000000000000000000000000000000 ñ 0 iñ 000000000000 00000000 ที่000 ஸñ 000000000000000000000000000000000000000000000000000000000000000 年 1. 000000000000000000000000000000000000000000000000000000000000000 00000000000000000000000000000000000000000000000000010140000000000

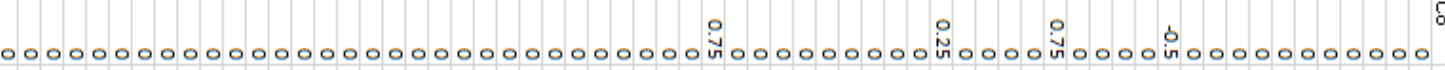

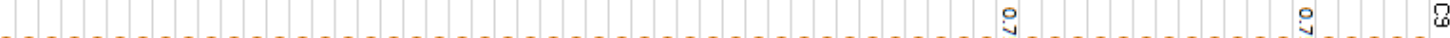
00000000000000000000000000000000000000000000 ที่000000000000 й 00000 0000000000000000000 inњ

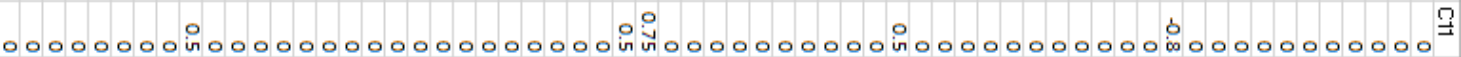
10.0015 000000000000000000000000000000000000000000000000000000000000000 $000000000000000000000000000000000000000000000000000000000000000 \frac{T}{\omega}$

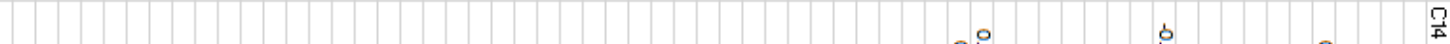
00000000000000000000000000000000000000000 in $000000000000000000000000000000000000000000000000000000000000000 \frac{?}{\sqrt[n]{n}}$ 1.

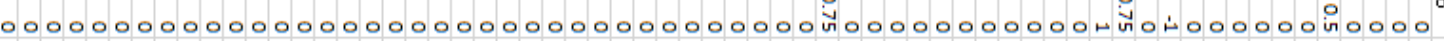
$000000000000000000000000000000000000000000000000000000000000000 \frac{7}{7}$

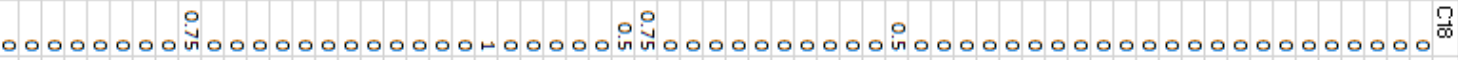
$000000000000000000000000000000000000000000000000000000000000000 \frac{\frac{\pi}{4}}{0}$ 10.000

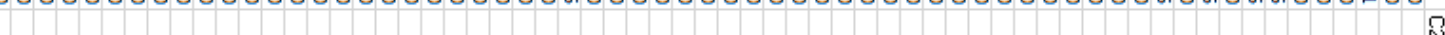
$000000000000000000000000000000000000000000000000000000000000000-1$

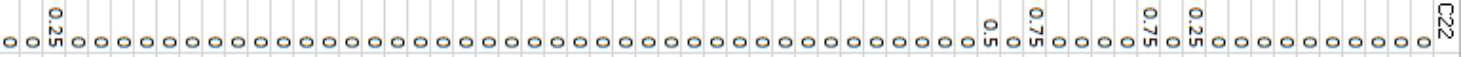

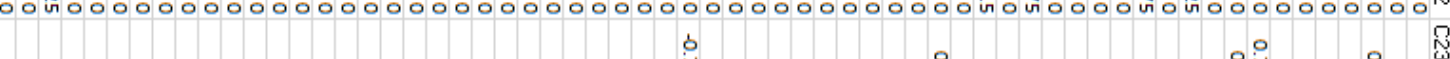

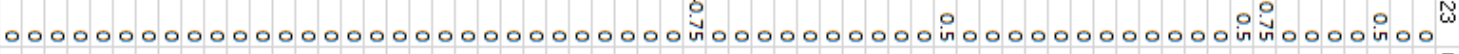
$000000000000000000000000000000000000000000000000000000000000000 \AA$ 000000000000000000000000000000000000000000000000000000000000000 N 0000000000000000010000000100000000000010 ib0000000000000000000000耳 $000000000000000000000000000000000000000000000000000000000000000-7$ $000000000000000000000000000000000000000000000000000000000000000 \$$ 00000000000000000000000000000000000 in 000000000000000 i⿱n 0000000000 $000000000000000000000000000000000000000000000000000000000000000 \frac{\mathscr{W}}{\mathscr{S}}$ 000 iñ 00000000000000000000000 ññ $000000000000000000000000000000000 \underline{B}$ $000000000000000000000000000000000000000000000000000000000000000 N$ $000000000000000000000000000000000000000000000000000000000000000 \omega$ $000000000000000000000000000000000000000000000000000000000000000 \AA$

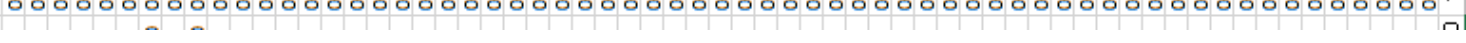

\section{Table E - 15 Elderly Woman (P8) Adjacency Matrix (first half)}




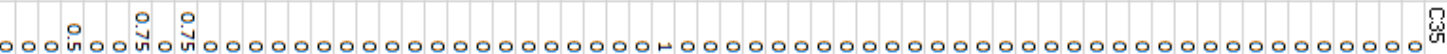
\begin{tabular}{l} 
Z \\
\hline
\end{tabular} 000000000000000000000000000000000000000000000000000000000000000 00000000 iñ 00000000000000000 iñ ○ $000000000000000000000000000000000000000000000000000000000000000 \Phi$ 0000000000000000000000000000000000000000000000000001000000000000 | 000000000000000000000000000000000000000000000000000000000000000 $000000000000000000000000000000000000000000000000000000000000000 \frac{n}{n}$ 00000000 iñ 00000000000000000 iñ $10000000000000000000000000000000000 \omega$ $000000000000000000000000000000000000000000000000000000000000000 \$$ 000000000000000000000000000000000000000000000000000000000000000 $000000000000000000000000000000000000000000000000000000000000000 \frac{0}{1}$ $000000000000000000000000000000000000000000000000000000000000000 \frac{f}{1}$

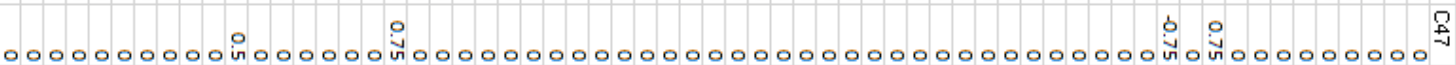
00000000000 iñ 0000 in 0000000000000000000000000000000000 ที่000000000

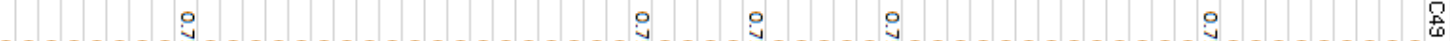

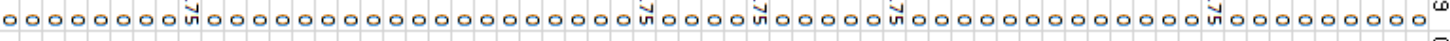
000000000000000000000000000000000000000000000000000000000000000 !n 0000000000000000000000000000000000000000000000000000000000000009 000000000000000000000000000000000000000000000000000000000000000 Mn

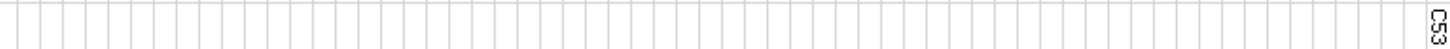
000000000000000000000000000000000000000000000000000000000000000 $000000000000000000000000000000000000000000000000000000000000000 \frac{M^{1}}{1}$ $000000000000000000000000000000000000000000000000000000000000000 \mathfrak{n n}^{0}$ 000000000000000000000000000000000000000000000000000000000000000 每 $000000000000000000000000000000000000000000000000000000000000000 \min ^{-1}$ 0000000 iñoinooñño0000000000000000000000000000000000000000000000000

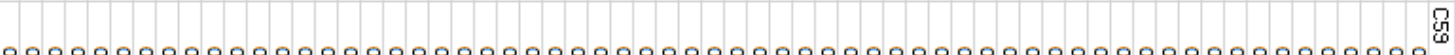
0000000000000000000000000000000000000000000000000000000000000009 0000000000000000000000000000000000000000000000000000000000000009 $000000000000000000000000000000000000000000000000000000000000000 \mathrm{R}$ $000000000000000000000000000000000000000000000000000000000000000 \$$

\section{Table E - 16 Elderly Woman (P8) Adjacency Matrix (second half)}

The elderly woman (P9) was not available to take the survey and hence the data set is missing her input. 

0000000000000000000000000000000000000000000000000000000000010 Uั 00000000000000000000000000000000000000100000000000 in 00000000

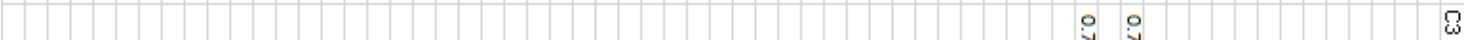
00000000000000000000000000000000000000000000000 Vี 0 ñ 0000000000000 in

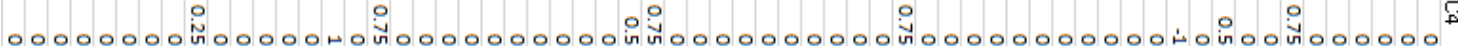
ñ 000000000000000000000000000000000000000000000000000000000000000 00000000000000000000000000000000000000000000000000000000000000 0000000000000000000000000000000000000000000000000 inj

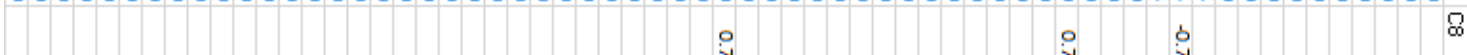

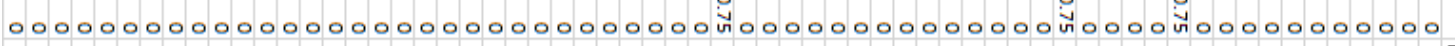
00000000000000000000000000000000000000000000 ที่000000000000 ñ 00000 S ○ 0000000000000000 in in in 000000000000000000 जै in iñ iñ iñ

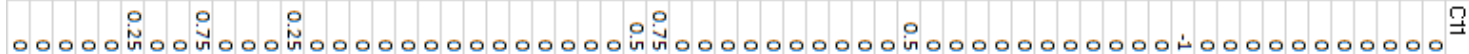
$00000000000000000000000000000000000000000000000000000000000000 \frac{\pi}{n}$ $00000000000000000000000000000000000000000000000000000000000000 \frac{N}{2}$ $000000000000000000000000000000000000000000000000000000000000000 \frac{0}{\omega}$

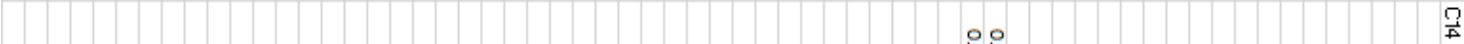
00000000000000000000000000000000000000000 Uี $000000000000000000000000000000000000000000000000000000000000000 \frac{?}{\sqrt{n}}$ 1. 00000000000000000000000000000000000 ṽ 0000000000 iñ $000000000000000000000000000000000000000000000000000000000000000=\frac{7}{3}$

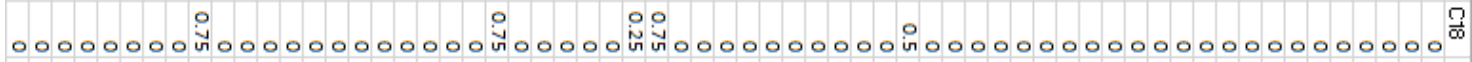
$00000000000000000000000000000000000000000000000000000000000000 \frac{7}{\frac{1}{4}}$ 10 . 000000000000000000000000010000000000000000000000000 iñ $000000000000000000000000000000000000000000000000000000000000000-1$ 00 iño000000000000000000000000000000000000000inoiño000 in 0 iño000000000 N

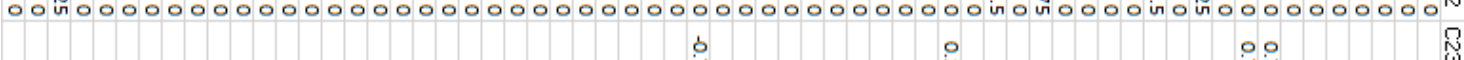
000000000000000000000000000000 ñ.0000000000 ñ 000000000000 ที่ $000000000000000000000000000000000000000000000000000000000000000 \AA$ 00000000000000000000000000000000000000000000000000000000000000 Nn

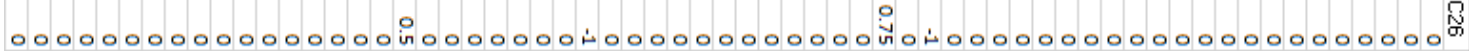
$000000000000000000000000000000000000000000000000000000000000000-1$ 0000000000000000000000000000000000000000000000000000000000000000

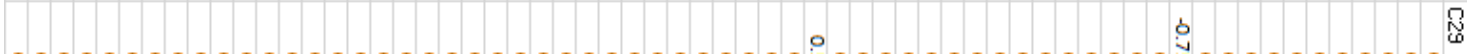
00000000000000000000000000000000000 in 000000000000000400000000000 $000000000000000000000000000000000000000000000000000000000000000 \underline{\mathscr{S}}$

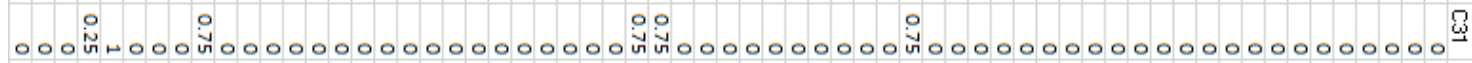
$000000000000000000000000000000000000000000000000000000000000000 N$ $00000000000000000000000000000000000000000000000000000000000000 \$$ $000000000000000000000000000000000000000000000000000000000000000 \AA$

Table E - 17 Elderly Woman (P10) Adjacency Matrix (first half) 


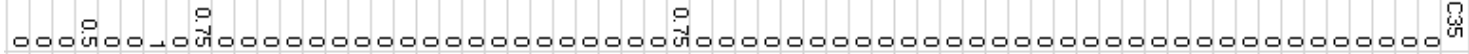
$000000000000000000000000000000000000000000000000000000000000000 \%$

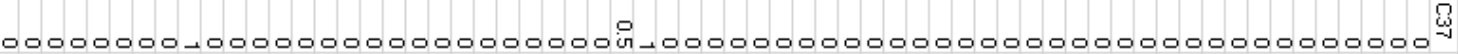
总 ऽ $000000000000000000000000000000000000000000000000000000000000000^{\Phi}$

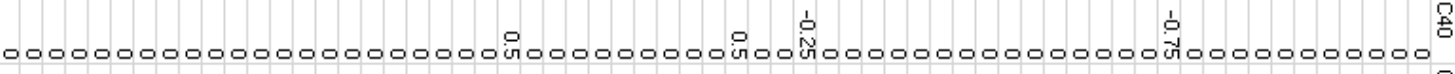

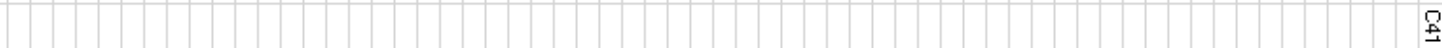
000000000000000000000000000000000000000000000000000000000000000 $000000000000000000000000000000000000000000000000000000000000000 \frac{0}{N}$

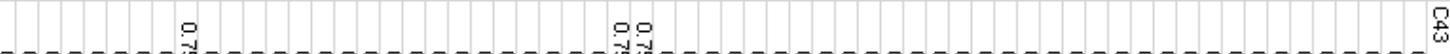

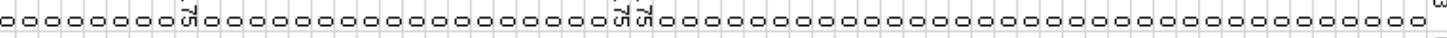
000000000000000000000000000000000000000000000000000000000000000 f $000000000000000000000000000000000000000000000000000000000000000^{\text {fin }}$ $000000000000000000000000000000000000000000000000000000000000000^{\text {f }}$

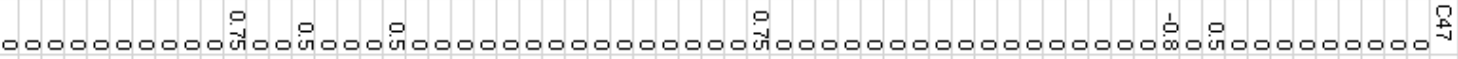

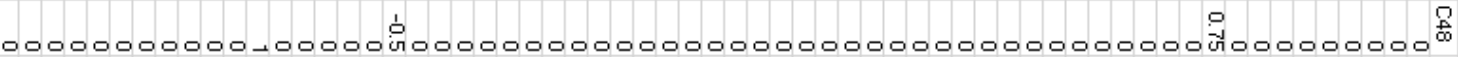

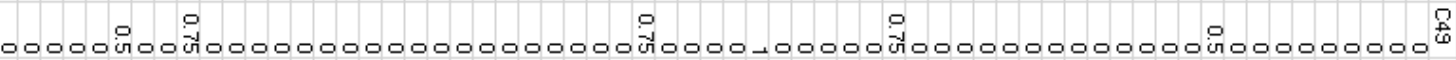
000000000000000000000000000000000000000000000000000000000000000 . T 000000000000000000000000000000000000000000000000000000000000000 S 000000000000000000000000000000000000000000000000000000000000000 N $000000000000000000000000000000000000000000000000000000000000000 \%$ $000000000000000000000000000000000000000000000000000000000000000^{\text {Tin }}$ $000000000000000000000000000000000000000000000000000000000000000^{\text {N }}$

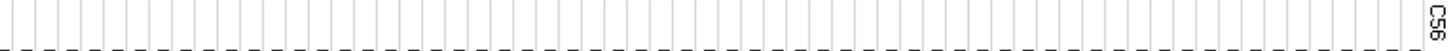
000000000000000000000000000000000000000000000000000000000000000 000000000000000000000000000000000000000000000000000000000000000 ר

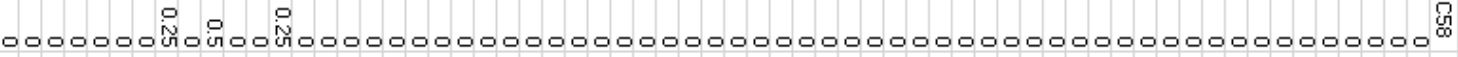
000000000000000000000000000000000000000000000000000000000000000 赑 000000000000000000000000000000000000000000000000000000000000000 \% 000000000000000000000000000000000000000000000000000000000000000 疋 000000000000000000000000000000000000000000000000000000000000000 忽 000000000000000000000000000000000000000000000000000000000000000\%

Table E - 18 Elderly Woman (P10) Adjacency Matrix (second half) 

00000000000000000000000000000000000000000000000000000000000 -

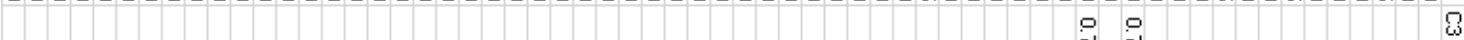
$00000000000000000000000000000000000000000000000 \operatorname{n̆}_{0} 00000000000000$

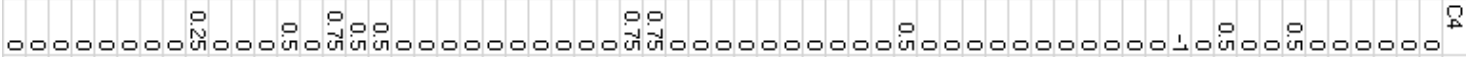
0000000000000000000000000000000000000000000000000000000

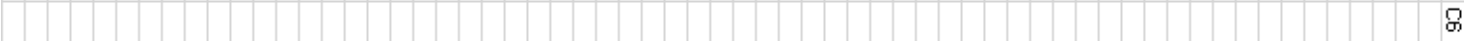
000000000000000000000000000000000000000000000000000000000000000

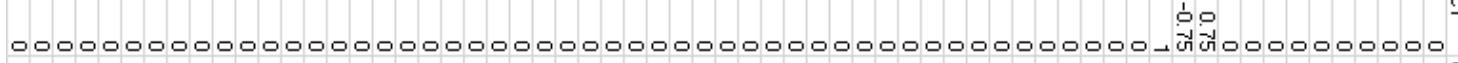

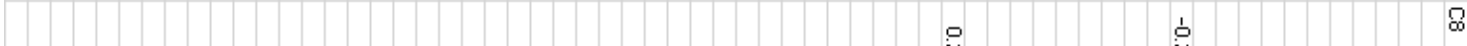

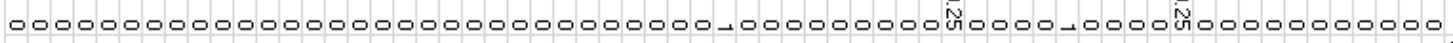

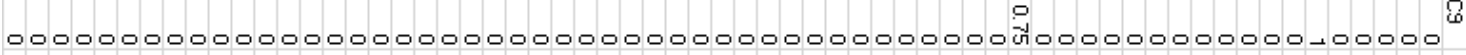

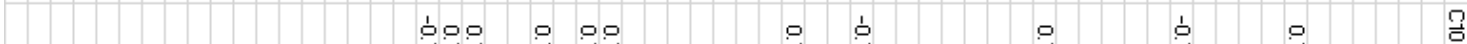

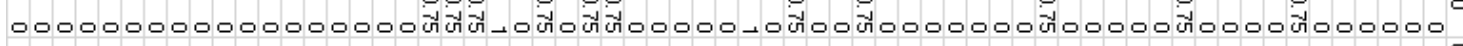
花 Un, $000000000000000000000000000000000000000000000000000000000000000 \frac{\vec{N}}{2}$ 000000000000000000000000000000000000000000000000000000000000000 , 0000000000000000000000000000000000000 $000000000000000000000000000000000000000000000000000000000000000 \frac{7}{11}$

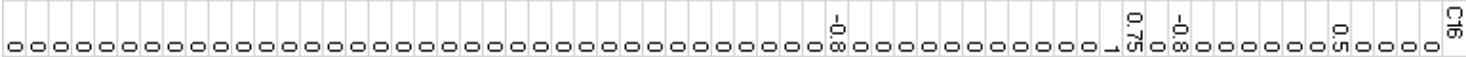
00000000000000000000000000000000000000000000000000000000000

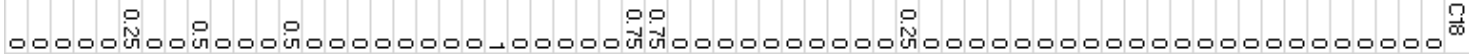

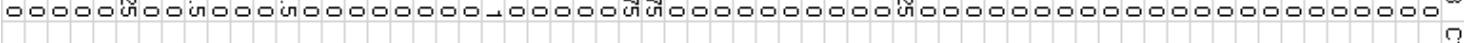
$000000000000000000000000000000000000000000000000000000000000000 \frac{0}{\omega}$

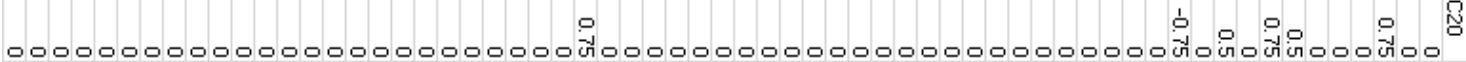
000000000000000000000000000000000000000000000000000000000000000 N ० ำ 每 000000000000000000000000000000 $00000000000000000000000000000000000000000000000000000000000000+$ $00000000000000000000000000000000000000000000000000000000000000 \hat{N}$

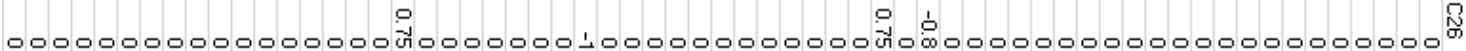
0000000000000000000000000000000000000000000000000000000000000007 0000000000000000000000000000000000000000000000000000000000000000

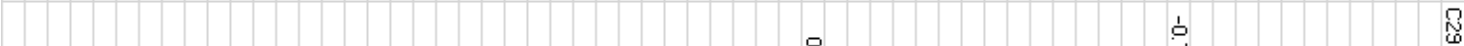
0000000000000000000000000000000000000000000000000000100000000000 $000000000000000000000000000000000000000000000000000000000000000 \mathscr{W}$

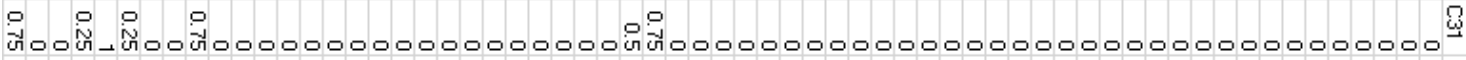
000000000000000000000000000000000000000000000000000000000000000 W 000000000000000000000000000000000000000000000000000000000000000 $\tilde{\omega}$ $000000000000000000000000000000000000000000000000000000000000000+$

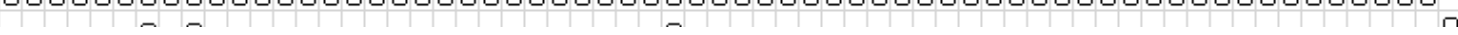

\section{Table E - 19 Elderly Woman (P11) Adjacency Matrix (first half)}




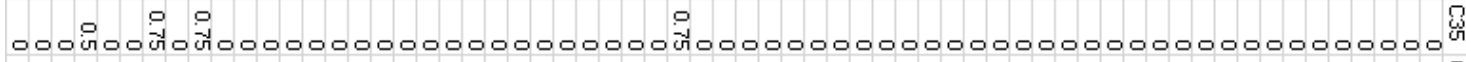
$000000000000000000000000000000000000000000000000000000000000000^{\mathscr{W}}$

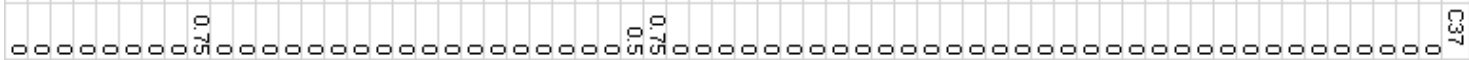
0

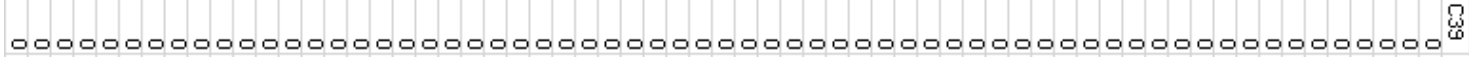

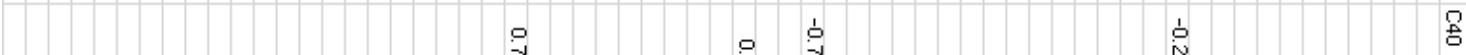
0000000000000000000000 ज̆ 000000000 in 00 ज̆ $000000000000000 \hat{n} 00000000000$

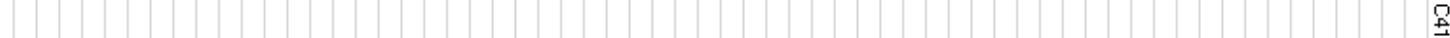
000000000000000000000000000000000000000000000000000000000000000

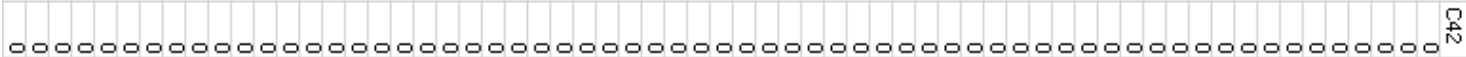

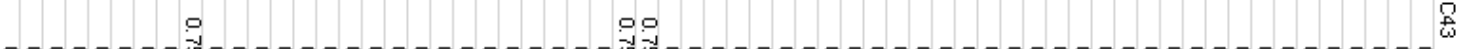

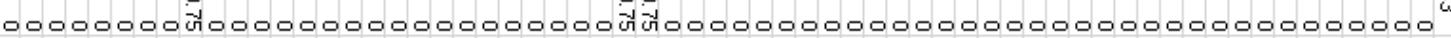
00000000000000000000000000000000000000000000000000000000000000 000000000000000000000000000000000000000000000000000000000000000 0000000000000000000000000000000000000000000000000000000000000 है

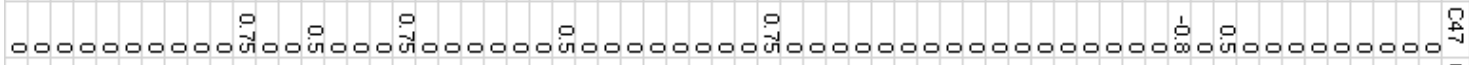

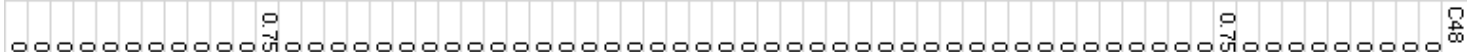

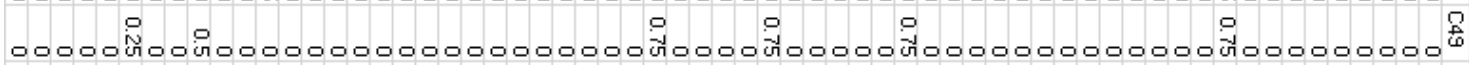

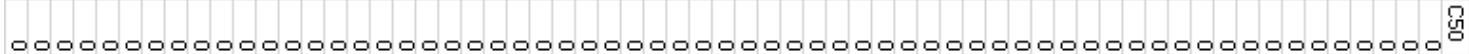
00000000000000000000000000000000000000000000000000000000000000

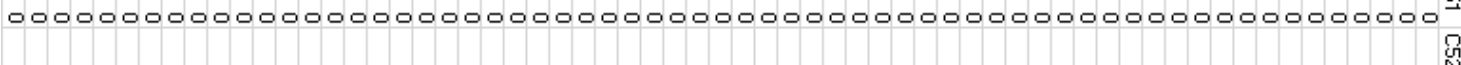
$000000000000000000000000000000000000000000000000000000000000000 N$

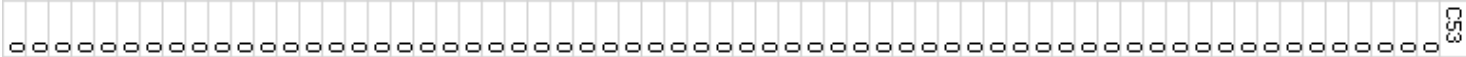
0000000000000000000000000000000000000000000000000000000000000 帒 0000000000000000000000000000000000000000000000000000000000000

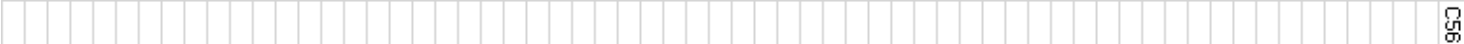
000000000000000000000000000000000000000000000000000000000000000

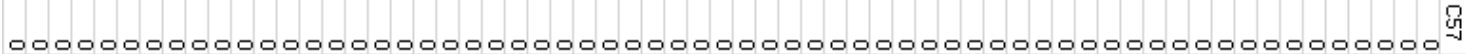

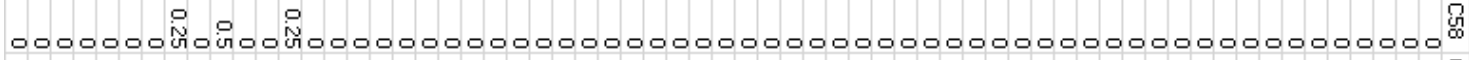
000000000000000000000000000000000000000000000000000000 గî́

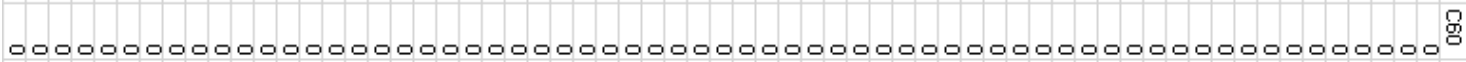

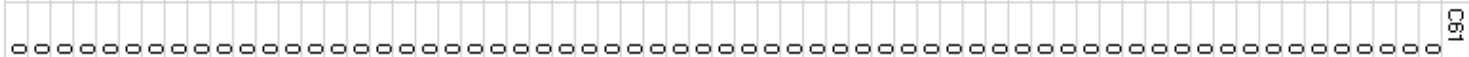
000000000000000000000000000000000000000000000000000000000000000 000000000000000000000000000000000000000000000000000000000000000 N

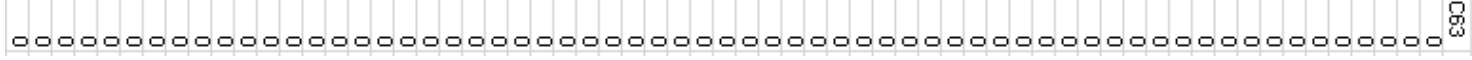

Table E - 20 Elderly Woman (P11) Adjacency Matrix (second half) 

0000000000000000000000000000000000000000000000000000000000000000 0000000000000000000000000000000000000000

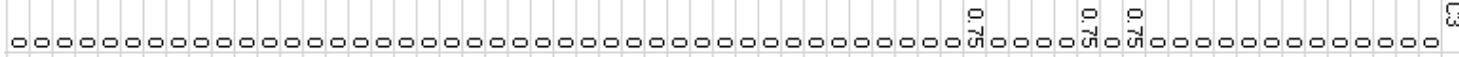

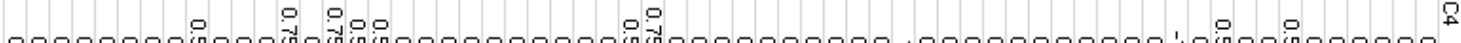

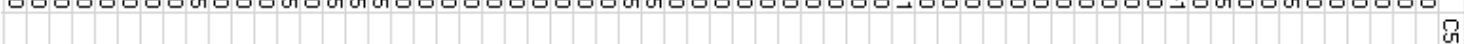
000000000000000000000000000000000000000000000000000000000000000 00000000000000000000000000000000000000000000000000000000000000 $00000000000000000000000000000000000000000000000000 \dot{0} 0000000000$

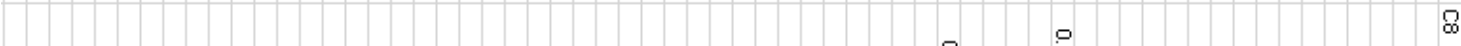
0000000000000000000000000000000-0000000000ั0000ǘ0000000000000000

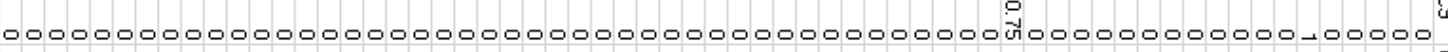

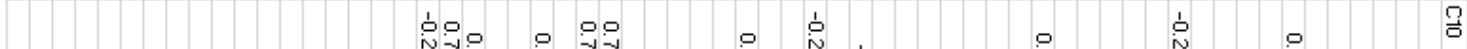

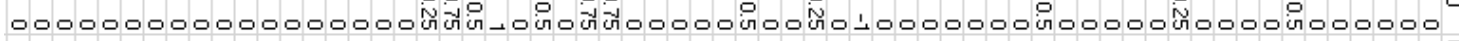

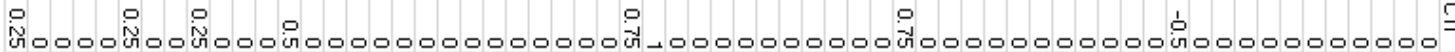

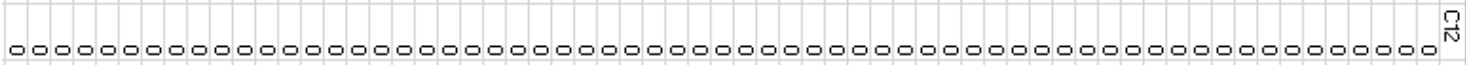

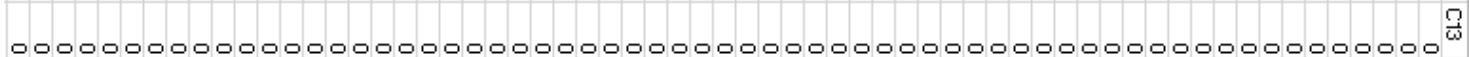
? ? ? ? ? ?

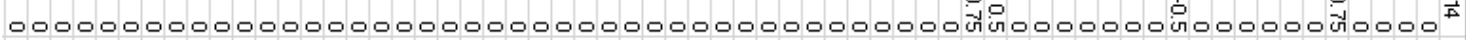
$00000000000000000000000000000000000000000000000000000000000000 \frac{?}{4}$

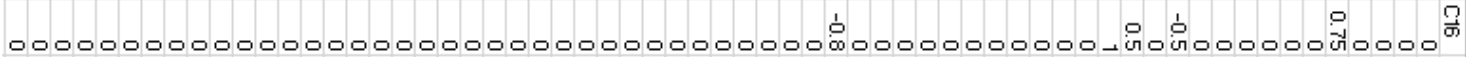

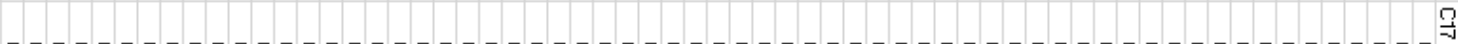
0000000000000000000000000000000000000000000000000000000000000007

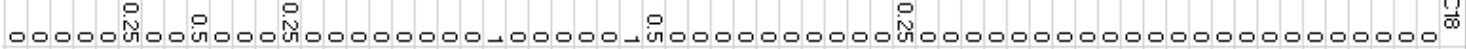
$000000000000000000000000000000000000000000000000000000000000000 \frac{T}{1}$

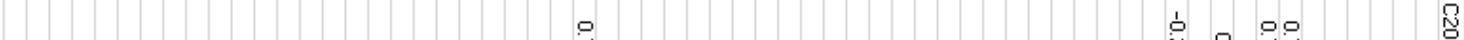

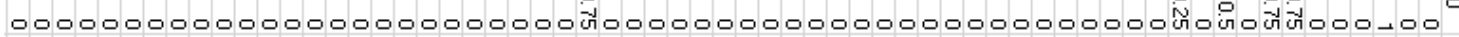
00000000000000000000000000000000000000000000000000000000000000

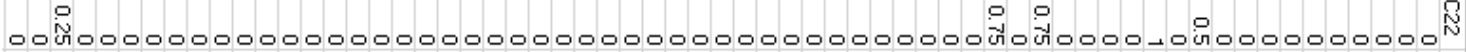

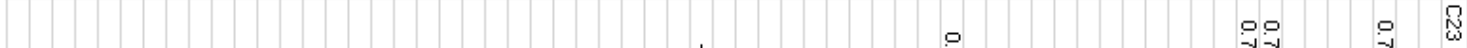

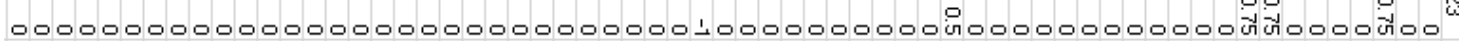
$00000000000000000000000000000000000000000000000000000000000000 \AA$ 00000000000000000000000000000000000000000000000000000000000000

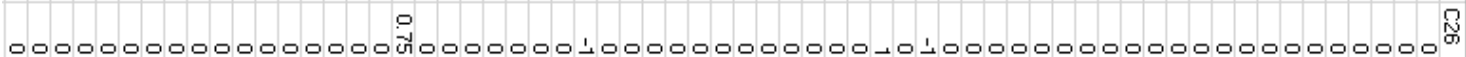

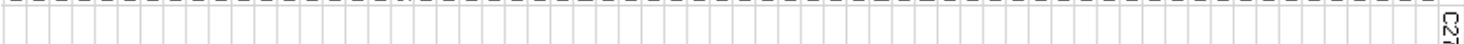
$000000000000000000000000000000000000000000000000000000000000000-1$ $0000000000000000000000000000000000000000000000000000000000000 \%$

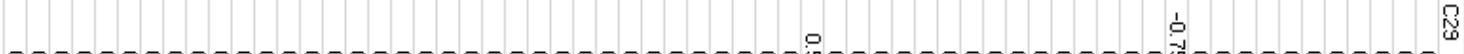
00000000000000000000000000000000000 in $00000000000000 \hat{n} 00000000000$ 000000000000000000000000000000000000000000000000000000000000000

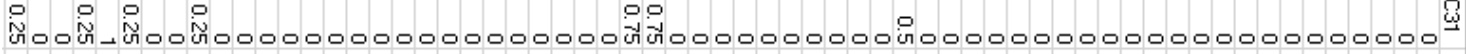
$000000000000000000000000000000000000000000000000000000000000000 \mathscr{W}^{3}$ $00000000000000000000000000000000000000000000000000000000000000 \mathscr{W}$ $00000000000000000000000000000000000000000000000000000000000000+$ $00000000000000000000000000000000000000000000000000000000000000+1$

\section{Table E - 21 Elderly Woman (P12) Adjacency Matrix (first half)}




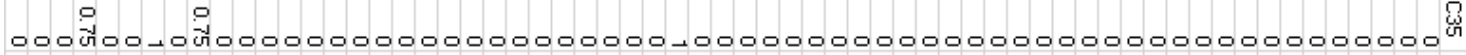
000000000000000000000000000000000000000000000000000000000000000

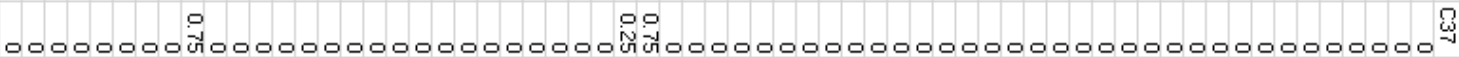

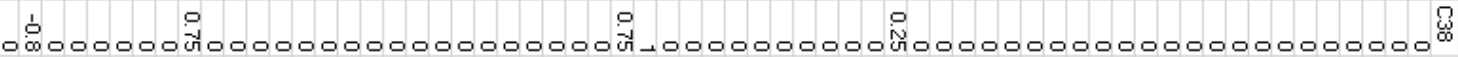
00000000000000000000000000000000000000000000000000000000000000 $\begin{array}{llll}1 & 1\end{array}$

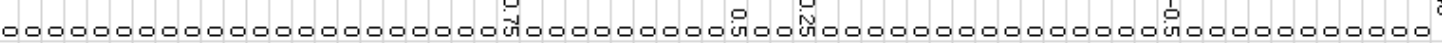

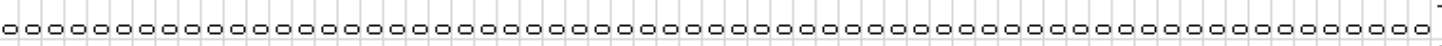
000000000000000000000000000000000000000000000000000000000000 代 (

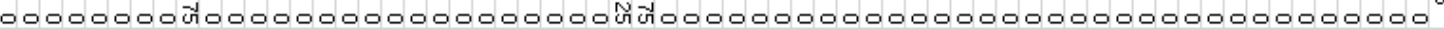
00000000000000000000000000000000000000000000000000000000000000 00000000000000000000000000000000000000000000000000000000000000

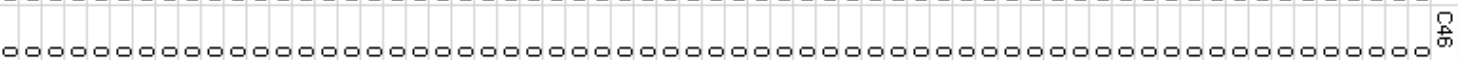

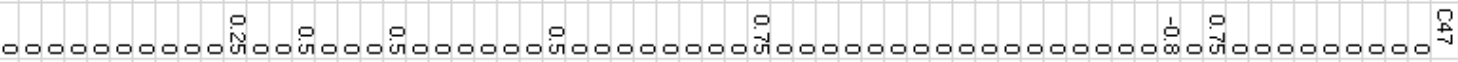
0 0000000000

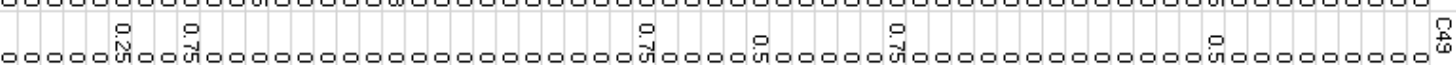

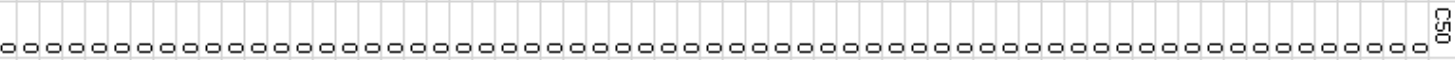
0000000000000000000000000000000000000000000000000000000000000000 0000000000000000000000000000000000000000000000000000000000 $00000000000000000000000000000000000000000000000000000000000000 N$

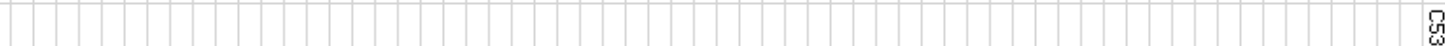
000000000000000000000000000000000000000000000000000000000000000 00000000000000000000000000000000000000000000000000000000 张 ०000000000000000000000000000000000000000000000000000000000000 0000000000000000000000000000000000000000000000000000000000000 $000000000000000000000000000000000000000000000000000000000000000-1$

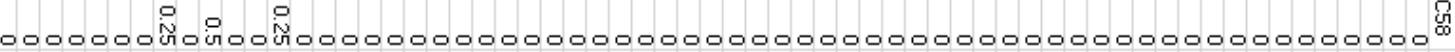

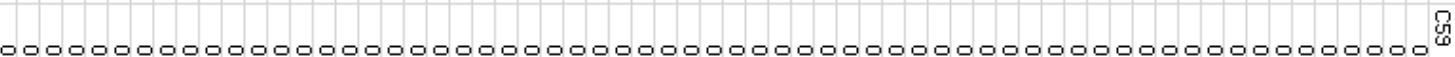
000000000000000000000000000000000000000000000000000000000000 ด

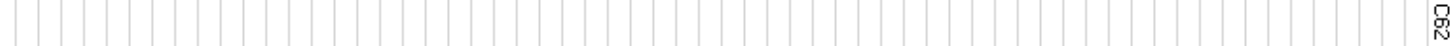
0000000000000000000000000000000000000000000000000000000000000 $000000000000000000000000000000000000000000000000000000000000000 \AA$

Table E - 22 Elderly Woman (P12) Adjacency Matrix (second half) 

$0000000000000000000000000000000000000000000000000000000000000 \tilde{n}^{0}$

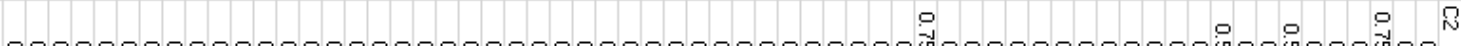
00000000000000000000000000000000000000000000000

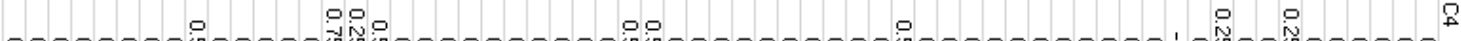

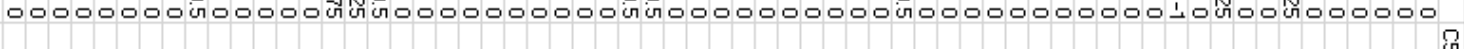
000000000000000000000000000000000000000000000000000000000000000

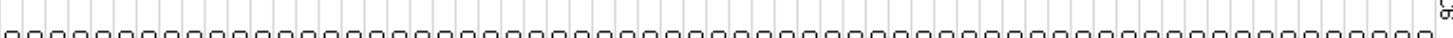
000000000000000000000000000000000000000000000000000000000000000

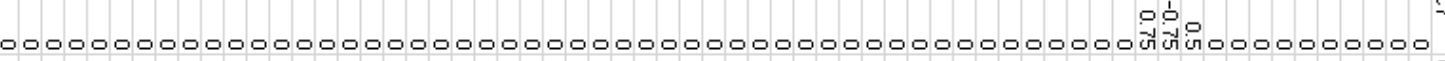

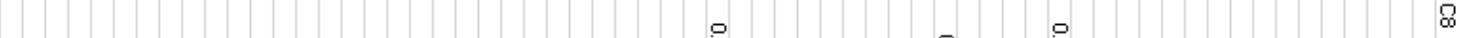

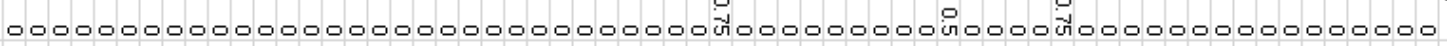

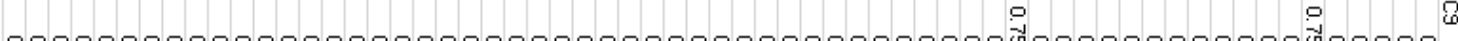
$00000000000000000000000000000000000000000000 \hat{1} 000000000000 \hat{1} 00000$

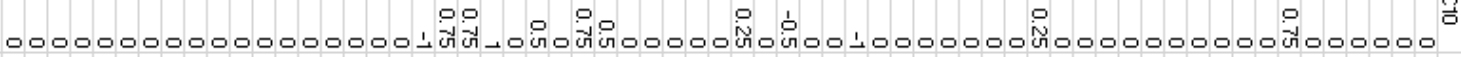

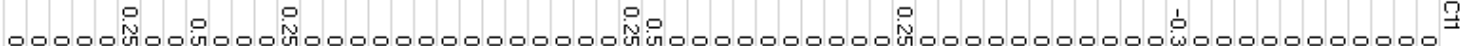
13 00000000000000000000000000000000000000000000000000000000000000012 $000000000000000000000000000000000000000000000000000000000000000 \frac{a}{\omega}$ (1) 0000000000000000000000000000000000000000000 iñ $000001000000 \hat{n} 0000$ $000000000000000000000000000000000000000000000000000000000000000 \frac{?}{\pi}$

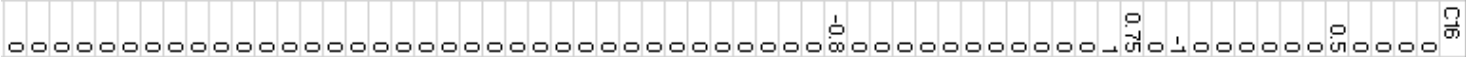

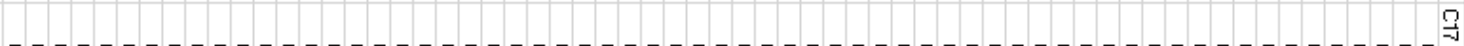
0000000000000000000000000000000000000000000000000000000000000000

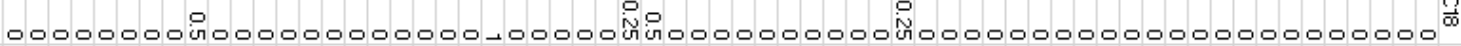
$000000000000000000000000000000000000000000000000000000000000000 \frac{?}{\omega}$

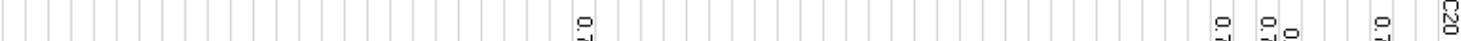

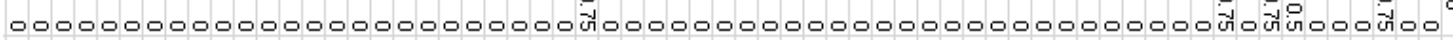
$00000000000000000000000000000000000000000000000000000000000000 \pm$ 0 0

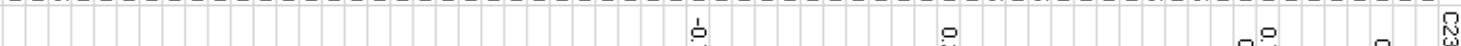

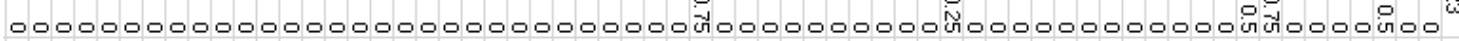
$00000000000000000000000000000000000000000000000000000000000000 \AA$ $00000000000000000000000000000000000000000000000000000000000000 \hat{N}$

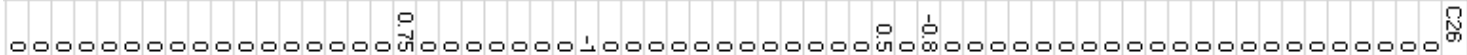
$00000000000000000000000000000000000000000000000000000000000000=1$ $00000000000000000000000000000000000000000000000000000000000000 \%$

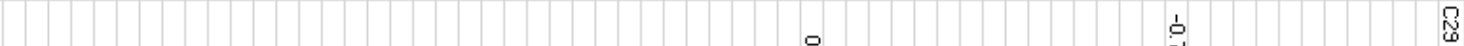
00000000000000000000000000000000000 iñ $00000000000000 \hat{1} 00000000000$ $000000000000000000000000000000000000000000000000000000000000000 \mathscr{\mathscr { S }}$

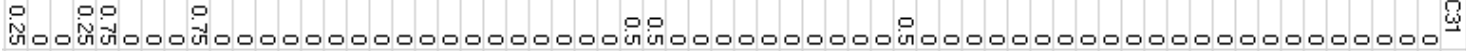
000000000000000000000000000000000000000000000000000000000000000 岕 $00000000000000000000000000000000000000000000000000000000000000 \$$

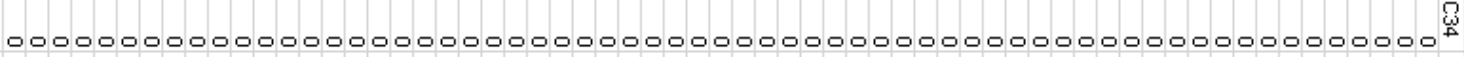

\section{Table E - 23 Elderly Woman (P13) Adjacency Matrix (first half)}




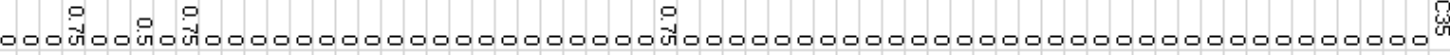
$000000000000000000000000000000000000000000000000000000000000000 \%$

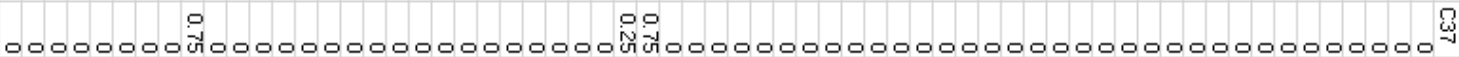
。 000000000000000000000000000000000000000000000000000000000000000 (2)

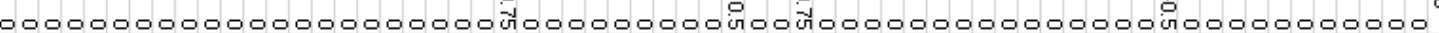

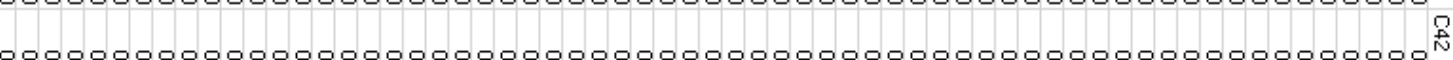

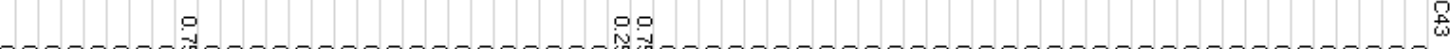
( 000000000000000000000000000000000000000000000000000000000000000 f 000000000000000000000000000000000000000000000000000000000000000 în 000000000000000000000000000000000000000000000000000000000000000 f

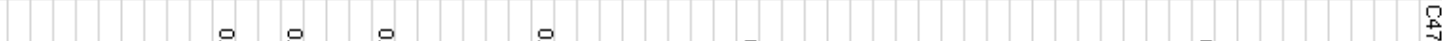

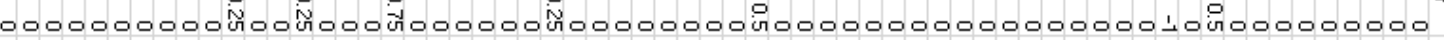

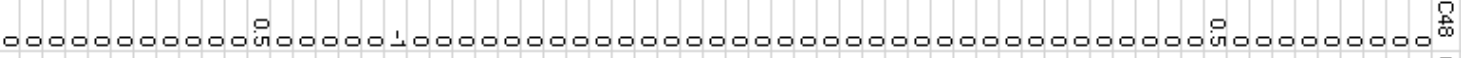

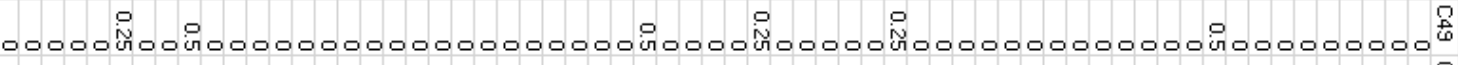

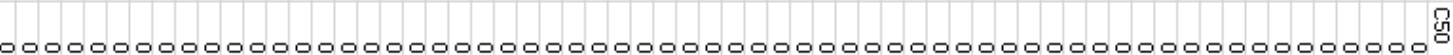
0000000000000000000000000000000000000000000000000000000000000009 9 $000000000000000000000000000000000000000000000000000000000000000^{0}$

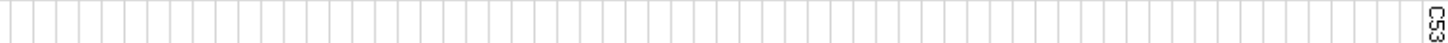
000000000000000000000000000000000000000000000000000000000000000 000000000000000000000000000000000000000000000000000000000000000 行 000000000000000000000000000000000000000000000000000000000000000 亿̆

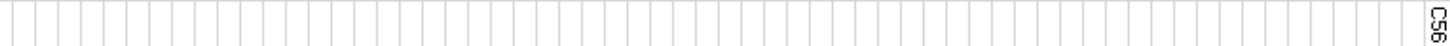
000000000000000000000000000000000000000000000000000000000000000 $000000000000000000000000000000000000000000000000000000000000000^{0}$

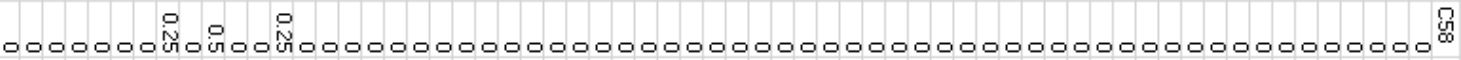
000000000000000000000000000000000000000000000000000000000000000 行

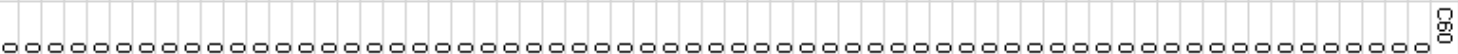
000000000000000000000000000000000000000000000000000000000000000 魚

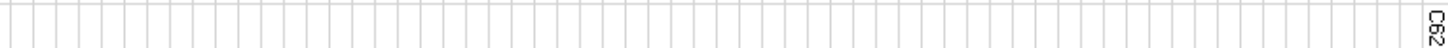
000000000000000000000000000000000000000000000000000000000000000 000000000000000000000000000000000000000000000000000000000000000

Table E - 24 Elderly Woman (P13) Adjacency Matrix (second half)

The elderly woman (P14) was no longer able to take part in the research and hence the data set is missing her input. 


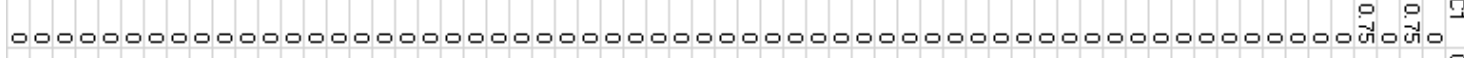
$0000000000000000000000000000000000000000 \frac{1}{0}$

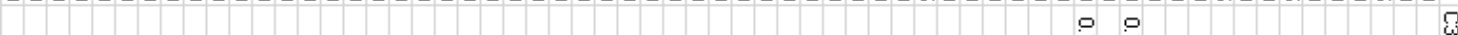

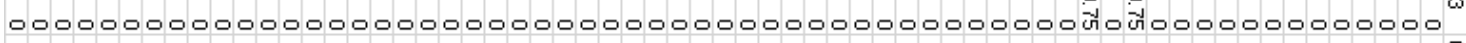

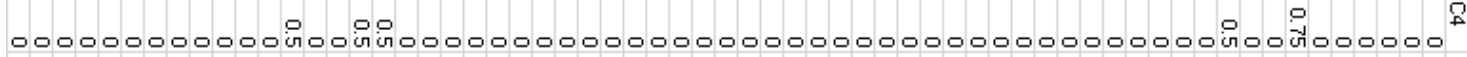
0000000000000000000000000000

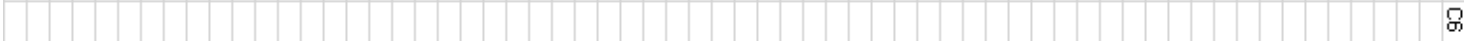
000000000000000000000000000000000000000000000000000000000000000 $00000000000000000000000000000000000000000000000000 \operatorname{lin}_{0}^{0} 0000000000$

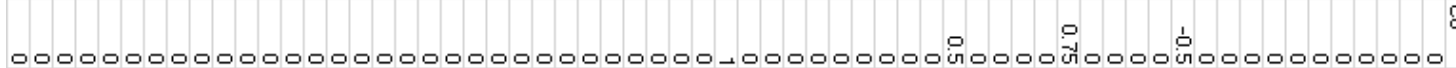
(

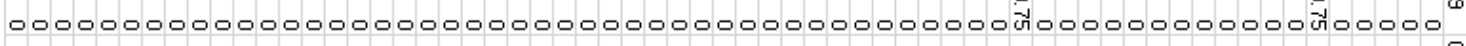

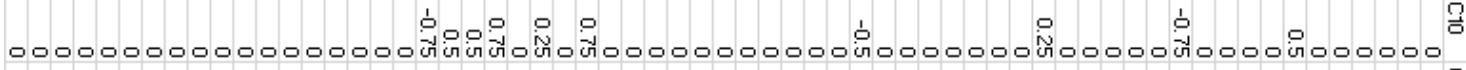

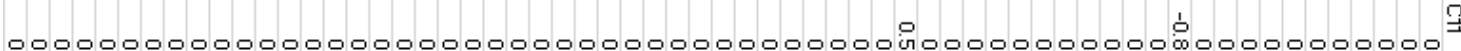

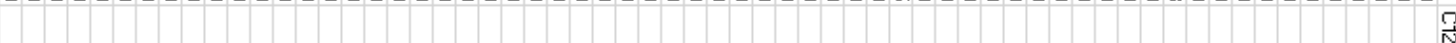
$000000000000000000000000000000000000000000000000000000000000000 \frac{1}{3}$ $000000000000000000000000000000000000000000000000000000000000000 \frac{3}{\omega}$

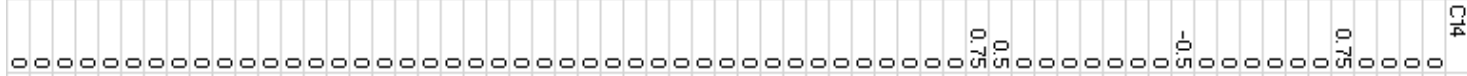
000000000000000000000000000000000000000000000000000000000000000

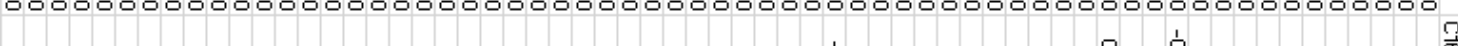

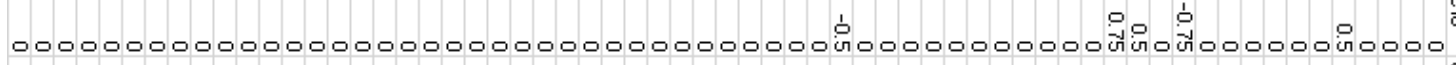
$000000000000000000000000000000000000000000000000000000000000000 \frac{7}{3}$

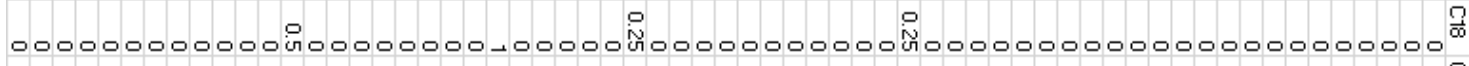
000000000000000000000000000000000000000000000000000000000000000

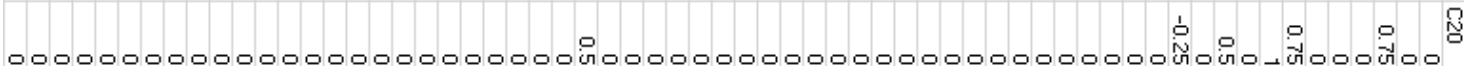

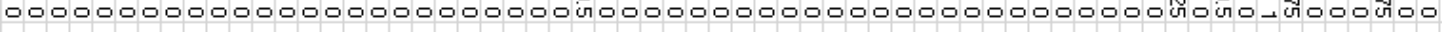
000000000000000000000000000000000000000000000000000000000000000 N

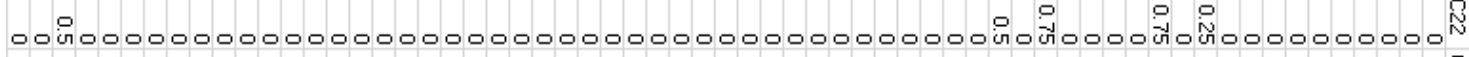

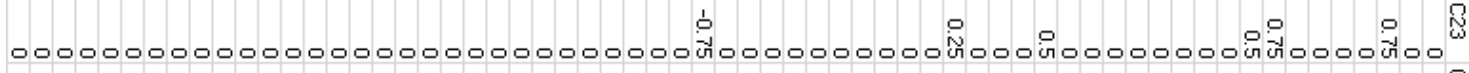
000000000000000000000000000000000000000000000000000000000000000+ 000000000000000000000000000000000000000000000000000000000000000000

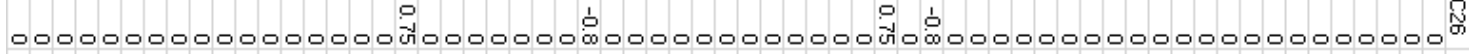

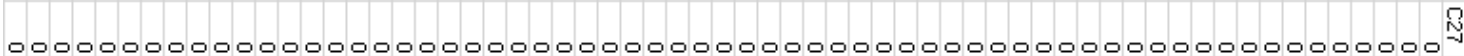
$00000000000000000000000000000000000000000000000000000000000000 \times$ 000000000000000000000000000000000000000000000000 $000000000000000000000000000000000000000000000000000000000000000 \dot{W}$

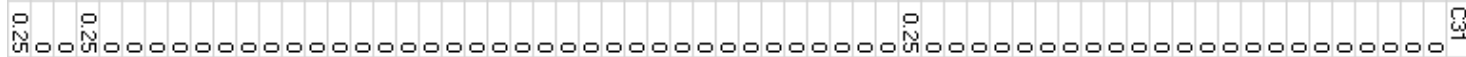

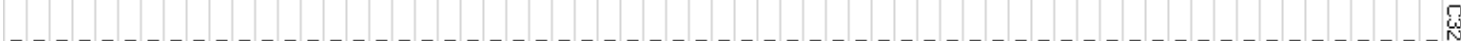
$000000000000000000000000000000000000000000000000000000000000000 \mathrm{~N}$ $00000000000000000000000000000000000000000000000000000000000000 \AA$ $000000000000000000000000000000000000000000000000000000000000000 \AA$

Table E - 25 Elderly Woman (P15) Adjacency Matrix (first half) 
๑ำ 000000000000000000000000000000000000000000000000000000000000 00000000000000000000000000000000000000000000000000000000000000

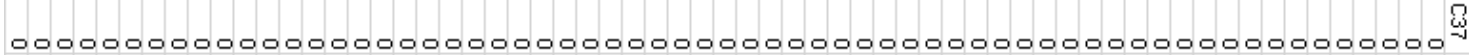

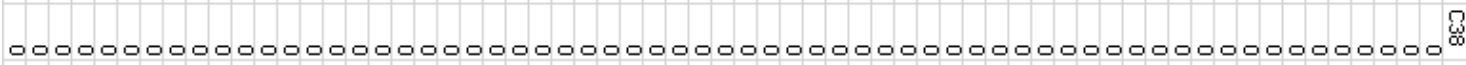

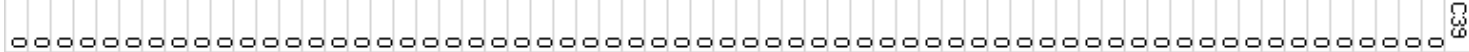

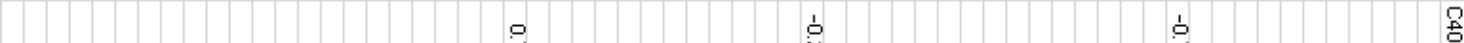

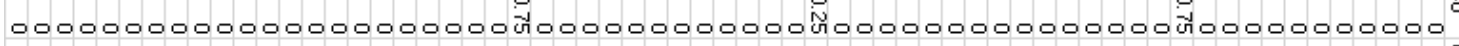
00000000000000000000000000000000000000000000000000000000

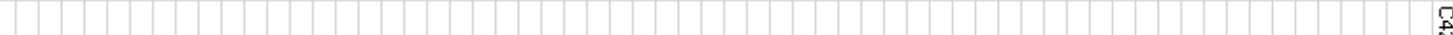
$000000000000000000000000000000000000000000000000000000000000000 \hat{N}$ 00000000000000000000000000000000000000000000000000000000000 000000000000000000000000000000000000000000000000000000000000 吉

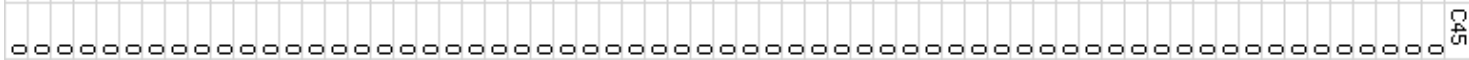

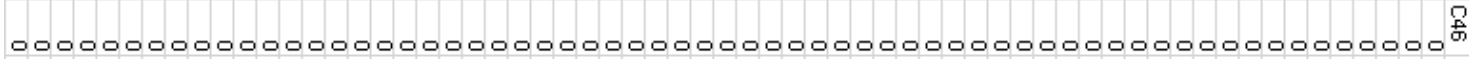
(2) D

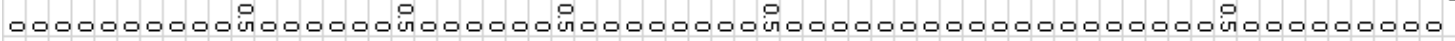

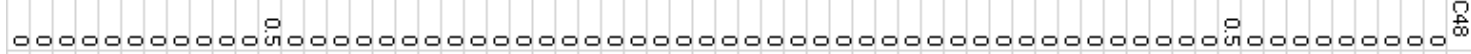
00000000000000000000000000000000 0000000000000000000000000000000000000000000000000000000000000 00000000000000000000000000000000000000000000000000000000 0000000000000000000000000000000000000000000000000000000000000 行

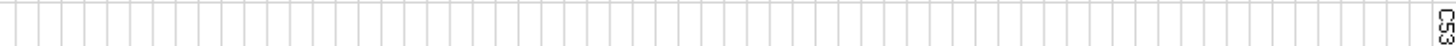
000000000000000000000000000000000000000000000000000000000000000 0000000000000000000000000000000000000000000000000000000000000 代 00000000000000000000000000000000000000000000000000000000000000 亿̂n

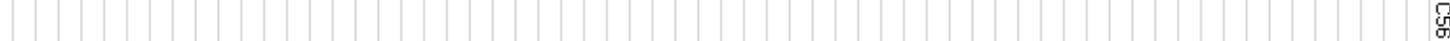
000000000000000000000000000000000000000000000000000000000000000 000000000000000000000000000000000000000000000000000000000000

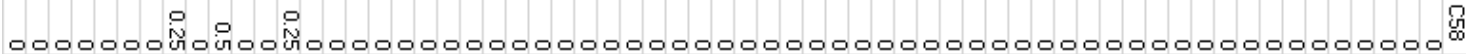
000000000000000000000000000000000000000000000000000000000000 0000000000000000000000000000000000000000000000000000000000000 总

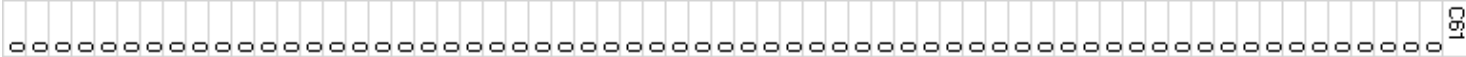

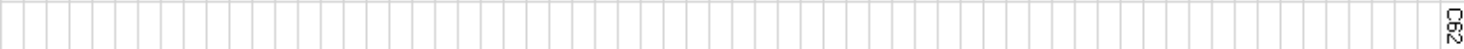
00000000000000000000000000000000000000000000000000000000000000 000000000000000000000000000000000000000000000000000000000000

Table E - 26 Elderly Woman (P15) Adjacency Matrix (second half) 


\section{E.III. Clustering FCM Algorithm (Aminpour, 2018)}

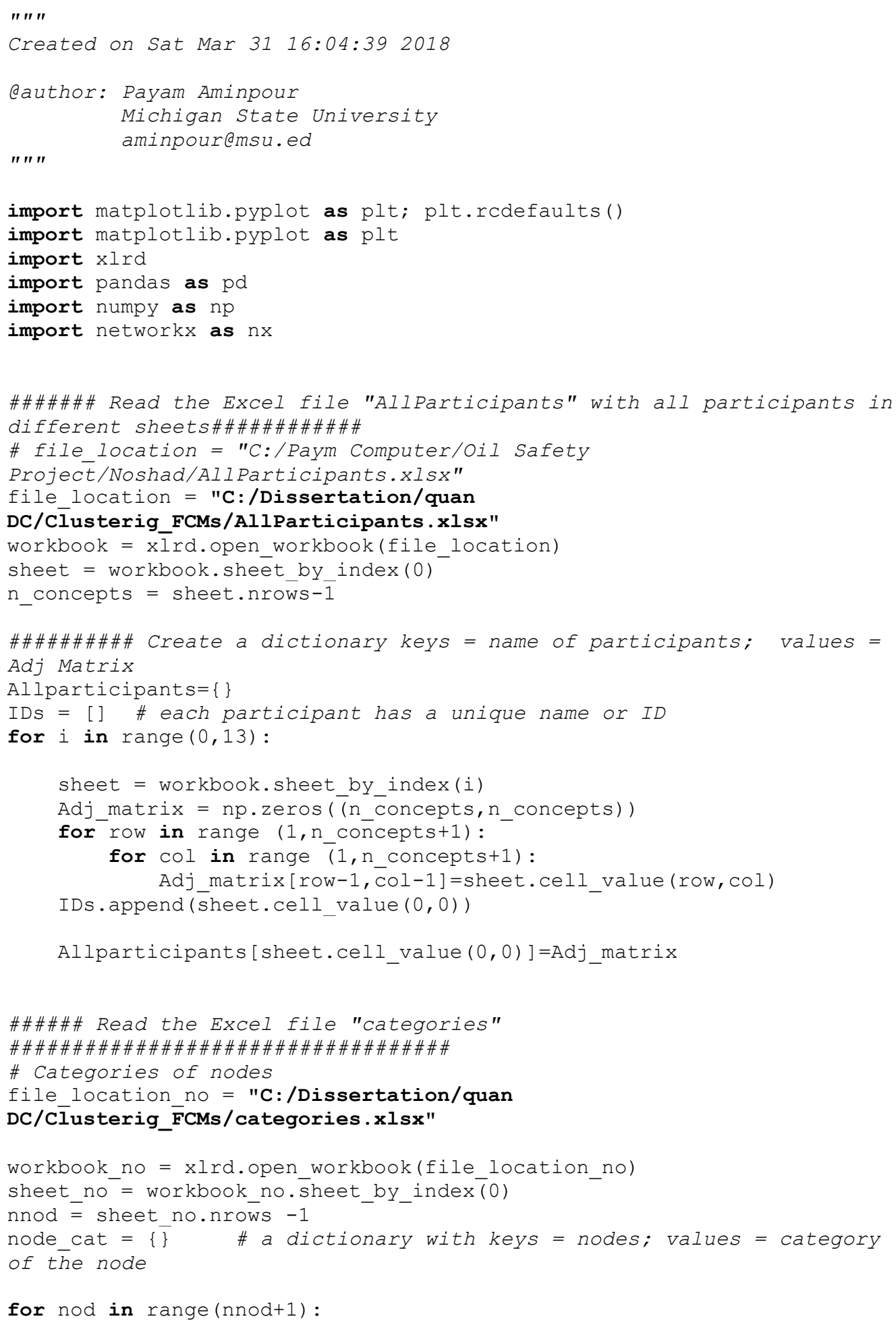




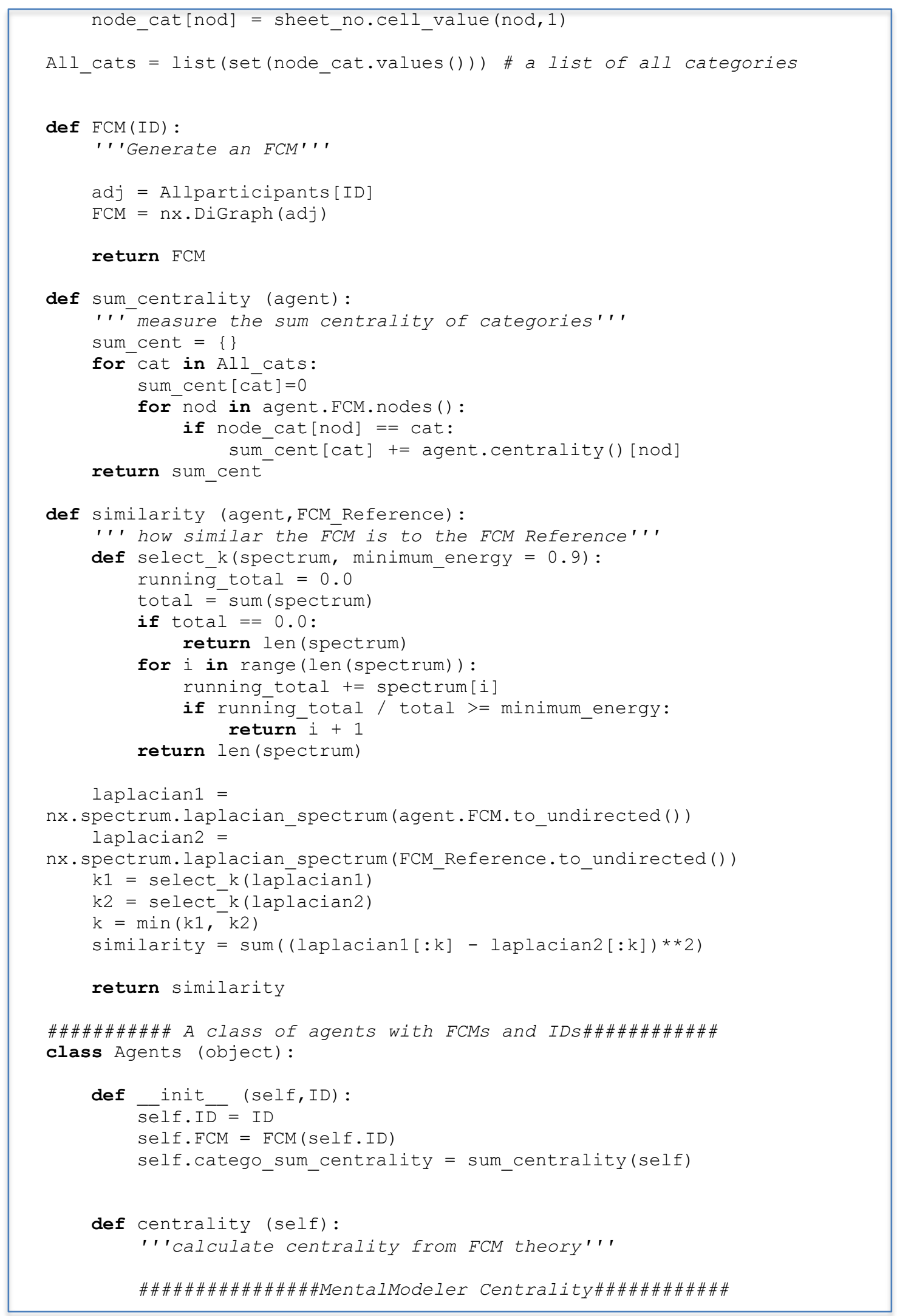




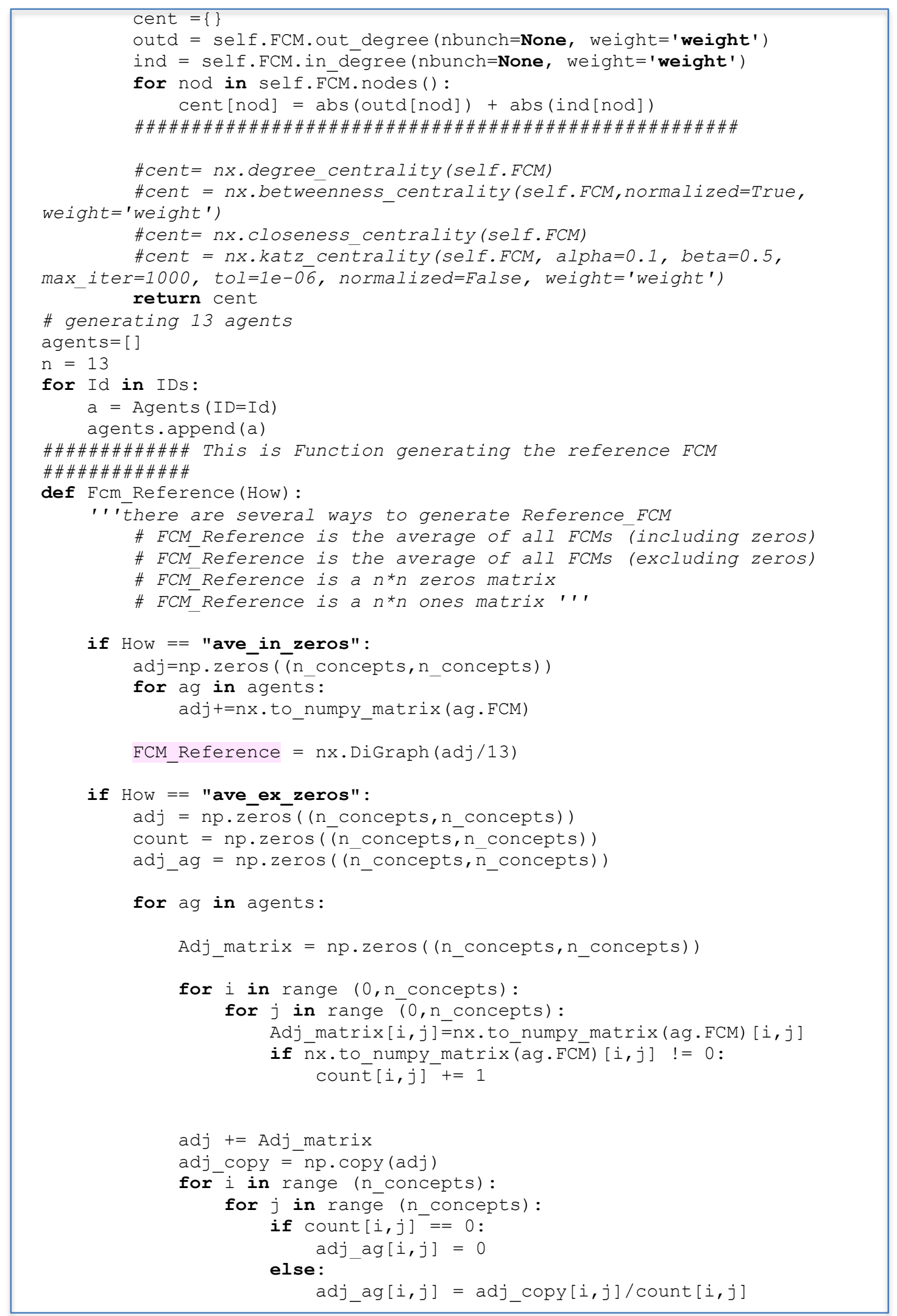




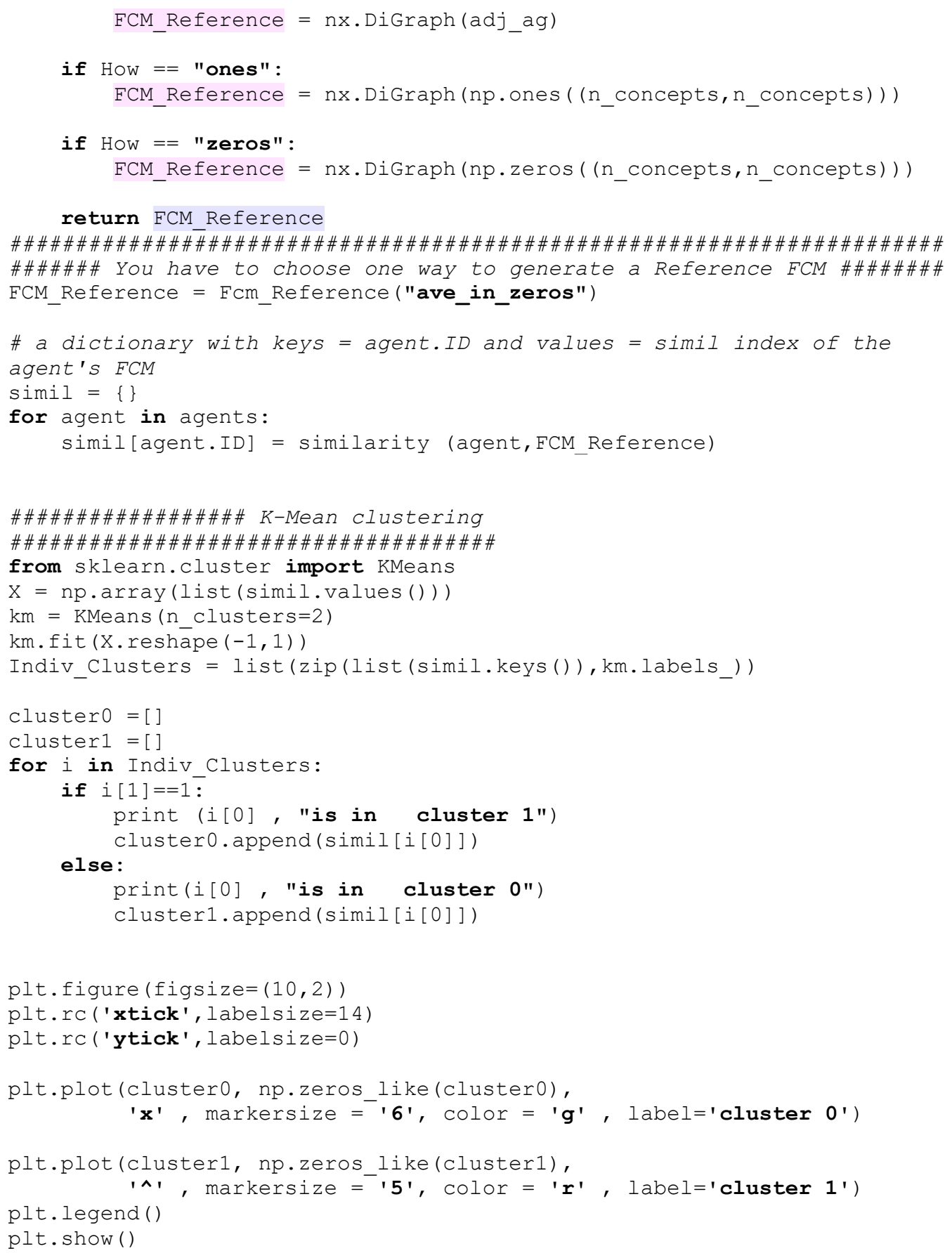




\section{E.IV. Total Population Average Adjacency Matrices}

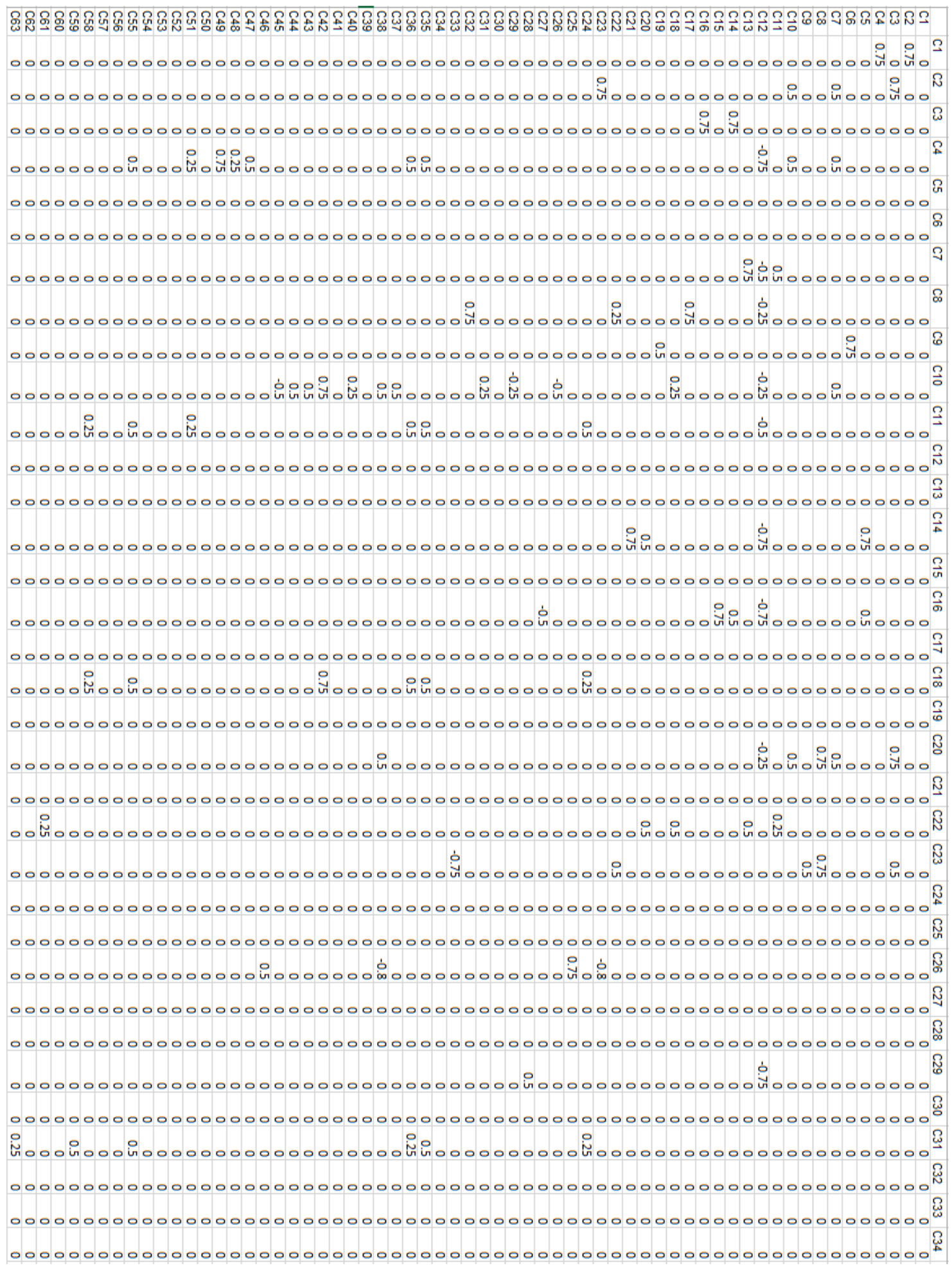

Table E - 27 Total population adjacency matrix (first half) 


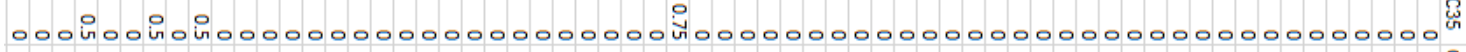
$000000000000000000000000000000000000000000000000000000000000000 \%$

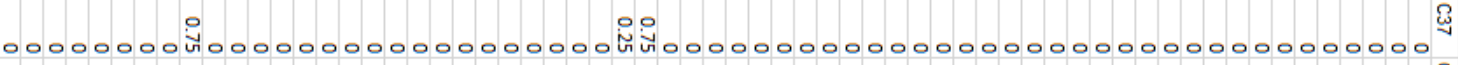

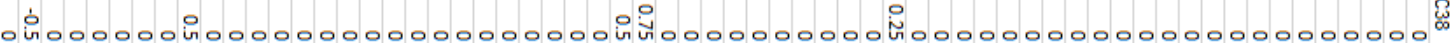
$000000000000000000000000000000000000000000000000000000000000000 \%$ 0000000000000000000000 ino00000000îno iñ 00000000000000 in 00000000000 f

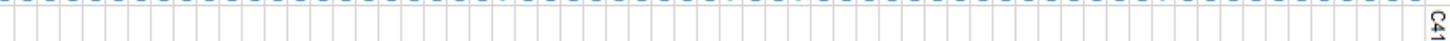
000000000000000000000000000000000000000000000000000000000000000 $000000000000000000000000000000000000000000000000000000000000000 \frac{\AA}{8}$

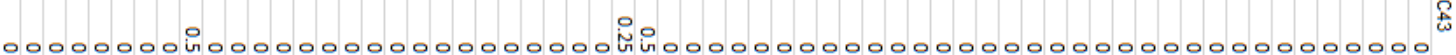
$00000000000000000000000000000000000000000000000000000000000000 \$$ $000000000000000000000000000000000000000000000000000000000000000 \hat{f}$ $00000000000000000000000000000000000000000000000000000000000000 \AA$

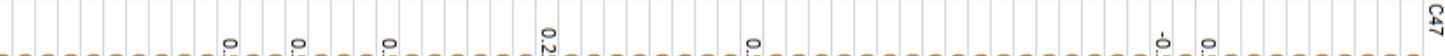
0000000000ino0ino00ino00000îno0000000ino0000000000000000inoino00000000 00000000000 iño000i்0000000000000000000000000000000000in000000000 (0) $000000000000000000000000000000000000000000000000000000000000000 \%$ 0000000000000000000000000000000000000000000000000000000000000000 000000000000000000000000000000000000000000000000000000000000000 向 000000000000000000000000000000000000000000000000000000000000000 至

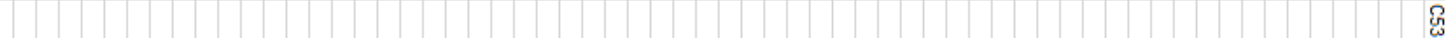
000000000000000000000000000000000000000000000000000000000000000 $000000000000000000000000000000000000000000000000000000000000000 \$$ 00000000000000000000000000000000000000000000000000000000000000 乩

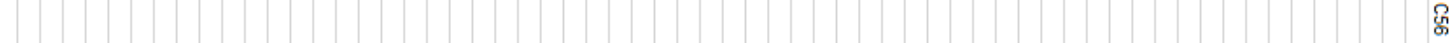
000000000000000000000000000000000000000000000000000000000000000 $000000000000000000000000000000000000000000000000000000000000000 \hat{~}$ 0000000 iñoino 0 iño000000000000000000000000000000000000000000000000 $000000000000000000000000000000000000000000000000000000000000000 \emptyset$ 0000000000000000000000000000000000000000000000000000000000000000 00000000000000000000000000000000000000000000000000000000000000

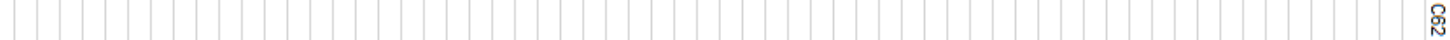
000000000000000000000000000000000000000000000000000000000000000 $000000000000000000000000000000000000000000000000000000000000000 \AA$

\section{Table E - 28 Total population adjacency matrix (second half)}




\section{E.V. Adjacency Matrix of FCMconnected}

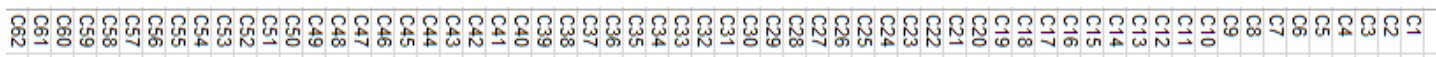
0000000000000000000000000000000000000000000000000000000000 V̆ $_{0} 0^{3}$

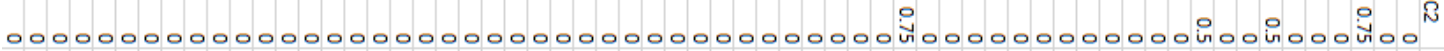
0000000000000000000000000000000000000000000000 ĭ 0 ज̃ 0000000000000 W

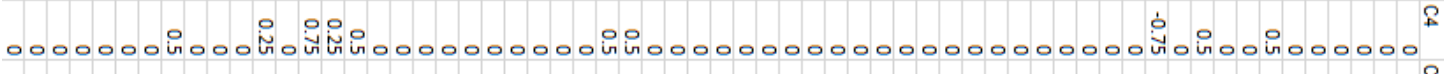
00000000000000000000000000000000000000000000000000000000000000 in S 000000000000000000000000000000000000000000000000000000000000009 0000000000000000000000000000000000000000000000000 iñ

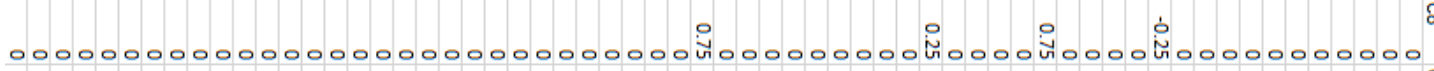
0000000000000000000000000000000000000000000 in 000000000000 Ŭ̉00000

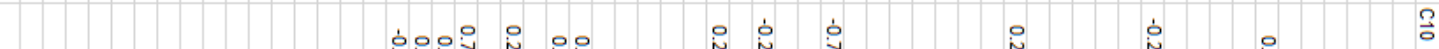

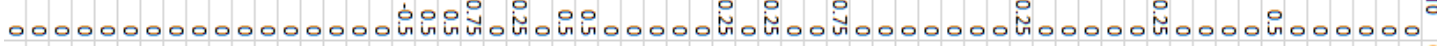

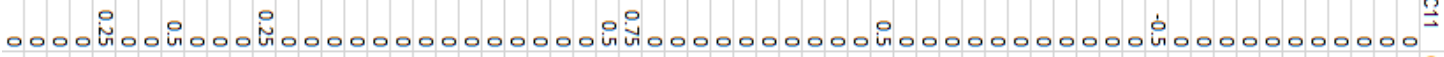
$00000000000000000000000000000000000000000000000000000000000000 \frac{\frac{3}{N}}{3}$ $00000000000000000000000000000000000000000000000000000000000000 \frac{?}{\omega}$

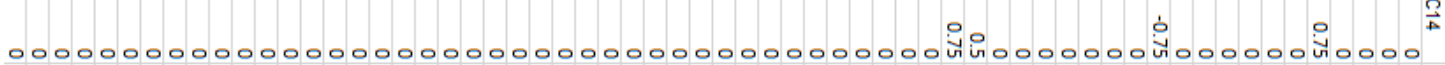
$00000000000000000000000000000000000000000000000000000000000000 \frac{?}{m}$ 垈

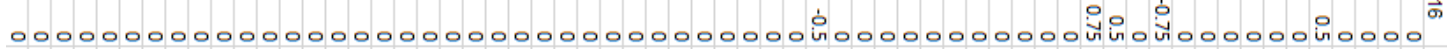
$00000000000000000000000000000000000000000000000000000000000000 \bar{\exists}$ 0000 $00000000000000000000000000000000000000000000000000000000000000 \frac{\vec{\omega}}{}$

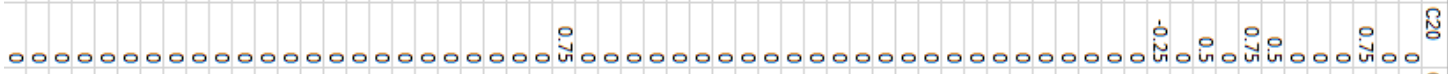
$00000000000000000000000000000000000000000000000000000000000000 \frac{N}{3}$

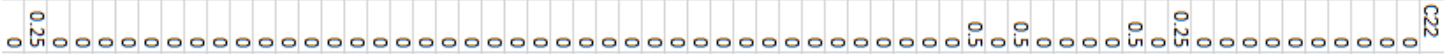

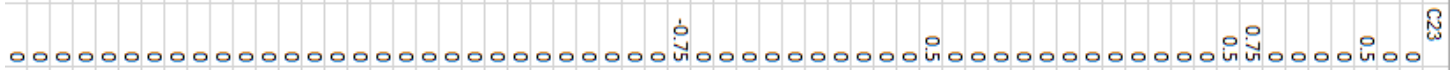
$00000000000000000000000000000000000000000000000000000000000000 \frac{N}{N}$ $00000000000000000000000000000000000000000000000000000000000000 \hat{n}$

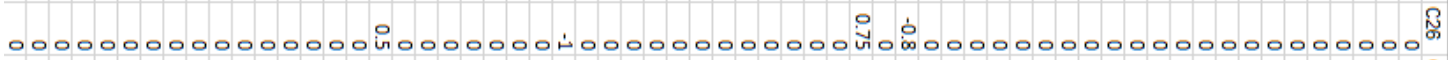
$00000000000000000000000000000000000000000000000000000000000000 \mathbb{N}^{3}$ $00000000000000000000000000000000000000000000000000000000000000 \%$

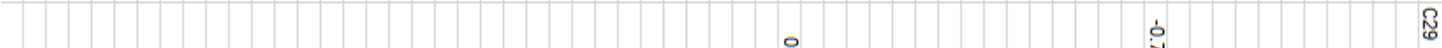
0000000000000000000000000000000000 iñ 00000000000000 जั00000000000 $00000000000000000000000000000000000000000000000000000000000000 \%$

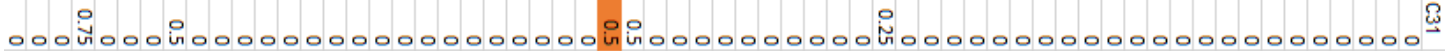
$00000000000000000000000000000000000000000000000000000000000000 \mathbb{N}^{2}$

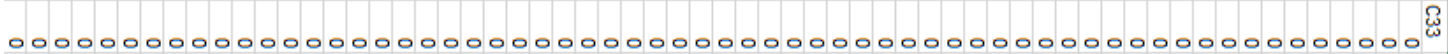
$00000000000000000000000000000000000000000000000000000000000000 \$$

\section{Table E - 29 FCMConnected's adjacency matrix (first half)}




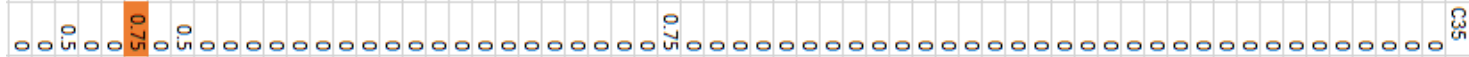
$00000000000000000000000000000000000000000000000000000000000000 \%$ 00000000000000000000000000000000000000000000000000000000000000

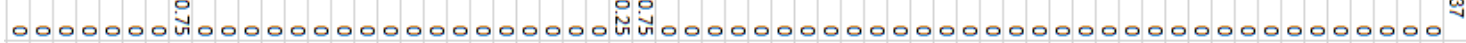

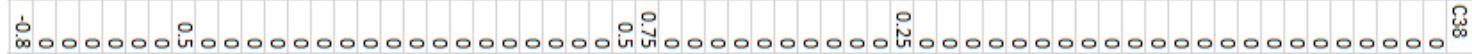
00000000000000000000000000000000000000000000000000000000000000 [ 年 000000000000000000000 in 000000000 Ninon 000000000000000 in 00000000000 00000000000000000000000000000000000000000000000000000000000000 $00000000000000000000000000000000000000000000000000000000000000 \frac{8}{1}$ 0000000 ज்̃ 00000000000000000 iñ

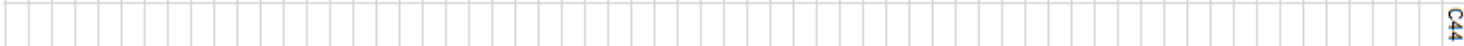
00000000000000000000000000000000000000000000000000000000000000 00000000000000000000000000000000000000000000000000000000000000 解 00000000000000000000000000000000000000000000000000000000000000 f 00000000000000000000000000000000000000000000000000000000000000

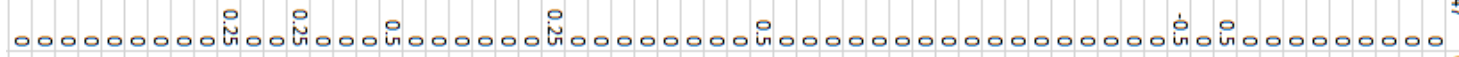

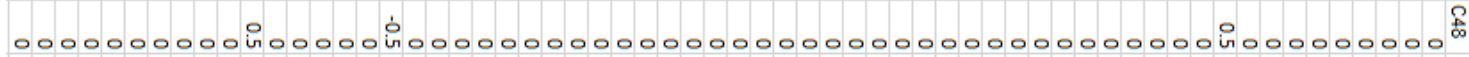
1.

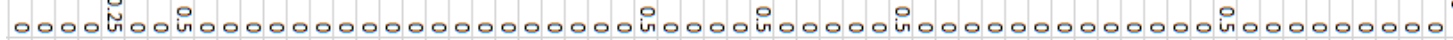
00000000000000000000000000000000000000000000000000000000000000 每 $00000000000000000000000000000000000000000000000000000000000000 \frac{\pi}{3}$ 00000000000000000000000000000000000000000000000000000000000000 负

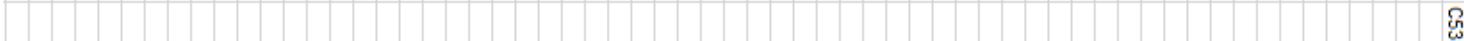
00000000000000000000000000000000000000000000000000000000000000 $00000000000000000000000000000000000000000000000000000000000000 \AA$ 00000000000000000000000000000000000000000000000000000000000000 无

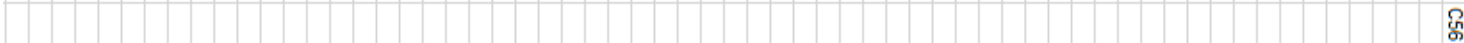
00000000000000000000000000000000000000000000000000000000000000 00000000000000000000000000000000000000000000000000000000000000 f 000000 $000000 \mathrm{k} 0 \mathrm{k} 00 \mathrm{k} 0000000000000000000000000000000000000000000000000$ 00000000000000000000000000000000000000000000000000000000000 000000000000000000000000000000000000000000000000000000000000008 $0000000000000000000000000000000000000000000000000000000000000 \AA$

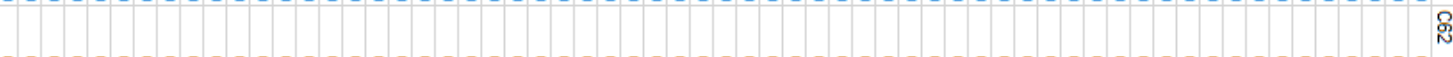

Table E - 30 FCM Connected's adjacency matrix (second half) 


\section{E.VI. Adjacency Matrix of FCM Isolated}

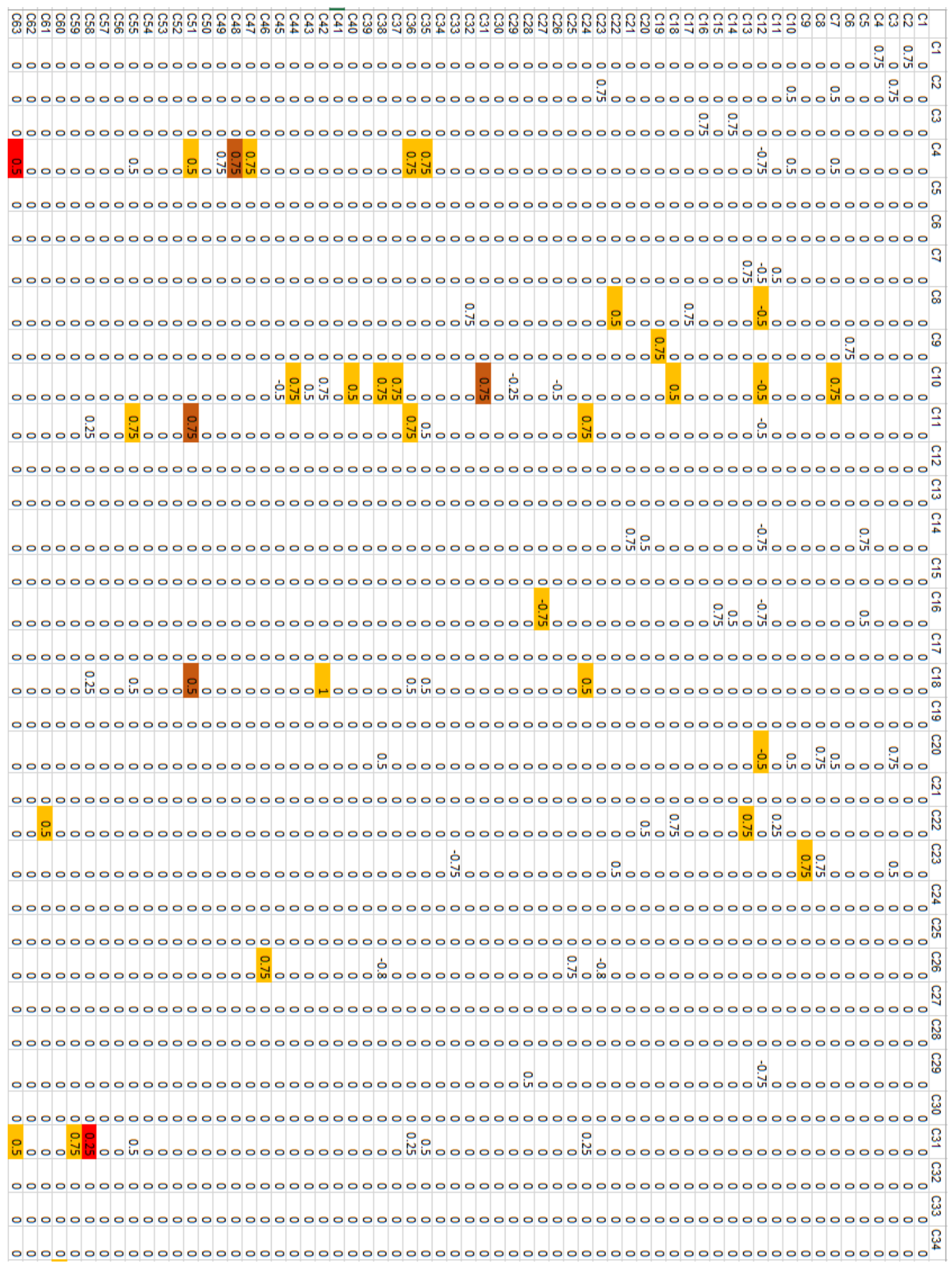

Table E - 30 FCM Isolated's adjacency matrix (first half) 


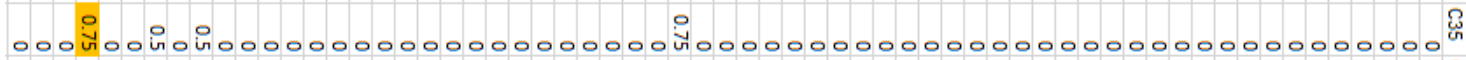
$000000000000000000000000000000000000000000000000000000000000000 \%$

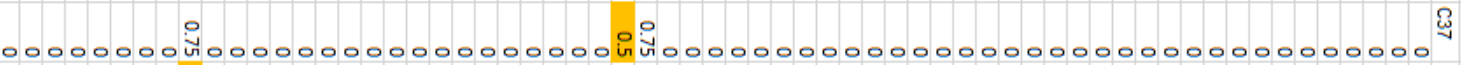

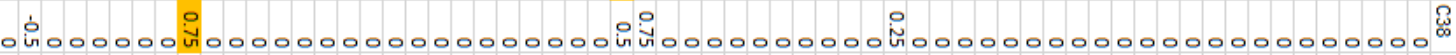
000000000000000000000000000000000000000000000000000000000000000\%

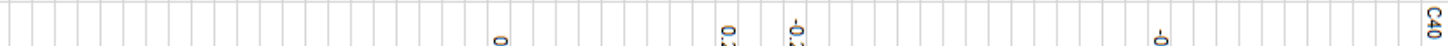
0000000000000000000000 ino 00000000 V̆́00

000000000000000000000000000000000000000000000000000000000000000 A $000000000000000000000000000000000000000000000000000000000000000 \frac{f}{k}$ 每 00000000 ที่000000000000000000 ที่ $000000000000000000000000000000000000000000000000000000000000000 \$$ $000000000000000000000000000000000000000000000000000000000000000 \frac{f}{n}$ $000000000000000000000000000000000000000000000000000000000000000 \AA$ f

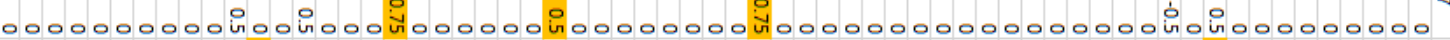

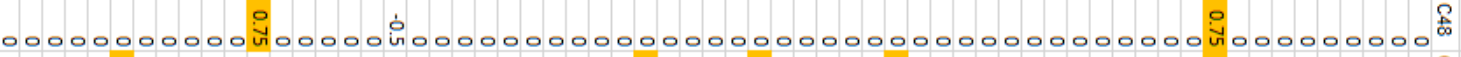

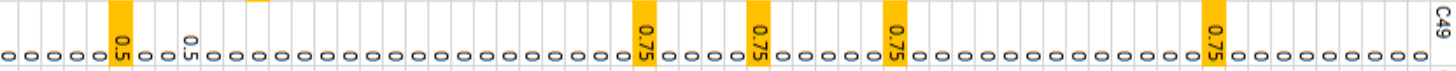
000000000000000000000000000000000000000000000000000000000000000 in $000000000000000000000000000000000000000000000000000000000000000^{\text {Th }}$ 000000000000000000000000000000000000000000000000000000000000000 赑

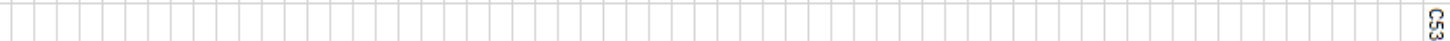
000000000000000000000000000000000000000000000000000000000000000 $000000000000000000000000000000000000000000000000000000000000000 \AA$ 000000000000000000000000000000000000000000000000000000000000000 fin | 000000000000000000000000000000000000000000000000000000000000000 000000000000000000000000000000000000000000000000000000000000000 f 0000000 000000000000000000000000000000000000000000000000000000000000000 ⿸尸匕 0000000000000000000000000000000000000000000000000000000000000008 $00000000000000000000000000000000000000000000000000000000000000 \frac{8}{3}$

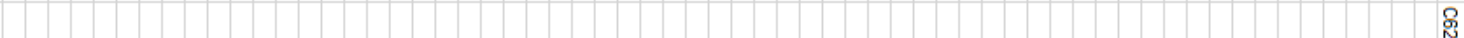
000000000000000000000000000000000000000000000000000000000000000 $000000000000000000000000000000000000000000000000000000000000000 \AA$

Table E - 31 FCM Isolated's adjacency matrix (second half) 
Appendix F - Quantitative (quan) Analysis Supporting Material

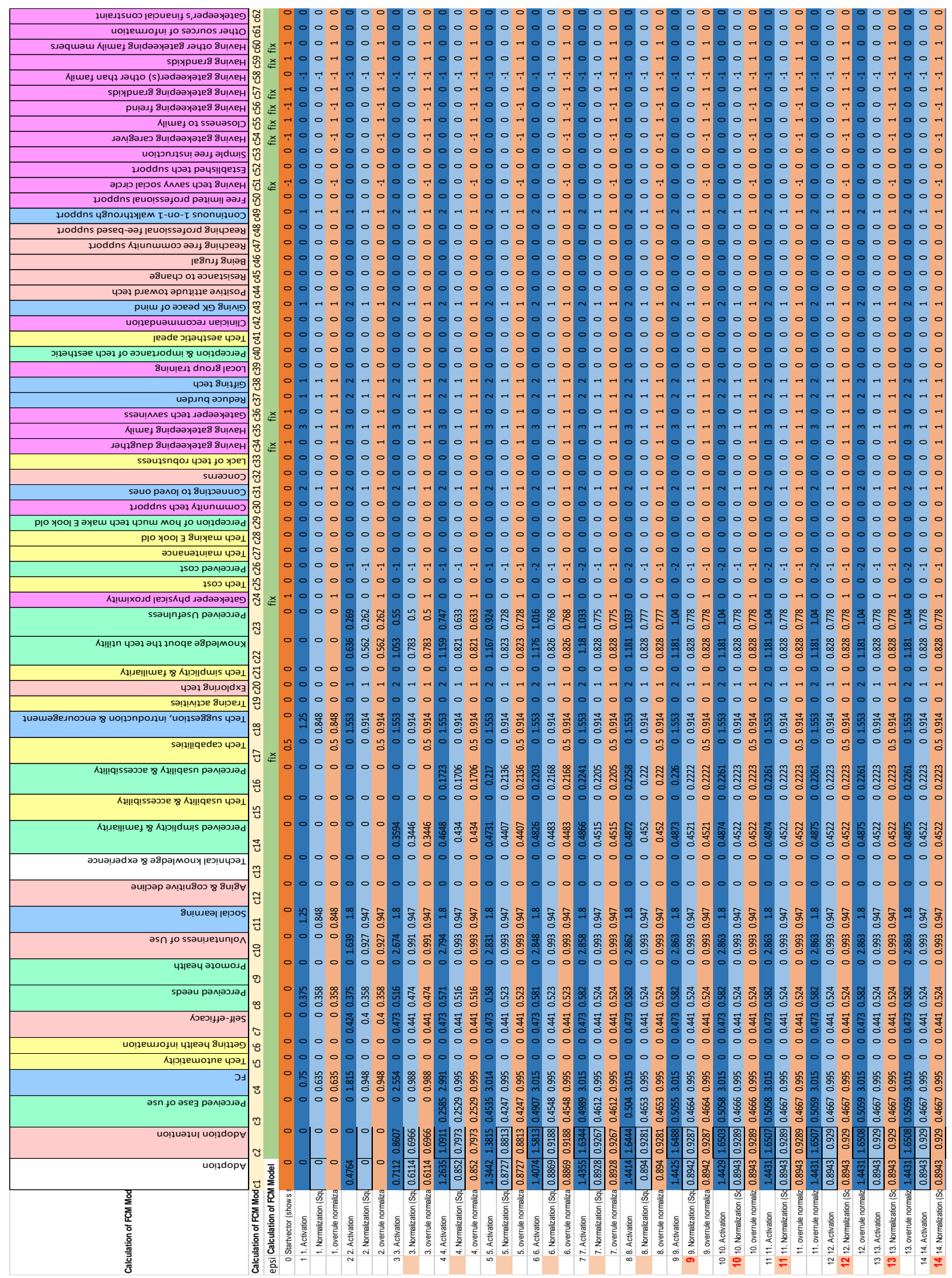

Figure F - 1 Typical FCM Simulation using Excel 
Appendix G - Results

\section{Common Ontology (Codebook)}

\begin{tabular}{|c|c|c|c|}
\hline \# & C\# & Concept Name & Concept Description \\
\hline \multicolumn{4}{|c|}{ Technology Characteristics Concepts } \\
\hline 1 & C6 & $\begin{array}{l}\text { Getting health } \\
\text { information }\end{array}$ & $\begin{array}{l}\text { The degree in which the technology provides useful health } \\
\text { information }\end{array}$ \\
\hline 2 & C33 & $\begin{array}{l}\text { Lack of tech } \\
\text { robustness }\end{array}$ & $\begin{array}{l}\text { The degree in which the technology fail to operate as it's } \\
\text { expected }\end{array}$ \\
\hline 3 & C41 & $\begin{array}{l}\text { Tech aesthetic } \\
\text { appeal }\end{array}$ & $\begin{array}{l}\text { General tech aesthetic appeal, from its physical look appeal } \\
\text { to the feeling of being young and up to date with technology }\end{array}$ \\
\hline 4 & C5 & $\begin{array}{l}\text { Tech } \\
\text { automaticity }\end{array}$ & $\begin{array}{l}\text { The degree in which the technology is automatic and } \\
\text { doesn't require human intervention to operate (pressing } \\
\text { button, etc.) }\end{array}$ \\
\hline 5 & C17 & Tech capabilities & $\begin{array}{l}\text { Tech features that provide safety (like fall detection), } \\
\text { independence \& needed health information }\end{array}$ \\
\hline 6 & $\mathrm{C} 25$ & Tech cost & The out of pocket price of the technology \\
\hline 7 & $\mathrm{C} 27$ & $\begin{array}{l}\text { Tech } \\
\text { maintenance }\end{array}$ & $\begin{array}{l}\text { The accumulative efforts to keep the technology up and } \\
\text { running (the frequency of charging, whether its waterproof } \\
\text { or needs to be taken off and on) }\end{array}$ \\
\hline 8 & $\mathrm{C} 28$ & $\begin{array}{l}\text { Tech making E } \\
\text { look old }\end{array}$ & $\begin{array}{l}\text { The degree in which wearing the technology make the } \\
\text { elderly look old }\end{array}$ \\
\hline 9 & C21 & $\begin{array}{l}\text { Tech simplicity \& } \\
\text { familiarity }\end{array}$ & $\begin{array}{l}\text { How simple it is to operate the technology and how familiar } \\
\text { it looks }\end{array}$ \\
\hline 10 & C15 & $\begin{array}{l}\text { Tech usability \& } \\
\text { accessibility }\end{array}$ & $\begin{array}{l}\text { The degree in which the technology is designed } \\
\text { ergonomically suitable so it can be easily used by elderly } \\
\text { with cognitive decline and special needs }\end{array}$ \\
\hline 11 & C19 & Tracing activities & Tech ability to track activities (e.g. distance, elevation, etc.) \\
\hline \multicolumn{4}{|c|}{ Elderly Personal traits, views and intention Concepts } \\
\hline 12 & $\mathrm{C} 2$ & $\begin{array}{l}\text { Adoption } \\
\text { Intention }\end{array}$ & Elderly's intention to adopt the technology \\
\hline 13 & C12 & $\begin{array}{l}\text { Aging \& cognitive } \\
\text { decline }\end{array}$ & $\begin{array}{l}\text { The physical \& emotional effects of aging (e.g. cognitive } \\
\text { decline) that influence elderly's technology usage }\end{array}$ \\
\hline 14 & C46 & Being frugal & $\begin{array}{l}\text { The degree in which the elderly is economical with regards } \\
\text { to purchasing technology or paying for technical support }\end{array}$ \\
\hline 15 & C32 & Concerns & $\begin{array}{l}\text { Elderly concerns and needs that can be alleviated using the } \\
\text { technology under study. Such as independency, and health } \\
\text { \& safety concerns. }\end{array}$ \\
\hline 16 & $\mathrm{C} 20$ & Exploring tech & $\begin{array}{l}\text { Elderly gradually trying, exploring and domesticating the } \\
\text { technology }\end{array}$ \\
\hline
\end{tabular}




\begin{tabular}{|c|c|c|c|}
\hline 17 & C44 & $\begin{array}{l}\text { Positive attitude } \\
\text { toward tech }\end{array}$ & $\begin{array}{l}\text { Accumulative effects of all the personal perspectives, } \\
\text { opinion and motivation that positively affect elderly } \\
\text { women's Voluntariness of tech use }\end{array}$ \\
\hline 18 & $\mathrm{C} 47$ & $\begin{array}{l}\text { Reaching free } \\
\text { community } \\
\text { support }\end{array}$ & $\begin{array}{l}\text { The degree in which free community support is available to } \\
\text { elderly and she reaches for it }\end{array}$ \\
\hline 19 & $\mathrm{C} 48$ & $\begin{array}{l}\text { Reaching } \\
\text { professional fee- } \\
\text { based support }\end{array}$ & $\begin{array}{l}\text { The degree in which fee-based professional support is } \\
\text { available to elderly and she reaches for it }\end{array}$ \\
\hline 20 & C45 & $\begin{array}{l}\text { Resistance to } \\
\text { change }\end{array}$ & $\begin{array}{l}\text { Accumulative effects of all the personal perspectives, } \\
\text { opinion and motivation that causes elderly resistance to } \\
\text { change her current habits or start a new one to use } \\
\text { technology }\end{array}$ \\
\hline 21 & $\mathrm{C} 7$ & Self-efficacy & $\begin{array}{l}\text { Elderly's ability and confidence in successfully using the } \\
\text { technology }\end{array}$ \\
\hline 22 & C10 & $\begin{array}{l}\text { Voluntariness of } \\
\text { Use }\end{array}$ & Elderly's inherent willingness to use the technology \\
\hline \multicolumn{4}{|c|}{ Elderly Perceptional Concepts } \\
\hline 23 & $\mathrm{C} 22$ & $\begin{array}{l}\text { Knowledge about } \\
\text { the tech utility }\end{array}$ & Elderly's product awareness \\
\hline 24 & $\mathrm{C} 26$ & Perceived cost & $\begin{array}{l}\text { Elderly's perception of the relative cost vs. benefit of the } \\
\text { technology }\end{array}$ \\
\hline 25 & C3 & $\begin{array}{l}\text { Perceived Ease of } \\
\text { use }\end{array}$ & Elderly's perception of how easy it is to use the technology \\
\hline 26 & $\mathrm{C} 8$ & Perceived needs & $\begin{array}{l}\text { Perceived needs that are not delivered w existing familiar } \\
\text { products }\end{array}$ \\
\hline 27 & C14 & $\begin{array}{l}\text { Perceived } \\
\text { simplicity \& } \\
\text { familiarity }\end{array}$ & $\begin{array}{l}\text { Elderly's perception of how simple and familiar the } \\
\text { technology is (e.g. the number of steps taken to use the tech } \\
\text { and how familiar the tech is) }\end{array}$ \\
\hline 28 & C16 & $\begin{array}{l}\text { Perceived } \\
\text { usability \& } \\
\text { accessibility }\end{array}$ & $\begin{array}{l}\text { Elderly's perception of how ergonomically friendly the } \\
\text { technology is (e.g. it's comfortable to wear, see information } \\
\text { and use). This is both in general and in particular if elderly } \\
\text { has a disability (Tremor, low vision, etc.) }\end{array}$ \\
\hline 29 & $\mathrm{C} 23$ & $\begin{array}{l}\text { Perceived } \\
\text { Usefulness }\end{array}$ & Elderly's perception of how useful the technology is \\
\hline 30 & $\mathrm{C} 40$ & $\begin{array}{l}\text { Perception \& } \\
\text { importance of } \\
\text { tech aesthetic } \\
\end{array}$ & $\begin{array}{l}\text { The perception of how aesthetically appealing the tech is } \\
\text { and how important that look is to the E (including look, cool } \\
\text { factor and pride to look tech savvy) }\end{array}$ \\
\hline 31 & $\mathrm{C} 29$ & $\begin{array}{l}\text { Perception of } \\
\text { how much tech } \\
\text { make E look old }\end{array}$ & $\begin{array}{l}\text { Perception of how much tech make E look old as if she } \\
\text { would need assistive device (i.e. walker effect) }\end{array}$ \\
\hline 32 & C9 & Promote health & $\begin{array}{l}\text { The degree in which the technology provides health benefits } \\
\text { in ways unavailable or hard otherwise }\end{array}$ \\
\hline & \multicolumn{3}{|c|}{ Environmental Concepts } \\
\hline
\end{tabular}




\begin{tabular}{|c|c|c|c|}
\hline 33 & C50 & $\begin{array}{l}\text { Free limited } \\
\text { professional } \\
\text { support }\end{array}$ & $\begin{array}{l}\text { The limited free support given by store staff to promote } \\
\text { their sale. Like Apple Genius diagnosis service or call center, } \\
\text { or the company store(Apple) or other store (e.g. REI) }\end{array}$ \\
\hline 34 & C51 & $\begin{array}{l}\text { Having tech } \\
\text { savvy social circle }\end{array}$ & $\begin{array}{l}\text { the degree in which } E \text { is surrounded by tech savvy family } \\
\text { and other social circle }\end{array}$ \\
\hline 35 & $\mathrm{C} 42$ & $\begin{array}{l}\text { Clinician } \\
\text { recommendation }\end{array}$ & $\begin{array}{l}\text { E's medical professionals recommending the usage of the } \\
\text { technology }\end{array}$ \\
\hline 36 & C55 & $\begin{array}{l}\text { Closeness to } \\
\text { family }\end{array}$ & $\begin{array}{l}\text { The degree in which the } \mathrm{E} \text { is emotionally close to and in } \\
\text { contact with her family }\end{array}$ \\
\hline 37 & C30 & $\begin{array}{l}\text { Community tech } \\
\text { support }\end{array}$ & $\begin{array}{l}\text { Free available tech support that is available and accessible } \\
\text { to E upon her reaching. (e.g. retirement community } \\
\text { support) }\end{array}$ \\
\hline 38 & C52 & $\begin{array}{l}\text { Established tech } \\
\text { support }\end{array}$ & $\begin{array}{l}\text { Having trusted for fee professional support whom E can } \\
\text { reach }\end{array}$ \\
\hline 39 & $\mathrm{C} 24$ & $\begin{array}{l}\text { Gatekeeper } \\
\text { physical } \\
\text { proximity }\end{array}$ & How close to elderly gatekeeper lives \\
\hline 40 & C36 & $\begin{array}{l}\text { Gatekeeper tech } \\
\text { savviness }\end{array}$ & How tech savvy E's gatekeeper is \\
\hline 41 & C62 & $\begin{array}{l}\text { Gatekeeper's } \\
\text { financial } \\
\text { constraint }\end{array}$ & $\begin{array}{l}\text { E's gatekeeper's inability or limited ability to financially } \\
\text { support E's health technology adoption }\end{array}$ \\
\hline 42 & C58 & $\begin{array}{l}\text { Having } \\
\text { gatekeeper(s) } \\
\text { other than family }\end{array}$ & $\begin{array}{l}\text { Having other people who serve as E's main point of contact } \\
\text { for anything related to technology (anything from modeling, } \\
\text { suggesting, becoming the reason to use, to helping, training } \\
\text { and supporting her tech use) }\end{array}$ \\
\hline 43 & C54 & $\begin{array}{l}\text { Having } \\
\text { gatekeeping } \\
\text { caregiver }\end{array}$ & $\begin{array}{l}\text { Having a caregiver who becomes E's main point of contact } \\
\text { for tech support or her door to the world of technology } \\
\text { (anything from modeling, suggesting, to helping, training } \\
\text { and supporting her tech use) }\end{array}$ \\
\hline 44 & C34 & $\begin{array}{l}\text { Having } \\
\text { gatekeeping } \\
\text { daughter }\end{array}$ & $\begin{array}{l}\text { E's daughter being the main point of contact for anything } \\
\text { related to the technology; i.e. she opens the door to the } \\
\text { world of technology for mom (anything from modeling, } \\
\text { suggesting, becoming the reason to use, to helping, training } \\
\text { and supporting her tech use) }\end{array}$ \\
\hline 45 & C35 & $\begin{array}{l}\text { Having } \\
\text { gatekeeping } \\
\text { family }\end{array}$ & $\begin{array}{l}\text { E's children being the main point of contact for anything } \\
\text { related to the technology; i.e. the family opening the door } \\
\text { to the world of technology for mom (anything from } \\
\text { modeling, suggesting, becoming the reason to use, to } \\
\text { helping, training and supporting her tech use) }\end{array}$ \\
\hline 46 & C56 & $\begin{array}{l}\text { Having } \\
\text { gatekeeping } \\
\text { friend }\end{array}$ & $\begin{array}{l}\text { Having a friend who serves as E's main point of contact for } \\
\text { anything related to technology (anything from modeling, } \\
\text { suggesting, becoming the reason to use, to helping, training } \\
\text { and supporting her tech use) }\end{array}$ \\
\hline
\end{tabular}




\begin{tabular}{|c|c|c|c|}
\hline 47 & C57 & $\begin{array}{l}\text { Having } \\
\text { gatekeeping } \\
\text { grandkids }\end{array}$ & $\begin{array}{l}\text { Having one or more grandchildren who serve as E's main } \\
\text { point of contact for anything related to technology } \\
\text { (anything from modeling, suggesting, becoming the reason } \\
\text { to use, to helping, training and supporting her tech use) }\end{array}$ \\
\hline 48 & C59 & Having grandkids & $\begin{array}{l}\text { Having grandkids (who often create deep motivation to } \\
\text { connect to often by using technology) }\end{array}$ \\
\hline 49 & $\mathrm{C} 60$ & $\begin{array}{l}\text { Having other } \\
\text { gatekeeping } \\
\text { family members }\end{array}$ & $\begin{array}{l}\text { Having family member(s) than daughter or grandchild who } \\
\text { serve as E's main point of contact for anything related to } \\
\text { technology (anything from modeling, suggesting, becoming } \\
\text { the reason to use, to helping, training and supporting her } \\
\text { tech use) }\end{array}$ \\
\hline 50 & C39 & $\begin{array}{l}\text { Local group } \\
\text { training }\end{array}$ & $\begin{array}{l}\text { Free group classes and training offered in E's community to } \\
\text { facilitate her tech use. E.g. Local classes in the library or } \\
\text { retirement community }\end{array}$ \\
\hline 51 & C61 & $\begin{array}{l}\text { Other sources of } \\
\text { information }\end{array}$ & $\begin{array}{l}\text { Learning about new technologies and their utility from } \\
\text { sources other than social learning (e.g. TV or paper ads) }\end{array}$ \\
\hline 52 & C53 & $\begin{array}{l}\text { Simple free } \\
\text { instruction }\end{array}$ & $\begin{array}{l}\text { Simple jargon free written or video instructions. The simpler } \\
\text { step by step written the better; and more visual ones tend } \\
\text { to be most popular }\end{array}$ \\
\hline 53 & $\mathrm{C} 63$ & Social circle & $\begin{array}{l}\text { The degree in which } \mathrm{E} \text { is surrounded by people other than } \\
\text { her own family members }\end{array}$ \\
\hline \multicolumn{4}{|c|}{ Environmental Influence Concepts } \\
\hline 54 & C31 & $\begin{array}{l}\text { Connecting to } \\
\text { loved ones }\end{array}$ & $\begin{array}{l}\text { Desire to connect to family (esp. grandchildren) \& to a much } \\
\text { less degree friends and her social circle by using the tech }\end{array}$ \\
\hline 55 & C49 & $\begin{array}{l}\text { Continuous 1-on- } \\
1 \text { walkthrough } \\
\text { support }\end{array}$ & $\begin{array}{l}\text { Access to continuous 1-on-1 walkthrough support that is } \\
\text { normally provided by a family member or someone whom E } \\
\text { is very comfortable with and preferring to ask questions } \\
\text { from first }\end{array}$ \\
\hline 56 & $\mathrm{C} 4$ & $\begin{array}{l}\text { Effectivity, } \\
\text { Continuity \& } \\
\text { Sufficiency of } \\
\text { Support \& } \\
\text { Training } \\
\end{array}$ & $\begin{array}{l}\text { The effectivity \& sufficiency of Support \& Training for the } \\
\text { elderly to successfully use and continue to use the } \\
\text { technology }\end{array}$ \\
\hline 57 & C38 & Gifting tech & $\begin{array}{l}\text { Given the tech free of cost to E usually only by family } \\
\text { members and could be both new or hands me down tech }\end{array}$ \\
\hline 58 & $\mathrm{C} 43$ & $\begin{array}{l}\text { Giving GK peace } \\
\text { of mind }\end{array}$ & $\begin{array}{l}\text { E's reassuring and giving peace of mind to her loved ones by } \\
\text { using the technology }\end{array}$ \\
\hline 59 & C37 & Reduce burden & $\begin{array}{l}\text { Reducing burden from the shoulder of E's family and other } \\
\text { loved ones by using technology }\end{array}$ \\
\hline 60 & $\mathrm{C} 11$ & Social learning & $\begin{array}{l}\text { Learning about the technology use by seeing the technology } \\
\text { use (from modeling) }\end{array}$ \\
\hline 61 & C18 & $\begin{array}{l}\text { Tech suggestion, } \\
\text { introduction \& } \\
\text { encouragement }\end{array}$ & $\begin{array}{l}\text { The act of suggesting, introducing \& encouraging the } \\
\text { adoption of the tech by people who E is close and trust in } \\
\text { their intention and tech savviness }\end{array}$ \\
\hline
\end{tabular}


Tech Adoption Concepts

\begin{tabular}{|l|l|l|l}
62 & C1 & Adoption & Elderly's acceptance and usage of the technology
\end{tabular}

63 C13 experience $\quad$ past career and the resulting technical knowledge 


\section{Resulted FCM connected Model}

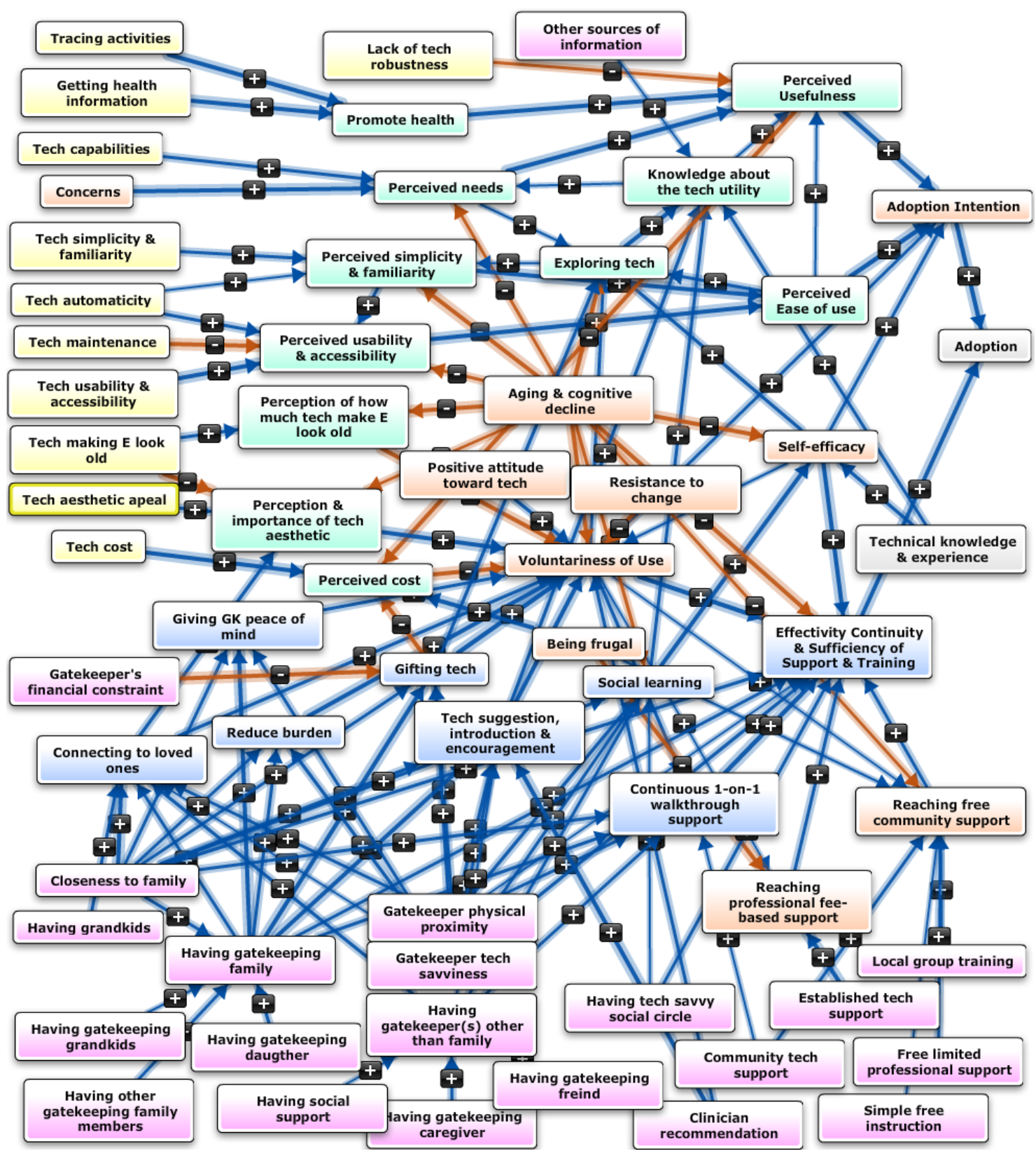




\section{Resulted FCM Isolated Model}

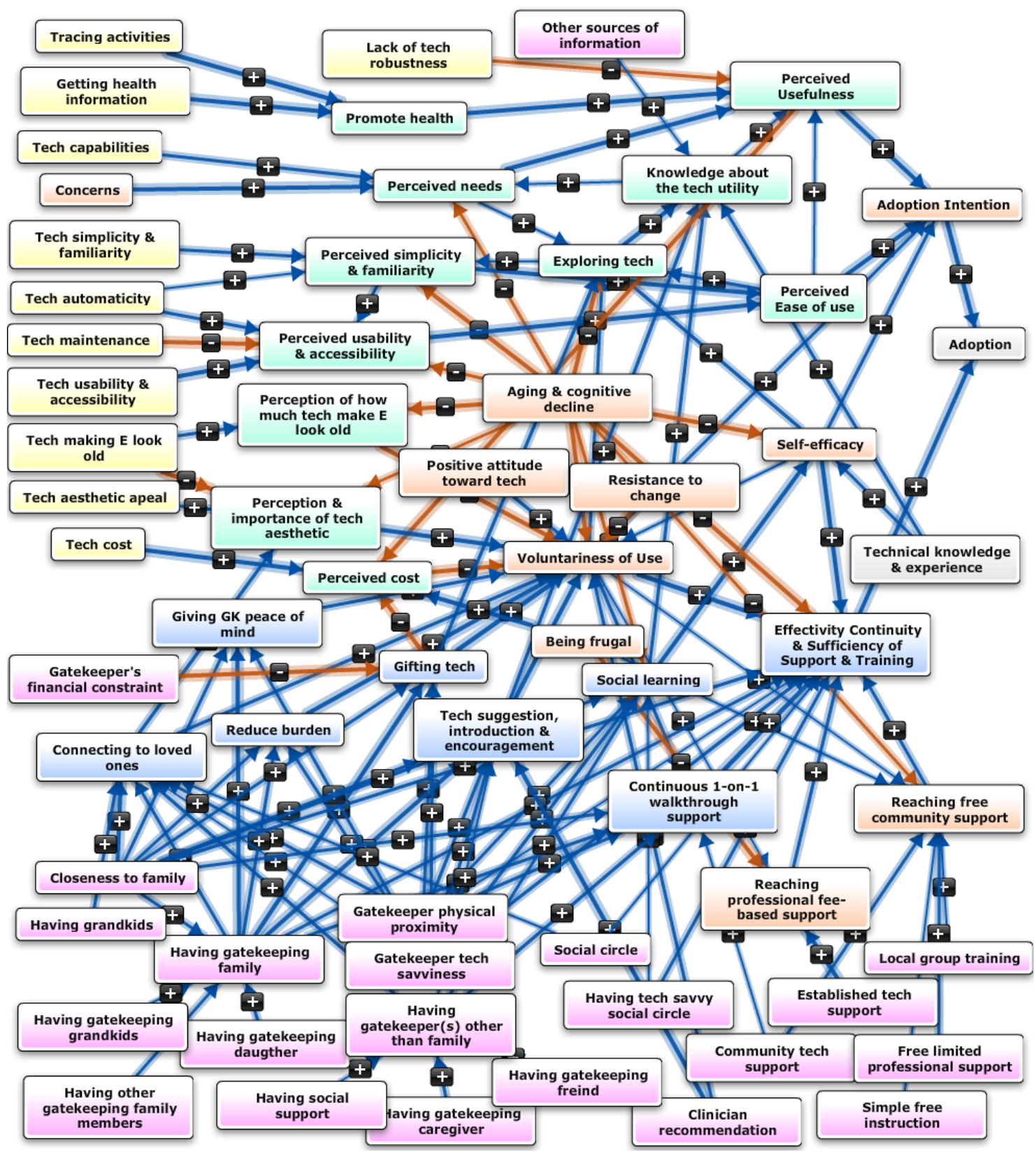




\section{Appendix H - Case Study Participants' Profiles}

\section{Elderly Woman \#1}

\begin{tabular}{|c|c|}
\hline Elderly Profile & P1 \\
\hline Interview Duration & $56 \mathrm{~min}$ \\
\hline Age & 83 \\
\hline Health Condition & OK \\
\hline Living condition & Active elderly living by herself (with a cat) in her owned house \\
\hline Attitude toward Tech & Negative \\
\hline Self-efficacy & Ok \\
\hline Experience & some \\
\hline Driven by & Need, prudence \\
\hline Has grandchildren? & $\mathrm{N}$ \\
\hline Has \& close to family & $\sim$ (has a daughter but not too close) \\
\hline Gatekeeper & Daughter (46 min) \\
\hline $\begin{array}{l}\text { Gatekeeper tech } \\
\text { savviness }\end{array}$ & No \\
\hline Degree of influence & Little \\
\hline Influence & Giving GK peace of mind, Desire to connect, \\
\hline $\begin{array}{l}\text { Perceived Usefulness } \\
\text { of the wearable }\end{array}$ & It doesn't work well, it doesn't work outside and not reliable \\
\hline $\begin{array}{l}\text { Perceived Ease of } \\
\text { Use of the wearable }\end{array}$ & $\begin{array}{l}\text { It's relatively easy to use but I wish it was waterproof, I have to } \\
\text { take it out }\end{array}$ \\
\hline $\begin{array}{l}\text { Will she adopt if } \\
\text { free? }\end{array}$ & Maybe \\
\hline $\begin{array}{l}\text { Will she buy to } \\
\text { adopt? }\end{array}$ & $\mathbf{N}$ \\
\hline Dominant Theme1 & E identifies Need as the most important driver of HTA \\
\hline Dominant Theme2 & In vivo: "I forget how to use it"; E needs a continuous support \\
\hline Dominant Theme3 & $\begin{array}{l}\text { I need the knowledge but need continuous support and repeating } \\
\text { instruction or print out so I can go back to it }\end{array}$ \\
\hline
\end{tabular}




\begin{tabular}{|l|l|} 
Dominant Theme4 & $\begin{array}{l}\text { She enjoys volunteering for OHSU and believe in helping and } \\
\text { enjoys learning but not really willing to initiate learning tech and } \\
\text { need familiar continuous FC }\end{array}$ \\
\hline Discovery & $\begin{array}{l}\text { I need the knowledge but need continuous support and repeating } \\
\text { instruction or print out so I can go back to it }\end{array}$ \\
\hline GK & $\begin{array}{l}\text { She enjoys volunteering for OHSU and believe in helping and } \\
\text { enjoys learning but not really willing to initiate learning tech and } \\
\text { need familiar continuous FC }\end{array}$ \\
\hline
\end{tabular}

Table H - 1 Elderly woman \#1 profile 


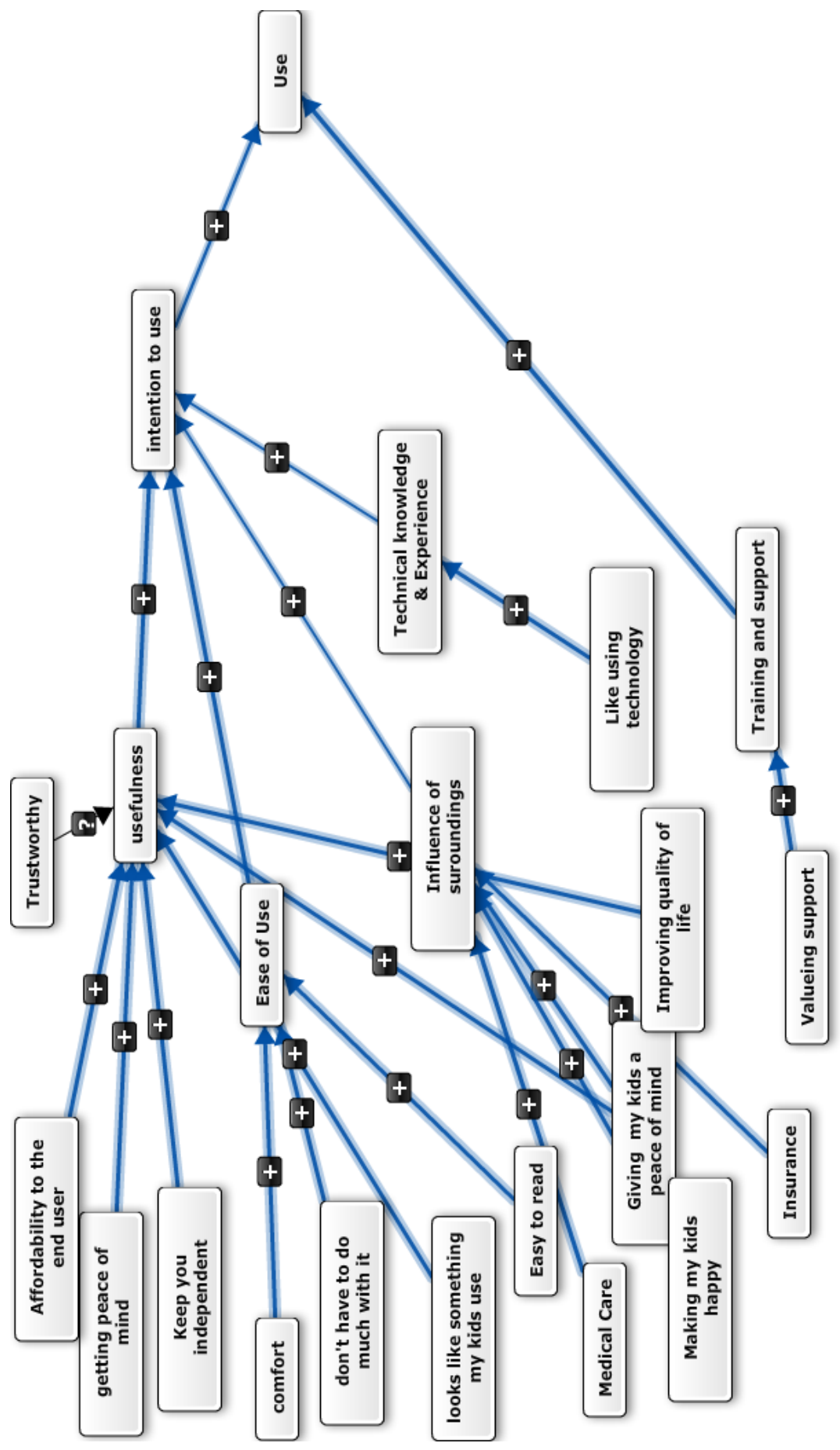

Figure H - 1 EW \#1 initial cognitive map 


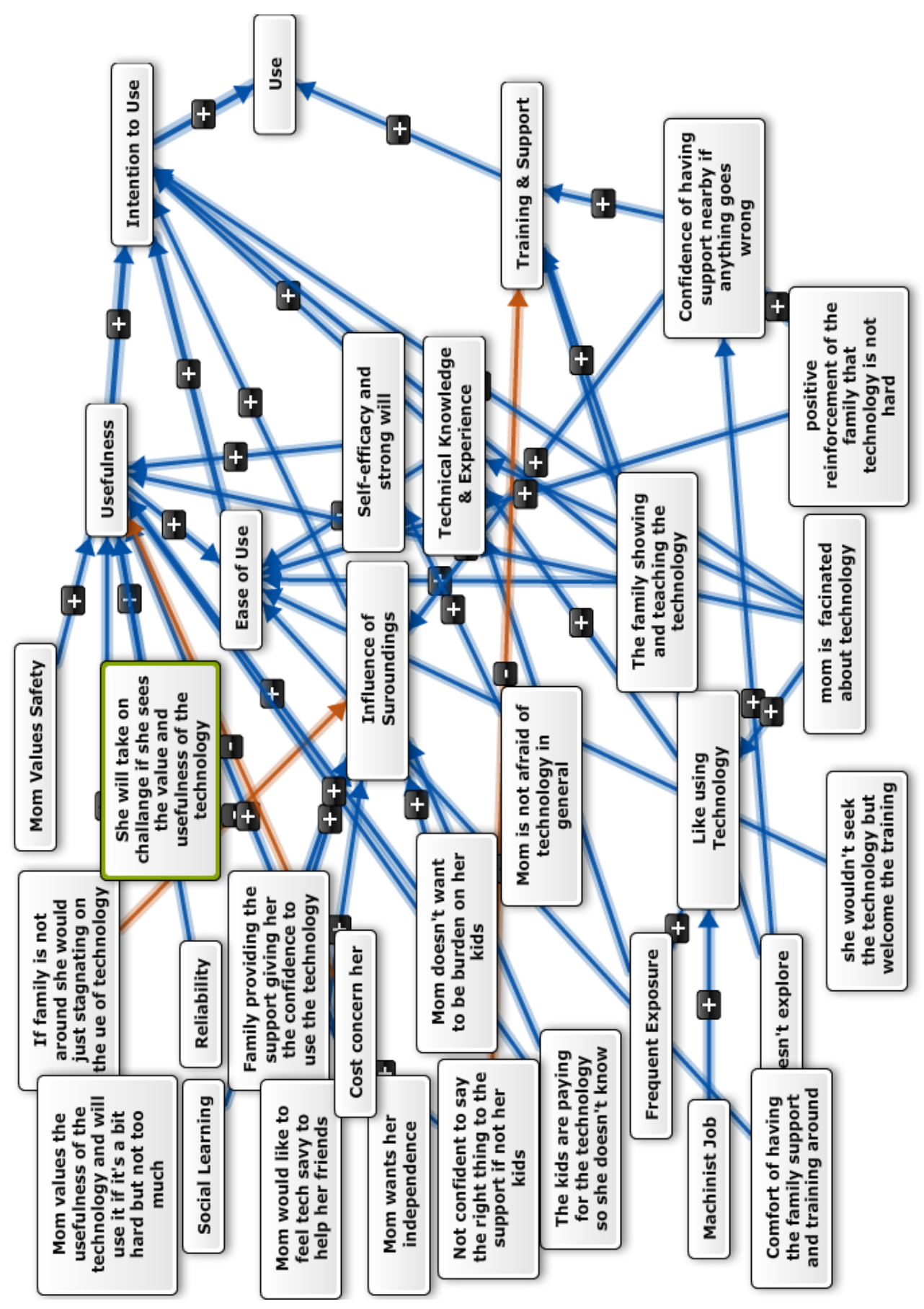

Figure H - 2 Gatekeeper \#1 initial cognitive map 


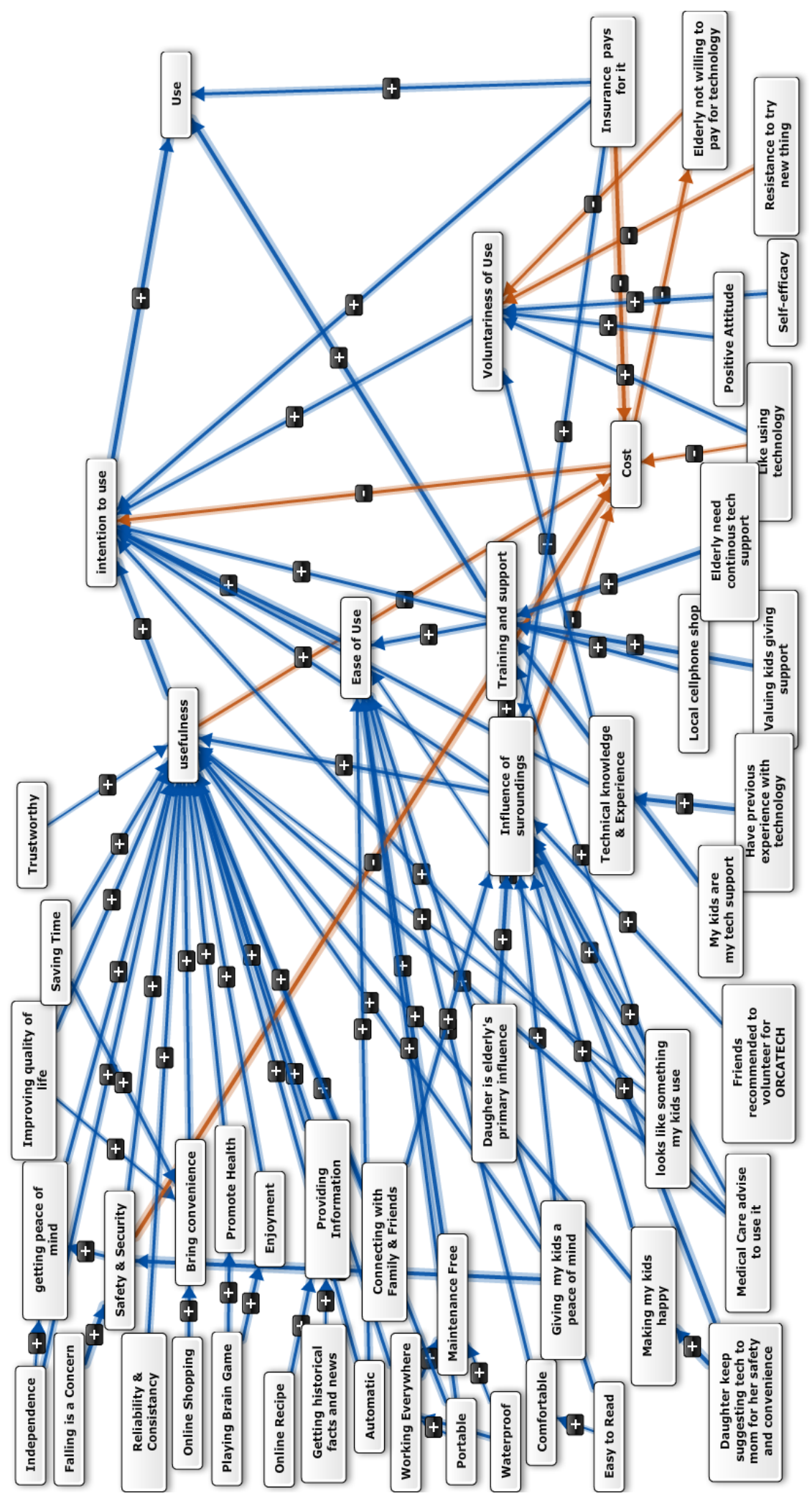

Figure H - 3 Elderly Gatekeeper pair \#1 integrated cognitive map 


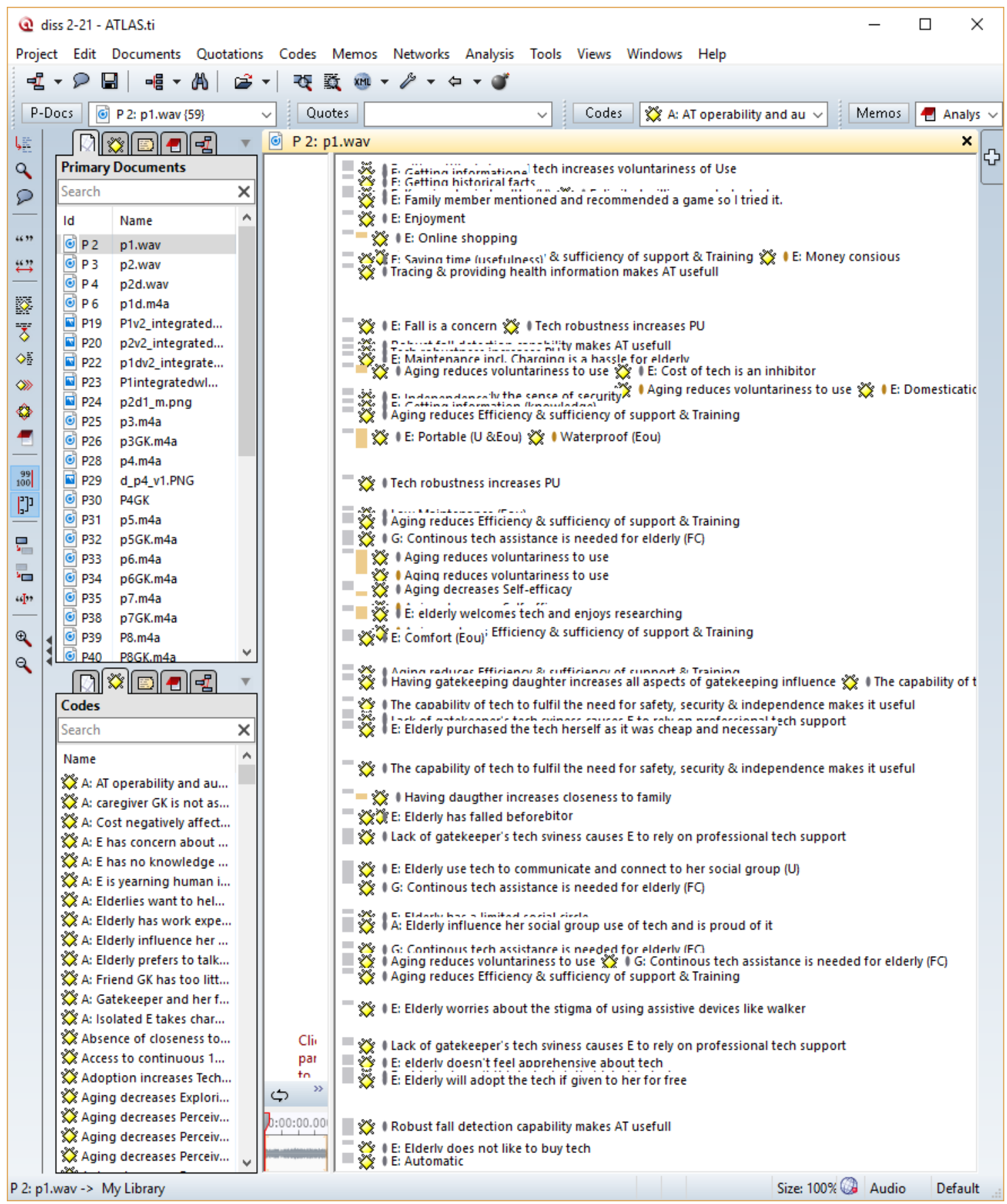

\section{Figure H - 4 EW\#1 interview open coding in Atlas.ti}




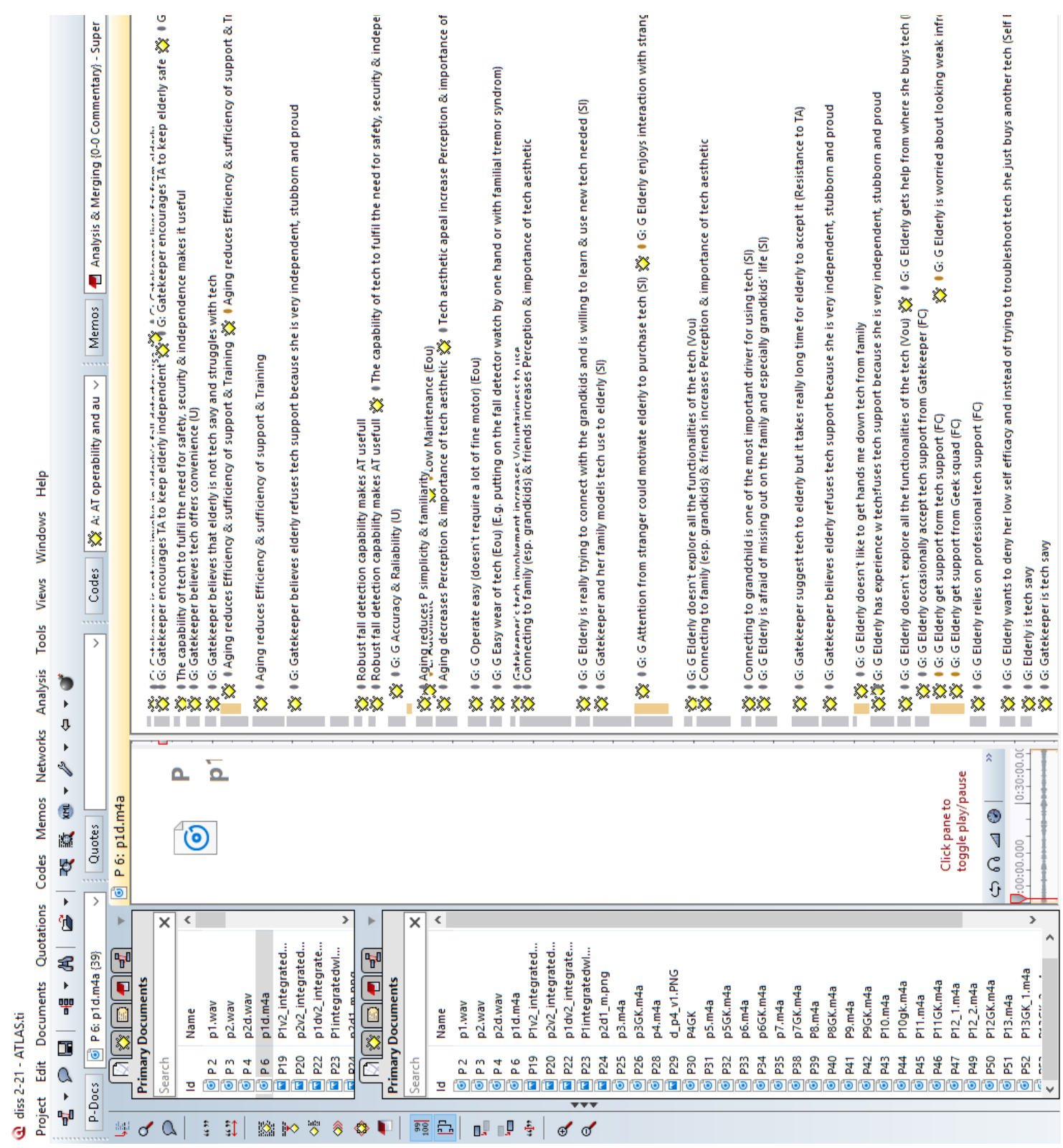

Figure H - 5 Gatekeeper \#1 interview open coding in Atlas.ti 


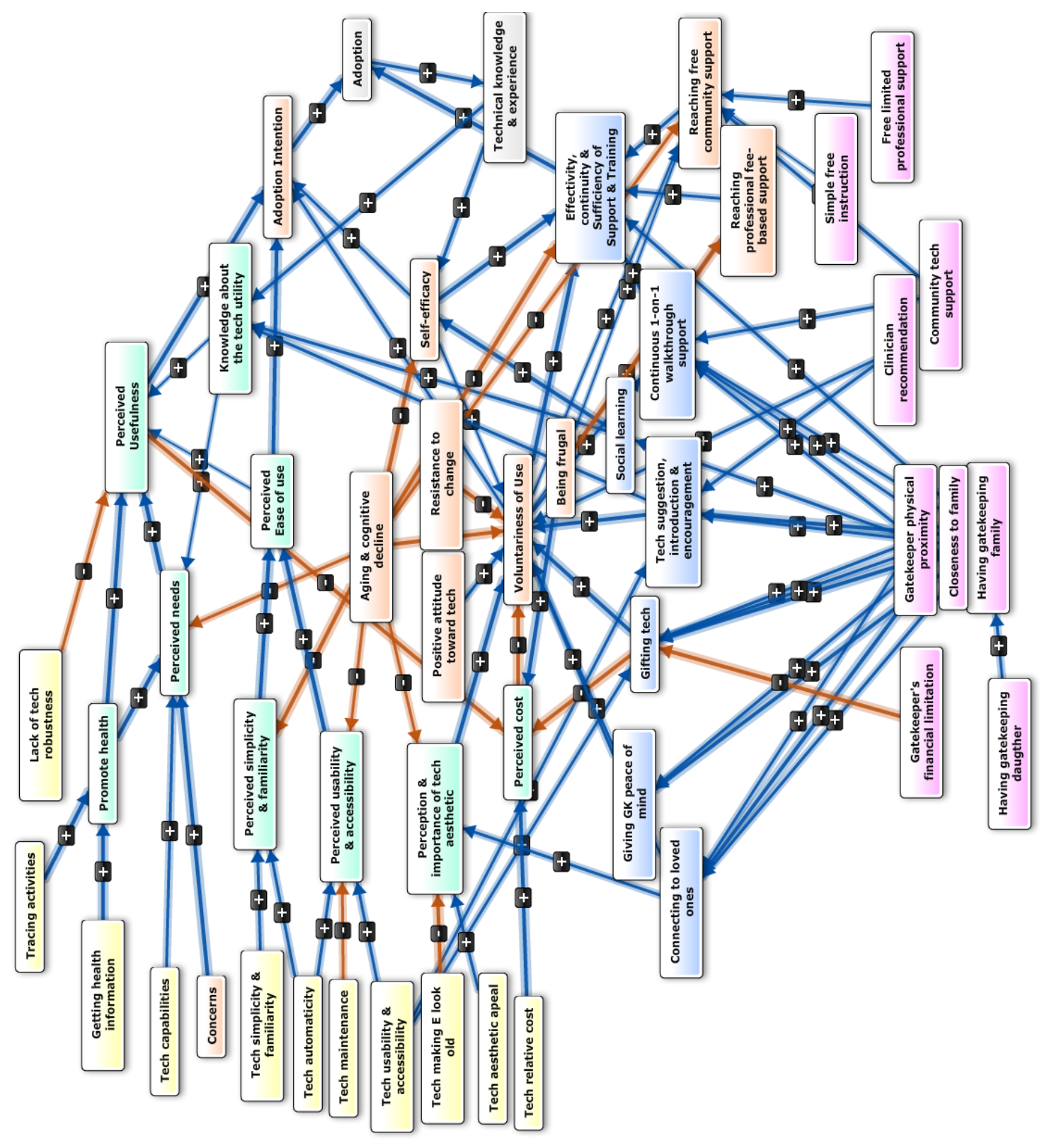

Figure H - 6 EW \#1 integrated \& standardized cognitive map 


\section{Elderly Woman \#2}

\begin{tabular}{|c|c|}
\hline Elderly Profile & $\mathrm{P} 2$ \\
\hline Interview Duration & $77 \mathrm{~min}$ \\
\hline Age & 69 \\
\hline Health Condition & Hand problem (Tremor) \\
\hline Living condition & Retirement Community (RC) \\
\hline Isolated & Semi (live in RC, have two daughters live far) \\
\hline Attitude toward Tech & Positive \\
\hline Experience & some \\
\hline Hobbies & Reading, Crochet, (TV to an extent) \\
\hline Driven by & Need, Connecting to the grandkids, Health \\
\hline Has grandchildren? & $Y($ primary driver of TA) \\
\hline Has \& close to family & $\mathrm{Y}$ \\
\hline Gatekeeper & Grand daughter and daughter (53 $\mathrm{min}$ ) \\
\hline $\begin{array}{l}\text { Gatekeeper tech } \\
\text { savviness }\end{array}$ & Y \\
\hline Degree of influence & Strong \\
\hline Influence & All \\
\hline $\begin{array}{l}\text { Perceived Usefulness } \\
\text { of the wearable }\end{array}$ & It is not robust, I need something better \\
\hline $\begin{array}{l}\text { Perceived Ease of } \\
\text { Use of the wearable }\end{array}$ & It's not hard as it is not intrusive but it's too big \\
\hline $\begin{array}{l}\text { Will she adopt if } \\
\text { free? }\end{array}$ & $\mathrm{y}$ \\
\hline $\begin{array}{l}\text { Will she buy to } \\
\text { adopt? }\end{array}$ & $\mathrm{N}$ \\
\hline Dominant Theme1 & $\begin{array}{l}\text { She is driven by perceived need, and unless domesticated through } \\
\text { family she doesn't think she needs the tech. }\end{array}$ \\
\hline Dominant Theme2 & $\begin{array}{l}\text { E is frugal and minimalistic and often tech has a high cost of } \\
\text { ownership and since E doesn't have the perceived need for the new } \\
\text { tech, she resist tech. This cycle only breaks with the family } \\
\text { intervening by giving her gift }\end{array}$ \\
\hline
\end{tabular}

Table H - 2 Elderly woman \#2 profile 


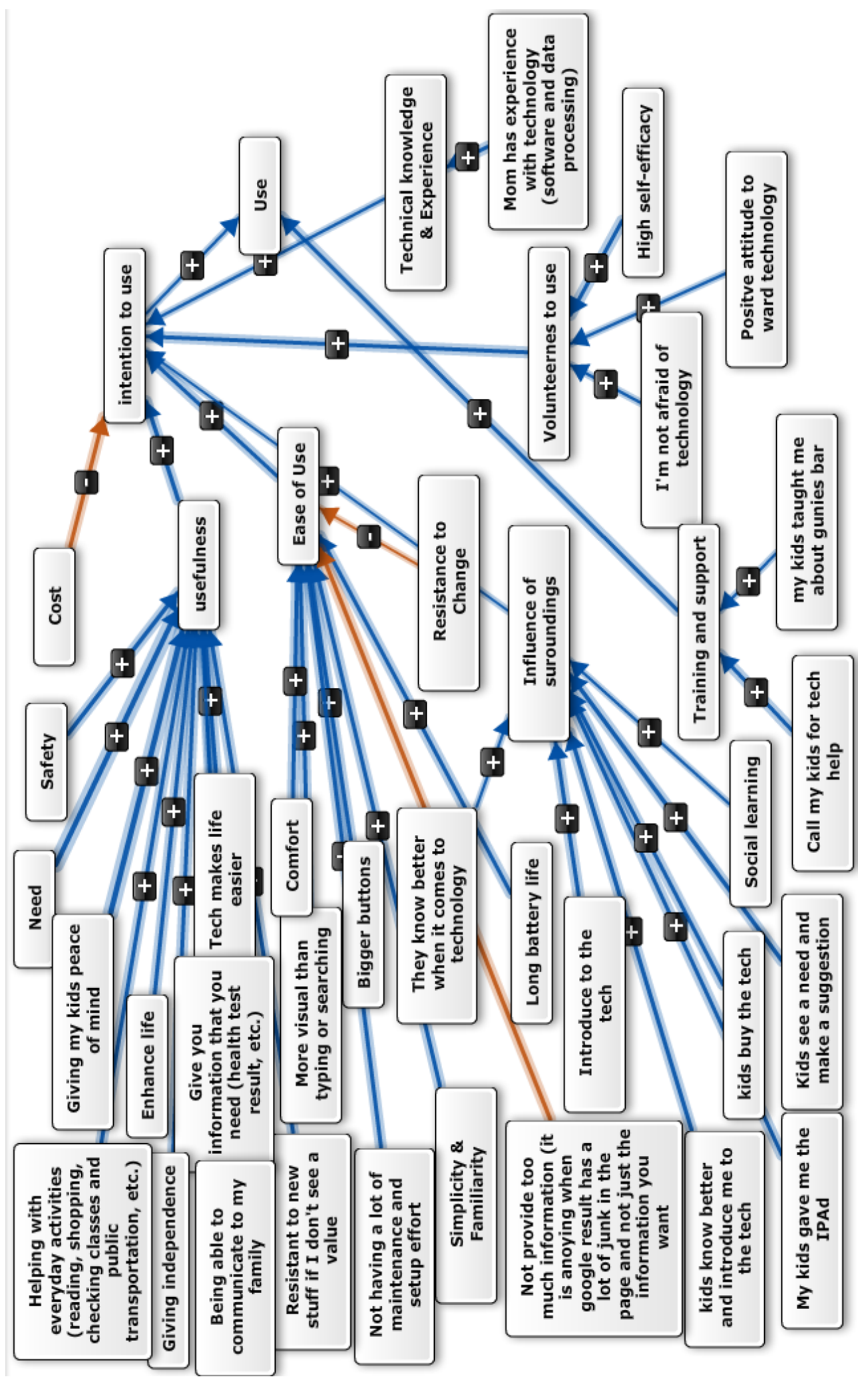

Figure H - 7 EW \#2 initial cognitive map 


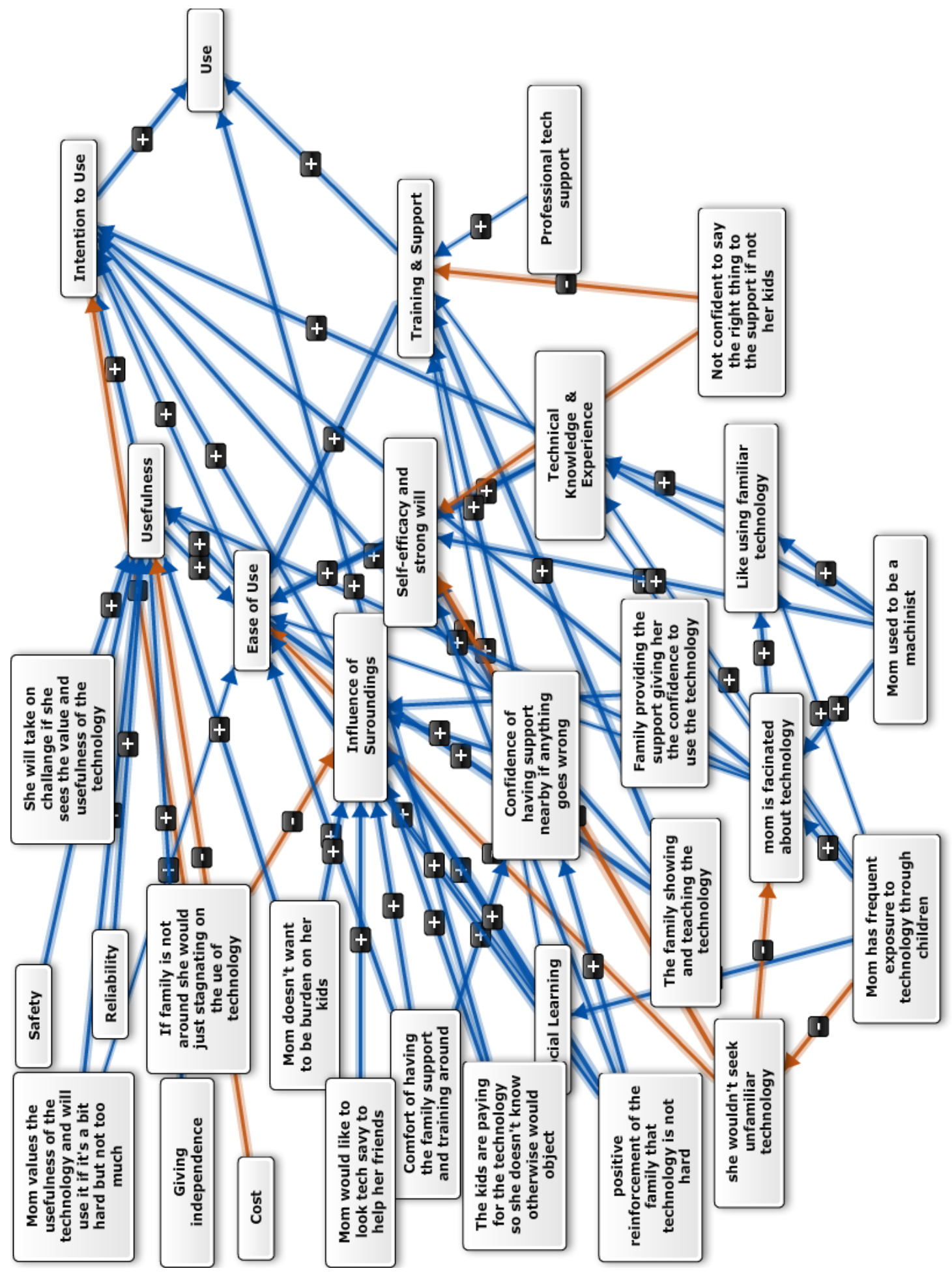

Figure H - 8 Gatekeeper \#2 initial cognitive map 


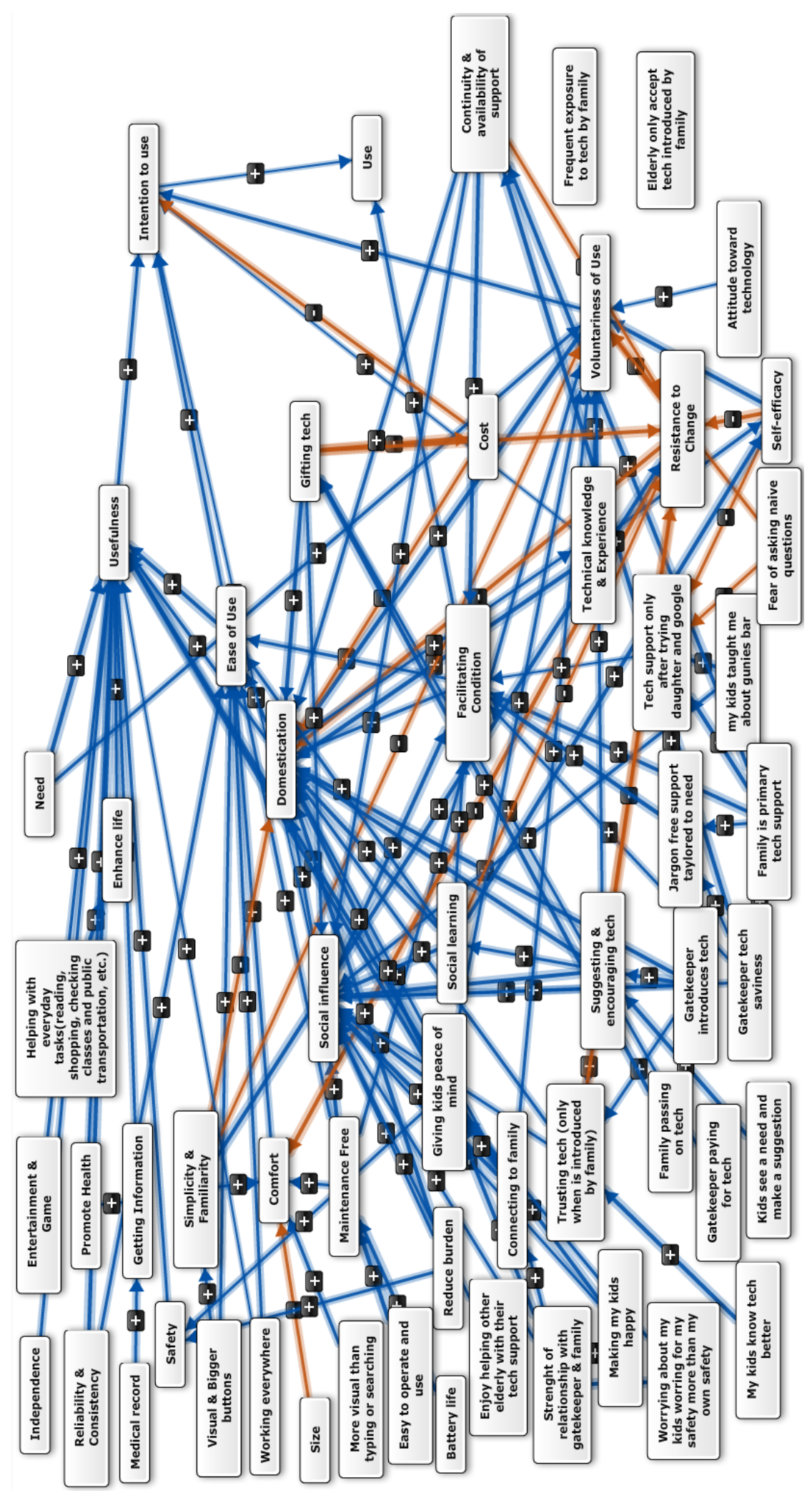

Figure H - 9 Elderly Gatekeeper pair \#2 integrated cognitive map 459 
Q diss 2-21 - ATLAS.ti

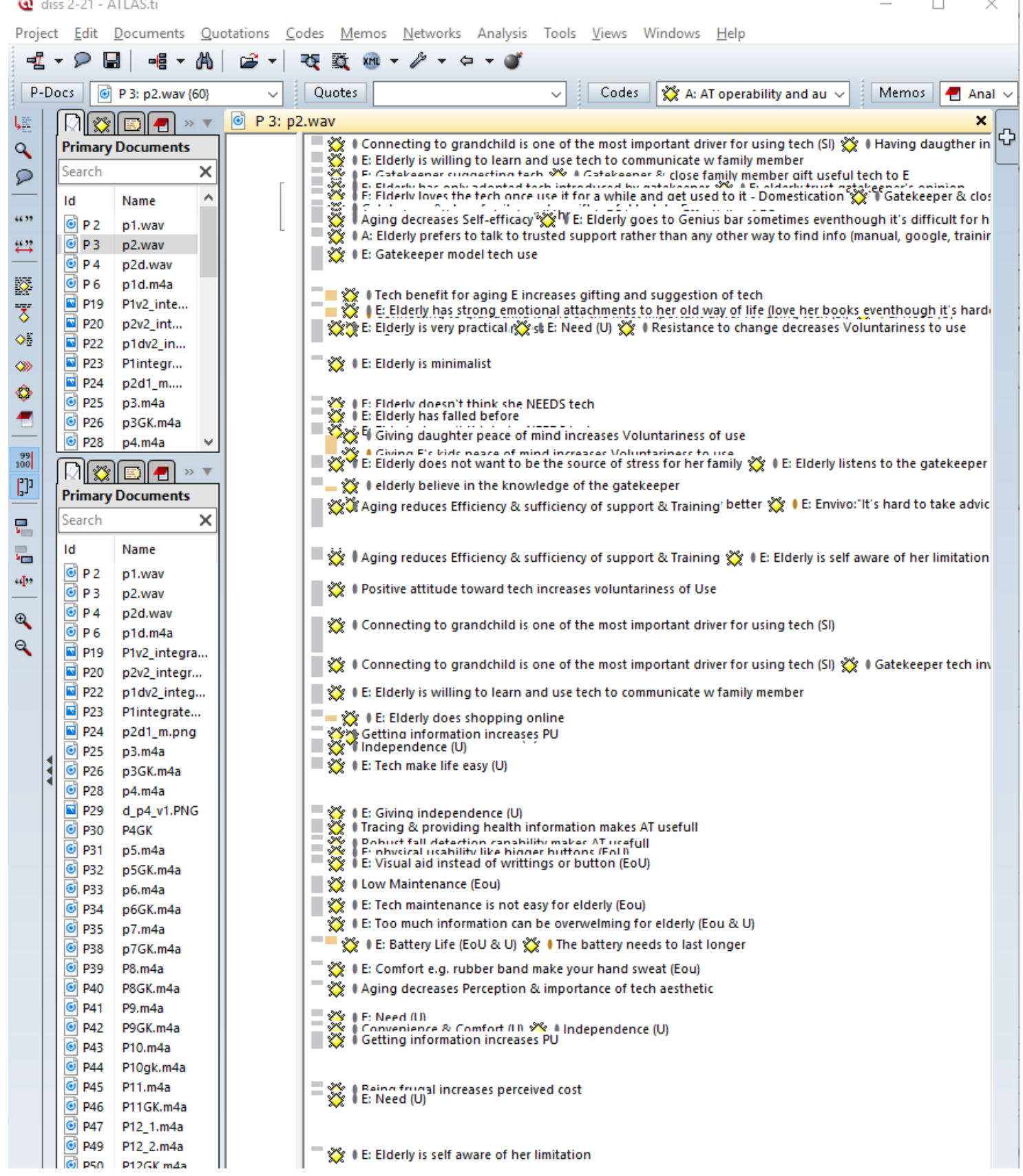

Figure H - 10 EW\#2 interview open coding in Atlas.ti 


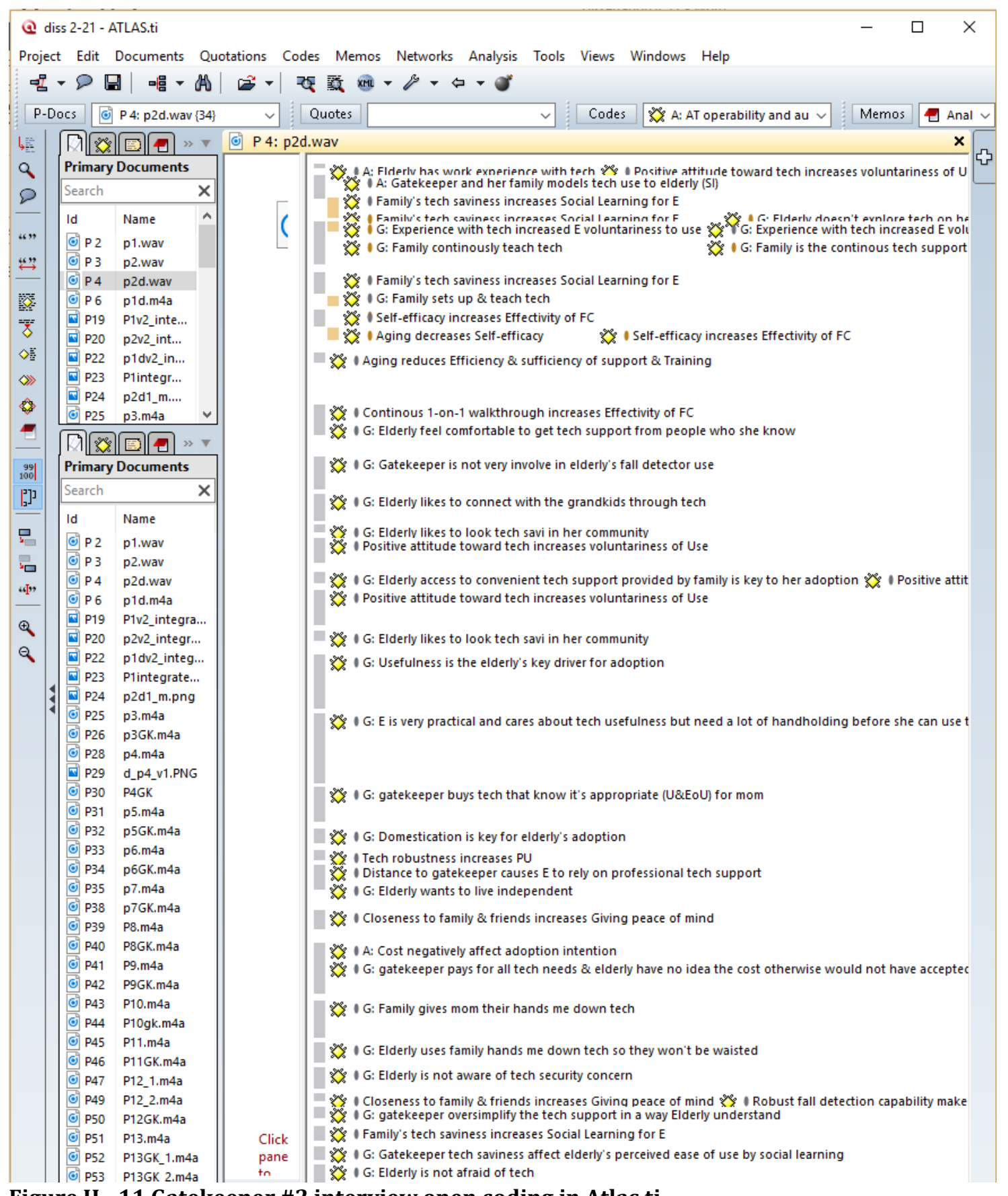

\section{Figure H - 11 Gatekeeper \#2 interview open coding in Atlas.ti}




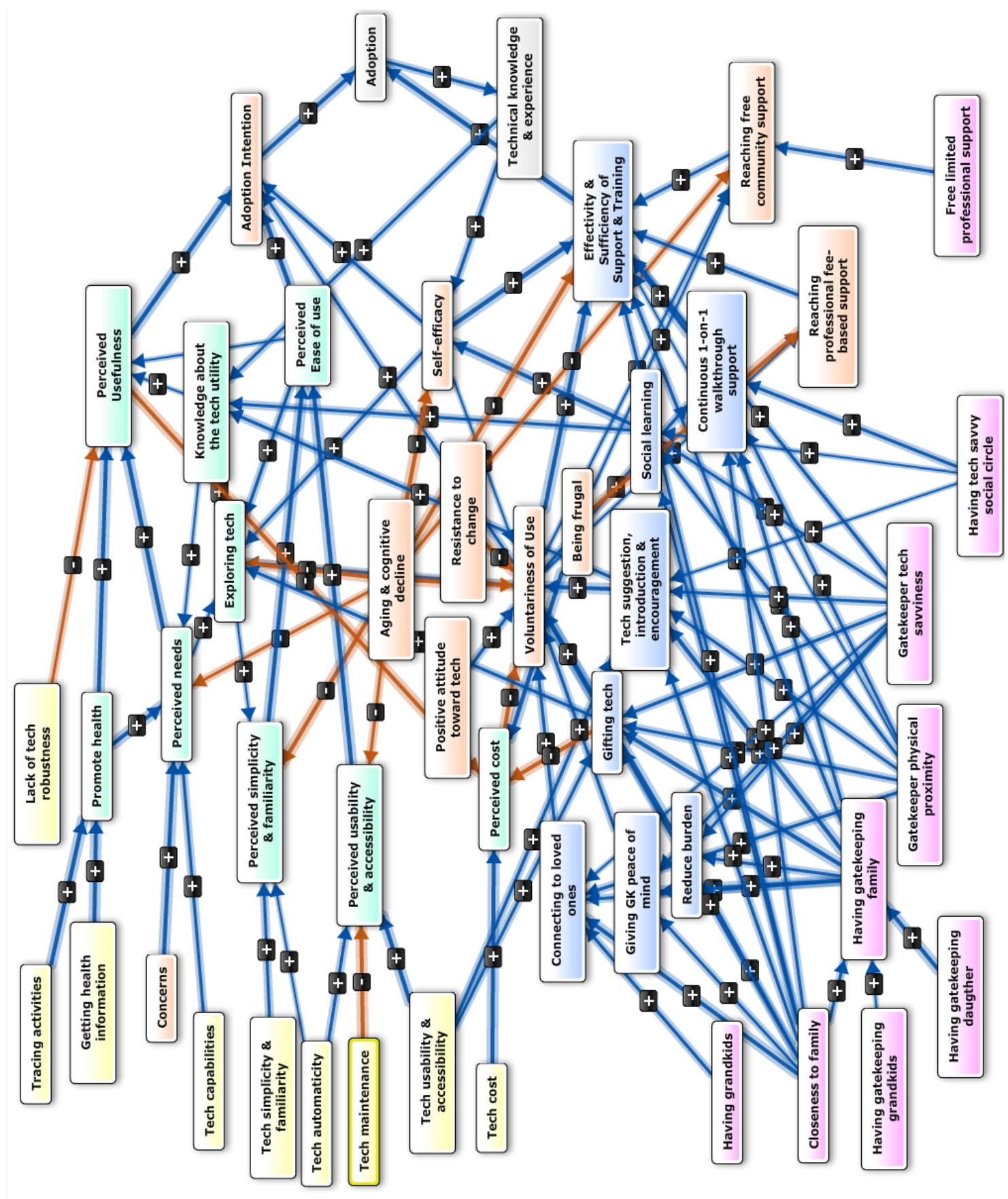

Figure H - 12 EW \#2 integrated \& standardized cognitive map 


\section{Elderly Woman \#3}

\begin{tabular}{|c|c|}
\hline Elderly Profile & P3 \\
\hline Interview Duration & $58 \mathrm{~min}$ \\
\hline Age & 80 \\
\hline Health Condition & OK \\
\hline Living condition & Retirement community \\
\hline Isolated & $\mathrm{Y}$ \\
\hline $\begin{array}{l}\text { Attitude toward } \\
\text { Tech }\end{array}$ & Very Negative \\
\hline Experience & some \\
\hline Driven by & Need, Simplicity \& Familiarity \\
\hline Has grandchildren? & $\mathrm{N}$ \\
\hline Has \& close to family & $\mathrm{N}$ \\
\hline Gatekeeper & Retirement community Volunteer Tech support (70 min) \\
\hline $\begin{array}{l}\text { Gatekeeper tech } \\
\text { savviness }\end{array}$ & Y \\
\hline Degree of influence & little \\
\hline Influence & Some tech support \\
\hline $\begin{array}{l}\text { Perceived } \\
\text { Usefulness of the } \\
\text { wearable }\end{array}$ & I don't need it \\
\hline $\begin{array}{l}\text { Perceived Ease of } \\
\text { Use of the wearable }\end{array}$ & It's not hard as it is not intrusive but it's too big \\
\hline $\begin{array}{l}\text { Facilitating } \\
\text { Condition }\end{array}$ & Community volunteers \& professionals \\
\hline $\begin{array}{l}\text { Will she adopt if } \\
\text { free? }\end{array}$ & $\mathrm{N}$ \\
\hline $\begin{array}{l}\text { Will she buy to } \\
\text { adopt? }\end{array}$ & $\mathrm{N}$ \\
\hline Note & $\begin{array}{l}\text { She does have a family who live in East Coast (no FC which } \\
\text { resulted in tech abandonment) their influence is very negligible } \\
\text { since they live far and not very emotionally close to E. }\end{array}$ \\
\hline Dominant Theme1 & $\begin{array}{l}\text { E thinks she is healthy and does not think she needs tech, \& } \\
\text { identifies Need as the most important driver of HTA. }\end{array}$ \\
\hline Dominant Theme2 & $\begin{array}{l}\text { In vivo: "Tech is not worth my time", tech is only good for } \\
\text { communication; In vivo: "They better not give me anything } \\
\text { techy!" }\end{array}$ \\
\hline Dominant Theme3 & $\begin{array}{l}\text { E has a strong attachment to her old simple and familiar way of } \\
\text { life. E.g. she googles and print the direction from computer and } \\
\text { not her smartphone that she has but never uses! }\end{array}$ \\
\hline Dominant Theme4 & $\begin{array}{l}\text { E is keep saying I don't need tech, but what she really is doing is } \\
\text { that she is using many technologies that have become familiar } \\
\text { over years (computer, printer, internet, social media (FB), phone }\end{array}$ \\
\hline
\end{tabular}


functionality of her smartphone, GPS, kindle). Her negative attitude is toward new technologies.

Table H - 3 Elderly woman \#3 profile 


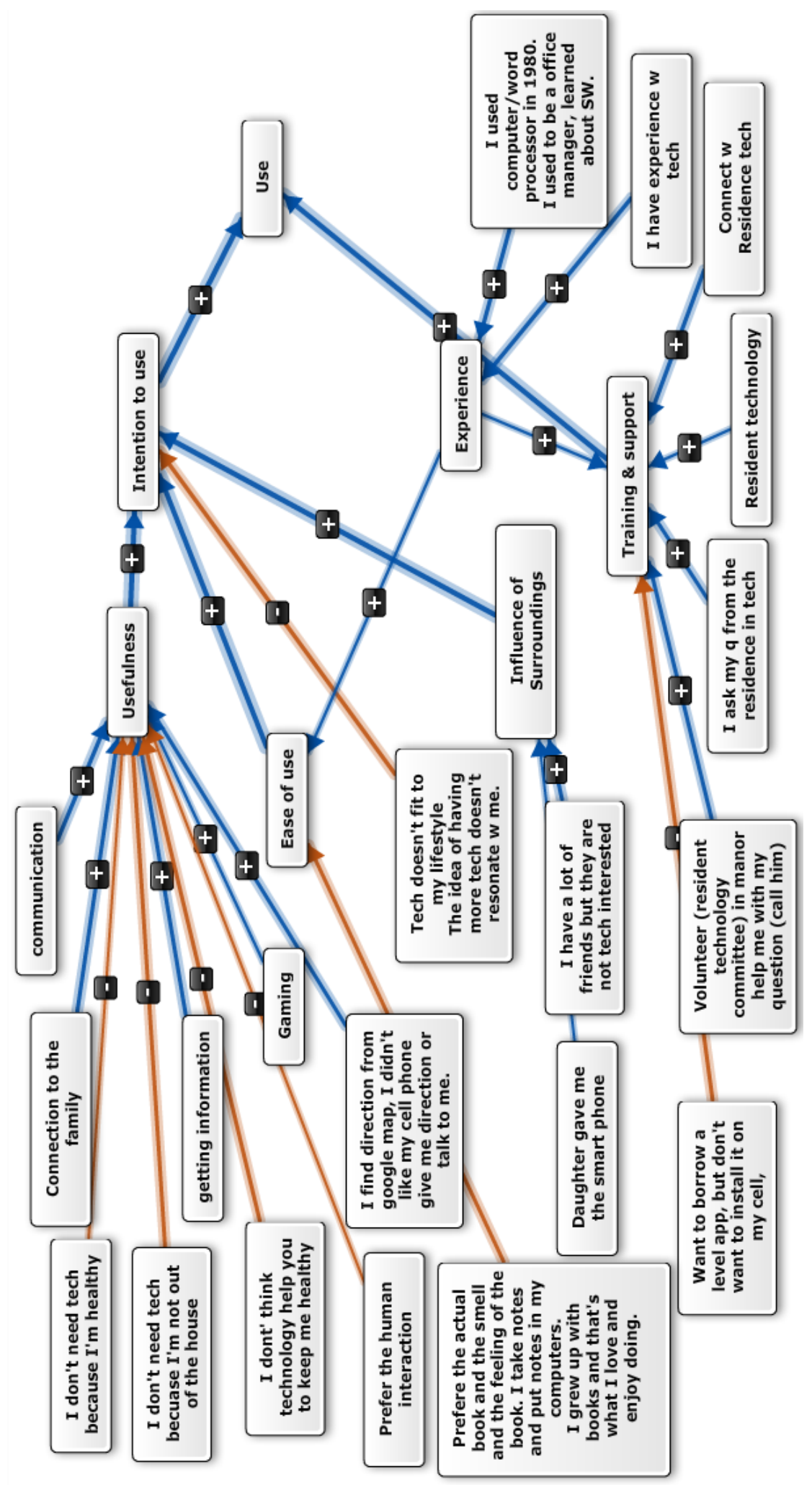

Figure H - 13 EW \#3 initial cognitive map 


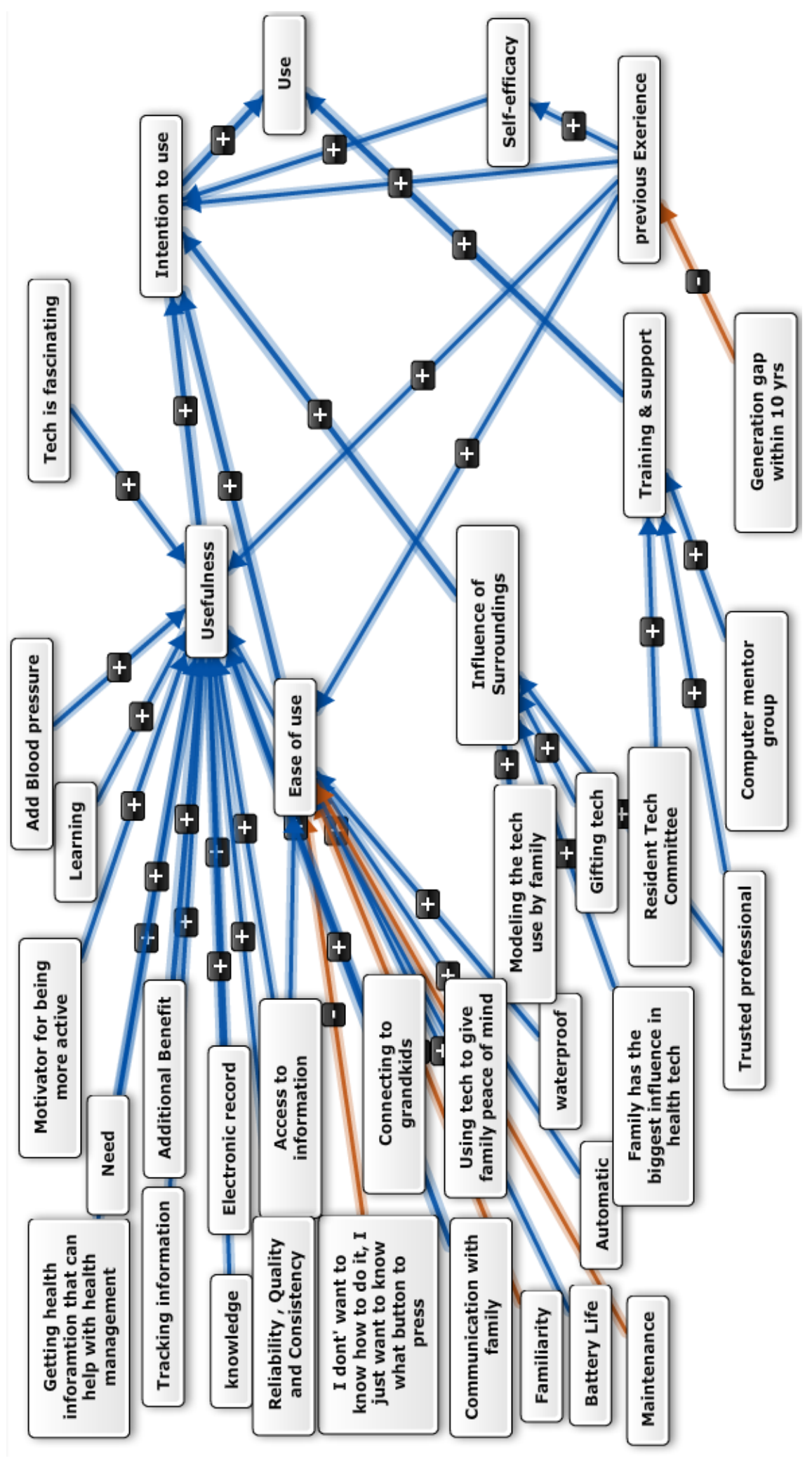

Figure H - 14 Gatekeeper \#3 initial cognitive map

466 


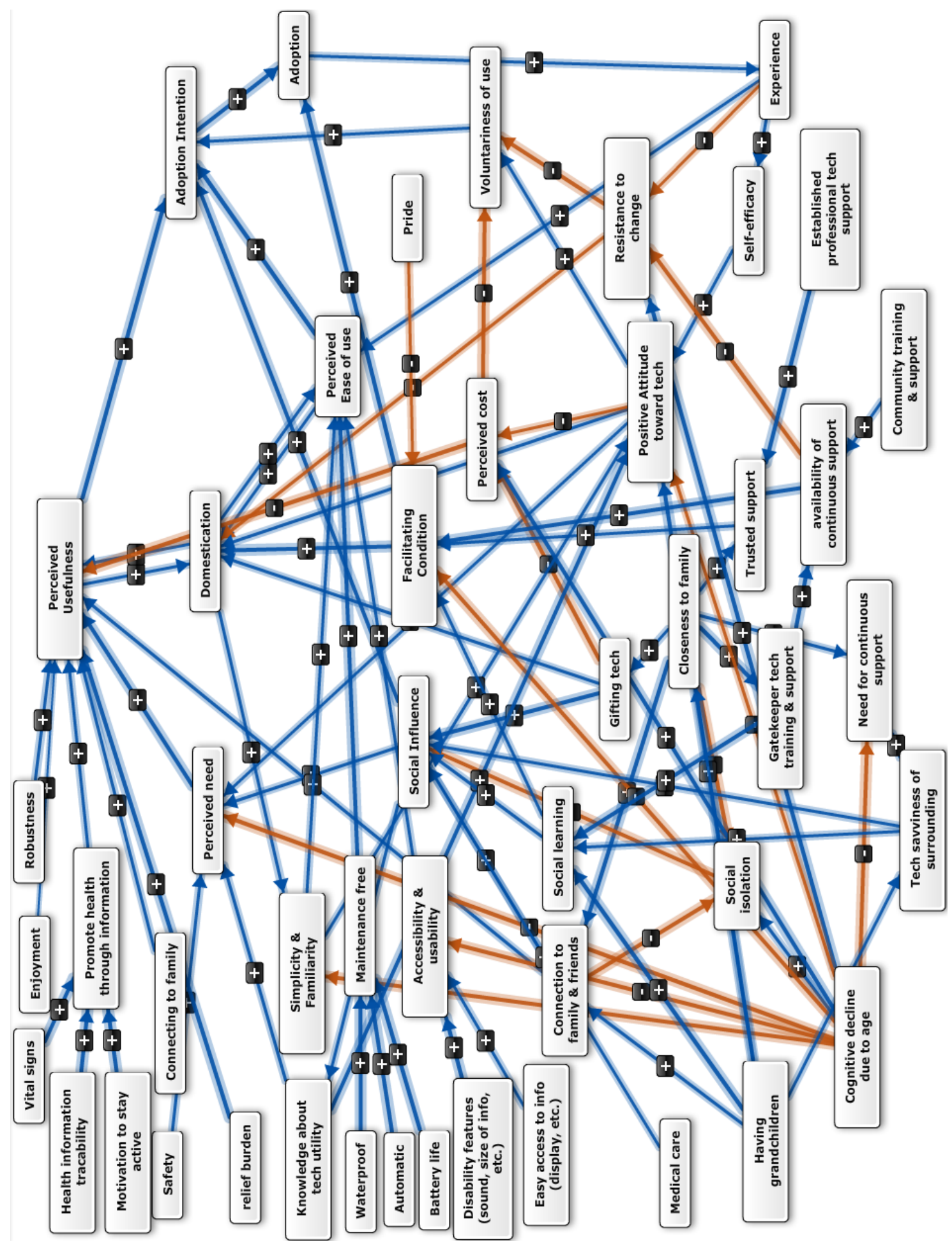

Figure H - 15 Elderly Gatekeeper pair \#3 integrated cognitive map 


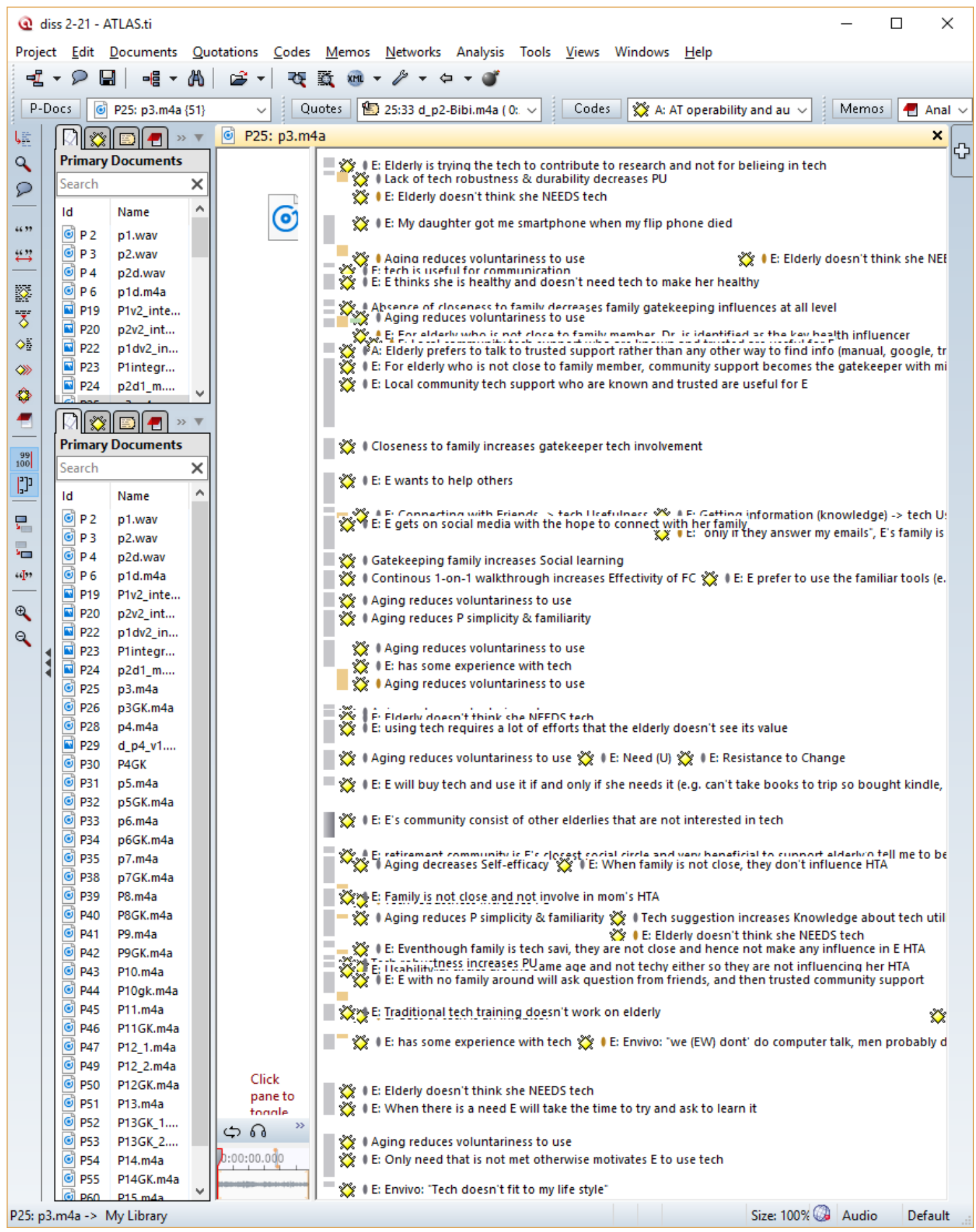

\section{Figure H - 16 EW\#3 interview open coding in Atlas.ti}




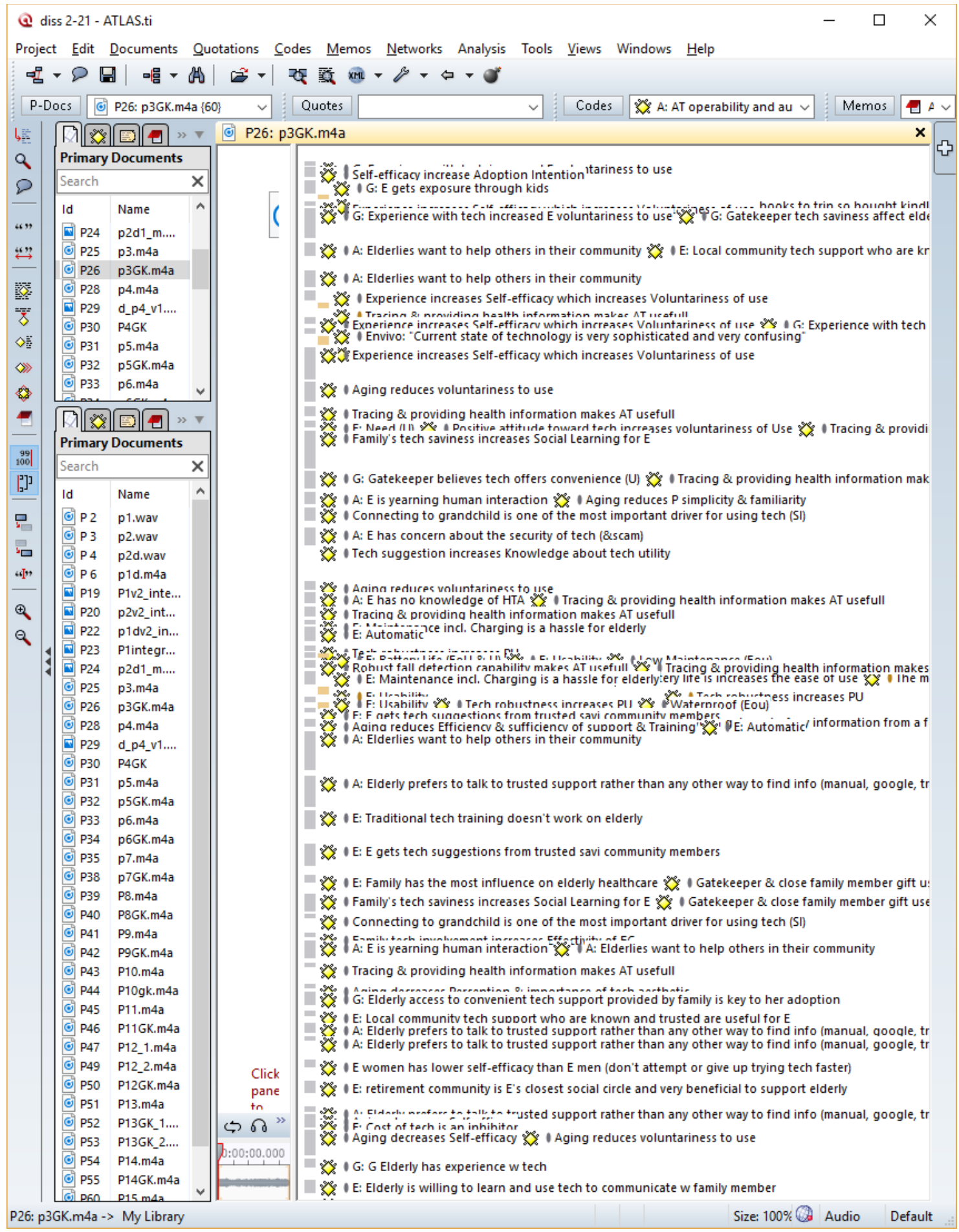

\section{Figure H - 17 Gatekeeper \#3 interview open coding in Atlas.ti}




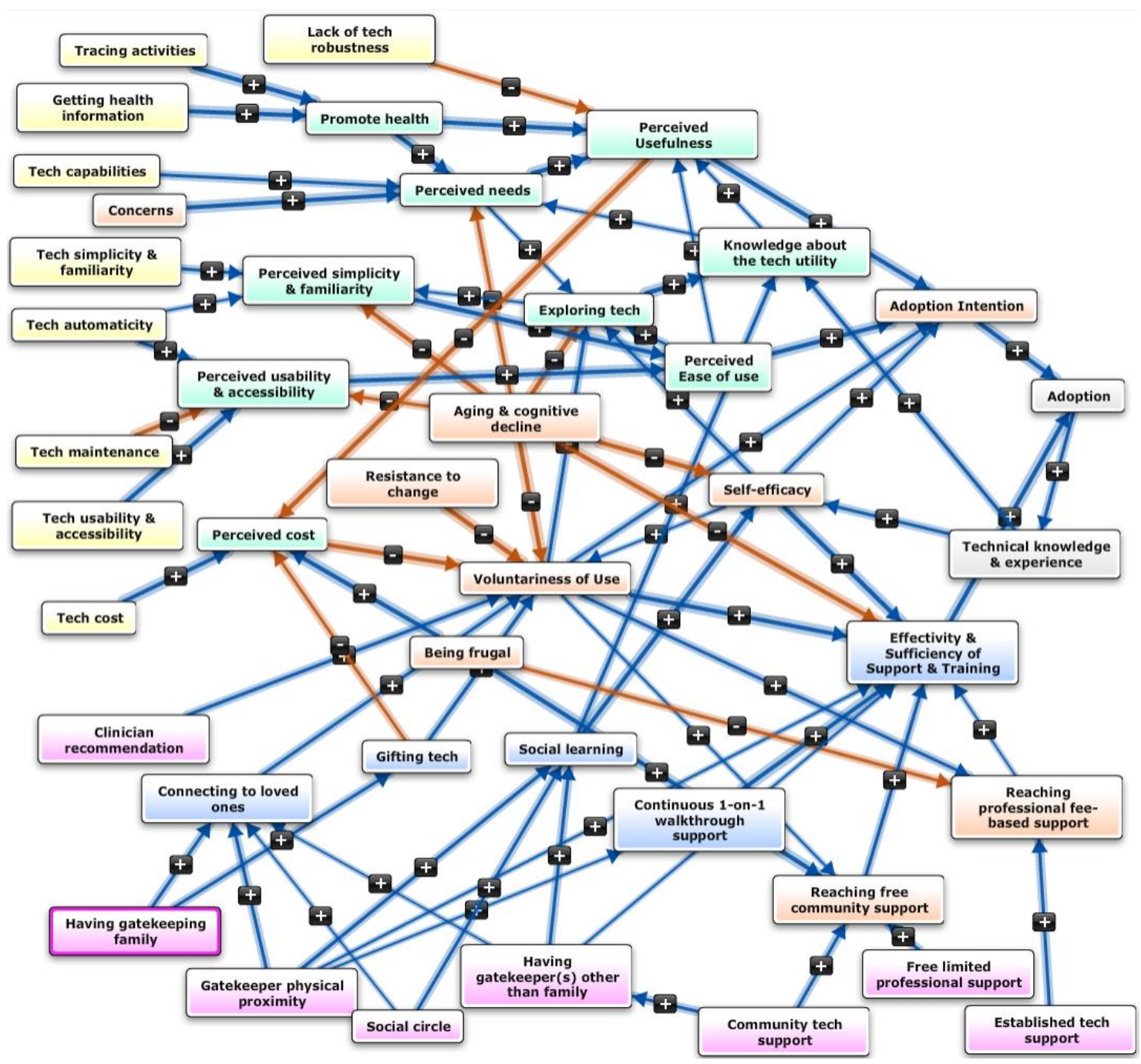

Figure H - 18 EW \#3 integrated \& standardized cognitive map 


\section{Elderly Woman \#4}

\begin{tabular}{|c|c|}
\hline Elderly Profile & p4 \\
\hline Interview Duration & $55 \mathrm{~min}$ \\
\hline Age & 94 \\
\hline Health Condition & Blind, Aphasia \\
\hline Living condition & Retirement Community \\
\hline Isolated & Y \\
\hline Attitude toward Tech & Very positive \\
\hline Self-efficacy & Good \\
\hline Experience & some \\
\hline Driven by & Learning, Staying healthy, Connecting to the world \\
\hline Has grandchildren? & $\mathrm{N}$ \\
\hline Has \& close to family & $\mathrm{N}$ \\
\hline Gatekeeper & Caregiver \\
\hline $\begin{array}{l}\text { Gatekeeper tech } \\
\text { savviness }\end{array}$ & little \\
\hline Degree of influence & some (FC, Suggestion) \\
\hline Influence & some \\
\hline $\begin{array}{l}\text { Perceived Usefulness } \\
\text { of the wearable }\end{array}$ & It could be useful \\
\hline $\begin{array}{l}\text { Perceived Ease of } \\
\text { Use of the wearable }\end{array}$ & It's really hard I can't see anything \\
\hline $\begin{array}{l}\text { Will she adopt if } \\
\text { free? }\end{array}$ & $y$ \\
\hline $\begin{array}{l}\text { Will she buy to } \\
\text { adopt? }\end{array}$ & $\mathrm{N}$ \\
\hline Dominant Theme 1 & $\begin{array}{l}\text { Tech enables this blind elderly, she enjoys and find way to connect } \\
\text { to the world outside through technology }\end{array}$ \\
\hline Dominant Theme 2 & Tech helps alleviating Social Isolation \\
\hline Dominant Theme 3 & $\begin{array}{l}\text { E finds tech empowering, especially that as a blind woman tech } \\
\text { helps her to read, write and do everyday activities }\end{array}$ \\
\hline Dominant Theme 4 & $\begin{array}{l}\text { Feels strong sense of need for technology, as tech helps her with } \\
\text { everyday life, and gives her sense of purpose and existence }\end{array}$ \\
\hline
\end{tabular}

Table H - 4 Elderly woman \#4 profile 


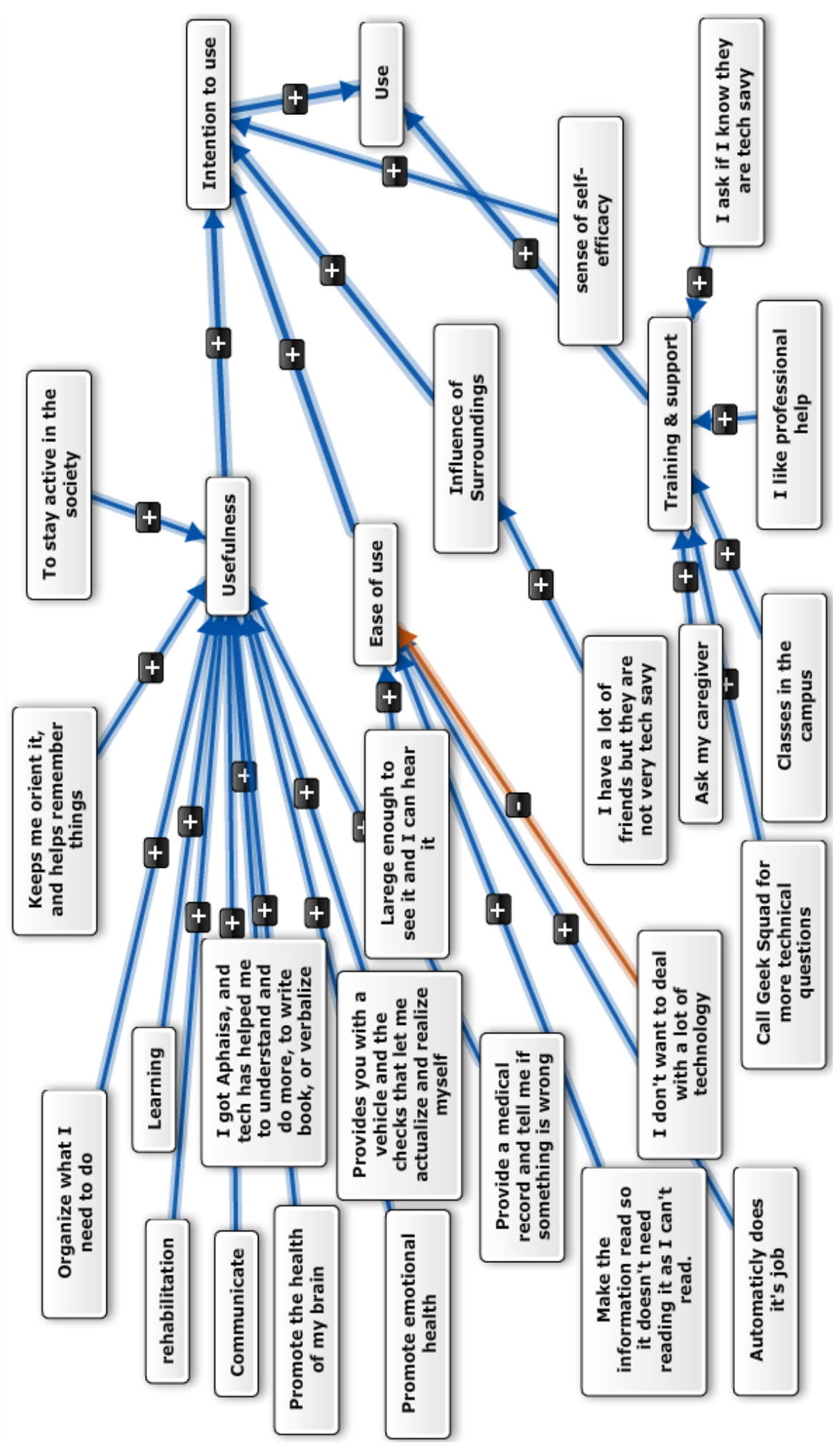

Figure H - 19 EW \#4 initial cognitive map 


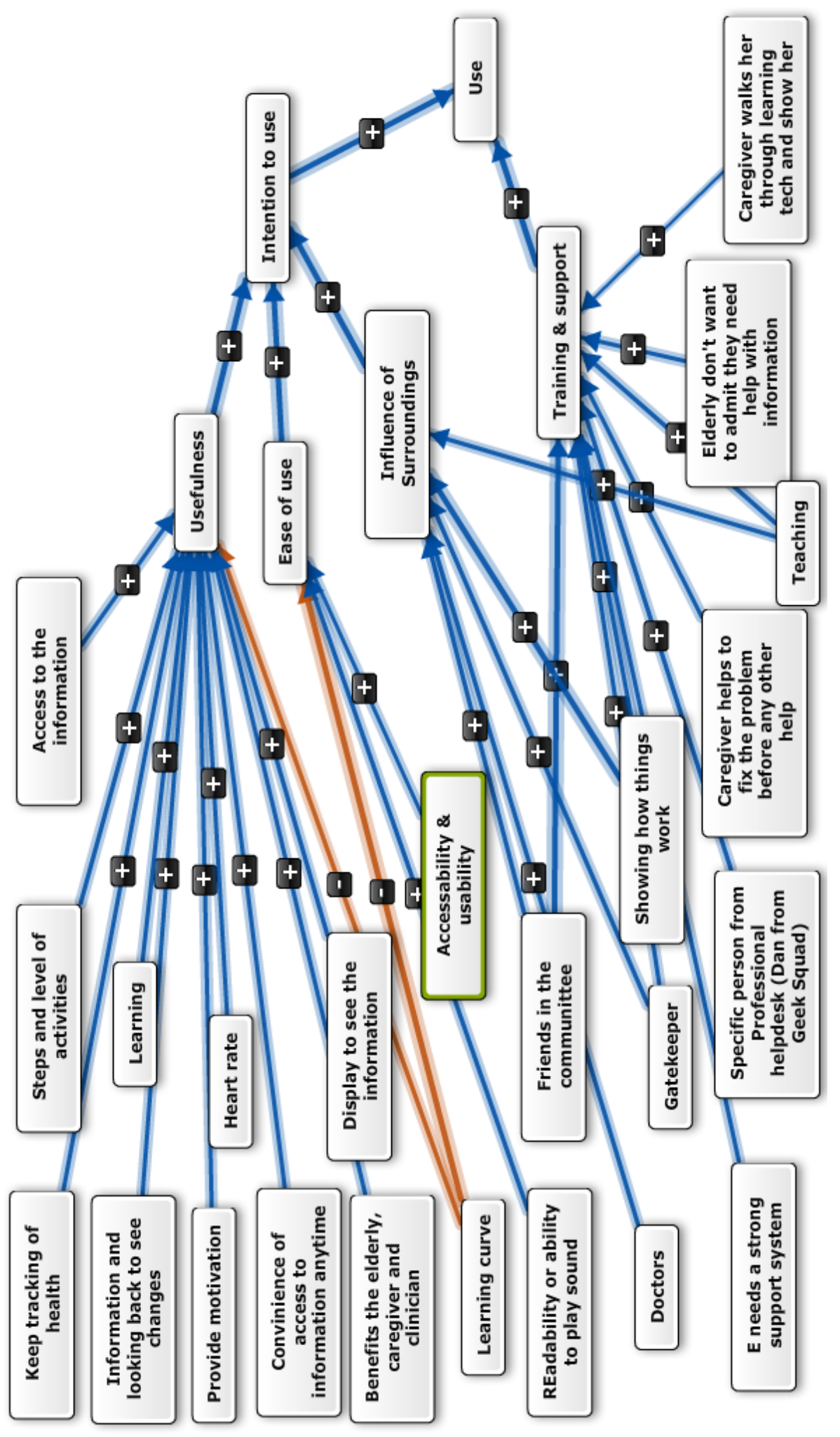

Figure H - 20 Gatekeeper \#4 initial cognitive map 


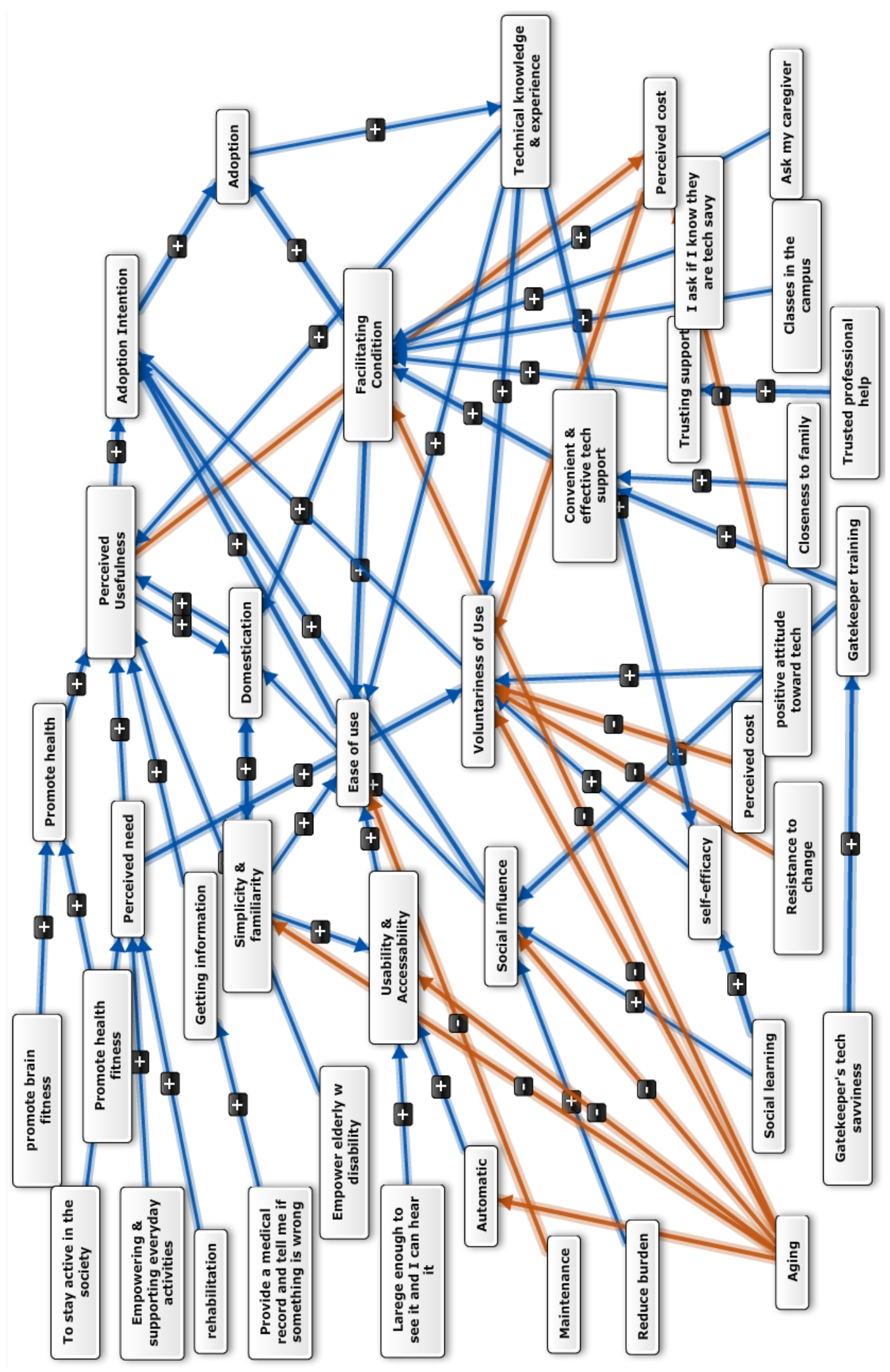

Figure H - 21 Elderly Gatekeeper pair \#4 integrated cognitive map

474 


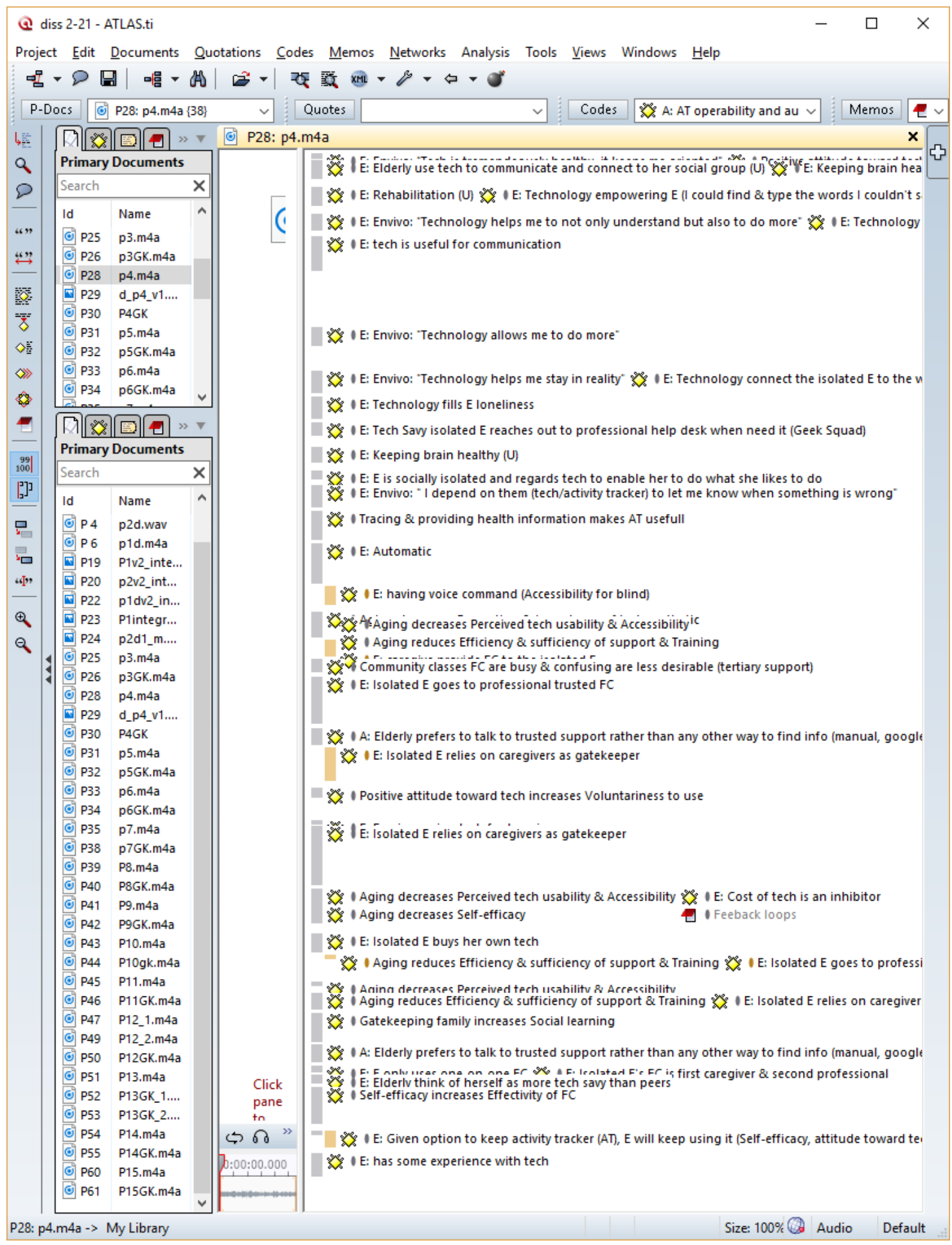

Figure H - 22 EW\#4 interview open coding in Atlas.ti 


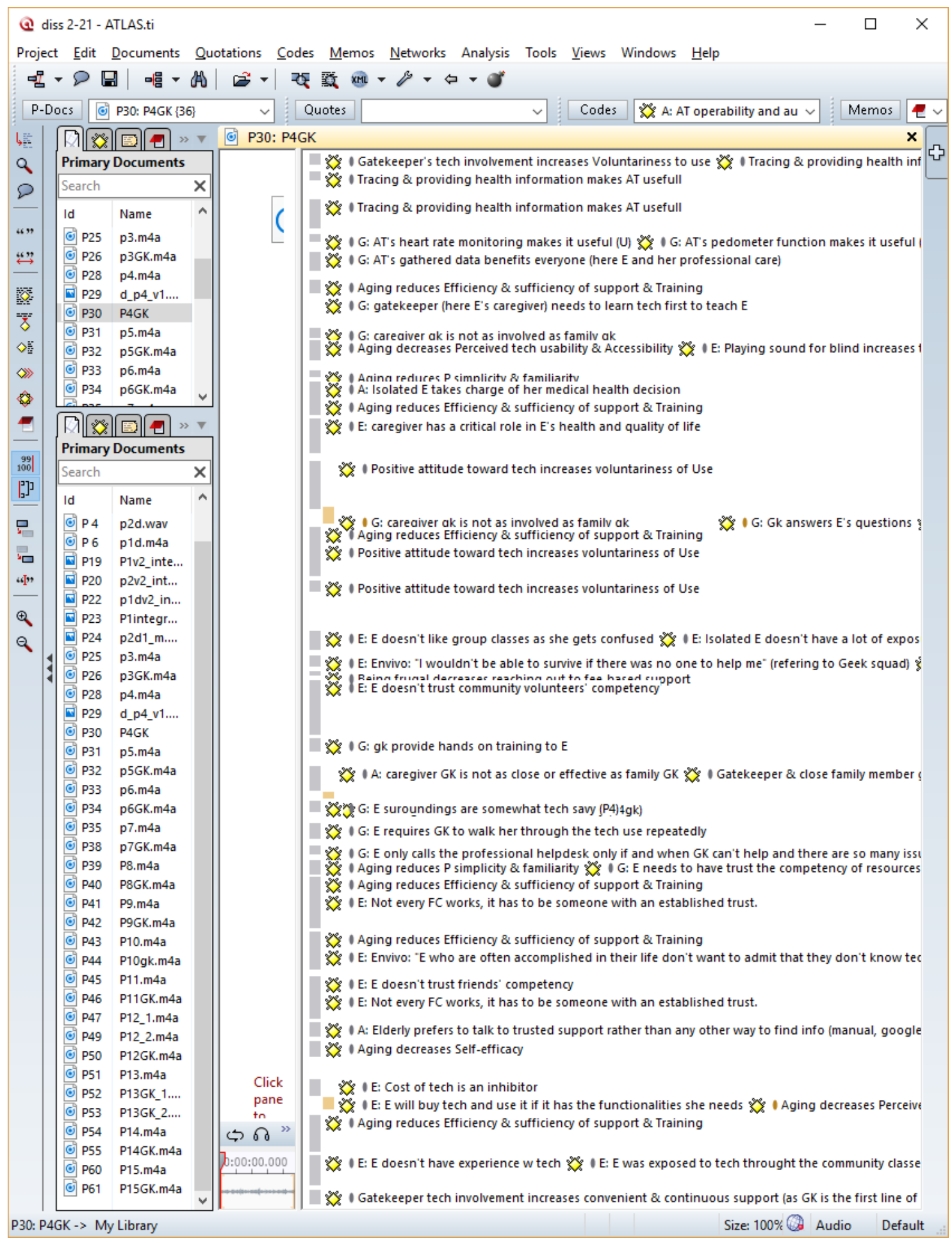

Figure H - 23 Gatekeeper \#4 interview open coding in Atlas.ti 


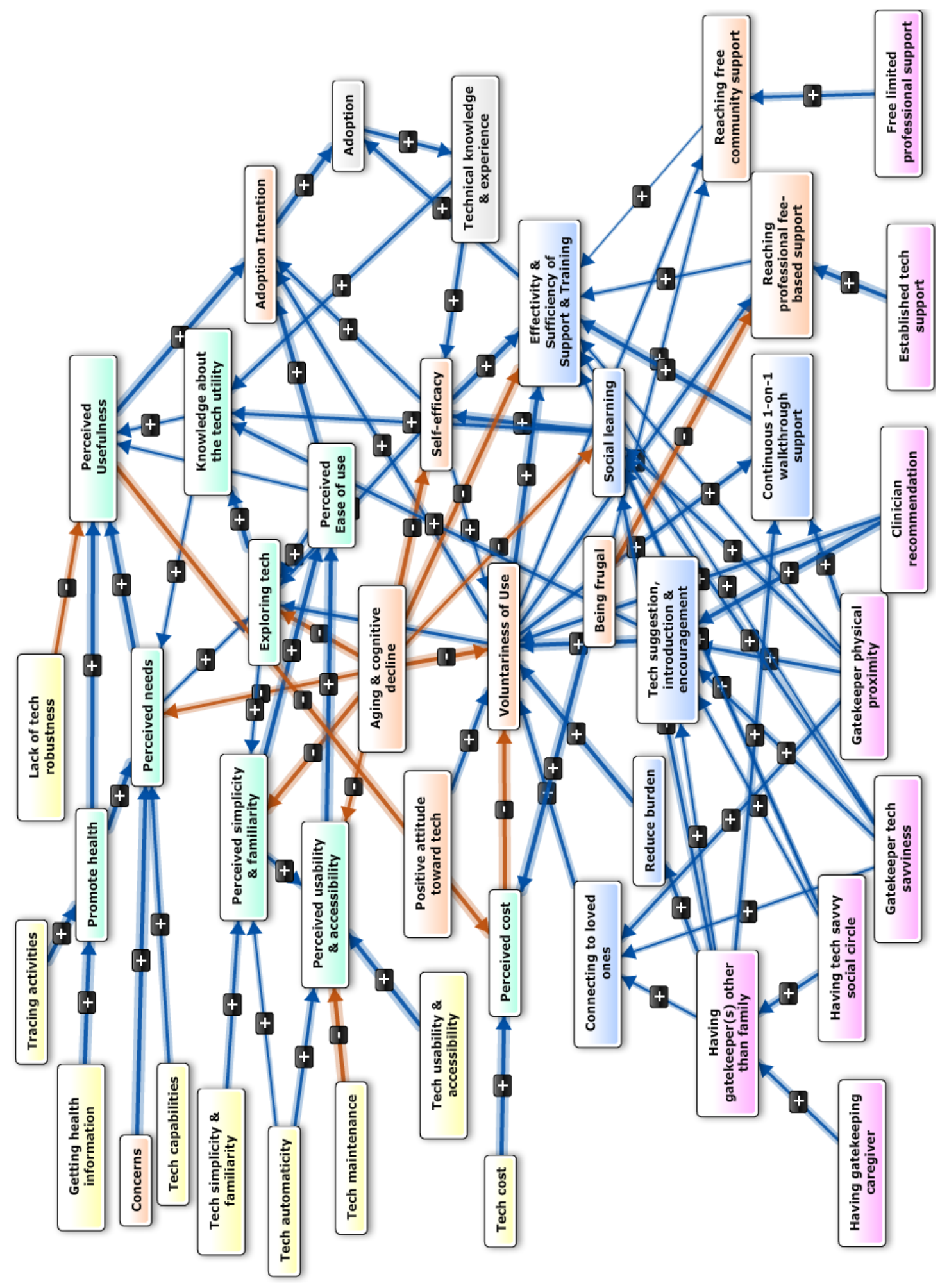

Figure H - 24 EW \#4 integrated \& standardized cognitive map 


\section{Elderly Woman \#5}

\begin{tabular}{|c|c|}
\hline Elderly Profile & p5 \\
\hline Interview Duration & $47 \mathrm{~min}$ \\
\hline Age & 90 \\
\hline Health Condition & Ok but rapidly cognitively and physically declining \\
\hline Living condition & Retirement Community \\
\hline Isolated & $\mathrm{N}$ \\
\hline Attitude toward Tech & Positive \\
\hline Self-efficacy & OK \\
\hline Experience & some \\
\hline Driven by & Cost, stay connected to especially family \& somewhat friends. \\
\hline Has grandchildren? & $\mathrm{N}$ \\
\hline Has \& close to family & $\mathrm{Y}$ \\
\hline Gatekeeper & Daughter (first line of tech support) (52 min) \\
\hline $\begin{array}{l}\text { Gatekeeper tech } \\
\text { savviness }\end{array}$ & $\begin{array}{l}\text { moderate (but has a son who is far but make suggestion and buys } \\
\text { tech for } E \text { ) }\end{array}$ \\
\hline Degree of influence & $\mathrm{Y}$ (all by GK, and suggestion and gifting by the remote) \\
\hline Influence & Y (All but gatekeeper is financially constraint) \\
\hline $\begin{array}{l}\text { Perceived Usefulness } \\
\text { of the wearable }\end{array}$ & It doesn't work \\
\hline $\begin{array}{l}\text { Perceived Ease of Use } \\
\text { of the wearable }\end{array}$ & It's not waterproof and doesn't show information \\
\hline Will she adopt if free? & $\mathrm{N}$ \\
\hline Will she buy to adopt? & $\mathrm{N}$ \\
\hline Note & $\begin{array}{l}\text { Although E has a positive view of tech she only uses tech when } \\
\text { it's accompanied with a lot of continuous support and training }\end{array}$ \\
\hline Dominant Theme 1 & $\begin{array}{l}\mathrm{CD} \text { (Cognitive Decline) is rapidly reducing E's willingness to try } \\
\text { new tech }\end{array}$ \\
\hline Dominant Theme 2 & $\begin{array}{l}\text { Aging increases financial prudence in } \mathrm{E} \text { and she just want to read, } \\
\text { enjoy and connect }\end{array}$ \\
\hline Dominant Theme 3 & $\begin{array}{l}\text { Although cost is the biggest inhibitor, when savy family member } \\
\text { suggest and often buys tech for her, she pays for it and uses it }\end{array}$ \\
\hline Dominant Theme 4 & $\begin{array}{l}\text { She keeps complaining that she doesn't remember things and } \\
\text { needs a lot of practice and continuous support to use tech }\end{array}$ \\
\hline $\begin{array}{l}\text { Dominant Theme } \\
\text { raised by GK }\end{array}$ & G: E heavily relies on habit when it comes to the everyday activity \\
\hline In vivo & $\begin{array}{l}\text { E: In vivo "I would love it if I had a little support system" (Minute } \\
18 \text { on p5 audio) }\end{array}$ \\
\hline
\end{tabular}




\begin{tabular}{|l|l|} 
Discovery & $\begin{array}{l}\text { G: When it comes to GK influence proximity and having daughter } \\
\text { trumps tech savviness (GK i.e. daughter is the primary GK since } \\
\text { lives close, even though son is more tech savvy and gift techs } \\
\text { from distance) }\end{array}$ \\
\hline
\end{tabular}

Table H - 5 Elderly woman \#5 profile

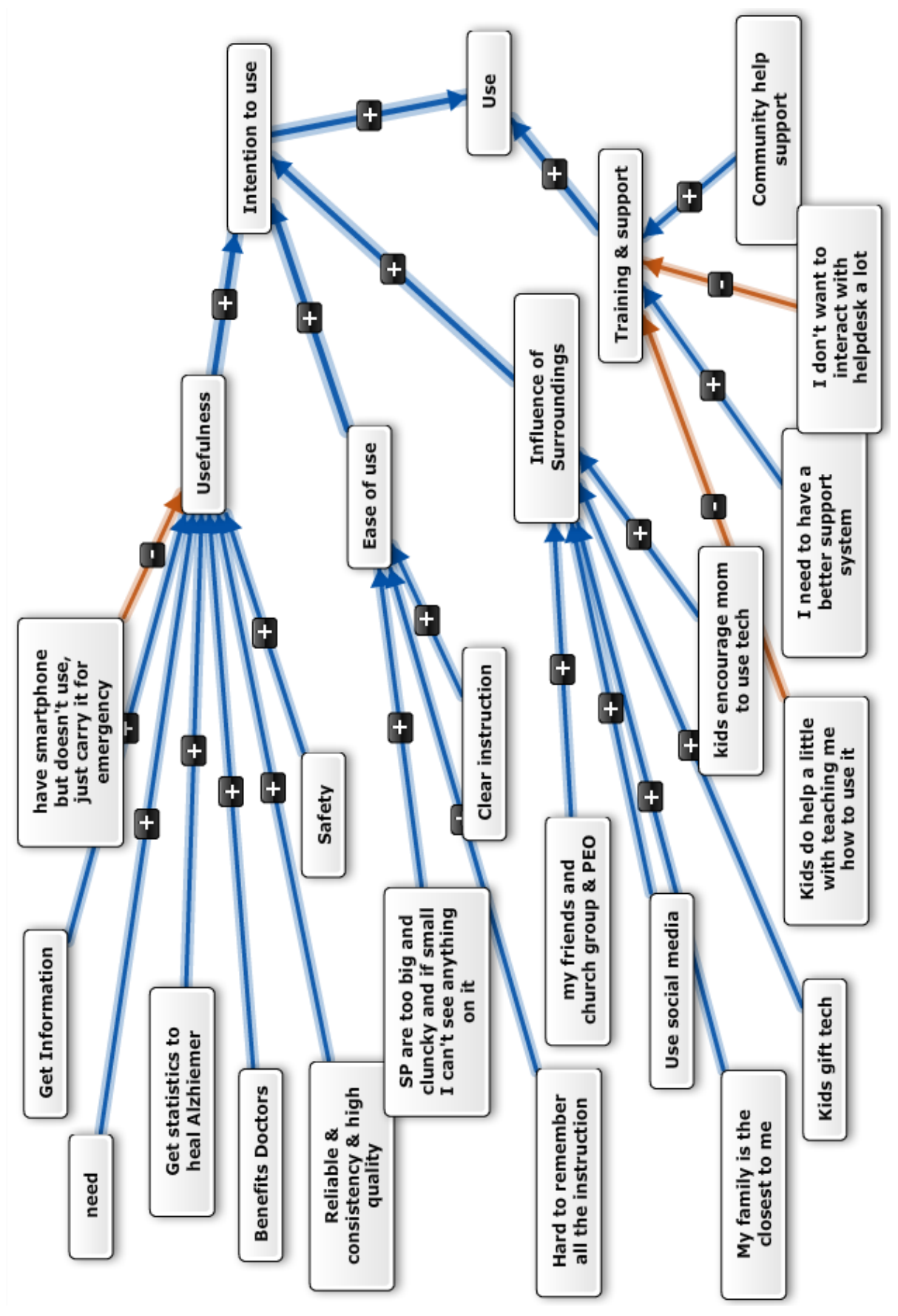

Figure H - 25 EW \#5 initial cognitive map 


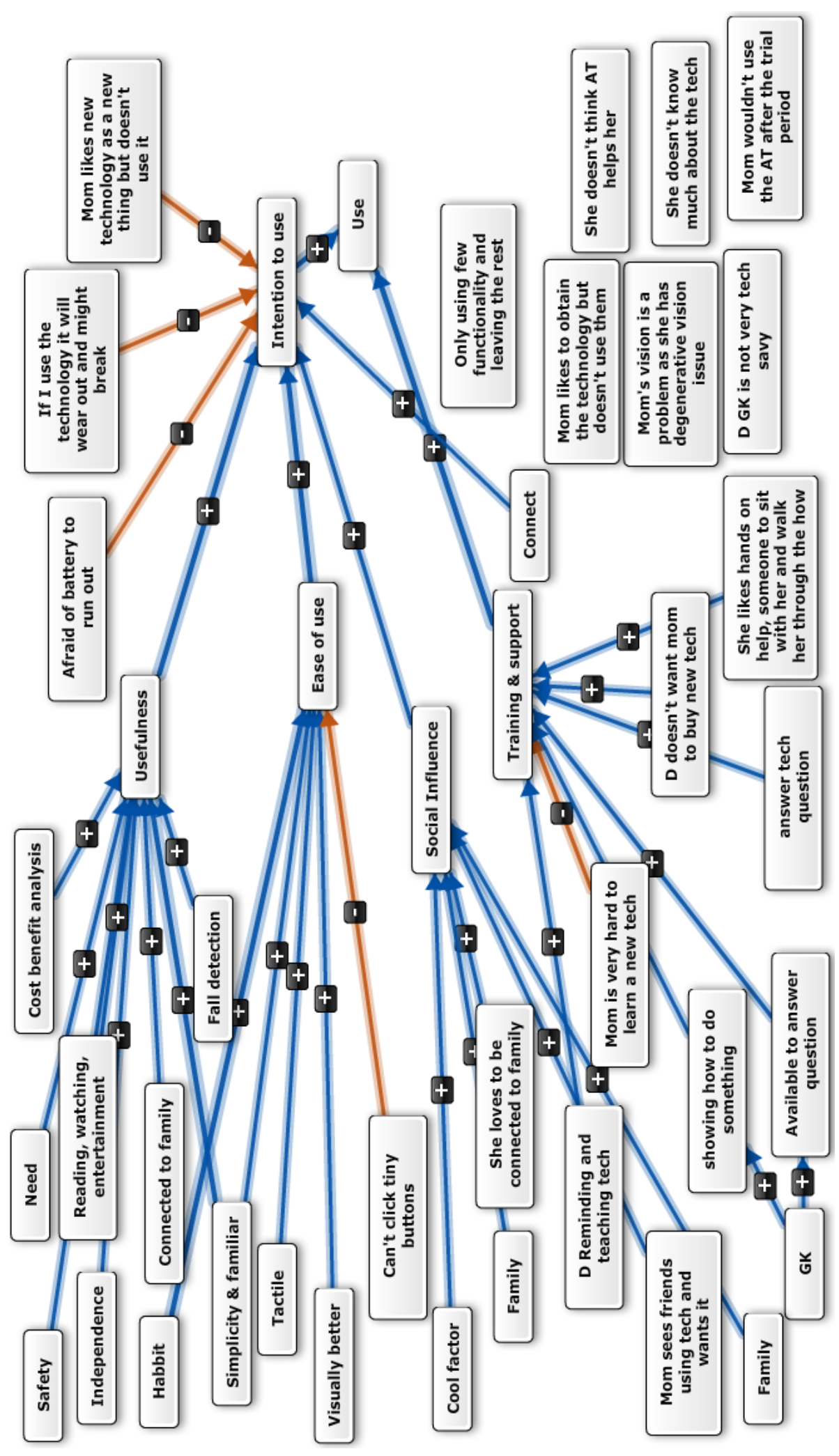

Figure H - 26 Gatekeeper \#5 initial cognitive map

480 


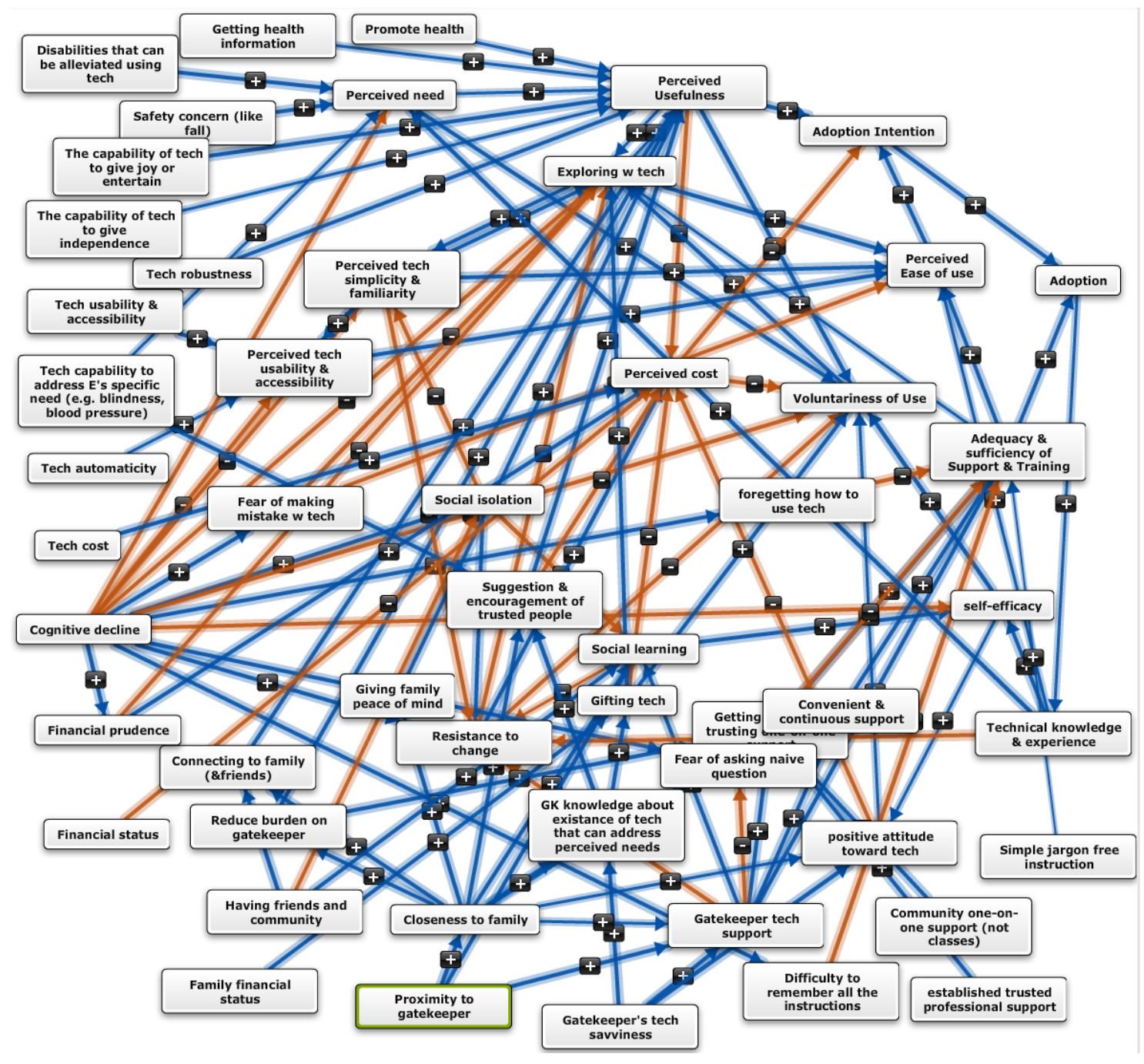

Figure H - 27 Elderly Gatekeeper pair \#5 integrated cognitive map 


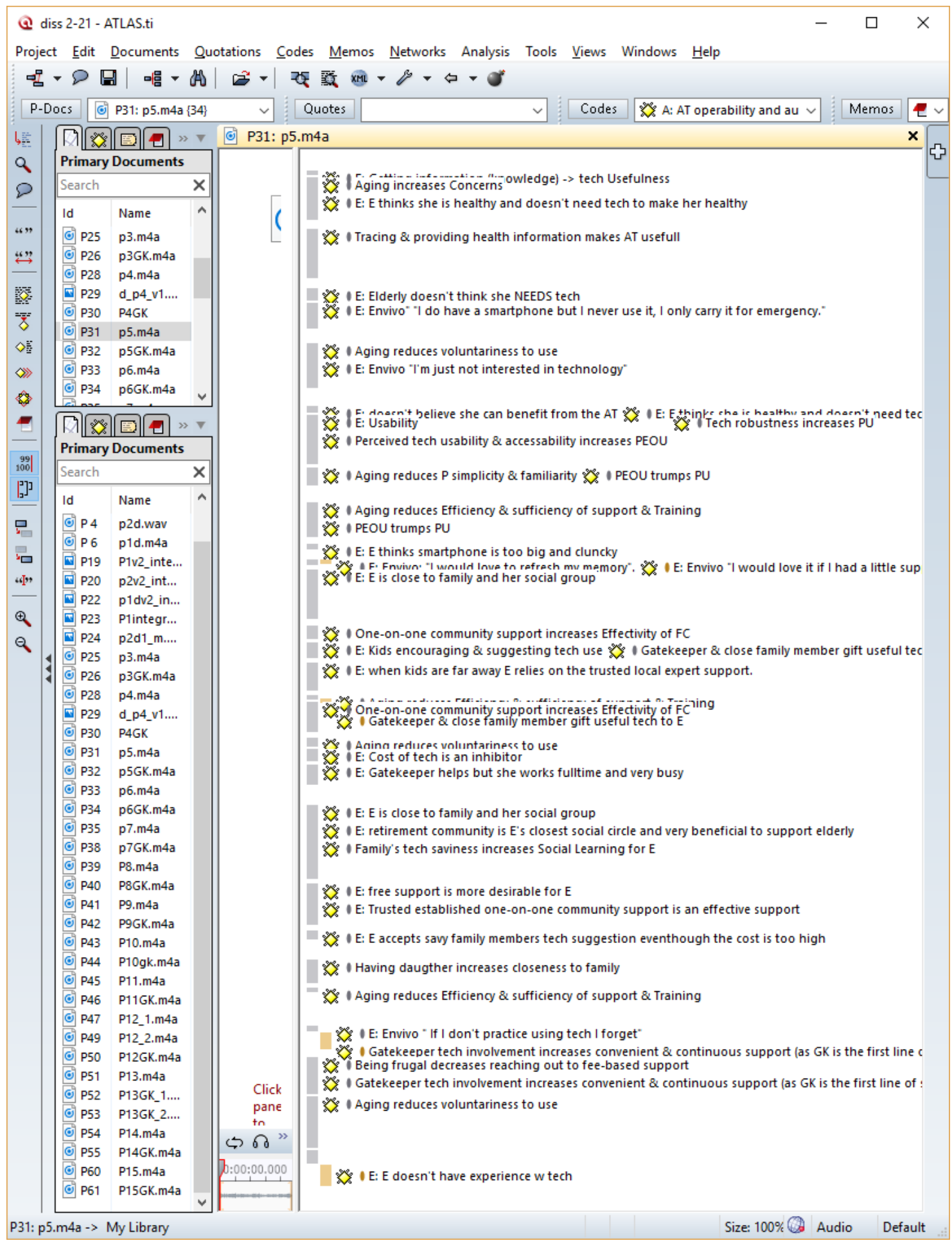

Figure H - 28 EW\#5 interview open coding in Atlas.ti 


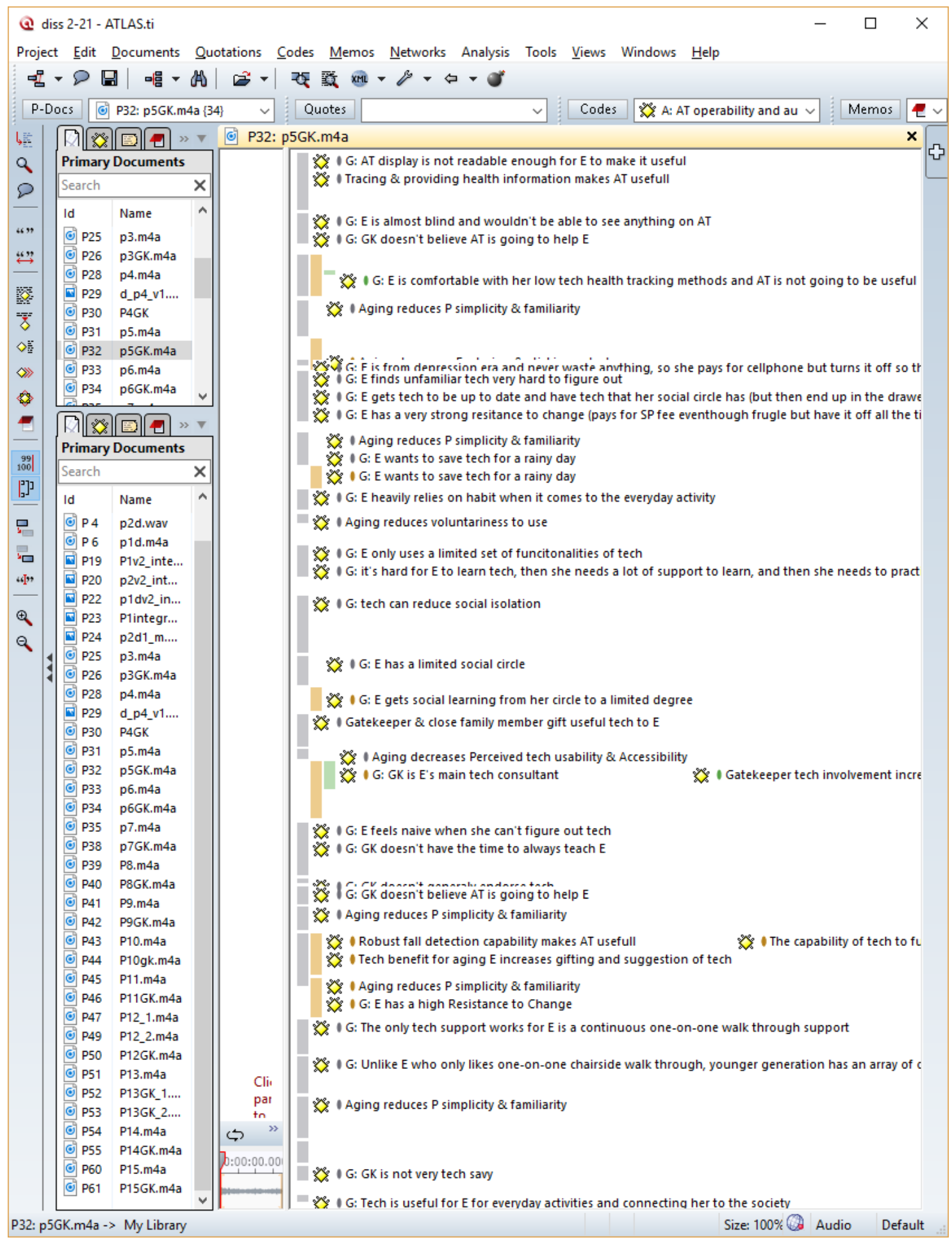

Figure H - 29 Gatekeeper \#5 interview open coding in Atlas.ti 


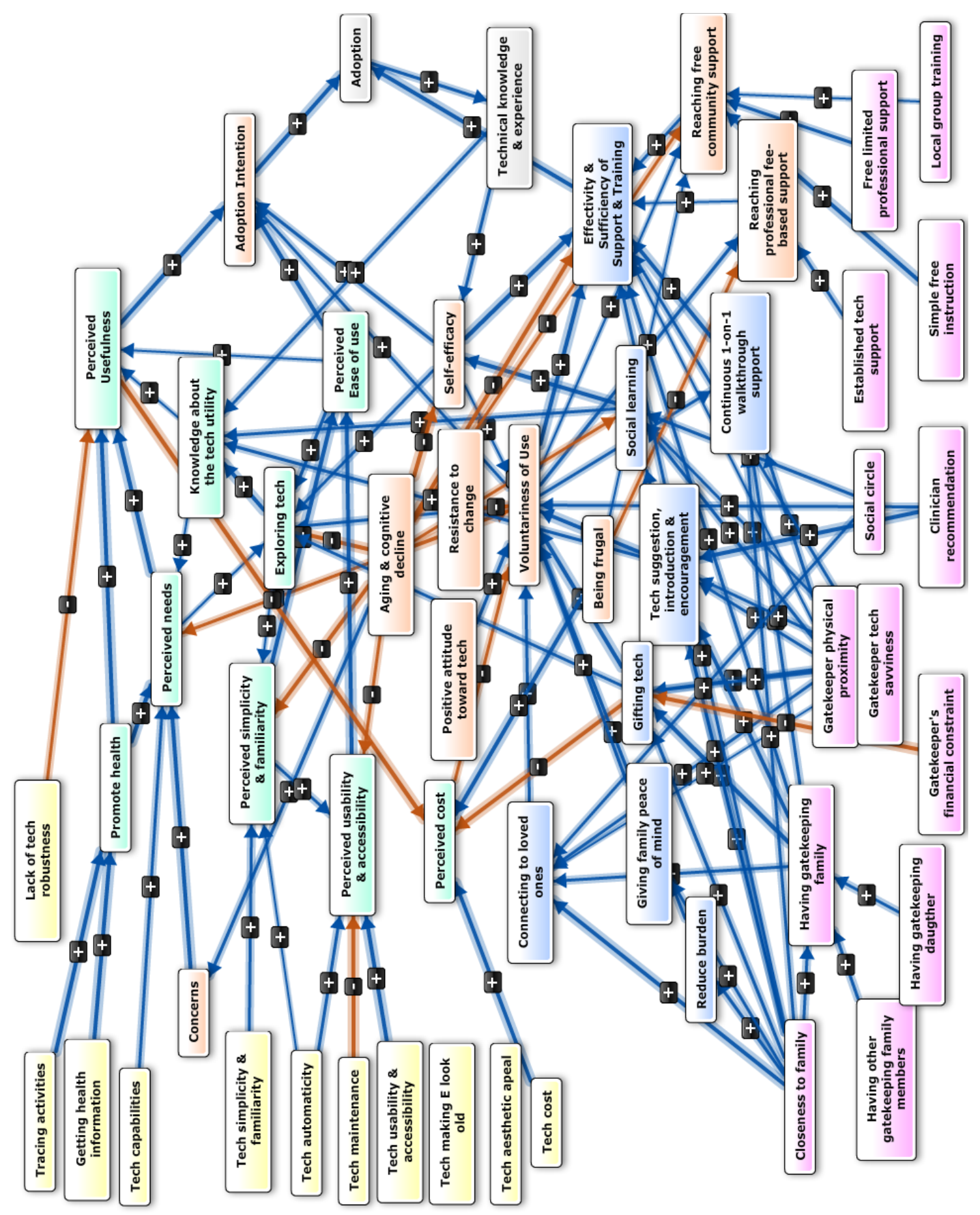

Figure H - 30 EW \#5 integrated \& standardized cognitive map 


\section{Elderly Woman \#6}

\begin{tabular}{|c|c|}
\hline Elderly Profile & p6 \\
\hline Interview Duration & $49 \mathrm{~min}$ \\
\hline Age & 95 \\
\hline Health Condition & hearing and vison loss and declining \\
\hline Living condition & Retirement Community \\
\hline Attitude toward Tech & OK \\
\hline Self-efficacy & $\mathrm{N}$ \\
\hline Experience & No \\
\hline Driven by & Cost, pressing need \\
\hline Has grandchildren? & $\mathrm{N}$ \\
\hline Has \& close to family & $\mathrm{N}$ \\
\hline Gatekeeper & Friend (little influence) (36 min) \\
\hline $\begin{array}{l}\text { Gatekeeper tech } \\
\text { savviness }\end{array}$ & $\mathrm{N}$ \\
\hline Degree of influence & Low \\
\hline Influence & $\begin{array}{l}\text { Little (E: E with no family is influenced by friends through social } \\
\text { learning) }\end{array}$ \\
\hline $\begin{array}{l}\text { Perceived Usefulness } \\
\text { of the wearable }\end{array}$ & It's not reliable \\
\hline $\begin{array}{l}\text { Perceived Ease of } \\
\text { Use of the wearable }\end{array}$ & It's too clunky \\
\hline $\begin{array}{l}\text { Will she adopt if } \\
\text { free? }\end{array}$ & $\mathrm{N}$ \\
\hline $\begin{array}{l}\text { Will she buy to } \\
\text { adopt? }\end{array}$ & $\mathrm{N}$ \\
\hline Dominant Theme 1 & $\begin{array}{l}\text { Elderly although says has a positive attitude toward tech has no } \\
\text { desire to try tech and CD is only making this high resistance to } \\
\text { change higher. }\end{array}$ \\
\hline \multirow[t]{2}{*}{ Dominant Theme 2} & $\begin{array}{l}\text { E is intimidated by tech and fear of breaking it, she states that she } \\
\text { can't figure our tech on her own }\end{array}$ \\
\hline & $\begin{array}{l}\text { GK who is an EW herself }(93) \text { and both (GK \& E) are only using } \\
\text { simple and familiar tech that they had adopted long ago when they } \\
\text { were cognitively more healthy }\end{array}$ \\
\hline Dominant Theme 3 & $\begin{array}{l}\text { There are hardly any tech gatekeeping in E's life except social } \\
\text { learning from limited social circle in her retirement community }\end{array}$ \\
\hline Dominant Theme 4 & $\begin{array}{l}\text { There is a huge distance between Adoption Intention and Adoption, } \\
\text { e.g. she has been interested in getting a tablet for a while but have } \\
\text { not acted on it }\end{array}$ \\
\hline
\end{tabular}




\begin{tabular}{|c|c|}
\hline & $\begin{array}{l}\text { Not having a very tech savy gatekeeper and family, there are hardly } \\
\text { any influences important in tech adoption; as the result tech has } \\
\text { hardly a place in E's life. This further contributes to E's social } \\
\text { isolation }\end{array}$ \\
\hline Discovery & $\begin{array}{l}\text { E: The huge hurdle of ETA can only be overcome by tech addressing } \\
\text { an essential need that can't be addressed otherwise (w existing } \\
\text { ways) and is made known and advocated and supported to E }\end{array}$ \\
\hline GK & $\begin{array}{l}\text { G: GK who is also an E, has a negative view of tech, she thinks both } \\
\text { her and } E \text { under study are healthy and can track their own health } \\
\text { and don't need the tech to keep them healthy }\end{array}$ \\
\hline Discovery & $\begin{array}{l}\text { Interestingly enough the GK was also an EW ( } 93 \text { yrs friend in the } \\
\text { retirement community), while she was keep insisting that the } \\
\text { reason behind her lack of adoption was lack of need, she mentioned } \\
\text { that she is terrible with technology and can never figure out how to } \\
\text { use tech and always need a walk through; it seems that while PEOU } \\
\text { is low, E indicates that PU is low; this could be due to being too } \\
\text { proud to admit that she can't figure out tech, or look naive. PEOU } \\
\text { remains to be the most important factor for EWHTA }\end{array}$ \\
\hline
\end{tabular}

Table H - 6 Elderly woman \#6 profile 


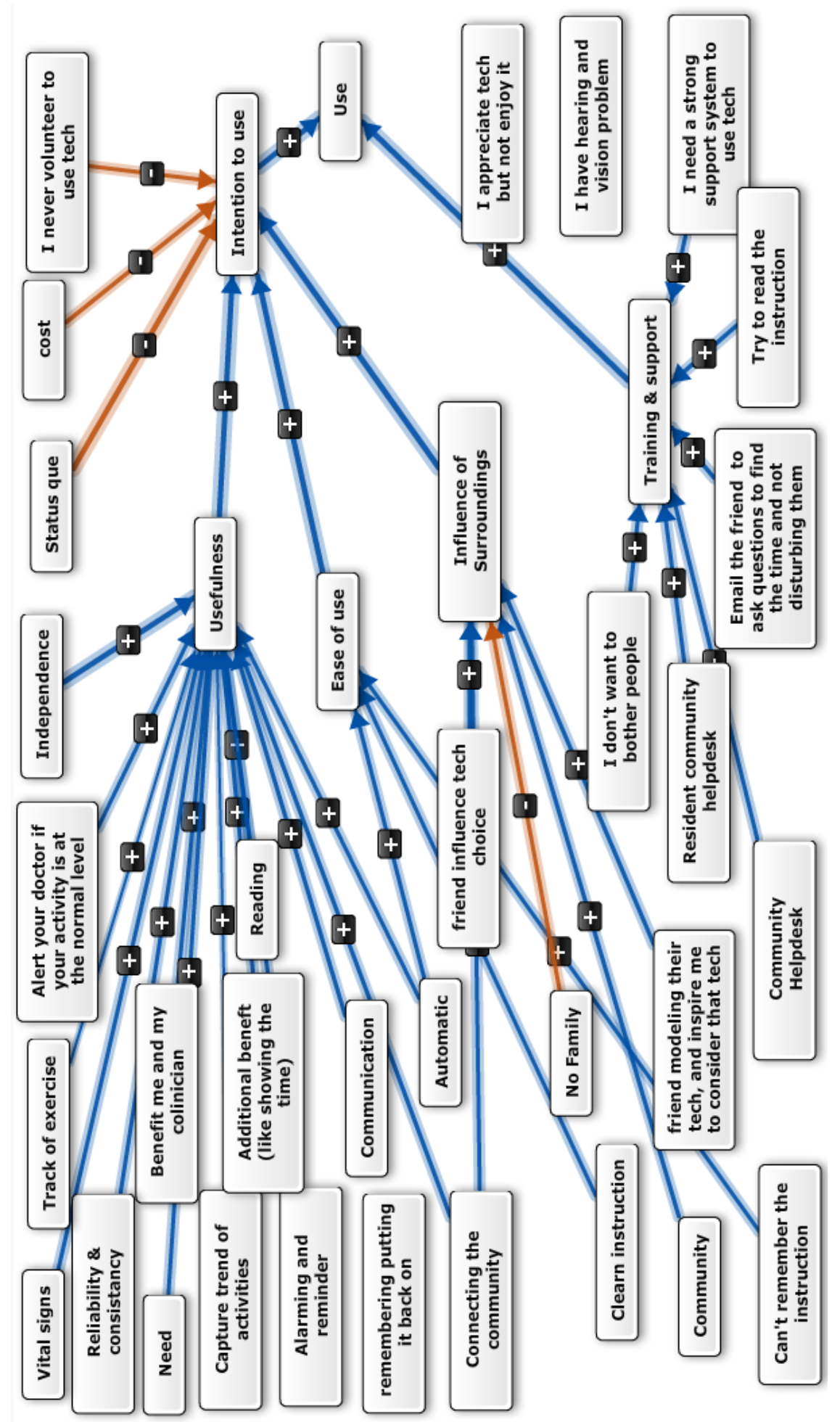

Figure H - 31 EW \#6 initial cognitive map 


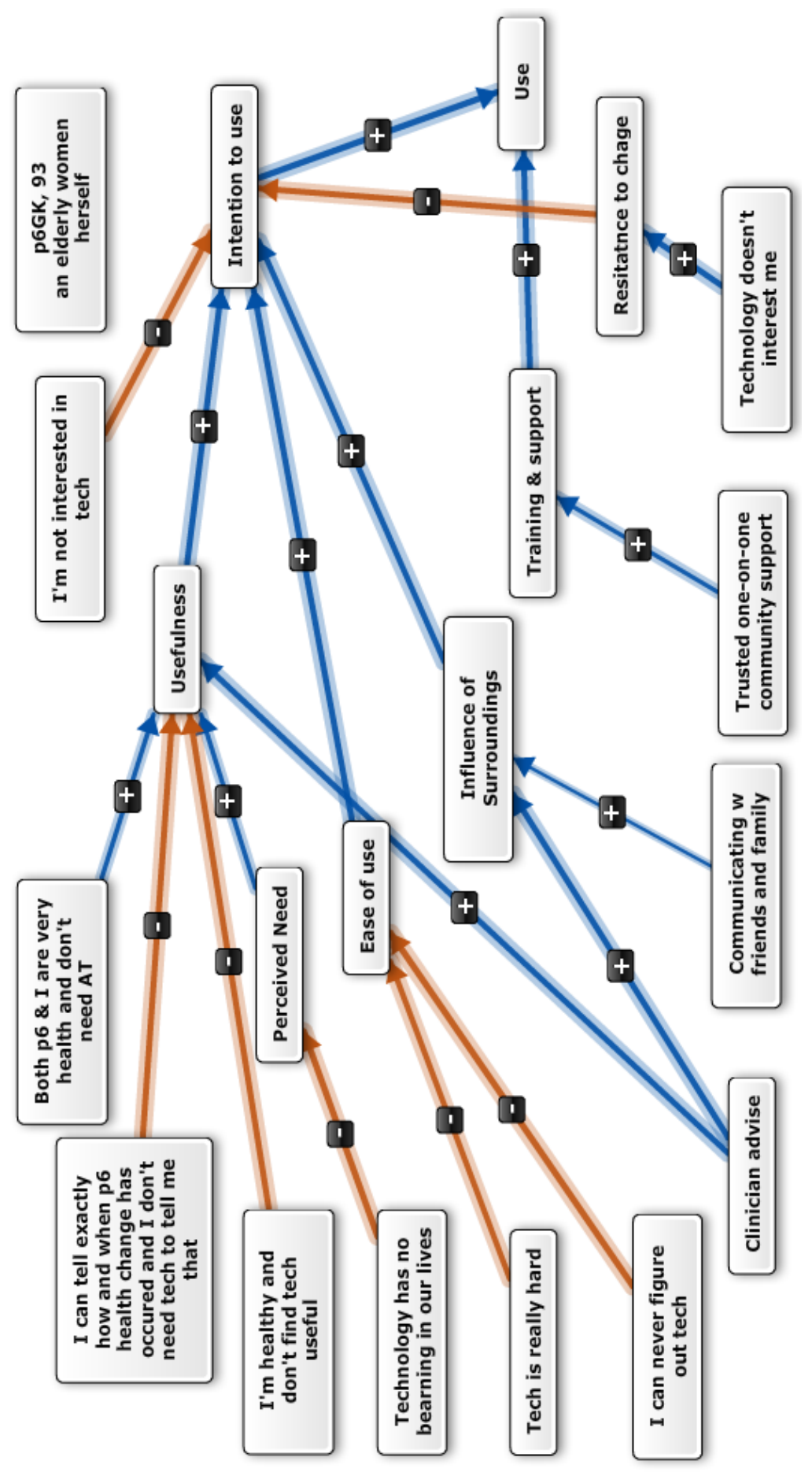

Figure H - 32 Gatekeeper \#6 initial cognitive map 


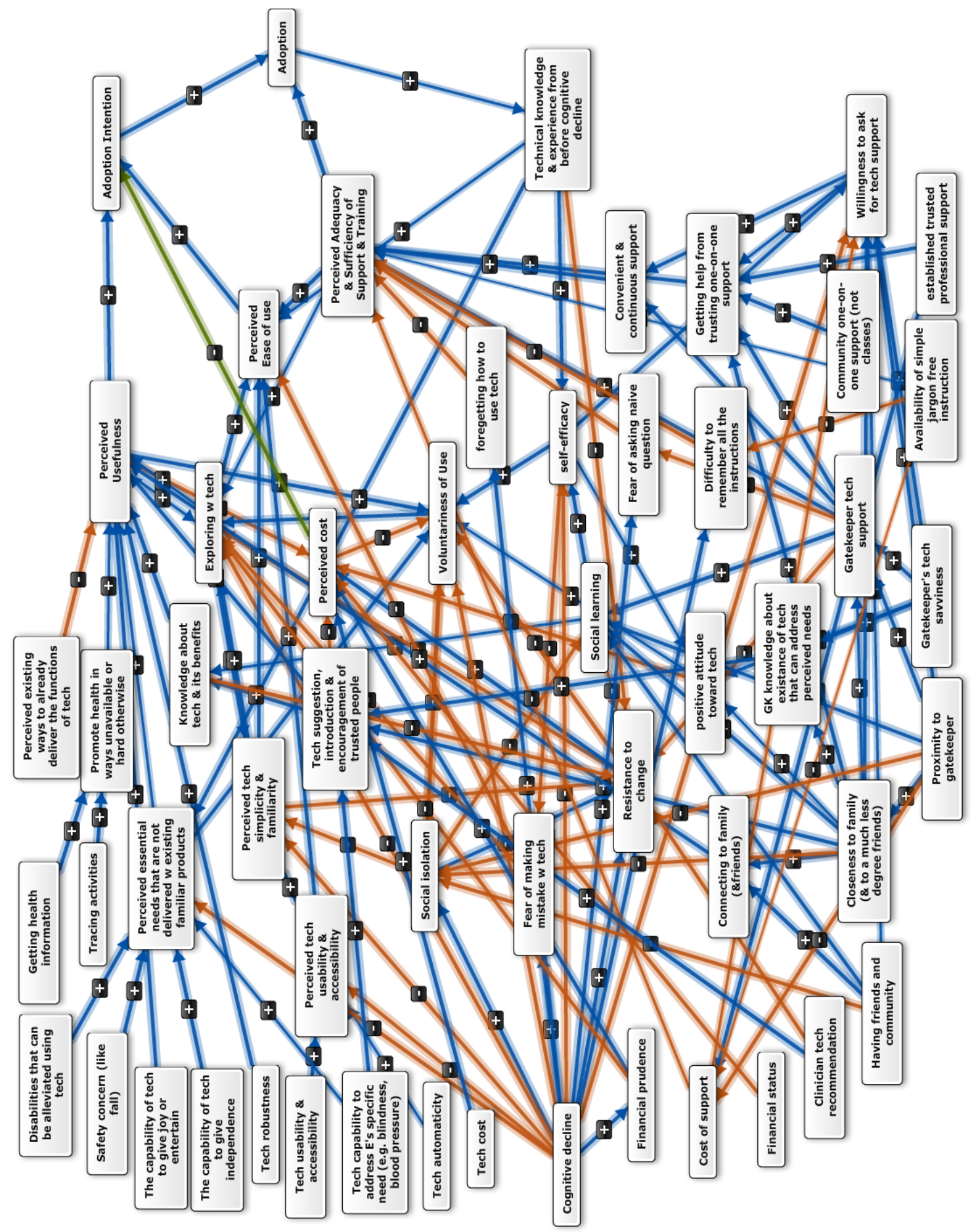

Figure H - 33 Elderly Gatekeeper pair \#6 integrated cognitive map 


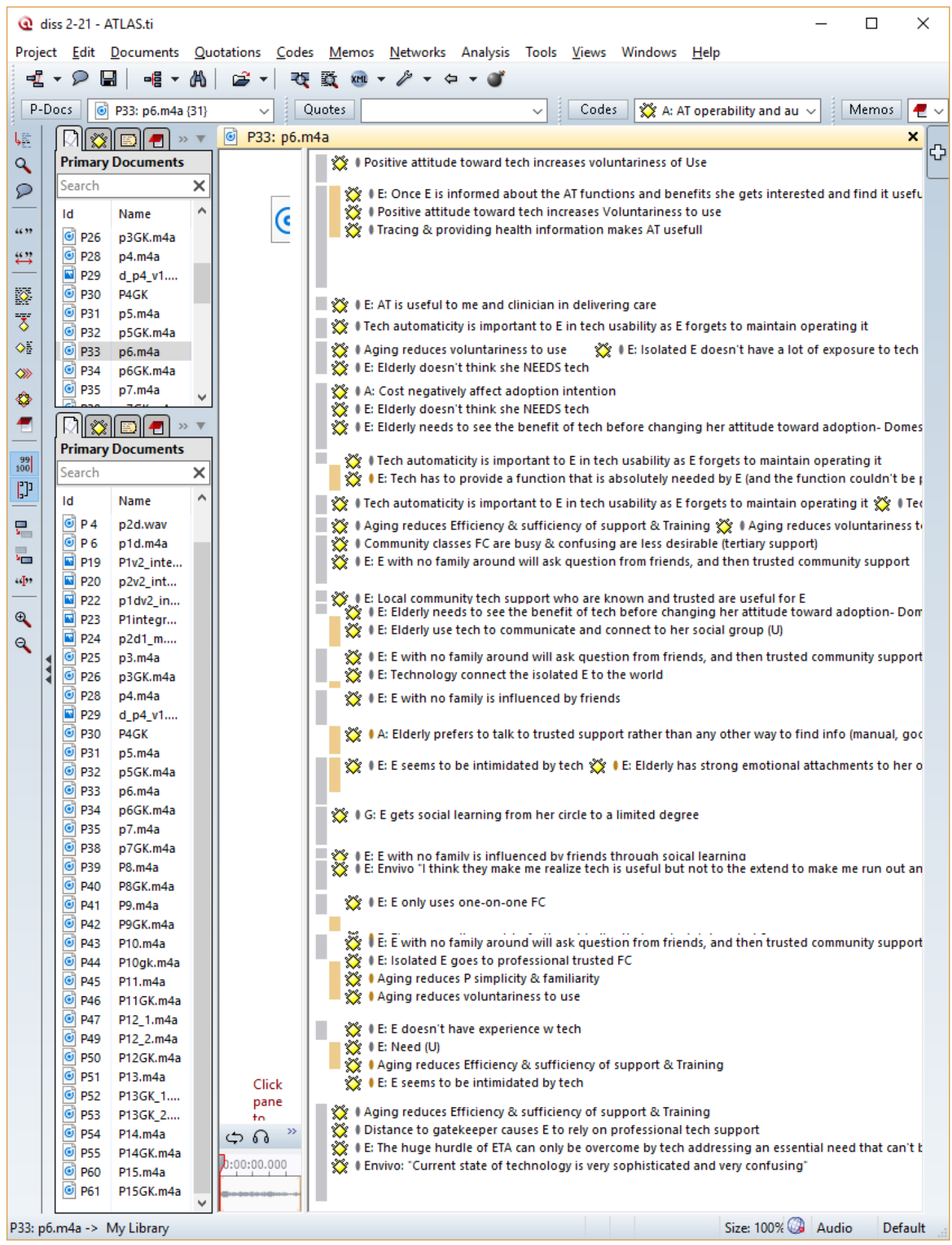

Figure H - 34 EW\#6 interview open coding in Atlas.ti 


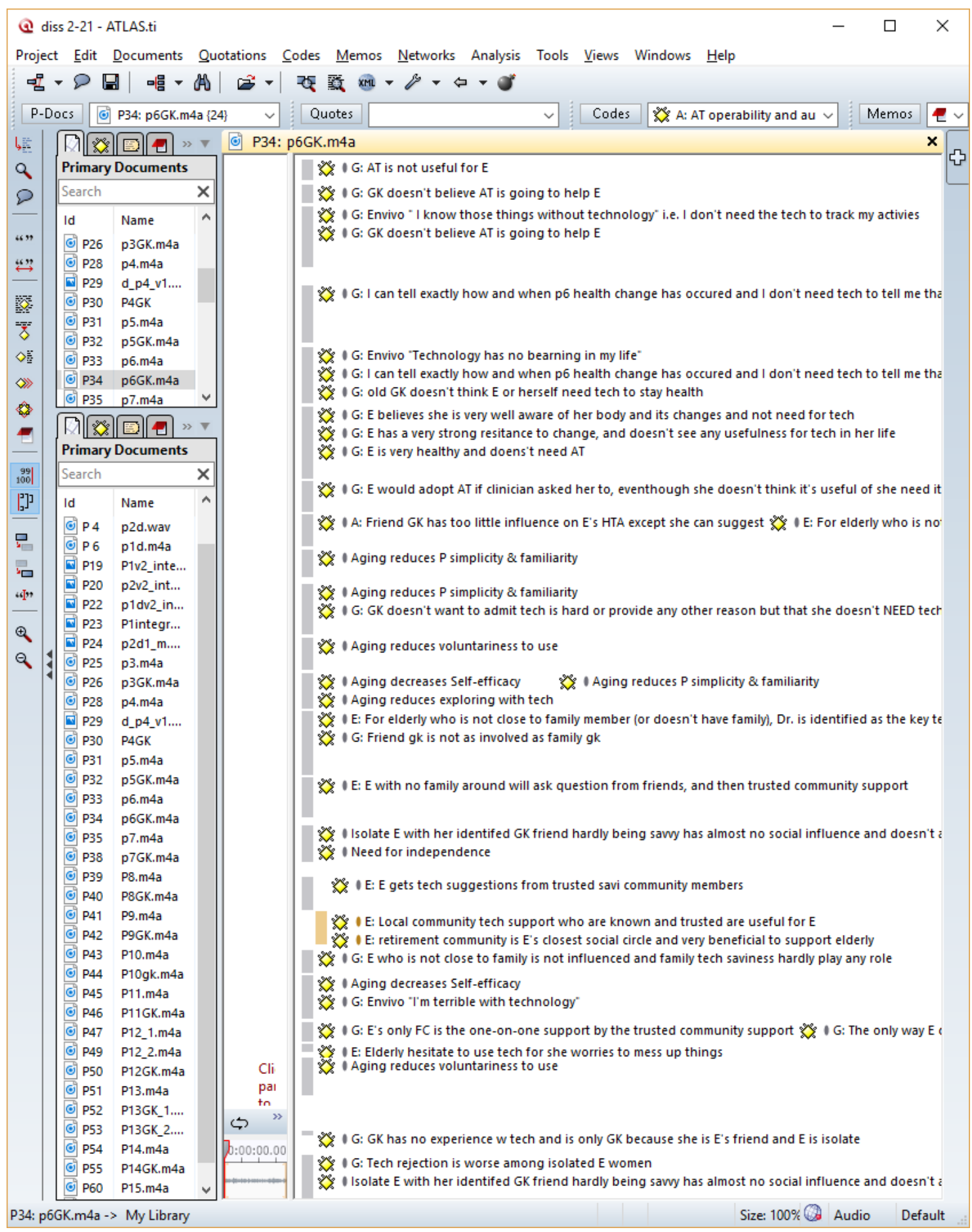

Figure H - 35 Gatekeeper \#6 interview open coding in Atlas.ti 


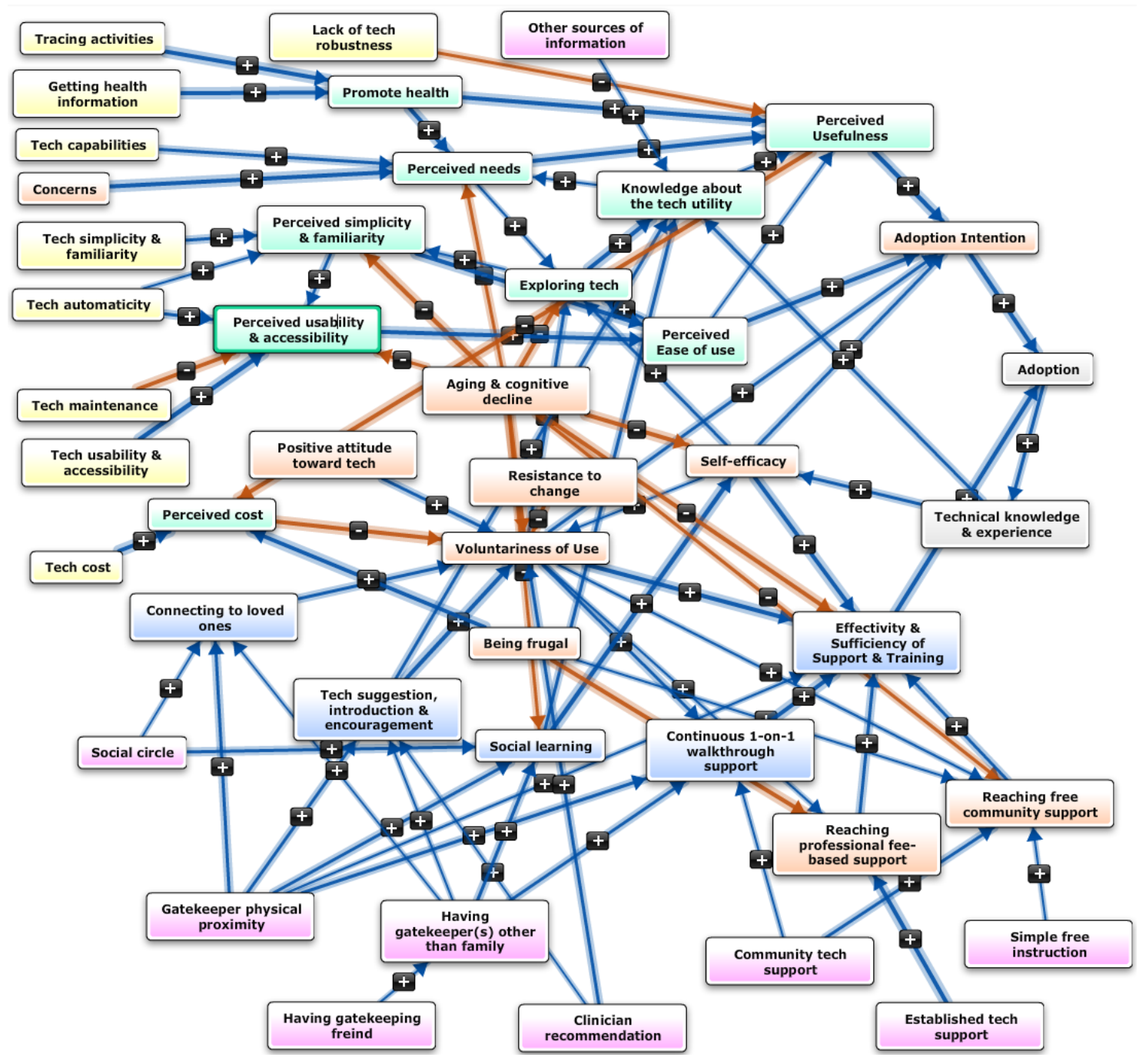

Figure H - 36 EW \#6 integrated \& standardized cognitive map 


\section{Elderly Woman \#7}

\begin{tabular}{|c|c|}
\hline Elderly Profile & p7 \\
\hline Interview Duration & $79 \mathrm{~min}$ \\
\hline Age & 82 \\
\hline Health Condition & Familial Tremor \\
\hline Living condition & Retirement Community \\
\hline Attitude toward Tech & Positive \\
\hline Self-efficacy & $\mathrm{Y}$ \\
\hline Experience & $\mathrm{Y}$ \\
\hline Driven by & Social \\
\hline Has grandchildren? & Y \\
\hline Has \& close to family & $\sim$ \\
\hline Gatekeeper & Daughter (46 min) \\
\hline $\begin{array}{l}\text { Gatekeeper tech } \\
\text { savviness }\end{array}$ & y \\
\hline Degree of influence & $\begin{array}{l}\text { Low. } E \text { is not close to her family and as aging, gets more depressed } \\
\text { and distance herself from family. }\end{array}$ \\
\hline Influence & $\begin{array}{l}\text { E doesn't reach out to the tech savvy family and refuse to accept } \\
\text { gift or suggestion. (however Connecting and social learning } \\
\text { influences are still present according to GKs but not E) }\end{array}$ \\
\hline $\begin{array}{l}\text { Perceived Usefulness } \\
\text { of the wearable }\end{array}$ & It's not good for the prime time yet \\
\hline $\begin{array}{l}\text { Perceived Ease of } \\
\text { Use of the wearable }\end{array}$ & It's OK \\
\hline $\begin{array}{l}\text { Will she adopt if } \\
\text { free? }\end{array}$ & $\mathrm{N}$ \\
\hline $\begin{array}{l}\text { Will she buy to } \\
\text { adopt? }\end{array}$ & May be but it has to much better than the one tried \\
\hline Note & $\begin{array}{l}\text { Overall, both the E and GK family are tech savvy and financially } \\
\text { sound which results in having variety of techs, however as the E is } \\
\text { aging, her ability to figure out tech and use new tech depletes. }\end{array}$ \\
\hline Dominant Theme 1 & $\begin{array}{l}\text { Eventhoght E portraits herself as a tech savvy, as ages her tech use } \\
\text { gets more and more limited, and she is quicker to abandon new } \\
\text { tech and less challenge herself to stick with a tech. }\end{array}$ \\
\hline Dominant Theme 2 & $\begin{array}{l}\text { E is very prideful and is so worried about her image that she refuses } \\
\text { to ask family for help and goes to professional FC }\end{array}$ \\
\hline
\end{tabular}




\begin{tabular}{|l|l|} 
Dominant Theme 3 & $\begin{array}{l}\text { E has a good self-efficacy and therefore willing to try and explore } \\
\text { tech however according to daughter, her cognitive decline is making } \\
\text { less patient with tech which results in keep buying and then } \\
\text { abandoning tech }\end{array}$ \\
\hline Dominant Theme 4 & $\begin{array}{l}\text { She was quick to buy an earlier version of AT, however abandoned } \\
\text { it due to not being easy to use. In general, E buys a new tech as } \\
\text { opposed to try to troubleshoot the owned tech. This downward } \\
\text { spiral continue and get worsen with cognitive decline }\end{array}$ \\
\hline Discovery & $\begin{array}{l}\text { As cognitive decline decreases E's tech savviness, FC becomes key } \\
\text { in E's ability to keep using tech }\end{array}$ \\
\hline GK & $\begin{array}{l}\text { Although it doesn't appear at the beginning, cognitive decline is } \\
\text { weakening even savvy E's perception of tech simplicity \& familiarity }\end{array}$ \\
\hline
\end{tabular}

Table H - 7 Elderly woman \#7 profile 


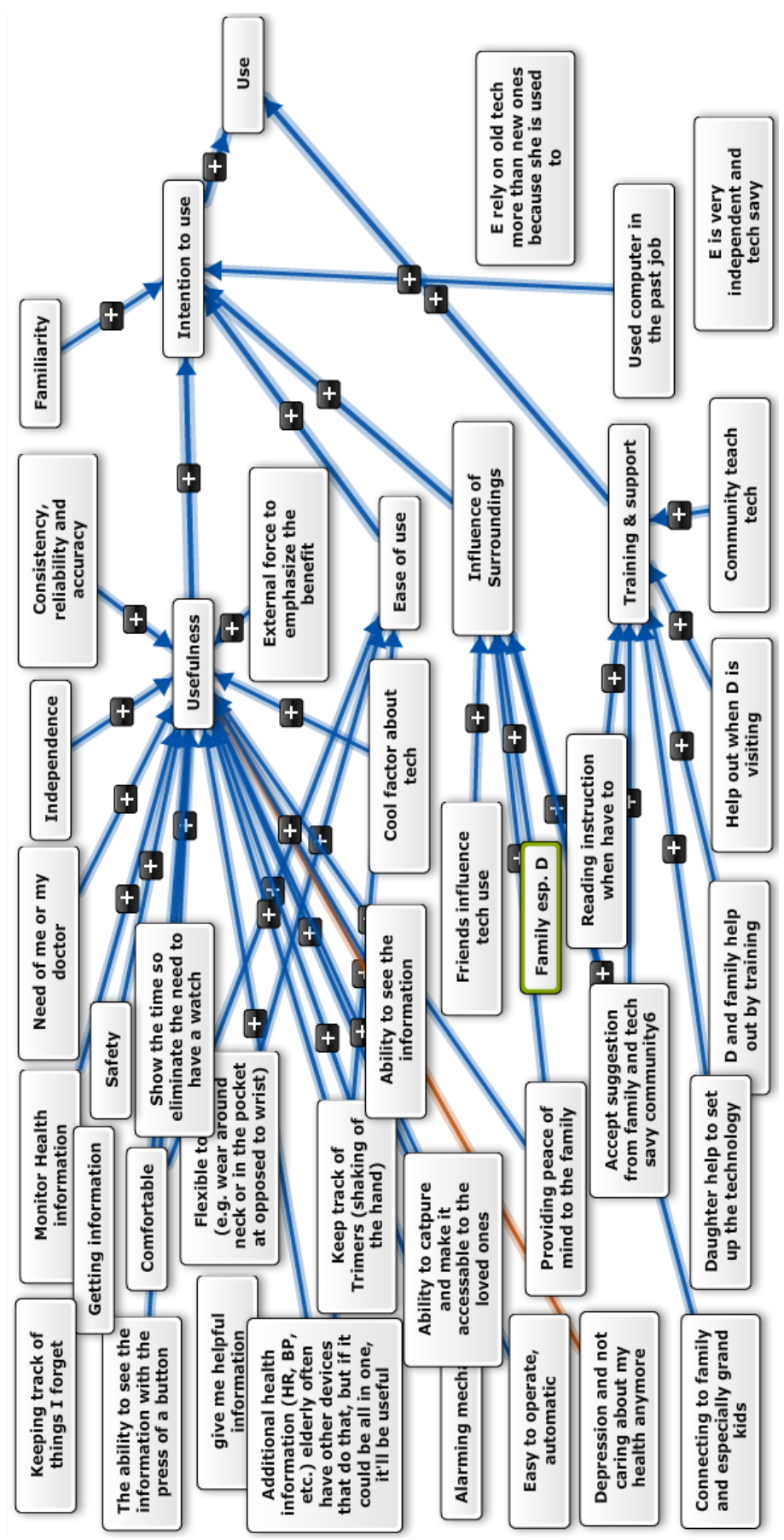

Figure H - 37 EW \#7 initial cognitive map 


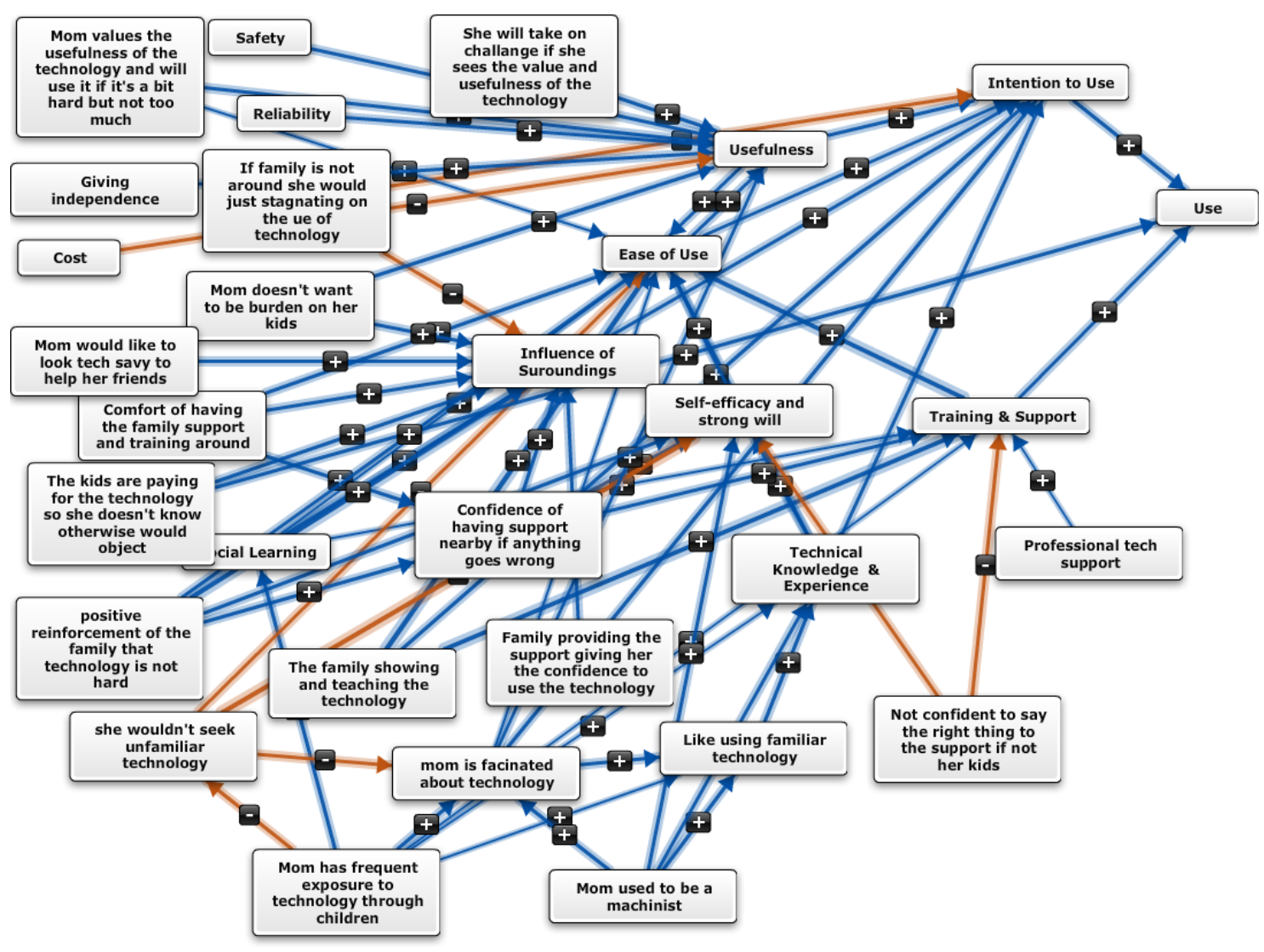

Figure H - 38 Gatekeeper \# 7 initial cognitive map 


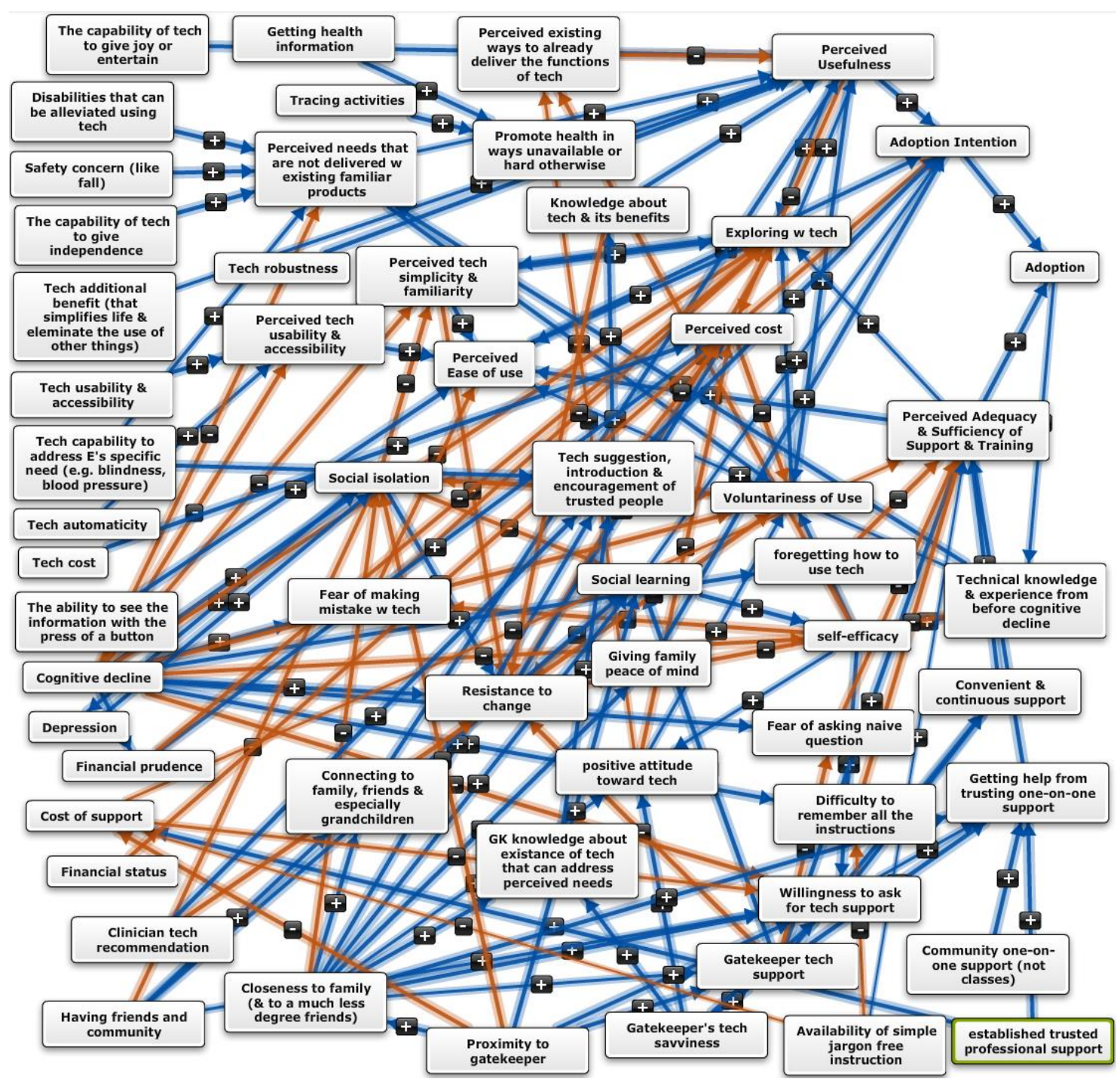

Figure H - 39 Elderly Gatekeeper pair \#7 integrated cognitive map 


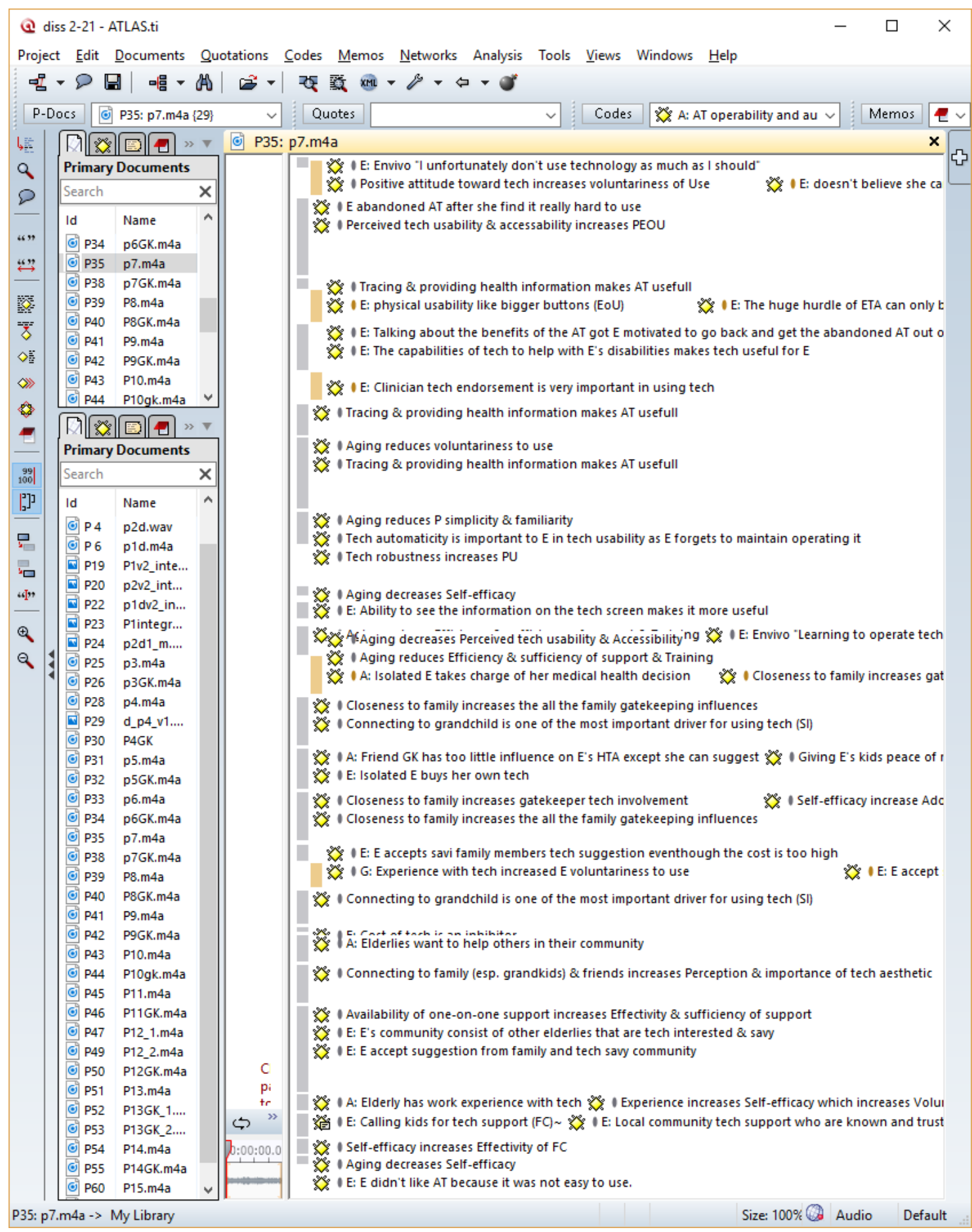

\section{Figure H - 40 EW\#7 interview open coding in Atlas.ti}




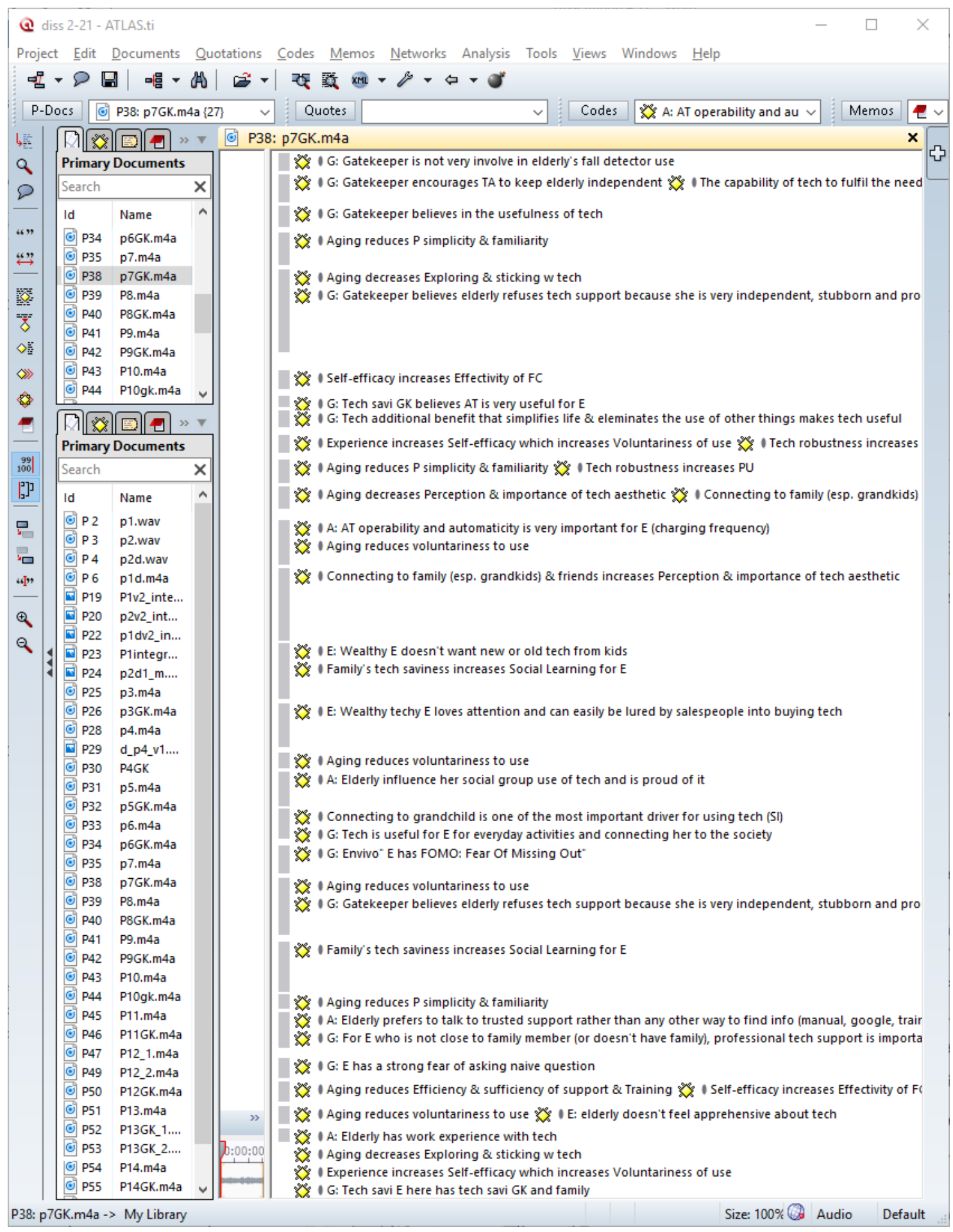

Figure H - 41 Gatekeeper \#7 interview open coding in Atlas.ti 


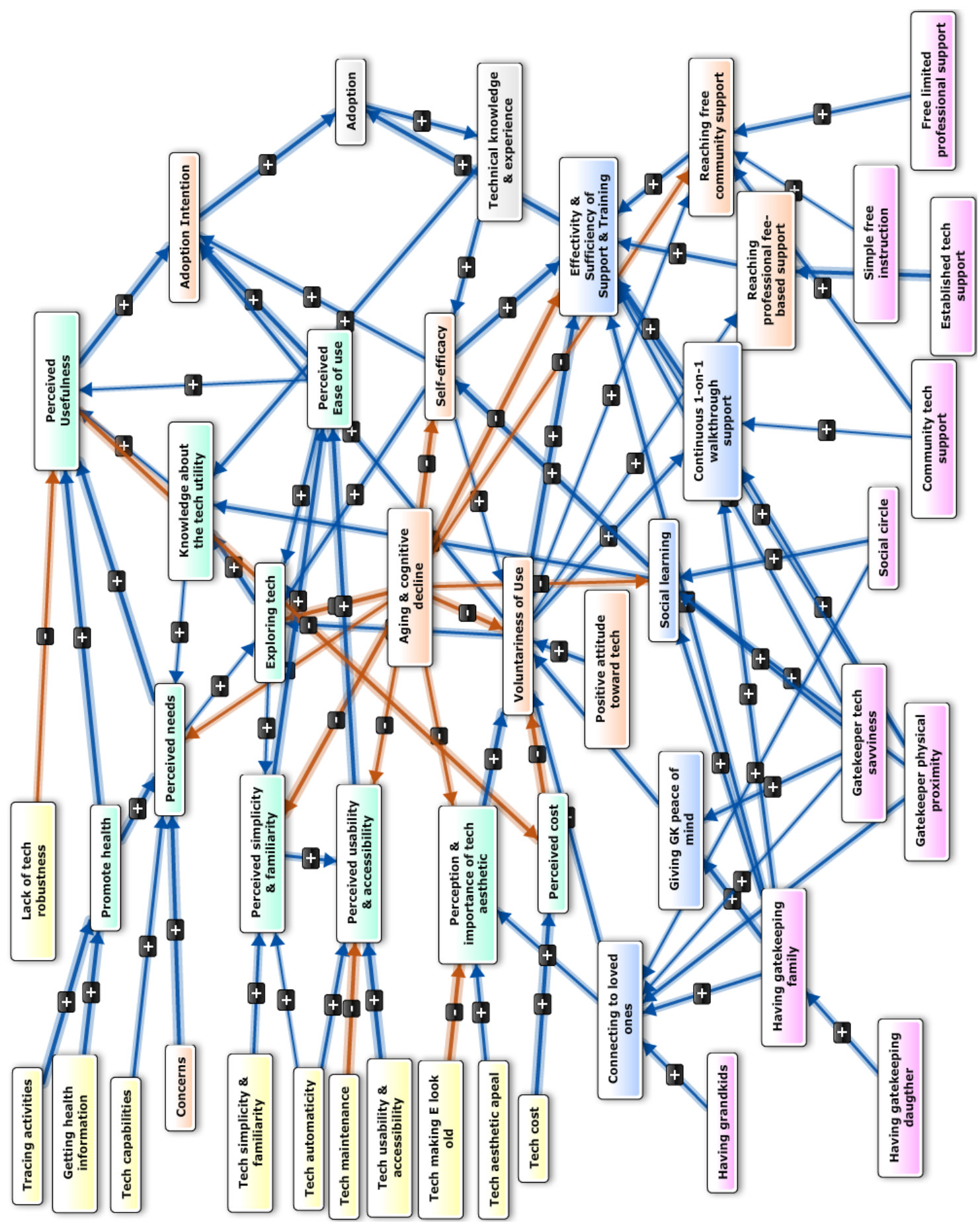

Figure H - 42 EW \#7 integrated \& standardized cognitive map 


\section{Elderly Woman \#8}

\begin{tabular}{|c|c|}
\hline Elderly Profile & P8 \\
\hline Interview Duration & $57 \mathrm{~min}$ \\
\hline Age & 90 \\
\hline Health Condition & Ok \\
\hline Living condition & Retirement Community \\
\hline $\begin{array}{l}\text { Attitude toward } \\
\text { Tech }\end{array}$ & Negative, $\sim$ I don't need tech \\
\hline Self-efficacy & Low \\
\hline Experience & No \\
\hline Driven by & Need and Ease of Use \\
\hline Has grandchildren? & $\mathrm{Y}$ \\
\hline $\begin{array}{l}\text { Has \& close to } \\
\text { family }\end{array}$ & $\mathrm{Y}$ \\
\hline Gatekeeper & Daughter (68 min) \\
\hline $\begin{array}{l}\text { Gatekeeper tech } \\
\text { savviness }\end{array}$ & Low \\
\hline Degree of influence & Moderate \\
\hline Influence & $\begin{array}{l}\text { FC, gifting, Closeness to family, Connect to grandparents, } 1 \text {-on-1 } \\
\text { walk through support, Suggesting \& encouraging (fading) }\end{array}$ \\
\hline $\begin{array}{l}\text { Perceived } \\
\text { Usefulness of the } \\
\text { wearable }\end{array}$ & It don't know \\
\hline $\begin{array}{l}\text { Perceived Ease of } \\
\text { Use of the wearable }\end{array}$ & It's OK \\
\hline $\begin{array}{l}\text { Will she adopt if } \\
\text { free? }\end{array}$ & $\mathrm{N}$ \\
\hline $\begin{array}{l}\text { Will she buy to } \\
\text { adopt? }\end{array}$ & $\mathrm{N}$ \\
\hline Note & I have everything I need, I don't long for anything \\
\hline Dominant Theme 1 & $\begin{array}{l}\text { In vivo "when one's get old, don't put new things in your elderly } \\
\text { parents' lives, it not blessing it causes a lot of anxiety, because we } \\
\text { don't adapt to new things" }\end{array}$ \\
\hline Dominant Theme 2 & Trying new things are not fun as you get old. \\
\hline Dominant Theme 3 & $\begin{array}{l}\text { E complains that her cognitive ability has declined greatly past few } \\
\text { years. }\end{array}$ \\
\hline Dominant Theme 4 & $\begin{array}{l}\text { People don't want to admit but as you get old working with } \\
\text { technology gets really hard }\end{array}$ \\
\hline Discovery & $\begin{array}{l}\text { I don't want new gadget, unless someone convince me that it's } \\
\text { interesting and easy. }\end{array}$ \\
\hline
\end{tabular}




\section{Table H - 8 Elderly woman \#8 profile}

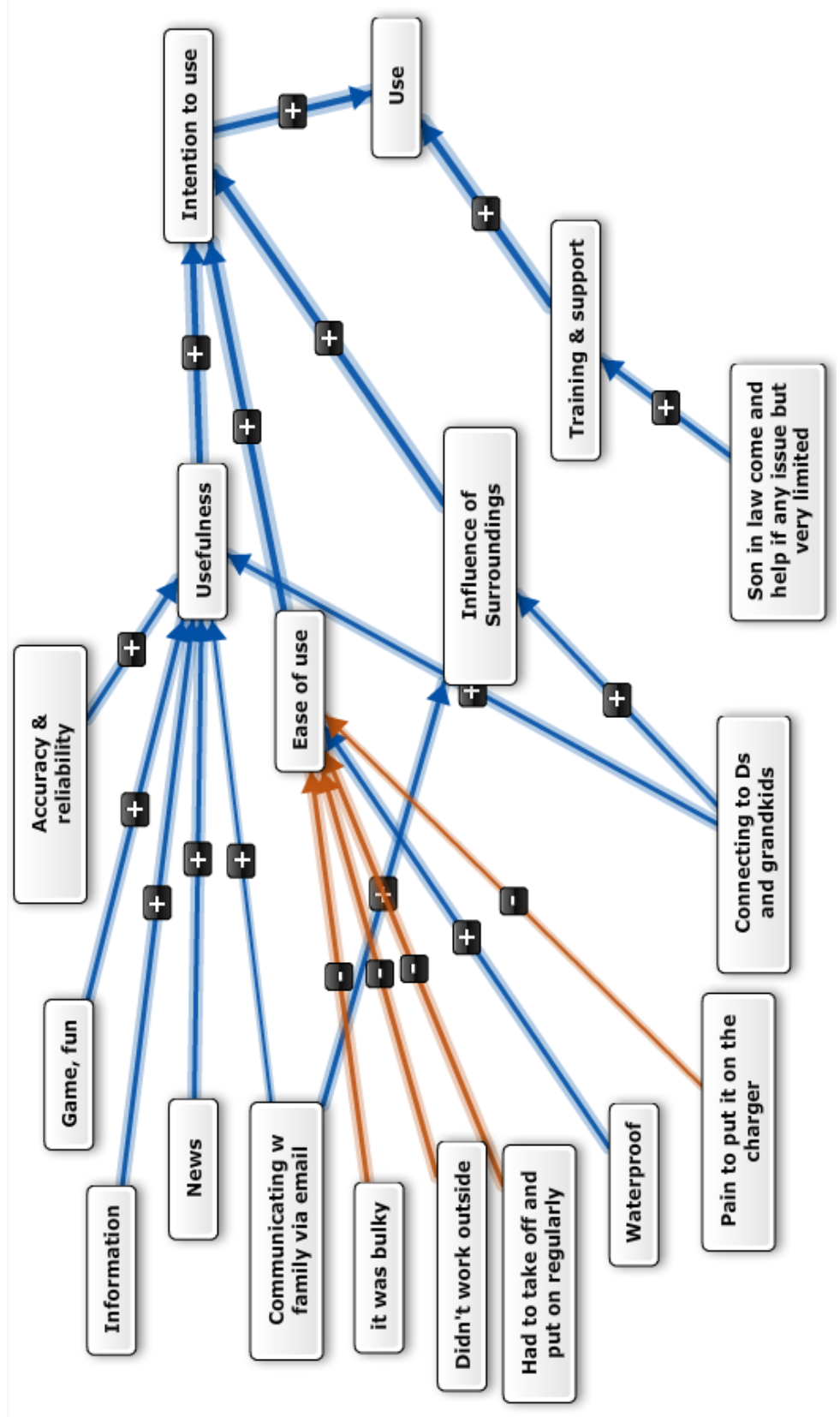

Figure H - 43 EW \#8 initial cognitive map 


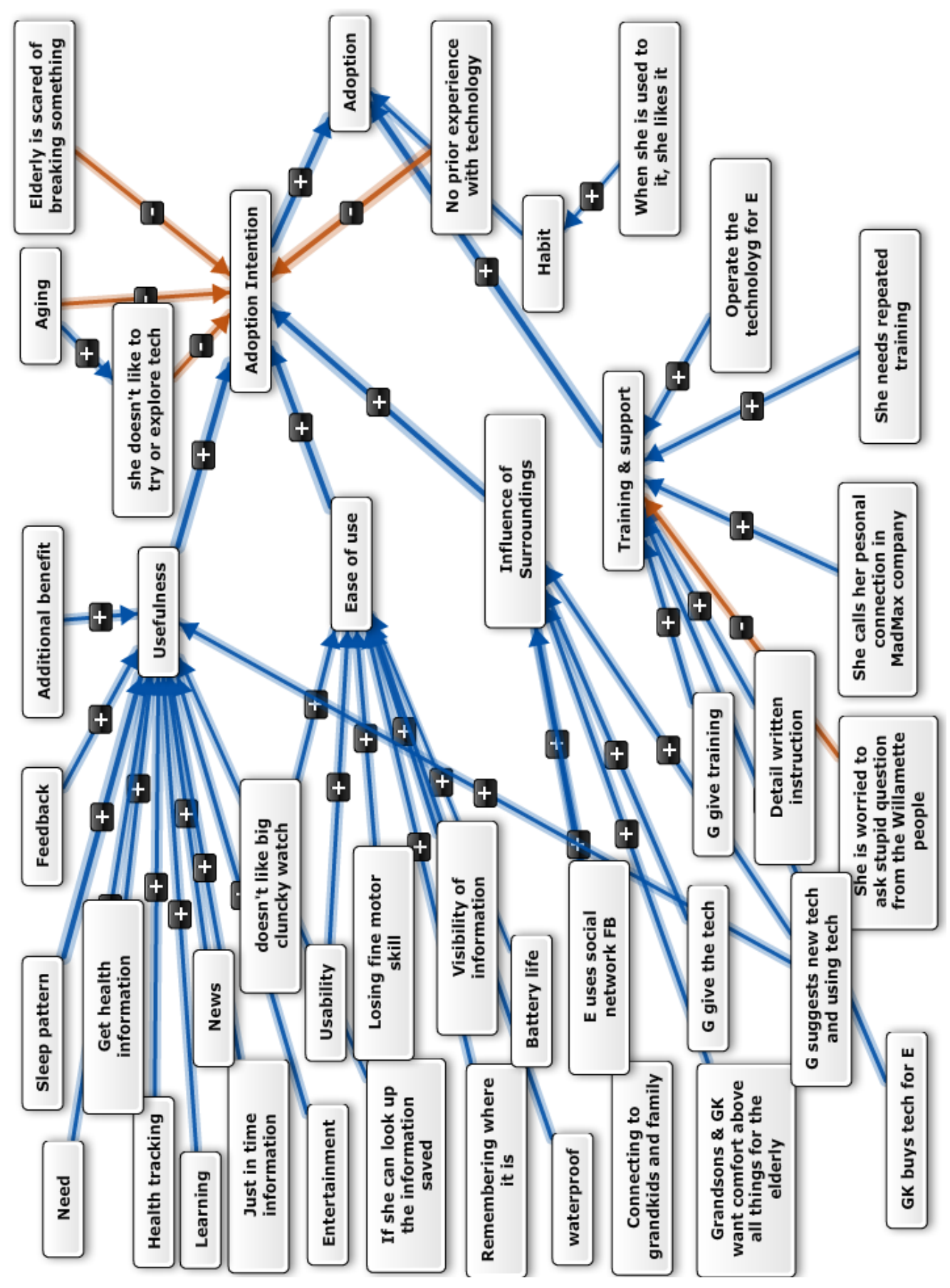

Figure H - 44 Gatekeeper \#8 initial cognitive map 


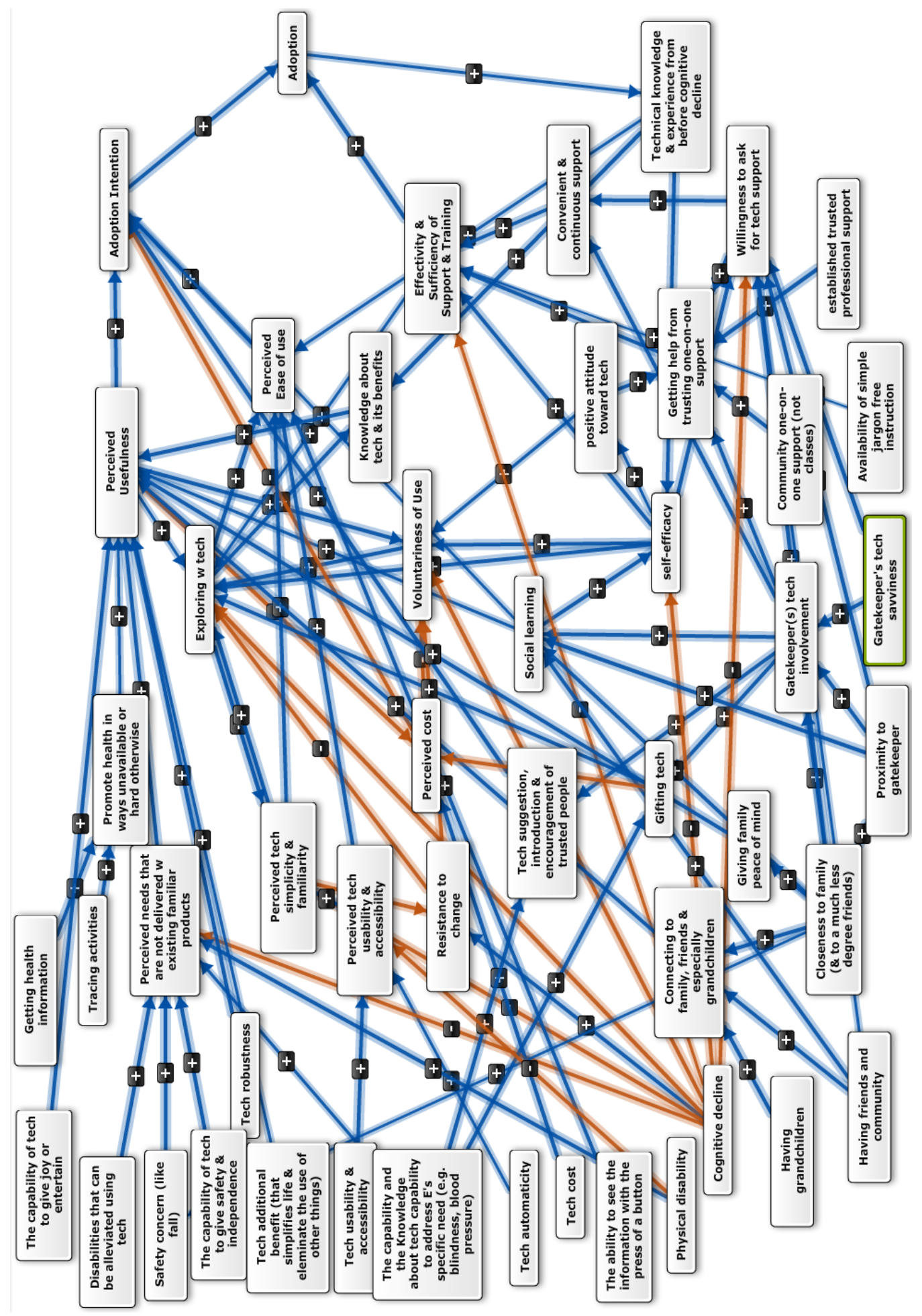

Figure H - 45 Elderly Gatekeeper pair \#8 integrated cognitive map 


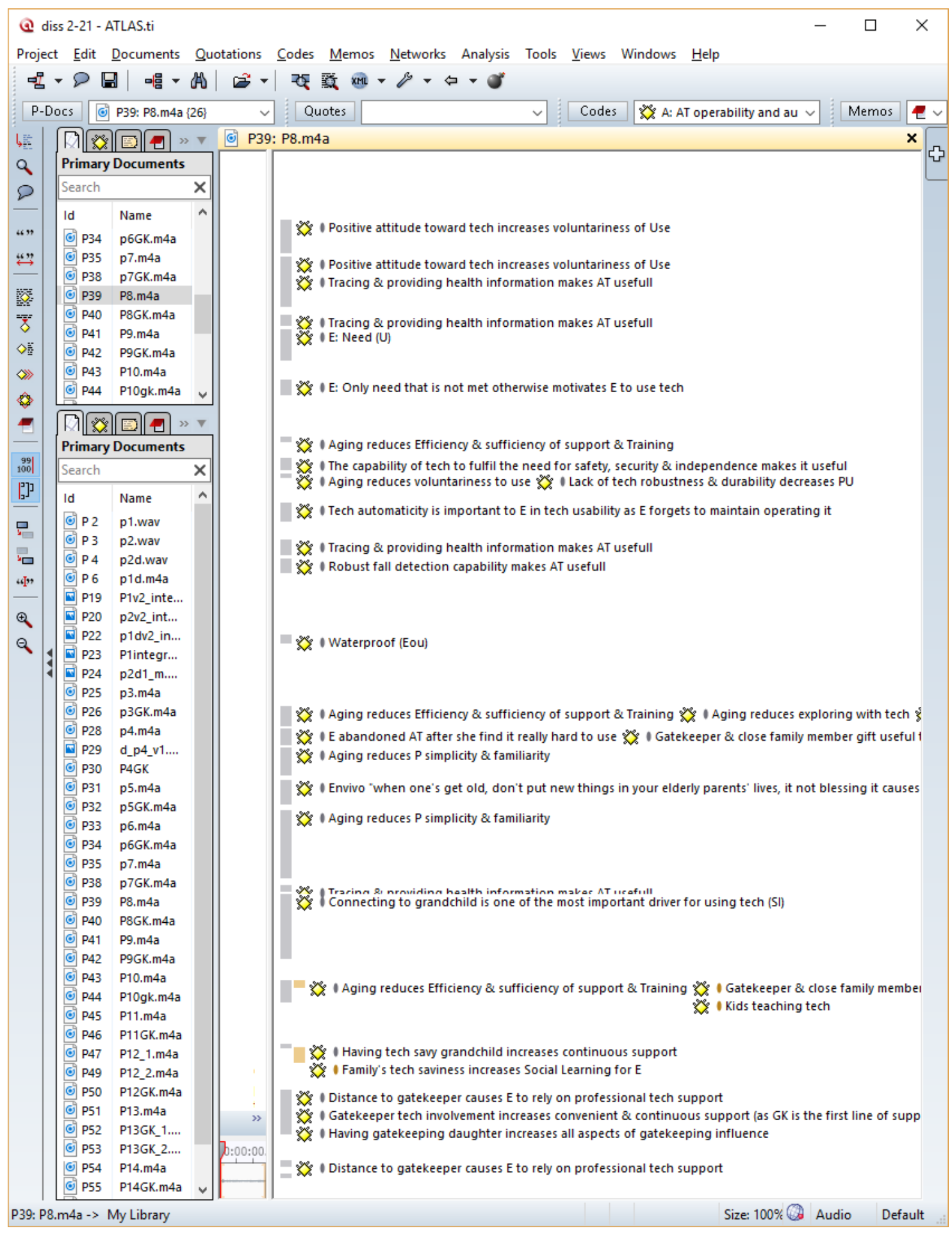

Figure H - 46 EW\#8 interview open coding in Atlas.ti 


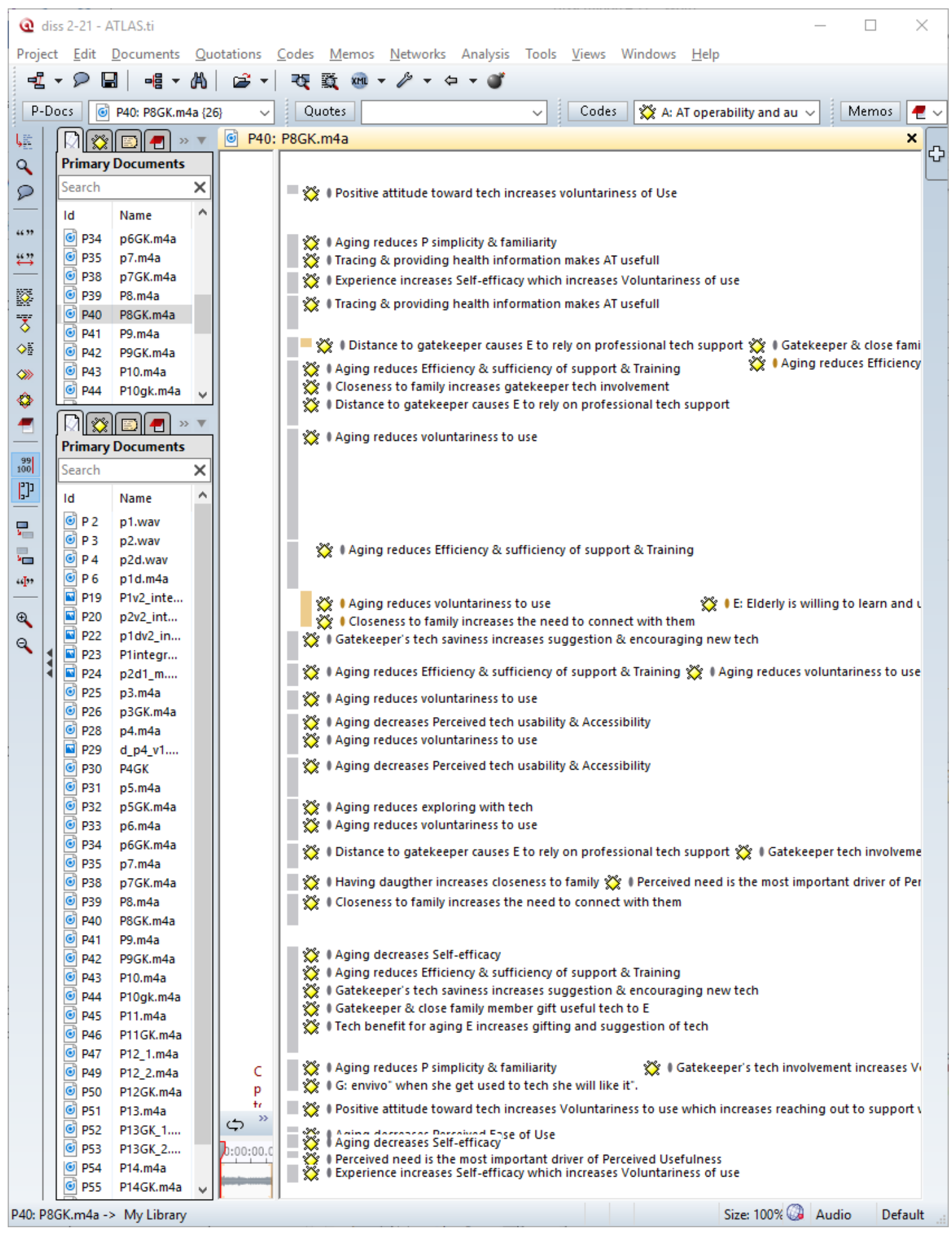

Figure H - 47 Gatekeeper \#8 interview open coding in Atlas.ti 


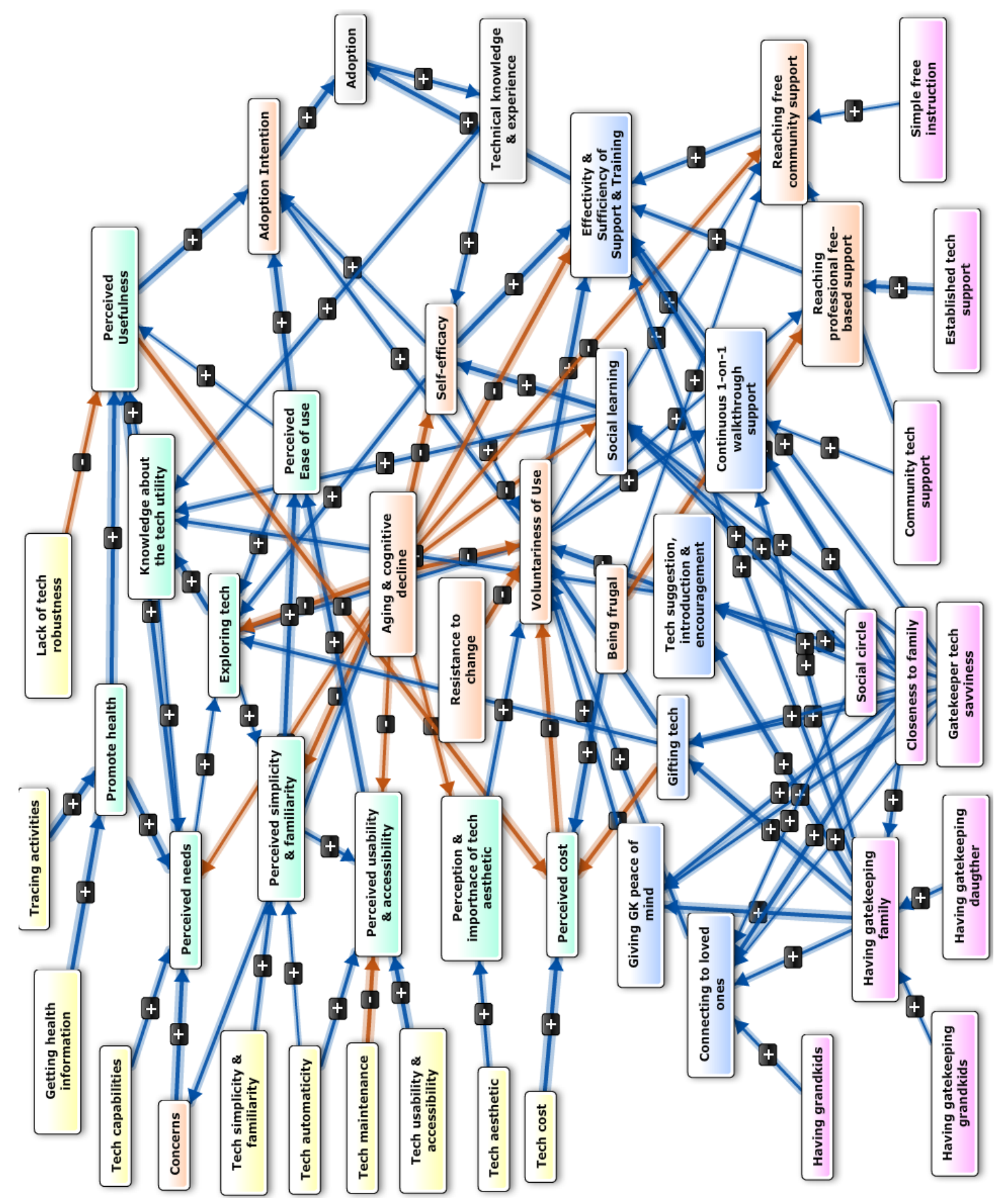

Figure H - 48 EW \#8 integrated \& standardized cognitive map 


\section{Elderly Woman \#9}

\begin{tabular}{|c|c|}
\hline Elderly Profile & P9 \\
\hline Interview Duration & $48 \mathrm{~min}$ \\
\hline Age & 68 \\
\hline Health Condition & Very active $\&$ healthy \\
\hline Living condition & Retirement Community \\
\hline Isolated & Y \\
\hline Attitude toward Tech & Negative \\
\hline Self-efficacy & $\mathrm{N}$ \\
\hline Experience & No \\
\hline Driven by & Ease of Use \\
\hline Has grandchildren? & $\mathrm{N}$ \\
\hline Has \& close to family & $\begin{array}{l}\text { Y (has a brother but not close; E lost her wife and kind of isolated } \\
\text { after that) }\end{array}$ \\
\hline Gatekeeper & Friend (and not too close) GK herself is a 72 yrs old $\mathrm{E}$ ( $45 \mathrm{~min}$ ) \\
\hline $\begin{array}{l}\text { Gatekeeper tech } \\
\text { savviness }\end{array}$ & not really \\
\hline Degree of influence & Low \\
\hline Influence & $\begin{array}{l}\text { The GK friend doesn't influence a lot other than limited social } \\
\text { learning, rare tech suggestion; however the innovative charter } \\
\text { school collaborates in a win-win program that E read w the kids and } \\
\text { kids help E with their technology "how to do" questions and } \\
\text { walkthroughs. E finds them (=free community tech support) helpful }\end{array}$ \\
\hline $\begin{array}{l}\text { Perceived Usefulness } \\
\text { of the wearable }\end{array}$ & Not sure, I don't need it yet \\
\hline $\begin{array}{l}\text { Perceived Ease of } \\
\text { Use of the wearable }\end{array}$ & I don't like technology \\
\hline $\begin{array}{l}\text { Will she adopt if } \\
\text { free? }\end{array}$ & $\mathrm{N}$ \\
\hline $\begin{array}{l}\text { Will she buy to } \\
\text { adopt? }\end{array}$ & $\begin{array}{l}\text { Not this one; Maybe one in future but it has to be much better than } \\
\text { the one tried }\end{array}$ \\
\hline Note & $\begin{array}{l}\text { Already has an activity tracker, a super simple pedometer that } \\
\text { clips/fits in a pocket; so long new AT is not as simple and more } \\
\text { useful, she is not going to adopt a new AT }\end{array}$ \\
\hline
\end{tabular}




\begin{tabular}{|c|c|}
\hline Dominant Theme 1 & $\begin{array}{l}\text { As E age her need for technology that can alleviate the effects of } \\
\text { aging increases however her perception of such needs decreases } \\
\text { (due to many factors including lack of experience, low self-efficacy } \\
\text { \& exposure and knowledge about the tech usefulness) and her } \\
\text { confidence about the ability to use tech diminishes. If this vicious } \\
\text { cycle is not intervened by convenient one-on-one accommodations } \\
\text { (encouragement, continuous walk-through support \& instruction, } \\
\text { and removing other barriers to adoption (gifting, etc.)) tech } \\
\text { adoption becomes unlikely. Usually, these interventions are only } \\
\text { done by E's gatekeeping family and effective when it's continuous } \\
\text { and simplified. }\end{array}$ \\
\hline Dominant Theme 2 & $\begin{array}{l}\text { E has a negative attitude toward new tech while happily using } \\
\text { familiar one to a limited scope that she is used to it }\end{array}$ \\
\hline Dominant Theme 3 & $\begin{array}{l}\text { A lot of social factors are missing from the life of an isolated } E \text { that } \\
\text { makes technology irrelevant which in turn decreases perceived } \\
\text { need, PU }\end{array}$ \\
\hline Dominant Theme 4 & $\begin{array}{l}\text { Technology is disrupting a lot of familiar things for E who has grown } \\
\text { up with them, find joy in, familiar with and have a strong longing to, } \\
\text { for this reasons E resist to change, hence casual relation: Aging } \\
\text { reduces voluntariness to. }\end{array}$ \\
\hline Discovery & $\begin{array}{l}\text { - Wealth or education (pre 1970) doesn't create tech savviness; the } \\
\text { E and GK here are both wealthy and educated but not savvy as their } \\
\text { education are from before tech area. This case does not contradict } \\
\text { the previous learning that experience increases the chance of } \\
\text { adoption } \\
\text { - As they say: "it takes a village". In most of the Elderly women cases } \\
\text { here (particularly the ones without close gatekeeping family), } \\
\text { gatekeeping role is not necessarily carried by one person, it's often } \\
\text { a network of social connection that each potentially influence one } \\
\text { or many or none of aspect(s) of the } 8 \text { category of influences } \\
\text { discovered here. The accumulation of all these influences emerge as } \\
\text { the most important state (vs. trait) factors in EWHTA. }\end{array}$ \\
\hline
\end{tabular}

Table H - 9 Elderly woman \#9 profile 


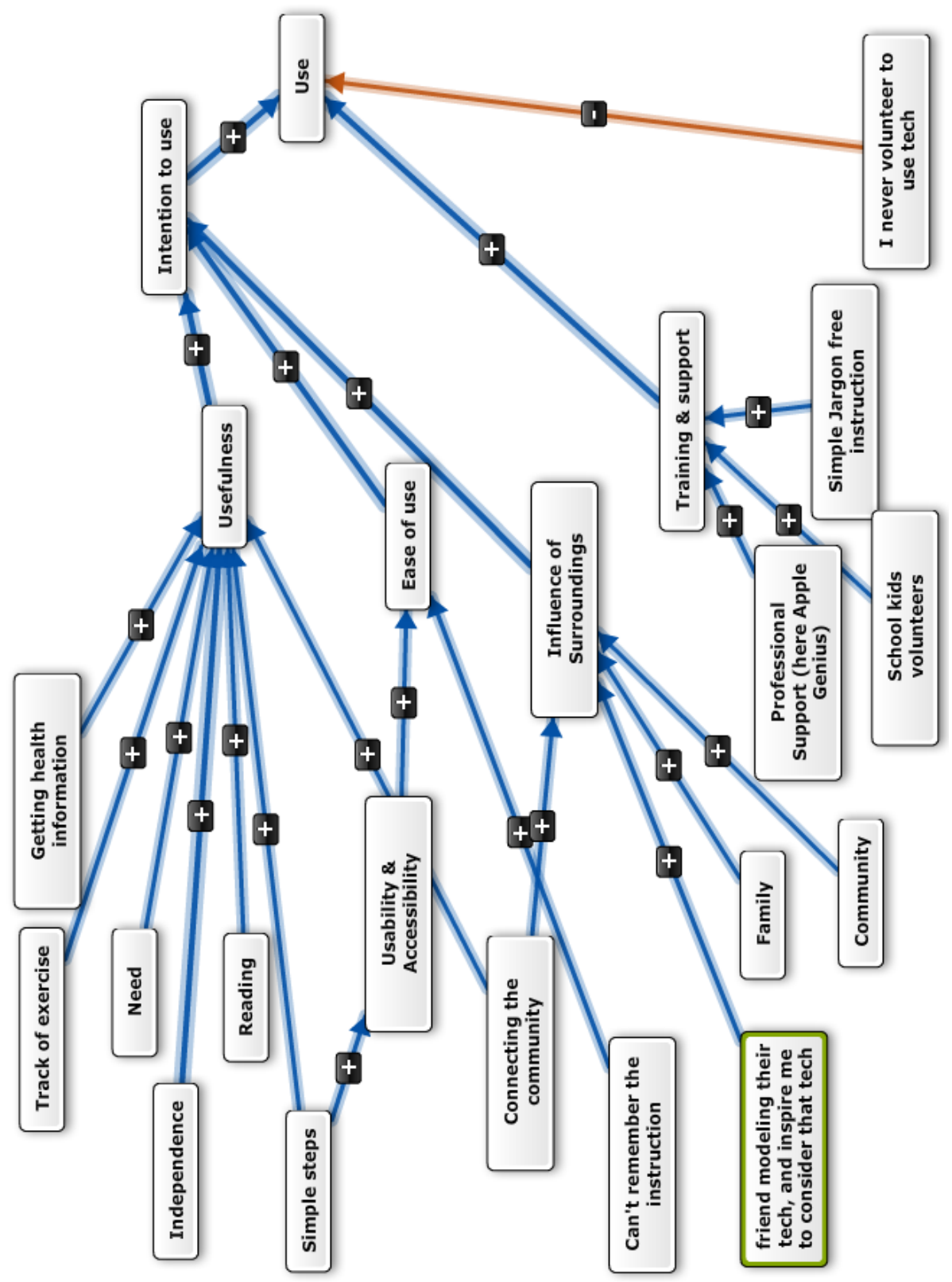

Figure H - 49 EW \#9 initial cognitive map 


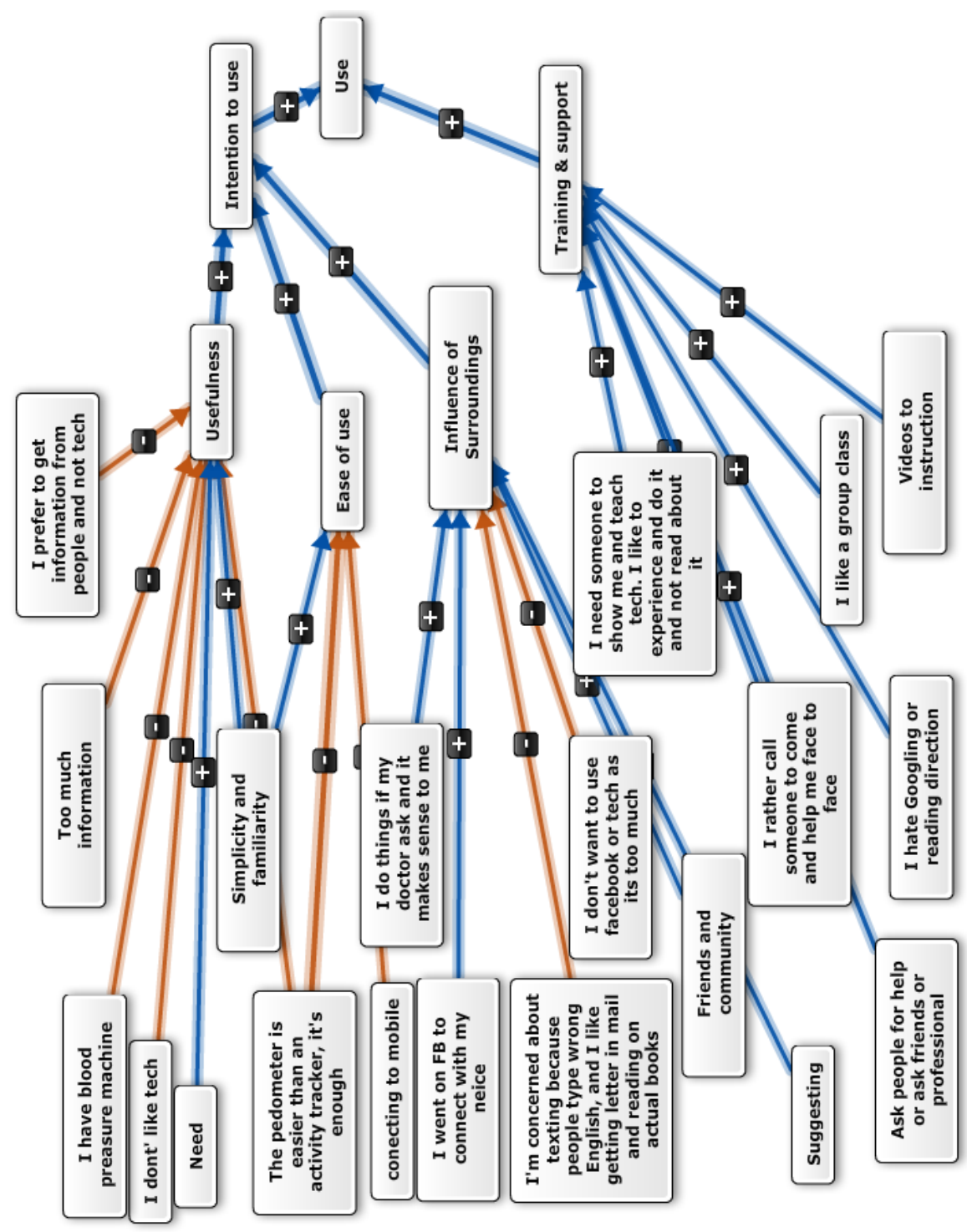

Figure H - 50 Gatekeeper \#9 initial cognitive map 


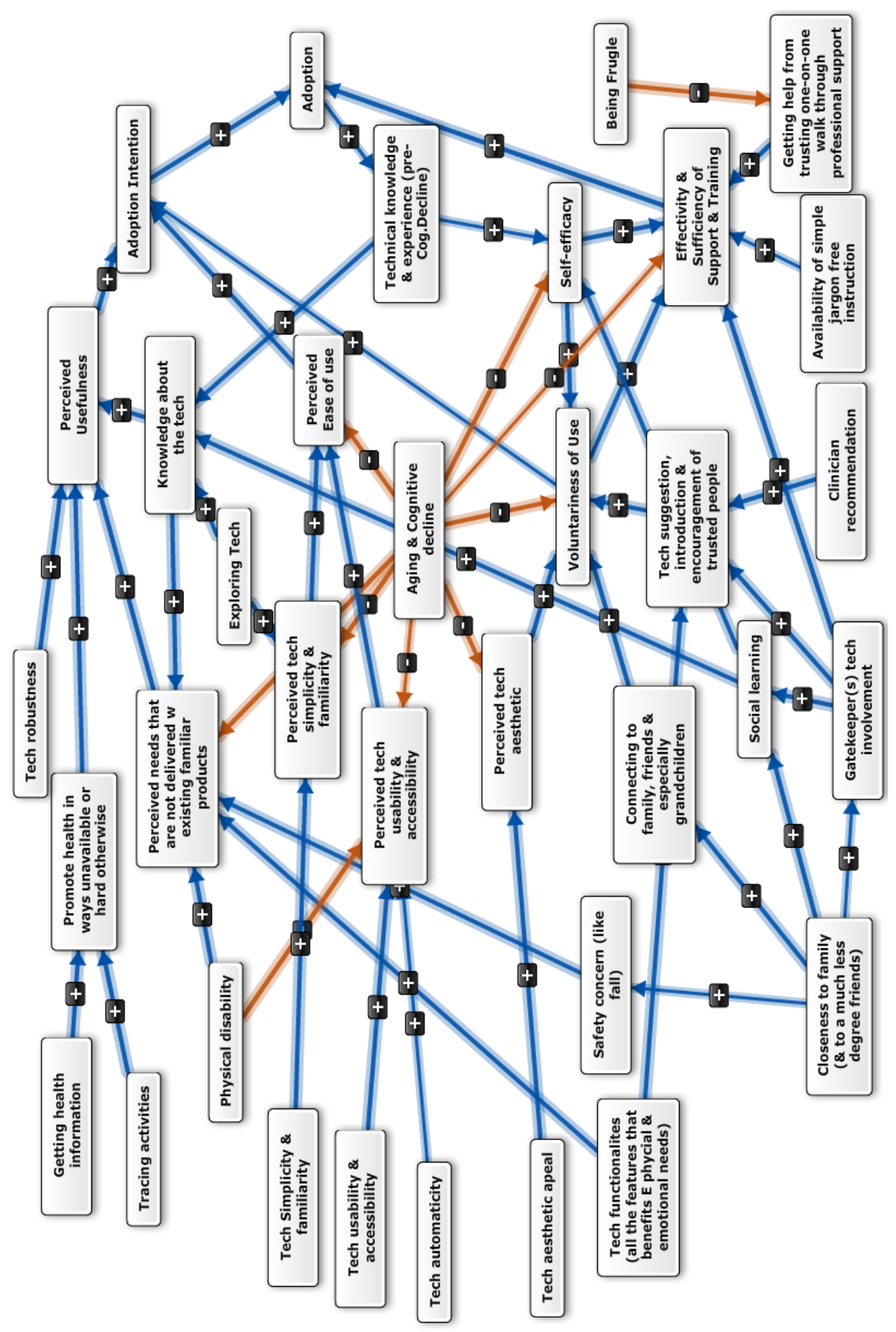

Figure H - 51 Elderly Gatekeeper pair \#9 integrated cognitive map 


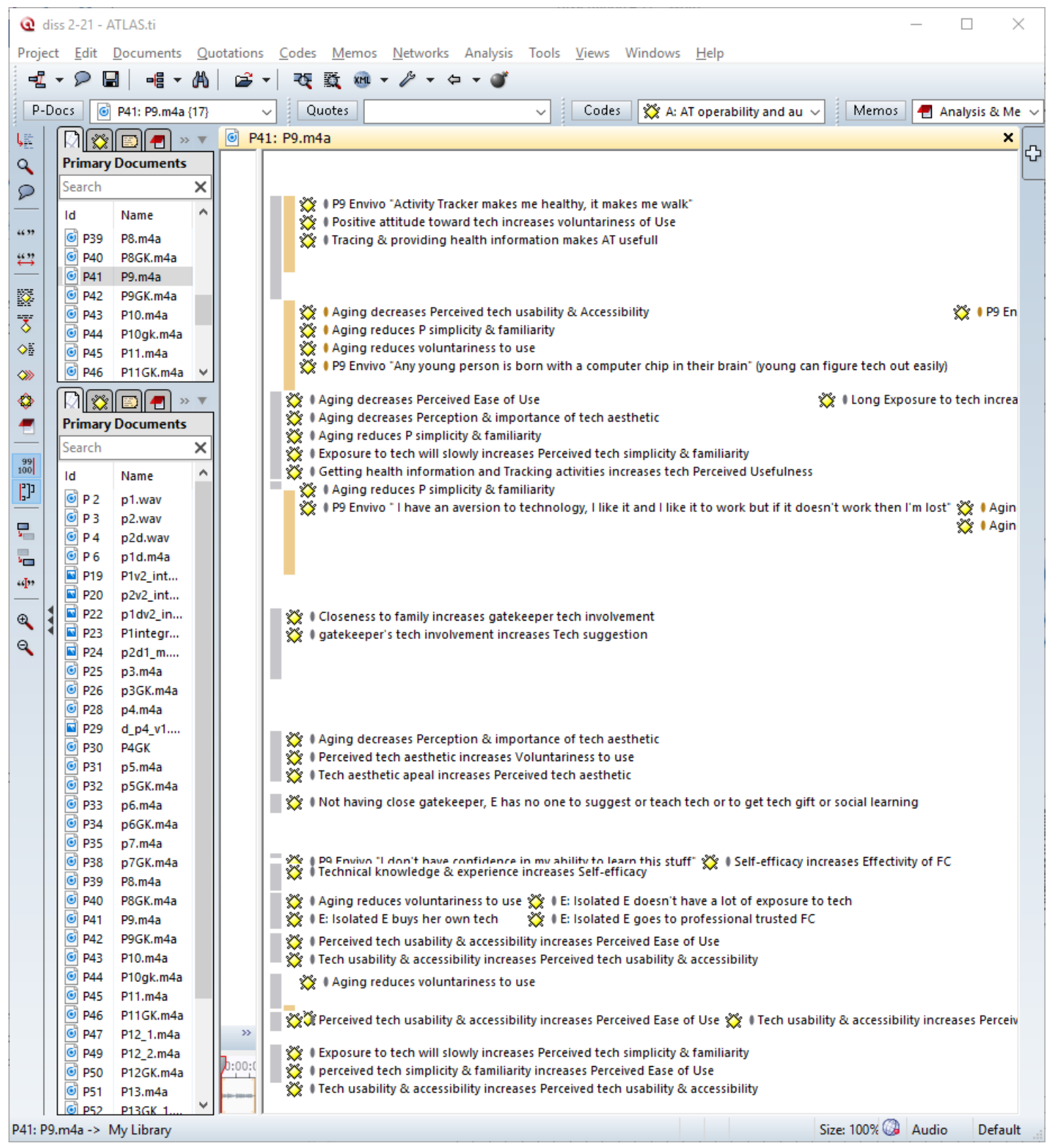

Figure H - 52 EW\#9 interview open coding in Atlas.ti 


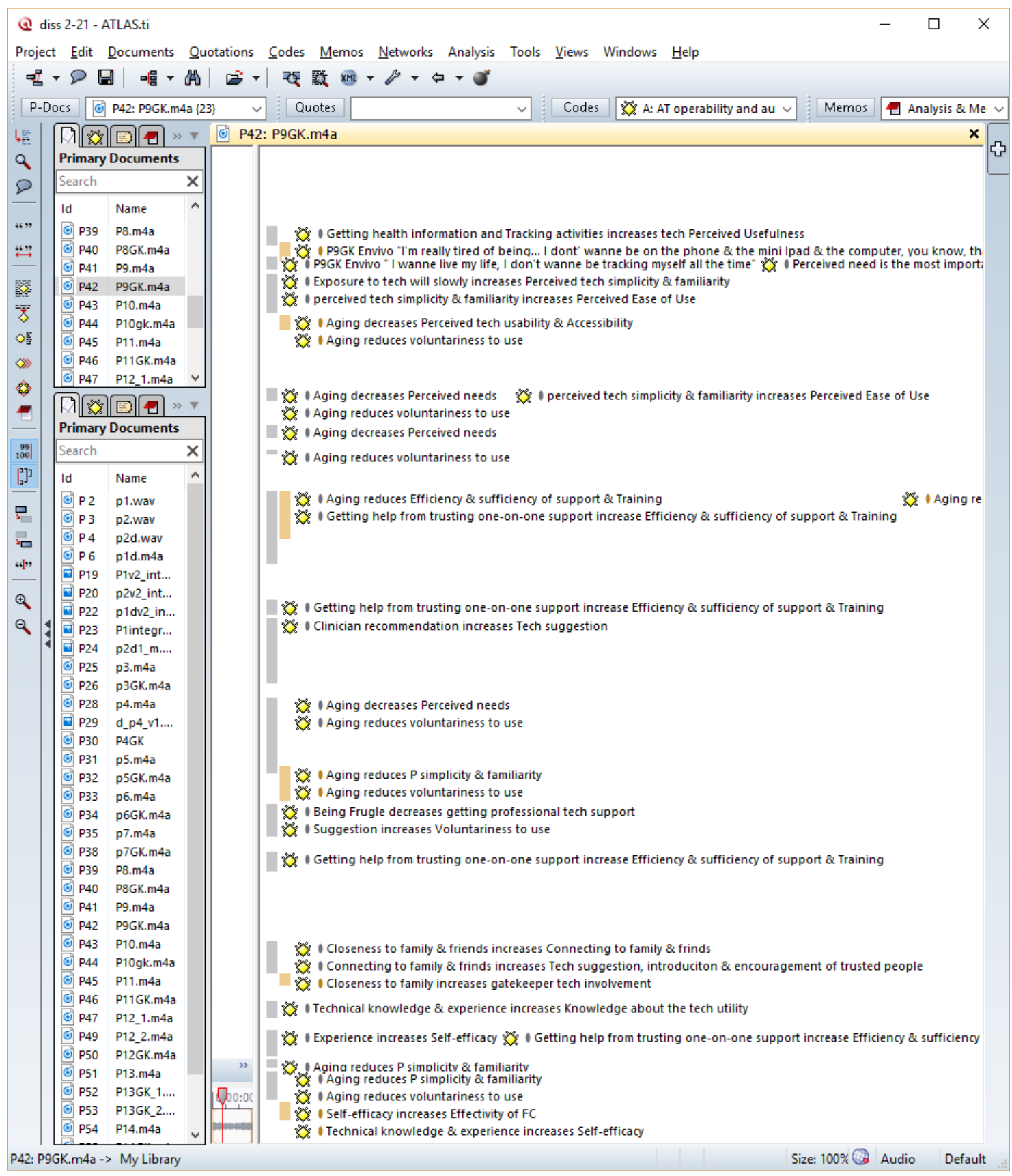

Figure H - 53 Gatekeeper \#9 interview open coding in Atlas.ti 


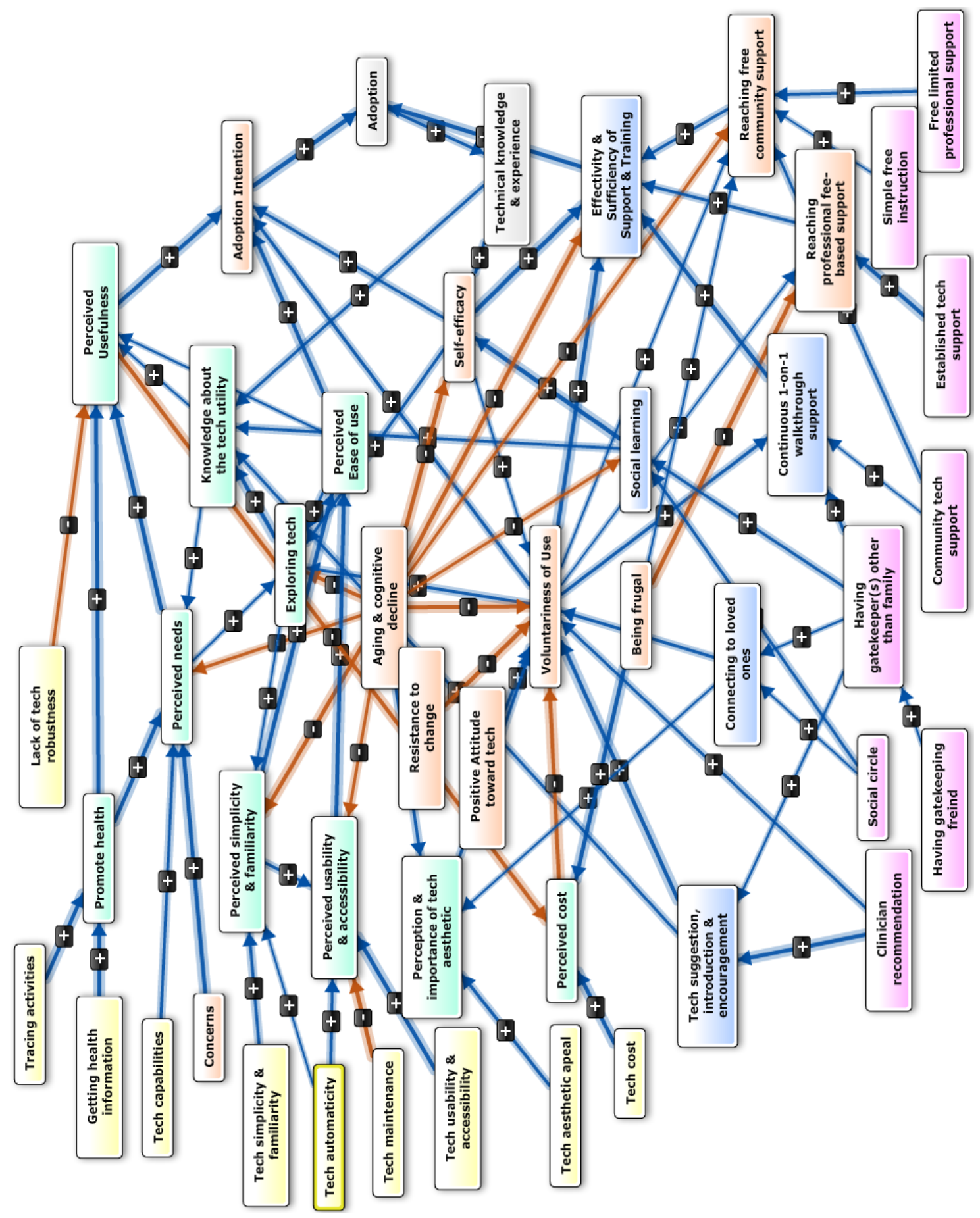

Figure H - 54 EW \#9 integrated \& standardized cognitive map 


\section{Elderly Woman \#10}

\begin{tabular}{|c|c|}
\hline Elderly Profile & P10 \\
\hline Interview Duration & $43 \mathrm{~min}$ \\
\hline Age & 84 \\
\hline Health Condition & OK \\
\hline living condition & Retirement Community \\
\hline Isolated & No \\
\hline Attitude toward Tech & Positive \\
\hline Self-efficacy & $\mathrm{Y}$ \\
\hline Experience & $\begin{array}{l}\text { Little (librarian) (GK said no, but E said she had worked w computer } \\
\text { for billing/reporting) }\end{array}$ \\
\hline Driven by & $\begin{array}{l}\text { Connecting to grandkids, personal trait ("Tenacious" as GK calls E) } \\
=>\text { not shy about asking for help w tech use }\end{array}$ \\
\hline Has grandchildren? & $Y$ \\
\hline Has \& close to family & Y \\
\hline Gatekeeper & Daughter (77 min) \\
\hline $\begin{array}{l}\text { Gatekeeper tech } \\
\text { savviness }\end{array}$ & $Y$ \\
\hline Degree of influence & High \\
\hline Influence & $\begin{array}{l}\text { All (Social learning + Suggestion + gifting + Access to continues 1-on- } \\
1 \text { support + increasing effective support by creating the comfort for } \\
\text { E that she waits and prefer her support over other supports+ } \\
\text { wanting to connect+ Reduce burden+ Giving peace of mind) }\end{array}$ \\
\hline $\begin{array}{l}\text { Perceived Usefulness } \\
\text { of the wearable }\end{array}$ & It seems useful \\
\hline $\begin{array}{l}\text { Perceived Ease of } \\
\text { Use of the wearable }\end{array}$ & It's OK \\
\hline $\begin{array}{l}\text { Will she adopt if } \\
\text { free? }\end{array}$ & $\mathrm{N}$ \\
\hline $\begin{array}{l}\text { Will she buy to } \\
\text { adopt? }\end{array}$ & Maybe, yes. \\
\hline Note & $\begin{array}{l}\text { GK is very interested in the resulting model and would like to get a } \\
\text { copy once available. }\end{array}$ \\
\hline Dominant Theme 1 & $\begin{array}{l}\text { P10 In vivo: "I use facebook, yah, I eavesdrop on the grandchildren } \\
\text { \& children" }\end{array}$ \\
\hline
\end{tabular}




\begin{tabular}{|c|c|}
\hline Dominant Theme 2 & $\begin{array}{l}\text { P10 In vivo: "It makes her seem, you know, up to date, and that is } \\
\text { the appearance of it being up-to-date for the grandkids. So, in that } \\
\text { case, it's not so much that it's cool but that it might impress them, } \\
\text { and so again it's: her doing pretty much anything to maintain that } \\
\text { connection" }\end{array}$ \\
\hline Dominant Theme 3 & $\begin{array}{l}\text { P10 is a successful EWHTA story. She is tenacious retired librarian } \\
\text { who moved to Portland to live close to her family. She has a family } \\
\text { with kids (daughter) and grandkids who are tech savvy and close to } \\
\text { her, that have become her gatekeeping network. They actively help } \\
\text { and support her with TA and influence at all } 8 \text { levels of my emerged } \\
\text { influences). More than anything, she is an optimistic grandmother } \\
\text { who has left her past life and moved to a new city just so she can be } \\
\text { close and connect with them. She sees tech as a way to be up-to- } \\
\text { date to impress and connect with the grandkids. She is also practical } \\
\text { and frugal and not shy about asking for tech support from anyone, } \\
\text { so uses her tech support network effectively to use tech (Family + } \\
\text { Community tech support) }\end{array}$ \\
\hline \multirow[t]{2}{*}{ GK } & $\begin{array}{l}\text { P10 GK In vivo: "I think if she (E) didn't have a good network of } \\
\text { support, I don't think she would... I think she would end up not } \\
\text { using it" }\end{array}$ \\
\hline & $\begin{array}{l}\text { This is the case of an elderly with almost no prior tech experience } \\
\text { whom with a strong GK network has been able to continuously } \\
\text { adopt previous tech. It seems like the GK influences are the } \\
\text { strongest }\end{array}$ \\
\hline Discovery & $\begin{array}{l}\text { Reduce burden (Elderly has a strong desire to connect to family, at } \\
\text { the same time, she is very considerate and doesn't want to be a } \\
\text { burden to the them and will use tech that provide that benefit) }\end{array}$ \\
\hline
\end{tabular}

Table H - 10 Elderly woman \#10 profile 


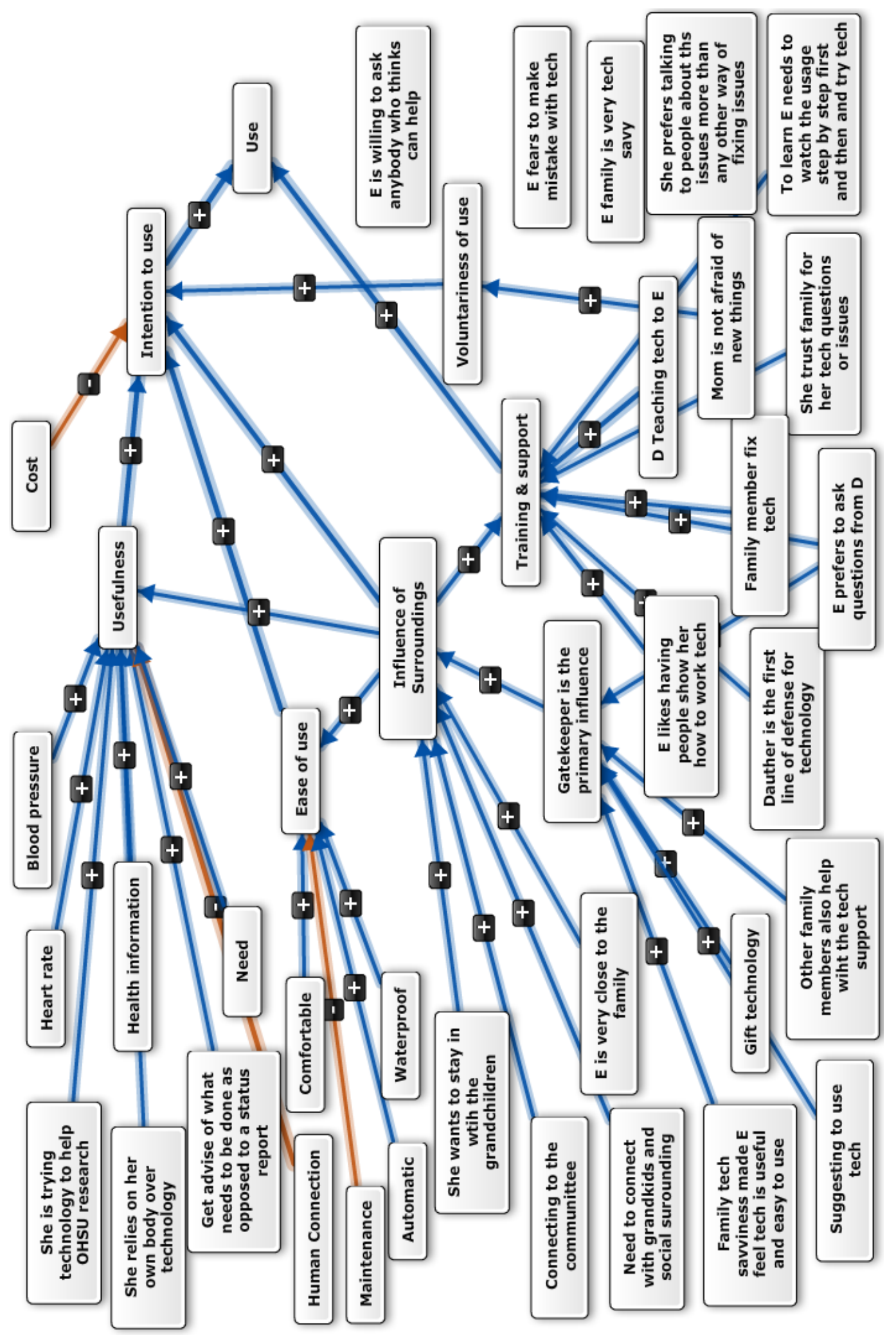

Figure H - 55 EW \#10 initial cognitive map 


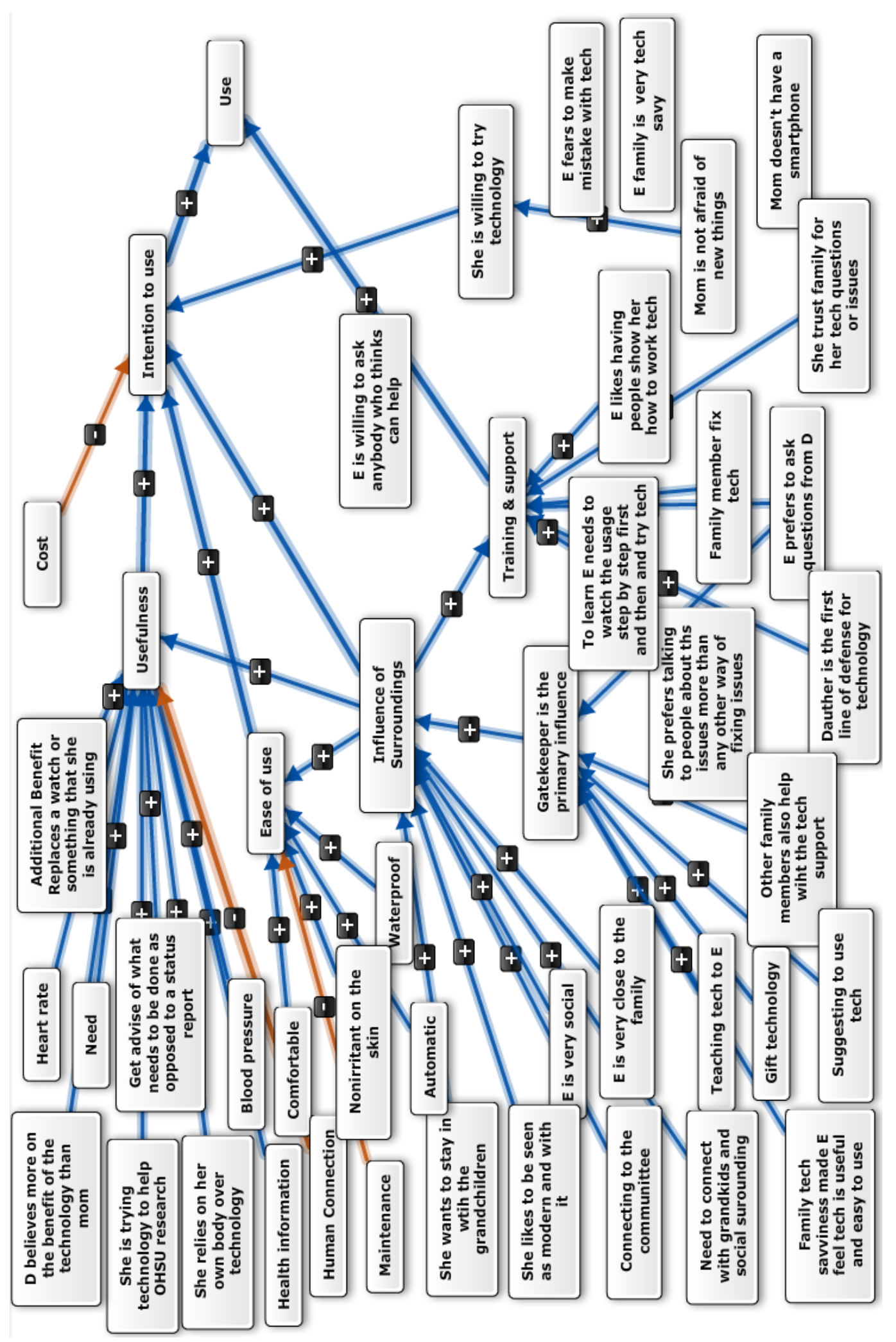

Figure H - 56 Gatekeeper \#10 initial cognitive map 


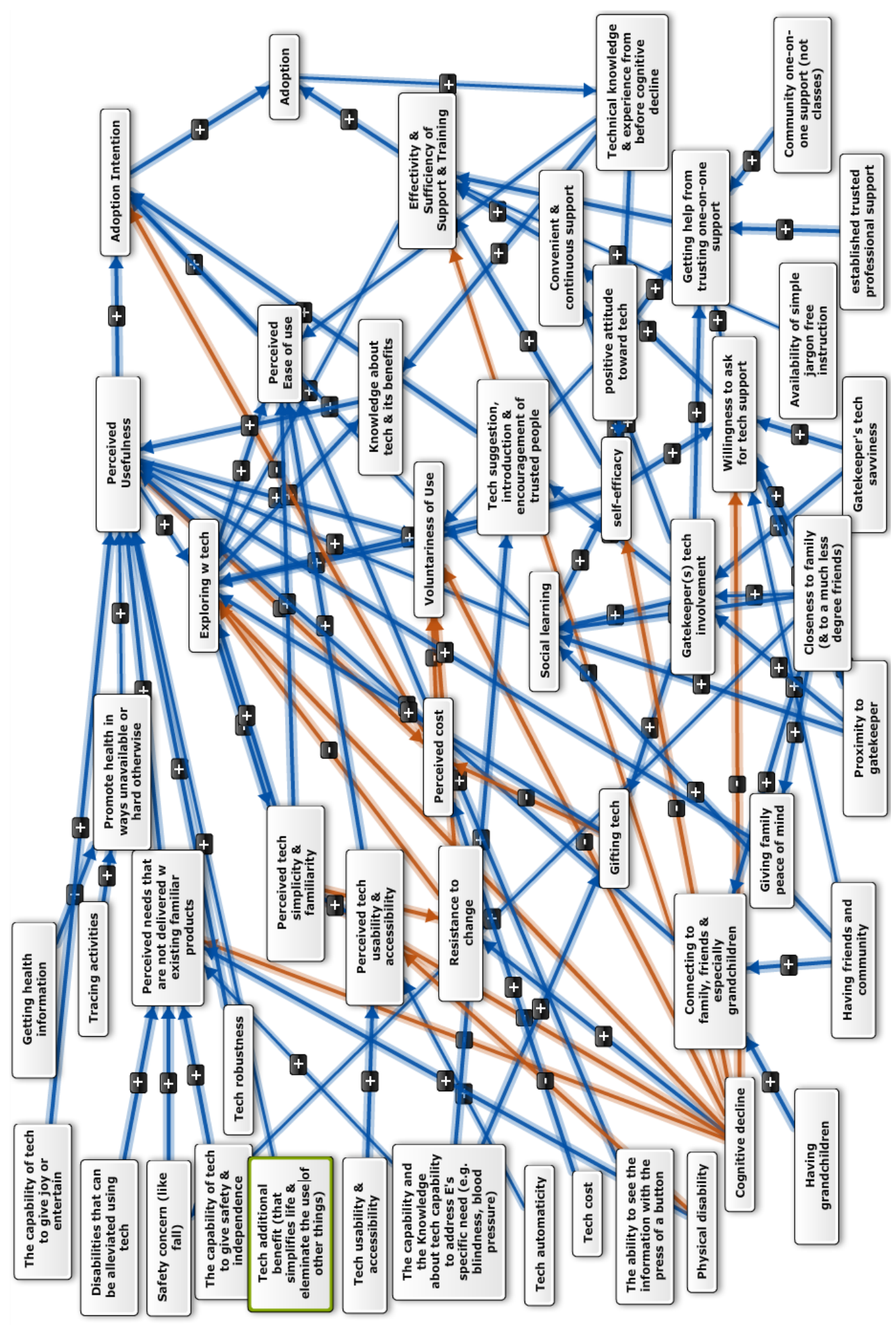

Figure H - 57 Elderly Gatekeeper pair \# 10 integrated cognitive map 


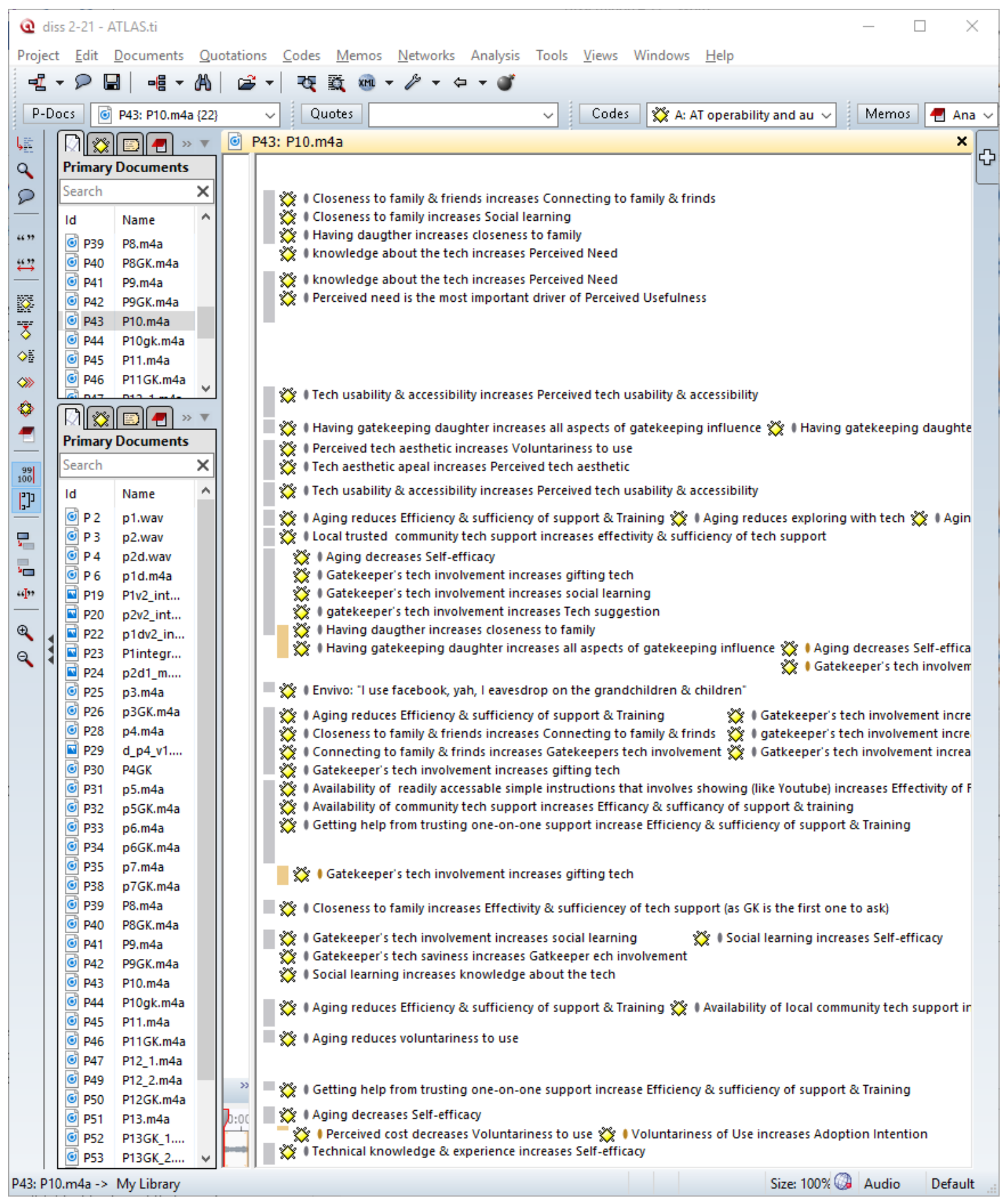

Figure H - $58 \mathrm{EW \# 10} \mathrm{interview} \mathrm{open} \mathrm{coding} \mathrm{in} \mathrm{Atlas.ti}$ 


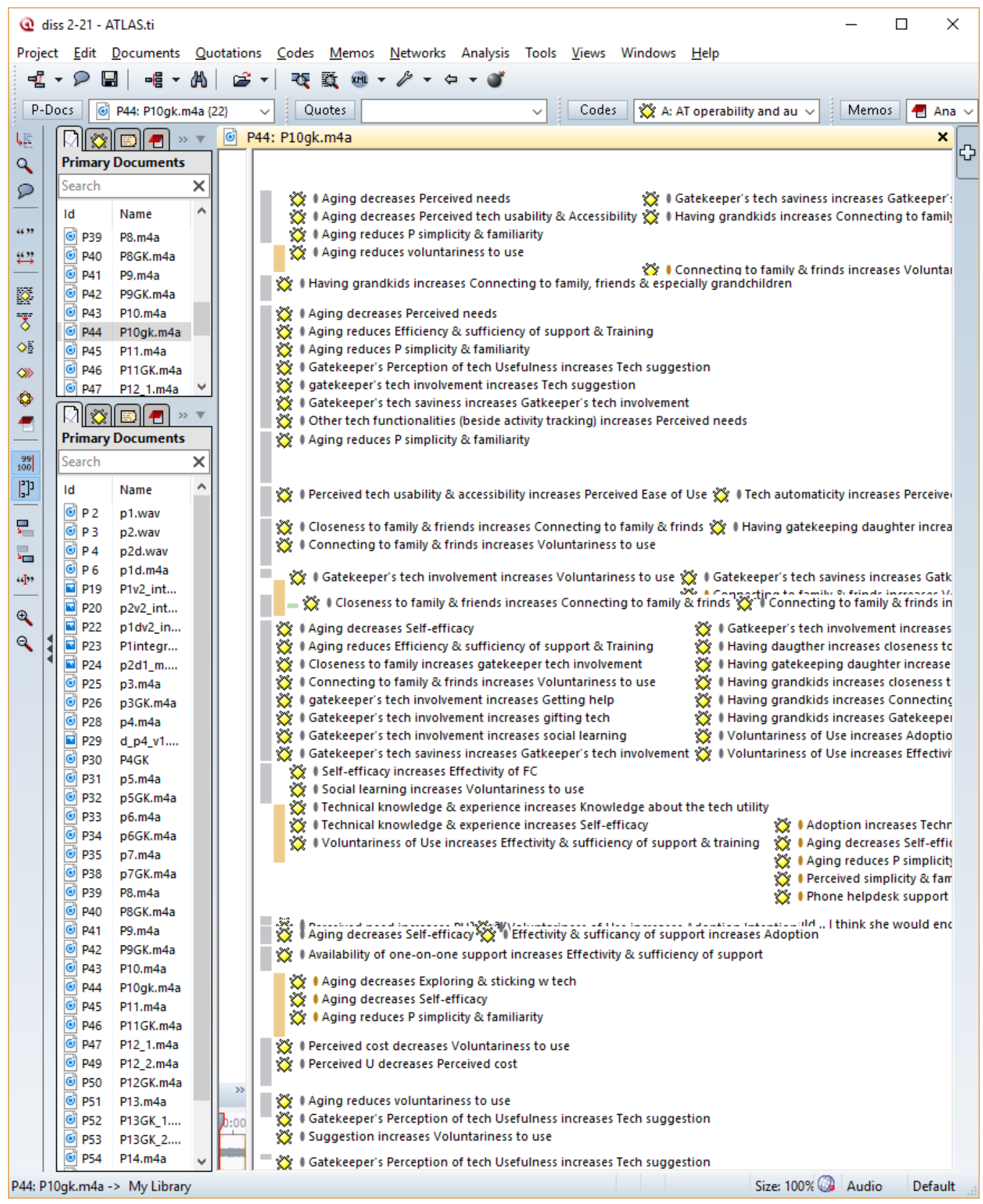

\section{Figure H - 59 Gatekeeper \#10 interview open coding in Atlas.ti}




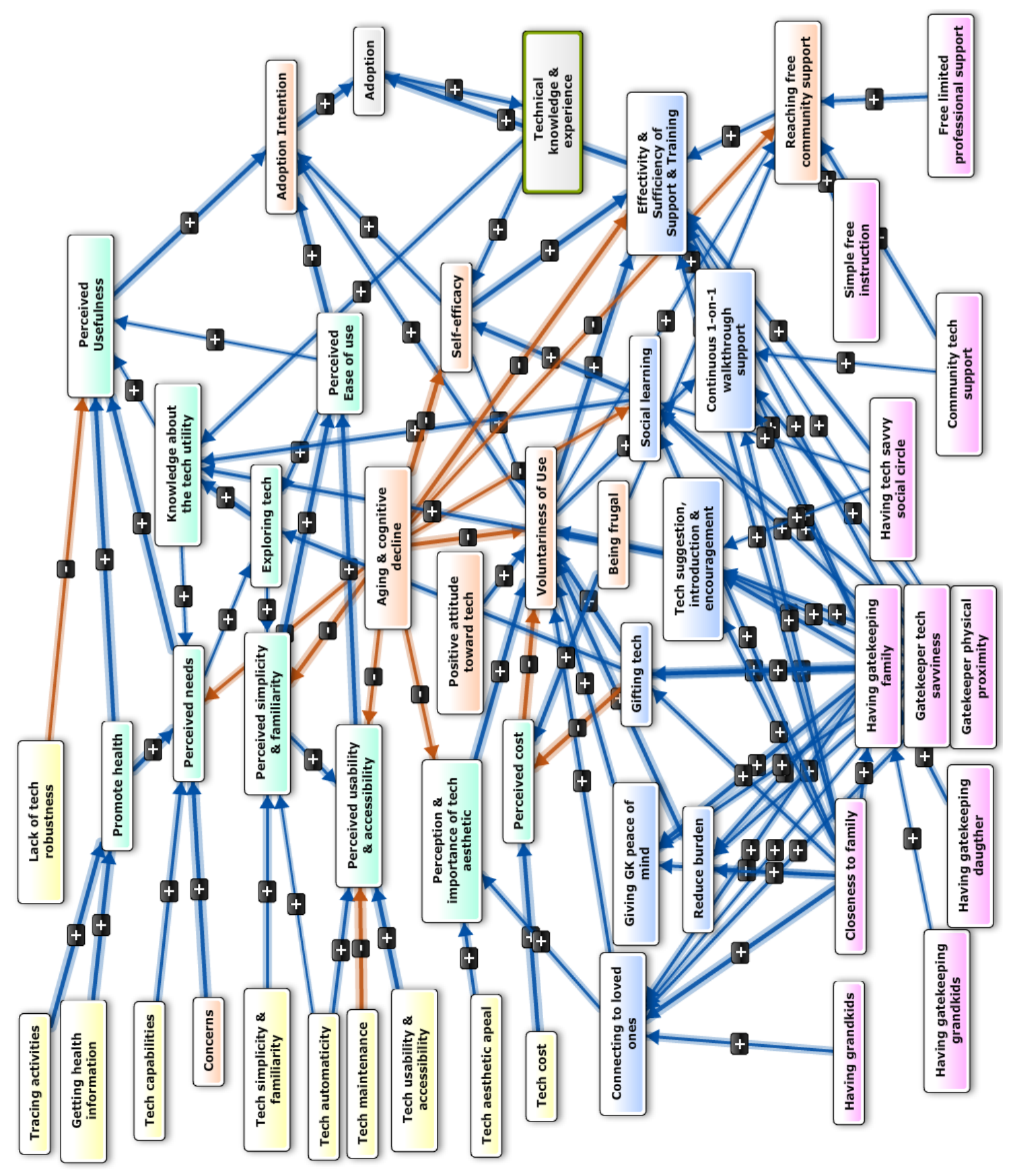

Figure H - 60 EW \#10 integrated \& standardized cognitive map 


\section{Elderly Woman \#11}

\begin{tabular}{|c|c|}
\hline Elderly Profile & P11 \\
\hline Interview Duration & $81 \mathrm{~min}$ \\
\hline Age & 67 \\
\hline Health Condition & OK \\
\hline Living condition & Living in her house $w$ husband \\
\hline Isolated & No \\
\hline Attitude toward Tech & Positive (In vivo: "We can't live without technology.") \\
\hline Self-efficacy & $\mathrm{Y}$ \\
\hline Experience & Y, Electrical Engineer with 30 years of experience \\
\hline Driven by & $\begin{array}{l}\text { Tech utility \& health information \& desire to stay young and } \\
\text { healthy, - cost }\end{array}$ \\
\hline Has grandchildren? & $\mathrm{N}$ \\
\hline Has \& close to family & Y \\
\hline Gatekeeper & $\begin{array}{l}\text { Husband } 67 \text { yrs. (Not savvy and not believe in AT usefulness) ( } 67 \\
\text { min) }\end{array}$ \\
\hline $\begin{array}{l}\text { Gatekeeper tech } \\
\text { savviness }\end{array}$ & $\mathrm{N}$ \\
\hline Degree of influence & Very low \\
\hline Influence & Suggestion \& showing tech (social learning) \\
\hline $\begin{array}{l}\text { Perceived Usefulness } \\
\text { of the wearable }\end{array}$ & Yes, I like it \\
\hline $\begin{array}{l}\text { Perceived Ease of } \\
\text { Use of the wearable }\end{array}$ & It should be easy to figure out \\
\hline $\begin{array}{l}\text { Will she adopt if } \\
\text { free? }\end{array}$ & Y \\
\hline $\begin{array}{l}\text { Will she buy to } \\
\text { adopt? }\end{array}$ & $\mathrm{N}$ \\
\hline Note & $\begin{array}{l}\text { E is very practical, driven by need. She has a lot of technical } \\
\text { knowledge \& experience, high self-efficacy, and tech savvy, } \\
\text { however very frugal. }\end{array}$ \\
\hline Background & Experience \& Positive attitude toward tech \\
\hline Dominant Theme 2 & $\begin{array}{l}\text { E is very practical, driven by need. She has a lot of technical } \\
\text { knowledge \& experience, high self-efficacy, and very tech savvy. }\end{array}$ \\
\hline
\end{tabular}




\begin{tabular}{|l|l|} 
Dominant Theme 3 & $\begin{array}{l}\text { P11 is also another successful EWHTA story. She is tenacious retired } \\
\text { electrical engineer immigrant with high self-efficacy and a lot of } \\
\text { technical experience. While p10's success was rooted in her family } \\
\text { and strong gatekeeping network, p11's is her drive to stay young } \\
\text { and healthy. This motivates her to learn the tech even though she is } \\
\text { not exposed to many technologies. She reaches out to every one- } \\
\text { on-one free resource she can to learn \& use technology. Even } \\
\text { though, she doesn't have a savvy gatekeeper, her positive personal } \\
\text { trait are strong driver of adoption. }\end{array}$ \\
\hline Dominant Theme 4 & $\begin{array}{l}\text { Young E cares about tech aesthetic and doesn't use a tech that } \\
\text { makes her look old. }\end{array}$ \\
\hline Discovery & $\begin{array}{l}\text { For E's with no strong gatekeeping network, good self-efficacy and } \\
\text { positive attitude toward tech is key to paving TA path }\end{array}$ \\
\hline
\end{tabular}

Table H - 11 Elderly woman \#11 profile 


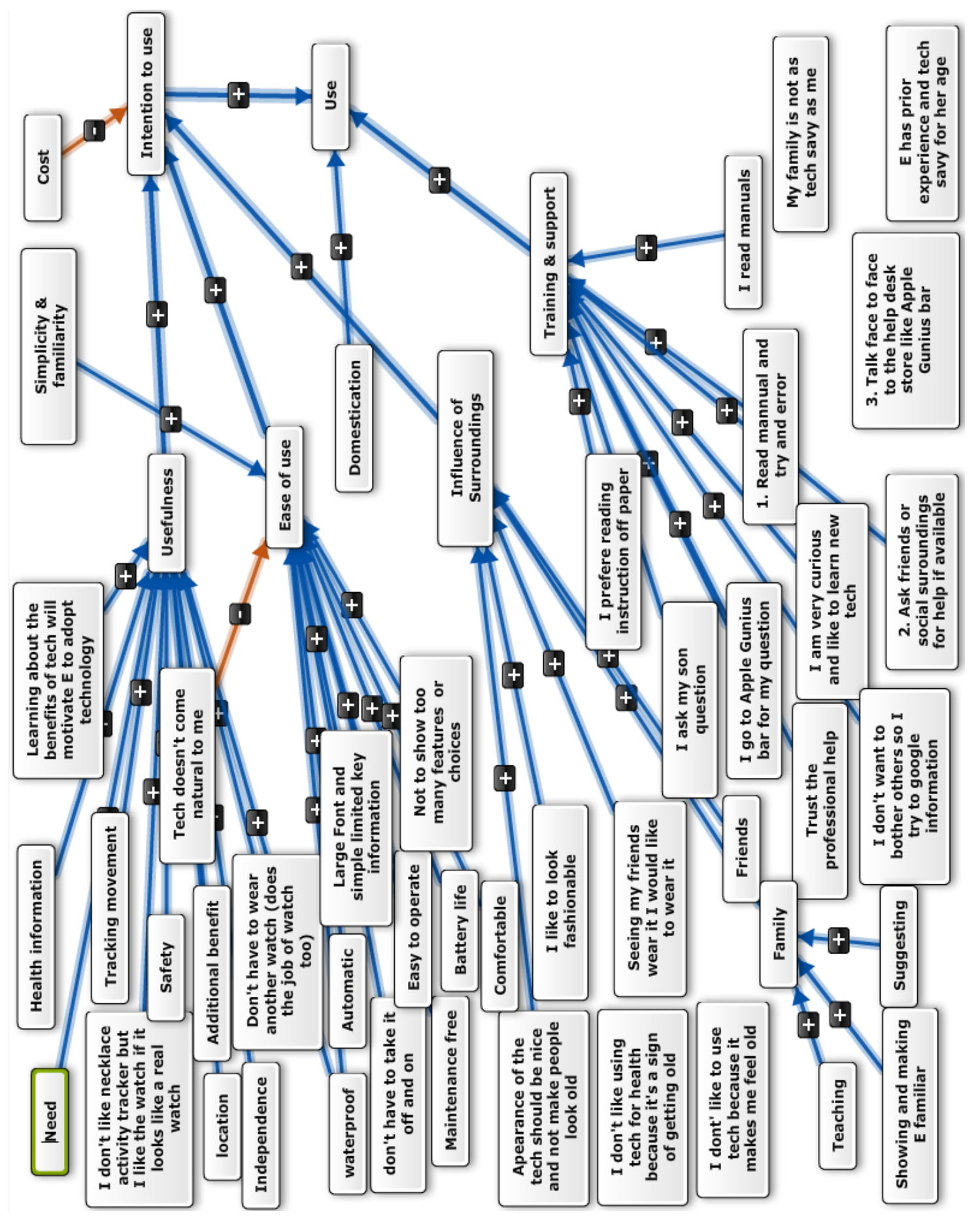

Figure H - 61 EW \#11 initial cognitive map 


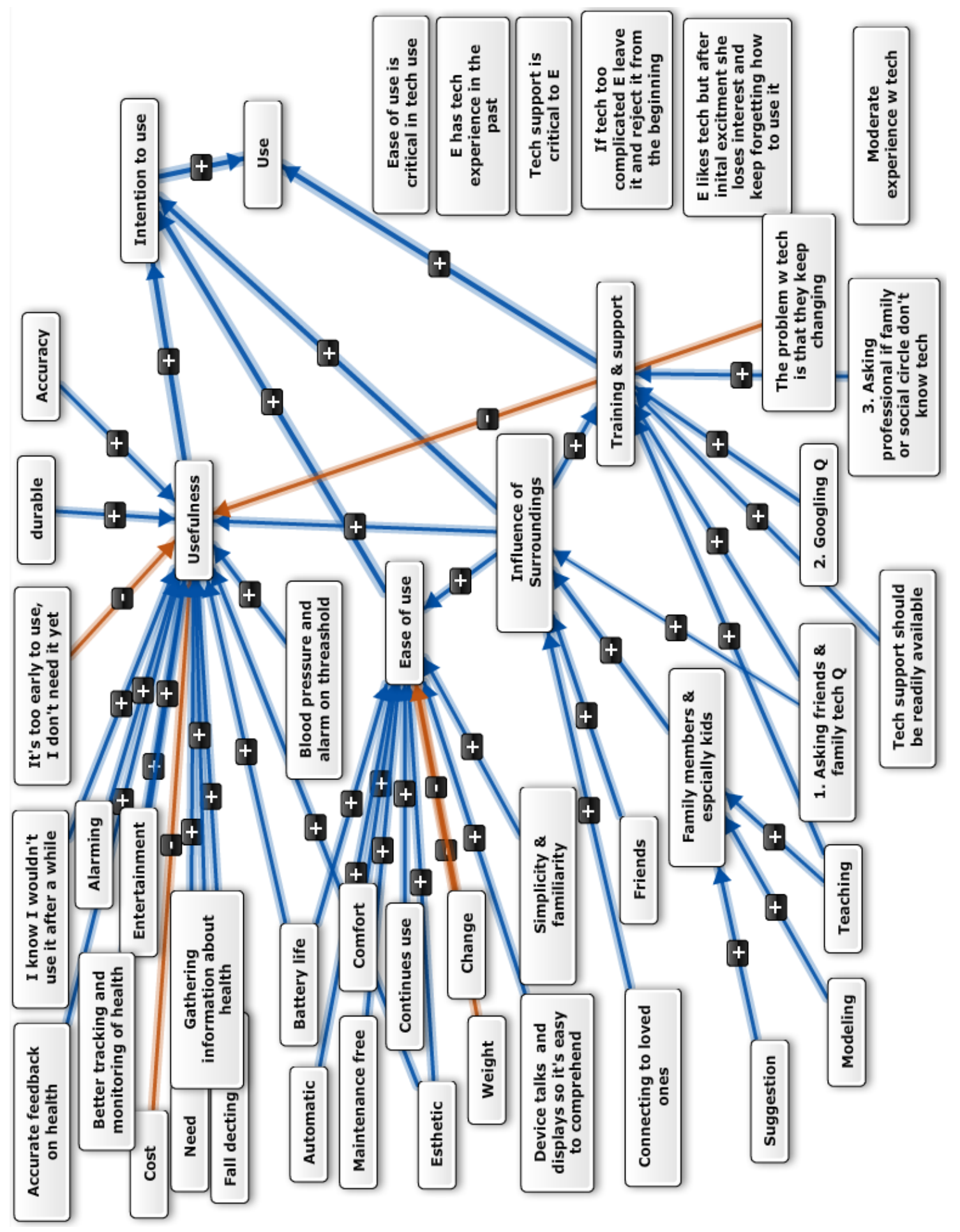

Figure H - 62 Gatekeeper \#11 initial cognitive map 


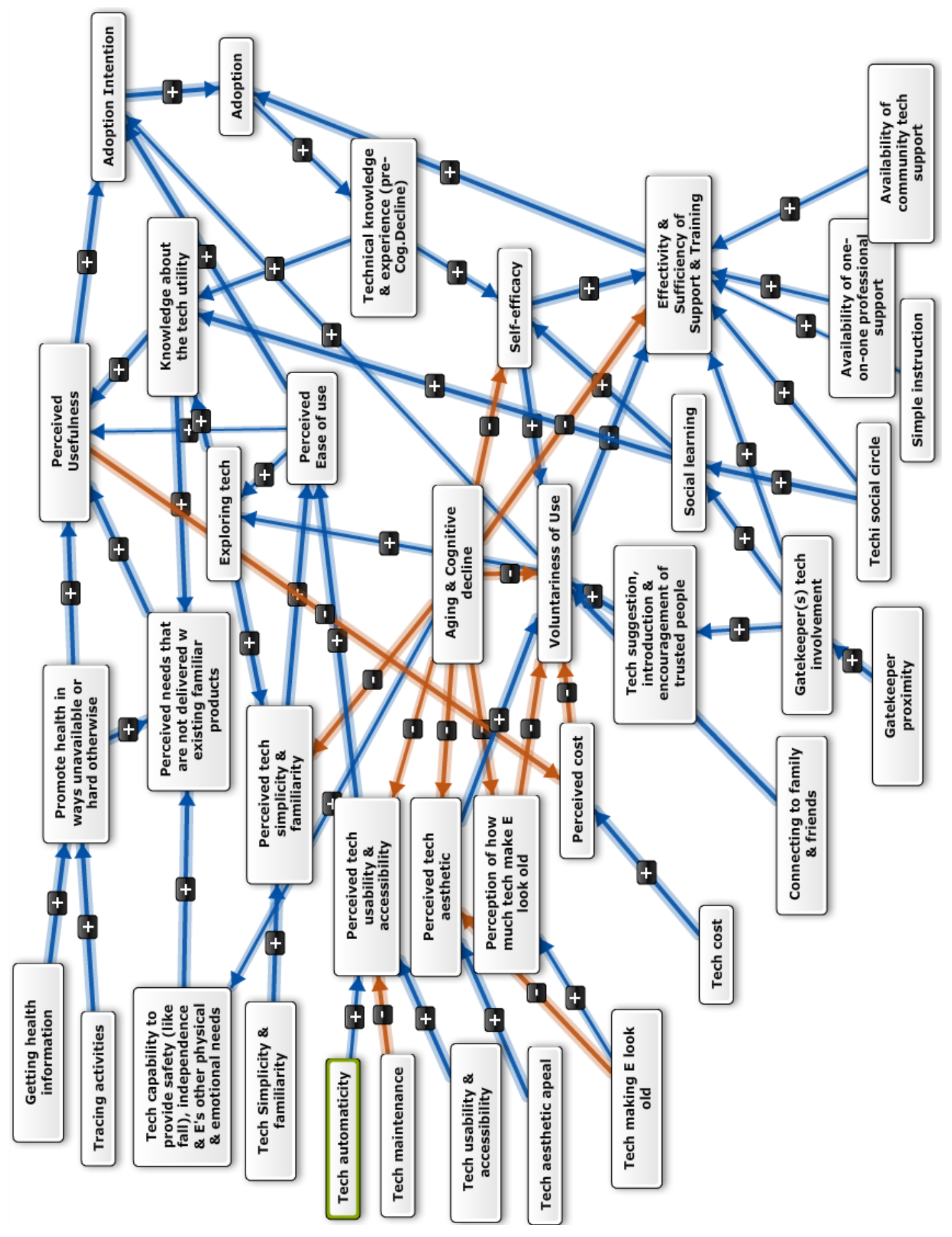

Figure H - 63 Elderly Gatekeeper pair \# 11 integrated cognitive map 


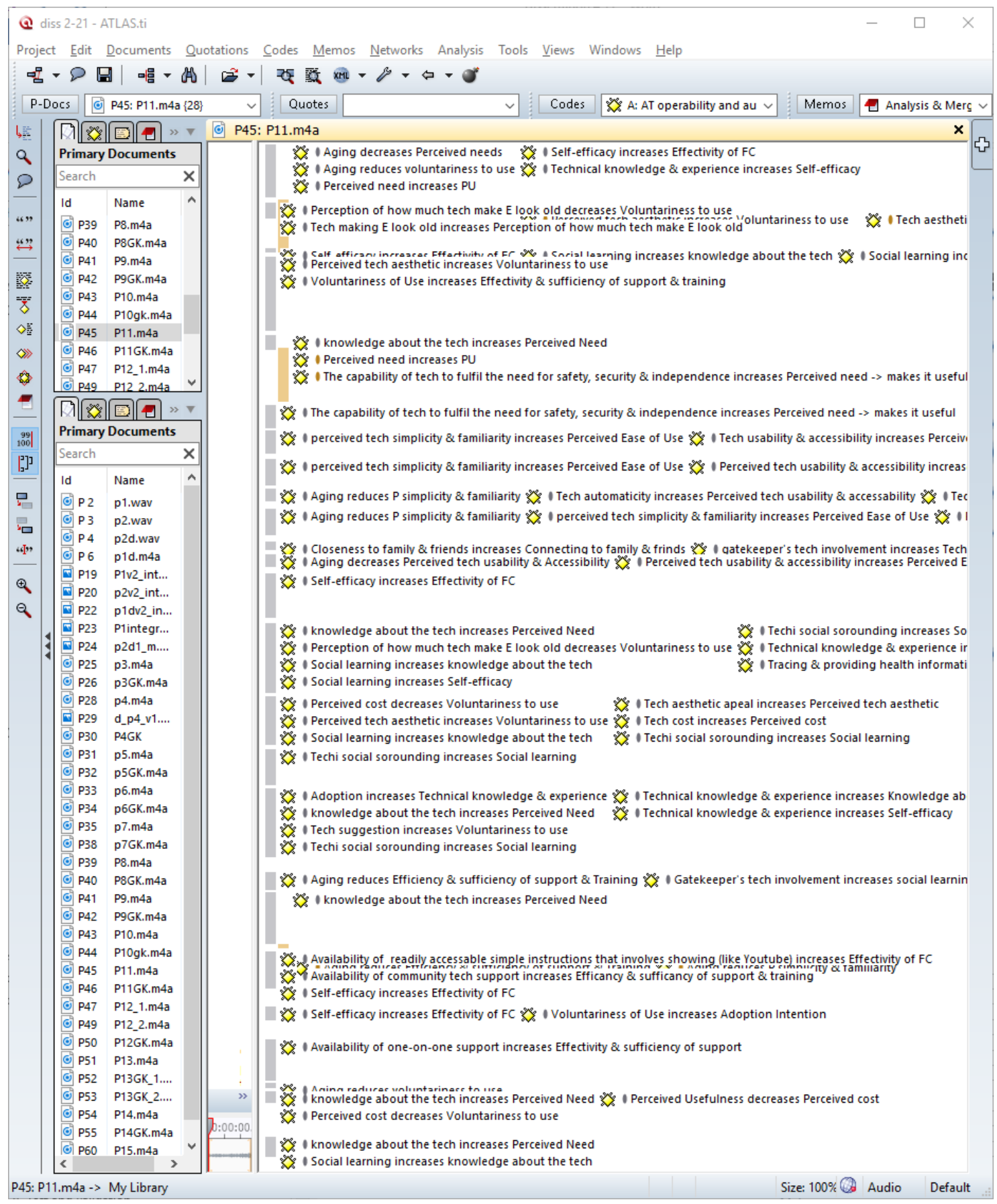

Figure H - 64 EW\#11 interview open coding in Atlas.ti 


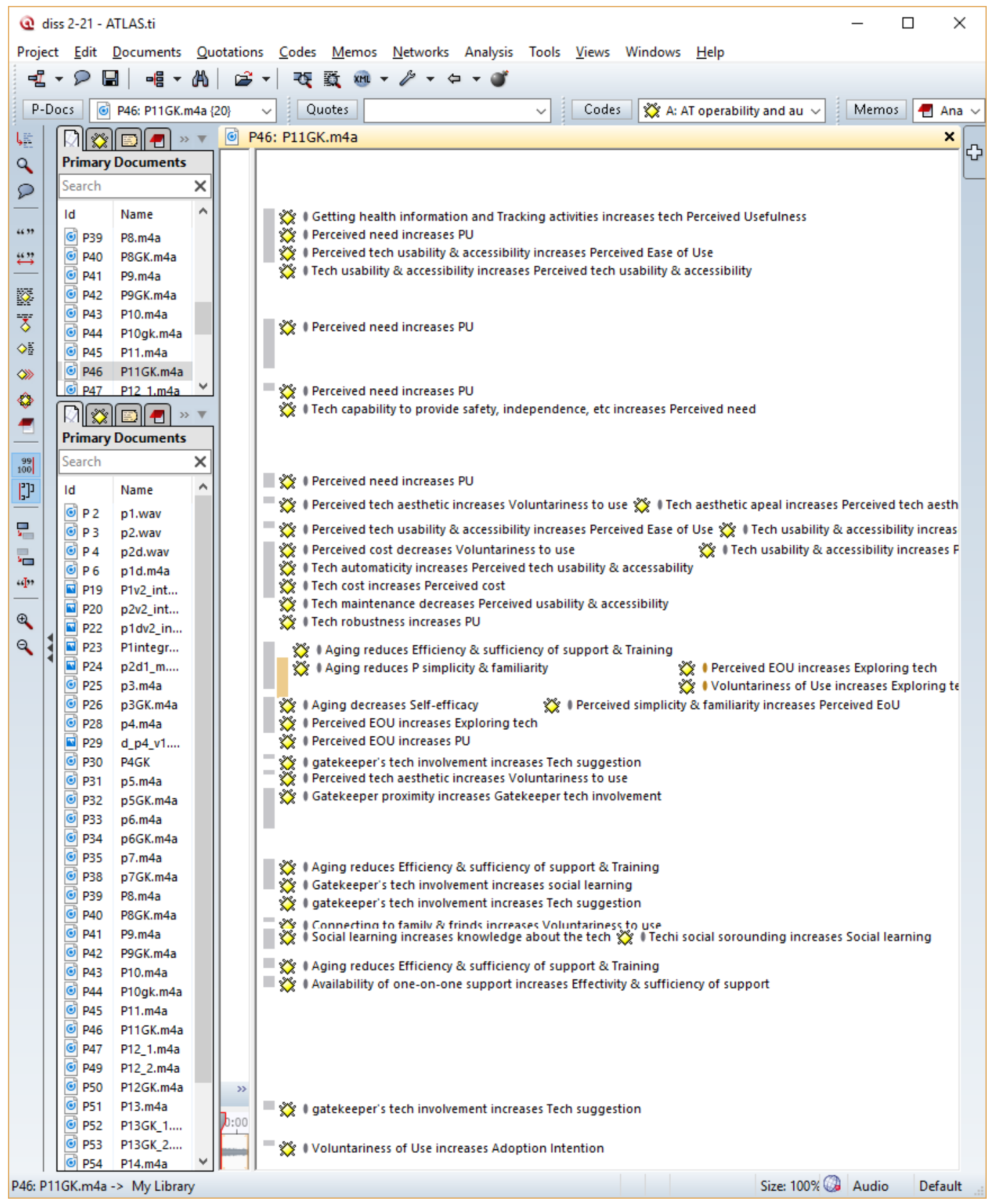

Figure H - 65 Gatekeeper \#11 interview open coding in Atlas.ti 


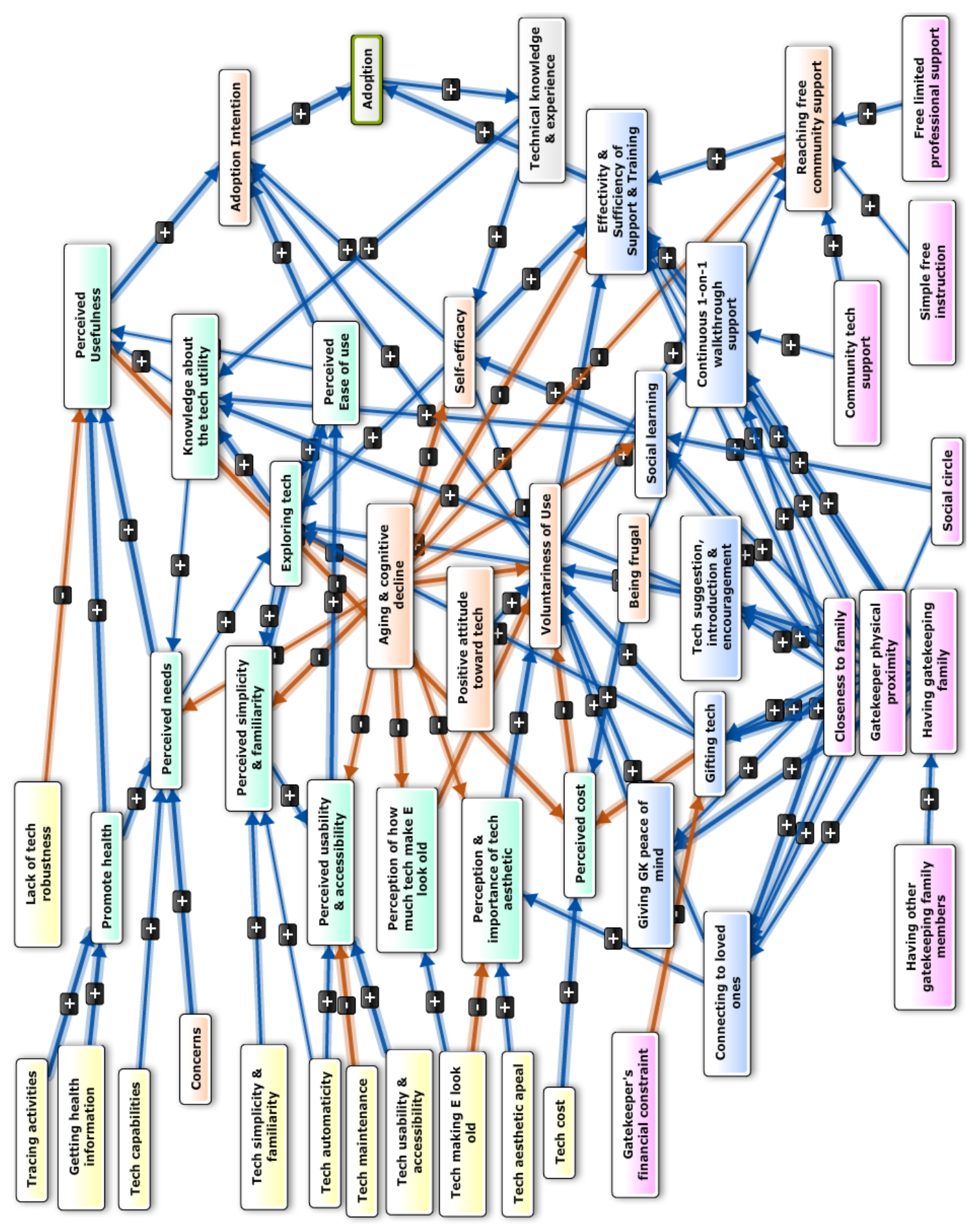

Figure H - 66 EW \#11 integrated \& standardized cognitive map 


\section{Elderly Woman \#12}

\begin{tabular}{|c|c|}
\hline Elderly Profile & $\mathrm{P} 12$ \\
\hline Interview Duration & $82 \mathrm{~min}$ \\
\hline Age & 65 \\
\hline Health Condition & OK, but impaired hearing \\
\hline Living condition & $\begin{array}{l}\text { Living in her house w husband, frequently travel to visit kids and } \\
\text { grandkids }\end{array}$ \\
\hline Isolated & No \\
\hline Attitude toward Tech & Positive \\
\hline Self-efficacy & $\mathrm{N}$ \\
\hline Experience & No \\
\hline Driven by & Health promotion, connecting to grandchildren \\
\hline Has grandchildren? & $\mathrm{Y}$ \\
\hline Has \& close to family & $\mathrm{Y}$ \\
\hline Gatekeeper & Daughter ( 36 yrs old, Interview duration: $56.5 \mathrm{~min}$ ) \\
\hline $\begin{array}{l}\text { Gatekeeper tech } \\
\text { savviness }\end{array}$ & $\mathrm{Y}$ \\
\hline Degree of influence & High \\
\hline Influence & $\begin{array}{l}\text { All (Social learning + Suggestion + gifting + effective support+ } \\
\text { wanting to connect+ Reduce burden + Giving Peace of mind) }\end{array}$ \\
\hline $\begin{array}{l}\text { Perceived Usefulness } \\
\text { of the wearable }\end{array}$ & It seems very useful \\
\hline $\begin{array}{l}\text { Perceived Ease of } \\
\text { Use of the wearable }\end{array}$ & I'm not sure, but my daughter can help me learn to use it \\
\hline $\begin{array}{l}\text { Will she adopt if } \\
\text { free? }\end{array}$ & Y \\
\hline $\begin{array}{l}\text { Will she buy to } \\
\text { adopt? }\end{array}$ & $\mathrm{N}$ \\
\hline Note & $\begin{array}{l}\text { E has a network of tech savvi people around that help her with tech } \\
\text { use. }\end{array}$ \\
\hline Dominant Theme 1 & $\begin{array}{l}\text { Young E cares about tech aesthetic and doesn't use a tech that } \\
\text { makes her look old. }\end{array}$ \\
\hline Dominant Theme 2 & $E$ is very practical, driven by need. But only adopt if it's easy to use \\
\hline Dominant Theme 3 & $\begin{array}{l}\text { P12 is also another successful EWHTA story. Although she has no } \\
\text { previous tech experience and has low self-efficacy, she is } \\
\text { surrounded by tech savvy family who are close and influence her } \\
\text { adoption at all levels. E's success is again rooted in her family and } \\
\text { strong gatekeeping network }\end{array}$ \\
\hline Dominant Theme 4 & Being frugal reduces the "Reaching professional fee-based support" \\
\hline
\end{tabular}




\begin{tabular}{|l|l|} 
Discovery & $\begin{array}{l}\text { Strong family gatekeeping network support, more than makes up } \\
\text { for lack of experience (low self-efficacy), nonetheless, P EOU remain } \\
\text { to be the most important factor in EWHTA. }\end{array}$ \\
\hline GK & $\begin{array}{l}\text { P12GK In vivo: "It's about east of use, 100\%, if she knows it } \\
\text { becomes easy for her to use, she is not gone resist it at all" }\end{array}$ \\
\hline
\end{tabular}

Table H - 12 Elderly woman \#12 profile 


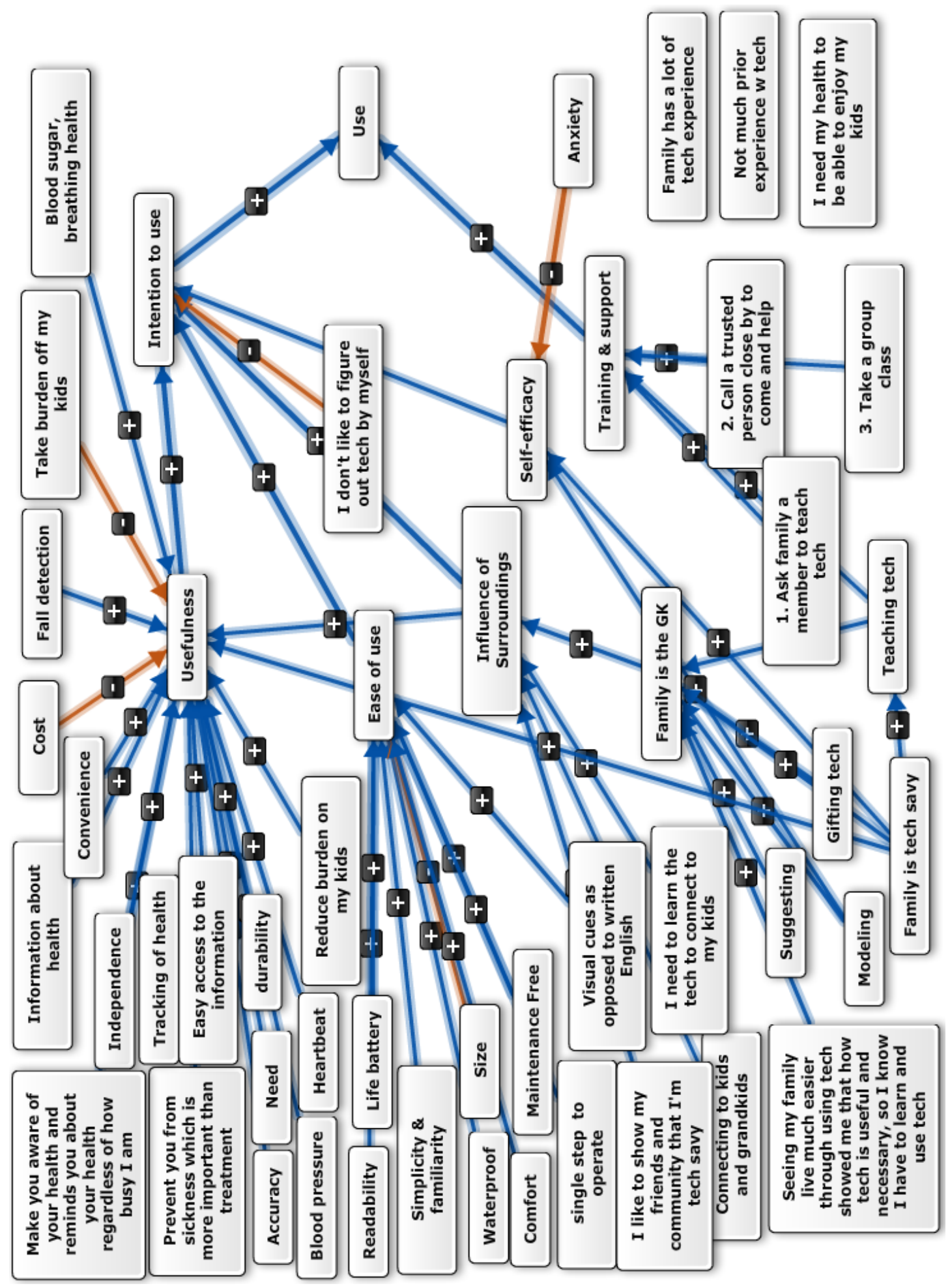

Figure H - 67 EW \#12 initial cognitive map 


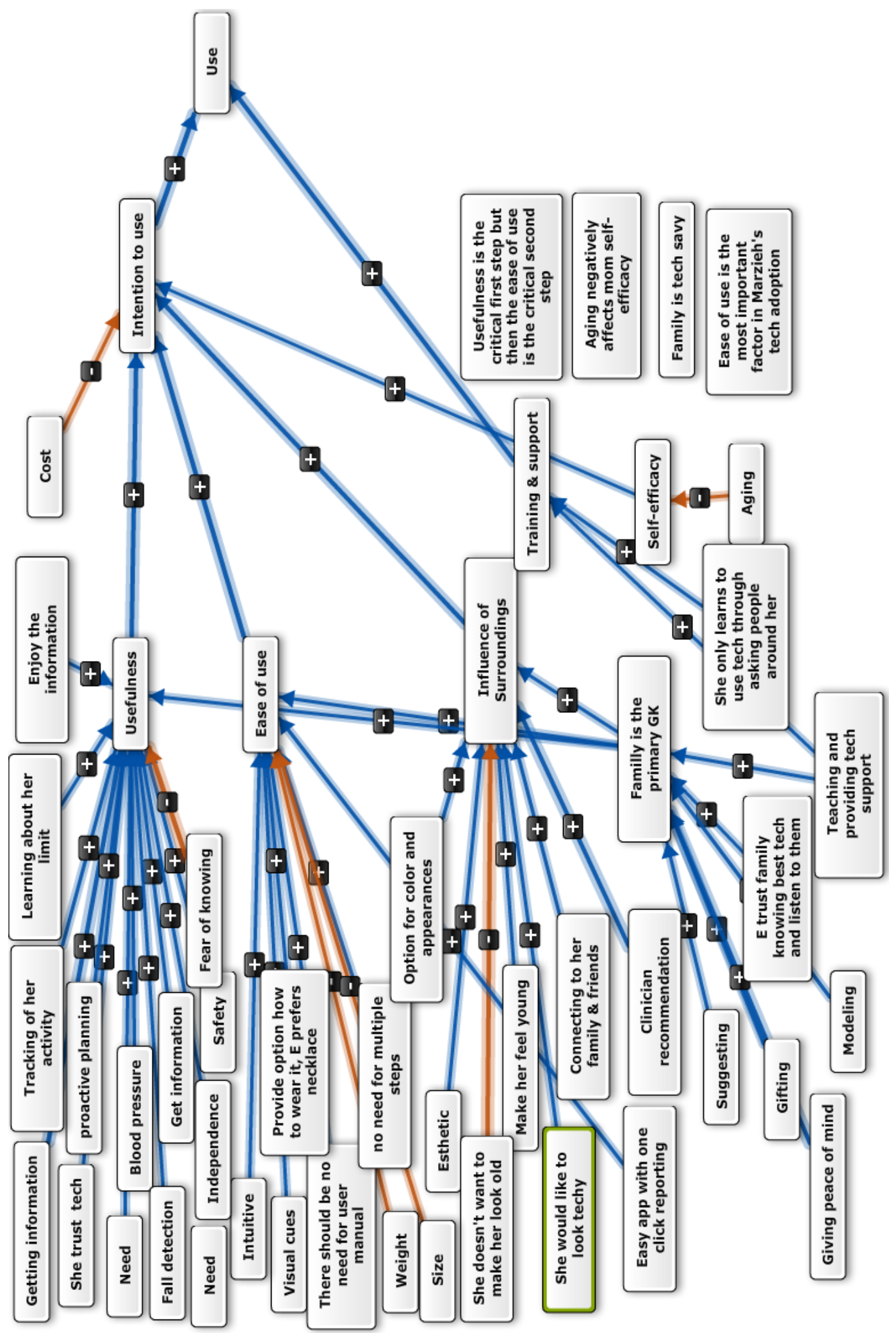

Figure H - 68 Gatekeeper \#12 initial cognitive map

535 


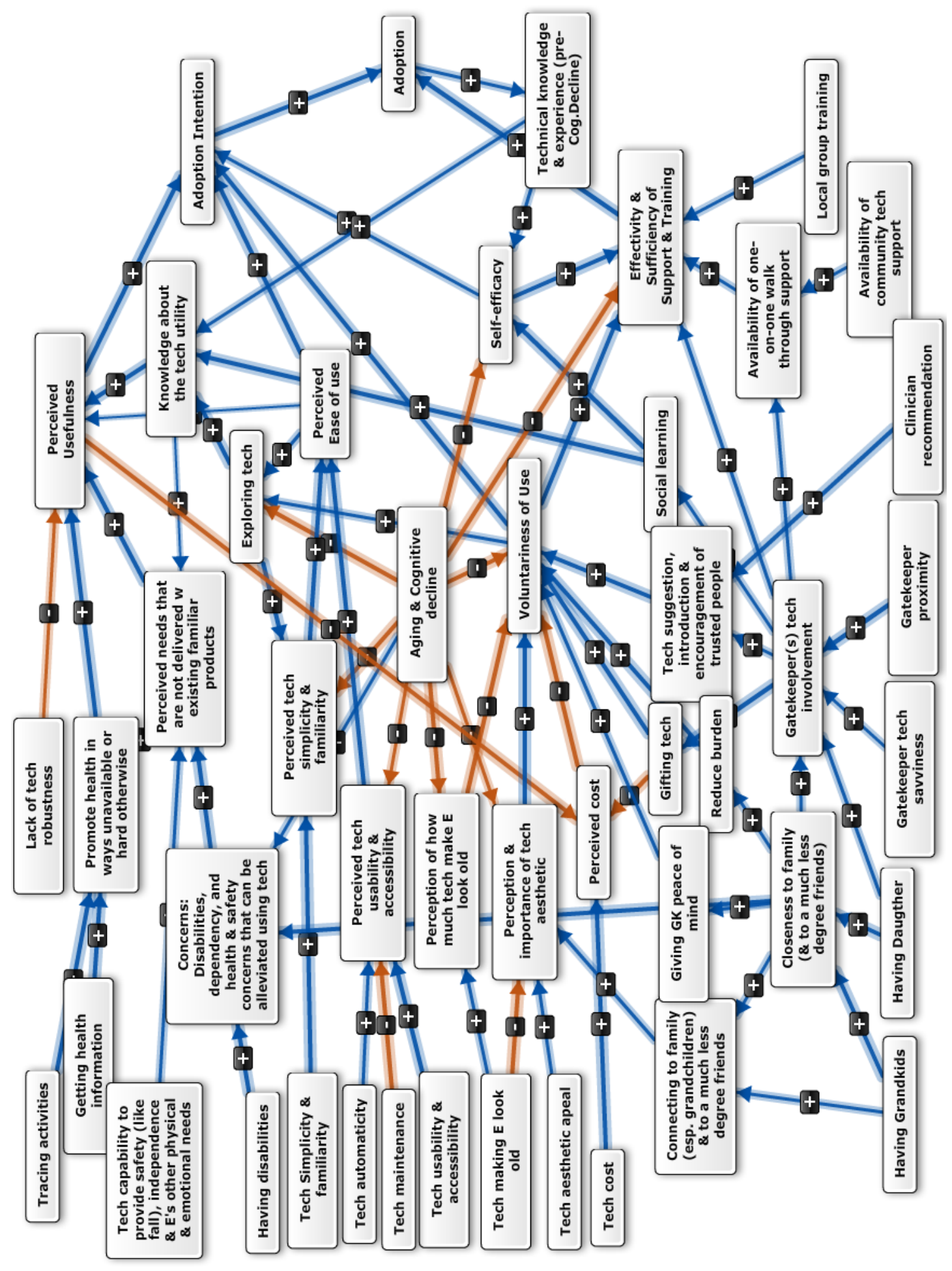

Figure H - 69 Elderly Gatekeeper pair \#12 integrated cognitive map 


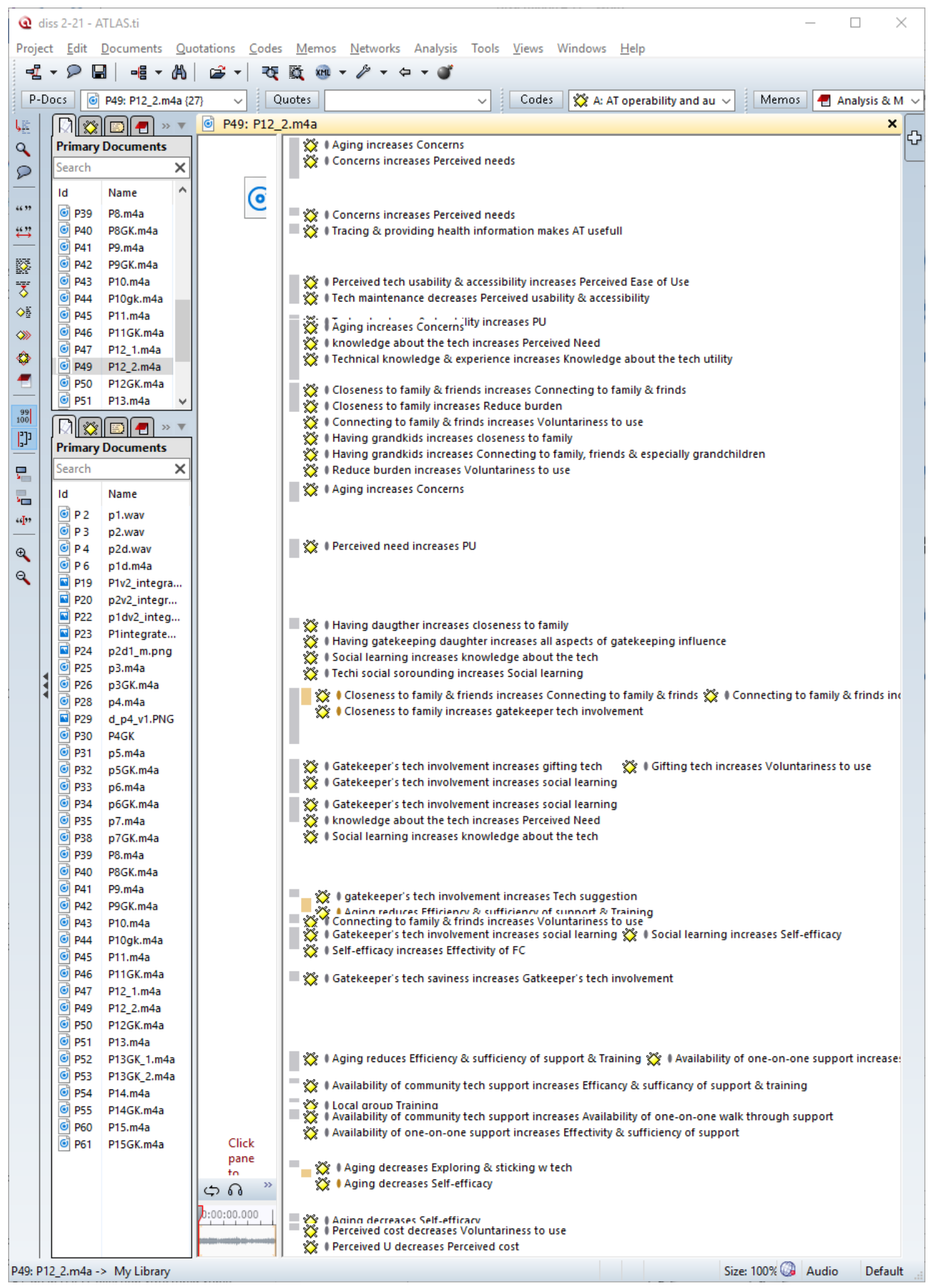

\section{Figure $\mathrm{H}$ - $70 \mathrm{EW} \# 12$ interview open coding in Atlas.ti}




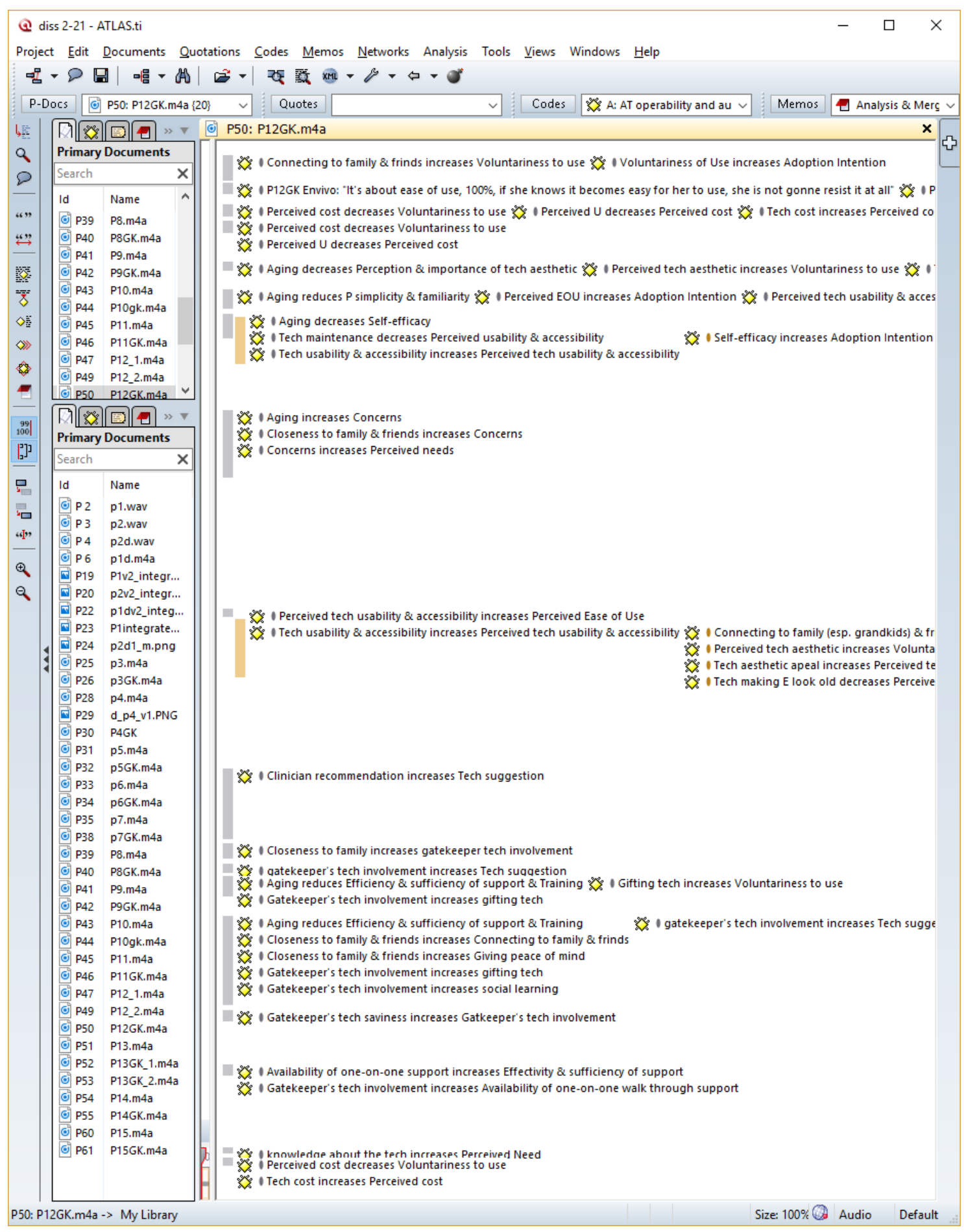

Figure H - 71 Gatekeeper \#12 interview open coding in Atlas.ti 


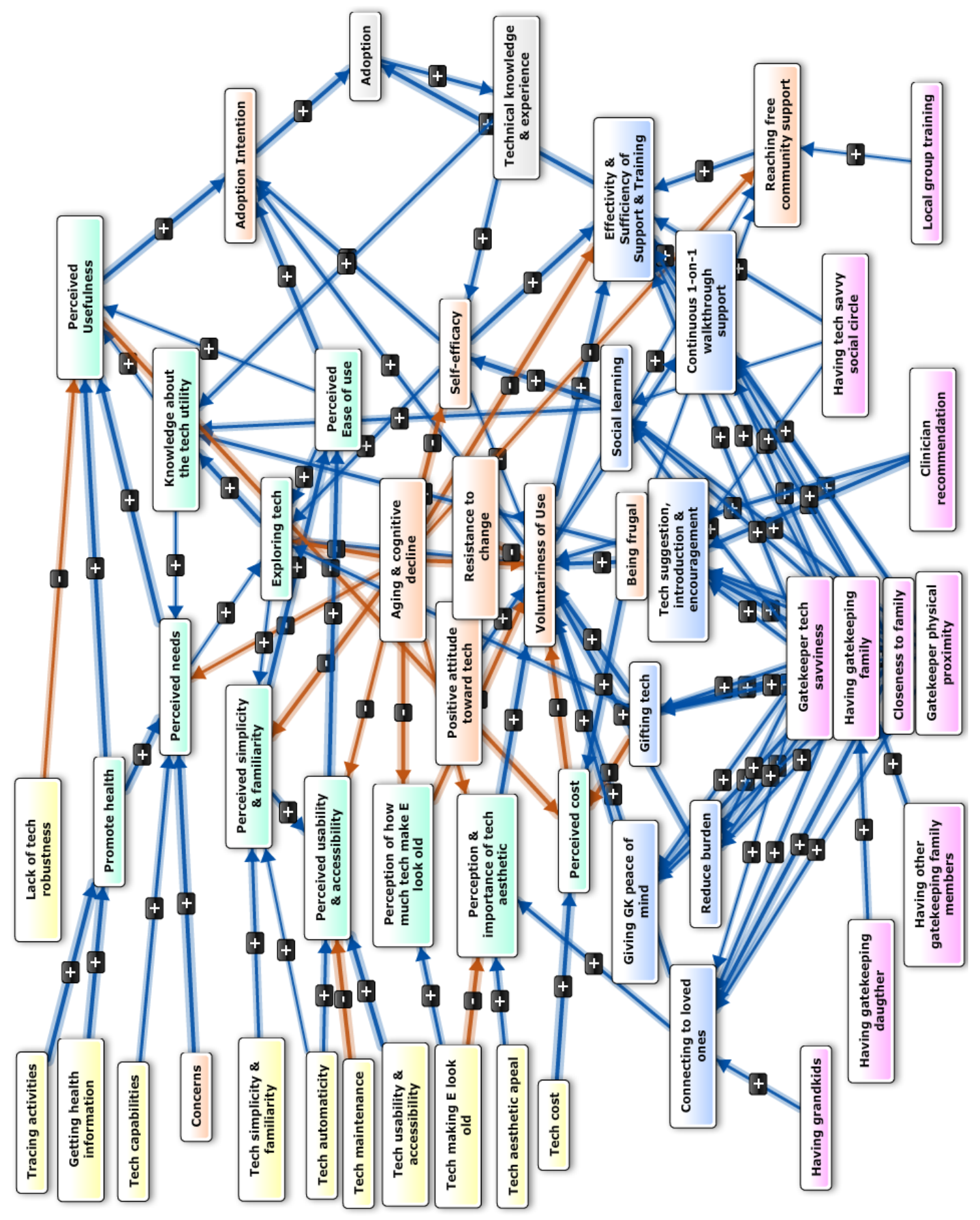

Figure H - 72 EW \#12 integrated \& standardized cognitive map 


\section{Elderly Woman \#13}

\begin{tabular}{|c|c|}
\hline Elderly Profile & P13 \\
\hline Interview Duration & $42 \mathrm{~min}$ \\
\hline Age & 71 \\
\hline Health Condition & OK \\
\hline Living condition & Living in her house w husband, Daughter lives in the same city \\
\hline Isolated & No \\
\hline Attitude toward Tech & Very positive for her age. Technology enthusiast \\
\hline Self-efficacy & Moderate \\
\hline Experience & No \\
\hline Driven by & Health promotion and information, staying young \\
\hline Has grandchildren? & Y \\
\hline Has \& close to family & Y \\
\hline Gatekeeper & Daughter (43 yrs old, Interview duration: 63 ) \\
\hline $\begin{array}{l}\text { Gatekeeper tech } \\
\text { savviness }\end{array}$ & $\begin{array}{l}\text { Y (More of a network of gatekeeping family with the daughter being } \\
\text { at the center and first point of contact) }\end{array}$ \\
\hline Degree of influence & High \\
\hline Influence & $\begin{array}{l}\text { All (Social learning + Suggestion + gifting + effective support+ } \\
\text { Available 1-on-1 + wanting to connect+ Reduce burden + Giving } \\
\text { Peace of mind) }\end{array}$ \\
\hline $\begin{array}{l}\text { Perceived Usefulness } \\
\text { of the wearable }\end{array}$ & It's very useful \\
\hline $\begin{array}{l}\text { Perceived Ease of } \\
\text { Use of the wearable }\end{array}$ & It's not easy \\
\hline $\begin{array}{l}\text { Will she adopt if } \\
\text { free? }\end{array}$ & Y \\
\hline $\begin{array}{l}\text { Will she buy to } \\
\text { adopt? }\end{array}$ & $\mathrm{Y}$ \\
\hline Note & $\begin{array}{l}\text { E has a strong desire to learn and stay young and think of } \\
\text { technology as enabling that desire. She has her own blog and very } \\
\text { active online. She ask questions from everyone in the family and the } \\
\text { social circle and loves to stay connected. E has a network of tech } \\
\text { savvy people around that help her with tech use. }\end{array}$ \\
\hline Dominant Theme 1 & $\begin{array}{l}\text { E's desire for life and staying young derives her enthusiasm for } \\
\text { learning in general and learning technology in particular }\end{array}$ \\
\hline
\end{tabular}




\begin{tabular}{|l|l|} 
Dominant Theme 2 & $\begin{array}{l}\text { E is very practical, driven by need. But only adopt if it's easy to use } \\
\text { and doesn't make her look old }\end{array}$ \\
\hline Dominant Theme 3 & $\begin{array}{l}\text { P13 is also another successful EWHTA story. She is tenacious retired } \\
\text { school teacher who loves to stay young and enjoy life. She focuses a } \\
\text { lot on her health and staying active and generally very enthusiastic } \\
\text { about technology. She likes to use beautiful colorful technology. } \\
\text { This success is made possible by her tech savvy family who are } \\
\text { strong gatekeeping network and provide all 7 category of emerged } \\
\text { influences. }\end{array}$ \\
\hline Dominant Theme 4 & $\begin{array}{l}\text { Young E cares about tech aesthetic and doesn't use a tech that } \\
\text { makes her look old }\end{array}$ \\
\hline Discovery & $\begin{array}{l}\text { The most important category of gatekeeping influence is the } \\
\text { availability, simplicity and continuous one-on-one support \& } \\
\text { training }\end{array}$ \\
\hline GK & $\begin{array}{l}\text { Daughter (even if she is not as savvy as the son) and Living close is } \\
\text { chosen as the primary GK over having son who is very tech savvy } \\
\text { but live far }\end{array}$ \\
\hline
\end{tabular}

Table H - 13 Elderly woman \#13 profile 


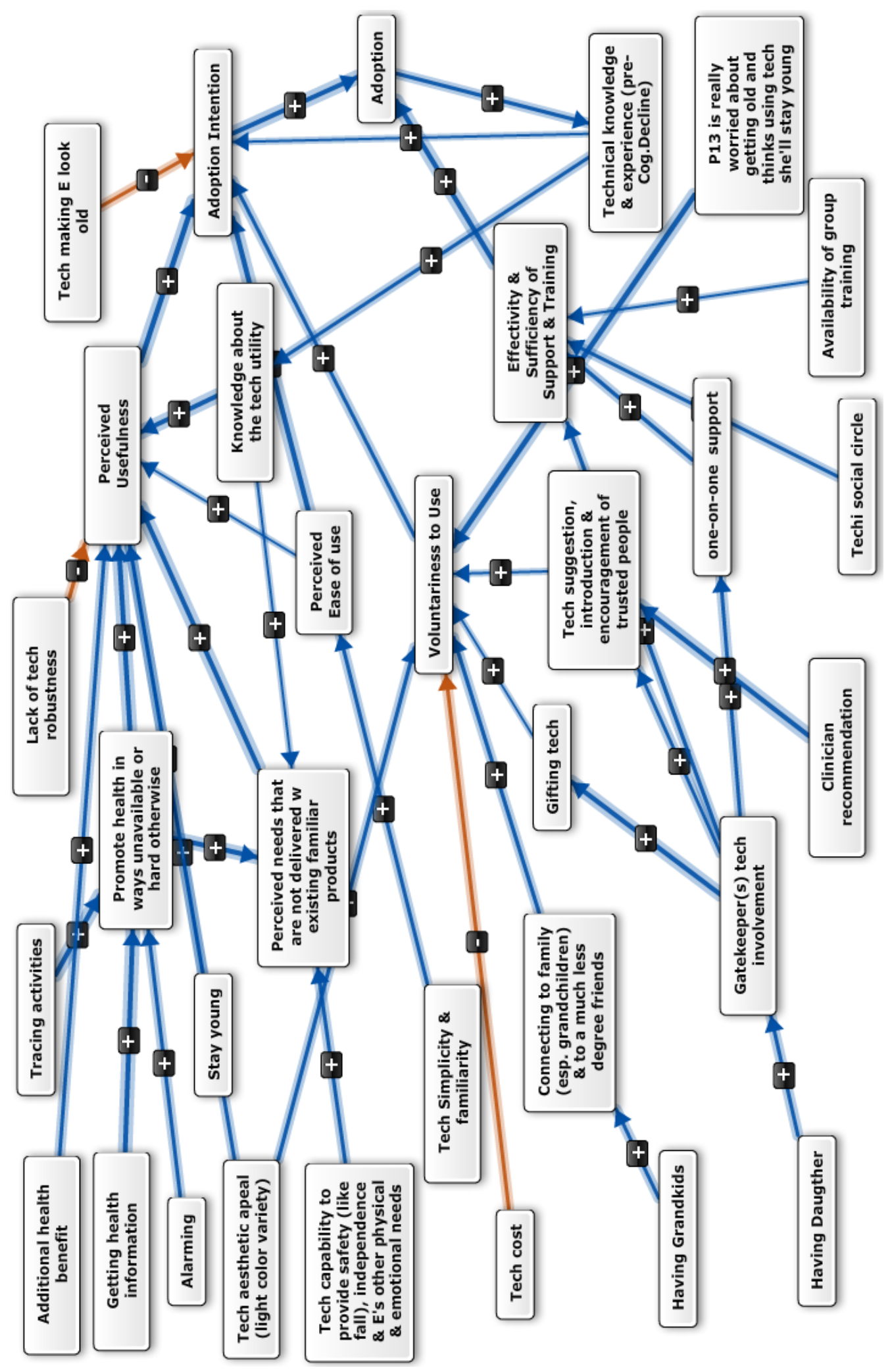

Figure H - 73 EW \#13 initial cognitive map 


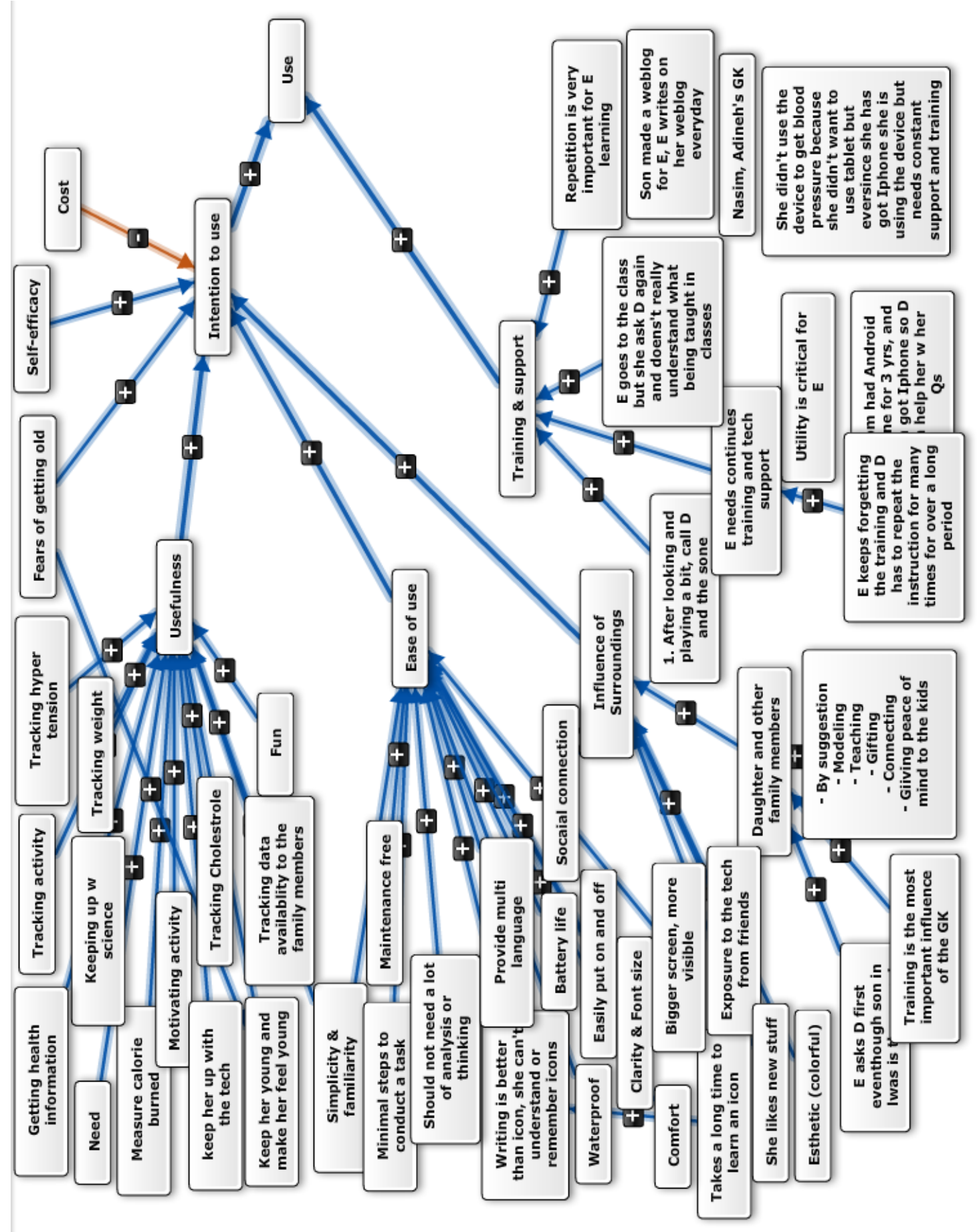

Figure H - 74 Gatekeeper \#13 initial cognitive map 


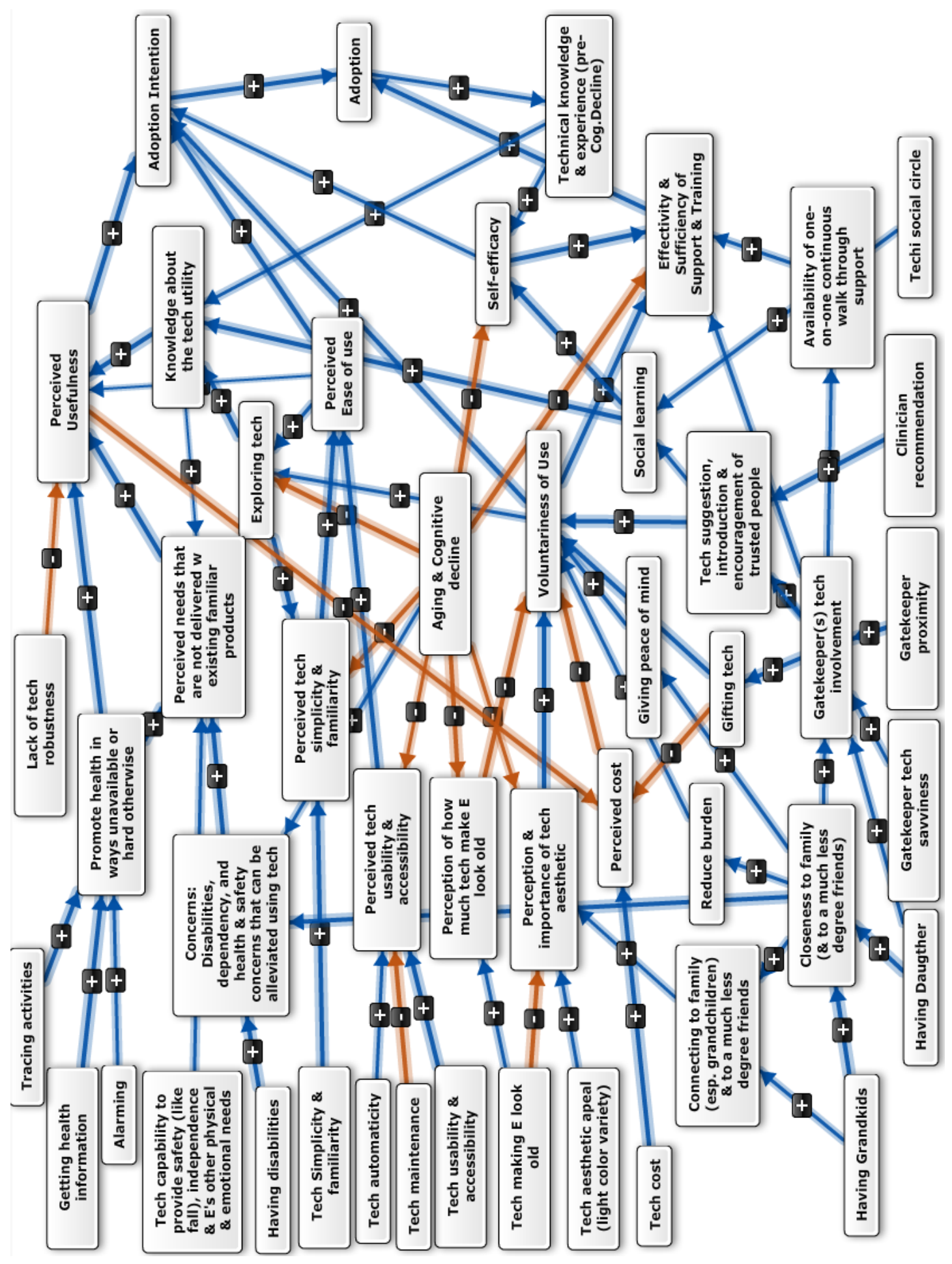

Figure H - 75 Elderly Gatekeeper pair \#13 integrated cognitive map 


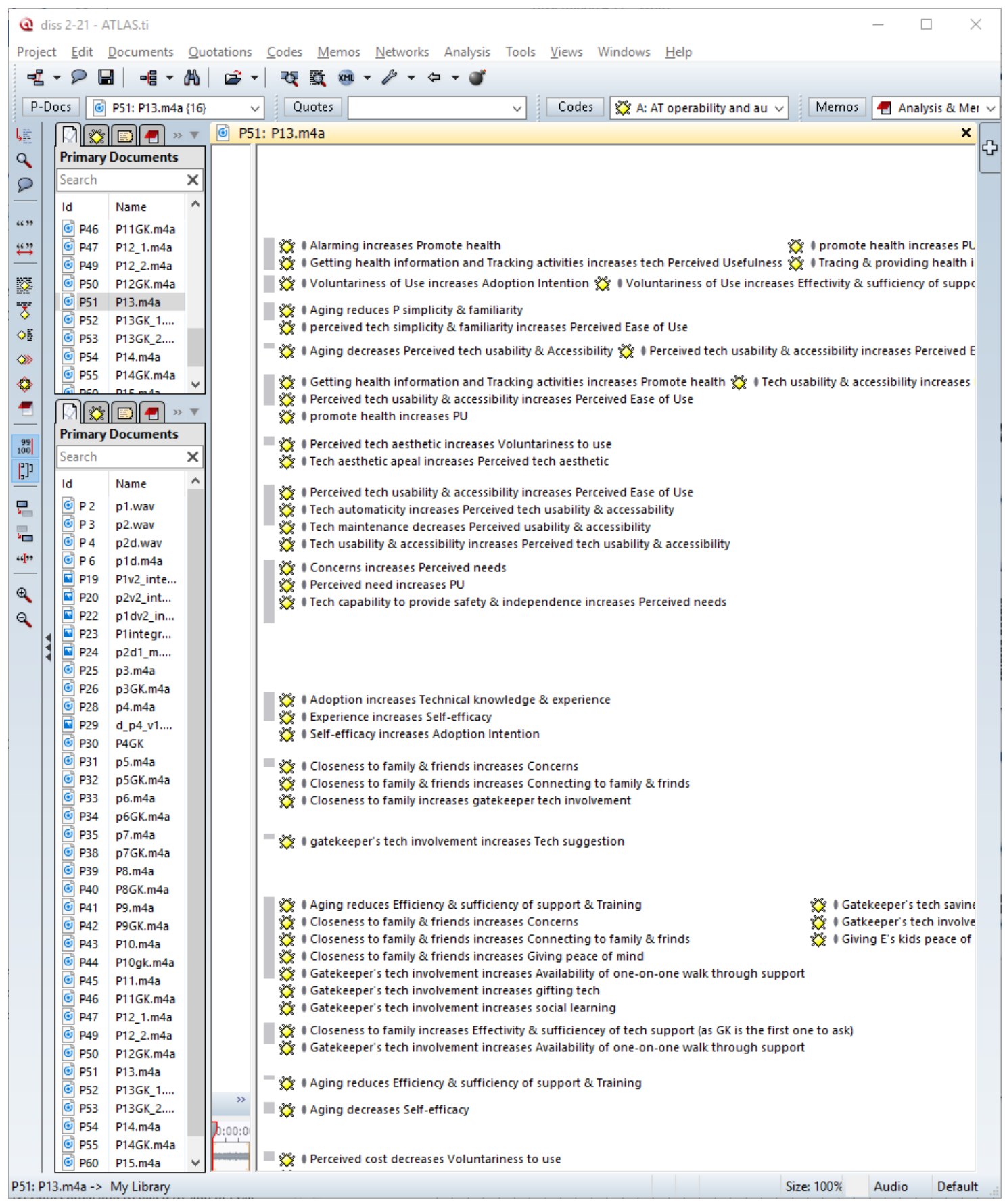

Figure H - 76 EW\#13 interview open coding in Atlas.ti 


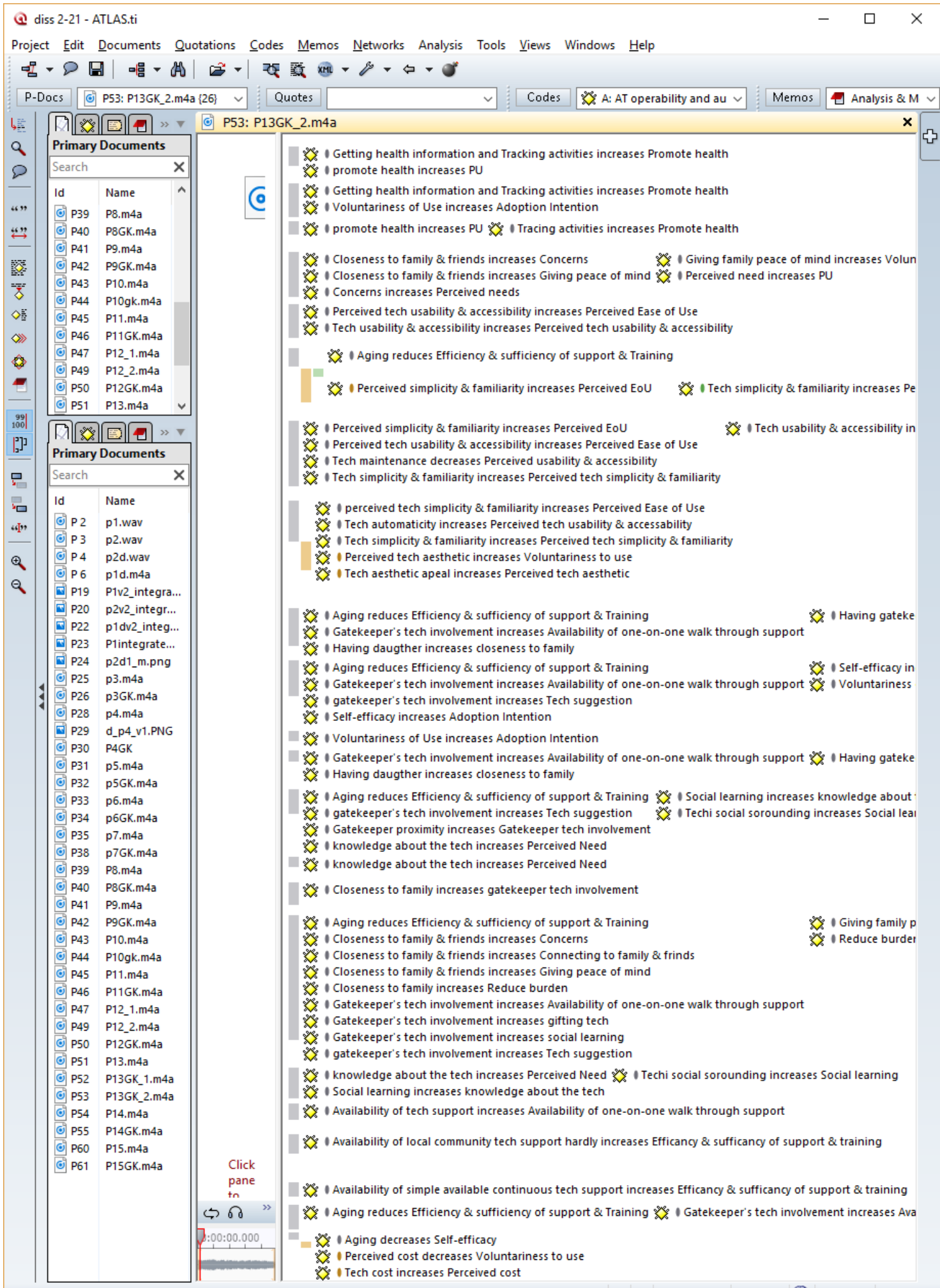

\section{Figure H - 77 Gatekeeper \#13 interview open coding in Atlas.ti}




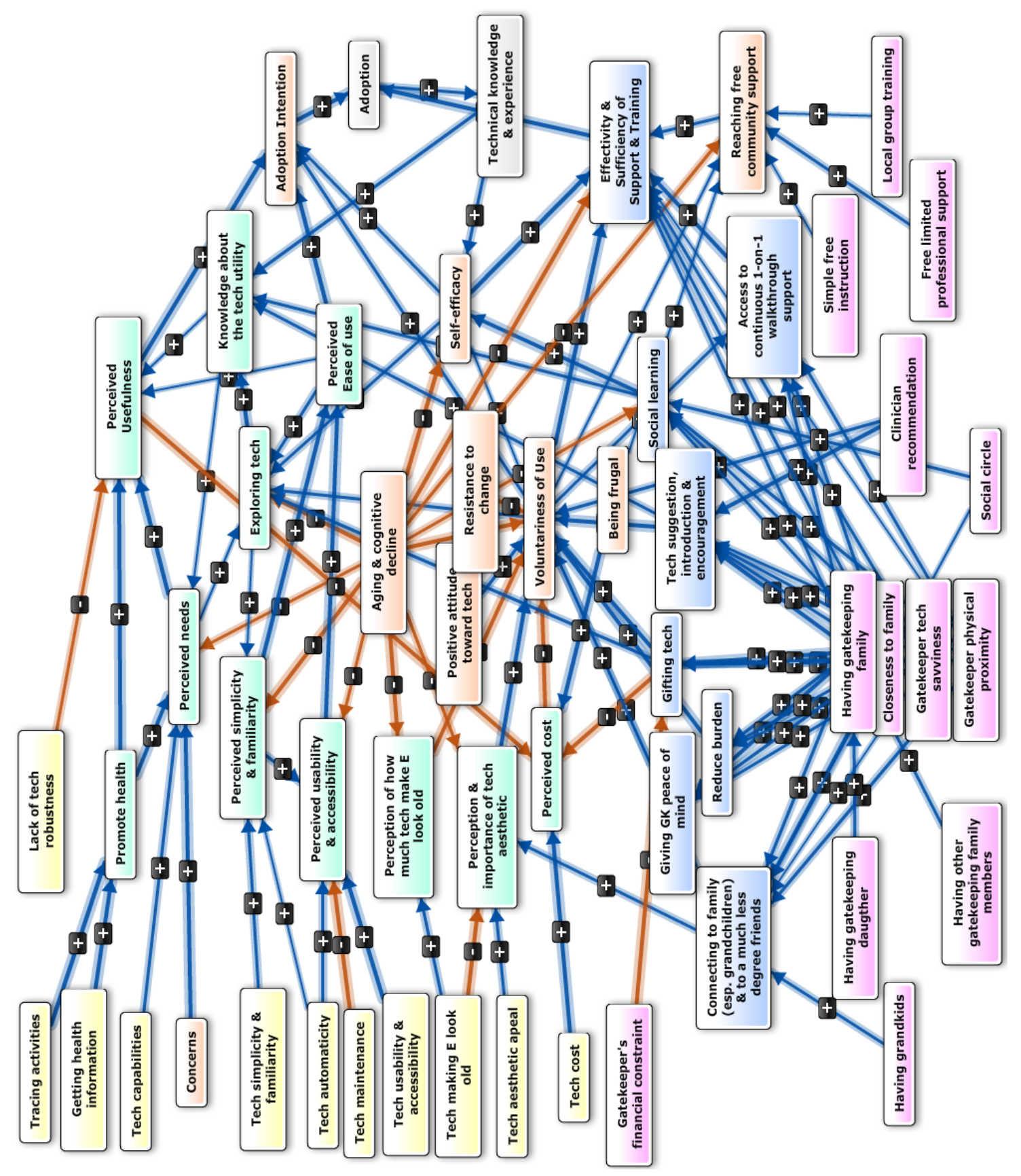

Figure H - 78 EW \#13 integrated \& standardized cognitive map 


\section{Elderly Woman \#14}

\begin{tabular}{|c|c|}
\hline Elderly Profile & P14 \\
\hline Interview Duration & $57 \mathrm{~min}$ \\
\hline Age & 78 \\
\hline Health Condition & Great, hiker, swimmer. \\
\hline Living condition & Living in a retirement community \\
\hline Isolated & $\mathrm{N}$ \\
\hline Attitude toward Tech & Negative, yet wearing 3 ATs ( 2 owned, 1 trial) \\
\hline Self-efficacy & $\mathrm{Y}$ \\
\hline Experience & $\mathrm{Y}$ (master degree, librarian, worked with technology from 60's) \\
\hline Driven by & Health promotion and information \\
\hline Has grandchildren? & Y \\
\hline Has \& close to family & Y, Not close \& Low tech influence (in East Coast) \\
\hline Gatekeeper & Friend ( 58 yrs old female college professor) $(81 \mathrm{~min})$ \\
\hline $\begin{array}{l}\text { Gatekeeper tech } \\
\text { savviness }\end{array}$ & $\begin{array}{l}\text { Moderate (Network of highly educated athletic elderly women with } \\
\text { the latest techs) }\end{array}$ \\
\hline Degree of influence & Moderate \\
\hline Influence & $\begin{array}{l}\text { The social circle influence by Social learning + some effective } \\
\text { support; and distance family by mainly Gifting and Wanting to } \\
\text { connect + some Suggestion + when E visit by effective walk-through } \\
\text { support and + Social learning) }\end{array}$ \\
\hline $\begin{array}{l}\text { Perceived Usefulness } \\
\text { of the wearable }\end{array}$ & It is not useful \\
\hline $\begin{array}{l}\text { Perceived Ease of } \\
\text { Use of the wearable }\end{array}$ & It's not too hard to figure it out \\
\hline $\begin{array}{l}\text { Will she adopt if } \\
\text { free? }\end{array}$ & $\mathrm{N}$ \\
\hline $\begin{array}{l}\text { Will she buy to } \\
\text { adopt? }\end{array}$ & $\mathrm{N}$ \\
\hline Note & $\begin{array}{l}\text { P14 In vivo: "It helps to have a grandchildren you see, grandma let } \\
\text { me show you this app" }\end{array}$ \\
\hline Dominant Theme 1 & Main influencers, Grandkids and social circle. \\
\hline Dominant Theme 2 & $\begin{array}{l}\text { High self-efficacy (increased by tech experience) and need for } \\
\text { independency (lives alone) who increases Perceived tech usefulness } \\
\text { can compensate for lack of full gatekeeper's involvement. }\end{array}$ \\
\hline
\end{tabular}




\begin{tabular}{|l|l|} 
Dominant Theme 3 & $\begin{array}{l}\text { P14 is yet another successful EWHTA story. In other cases, most of } \\
\text { successful EWHTAs were due to having close family around who } \\
\text { played strong gatekeeping effects. P14 success is due to her: 1) } \\
\text { Strong self-efficacy rooted in her tech experience, 2) strong techy } \\
\text { social circle who model, help and inspire her to use tech, and 3) her } \\
\text { strong personal characteristic of being athletic and wanting to be } \\
\text { healthy which compensate for not having strong gatekeeping } \\
\text { presence and drive her reaching out to using secondary support. }\end{array}$ \\
\hline Dominant Theme 4 & $\begin{array}{l}\text { It's fascinating that P14 is keep talking negative about tech } \\
\text { (skeptical about its performance), yet she is the most equipped w } \\
\text { tech E I've interviewed. Re-listening to the interview, high self- } \\
\text { efficacy increases Adoption Intention and getting support as she ask } \\
\text { everyone for tech support (as she said experience has caused her to } \\
\text { have no fear of tech -> high self-efficacy) }\end{array}$ \\
\hline Discovery & $\begin{array}{l}\text { The most important gatekeeping influence is the availability, } \\
\text { simplicity and continuous one-on-one support \& training. When } \\
\text { family is not close by, if E has a techy social circle, they take an } \\
\text { active GK role in E's HTA by social learning and more importantly } \\
\text { provide that continuous jargon free support she immensely need. }\end{array}$ \\
\hline
\end{tabular}

Table H - 14 Elderly woman \#14 profile 


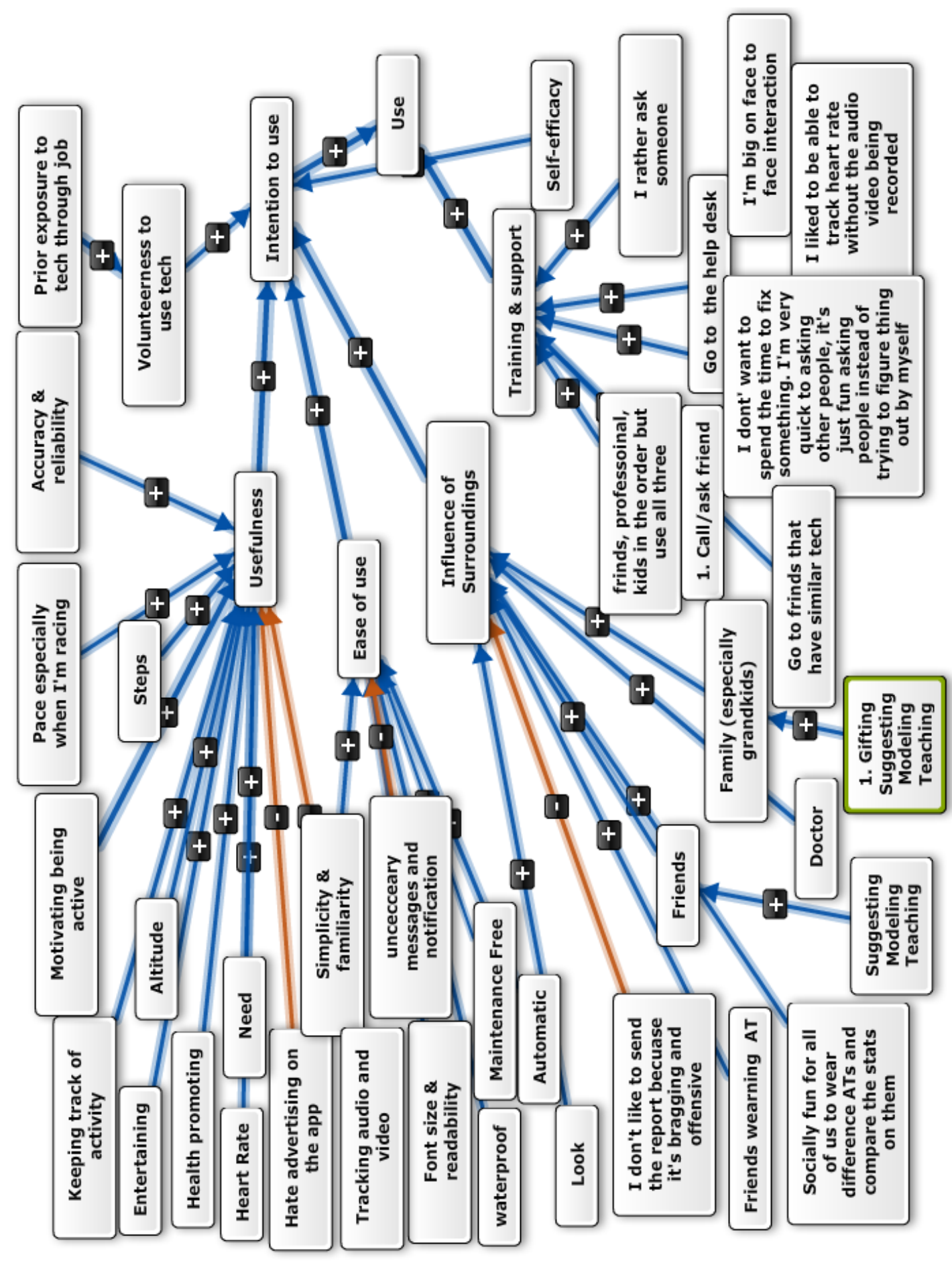

Figure H - 79 EW \#14 initial cognitive map 


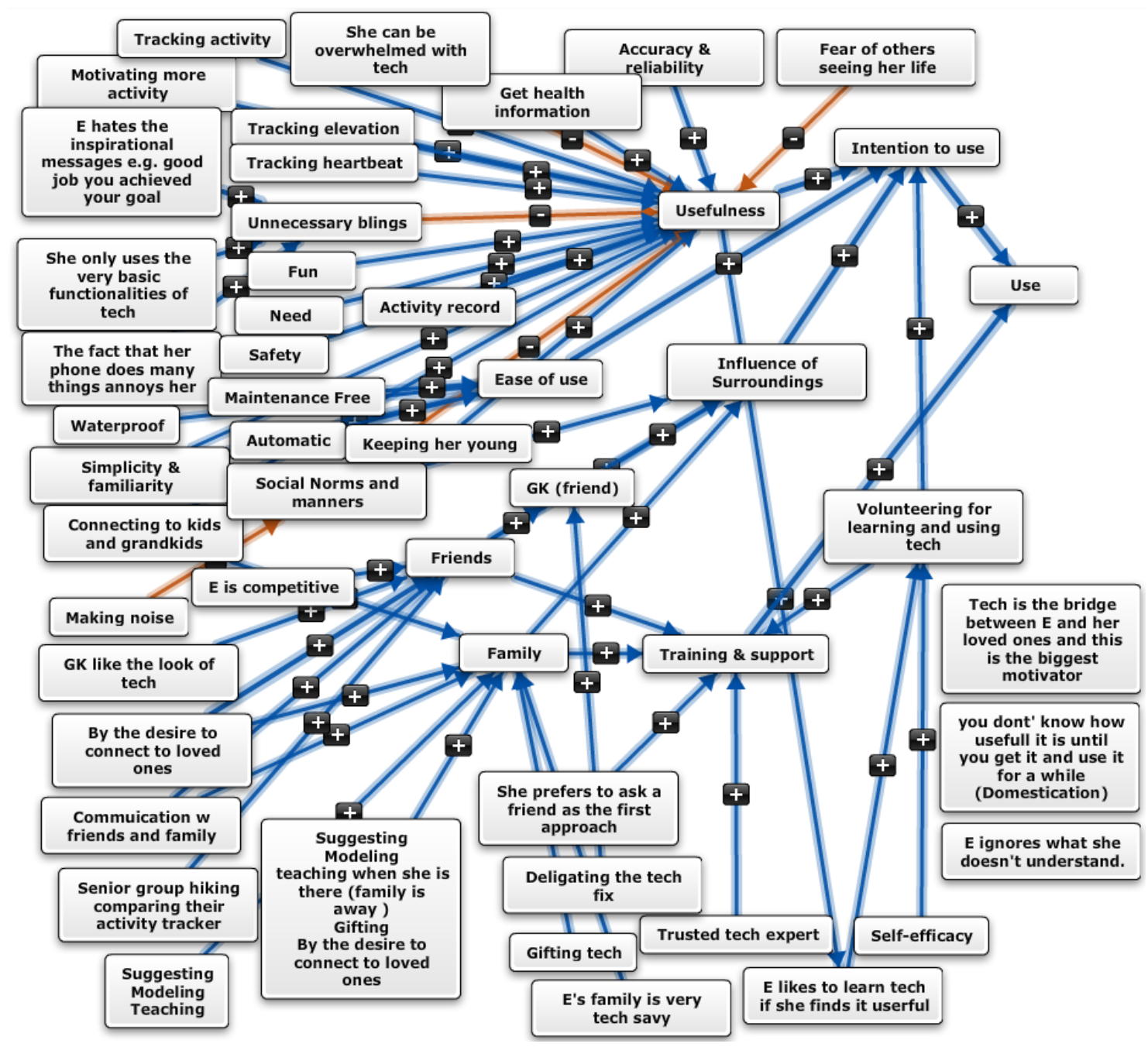

Figure H - 80 Gatekeeper \#14 initial cognitive map 


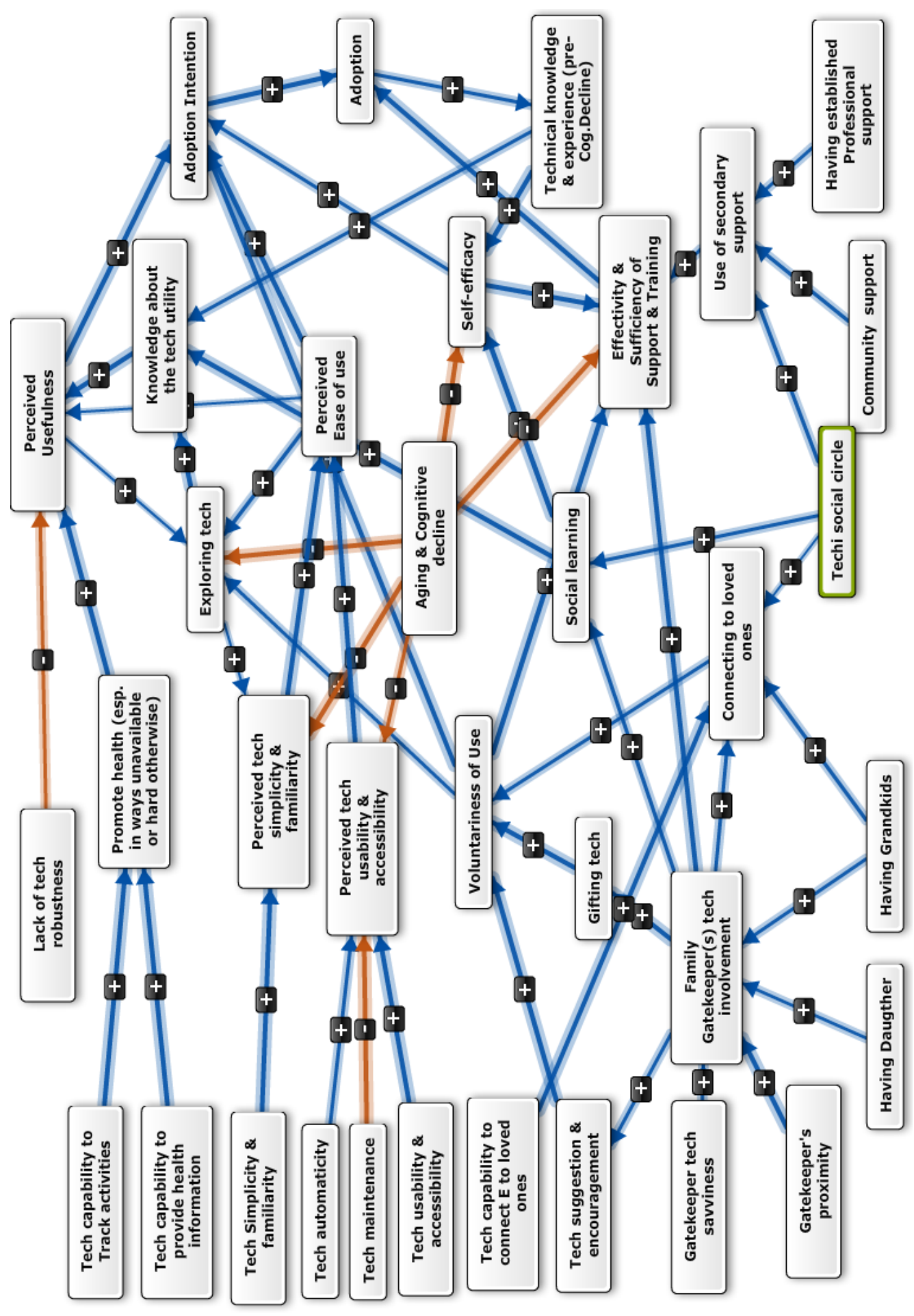

Figure H - 81 Elderly Gatekeeper pair \#14 integrated cognitive map 


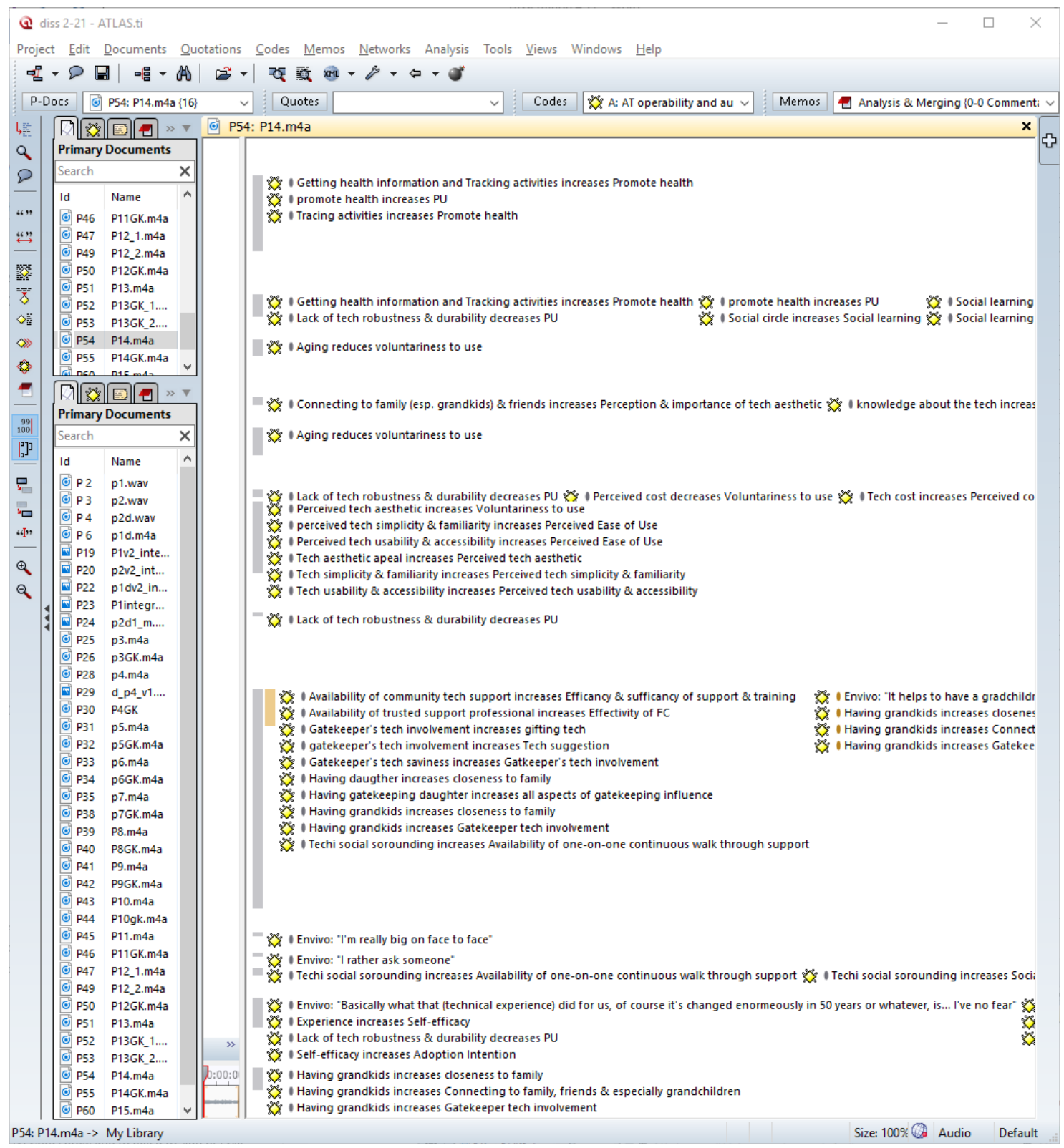

Figure H - 82 EW\#14 interview open coding in Atlas.ti 


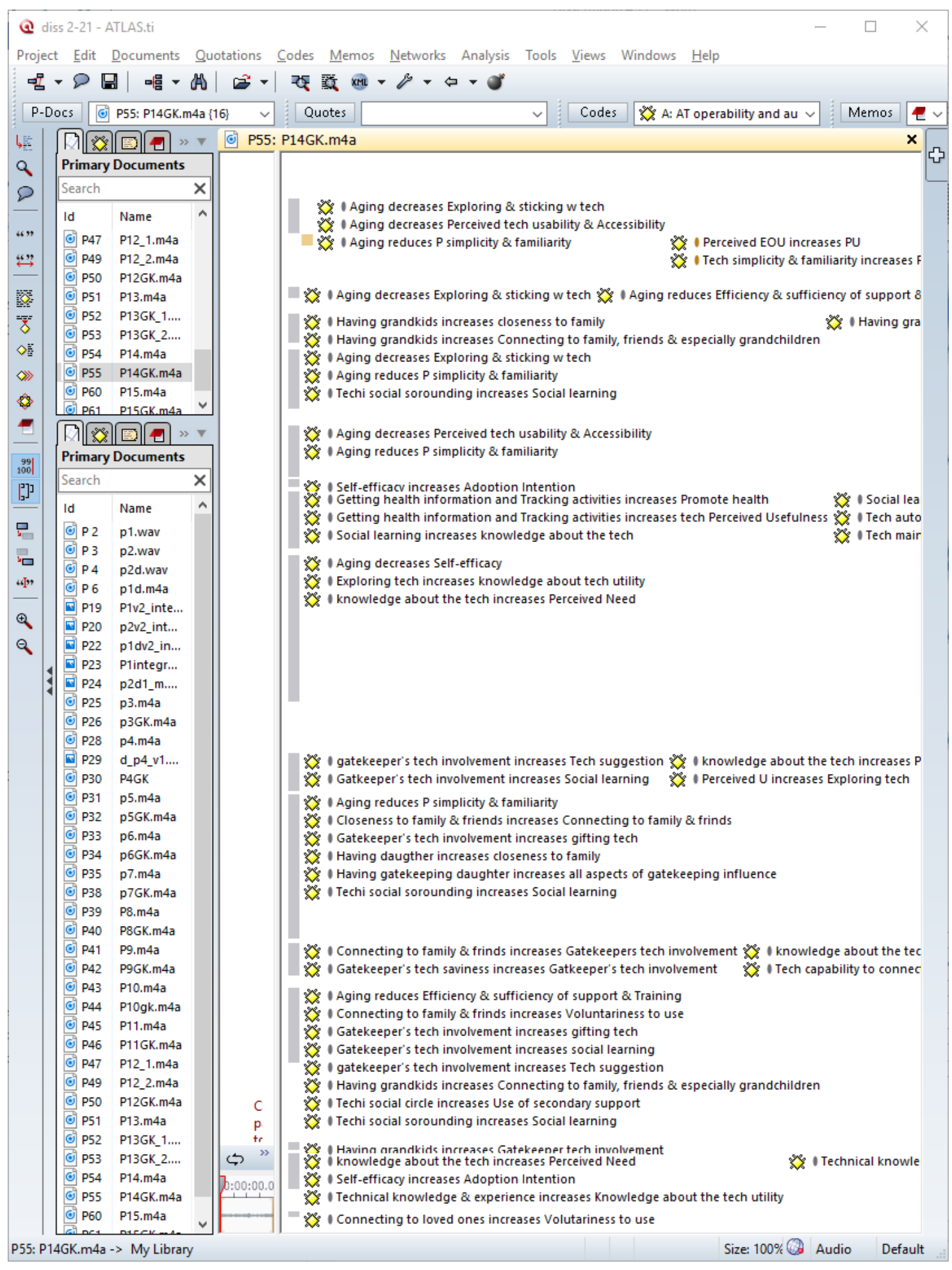

\section{Figure H - 83 Gatekeeper \#14 interview open coding in Atlas.ti}




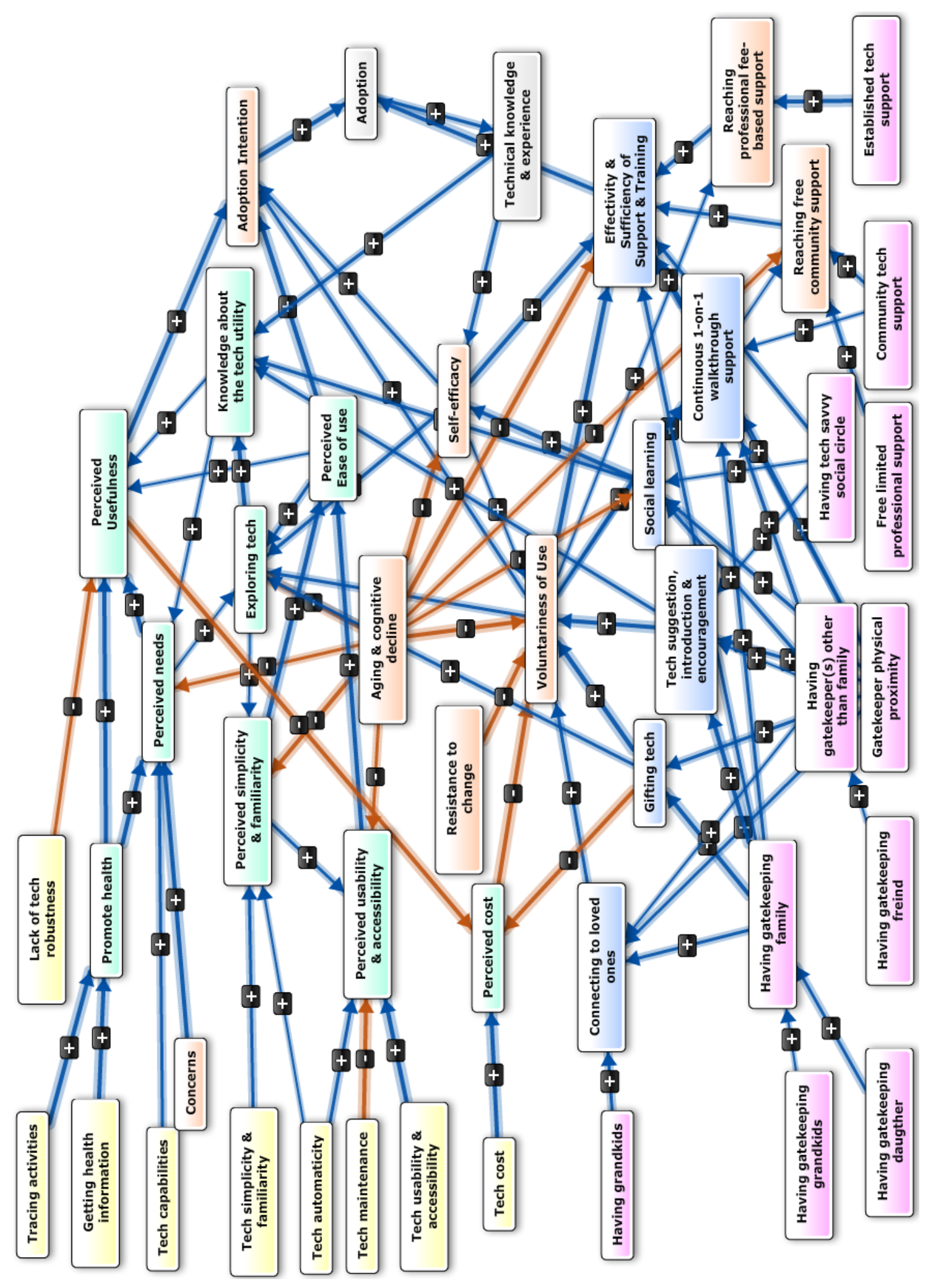

Figure H - 84 EW \#14 integrated \& standardized cognitive map 


\section{Elderly Woman \#15}

\begin{tabular}{|c|c|}
\hline Elderly Profile & P15 \\
\hline Interview Duration & $54 \mathrm{~min}$ \\
\hline Age & 94 \\
\hline Health Condition & OK \\
\hline Living condition & Living in a retirement community \\
\hline Isolated & $\mathrm{Y}$ \\
\hline Attitude toward Tech & Positive \\
\hline Self-efficacy & $\mathrm{Y}$ \\
\hline Experience & Y (Banker, worked w computer since 80's) \\
\hline Driven by & Health promotion and information \\
\hline Has grandchildren? & $\mathrm{N}$ \\
\hline Has \& close to family & $\sim$ (has some distant family) \\
\hline Gatekeeper & $\begin{array}{l}\text { Neighbor friend in the retirement community ( } 78 \text { yrs old female ; } \\
\text { interview duration } 51.5 \mathrm{~min} \text { ) }\end{array}$ \\
\hline $\begin{array}{l}\text { Gatekeeper tech } \\
\text { savviness }\end{array}$ & Low \\
\hline Degree of influence & Very low \\
\hline Influence & Connecting to family \& SC, Social learning \\
\hline $\begin{array}{l}\text { Perceived Usefulness } \\
\text { of the wearable }\end{array}$ & It seems useful \\
\hline $\begin{array}{l}\text { Perceived Ease of } \\
\text { Use of the wearable }\end{array}$ & It's OK \\
\hline $\begin{array}{l}\text { Will she adopt if } \\
\text { free? }\end{array}$ & Y \\
\hline $\begin{array}{l}\text { Will she buy to } \\
\text { adopt? }\end{array}$ & $\mathrm{N}$ \\
\hline Note & $\begin{array}{l}\text { P15 states since there are no family around she relies on the friends } \\
\text { circle for emergency }\end{array}$ \\
\hline Note & $\begin{array}{l}\text { Distant family (sister) is not tech savvy and therefore tech wouldn't } \\
\text { connect them }\end{array}$ \\
\hline
\end{tabular}




\begin{tabular}{|l|l|} 
Note & $\begin{array}{l}\text { P15 has a strong motivation to serve and help others. The fact that } \\
\text { at the age of 94 and for the past 16 years, she has been } \\
\text { volunteering in the airport to help people get around, shows how } \\
\text { much she likes to help others. This is one of the drivers for her TA, } \\
\text { beside need to stay independent and be an active member of the } \\
\text { society. Having prior experience has given her the high self-efficacy } \\
\text { she needs to both figuring out how to use tech (making FC } \\
\text { effective) as well as intending to adopt (increasing Adoption } \\
\text { Intention) }\end{array}$ \\
\hline Dominant Theme 1 & $\begin{array}{l}\text { In the absence of family, the social E makes a family out of the } \\
\text { retirement community }\end{array}$ \\
\hline Dominant Theme 2 & $\begin{array}{l}\text { E's family live far and they are not tech savvy at all (no computer, } \\
\text { etc.) }\end{array}$ \\
\hline Dominant Theme 3 & $\begin{array}{l}\text { E TA is heavily influenced by her participation in OHSU research and } \\
\text { have learned a lot from it }\end{array}$ \\
\hline Discovery & $\begin{array}{l}\text { P15 is another relatively successful HTA story. Although E is socially } \\
\text { isolate and really old, thanks to her prior experience w tech } \\
\text { combined with her strong need for independency and social } \\
\text { learning she has become moderately tech savvy for her age. For the } \\
\text { past 16 years she volunteers to Pdx airport and continue to use } \\
\text { technology. She enjoys a high level of self-efficacy as a result. }\end{array}$ \\
\hline GK & $\begin{array}{l}\text { High self-efficacy (increased by tech experience) and need for } \\
\text { independency (lives alone) who increases Perceived tech usefulness } \\
\text { can moderately compensate for lack of some of key gatekeeper's } \\
\text { influences (1-on-1 FC, and gifting). }\end{array}$ \\
\hline $\begin{array}{l}\text { Now that GK talks about E's being frugal, it becomes clear why E } \\
\text { tries hard to use instruction and not having professional help. }\end{array}$ \\
\hline Dominant Theme 4
\end{tabular}

Table H - 15 Elderly woman \#15 profile 


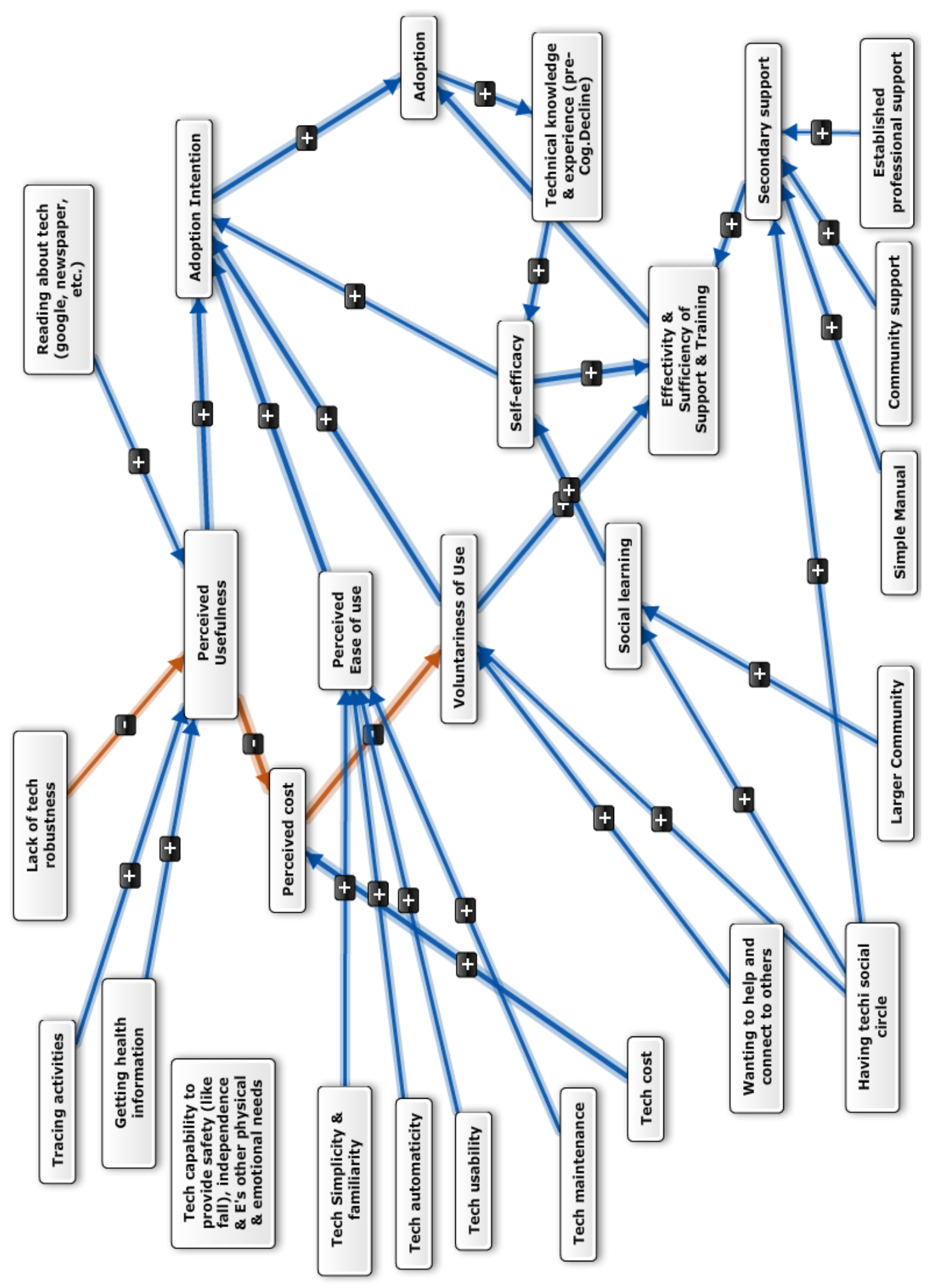

Figure H - 85 EW \#15 initial cognitive map 


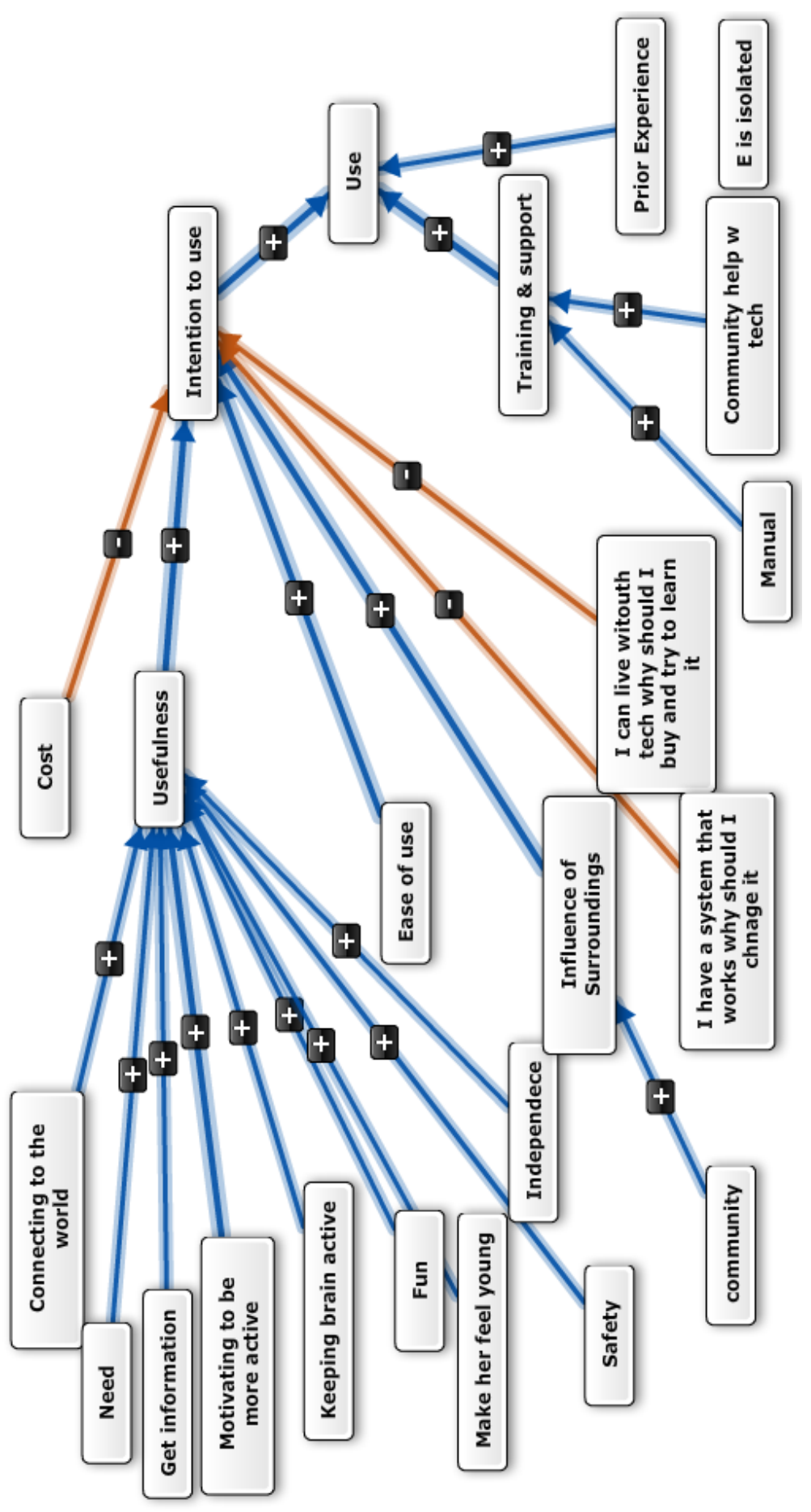

Figure H - 86 Gatekeeper \#15 initial cognitive map 


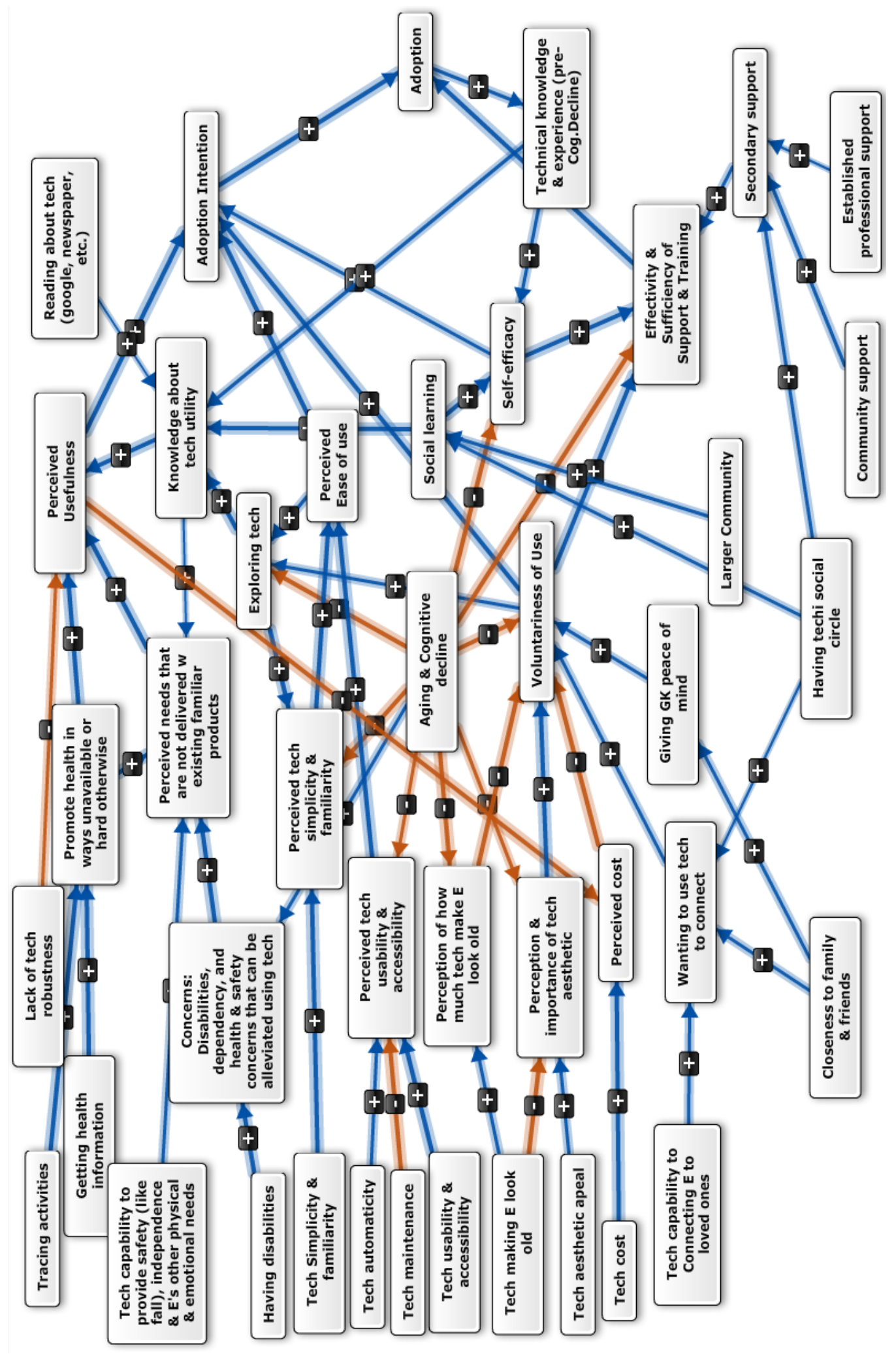

Figure H - 87 Elderly Gatekeeper pair \#15 integrated cognitive map 560 


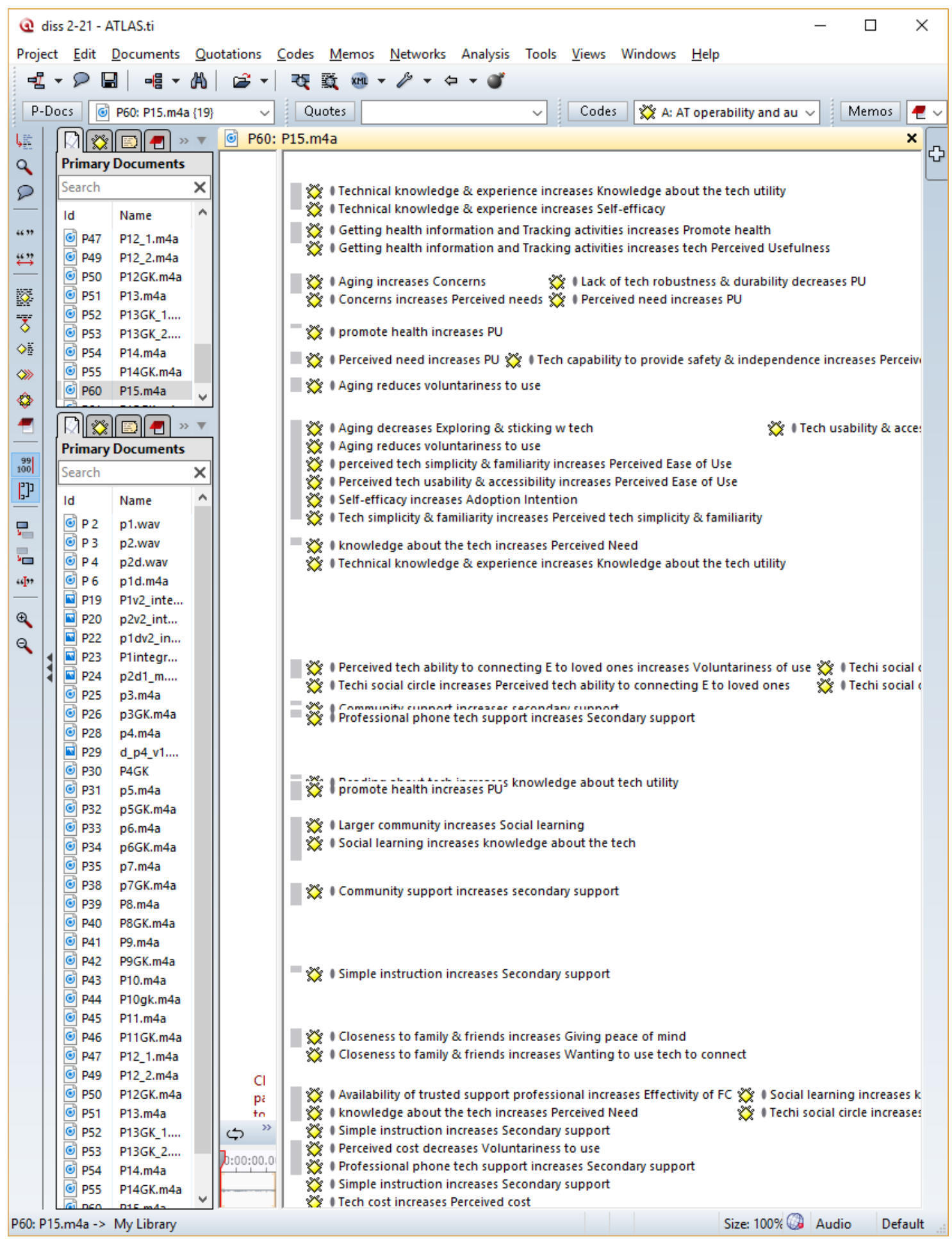

Figure H - $88 \mathrm{EW \# 15}$ interview open coding in Atlas.ti 


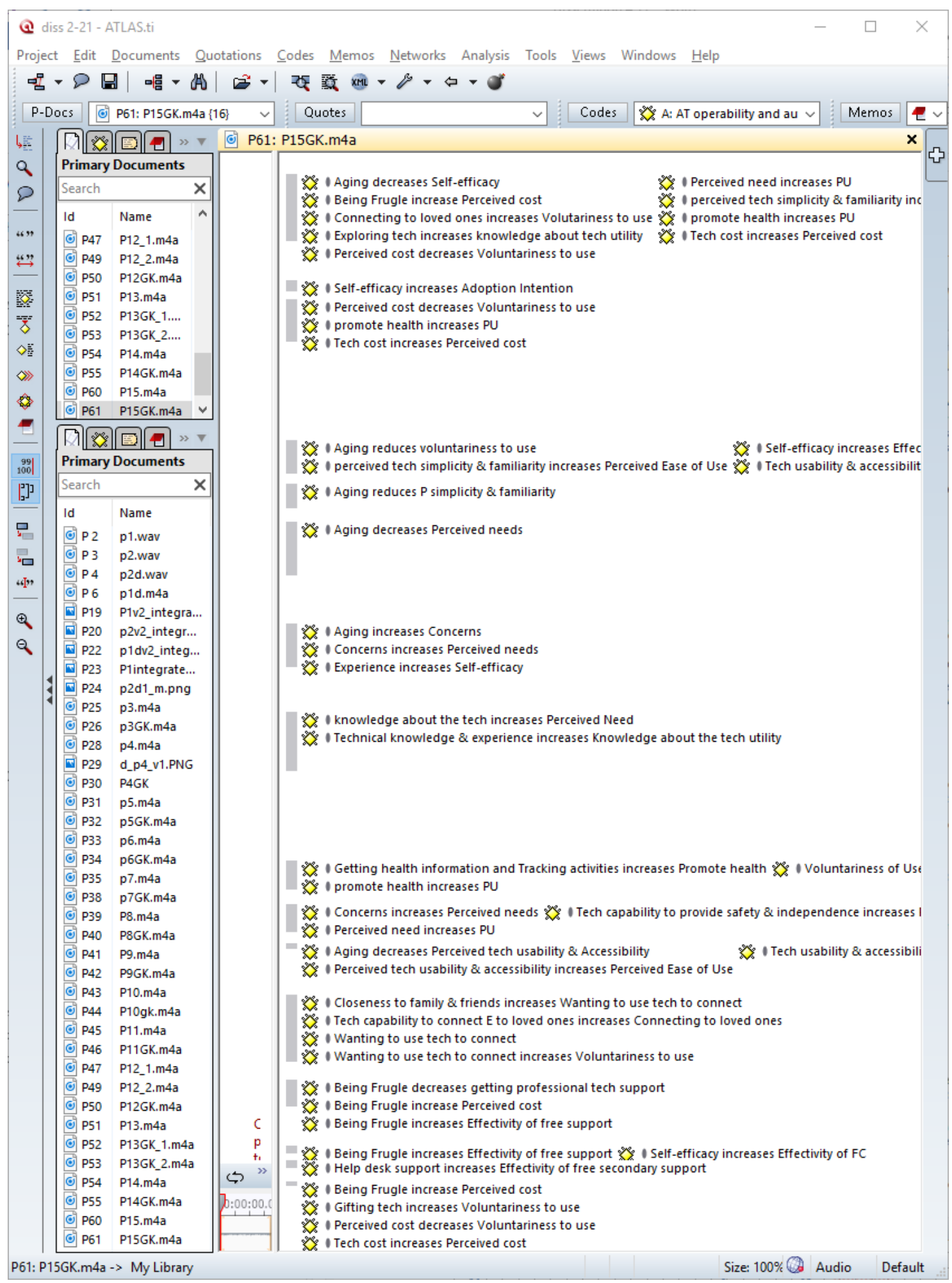

Figure H - 89 Gatekeeper \#15 interview open coding in Atla s.ti 


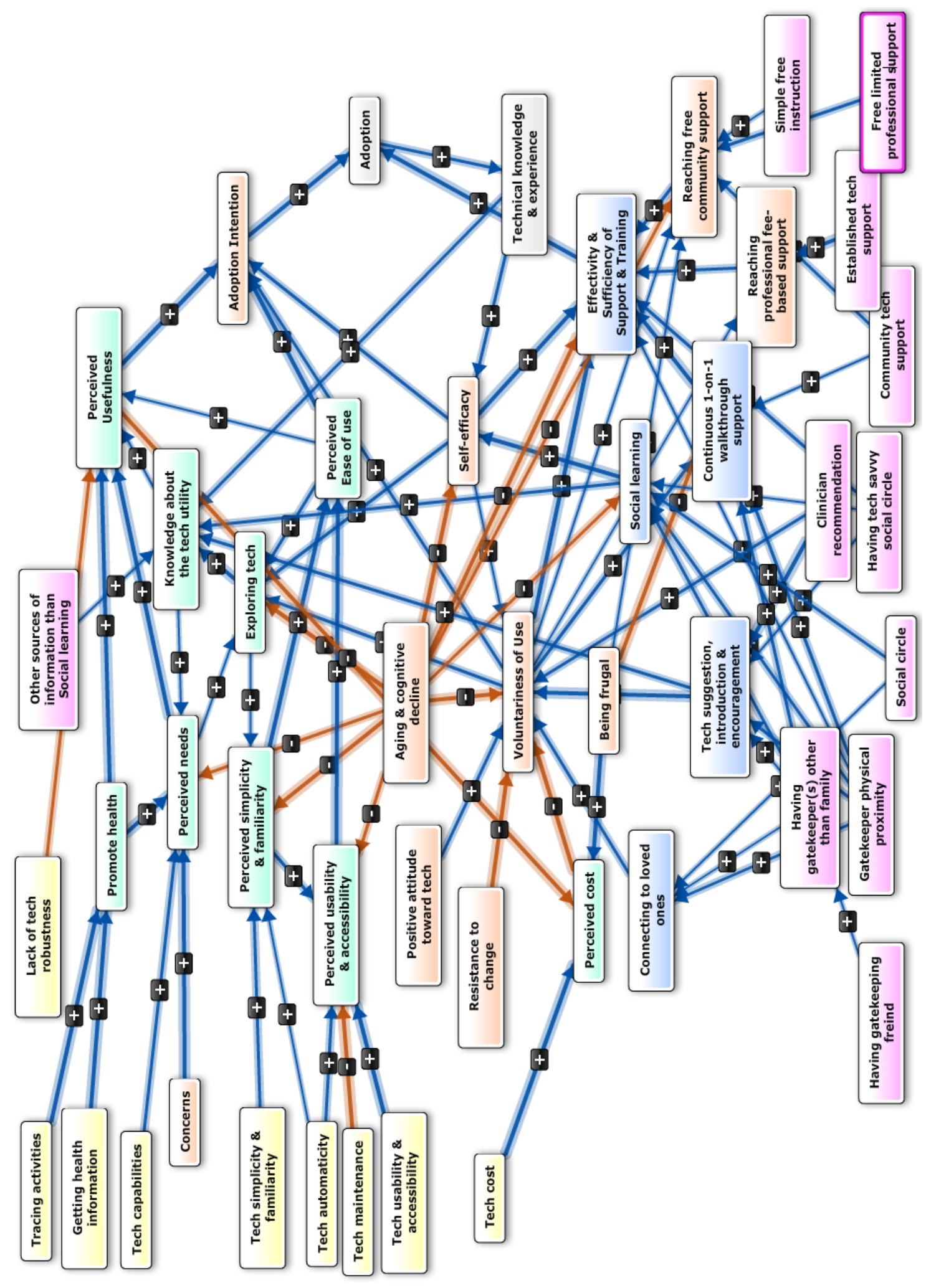

Figure H - 90 EW \#15 integrated \& standardized cognitive map

563 